\title{
ASTA
}

\section{Proposal for an Accelerator R\&D User Facility at Fermilab's Advanced Superconducting Test Accelerator (ASTA)

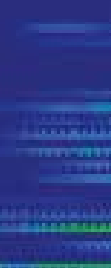

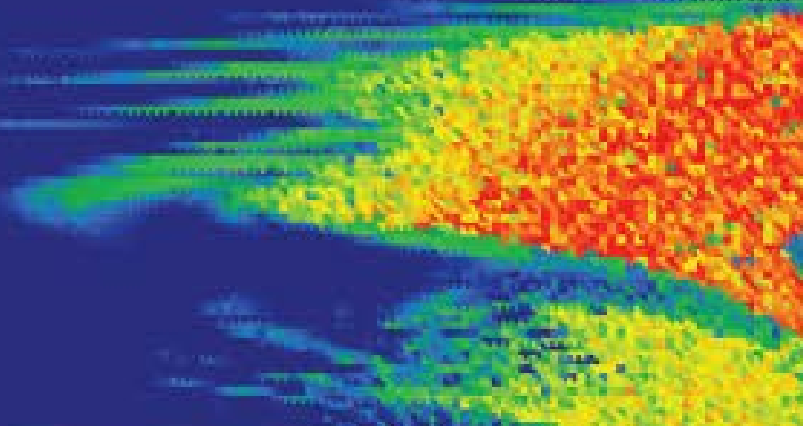


Proposal for an

\section{Accelerator R\&D User Facility at Fermilab's Advanced Superconducting Test Accelerator (ASTA)}

October 2013

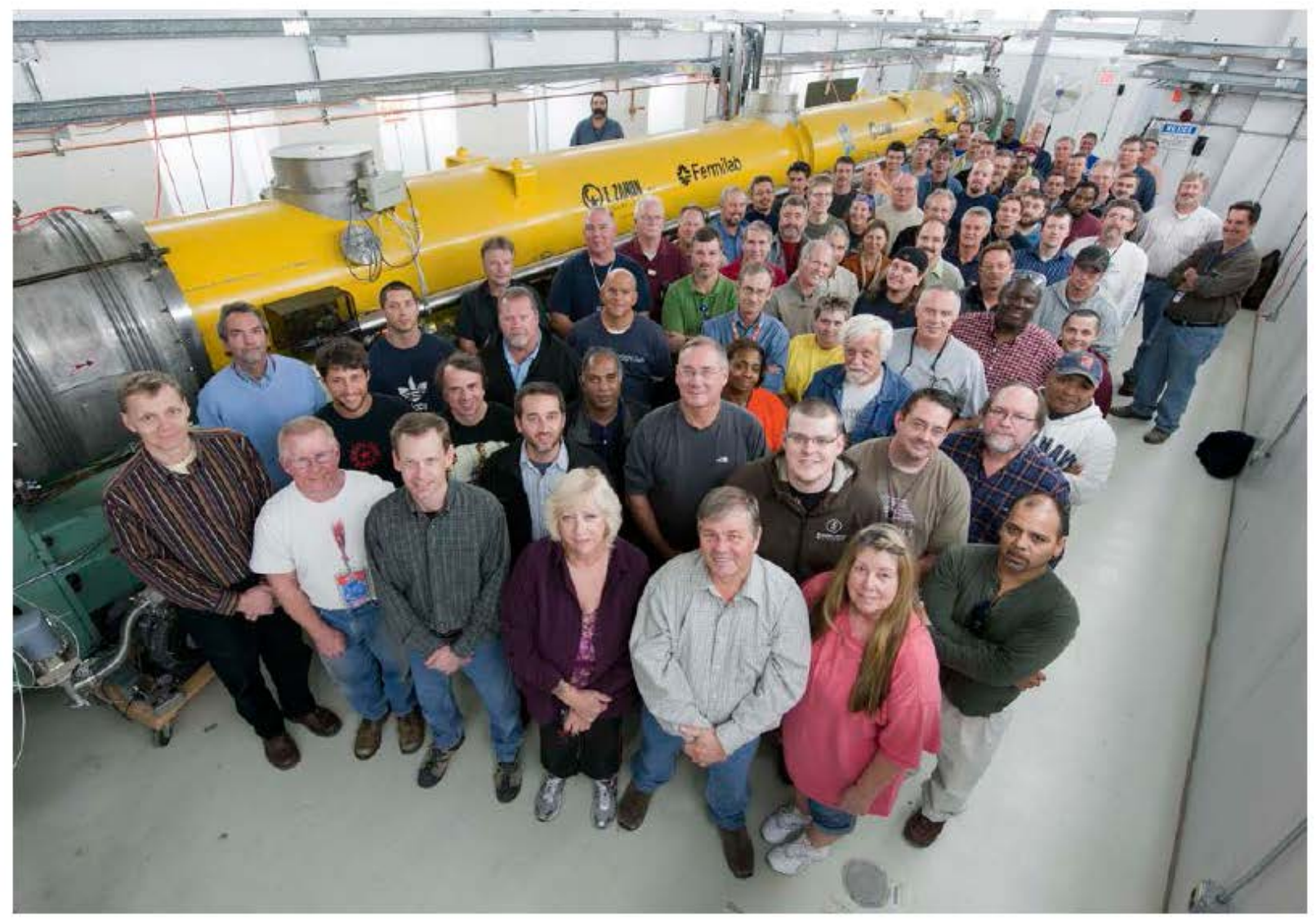

\section{荤 Fermilab}

(7) ENERGY 1 Scincere

Managed by Fermi Research Alliance, LLC for the Department of Energy Cover: Simulation of stable (blue) and chaotic (red) frequency regions in phase space. 


\section{List of Authors and Contributors}

\section{ASTA Facility}

M. Church ${ }^{1}$, H. Edwards ${ }^{1}$, E. Harms ${ }^{1}$, S. Henderson ${ }^{1}$, S. Holmes ${ }^{1}$, A. Lumpkin ${ }^{1}$, R. Kephart ${ }^{1}$, V. Lebedev ${ }^{1}$, J. Leibfritz ${ }^{1}$, S. Nagaitsev ${ }^{1}$, P. Piot ${ }^{1,2}$, C. Prokop ${ }^{2}$, V. Shiltsev ${ }^{1}$, Y.E. Sun ${ }^{11}$,

A. Valishev ${ }^{1}$

\section{Scientific Case}

R. Abrams ${ }^{3}$, G.N. Allen ${ }^{15}$, C. Ankenbrandt ${ }^{3}$, D.P. Arnold ${ }^{14}$, B. Beaudoin ${ }^{16}$, S. Biedron ${ }^{18}$,

B. Blomberg ${ }^{2}$, S. Boucher ${ }^{9}$, C. Brau ${ }^{5}$, D. Bruhwiler ${ }^{13}$, J. Byrd ${ }^{6}$, L.W. Cambell ${ }^{17}$, B. Carlsten ${ }^{4}$,

B. Chase ${ }^{1}$, D. Christian ${ }^{1}$, M. Chung ${ }^{1}$, M. Church ${ }^{1}$, J. Corlett ${ }^{6}$, M.A. Cummings ${ }^{3}$, V. Danilov ${ }^{7}$, Ya. Derbenev ${ }^{8}$, B. Digiovine ${ }^{11}$, N. Eddy ${ }^{1}$, H. Edwards ${ }^{1}$, P. Emma ${ }^{6}$, B. Erdelyi $^{2}$, J.E. Fast $^{17}$,

G. Flanagan ${ }^{3}$, A. Garraud ${ }^{14}$, A. Grabenhofer ${ }^{2}$, D. Henderson ${ }^{11}$, R. Holt ${ }^{11}$, P. Hurh ${ }^{1}$, R. Johnson ${ }^{3}$,

G. Kazakevitch ${ }^{3}$, Y.K. Kim ${ }^{12}$, R. Kishek ${ }^{16}$, T. Koeth ${ }^{16}$, G. Krafft ${ }^{8}$, F. Lemery ${ }^{2}$, A. Lumpkin ${ }^{1}$, V. Lebedev ${ }^{1}$, A. Malyzhenkov ${ }^{2,4}$, A. Marchionni ${ }^{1}$, D. Mihalcea ${ }^{2}$, S. Milton ${ }^{18}$, A. Morin $^{18}$, A. Murokh ${ }^{9}$, S. Nagaitsev ${ }^{1}$, E. Nissen ${ }^{8}$, M. Palmer ${ }^{1}$, B.A. Peterson ${ }^{15}$, P. Piot $^{1,2}$, E. Prebys ${ }^{1}$, J. Qiang ${ }^{6}$, K.E. Rehm ${ }^{11}$, M. Reinsch ${ }^{6}$, T. Roberts ${ }^{3}$, A. Robinson ${ }^{12}$, J. Ruan ${ }^{1}$, K. Ruisard ${ }^{16}$, V. Scarpine ${ }^{1}$, T. Sen ${ }^{1}$, V. Shiltsev ${ }^{1}$, Y.M. Shin ${ }^{1,2}$, E. Simakov ${ }^{4}$, N. Solyak ${ }^{1}$, A. Sonnenschein ${ }^{1}$, D. Still ${ }^{1}$, R. Suleiman ${ }^{8}$, Y.E. Sun ${ }^{11}$, D. Sutter ${ }^{16}$, J.C. Thangaraj ${ }^{1}$, R. Thurman-Keup ${ }^{1}$, Y. Tokpanov ${ }^{1}$, R. Tschirhart ${ }^{1}$, C. Ugalde ${ }^{12}$, A. Valishev ${ }^{1}$, M. Venturini ${ }^{6}$, N. Vinokurov $^{10}$, R. Wilcox $^{6}$, Tao Xu $^{2}$, V. Yakovlev ${ }^{1}$, N. Yampolsky ${ }^{4}$ Y. Zhang ${ }^{8}$, T. Zolkin $^{12}$, M. Zolotorev ${ }^{6}$, R. Zwaska ${ }^{1}$

\section{Editors}

P.H. Garbincius ${ }^{1}$, S. Henderson ${ }^{1}$, J. Leibfritz ${ }^{1}$, P. Piot ${ }^{1,2}$, V. Shiltsev ${ }^{1}$

${ }^{1}$ Fermi National Accelerator Laboratory

${ }^{2}$ Northern Illinois University

${ }^{3}$ Muons, Inc.

${ }^{4}$ Los Alamos National Laboratory

${ }^{5}$ Vanderbilt University

${ }^{6}$ Lawrence Berkeley National Laboratory

${ }^{7}$ Oak Ridge National Laboratory

${ }^{8}$ Thomas Jefferson National Laboratory

${ }^{9}$ RadiaBeam Technologies

${ }^{10}$ Budker Institute of Nuclear Physics

${ }^{11}$ Argonne National Laboratory

${ }^{12}$ University of Chicago

${ }^{13}$ University of Colorado

${ }^{14}$ University of Florida

${ }^{15}$ Georgia Institute of Technology

${ }^{16}$ University of Maryland

${ }^{17}$ Pacific Northwest National Laboratory

${ }^{18}$ Colorado State University 


\section{Table of Contents}

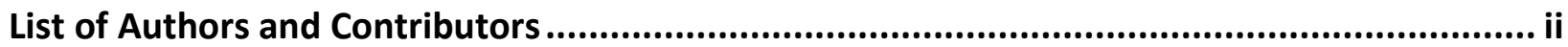

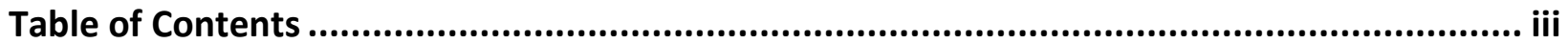

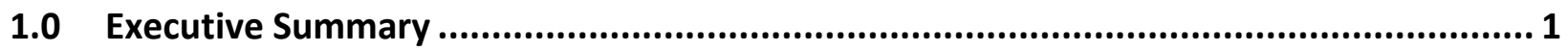

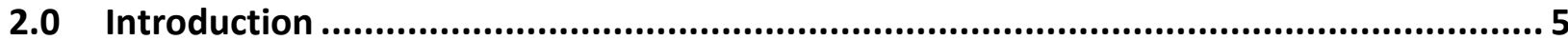

2.1 The Role of Particle Physics in Accelerator Development ............................................. 5

2.2 ASTA's Role in Furthering the HEP Mission ............................................................ 6

2.3 Overview of Scientific Opportunities at ASTA ........................................................... 8

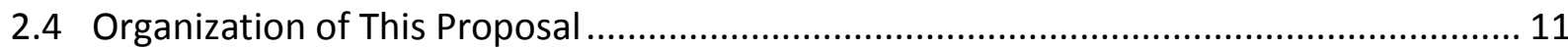

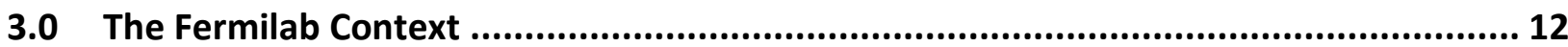

3.1 Connection to Fermilab's Mission ............................................................................ 12

3.2 Fermilab's Historical Contributions in Advanced Accelerator R\&D.............................. 14

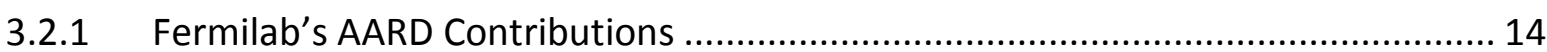

3.2.2 Selected AARD Publications from Fermilab....................................................... 15

3.2.3 Historical Achievements in Advanced Accelerator R\&D at the AO Photoinjector Facility (AOPI) .............................................................. 16

3.2.4 PhD Theses in Advanced Accelerator R\&D from Fermilab-based Research .......... 23

4.0 Scientific Drivers in Advanced Accelerator R\&D............................................... 24

5.0 Comparison with Other AARD Facilities ........................................................... 28

6.0 Management of the ASTA Scientific User Program ................................................. 34

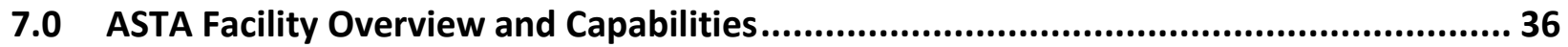

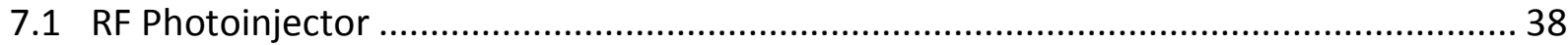

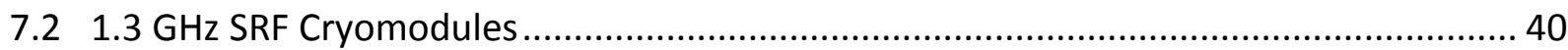

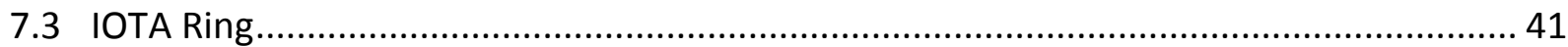

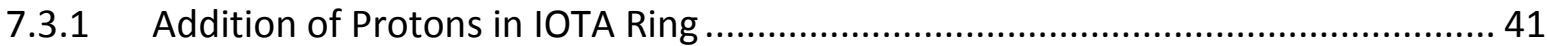

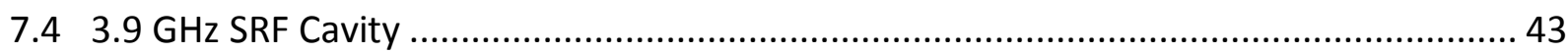

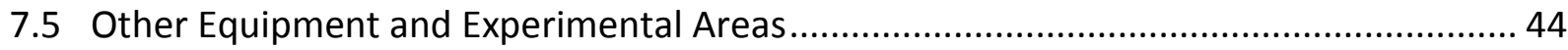

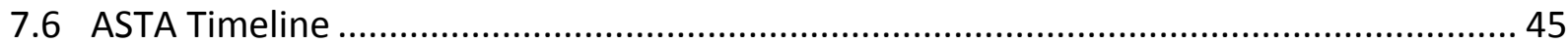

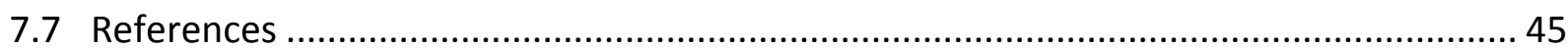

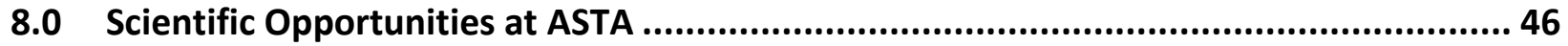

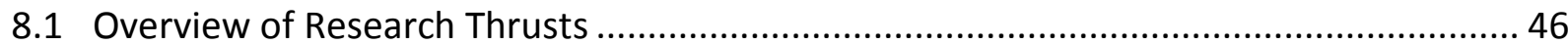

8.1.1 Accelerator R\&D for Particle Physics at the Intensity and Energy Frontiers ......... 46

8.1.2 Accelerator R\&D for Future SC RF Accelerators ............................................... 46

8.1.3 Accelerator R\&D for Novel Radiation Sources.................................................. 47

8.1.4 Accelerator R\&D for Stewardship and Applications ......................................... 47

8.2 Accelerator R\&D Proposals for Particle Physics at the Intensity Frontier ...................... 48

8.2.1 Experimental Demonstration of Integrable Optics Lattices at IOTA .................... 48

8.2.2 Space Charge Compensation in High Intensity Circular Accelerators ................... 61 
8.3 Accelerator R\&D Proposals for Particle Physics at the Energy Frontier ......................... 63

8.3.1 Advanced Phase Space Manipulations .......................................................... 63

8.3.2 Optical Stochastic Cooling Experiment at ASTA .............................................. 75

8.3.3 Flat-Beam-Driven Dielectric-Wakefield Acceleration in Slab Structures................ 83

8.4 Accelerator R\&D for Future SCRF Accelerators .................................................... 90

8.4.1 Demonstration of High Power High Gradient SRF Cryomodules with High Intensity Beams ........................................................................... 90

8.4.2 Proposed Long-Range Wakefield Measurements at ASTA................................ 98

8.4.3 Towards Ultra-Stable Operation of ASTA with Beam Based Feedback ................ 101

8.5 Accelerator R\&D for Novel Radiation Sources........................................................... 109

8.5.1 High-Brightness X-ray Channeling Radiation Source ........................................ 109

8.5.2 Inverse Compton Scattering Gamma-Ray Source at ASTA ................................. 117

8.5.3 Application of High-Flux Inverse Compton Scattered Photons to Nuclear Astrophysics .............................................................................. 122

8.6 Accelerator R\&D for Stewardship and Applications .............................................. 128

8.6.1 Demonstration of Techniques to Generate and Manipulate Ultra-Low Emittance Beams for Future Hard X-ray Free-Electron Lasers............ 128

8.6.2 Beam Dechirper for Free-Electron-Laser Linacs ............................................ 136

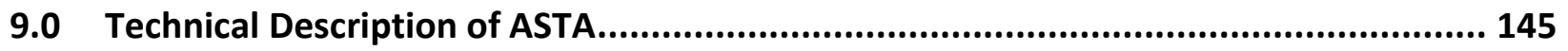

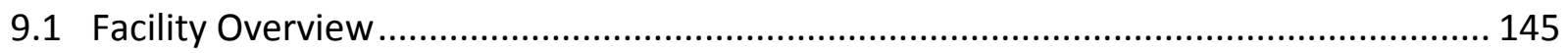

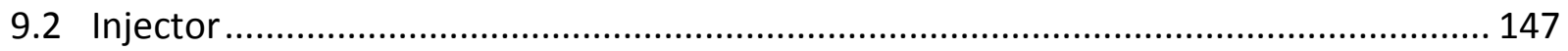

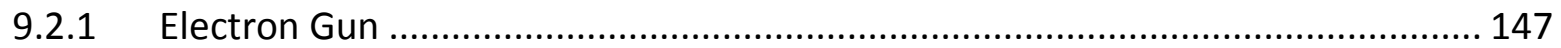

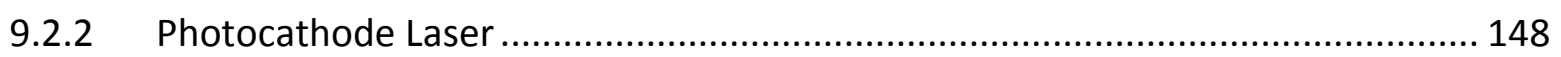

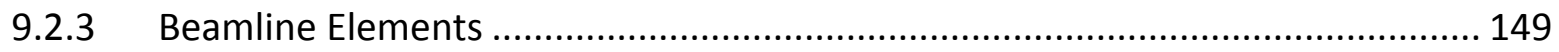

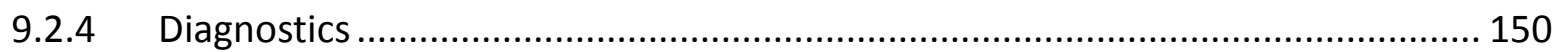

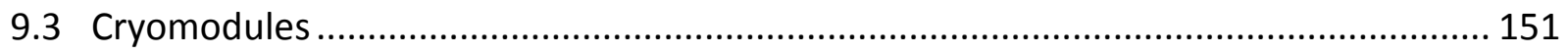

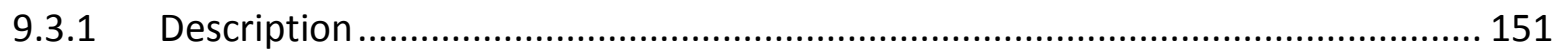

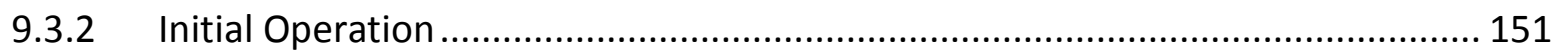

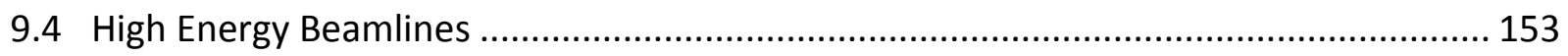

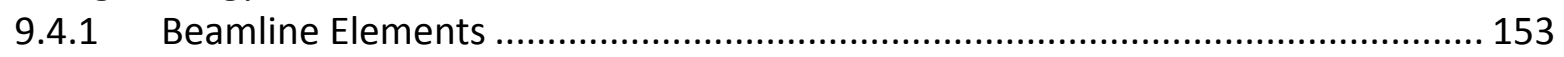

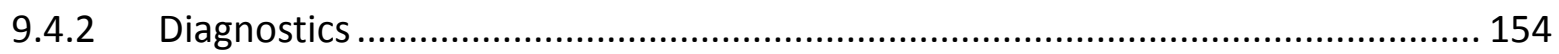

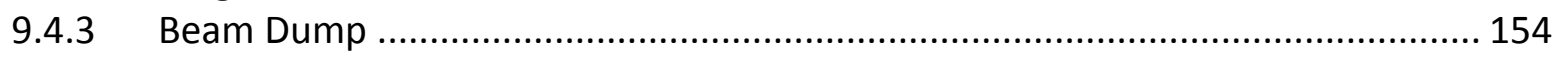

9.5 Beam Dynamics Calculations and Simulations .................................................... 154

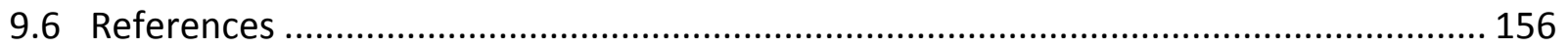

10.0 Accelerator Science Education at ASTA and Collaboration with the Program of Technology Development of the Illinois Accelerator Research Center (IARC) ............. 158

11.0 Scope, Cost, Schedule, and Management ....................................................... 161

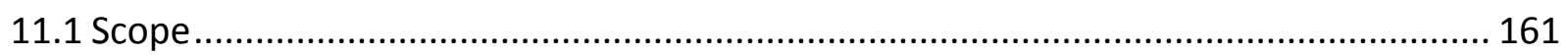

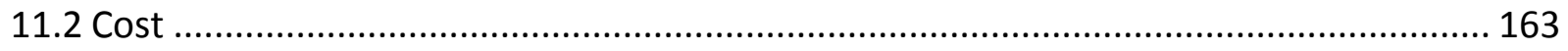

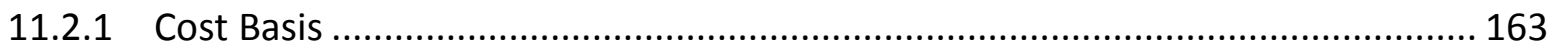

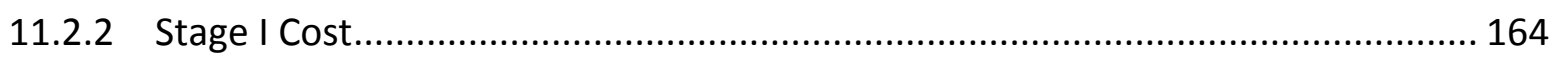

11.2.3 Stage II Cost.......................................................................................... 166

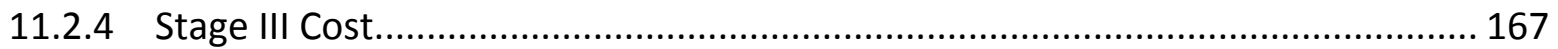

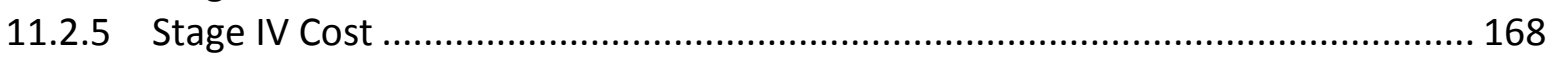

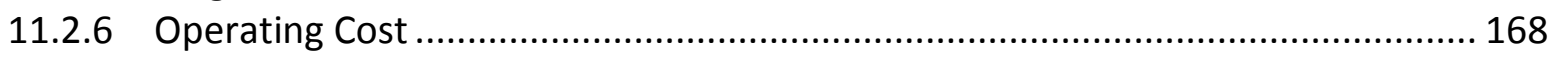




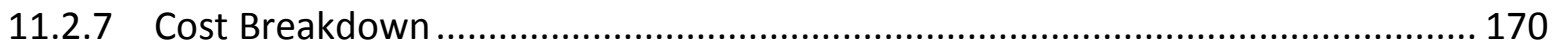

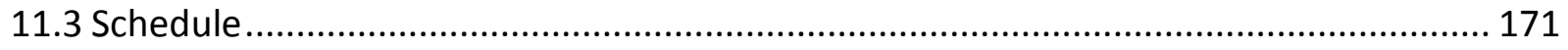

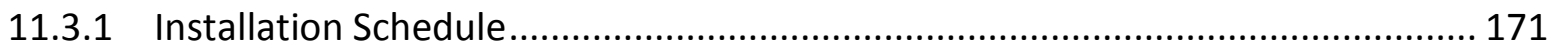

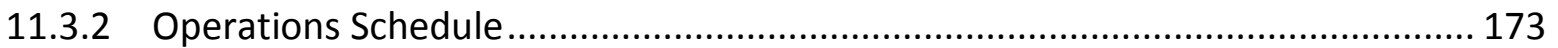

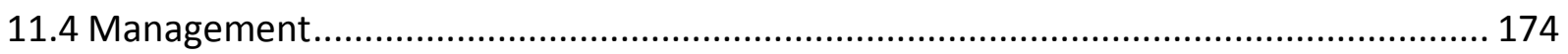

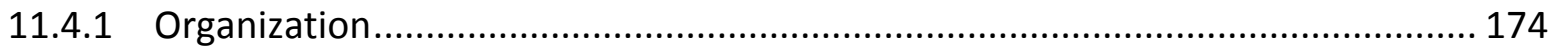

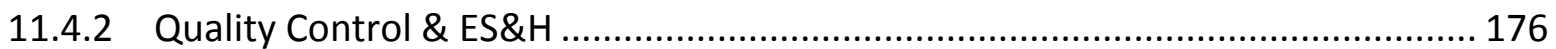

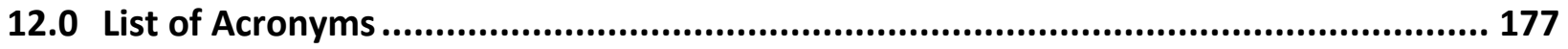

A.0 Other Expressions of Interest and Concepts for Experimental Studies ...................... 184

A.1 Studies Utilizing ASTA Capabilities in SCRF Technology .......................................... 184

A.1.1 Artificial Intelligence-based LLRF Control ........................................................ 184

A.1.2 Experimental Test of CW Operation of the ASTA SRF Cryomodule \#1................ 186

A.1.3 A Magnetron Transmitter Test at the ASTA SRF Linac.................................... 189

A.1.4 Feasibility of an XUV FEL Oscillator at ASTA ................................................ 195

A.1.5 LBNE Targetry Experiments at ASTA .......................................................... 209

A.1.6 Potential for Critical Laser-Induced Microbunching Studies with the High-Micropulse-Repetition-Rate Electron Beams at ASTA.......................... 213

A.1.7 Generation of Parametric X-rays at ASTA .................................................... 225

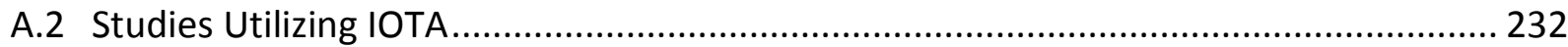

A.2.1 Electron Wave Function Size Measurements in IOTA Ring ............................... 232

A.2.2 Multi-Pickup Beam Profile Monitor for IOTA .................................................. 234

A.2.3 Conservative Relaxation and Echo Studies with IOTA ...................................... 237

A.3 Studies Utilizing ASTA Capabilities for Phase Space Manipulation ............................. 241

A.3.1 Miniaturized Magnetic Undulators for X-Ray Generation.................................. 241

A.3.2 Electron Beam Shaping and High Gradient, High Transformer Ratio Acceleration in a Dielectric Tube ............................................................ 246

A.3.3 Beam-Beam Kicker for an Electron-Ion Collider ........................................... 251

A.3.4 Measuring Coherent Synchrotron Radiation (CSR) Effects on High Brightness Electron Beams ..................................................................................... 256

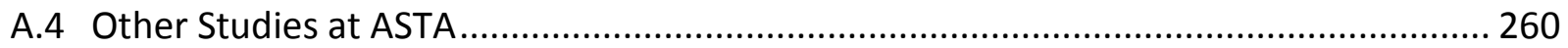

A.4.1 Nano-Crystal Channeling Acceleration Experiment at ASTA .............................. 260

A.4.2 A Tagged Photon Beam at ASTA for Detector R\&D ........................................ 269

A.4.3 Tunable, Nearly Monoenergetic Gamma Ray Beams for Special Nuclear Material (SNM) Interrogation ................................................. 271

A.4.4 Proposed Coherent Diffraction Radiation Measurements

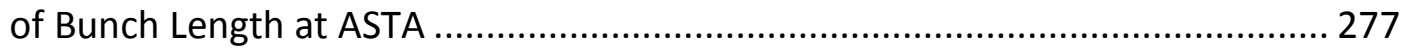

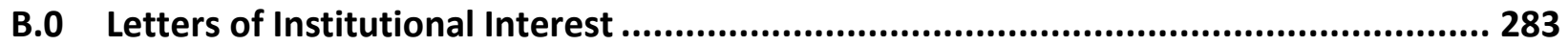




\subsection{Executive Summary}

Fermilab is the nation's particle physics laboratory, supported by the DOE Office of High Energy Physics (OHEP). Fermilab is a world leader in accelerators, with a demonstrated track-recordspanning four decades- of excellence in accelerator science and technology. We describe the significant opportunity to complete, in a highly leveraged manner, a unique accelerator research facility that supports the broad strategic goals in accelerator science and technology within the OHEP. While the US accelerator-based HEP program is oriented toward the Intensity Frontier, which requires modern superconducting linear accelerators and advanced highintensity storage rings, there are no accelerator test facilities that support the accelerator science of the Intensity Frontier. Further, nearly all proposed future accelerators for Discovery Science will rely on superconducting radiofrequency (SRF) acceleration, yet there are no dedicated test facilities to study SRF capabilities for beam acceleration and manipulation in prototypic conditions. Finally, there are a wide range of experiments and research programs beyond particle physics that require the unique beam parameters that will only be available at Fermilab's Advanced Superconducting Test Accelerator (ASTA). To address these needs we submit this proposal for an Accelerator R\&D User Facility at ASTA.

The ASTA program is based on the capability provided by an SRF linac (which provides electron beams from $50 \mathrm{MeV}$ to nearly $1 \mathrm{GeV}$ ) and a small storage ring (with the ability to store either electrons or protons) to enable a broad range of beam-based experiments to study fundamental limitations to beam intensity and to develop transformative approaches to particle-beam generation, acceleration and manipulation which cannot be done elsewhere. It will also establish a unique resource for R\&D towards Energy Frontier facilities and a test-bed for SRF accelerators and high brightness beam applications in support of the OHEP mission of Accelerator Stewardship.

ASTA is unique among accelerator user facilities in six principal ways:

- High repetition-rate: ASTA accelerates $1 \mathrm{msec}$ long bunch trains, with $3 \mathrm{MHz}$ micro-pulse repetition rate, with up to 3000 bunches per train

- High average power: ASTA accelerates $1 \mathrm{msec}$ long bunch trains at up to five pulses per second, providing the highest beam power and highest average brightness of any accelerator test facility in the U.S.

- High energy: ASTA accelerates beam to $\sim 1 \mathrm{GeV}$, which is important for a number of photon-science and FEL-related experiments

- Extremely stable beams: ASTA, based on ILC technology, is capable of providing exceptional beam stability, which has been demonstrated at the FLASH facility at DESY

- Superconducting technology: ASTA necessarily brings SRF and beams together. As nearly all future large scale accelerator facilities will rely on SRF technology, ASTA will be the only GeV-scale test facility that is capable of supporting R\&D in prototypical conditions.

- Storage ring: ASTA includes a small, very flexible storage ring, based on innovative optics, capable of supporting a broad range of ring-based advanced beam dynamics experiments. 
These unique features enable a broad program in advanced accelerator R\&D which cannot be carried out elsewhere. We propose to establish Fermilab's ASTA as an Office of Science User Facility. ASTA will further the mission of OHEP by addressing several needs:

- Furthering the strategic priorities for particle physics

- Strengthening the nation's Accelerator Test Facility infrastructure

- Establishing a test-bed for Superconducting RF Accelerators

- Fulfilling the Accelerator Stewardship Mission

- Educating and training the next generation of accelerator scientists

Construction of the ASTA Facility at the New Muon Lab (NML) building at Fermilab began in 2006 as part of the International Linear Collider/SRF R\&D Program and later the American Recovery and Reinvestment Act (ARRA) within the DOE OHEP. Construction of the facility was motivated by the goal of building, testing and operating a complete ILC RF unit to demonstrate industrial and laboratory capability for producing state-of-the-art superconducting linear accelerator components, and assembling them into a fully functioning system. ASTA, configured as a single ILC RF unit, would consist of an electron photo-injector, accelerating cavities, a bunch compressor, and three $1.3 \mathrm{GHz}$ ILC cryomodules powered together from a single high-power klystron. Each cryomodule provides $250 \mathrm{MeV}$ of energy gain for total output energy of approximately $800 \mathrm{MeV}$.

It was recognized early in the planning process that an electron beam meeting the demanding ILC performance parameters was itself a powerful resource of interest to the wider Advanced Accelerator R\&D (AARD) community as expressed at the Workshop "Future Directions for Accelerator R\&D at Fermilab" (May 2009, Lake Geneva, WI). For those reasons the ARRAfunded facility construction incorporated space for the installation of additional SRF cryomodules to increase the beam energy to a maximum of $1.5 \mathrm{GeV}$, space for multiple highenergy beamlines, as well as provisions for a small circular ring for the exploration of advanced accelerator concepts, for transporting laser light into and out of the accelerator enclosure, and an adequately-sized control room.

With the completion of the ILC R\&D Program in FY2012, DOE/OHEP has directed Fermilab to submit a proposal for funding the completion of the ASTA facility and establishing an accelerator R\&D user program there. This Proposal is submitted in recognition of the tremendous opportunity that the ASTA facility, based on a state-of-the-art superconducting linear accelerator, represents to the national and international Advanced Accelerator R\&D user community. 
The ASTA facility at NML presently consists of the following:

- A 47 meter long, 4,500 $\mathrm{ft}^{2}$ shielded accelerator enclosure housed inside the $12,000 \mathrm{ft}^{2}$ NML building.

- A 70 meter long, 8,000 $\mathrm{ft}^{2}$ newly constructed tunnel enclosure to the North of the NML building.

- An $2,800 \mathrm{ft}^{2}$ support building

- A control room

- A radio-frequency photo-injector system, including the drive laser system, which is scheduled to be commissioned in FY 2013

- Operating high-power and low-level RF systems, RF distribution system and controls

- Refrigeration system capable of 120 W @ 2K.

Two SRF cryomodules have been assembled and a third is presently under construction. All parts, including cavities, couplers, vacuum vessels, etc., for a total of 6 cryomodules have been purchased. ASTA experimental beamline equipment, including 154 magnets, all vacuum chambers, 55 beam position monitors, etc. have also been purchased. To date, a total of \$90 $\mathrm{M}$ has been spent to construct and operate the facility. The ARRA-funded construction of the facility has been completed at a total investment of approximately $\$ 18 \mathrm{M}$. Approximately $80 \%$ of the equipment and infrastructure has already been procured and installed, including $\$ 15 \mathrm{M}$ of in-kind contributions and reused equipment from other experiments and Laboratories.

To enable a broad range of scientific opportunities, the completion of the ASTA facility requires establishing beams to low-energy (50 MeV) and high-energy (300-800 MeV) experimental areas and to complete construction of the Integrable Optics Test Accelerator (IOTA), a 38-m circumference ring designed for the study of novel, non-linear accelerator lattices and highbrightness beam dynamics. With this proposal, we request funds totaling $\$ 18.6 \mathrm{M}$ plus $\$ 5.6 \mathrm{M}$ contingency for the completion of the ASTA facility, and provide an estimate of the annual operating cost to support a user program at ASTA of $\$ 3.9 \mathrm{M} / \mathrm{yr}$. This estimate assumes nine months of beam operation per year.

The completion of ASTA and operation of a user program builds upon Fermilab's strong history and experience in the operation of the AO Photoinjector and its associated R\&D program. That program has produced $9 \mathrm{PhDs}$ in AARD since its inception in the mid-1990's. The scientific strength of that program and the assembled staff which operates it and directs the R\&D program has resulted, just in the last few years, in two dozen high-quality publications in accelerator science.

The unique features of ASTA will enhance OHEP's activities in commercialization of accelerator technology through its new Stewardship Program. With the Illinois Accelerator Research Center (IARC) at Fermilab, partnerships are already developing with potential industrial users and collaborators. ASTA offers unique capabilities that will enable a range of industrial-related R\&D. 
With the substantial investment already made in the ASTA facility, and the additional funds requested to complete the facility, OHEP has the opportunity to support a unique, state-of-theart, modern AARD user facility which is expected to have a broad impact, influencing fields from particle physics to basic-energy sciences to national security. As the nation's particle physics laboratory, Fermilab intends to play a leading role in the development of tomorrow's accelerators. A key element of that vision is a world-leading user facility for accelerator science. ASTA will offer unique opportunity for accelerator education and training of students though the US Particle Accelerator School and University programs, and industrial developments enabled by the Illinois Accelerator Research Center (IARC). 


\subsection{Introduction}

\subsection{The Role of Particle Physics in Accelerator Development}

Since the invention of the cyclotron eighty years ago, the multitude of historic breakthroughs in our understanding of the subatomic world has been inextricably linked to advances in accelerator technologies. These advances have enabled the construction of a remarkably compact and, thus far, complete picture of the subatomic world. Beginning approximately three decades ago, accelerator technologies developed for particle and nuclear physics began to be deployed to further the understanding of materials, leading to a revolution in our understanding of the atomic and molecular worlds as well. Today, large user facilities support the research of thousands of biologists, chemists and materials scientists by exploiting the accelerator technologies developed for particle and nuclear physics.

Industry has come to rely on technologies such as the cyclotron and the electrostatic accelerator for myriad industrial processes ranging from curing plastics to sterilizing medical equipment. The health sciences field recognized the critical capabilities that accelerator technology could play in treating cancer, in producing valuable radioisotopes and in expanding medical imaging. The security field has recently deployed accelerators to serve a security function that would be impossible to achieve otherwise. Today, the total value of all goods and products touched by accelerators exceeds $\$ 500 \mathrm{~B}$ per year.

Nearly all the fundamental accelerator technology that has enabled these remarkable achievements of the last eight decades can be traced to the need to develop accelerators for particle physics with ever increasing energy, intensity and particle densities. In the U.S., DOE's Office of High Energy Physics (OHEP) (and its progenitor agencies) has supported the bulk of this development. Indeed, the importance of research in accelerator science and technology is made explicit in the Mission of the Department of Energy's Office of High Energy Physics:

"The mission of the High Energy Physics program is to understand how our universe works at its most fundamental level. We do this by discovering the most elementary constituents of matter and energy, exploring the basic nature of space and time itself, and probing the interactions between them. These fundamental ideas are at the heart of physics and hence all of the physical sciences. To enable these discoveries, HEP supports theoretical and experimental research in both elementary particle physics and fundamental accelerator science and technology. HEP underpins and advances the DOE missions and objectives through this research, and by the development of key technologies and trained manpower needed to work at the cutting edge of science."

The importance of basic research in accelerator science and technology in support of High Energy Physics and the larger national needs is widely recognized and acknowledged. The P5 Panel (2008) recognized this need and recommended a broad strategic program in accelerator R\&D: 
"The US must continue to make advances in accelerator and detector R\&D to maintain leadership at the Intensity and Cosmic Frontiers of particle physics; to allow for a return to the Energy Frontier in the US; and to develop applications for the benefit of society.

The panel recommends a broad strategic program in accelerator R\&D, including work on ILC technologies, superconducting RF, high-gradient normalconducting accelerators, neutrino factories and muon colliders, plasma and laser acceleration, and other enabling technologies, along with support of basic accelerator science."

Recently, the special role that HEP has traditionally played in expanding the frontiers of accelerator science and technology has been codified through OHEPs Accelerator Stewardship Mission, which charges OHEP with managing an accelerator R\&D program in accordance with the broader scientific and societal needs for accelerator technology. This Accelerator Stewardship Mission recognizes the pivotal role that accelerators have played and will continue to play in furthering Discovery Science, Medicine, Industry, National Security, and challenges confronting the nation in Energy and the Environment.

\subsection{ASTA's Role in Furthering the HEP Mission}

It is in this context that we propose completing the construction of the Advanced Superconducting Test Accelerator (ASTA) at Fermilab, and establishing a User Facility for Accelerator R\&D based upon it.

Construction of the Advanced Superconducting Test Accelerator (ASTA) Facility at NML began in 2006 as part of the International Linear Collider/SRF R\&D Program and later the American Recovery and Reinvestment Act (ARRA) within the Department of Energy, Office of High Energy Physics. Construction of the facility was motivated by the goal of building, testing and operating a complete ILC RF unit to demonstrate industrial and laboratory capability for producing state-of-the-art superconducting linear accelerator components, and assembling them into a fully functioning system. ASTA, configured as a single ILC RF unit, would consist of an electron photo-injector, accelerating cavities, a bunch compressor, and three $1.3 \mathrm{GHz}$ ILC cryomodules powered together from a single high-power klystron. Each cryomodule provides $250 \mathrm{MeV}$ of energy gain for total output energy of approximately $800 \mathrm{MeV}$.

It was recognized early in the planning process that an electron beam meeting the demanding ILC performance parameters was itself a powerful resource of interest to the wider Advanced Accelerator R\&D (AARD) community. For those reasons the ARRA-funded facility construction incorporated space for the installation of additional cryomodules to increase the beam energy to a maximum of $1.5 \mathrm{GeV}$, space for multiple high-energy beamlines, as well as provision for a small circular ring for the exploration of ring-based advanced accelerator concepts. The facility design incorporated features necessary for the operation of an AARD user program, including adequate experimental beamline and insertion space, an appropriately sized support building,

Proposal for an Accelerator R\&D User Facility at Fermilab’s Advanced Superconducting Test Accelerator 
provision for transporting laser light into and out of the accelerator enclosure, and an adequately-sized control room.

ASTA is unique among accelerator user facilities in the U.S. in six principal ways:

- High repetition-rate: ASTA accelerates $1 \mathrm{msec}$ long bunch trains, with $3 \mathrm{MHz}$ micro-pulse repetition rate, with up to 3000 bunches per train.

- High average power: ASTA accelerates $1 \mathrm{msec}$ long bunch trains at up to five pulses per second, providing the highest beam power and highest average brightness of any accelerator test facility in the U.S.

- High energy: ASTA accelerates beam to $\sim 1 \mathrm{GeV}$, which is important for a number of photon-science and FEL-related experiments.

- Extremely stable beams: ASTA, based on ILC technology, is capable of providing exceptional beam stability, which has been demonstrated at the FLASH facility at DESY.

- Superconducting technology: ASTA necessarily brings SRF and beams together. As nearly all future large scale accelerator facilities will rely on SRF technology, ASTA will be the only GeV-scale test facility which is capable of supporting R\&D in prototypical conditions.

- Storage ring: ASTA includes a small, very flexible storage ring, based on innovative optics, capable of supporting a broad range of ring-based advanced beam dynamics experiments.

A national User Facility for Accelerator R\&D based at Fermilab's ASTA will further the mission of OHEP by addressing several needs:

\section{Furthering the Strategic Priorities for Particle Physics:}

While the domestic accelerator-based HEP program is oriented toward the Intensity Frontier, there are no accelerator test facilities that support Intensity Frontier accelerator science. ASTA, with both a modern superconducting linear accelerator and a small storage ring (with the capability of storing either electrons or protons) enables a class of beam-based experiments which seek to study fundamental limitations to beam intensity which cannot be carried out elsewhere.

\section{Strengthening the Nation's Accelerator Test Facility Infrastructure:}

Historically, much of the accelerator R\&D needed to build the next generation accelerators and accelerator-based facilities was carried out at operating facilities. The last decade and a half has witnessed the shutdown of a substantial accelerator infrastructure for particle physics in the US (SLC, PEP-II, CESR, Fermilab's Tevatron and Antiproton Complex) and abroad (LEP, HERA, DAFNE). The P5 Panel recognized this need in their report:

"Finally, the field must address the critical lack of accelerator test facilities that $R \& D$ requires. Historically, much accelerator $R \& D$ took place using operating high-energy physics facilities. However, as the operating facilities have turned off 
in the US, the opportunities for experimental demonstrations have correspondingly decreased. Some dedicated facilities have been proposed; and, while such facilities tend to be expensive to operate, they are essential for progress."

\section{Establishing a Test-Bed for Superconducting RF Accelerators:}

Nearly all large-scale accelerator user facilities envisioned for the future are based on superconducting RF technology. This includes Project $\mathrm{X}$, a MW-class accelerator facility for Intensity Frontier Physics based on a high-power superconducting linac; the International Linear Collider; next generation light sources, a high repetition rate soft x-ray FEL based on ILC/TESLA technology; the Facility for Rare Isotope Beams (FRIB) at MSU, a superconducting linac for heavy-ions; Cornell's Energy Recovery Linac, a superconducting linac-based photon science facility. Despite the ambitions of the accelerator field to deploy SRF technology in myriad future projects, there does not exist on U.S. soil an SRF test accelerator to meet the R\&D needs of these future projects. ASTA will fill a need for a superconducting test accelerator based on state-of-the-art SRF technology. ASTA will deliver prototypic beam conditions that cannot be reproduced elsewhere.

\section{Fulfilling the Accelerator Stewardship Mission:}

While ASTA is intended to support the particle physics mission, it will strengthen OHEP's stewardship portfolio at the same time by supporting a broad range of accelerator R\&D which will have a broad impact, ranging from particle physics, to photon sciences, to security.

\section{Educating and Training the Next Generation of Accelerator Scientists:}

With the closure of many of the accelerators that accommodated substantial machine studies programs, there is a critical lack of experimental beam facilities to allow training the next generation of accelerator scientists. As an accelerator R\&D User Facility, ASTA could be expected to support the educational needs of dozens of Ph.D. students over a decade of operation.

\subsection{Overview of Scientific Opportunities at ASTA}

The unique features and capabilities of ASTA, as outlined above, will allow the exploration of a wide range of advanced accelerator R\&D topics, which are summarized in Table 1 and explained in detail in Section 8 below. ASTA will enable a world-leading program in AARD for HEP Discovery Science at both the Intensity and Energy Frontiers, making use of the capabilities of ASTA's state-of-the-art superconducting linac, and flexible storage ring. With its high energy, high brightness, high repetition-rate beams, and the capability for emittance manipulation built into the design, ASTA is an ideal platform for exploring novel approaches for producing bright photon beams, which aligns with OHEP's Stewardship and Applications mission.

Proposal for an Accelerator R\&D User Facility at Fermilab's Advanced Superconducting Test Accelerator 
The Science enabled by ASTA can be best understood along five principal research thrusts:

- Intensity Frontier of Particle Physics: The U.S. HEP accelerator-based program has as a priority developing world-leadership in the Intensity Frontier. This requires powerful accelerators with precise control of beam losses and mitigation of beam-intensity limiting phenomena. ASTA will enable study and exploration of new methods for overcoming intensity-limiting phenomena in accelerators that cannot be explored elsewhere.

- Energy Frontier of Particle Physics: The next energy frontier accelerator requires technologies not previously used in colliders. ASTA will enable exploration and demonstration of critical technologies and methods central to the Energy Frontier. In particular, demonstration and exploration of ILC technology in a beam-based experimental program is central to near-term world-wide plans for the Energy Frontier. ASTA will bring together for the first time realistic beam-based system tests and SRF cryomodules operating at high-gradient.

- Superconducting RF Accelerators for Science: Nearly all future particle accelerators for Discovery Science, whether in the near-term or far-term, will be based on superconducting radiofrequency accelerators. ASTA brings together state-of-the-art SRF and beams in prototypic conditions, enabling a host of studies of direct relevance to the accelerator-based user facilities of the future.

- Novel Radiation Sources: The demand for radiation sources spans Discovery Science, national security and industrial applications. The unique combination of high energy, high peak and average power and high brightness electron beams available at ASTA will enable exploration of new techniques in the generation of high-brightness photon beams spanning the range from keVs to tens of MeVs.

- Stewardship and Applications: ASTA's flexibility and unique features enable a widerange of accelerator-science studies of relevance both to industrial partners, as well as other programs within DOE Office of Science and other agencies.

The experiments and research topics described in this proposal are mapped according to their applicability to Particle Physics, as well as along the five research thrusts outlined above, together with the needed ASTA Stage, in Table 1.

Table 1: Proposed ASTA Experiments and Accelerator R\&D thrusts, with Required ASTA Stage. The red and blue plot at bottom shows the relative fraction of HEP vs. Applications. [Next Page] 


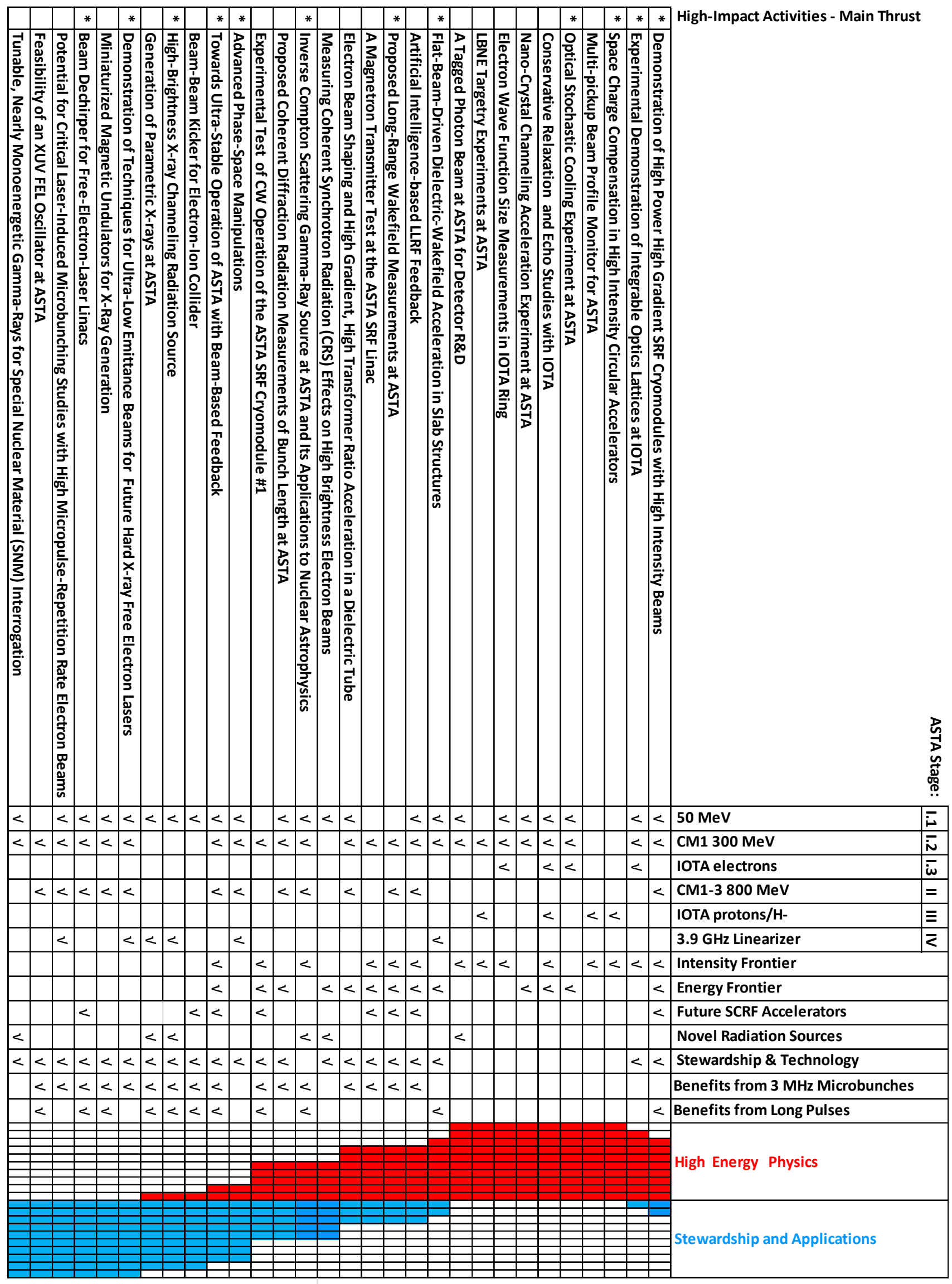




\subsection{Organization of This Proposal}

Section 3 describes the context of ASTA within Fermilab's broader mission, as well as Fermilab's historical contributions within the field of Advanced Accelerator R\&D.

Section 4 describes the scientific drivers in the field of advanced accelerator R\&D.

Section 5 presents a comparison of ASTA's parameters, capabilities, and costs with those of other advanced accelerator R\&D user facilities.

Section 6 describes the management of the Scientific User Program at the ASTA as an Office of Science (SC) user facility

Section 7 describes the ASTA Facility and its capabilities. A staged approach for build-out is described in which the linac beam energy is increased from an initial $300 \mathrm{MeV}$ with a single ILC cryomodule to $800 \mathrm{MeV}$ with the full build-out of three cryomodules. A small ring, the Integrable Optics Test Accelerator (IOTA), enables a broad range of AARD experiments already in the first stage.

Section 8 describes the scientific opportunities at ASTA. Those opportunities are aligned along research thrusts including Discovery Science at the Intensity and Energy Frontiers, development of SRF technology, novel radiation sources and Accelerator Stewardship and Applications. Taken together, ASTA provides a platform upon which a very broad range of accelerator science experiments can be carried out.

Section 9 provides a technical description of the ASTA linac and user facility.

Section 10 describes the role that ASTA will play in Accelerator Science education.

Section 11 presents the estimated costs to complete ASTA and establish the capability to support a user program, as well as the estimated costs to operate the facility in support of a user program.

Section 12 is a list of acronyms used in this proposal.

Finally, additional expressions of interest and concepts for experimental studies at ASTA utilizing the facilities unique capabilities in SRF, phase space manipulation and IOTA storage ring are presented in Attachment A.

Attachment B includes 21 Letters of Institutional Interest in the ASTA Facility from Laboratories, Accelerator R\&D Projects, Universities, and Corporations. 


\subsection{The Fermilab Context}

\subsection{Connection to Fermilab's Mission}

Since the invention of the cyclotron eighty years ago the multitude of historic breakthroughs in particle physics has been inextricably linked to advances in accelerator technologies. Much of the accelerator development in the U.S. over this period has taken place within the Office of High Energy Physics in the Department of Energy (and its progenitor agencies). OHEP's unique role is now formally recognized through the assumption of stewardship responsibility for longrange accelerator development within the U.S., independent of the final application.

Fermilab is the sole remaining U.S. laboratory devoted to providing facilities for acceleratorbased high energy physics. Since it's founding in the late 1960s Fermilab's history has been closely linked to leadership in the advancement of accelerator technologies, most notably the development of superconducting magnets for accelerator applications, but also including the development of large scale cryogenics systems, electron and stochastic cooling systems, and novel beam manipulations utilizing radiofrequency systems. All these advances have been supported by comprehensive integrated theoretical concepts and simulations of complete future facilities on both the energy and intensity frontiers. More recently Fermilab has established cutting edge capabilities and infrastructure for the development of superconducting radio-frequency acceleration based on its promise as an enabling technology for high energy physics. At present Fermilab (i) supplies integrated design concepts and technology development for a multi-MW proton source (Project $\mathrm{X}$ ) to support world-leading programs in long baseline neutrino and rare processes experiments; (ii) plays a leading role in the development of ionization cooling technologies required for muon storage ring facilities at the energy (multi-TeV Muon Collider) and intensity (Neutrino Factory) frontiers, and supplies integrated design concepts for these facilities; (iii) provides leadership on the development of next generation superconducting magnet technologies for application in upgrades to the Large Hadron Collider in Europe and a possible next generation hadron collider; and (iv) carries out a program of advanced accelerator R\&D (AARD) in the field of high quality beam sources, and novel beam manipulation techniques.

The advanced accelerator R\&D program described within this report builds upon accomplishments of the prior decade while leveraging multiple assets unique to Fermilab. Foremost among these assets is the Advanced Superconducting Test Accelerator, consisting of an ILC RF-unit, currently nearing completion in the New Muon Lab (NML) complex. The facility is configured to eventually produce electron beams with energies close to $1 \mathrm{GeV}$, and is unique in its ability to produce long pulse beams at GeV-scale energies. Fermilab envisions that this facility will eventually support a world-leading, vibrant user-driven research program in Accelerator Science and beyond. 
Beyond the NML complex Fermilab brings a number of additional assets in support of the ASTA program:

- Staff Capabilities and Core Competencies: Fermilab staff possesses unique capabilities and skills in accelerator design, construction, and operations, in leading edge accelerator technologies, and in advanced accelerator R\&D. In particular the staff is well experienced in superconducting RF and superconducting magnet development, in beam physics and simulations, in the management of significant accelerator projects, and in the construction and operations of accelerators. Personnel who work within the accelerator operations programs are available to support the ASTA program on either temporary or longer term bases via full or part time assignments.

- Infrastructure: Beyond the very significant infrastructure in place in the NML complex Fermilab has available very substantial cryogenics, RF power, assembly, testing, and electrical infrastructure within the laboratory.

- Accelerator Computation/Simulation Tools: A variety of advanced computation and simulation tools are available in the Accelerator Physics Center and via the SciDAC sponsored national accelerator computational program, which is led by Fermilab.

- Collaborative Connections: Fermilab has strong collaborative connections with a large number of national and international laboratories and universities within the ongoing accelerator development programs. In addition several local universities, plus Argonne National Laboratory, are active or potential participants in the Fermilab AARD program.

- Educational Programs: Fermilab has a long established accelerator PhD program that typically supports 5-10 students at any given time. Many leaders within the U.S. accelerator community have come through this program. In addition Fermilab hosts the U.S. Particle Accelerator School.

- Post-doctoral Program: The Peoples and Bardeen Fellowship programs bring the most accomplished post-doctoral accelerator scientists and engineers in the world to Fermilab, where they become vital contributors to the development and operations programs.

- Illinois Accelerator Research Center: IARC serves as a hub for industrial partnerships in accelerator technology, with a mission to develop commercialization opportunities for HEP-based technology.

ASTA contributes to Fermilab's Mission in at least two important ways. First, ASTA enables the exploration of several accelerator science topics of direct relevance to future Intensity Frontier facilities that cannot be carried out elsewhere. Second, as the national particle physics laboratory, Fermilab plays a unique role in ensuring that the accelerators of tomorrow can support the ambitions of the field. A vibrant Accelerator R\&D program is a requirement for serving that unique mission. 


\subsection{Fermilab's Historical Contributions in Advanced Accelerator R\&D}

\subsubsection{Fermilab's AARD Contributions}

Fermilab-as the leading US HEP laboratory and one of the leading accelerator institutions in the world-was always in the forefront of accelerator research and development in general (broadly understood as efforts toward improvement of existing accelerators and near future facilities), and advanced accelerator R\&D (AARD) in particular (which is focused on the next generation and beyond accelerators, fundamental beam physics issues, and novel accelerator technologies). The focus of the AARD efforts at Fermilab closely followed the priorities of HEP and sequentially was directed toward: in the mid-late 1990s-the Tevatron and Tevatron upgrades, LHC, VLHC and normal-conducting (NC) RF linear e+e- collider; in the 2000s-LHC, ILC and future multi-TeV lepton collider; in the early 2010s-on the next generation of intensity frontier accelerators, the LHC luminosity and energy upgrades, and multi-TeV Muon Colliders. It is to be noted that many AARD activities at Fermilab developed in separate national programs-e.g., LHC Accelerator Research Program (US-LARP), and, recently, the Muon Accelerator Program (US-MAP). Over the decades, the AARD activities had been carried out at the operational facilities at Fermilab (Tevatron, Main Injector, Booster), other labs (LHC), dedicated R\&D facilities elsewhere (CESR-TA, ATF, EMMA) and our own dedicated facilitiesthe AO Photoinjector, which operated since the mid-1990s and was closed last year, and ASTA at NML, which is being established at present. Results of the research are published in more than a hundred technical and peer-reviewed publications (the list of two dozen high-impact publications is given below). The research resulted in $11 \mathrm{PhDs}$ over the past decade and a half, several of whom have gone on to become today's leaders in accelerator science and technology.

The highlights of the past Fermilab AARD program include many important contributions to the physics of beams, e.g.:

- Development of high-brightness particle sources, such as RF photo-injectors, combined with SRF

- Development of the relativistic electron cooling method

- Pioneering research on the method of electron lenses for beam-beam compensation and beam collimation

- Novel beam optics techniques, e.g., round colliding beams, non-scaling FFAGs and integrable optics accelerators

- First ever demonstration of high ratio round-to-flat beam transformation and transverse-to-longitudinal emittance exchange

- Advanced beam collimation by bent crystals, include first time demonstration of volume-reflection collimation in colliders

- Studies of ground motion and vibration effects for future colliders

- Development of advanced beam diagnostics. 


\subsubsection{Selected AARD Publications from Fermilab}

[1] P. Piot, et al., Formation and Acceleration of uniformly-filled ellipsoidal electron bunches obtained via space-charge expansion from a Cesium-Telluride cathode, Phys. Rev ST-AB, 16, 010102 (2013).

[2] C.R. Prokop, P. Piot, B.E. Carlsten, M. Church, Beam dynamics performances and applications of a low-energy electron-beam magnetic bunch compressor, Nuclear Instruments and Methods in Physics Research A719, 17-28 (2013).

[3] P. Piot, et al., Generation and Characterization of Electron Bunches with Ramped Current Profiles in a Dual-Frequency Superconducting Linear Accelerator, Phys. Rev. Lett. 108, 034801 (2012).

[4] S. Machida et al., Acceleration in the linear non-scaling fixed-field alternating-gradient accelerator, Nature Physics (2012).

[5] T. Maxwell, et al., Synchronization and characterization of an ultrashort pulse laser for photoemission and electron-beam diagnostics studies at a radio frequency photoinjector JINST 7 T11001 (2012).

[6] J. Thangaraj, Experimental Studies on Coherent Synchrotron Radiation at an Emittance Exchange Line, Phys. Rev. ST-AB, 15, 110702 (2012).

[7] A.H. Lumpkin, J. Ruan, and R.M. Thurman-Keup, Synchroscan streak camera imaging at a 15-MeV photoinjector with emittance exchange, Nucl. Instr. and Methods, Section A, vol. 687, 92-100 (2012).

[8] A. Shishlo, et al., First Observation of Intrabeam Stripping of Negative Hydrogen in a Superconducting Linear Accelerator, Phys. Rev. Lett. 108, 114801 (2012).

[9] J. Thangaraj, et al., Demonstration of a real-time interferometer as a bunch-length monitor in a high-current electron beam accelerator, Rev. Sci. Instrum. 83, 043302 (2012).

[10] G. Stancari, et al., Collimation with Hollow Electron Beams, Phys. Rev. Lett. 107, 084802 (2011).

[11] J. Ruan, et al., First observation of the exchange of transverse and longitudinal emittances, Phys. Rev. Lett. 106, 244801 (2011).

[12] Y.-E. Sun, et al., Tunable Subpicosecond Electron-Bunch-Train Generation Using a Transverse-To-Longitudinal Phase-Space Exchange Technique, Phys. Rev. Lett. 105, 23480 (2010).

[13] V. Shiltsev, Observations of Random Walk of the Ground In Space and Time, Phys. Rev. Lett., v.104, 238501 (2010).

[14] V. Danilov, S. Nagaitsev, Nonlinear accelerator lattices with one and two analytic invariants, Phys. Rev. ST Accel. Beams 13, 084002 (2010).

[15] A. Lumpkin, et al., Coherent optical transition radiation and self-amplified spontaneous emission generated by chicane-compressed electron beams, Phys. Rev. ST Accel. Beams 12, 040704 (2009).

[16] V. Shiltsev, et al., Experimental Demonstration of Colliding-Beam-Lifetime Improvement by Electron Lenses, Phys. Rev. Lett. 99, 244801 (2007).

[17] S. Nagaitsev, et al., Experimental Demonstration of Relativistic Electron Cooling, Phys. Rev. Lett. 96, 044801 (2006). 
[18] P. Piot, et al., Photoinjector generation of a flat electron beam with transverse emittance ratio of 100, Phys. Rev. ST Accel. Beams 9, 031001 (2006).

[19] Y.-E Sun, et al., Generation of angular-momentum-dominated electron beams from a photoinjector, Phys. Rev. ST Accel. Beams 7, 123501 (2004).

[20] M. Thompson, et al., Status of the UCLA/NICADD Plasma Density Transition Trapping Experiment, AIP Conf. Proc. 737, 440 (2004).

[21] N. Barov, et al., Plasma Wakefield Experiments, AIP Conf. Proc. 647, 71 (2002).

[22] B. Aune, et al., Superconducting TESLA cavities, Phys. Rev. ST Accel. Beams 3, 092001 (2000).

[23] A. Burov, et al., Optical principles of beam transport for relativistic electron cooling, Phys. Rev. ST Accel. Beams 3, 094002 (2000).

[24] B. Baklakov, et al., Ground vibration measurements for Fermilab future collider projects, Phys. Rev. ST Accel. Beams 1, 031001 (1998).

[25] E. Colby, et al., Design of high brightness symmetric and asymmetric emittance RF photoinjectors for TESLA, AIP Conf. Proc. 335 (1995) 708-723.

\subsubsection{Historical Achievements in Advanced Accelerator R\&D at the A0 Photoinjector Facility (AOPI)}

In this section we summarize key results obtained at the AOPI facility, which was operated from 1998 to 2011 in the AO building on the Fermilab campus. Over its lifetime the facility assumed different beamline configurations. Most of the experiments were carried out in support of PhD research topics and included the development of new diagnostics, beam dynamics research on advanced phase space manipulation, and the demonstration of advanced-acceleration concepts. A description of the facility from its construction to c.a. 2004 appears in Ref. [1]. The facility was later reconfigured to accommodate advanced phase space manipulation and most notably to demonstrate transverse-to-longitudinal phase space exchange. A description of the reconfigured facility can be found in Ref. [2]. Some of the components associated with this facility were relocated to the ASTA photoinjector and the AOPI photoinjector in its current configuration only include an RF electron source. It is being used for photoemission and fieldemission studies along with other experiments on electron bunch shaping and R\&D on lowenergy radiation sources.

\subsubsection{Phase-Space Manipulations of Electron Beams}

In its early days, the AOPI included a low-energy magnetic bunch compressor chicane. The compressor was designed to support plasma-wakefield acceleration experiments led by UCLA. The bunch compressor demonstrated the generation of 2.8-kA peak current at $16 \mathrm{MeV}$ [3]. This low-energy compressed beam was used to investigate beam-driven plasma-wakefield acceleration.

The flat-beam technique consisting of producing beams with large-emittance ratio by applying a linear transformation to incoming canonical-angular-momentum dominated (magnetized) beams was proposed by Brinkmann et al. in 1999 [4]. The scheme has been experimentally 
demonstrated at the Fermilab A0 Photoinjector in 2001 [5]. Steady progress on understanding the dynamics associated with the photoinjector production of magnetized beams [6] and their conversion to flat beams led to the production of flat beams with record transverse emittance ratio of 100 [7]; see Figure 1. The flat beam techniques, if further improved or combined with other phase space transformation, could mitigate the requirements (and maybe the need) for an electron damping ring in future high-energy linear colliders. Flat beams produced via this simple transformation have parameters consistent with their possible use in beam-driven techniques based on asymmetric structures $[8,9]$ or to enhance the beam-surface-wave interaction in, e.g., Smith-Purcell free-electron laser [10].
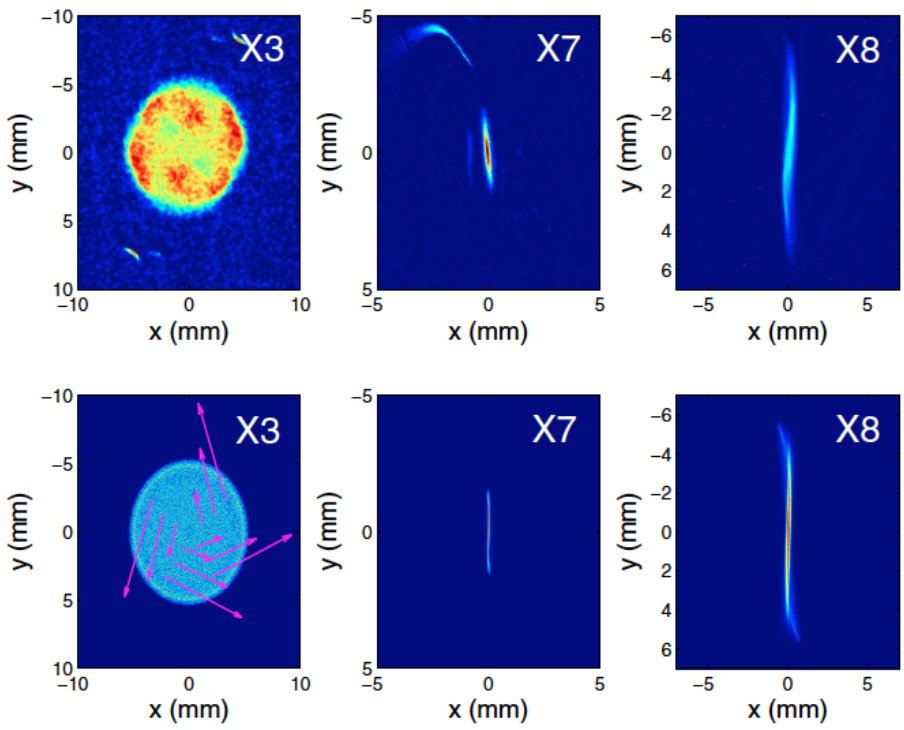

Figure 1: Flat beam generation at the $A 0$ photoinjector. Comparison of the measured (top row) and simulated (bottom row) beam distribution at the cathode (left column), just after the flat beam transformation (middle) and after a 2-m drift downstream of the flat beam transform (right column).

The other advanced phase-space manipulation pioneered at Fermilab's AOPI facility is the transverse-to-longitudinal phase space exchanger. A beamline capable of exchanging the horizontal and longitudinal phase space coordinate devised in [11] was constructed in 2005. The beamline supported the demonstration of near-ideal emittance exchange between the horizontal and longitudinal degrees of freedom [12] and was also used to show that this type of beamline can also serve as a pulse shaper $[13,14]$; see Figure 2 . The developed pulse-shaping techniques could be applied to the generation of high-charge electron bunches with current profiles optimized to enhance the transformer ratio associated to beam-driven high-energy accelerators (e.g. based on plasma or dielectric wakefield acceleration). Likewise, when combined with structured cathodes, e.g. such as field-emission tips, the phase-space-exchange method enables the generation of temporally-modulated beams with applications to compact, coherently-enhanced, radiation sources ranging from the $\mathrm{THz}$ to extreme ultra-violet wavelengths. The technique can also produce attosecond pulses for ultra-fast diffraction. 


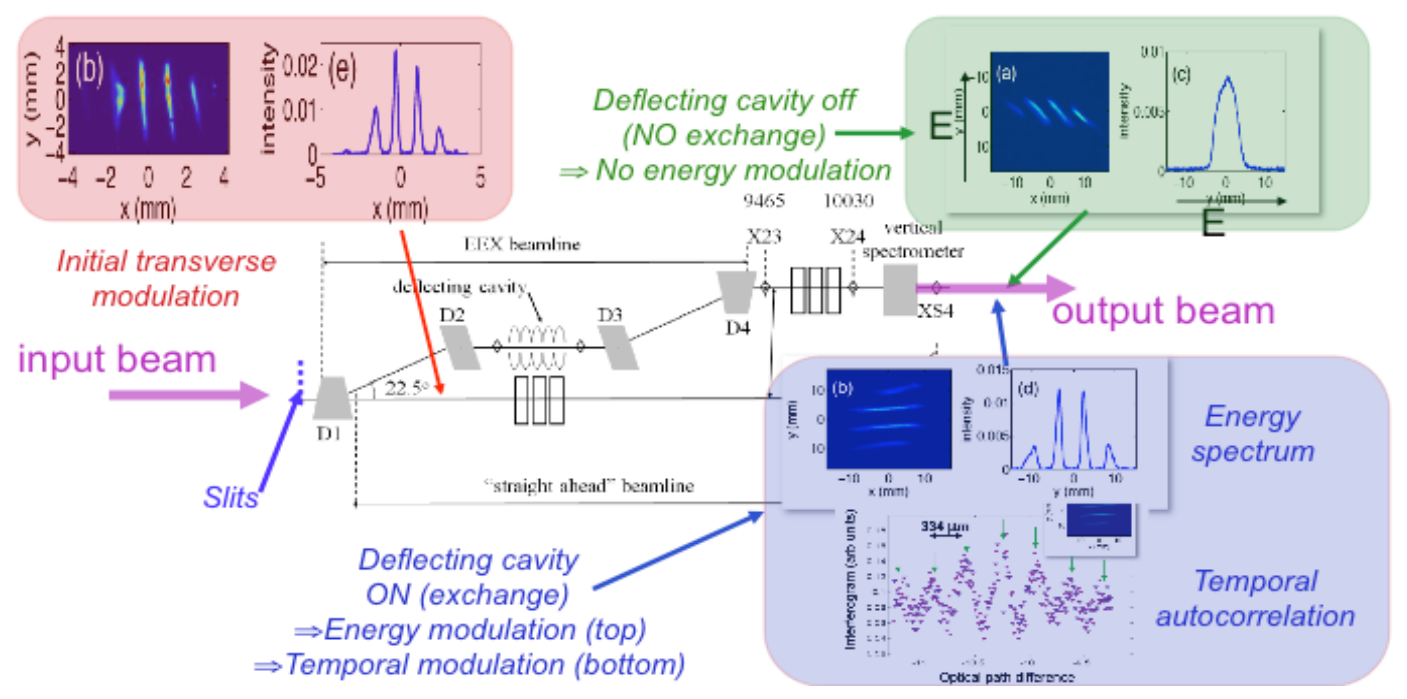

Figure 2: Principle of a pulse shaper based on a phase-space exchanger. In this experiment conducted at Fermilab [14, 15], an incoming transversely-segmented beam (distribution shown in the red-shaded area) is transformed into a temporally modulated (blue-shaded area) downstream of the phase space exchanger. Turning off the deflecting cavity (and therefore disabling the beamline to exchange phase spaces) does not exchange the horizontal and longitudinal phase-space distributions.
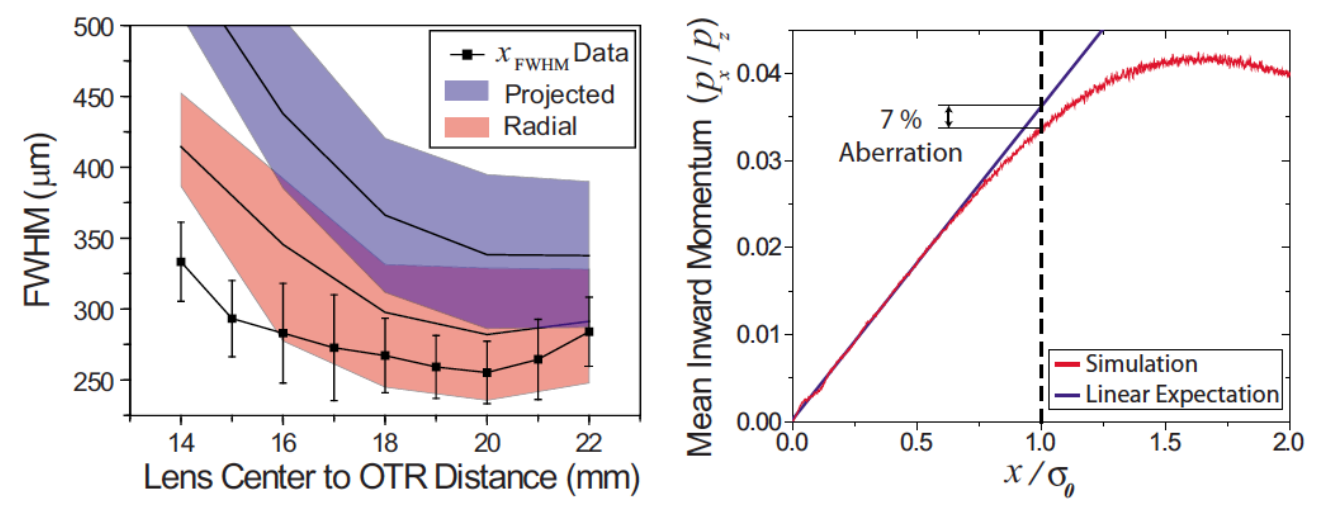

Figure 3: Comparison of focusing data with MAGIC simulation results. The width of the colored bands represents the range of values possible given the uncertainty on emittance measurement (left) and simulated average inward momentum imparted by the plasma lens on the beam (right).

The capability of the AOPI to generate high-charge bunch was also utilized to demonstrate the operation of a plasma lens in the underdense regime by UCLA [16]. In this experiment, focusing of the $15 \mathrm{MeV}$ electron bunch by a plasma lens operated at the threshold of the underdense 
regime was demonstrated. The strong, $1.7 \mathrm{~cm}$ focal length, plasma lens focused both transverse directions simultaneously and reduced the minimum area of the beam spot by a factor of 23 . It was shown through analytic analysis and simulation that the observed spherical aberration of this under dense lens, when expressed as the fractional departure of the focusing strength from its linear expectation, is $\Delta K / K=0.08 \pm 0.04$; see Figure 3 . This is significantly lower than the minimum theoretical value for the spherical aberration of an over-dense plasma lens.
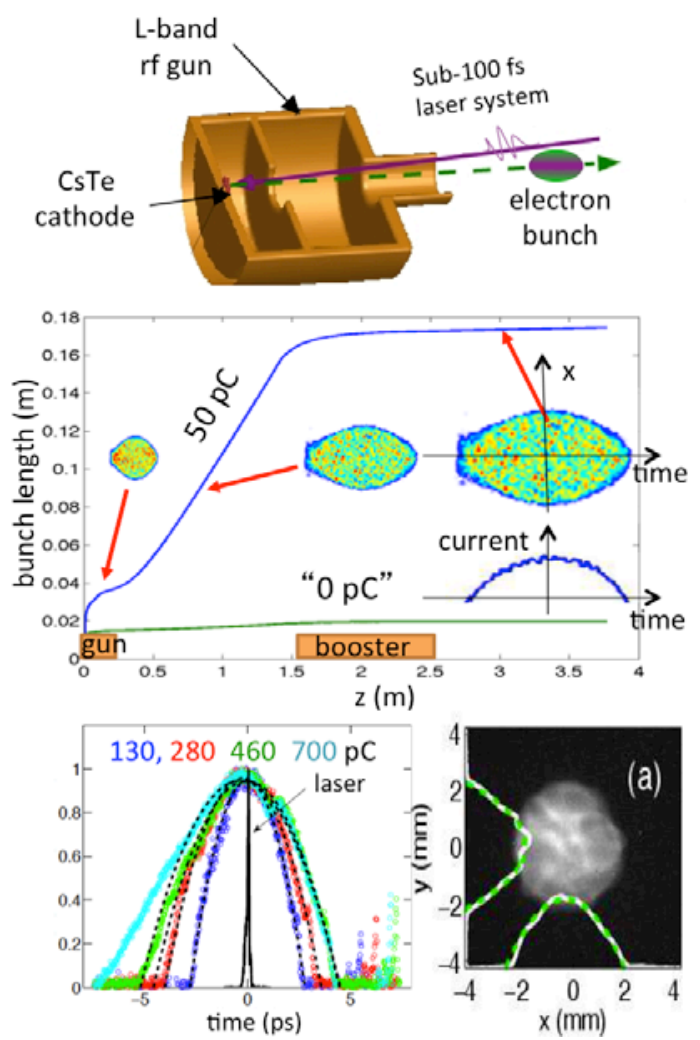

Figure 4: Principle of generation of ellipsoidal bunches using the "blow-out" regime (top) and simulated bunch length evolution at the AOPI (middle). Measured temporal (bottom left) and transverse (bottom right) beam distributions.

Finally, the AOPI also supported the R\&D on photoemission electron sources using highefficiency cathodes based on Cesium-Telluride. A recent example of such a study includes the experimental generation, acceleration, and characterization of a uniformly filled electron bunch obtained via space-charge-driven expansion (often referred to as "blow-out regime") in an Lband $(1.3-\mathrm{GHz})$ radiofrequency photoinjector. The beam was photoemitted from the cesiumtelluride semiconductor photocathode using a short $(<200 \mathrm{fs})$ ultraviolet laser pulse. The produced electron bunches were characterized with conventional diagnostics and the signatures of their ellipsoidal character was observed. The experiment especially demonstrated the production of ellipsoidal bunches with charges up to $\sim 0.5 \mathrm{nC}$ corresponding to a $\sim 20$-fold increase compared to previous experiments with metallic photocathodes [17]. An example of measurements appears in Figure 4 


\subsubsection{Advanced Acceleration Techniques}

The plasma wake-field mechanism can be used to couple energy at a high rate from a bunched electron beam into a plasma wave. At the Fermilab AOPI facility a 4-8-nC beam with an initial energy of $14 \mathrm{MeV}$ was passed through an $8-\mathrm{cm}$ length of plasma with $10^{14} \mathrm{~cm}^{-3}$ density. The beam emerged with a much broader energy spread, spanning from a low of $3 \mathrm{MeV}$ to a high of over $20 \mathrm{MeV}$. Such broadened energy spread implied the presence of a peak decelerating field with 140-MV/m amplitude, and a 72-MV/m acceleration gradient [18].

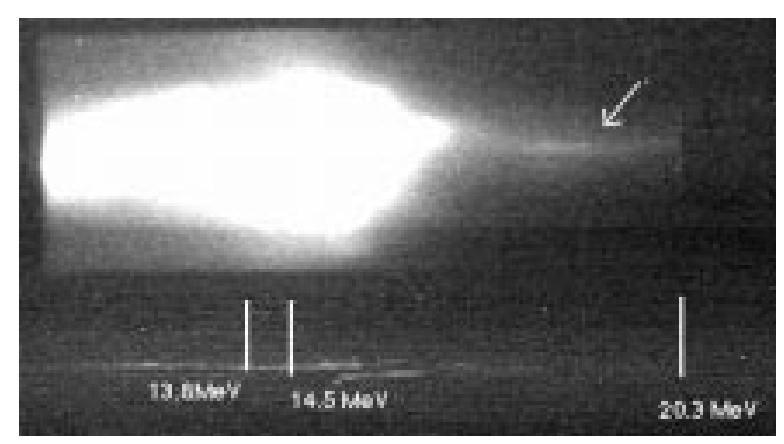

Figure 5: Demonstration of plasma-wakefield acceleration at the AOPI: spectrometer image with plasma on. The accelerated electrons are the whisker-like feature near the arrow.

\subsubsection{Beam Diagnostics R\&D}

AOPI hosted the first demonstration of time-domain electro-optical imaging of the velocity field of an electron bunch. Measurements of the electric field as a function of time as measured at a fixed location in the AOPI beam line were carried out. For a 12-nC bunch of duration 4.2 ps FWHM, the peak field was measured $>0.5 \mathrm{MV} / \mathrm{m}$. A modest time resolution of $\sim 5$ ps was achieved using this electro-optic sampling with a lithium tantalate $\left(\mathrm{LiTaO}_{3}\right)$ crystal and a ps infrared laser synchronized to the beam. The resolution was limited by the electron-beam energy and the laser pulse duration (which was limited to a 3-ps minimum duration) [19].

More recently, a similar technique was utilized to carry single-shot imaging of the coherent transition radiation excited as the bunch passes through an aluminum foil. The method was used to demonstrate the generation of a two-bunch beam by imaging an initially transverselysegmented beam at the cathode using the transverse-to-longitudinal phase space exchanger; see Figure 6 [20]. For these experiments an ultra-short Titanium-Sapphire laser was used so that higher time resolutions were achieved. 

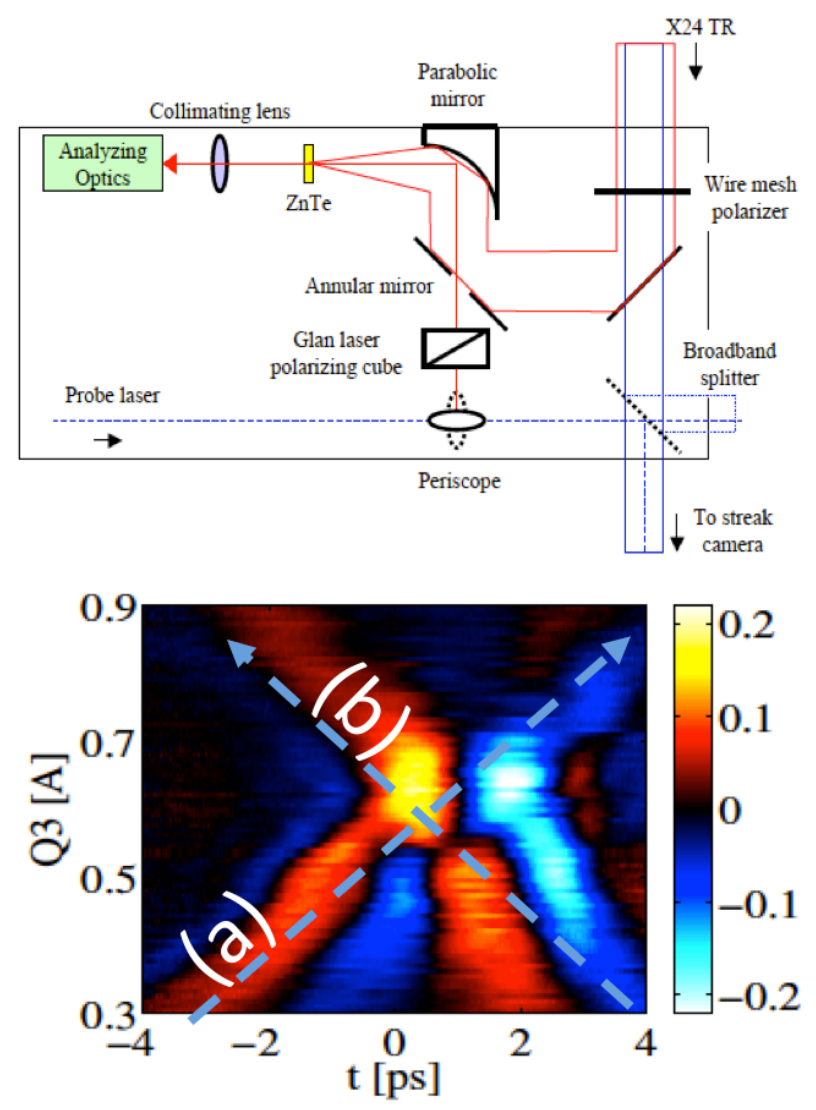

Figure 6: Electro-optical imaging of coherent transition radiation (top) and measured double-bunch distribution (bottom).
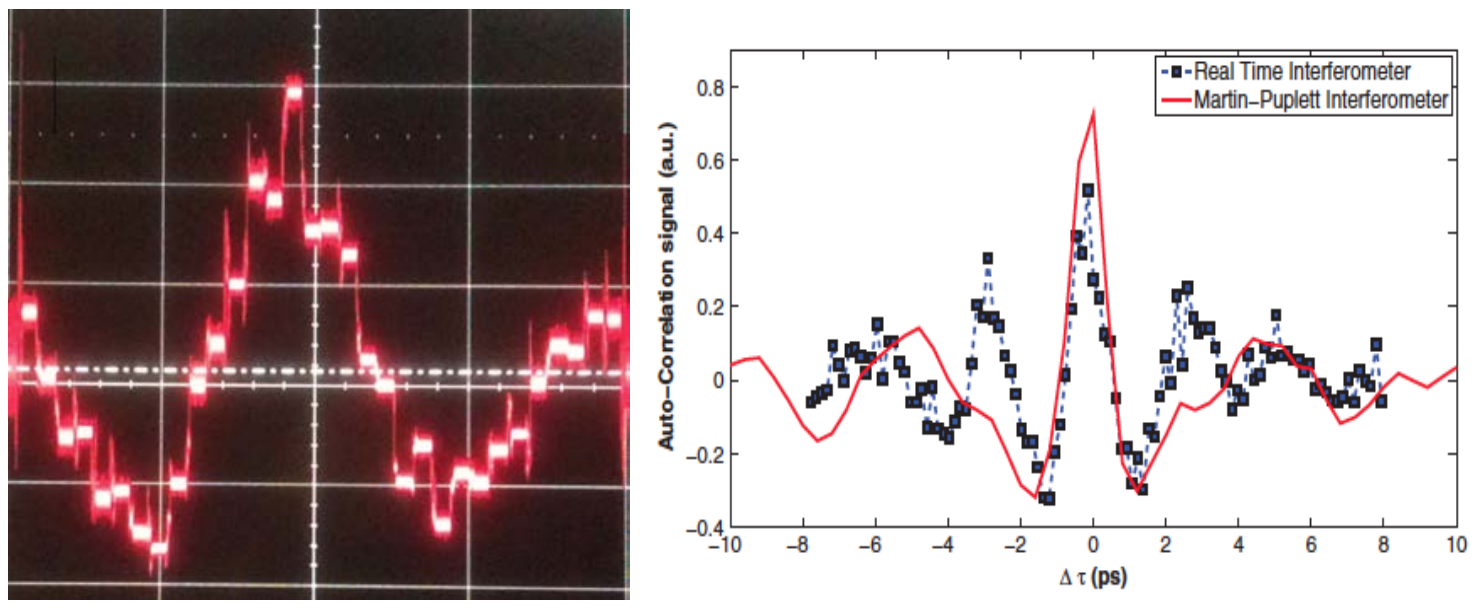

Figure 7: Raw (real-time) oscilloscope autocorrelation trace obtained from the 32channel detector array used in the RTI (left) and comparison of autocorrelation traces obtained with the RTI and a conventional scanning Martin-Puplett interferometer. The central peaks (representative of the electron-bunch duration) agree. The observed difference for time $|\Delta \tau|>2$ ps are due to difference in the limited responses associated to the two devices. 
Most recently, a real-time interferometer (RTI) developed by Radiabeam to monitor the bunch length of high-current electron beams in an accelerator was tested. The RTI employs spatial autocorrelation, reflective optics, and a fast response pyro-detector array to obtain a real-time autocorrelation trace of the coherent radiation from an electron beam, thus providing the possibility of online bunch-length diagnostics. A complete RTI system was commissioned at the A0 photoinjector facility to measure sub-mm bunches at $13 \mathrm{MeV}$. Bunch length variation (FWHM) between $0.8 \mathrm{ps}(\sim 0.24 \mathrm{~mm})$ and $1.5 \mathrm{ps}(\sim 0.45 \mathrm{~mm})$ has been measured and compared with a Martin-Puplett interferometer and a streak camera. The comparisons demonstrated that the RTI is a viable, complementary bunch length diagnostic for sub-mm high-current electron bunches [21]. An example of measurement appears in Figure 7.

\subsubsection{References}

[1] J.-P. Carneiro, N. Barov, H. Edwards, M. Fitch, W. Hartung, K. Flöttmann, S. Schreiber, and M. Ferrario, Phys. Rev. ST Accel. Beams 8, 040101 (2005).

[2] T. Koeth, "An Observation of a Transverse to Longitudinal Emittance Exchange at the Fermilab A0 Photoinjector," Doctoral dissertation, Rutgers, New Jersey (2010).

[3] M. J. Fitch, A. C. Melissinos, N. Barov, J.-P. Carneiro, H. T. Edwards, "compression of highcharge electron bunches," in Proceedings of the 2000 International Linear Accelerator Conference (LINAC2000), also available as preprint arXiv:physics/0008206 (2000).

[4] R. Brinkmann, Y. Derbenev, and K. Flöttmann, DESY Report No. TESLA 99-09, 1999; Phys. Rev. ST Accel. Beams 4, 053501 (2001).

[5] D. Edwards et al., in Proceedings of the 2001 Particle Accelerator Conference, Chicago, IL (IEEE, Piscataway, NJ, 2001), pp. 73-75.

[6] Y.-E Sun, P. Piot, K.-J. Kim, N. Barov, S. Lidia, J. Santucci, R. Tikhoplav, and J. Wennerberg, Phys. Rev. ST Accel. Beams 7, 123501 (2004).

[7] P. Piot, Y.-E Sun, and K.-J. Kim, Phys. Rev. ST Accel. Beams 9, 031001 (2006).

[8] D. Mihalcea, P. Piot, and P. Stoltz, Phys. Rev. ST Accel. Beams 15, 081304 (2012).

[9] A. Cahill, A. Fukusawa, P. Musumeci, B. Spataro, A. Yakub, and J. B. Rosenzweig, and A. Valloni, AIP Conf. Proc. , vol. 1507, p. 762, 2012.

[10] V. Kumar and K.-J. Kim, Phys. Rev. ST Accel. Beams 12, 070703 (2009).

[11] P. Emma, Z. Huang, K.-J. Kim, and P. Piot, Phys. Rev. ST Accel. Beams 9, 100702 (2006).

[12] J. Ruan, A. S. Johnson, A. H. Lumpkin, R. Thurman-Keup, H. Edwards, R. P. Fliller, T. W. Koeth, and Y.-E Sun, Phys. Rev. Lett. 106, 244801 (2011).

[13] P. Piot, Y.-E Sun, J. G. Power and M. Rihaoui, Phys. Rev. ST Accel \& Beams, 14022801 (2011)

[14] Y.-E Sun, P. Piot, A. Johnson, A.H. Lumpkin, T.J. Maxwell, J. Ruan, R. Thurman-Keup, Phys. Rev. Lett. 105, 234801 (2010)

[15] P. Piot, Y.-E Sun, T.J. Maxwell, A. H. Lumpkin, J. Ruan, M. M. Rihaoui, R. Thurman-Keup, T. J. Maxwell, J. Ruan, R. Thurman-Keup, Appl. Phys. Lett., 96261501 (2011).

[16] M. C. Thompson, H. Badakov, J. B. Rosenzweig, G. Travish, N. Barov, P. Piot, R. Fliller, G. M. Kazakevich, J. Santucci, J. Li, and R. Tikhoplav, Physics of Plasmas 17, 073105 (2010).

[17] P. Piot, Y.-E Sun, T. J. Maxwell, J. Ruan, E. Secchi, J. C. T. Thangaraj, Phys. Rev. ST Accel. Beams 16, 010102 (2013). 
[18] N. Barov, K. Bishofberger, J.B. Rosenzweig, J.P. Carneiro_, P. Colestock†, H. Edwards, M.J. Fitch, W. Hartung, J. Santucci, Proc. 2001 Particle Accelerator Conference (Chicago, IL), 126 (2001).

[19] M.J. Fitch, A.C. Melissinos, P.L. Colestock, J.-P. Carneiro, H.T. Edwards, W.H. Hartung, Phys. Rev. Lett. 87, 034801 (2001).

[20] T. J. Maxwell, J. Ruan, P. Piot, R. Thurman-Keup, FERMILAB-CONF-11-414-APC, arXiv:1205.0800 [physics.acc-ph] (2011).

[21] J. Thangaraj, G. Andonian, R. Thurman-Keup, J. Ruan, A. S. Johnson, A. Lumpkin, J. Santucci, T. Maxwell, A. Murokh, M. Ruelas, and A. Ovodenko. Review of Scientific Instruments 83, 043302 (2012).

\subsubsection{PhD Theses in Advanced Accelerator R\&D from Fermilab-based Research}

Alan Reilly Fry, 1996 University of Rochester, "Novel Pulse Train Glass Laser for RF Photoinjectors"

Eric Colby, 1997, UCLA, "Design, Construction, and Testing of a Radiofrequency Electron Photoinjector for the Next Generation Linear Collider"

Michael Fitch, 2000, University of Rochester, "Electro-optic Sampling of Transient Electric Fields from Charged Particle Beams"

Jean-Paul Carneiro, 2001, Paris XI, “Etude Experimentale du Photo-injecteur de Fermilab"

Matthew Thompson, 2004, UCLA, "Plasma Density Transition Trapping of Electrons in Plasma Wakefield Accelerators"

Kip Bishofberger, 2005, UCLA, "Tevatron Beam-Beam Compensation"

Sergei Seletskiy, 2005, University of Rochester, "Attainment of Electron Beam Suitable for Medium Energy Electron Cooling"

Yin-E Sun, 2005, University of Chicago, "Angular-momentum-dominated electron beams and flat beam generation"

Rodion Tikhoplav, 2006, University of Rochester, "Low Emittance Electron Beam Studies"

Pavel Snopok, 2007, Michigan State University, “Capture of a Large Phase Space Beam”

Timothy Koeth, 2009, Rutgers University, "The First Observation of a Transverse to Longitudinal Emittance Exchange"

Tim Maxwell, 2012, Northern Illinois University, "Measurement of sub-picosecond electron bunches via electro-optic sampling of coherent transition radiation" 


\subsection{Scientific Drivers in Advanced Accelerator R\&D}

The US domestic accelerator-based particle physics program is shifting focus from the Energy Frontier to the Intensity Frontier [1, 2]. The nation's and Fermilab's strategy has been laid out at the 2013 Community Summer Study "Snowmass-2013" [3] and is focused on development of the facilities to enable a world-leading program in Intensity Frontier Physics [4]. This will be accomplished by making use of the substantial existing infrastructure at Fermilab, and extending the capabilities of the accelerator complex by constructing a new high-power proton linear accelerator, called Project X [5]. Figure 1 shows Fermilab's accelerator complex, as it will be configured in 2020, with the construction of Project $X$ underway.

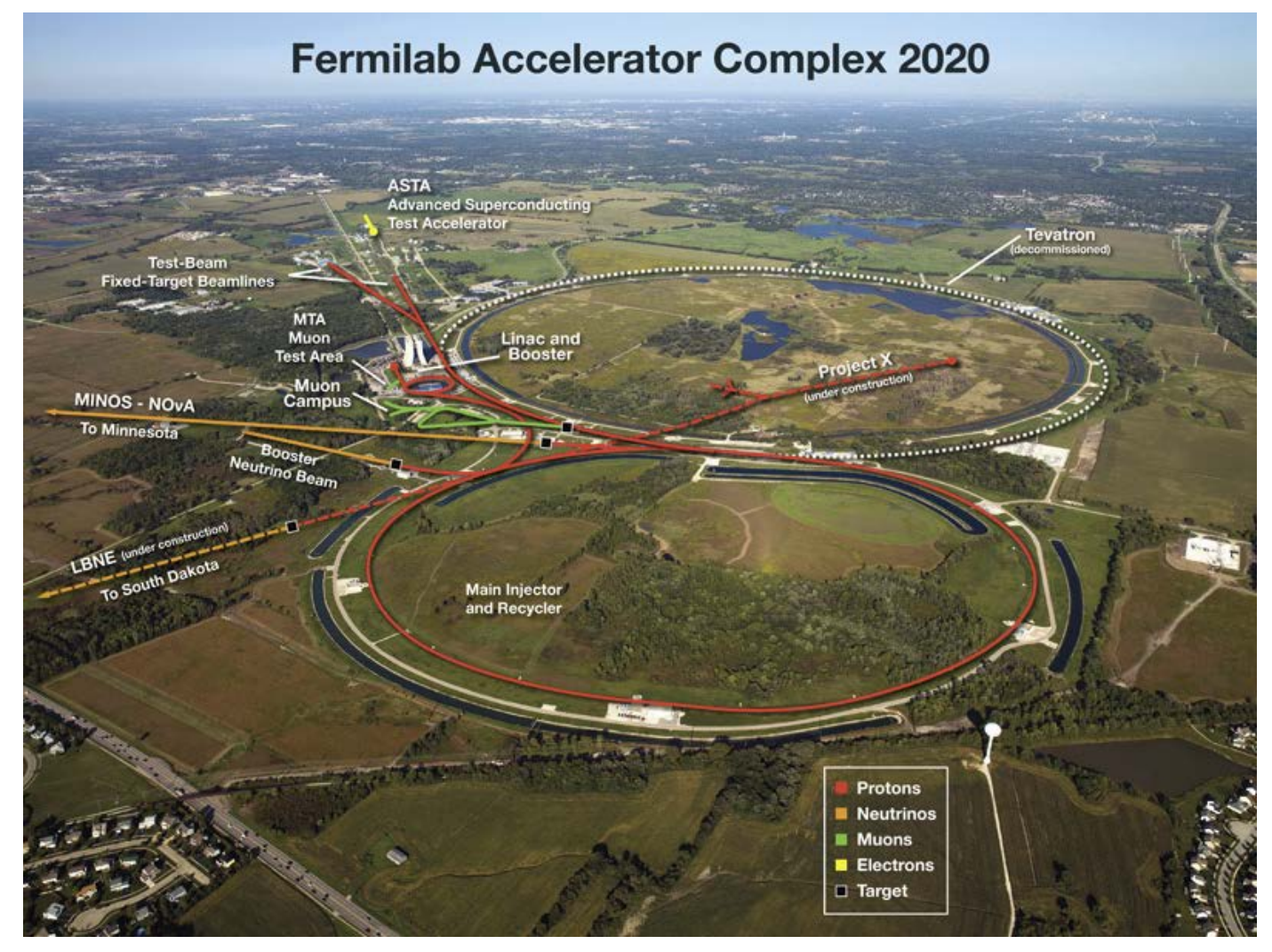

Figure 1: Fermilab's accelerator complex by the end of this decade [1].

The 2008 P5 report [2] specifically pointed out that “...advances in accelerator R\&D are critical for the United States to maintain leadership not only at the energy frontier of particle physics, but also in the wider context of applications of particle accelerators and detectors reaching across science and society." Three major considerations shape the directions and scope of the accelerator research in the country [6]: 
1. The recent Higgs discovery and the potential for discovering supersymmetry or other beyond standard-model physics (BSM) at the LHC will provide guidance for the next Energy Frontier accelerator:

- Perhaps a Higgs factory will be required to fully unravel its properties and couplings.

- Perhaps BSM results will motivate a multi-TeV lepton collider.

- Looking beyond, it is very difficult to probe well beyond LHC energies.

2. There are two approaches to probing mass-scales well beyond the LHC:

- Utilizing very intense beams to probe high mass-scales in rare decays and precision measurements [4].

- Achieve very high energies, well beyond that which is routinely discussed today, enabled by advanced concepts, such as acceleration in crystal-channels, solidstate plasmas driven by high brightness x-rays, or similar approaches [7].

3. Accelerator R\&D beyond the field of particle physics is motivated by the growing recognition that:

- Accelerators are essential tools for all fields of Discovery Science, including photon and neutron science, nuclear science and fusion energy science.

- In addition to their role in Discovery Science, accelerators are vital to industry, medicine, national security, and have potential in energy and environment [8].

- The path of accelerator development must be carried out in a socially and financially realistic way.

As the nation's particle physics laboratory, Fermilab has a responsibility to ensure that the United States has a handle on technology needed to build the next generation facilities for particle physics, and that we're doing the necessary long-range R\&D to support the ambitions of the field, and therefore the machines of the far future.

Regarding the Energy Frontier, there are several ideas to move into the few-TeV regime for lepton colliders which are presently being addressed by the AARD community (muon collider, two-beam and plasma-based e+e-colliders) [9], but beyond that, there is a great void in terms of direct searches beyond 10 times LHC energies. There is also a growing consensus that collisions of multi-TeV e+e- beams might not be feasible due to radiative energy losses and induced energy spreads which make such colliders ineffective-therefore, muons become the preferred particles for very high energy [7]. Correspondingly, there is a need to explore the issues associated with such a paradigm-muon sources, acceleration, manipulation, control, etc. - which is being addressed by the US Muon Accelerator Program [10].

Similarly, the Intensity Frontier facilities of the near- and far-future have many challenges that require advancing the state-of-the-art through a dedicated R\&D program. Key Challenges include [11]:

- Producing high-current, high-quality beams (high brightness)

- Accelerating high beam currents to high energy

- High-duty factor, high-power RF systems, structures and components 
- Transporting high-power beams with extremely low beam loss, in order to allow routine hands-on maintenance (minimizing beam loss is the over-riding design constraint as a typical beam loss requirements for a MW proton beam are $<1 \mathrm{Watt} / \mathrm{m},<1 \times 10^{-4}$ total beam loss in a ring)

- Acceleration of beams from keVs to GeVs preserving emittance and minimizing growth in large-amplitude particles ("beam halo")

- Target systems capable of handling extreme power densities and extreme radiation environments

- Producing intense, high-quality beams of secondary particles (muons, kaons, ...)

- Achieving highly efficient $\mathrm{H}$ - stripping for high-intensity, high-power CW beams;

- Overcoming beam dynamics issues of space-charge and instabilities, which ultimately limit all synchrotrons, accumulators, compressors and linacs

- Creating self-consistent beam distributions (i.e. linear space-charge force or spacecharge compensated beams)

- Generating large Landau-damping of coherent instabilities via non-linear (but stable) betatron motion

- Novel ways to cleanly achieve slow spills of high intensity beams

- More efficient non-standard methods of resonant extraction (e.g., can it be extended to higher orders? Can crystals be used for high-power proton extraction? Can electron beams be used to extract protons?)

- Instrumentation for precise halo (and emittance) measurement and connection to simulations in a reliable way.

One can see that the high intensity frontier accelerator research thrusts have direct connections to the high energy frontier development-for example, the Muon Collider concept has many similar challenging ingredients.

Other Discovery Science fields provide powerful motivation for advanced accelerator R\&D. Accelerators have become essential tools in biology, health sciences, energy sciences, materials science, geophysics, in addition to those that have traditionally relied on them (nuclear and particle physics). Indeed, five of the last 15 Nobel Prizes in Chemistry have been awarded based on work carried out at synchrotron radiation facilities. The need for compact and affordable sources of particle probes for materials science drives much research throughout the world in new sources of photons and neutrons. The need for tunable photon sources with higher brightness and temporal and spatial coherence drives much of the Free Electron Laser (FEL) R\&D that is carried out throughout the world.

Accelerator applications for industry, medicine, national security, energy, and environment bring an increasing emphasis on applied accelerator technology, as they have real potential to make an impact, which in turn is becoming yet another powerful driver for advanced accelerator R\&D [12].

Past developments have shown that the most effective way for AARD to address the scientific challenges faced by particle physics involves accelerator facilities. The 2008 P5 report 
recommendation summarized the situation: “... the field must address the critical lack of accelerator test facilities that $R \& D$ requires. Historically, much accelerator R\&D took place using operating high-energy physics facilities. However, as the operating facilities have turned off in the US, the opportunities for experimental demonstrations have correspondingly decreased. Some dedicated facilities have been proposed; and, while such facilities tend to be expensive to operate, they are essential for progress." [2]

\section{References}

[1] Fermilab: Plan for Discovery (Fermilab, 2012), see at https://www.fnal.gov/directorate/ plan for discovery/.

[2] "US Particle Physics: Scientific Opportunities A Strategic Plan for the Next Ten Years." Report of the Particle Physics Project Prioritization Panel (2008), at http://science. energy.gov/ /media/hep/pdf/files/pdfs/p5 report 06022008.pdf.

[3] 2013 APS-DPF Community Summer Study (Snowmass-2013), University of Minnesota, MN, July 29 - August 6, 2013, at http://www.snowmass2013.org/tiki-index.php.

[4] Proc. "Fundamental Physics at the Intensity Frontier" Workshop, Nov.-Dec., 2011 (Rockville, MD, USA).

[5] “Project X: Accelerator Reference Design, Physics Opportunities, Broader Impacts," Eds. S. Henderson, S. Holmes, A. Kronfeld, R. Tschirhart, FERMILAB-TM-2557 (2013).

[6] S. Henderson, "From Intensity Frontier to Energy Frontier," talk at the AAC-2012 Workshop, University of Texas, June 10-15, 2012 (Austin TX).

[7] V. Shiltsev, "High energy particle colliders: past 20 years, next 20 years and beyond," Phys. Uspekhy 55 (10) 977 (2012), also at http://arxiv.org/abs/1205.3087.

[8] "Accelerators for America's Future," Report of "The Symposium on Accelerators for America's Future," Washington, DC (October 26, 2009).

[9] V. Shiltsev, "Current Landscape of High Energy Particle Colliders," ICFA Beam Dynamics Newsletter \#58, pp.16-22 (August 2012).

[10] Muon Accelerator Program http://map.fnal.gov/.

[11] V. Shiltsev, S. Henderson, P. Hurh, I. Kourbanis, V. Lebedev, "Issues and R\&D Required for the Intensity Frontier Accelerators," report to the 2013 APS-DPF Community Summer Study (Snowmass-2013), arXiv:1305.6917 (2013).

[12] DOE Accelerator R\&D task Force report (2012), http://www.acceleratorsamerica.org/ report/accelerator task force report.pdf. 


\subsection{Comparison with Other AARD Facilities}

Particle physics research is strongly dependent on the use of high-energy beams provided by charged particle accelerators, storage rings and the associated detectors. Operating in the extreme domains that are essential for successful particle physics research demands very specialized technology that takes substantial time and expense to invent, design, build, maintain, and upgrade. It has long been recognized that historically, much of accelerator R\&D took place using operating high-energy physics facilities. However, only in the last decade, a great number of the operating frontier facilities have been turned off in the US and worldwide -SLC, LEP, HERA, PEP-II, CESR-c, Fermilab's Antiproton Accumulator, Debuncher and Tevatron, DAFNE, etc.-and the opportunities for experimental AARD have correspondingly decreased. The critical lack of accelerator R\&D test facilities has been appreciated and several dedicated facilities have been proposed and constructed, e.g., ATF at BNL [1], A0 Photoinjector at FNAL (recently closed and being moved to ASTA) [2], AWA at Argonne [3], BELLA at LBNL [4], FACET at SLAC [5], CESR-TA at Cornell University[6], UMER at the University of Maryland [7], and TTF at DESY (recently converted into FLASH, a high-gain free-electron laser) [8], CTF-3 at CERN [9], and ATF (ATF-II) at KEK [10]. Such facilities are essential for progress in the field, and in the US are supported primarily by the DOE Office of High Energy Physics (CESR-TA is an NSF-supported facility).

Among all the facilities, ASTA [11] has two unique features: (i) it allows broad R\&D in support of the program toward the intensity frontier accelerators and offers both linacs and a ring, and electrons and ions for accelerator research; (ii) due to employment of the advanced SRF technology, record high average electron beam power and beam brightness are available to users. Figure 1 shows the energy and beam brightness available at the various AARD facilities. 

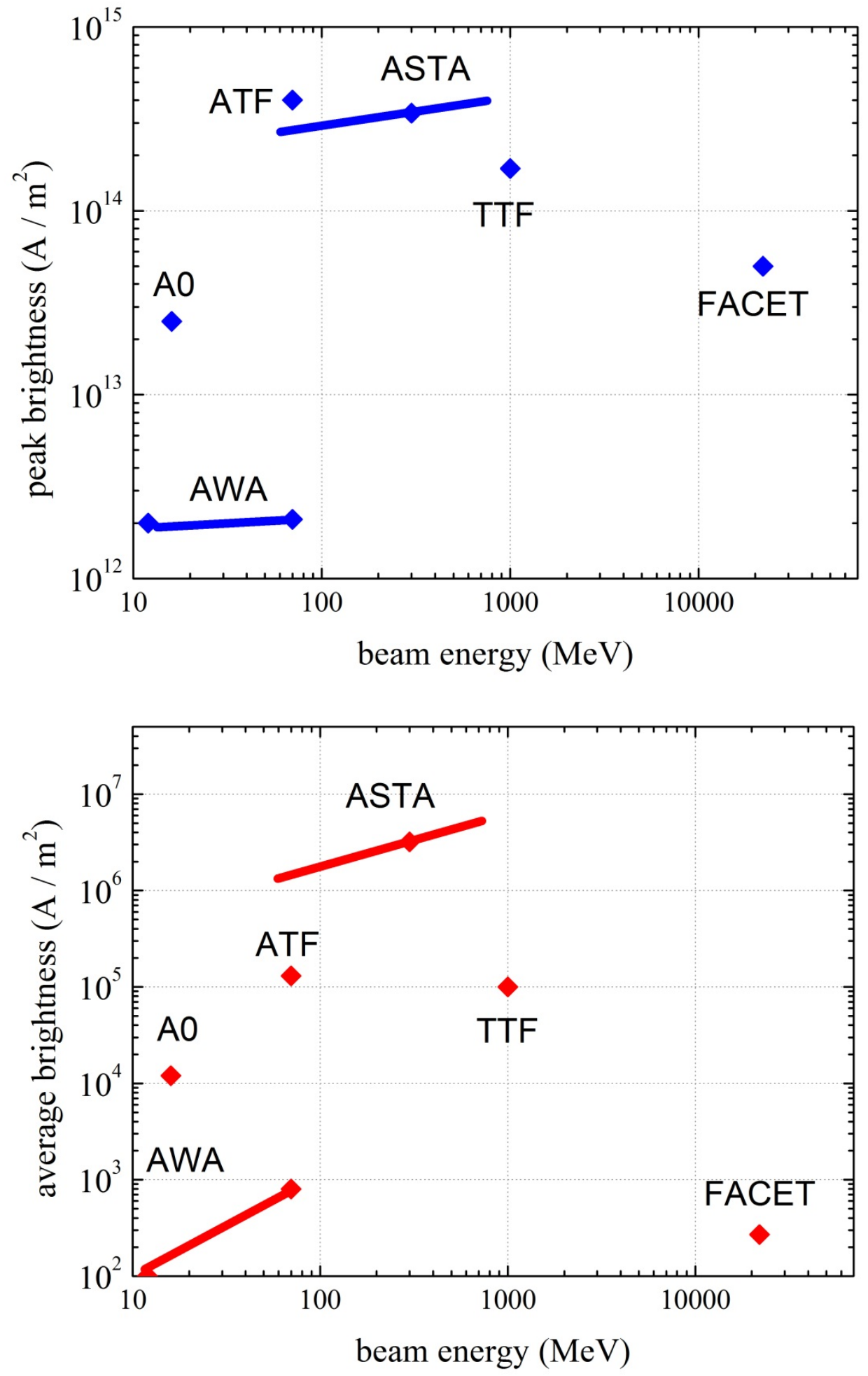

Figure 1: Comparison of beam parameters of AARD facilities. The peak brightness is computed as the ratio of peak current over the four-dimensional normalized transverse emittance, while the average uses the averaged beam current. 
The ASTA facility will offer variable energy electron beams, from ( 50 to $~ 800 \mathrm{MeV}$ ), highrepetition rate (1-ms trains), L-band SRF linac, small emittance photoinjector source, arbitrary emittance partition (with repartition of phase spaces to match final applications), and tailored current profiles. The Integrable Optics Test Accelerator (IOTA) ring will be capable of operating for a wide range of experiments with both 50-150 MeV electrons and 2.5 MeV ions/protons. Parameters of existing AARD facilities are shown in Table 1, together with those of ASTA.

Table 1: Beam parameters available to users at the accelerator R\&D facilities: US facilities (top), non-US facilities (middle), ASTA (bottom).

\begin{tabular}{|c|c|c|c|c|}
\hline Facility & Energy & Particles/sec & $\begin{array}{c}\text { Macropulse } \\
\text { duration }\end{array}$ & $\begin{array}{c}\text { Rep rate/ max \# } \\
\text { of bunches }\end{array}$ \\
\hline ATF & 25-70 MeV e- & $(3-10) \times 10^{11}$ & up to $3 \mu \mathrm{s}$ & $1-3 \mathrm{~Hz} / 200$ \\
\hline HBESL AO (FNAL) & $4.5 \mathrm{MeV}$ e- & $(0.5-2) \times 10^{11}$ & up to $100 \mu \mathrm{s}$ & $\sim 1 \mathrm{~Hz} /$ up to 100 \\
\hline AWA & 70 MeV e- & up to $2 \times 10^{13}$ & $2 \mu \mathrm{s}$ & $\sim 1 \mathrm{~Hz} / 32$ \\
\hline BELLA & 1-5 GeV e- & $\sim 0.1 \times 10^{11}$ & $<0.3 \mathrm{ps}$ & $<1 \mathrm{~Hz} / 1$ \\
\hline FACET & $21 \mathrm{GeV}$ e-, e+ & $0.6 \times 10^{11}$ & $0.1 \mathrm{ps}$ & $30 \mathrm{~Hz} / 1$ \\
\hline CESR-TA & $\begin{array}{c}\text { 1.8-5.3 GeV e- } \\
\mathrm{e}+\end{array}$ & $1.6 \times 10^{11} /$ bunch & ring & Ring / 45 \\
\hline UMER & $10 \mathrm{keV}$ e- & $(0.02-3) \times 10^{10}$ & 100 's turns & Ring/ 1 \\
\hline TTF & $1000 \mathrm{MeV}$, e- & $5 \times 10^{13}$ & $0.8 \mathrm{~ms}$ & $5-10 \mathrm{~Hz} / 800$ \\
\hline CTF-3 & $150 \mathrm{MeV}, \mathrm{e}-$ & $3 \times 10^{13}$ & ring/140 ns & $5 \mathrm{~Hz} / 1700$ \\
\hline ATF(ATF-II) & $1280 \mathrm{MeV}$, e- & $1 \times 10^{10} /$ bunch & ring & Ring / 20 \\
\hline \multirow{4}{*}{$\begin{array}{c}\text { ASTA Phase I.1 } \\
\text { Phase I.2 } \\
\text { Phase II } \\
\text { Phase III }\end{array}$} & \multirow{4}{*}{$\begin{array}{l}50 \mathrm{MeV} \text { e- } \\
300 \mathrm{MeV} \text { e- } \\
800 \mathrm{MeV} \text { e- } \\
2.5 \mathrm{MeV} \text { H- }\end{array}$} & $5 \times 10^{13}$ & $1 \mathrm{~ms}$ & $5 \mathrm{~Hz} / 3000$ \\
\hline & & $3 \times 10^{14}$ & $1 \mathrm{~ms}$ & $5 \mathrm{~Hz} / 3000$ \\
\hline & & $3 \times 10^{14}$ & $1 \mathrm{~ms}$ & $5 \mathrm{~Hz} / 3000$ \\
\hline & & $>10^{15}$ & $1 \mathrm{~ms}$ & $5 \mathrm{~Hz} / 1$ \\
\hline
\end{tabular}

All of the current Intensity Frontier facilities and many of the future ones are based on circular accelerators with high intensity and high brightness beams. Accelerator R\&D opportunities at the operational accelerators are practically non-existent; further, except for the University of Maryland Electron Ring (UMER) there is no other circulating beam (ring) facility in the US dedicated for R\&D. ASTA will address this need and will have a dual species (electrons and ions) IOTA storage ring. The possible experiments and tests cover a broad range of important subject: beam-loss control, beam dynamics, novel optics for space-charge compensation, collimation, and diagnostics. Together with studies enabled by SRF beam acceleration, ASTA promises to explore revolutionary techniques for the intensity frontier facilities, such as Fermilab accelerator complex upgrades, Project X, Neutrino Factory, etc.

The needs of all future Energy Frontier accelerators which are currently being considered in the US can and will be addressed by ASTA: future e+e- collider (SRF beam studies and tests, high brightness electron sources, phase-space manipulations, and positron production); the LHC upgrades (space-charge studies and compensation, optical stochastic cooling, and new beam 
collimation techniques); Muon Collider and ultra-high energy colliders of the far future (high beam loading SRF acceleration, space-charge effects and compensation, optical stochastic cooling, use of crystals for beam shaping/acceleration).

Accelerator Stewardship and Applications research and development will employ the unique features of the ASTA facility: high power SRF, high brightness electron source, and high energy, to test a variety of methods of relevance to future FEL accelerators. This includes production of very high energy gamma-rays via the Compton-scattering mechanism, SRF technology development and demonstration, materials, beam diagnostics and ultrafast choppers tests.

Table 2 summarizes relevance of the research proposed for ASTA and that of other facilities to the main thrusts of the DOE Office of HEP.

Table 2: Accelerator facilities and Accelerator R\&D thrusts: US facilities (top), non-US facilities (middle), ASTA (bottom).

\begin{tabular}{|c|c|c|c|c|}
\hline \multirow{2}{*}{ Facility } & \multirow{2}{*}{$\begin{array}{l}\text { Main } \\
\text { beams }\end{array}$} & \multicolumn{2}{|c|}{ HEP Discovery Science } & \multirow{2}{*}{ Applications } \\
\hline & & Intensity Frontier & Energy Frontier & \\
\hline ATF $\quad$ (BNL) & $\begin{array}{l}\mathrm{e}-, \\
\mathrm{CO}_{2} \text { laser }\end{array}$ & & $\begin{array}{l}\text { PWFA, LPWA } \\
\text { for e+e- LCs }\end{array}$ & $\begin{array}{l}\text { FEL, } \gamma^{\prime} s \text {, } \\
\text { medical laser-gas }\end{array}$ \\
\hline HBESL AO (FNAL) & e- & & e+e- LCs, PWFA & FEL \\
\hline AWA $\quad$ (ANL) & e- & & $\mathrm{e}+\mathrm{e}-\mathrm{LCS}, \mathrm{DWFA}$ & \\
\hline BELLA (LBNL) & laser & & e+e- LCs, LWFA & $\begin{array}{l}\text { FEL, } \gamma^{\prime} s \text {, } \\
\text { medical laser-gas }\end{array}$ \\
\hline FACET (SLAC) & e-, e+ & & $\mathrm{e}+\mathrm{e}-\mathrm{LCS}, \mathrm{PWFA}$ & \\
\hline $\begin{array}{l}\text { CESR-TA } \\
\text { (Cornell) }\end{array}$ & e-, e+ & e-cloud (for $\mathrm{e}+)$ & $\mathrm{e}+\mathrm{e}-\mathrm{LCs}$ & \\
\hline UMER (UMD) & e- & space charge & & \\
\hline TTF $\quad$ (DESY) & e- & & $\begin{array}{l}\text { Initially - e+e- } \\
\text { LC }\end{array}$ & FEL, SRF technology \\
\hline CTF-3 (CERN) & e- & & $\begin{array}{l}\text { Two-beam e+e- } \\
\text { NC LC }\end{array}$ & \\
\hline ATF(ATF-II) (KEK) & e- & & $\begin{array}{l}\text { ILC DR and } \\
\text { other e+e- LCs } \\
\text { R\&D }\end{array}$ & \\
\hline ASTA (Fermilab) & $\begin{array}{l}\text { e-p/ions, } \\
\text { laser }\end{array}$ & $\begin{array}{l}\text { Losses, beam } \\
\text { dynamics, novel } \\
\text { optics, } \\
\text { collimation, } \\
\text { space-charge } \\
\text { compensation, } \\
\text { diagnostics, SRF } \\
\text { control }\end{array}$ & $\begin{array}{l}\text { e+e- LCs, } \\
\text { e+ sources, } \\
\text { LHC upgrades, } \\
\text { Muon Collider } \\
\text { R\&D }\end{array}$ & $\begin{array}{l}\text { FEL, } \gamma^{\prime} s \text {, } \\
\text { SRF technology, } \\
\text { development \& } \\
\text { testing, material test }\end{array}$ \\
\hline
\end{tabular}


On average, approximately 9-12 FTEs are needed to operate each of the US AARD facilities, similar to what is expected for ASTA. The facility will be embedded in, and leverage resources and infrastructure from, the much larger operations and general technology development programs at Fermilab. These provide access to accelerator infrastructure (cryogenics, electrical, LCW, etc.), support functions (procurement, ESH, QA, accounting, etc.), skilled accelerator personnel (scientists, engineers, technicians), education programs closely connected with leading Universities and laboratories (IIT, NIU, UIUC, U of C, ANL, ICL, etc.), accelerator theory, computation and simulations tools (SciDAC, CHEF, etc.), and will be synergistic with IARC (Illinois Accelerator Research Center). Table 3 summarizes the construction costs and operations costs of the various AARD facilities.

Table 3: Cost of AARD Facilities: US facilities (top), non-US facilities (middle), ASTA (bottom).

\begin{tabular}{|c|c|c|c|c|}
\hline \multirow[b]{2}{*}{ Facility } & \multirow[b]{2}{*}{$\begin{array}{c}\text { Supporting } \\
\text { Agencies }\end{array}$} & \multicolumn{2}{|c|}{ Cost } & \multirow[b]{2}{*}{ Comments } \\
\hline & & $\begin{array}{c}\text { Total } \\
\text { Construction } \\
\text { Cost }\end{array}$ & $\begin{array}{l}\text { Operating } \\
\text { Cost }\end{array}$ & \\
\hline ATF $\quad(B N L)$ & HEP, BES & $\mathrm{n} / \mathrm{a}$ & $2.7 \mathrm{M} \$ / \mathrm{yr}$ & $\begin{array}{l}2.2 \mathrm{M} \$ / \mathrm{yr} \mathrm{HEP}+ \\
0.5 \mathrm{M} \$ / \mathrm{yr} \mathrm{BES} ; \\
120 \text { days/yr }\end{array}$ \\
\hline HBESL AO (FNAL) & HEP & $\mathrm{n} / \mathrm{a}$ & $1.7 \mathrm{M} \$ / \mathrm{yr}$ & $\begin{array}{l}\text { Year-round, } \\
\text { ca 2007-2008 }\end{array}$ \\
\hline AWA $\quad(A N L)$ & HEP, other & $\mathrm{n} / \mathrm{a}$ & $\sim 2 \mathrm{M} \$ / \mathrm{yr}$ & Year-round \\
\hline BELLA $\quad$ (LBNL) & HEP, other & $33.5 \mathrm{M} \$$ & $2 \mathrm{M} \$ / \mathrm{yr}$ & $\begin{array}{l}\text { Not incl. support } \\
\text { from DARPA, LDRD, } \\
\text { SBIR; year-round }\end{array}$ \\
\hline FACET $\quad$ (SLAC) & HEP, BES & $14.5 \mathrm{M} \$$ & $14.2 \mathrm{M} \$ / \mathrm{yr}$ & $\begin{array}{l}\text { Incl.6.2M\$/yr } \\
\text { from BES, } \\
\text { few months/yr }\end{array}$ \\
\hline CESR-TA (Cornell) & NSF & $9 \mathrm{M \$}$ & $4.25 \mathrm{M} \$ / \mathrm{yr}$ & Beam $\sim 80$ days/yr \\
\hline UMER (UMD) & $\begin{array}{l}\text { HEP, ONR, } \\
\text { DOD, JTO }\end{array}$ & $\mathrm{n} / \mathrm{a}$ & $\mathrm{n} / \mathrm{a}$ & Year-round \\
\hline TTF $\quad$ (DESY) & Germany & $>100$ MEuro & $\mathrm{n} / \mathrm{a}$ & Several months/yr \\
\hline CTF-3 (CERN) & CERN & $\mathrm{n} / \mathrm{a}$ & $\mathrm{n} / \mathrm{a}$ & Year-round \\
\hline ATF(ATF-II) (KEK) & Japan & $\mathrm{n} / \mathrm{a}$ & $\mathrm{n} / \mathrm{a}$ & Year-round \\
\hline $\begin{array}{l}\text { ASTA Stage I } \\
\text { (FNAL) Stages II-IV }\end{array}$ & HEP & $\begin{array}{l}\text { 9.7 M\$ + } \\
\text { 14.5 M\$ } \\
\text { including } \\
\text { contingency }\end{array}$ & $3.9 \mathrm{M} \$ / \mathrm{yr}$ & $>9$ months/yr \\
\hline
\end{tabular}




\section{References}

[1] BNL Accelerator Test Facility, http://www.bnl.gov/atf/.

[2] FNAL A0/NICADD Photoinjector Laboratory, http://nicadd.niu.edu/fnpl/.

[3] ANL Argonne Wakefield Accelerator Facility, http://www.hep.anl.gov/awa/.

[4] LBNL BELLA (Berkeley Laboratory Laser Accelerator), http://www.lbl.gov/Community/ BELLA/index.html.

[5] SLAC FACET (Facility for Advanced Accelerator Experimental Tests), http://facet.slac. stanford.edu/.

[6] CESR Damping Ring Test Accelerator, https://wiki.lepp.cornell.edu/ilc/bin/view/ Public/CesrTA/.

[7] University of Maryland Electron Ring/UMER, http://www.umer.umd.edu/.

[8] DESY Tesla Test Facility, http://tesla.desy.de/TTF intro.html.

[9] CTF-3 at CERN, https://espace.cern.ch/be-dep/OP/CTF3/default.aspx.

[10] ATF (ATF-II) at KEK, http://www-atf.kek.jp/atf/.

[11] ASTA at Fermilab (Proc. Int'I PAC'2012, New Orleans), http://accelconf.web.cern.ch/ AccelConf/IPAC2012/papers/mooac02.pdf. 


\subsection{Management of the ASTA Scientific User Program}

ASTA is intended to be operated as a scientific user facility for advanced accelerator research and development. We propose ASTA as an Office of Science (SC) user facility as all the characteristics of a national user facility (http://science.energy.gov/user-facilities/) will be in evidence in the operation of ASTA and its user program. Namely,

- The facility is open to all interested potential users without regard to nationality or institutional affiliation.

- Allocation of facility resources is determined by merit review of the proposed work.

- User fees are not charged for non-proprietary work if the user intends to publish the research results in the open literature. Full cost recovery is required for proprietary work.

- The facility provides resources sufficient for users to conduct work safely and efficiently.

- The facility supports a formal user organization to represent the users and facilitate sharing of information, forming collaborations, and organizing research efforts among users.

- The facility capability does not compete with an available private sector capability.

The user program will be proposal-driven and peer-reviewed in order to ensure that the facility focuses on the highest quality research. ASTA is open to all interested users without regard to national or institutional affiliation and researchers from national- and international universities and groups, the Department of Energy's National Laboratories, small business and industrial companies will carry out large and diverse research programs at ASTA. Proposals will be evaluated by an external Program Committee (the ASTA Program Advisory Committee), consisting of internationally recognized scientists. Proposals are solicited by a call for proposals that is communicated by emails, the internet, and personal communication ahead of the annual Users Meetings. The allocation of ASTA resources will be determined by merit review of the proposed work. Proposal evaluation will be carried out according to established merit review guidelines. The ASTA PAC will advise the ASTA Director on user proposal ranking. The ASTA Director approves the proposed experiments and is responsible for the management of the user program. In addition, ASTA will support a user's organization that will advise the facility management and be part of the larger Fermilab's Users Organization (http://www.fnal.gov/ orgs/fermilab users org/). ASTA users' meetings are held every year, in conjunction with the meetings of the ASTA PAC.

ASTA does not plan to charge fees for nonproprietary work, provided that the user intends to publish the research findings in the open literature. ASTA staff will provide expert support to user experiments by operating the accelerators and delivering beams to all three experimental areas, assisting with design and installation of the experiments, maintaining computer control system with flexible DAQ and user interface, and providing safety training and review of experiments.

Proposal for an Accelerator R\&D User Facility at Fermilab's Advanced Superconducting Test Accelerator 


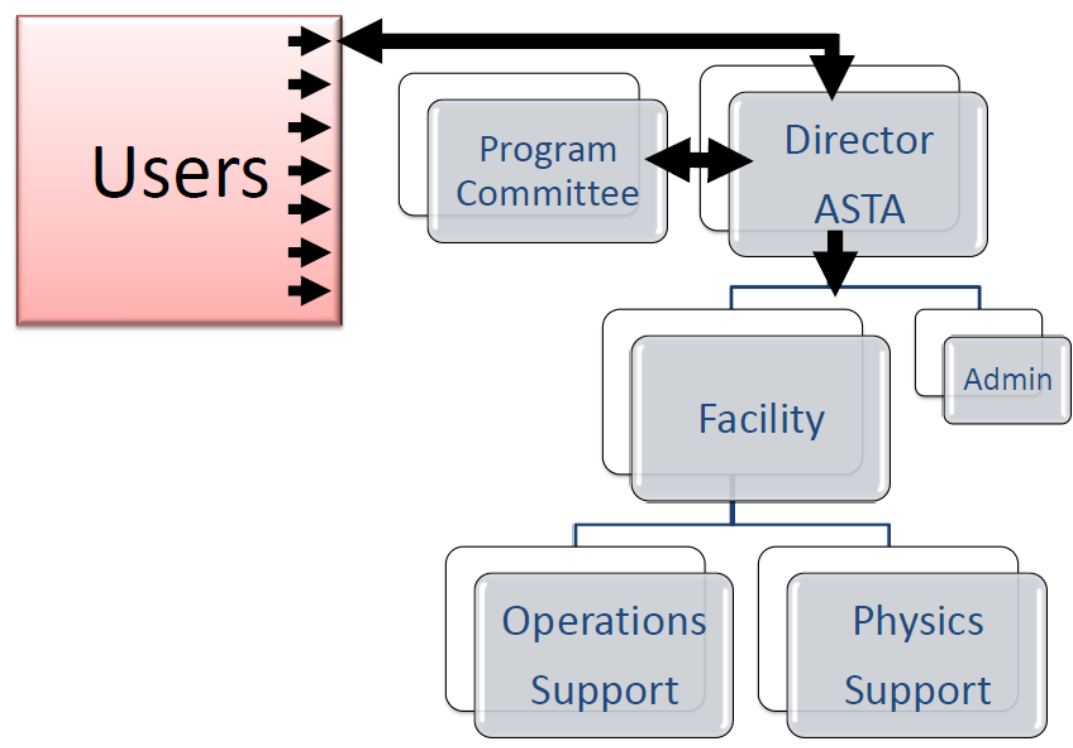

Figure 1: Proposed organizational structure of the ASTA as an Office of Science User Facility.

Organizationally, the ASTA Director will report to the Accelerator Physics Center Head, who has oversight responsibility for the ASTA Program. The ASTA Director regularly reports to the Fermilab Associate Lab Director for Accelerators. The beam operations and facility maintenance will be the responsibility of the Fermilab's Accelerator Division. The Program Advisory Committee is appointed by the ASTA Director. The meetings of the Program Advisory Committee are held every 12 months.

The current membership of the ASTA Program Advisory Committee is listed below.

\section{ASTA Director (Interim)}

Vladimir Shiltsev, FNAL

\section{Program Advisory Committee}

Gerry Dugan (chair), ILC GDE/Cornell University Michael Blaskiewicz, Brookhaven National Laboratory John Byrd, Lawrence Berkeley National Laboratory Georg Hoffstaetter, Cornell University Alexander Zholents, Argonne National Laboratory

The first ASTA Users meeting and ASTA Program Advisory Committee meeting have taken place July 23-24, 2013 at Fermilab; see https://indico.fnal.gov/conferenceDisplay.py?ovw= True\&confld=6822. 84 scientists were in attendance, two-thirds of them not from Fermilab. The facility status and 28 experimental proposals, including 4 new ones, were presented and discussed. 


\subsection{ASTA Facility Overview and Capabilities}

The ASTA User Facility will evolve over time to address the demands of the accelerator R\&D program. The main subsystems of ASTA include:

- Photoinjector source

- Linear accelerator based on superconducting RF cryomodules

- Electron storage ring (IOTA)

- Low- and high-power lasers

- Experimental areas for research with low-energy and high-energy beams.

Possibilities exist to further expand the capabilities of the R\&D program, including

- An H-source and RFQ accelerator to expand the IOTA program to protons

- 3.9-GHz SRF accelerating cavity to enhance the peak current of compressed electron bunches and provide opportunities for bunch-current shaping

- Additional SRF cryomodules to increase the beam energy.

We envision a multi-staged approach to ASTA completion as shown in Figures 1 and 2. The first stage enables a low-energy AARD program based on the photoinjector and a $300 \mathrm{MeV}$ program based on a single superconducting cryomodule, with associated beam transport lines and beam dumps. The first stage also enables one of the transformative beam dynamics experiments: exploration of novel, non-linear accelerator lattices in the Integrable Optics Test Accelerator (IOTA).

\section{Stage I}

I.1
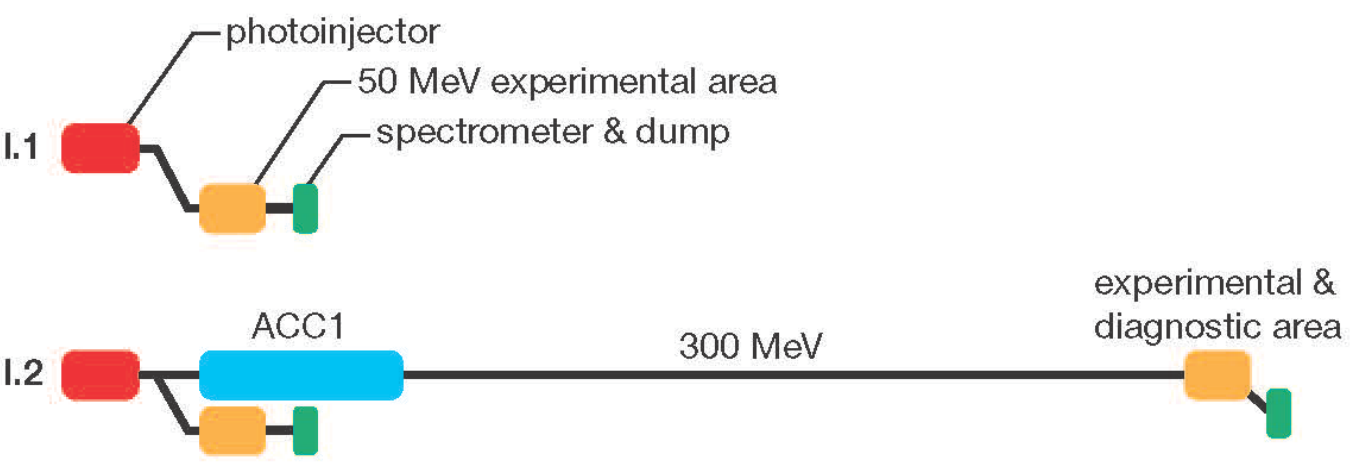

1.3

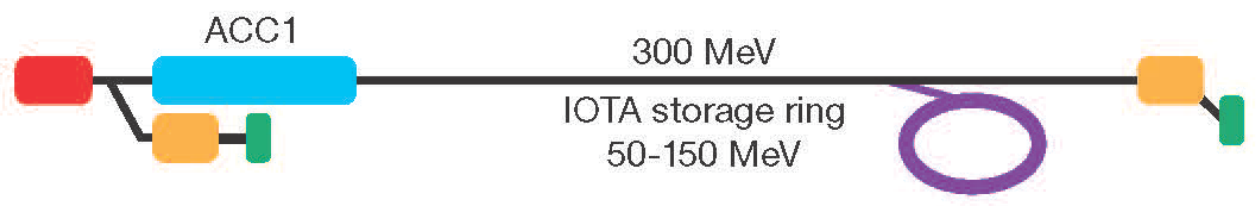

Figure 1: Stage I of the ASTA AARD User Facility construction and operation phases. 
Stages II, III and IV allow a vast expansion of the ASTA capabilities for advanced accelerator research, as shown in Figure 2 . In the second stage two additional cryomodules and associated subsystems are added to provide high-energy beam up to $800 \mathrm{MeV}$. The AARD program will be expanded significantly with the higher beam energy capability, as outlined below. The IOTA program will also be expanded in Stage III to support beam-dynamics experiments on lowenergy protons through the addition of proton beam injection capability for IOTA. Finally, Stage IV will allow a 3-fold increase of the electron peak current after installation of a linearizing 3.9$\mathrm{GHz}$ cavity in the photoinjector area.

The beam energy can be further increased through the installation of up to 3 additional cryomodules.
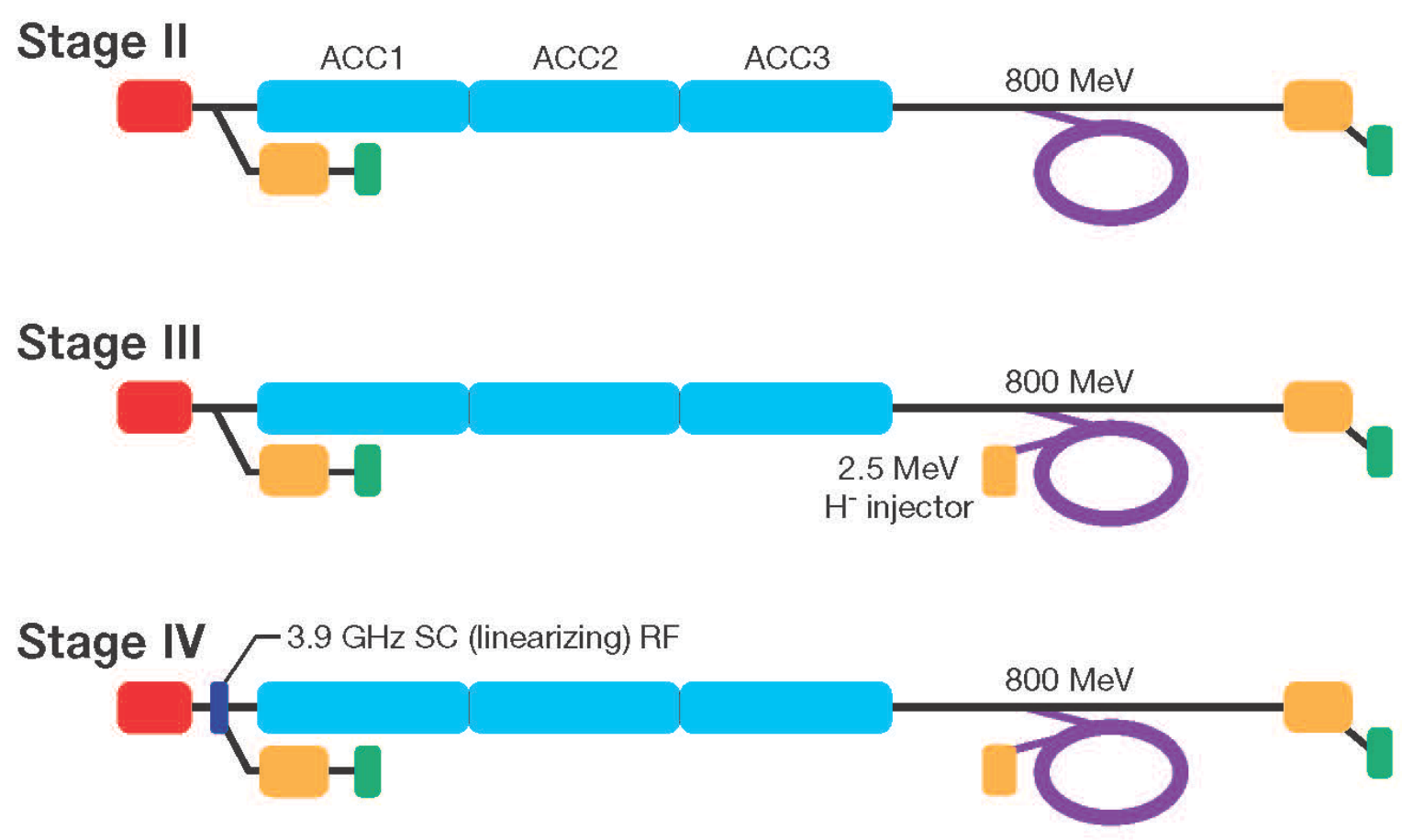

Figure 2: Stages II, III and IV of the ASTA AARD User Facility construction and operation.

The ASTA facility is located within NML and is adjacent to the newly constructed Cryomodule Test Facility (CMTF), as shown in Figure. 3. 


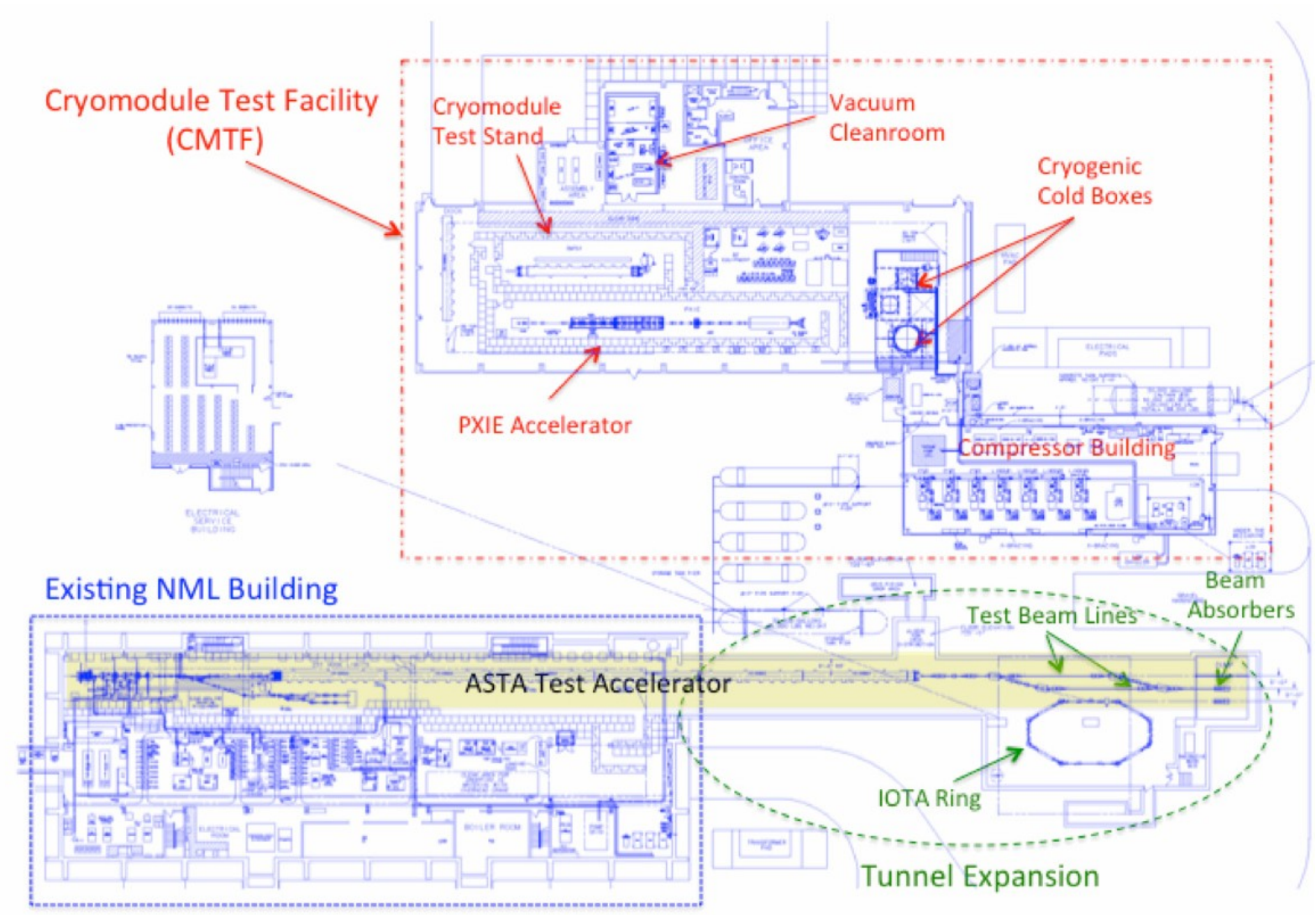

Figure 3: Layout of the ASTA Facility.

\subsection{RF Photoinjector}

The backbone of the ASTA facility is a radio-frequency (RF) photoinjector, shown in Figure 4. The electron source is a 1-1/2 cell cylindrical-symmetric RF gun with a $\mathrm{Cs}_{2} \mathrm{Te}$ photocathode. The cathode is illuminated by an ultraviolet (UV, $\lambda=263 \mathrm{~nm}$ ) laser pulse produced via frequency quadrupling of an amplified infrared (IR, $\lambda=1054 \mathrm{~nm}$ ) pulse [1]. The photocathode drive laser enables the generation of a train of bunches repeated at $3 \mathrm{MHz}$ within a 1-ms-duration macropulse-see Figure 5. The parameters associated with the pulse format are summarized in Table 1. The 5-MeV electron bunches exiting the RF gun are then accelerated with two superconducting radio-frequency (SRF) TESLA-type cavities (CAV1 and CAV2) to approximately $50 \mathrm{MeV}$. Downstream of this accelerating section the beamline includes quadrupole and steering dipole magnets, along with a four-bend magnetic compression chicane (BC1). The beamline also incorporates a round-to-flat-beam transformer (RTFB) capable of manipulating the beam to generate a high transverse-emittance ratio. In the early stages of operation, the bunches will be compressed in BC1 by operating CAV2 off crest. In this scenario the longitudinal phase space is strongly distorted and the achievable peak current limited to less than $3 \mathrm{kA}$. Eventually, a third-harmonic cavity (CAV39) operating at $3.9 \mathrm{GHz}$ will be added. The prime purpose of this cavity is to linearize the longitudinal phase space and thereby enhance the 
compression scheme. In addition such a cavity could also be used to shape the current profile of the electron bunch [2]. The bunch generation and acceleration was extensively simulated and optimized using a multi-objective genetic optimizer [3].

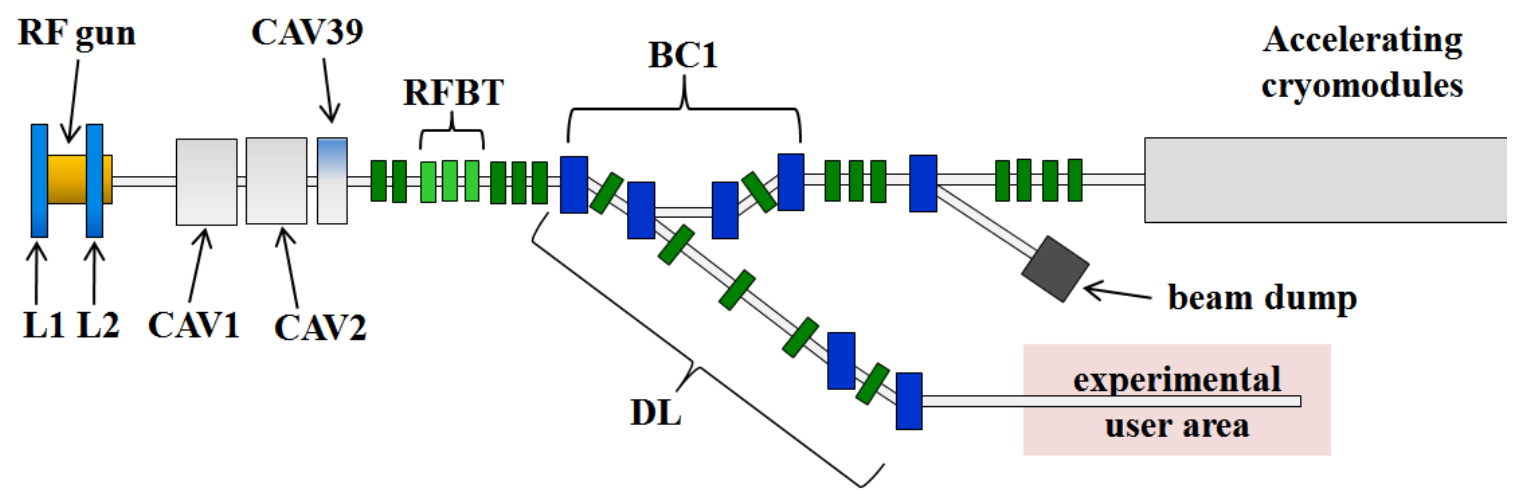

Figure 4: Overview of the ASTA photoinjector. The legend is L1, L2: solenoidal lenses, CAV1, CAV2 superconducting TESLA cavities, BC1: magnetic bunch compressor, DL: dogleg, green rectangles: quadrupole magnets, RFBT: round-to-flat-beam transformer.

Downstream of the accelerating section, the photoinjector includes an extensive diagnostics suite. In addition to conventional diagnostics (optical transition radiation screens, frequencydomain bunch-length measurement, etc.), a deflecting cavity with a spectrometer will provide single-shot measurement of the longitudinal phase space (LPS diagnostics). Further developments of single-shot electro-optical imaging methods initiated at the A0 photoinjector are also planned downstream of BC1 [4].

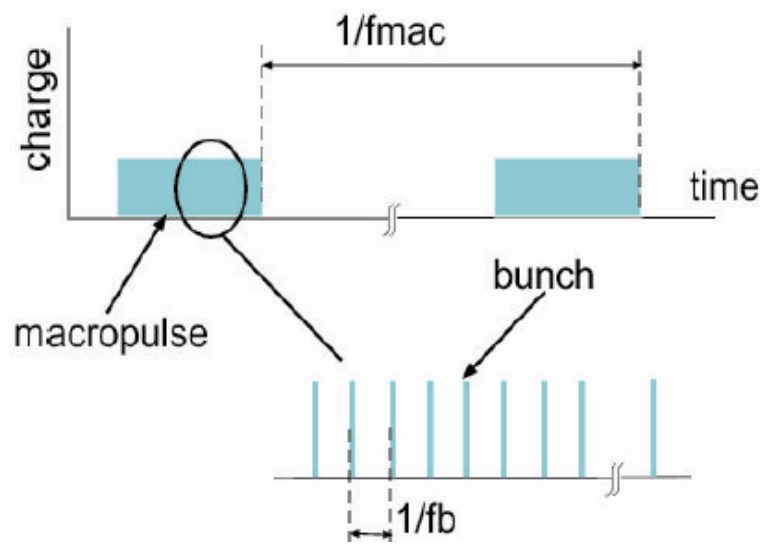

Figure 5: Time structure of the ASTA electron beam: macropulse rate $f_{\text {mac }}=5 \mathrm{~Hz}$, macropulse length $1 \mathrm{~ms}$, bunch frequency $\mathrm{f}_{\mathrm{b}} \sim 3 \mathrm{MHz}$ (369 ns bunch spacing). 
The photoinjector also includes an off-axis experimental beamline branching off at the second dipole of BC1 that will support beam physics experiments and diagnostics R\&D.

Table 1: ASTA electron beam pulse format.

\begin{tabular}{|l|c|c|}
\hline \multicolumn{1}{|c|}{ Parameter } & Value & Unit \\
\hline Bunch charge & $0.02-3.2$ & $\mathrm{nC}$ \\
\hline Bunch train duration & 1 & $\mathrm{~ms}$ \\
\hline Bunch frequency within train & 3 & $\mathrm{MHz}$ \\
\hline Number of bunches/train & $1-3000$ & \\
\hline Bunch train frequency & $1-5$ & $\mathrm{~Hz}$ \\
\hline
\end{tabular}

\section{2 $1.3 \mathrm{GHz}$ SRF Cryomodules}

The 50-MeV beam is injected into the superconducting linear accelerator, which will eventually consist of three, $12-\mathrm{m}$ long, $1.3 \mathrm{GHz}$ superconducting accelerating modules. The linac consists of TESLA/ILC-type cryomodules, each of which includes eight $1.3 \mathrm{GHz}$ nine-cell cavities. The first two cryomodules (CM1 and CM2) are a TESLA Type-III+ design, whereas the third (CM3), will be an ILC-Type IV design [5]. Together, these three cryomodules constitute a complete ILC RF Unit. It is expected that these three cryomodules will be capable of generating a beam energy gain of approximately $750 \mathrm{MeV}$. The installation of the cryomodules will be staged pending the completion of their construction as depicted in Figures 1 and 2. The first CM has already been tested in the ASTA Facility, as shown in Figure 6.

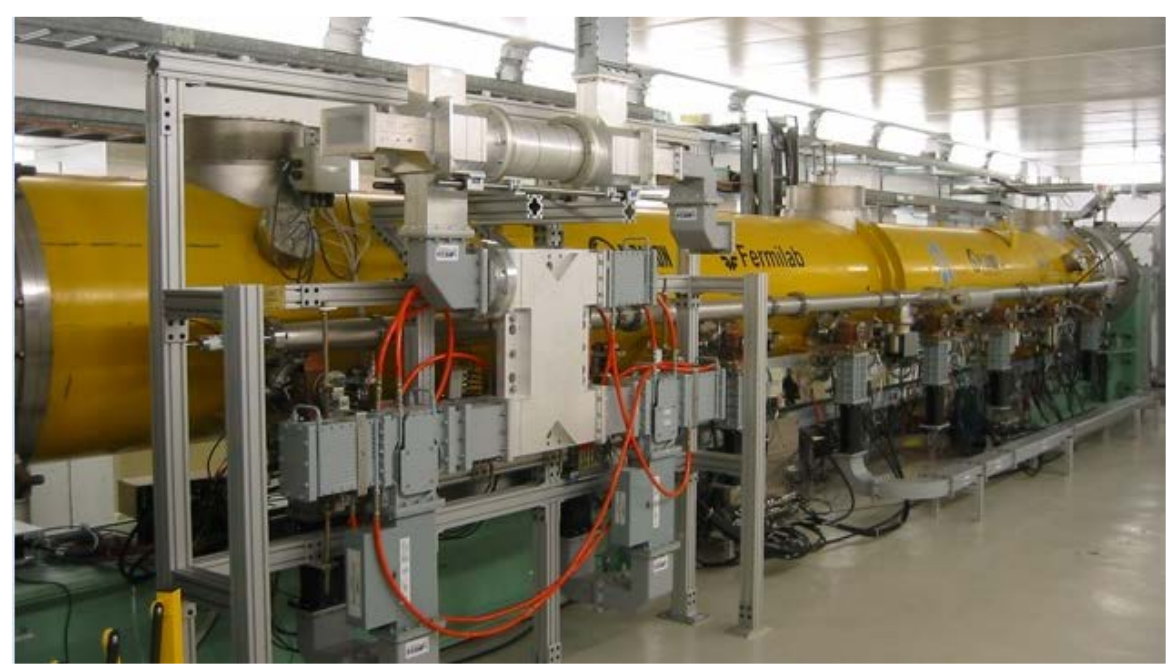

Figure 6: The first 1.3 GHz SRF cryomodule installed in the ASTA facility. 
Downstream of the linac is the test beam line section, which consists of an array of multiple high-energy beam lines that transport the electron beam from the accelerating cryomodules to one of two beam absorbers. Each absorber is capable of dissipating up to $75 \mathrm{~kW}$ of power and is surrounded by a large steel and concrete shielding dump [6]. In addition to testing the accelerator components, the intent of this facility is to also test the support systems required for a future SRF linac.

During the first high energy beam operation and commissioning (referred to as Phase 1.2 in Figure 1), only one cryomodule will be installed allowing for the production of bunches with energies up to $300 \mathrm{MeV}$. Eventually, the second and third cryomodules will be installed in Phase II. Together, the three cryomodules plus the RF power systems will make up one complete ILC RF unit. During Phase II operation the beam energy will reach approximately 800 $\mathrm{MeV}$. Beyond that stage several options are under consideration, including the installation of a $4^{\text {th }}$ cryomodule downstream of a phase-space-manipulation beamline (either a simple magnetic bunch compressor or a phase space exchanger similar to the ones discussed below).

\subsection{IOTA Ring}

The ASTA Facility was designed with the provision for incorporating a small storage ring to enable a ring-based AARD program in advanced beam dynamics of relevance to both Intensity and Energy Frontier accelerators. The Integrable Optics Test Accelerator (IOTA) ring is 39 meters in circumference and will be capable of storing electrons from 50 to $150 \mathrm{MeV}$ in energy. Figure 3 shows the placement of the ring in the ASTA facility layout. IOTA will be described in detail in Section 8.2.1.

\subsubsection{Addition of Protons in IOTA Ring}

It is planned to expand capabilities for AARD in ASTA by the installation of a $2.5 \mathrm{MeV}$ proton/HRFQ accelerator that was previously used for High Intensity Neutrino Source (HINS) research at Fermilab's Meson Detector Building (MDB) facility [7]. The HINS accelerator starts with a $50 \mathrm{kV}$, $40 \mathrm{~mA}$ proton (or $\mathrm{H}$ - ion) source followed by a 2-solenoid Low Energy Beam Transport (LEBT) line. The protons/ions are then accelerated by the pulsed $325 \mathrm{MHz}$ RFQ to $2.5 \mathrm{MeV}$ (with $1 \mathrm{~ms}$ pulse duration) before injection into IOTA.

Almost all of the required hardware exists at the HINS Facility, including:

- $8 \mathrm{~mA}$ proton source and LEBT

- $2.5 \mathrm{MeV} 325 \mathrm{MHz}$ RFQ

- Various beam transport elements

- $325 \mathrm{MHz}$ RF system consisting of

o Charging supply

o Modulator

o Pulse transformer

o $325 \mathrm{MHz}$ klystron

o Circulator and load

Proposal for an Accelerator R\&D User Facility at Fermilab’s Advanced Superconducting Test Accelerator 
Figure 7 shows the present HINS configuration, indicating which items will be relocated to ASTA. The details of the HINS beam line are shown in Figure 8. The spoke resonators will not be required for this project, so the plan is to condense the required beam line elements onto the first girder for the new location.

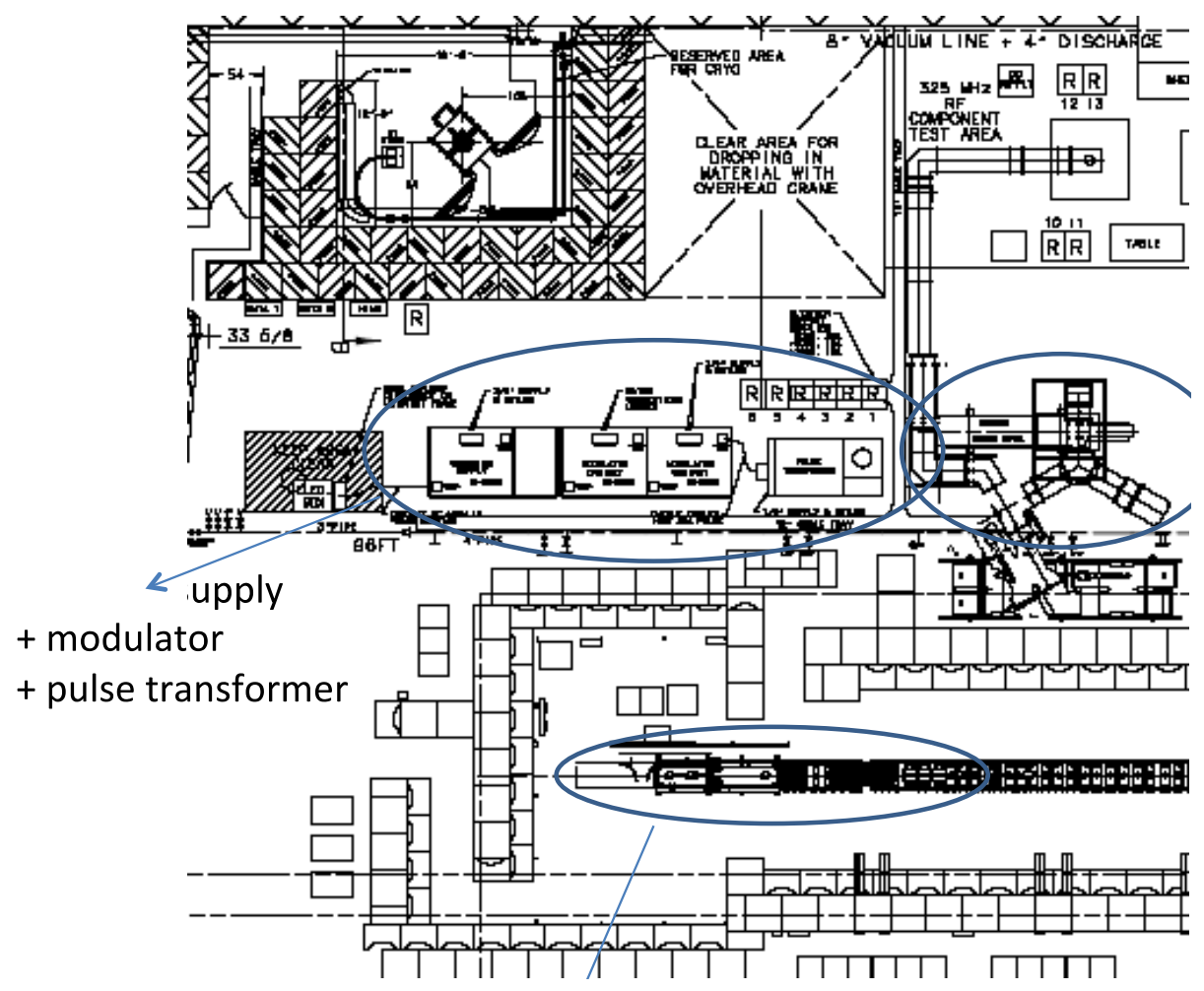

Figure 7: Existing layout of HINS facility, indicating equipment that will be moved to ASTA.

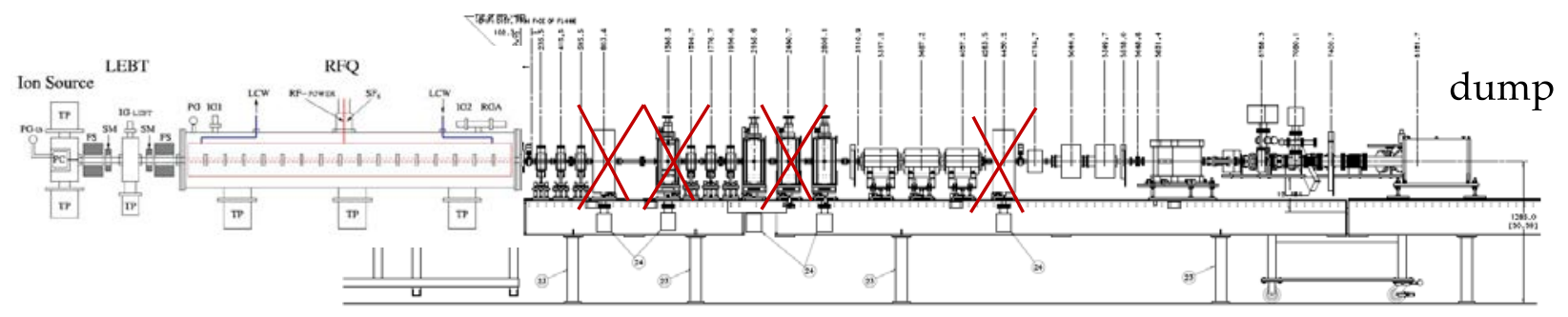

Figure 8: Detail of HINS beam line. Spoke resonator cavities will not be required for ASTA (indicated by red X's). 
Figure 9 shows the proposed layout of HINS in the ASTA facility. The RFQ and optics girder will be placed near the end of the electron beam line. A bending magnet will steer the proton beam into the injection insertion parallel to the electron beam transport line. The charging supply, modulator, pulse transformer and klystron are planned to be located inside the IOTA ring. It should be noted that because the magnet power supplies are unipolar, the polarity of the connections will have to be reversed during proton operation.

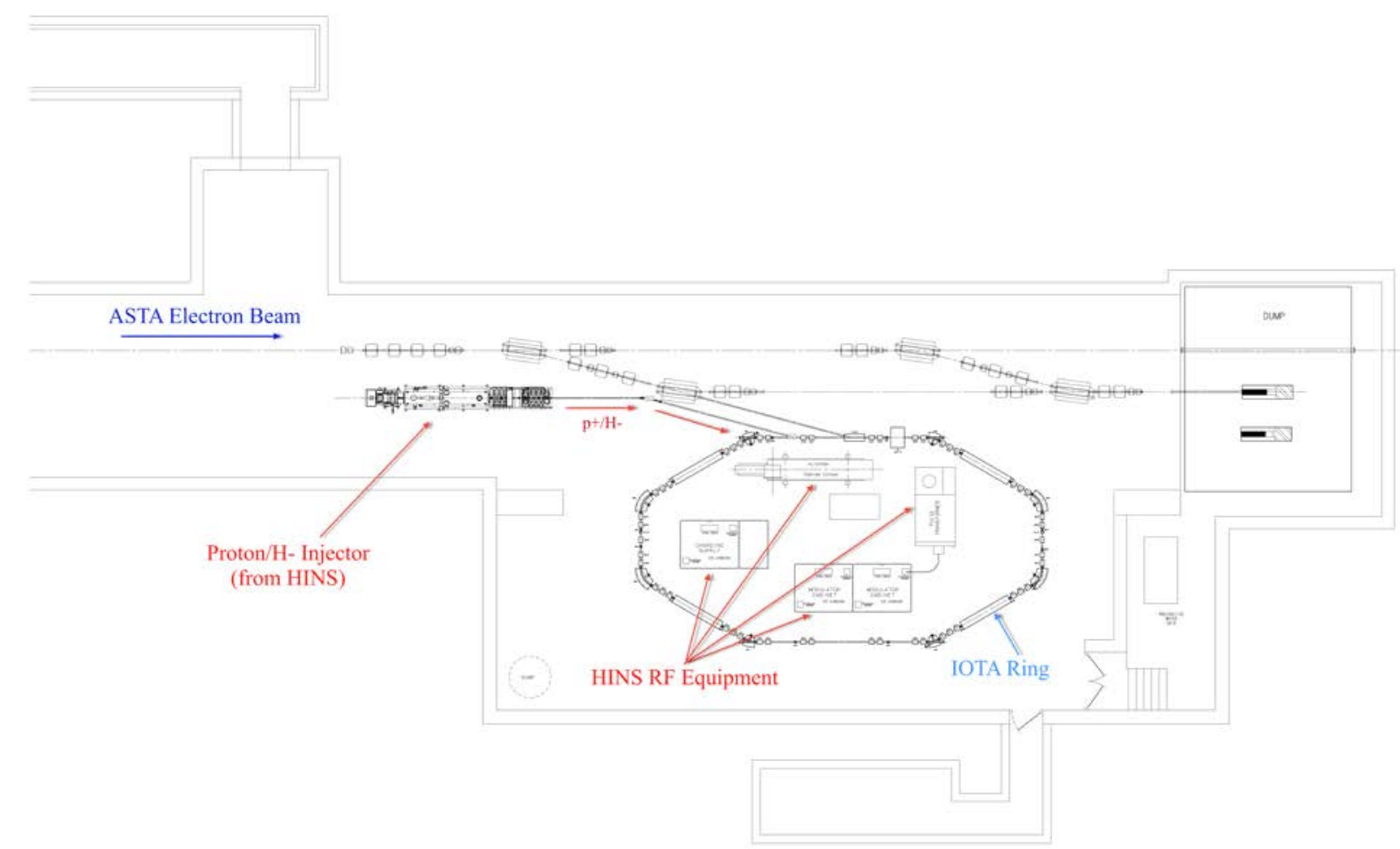

Figure 9: Proposed layout of HINS equipment at ASTA.

\section{4 $\quad$ 3.9 GHz SRF Cavity}

In the early phases of ASTA, the electron bunch will be compressed using one magnetic bunch compressor located in the photoinjector. Because of the relatively long electron bunch (several $\mathrm{mm}$ depending on the charge) formed before acceleration in CAV1 and CAV2, significant longitudinal phase space distortion occurs during acceleration in these two cavities; see illustration in Figure 10. The resulting compressed bunch consists of a peaked distribution with a long trailing electron population. The addition of a $3.9-\mathrm{GHz}$ harmonic cavity similar to the ones already developed at Fermilab and installed and operated at FLASH [8] would allow correcting for the longitudinal phase space nonlinearities and significantly enhancing the final peak current, as shown in Figure 10. 


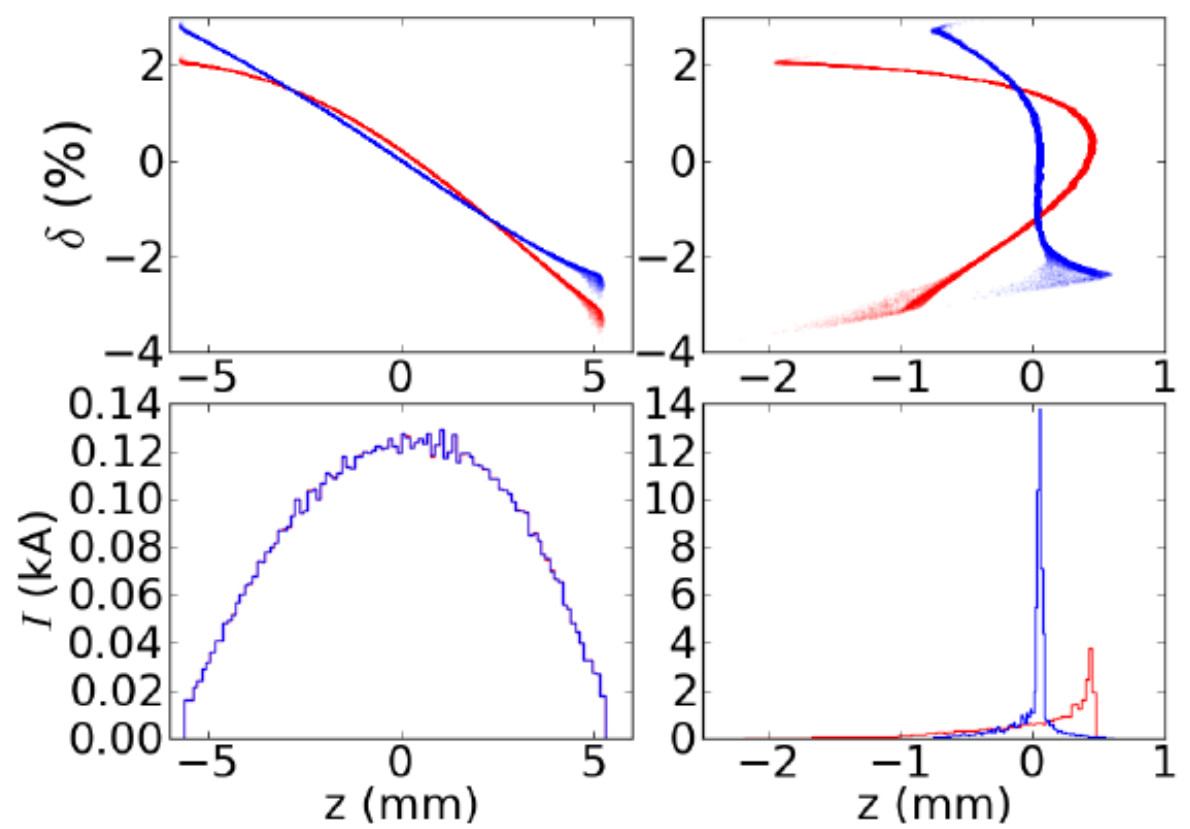

Figure 10: Longitudinal phase space distribution (top row) and associated current profiles (bottom row) before (left column) and after (right column) the BC1 bunch compressor. The red and blue traces correspond respectively to the cases of a nonlinearized and linearized longitudinal phase space upstream of BC1. The linearization is accomplished with a 3.9-GHz accelerating mode cavity and leads to a three-fold peak current increase [9]. Collective effects are not included in this plot.

\subsection{Other Equipment and Experimental Areas}

Operation and research at ASTA will require other equipment which will be provided by users (e.g., devices under test, specific beamline components, and high power lasers). There is significant space at ASTA reserved for as many as 6 experimental areas. Compared to the original NML building, there is $70 \mathrm{~m}$-long tunnel expansion to the North that essentially doubled the length of the test facility from $75 \mathrm{~m}$ to $140 \mathrm{~m}$. The expansion also includes a large $15 \mathrm{~m}$ wide area for the high-energy test beam lines at the downstream end of the accelerator, as shown in Figure 3. This provides ample space for the experimental areas, which is a critical requirement for an AARD User Facility. An enclosure to house the high-energy beam absorbers and dump is situated at the end of the test beam lines and contains a pass-thru beam line that preserves the capability for further expansion of the accelerator in the future. 


\subsection{ASTA Timeline}

The main purpose of ASTA is to serve a broader accelerator community and provide infrastructure for advanced research in accelerator science and technology. ASTA will offer a variety of beams and capabilities according to the timeline shown in Table 2 below.

Table 2: Timeline for ASTA.

Timeline for ASTA at Fermilab

ASTA

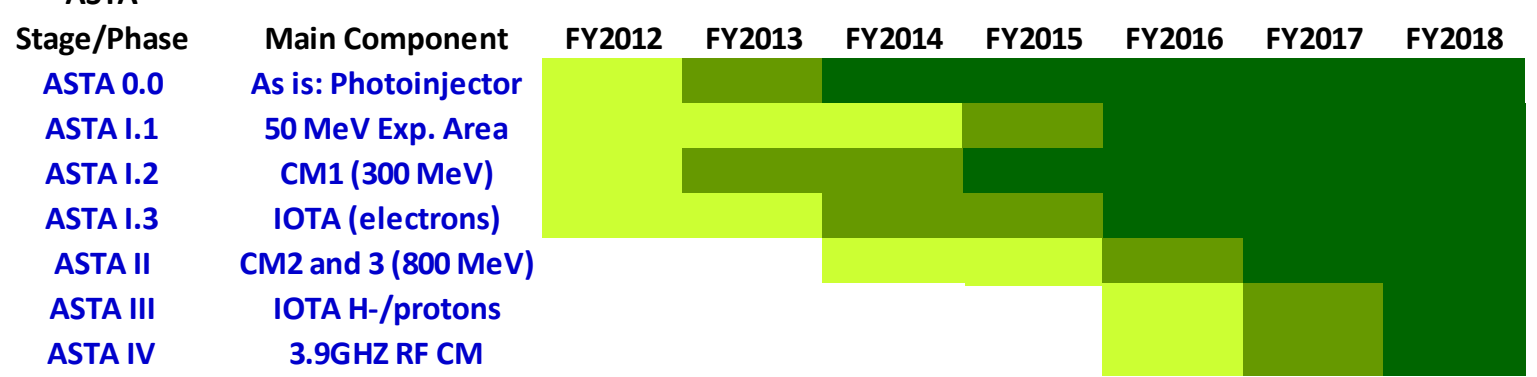

Building components

Installation and Commissioning

Operation, experiments

The program of accelerator beam experiments will be determined by the ASTA Program Advisory Committee and its timeline will depend on the requirements and support of the studies. The ASTA beams required for the experiments and research programs presented in Section 8 are summarized in Table 1 of Section 2.3.

\subsection{References}

[1] J. Ruan, H. Edwards, R. P. Fliller III, J. K. Santucci, Proceedings of PAC07, Albuquerque, New Mexico, USA, 470 (2007).

[2] P. Piot, Phys. Rev. Lett. 108, 034801 (2012).

[3] P. Piot, Proc. IPAC'10, Kyoto, Japan, (23-28 May 2010), p. 4316.

[4] T. Maxwell, PhD Thesis (NIU, 2012).

[5] T. Arkan et al., Proc. LINAC'10, Tsukuba, Japan, September 2010.

[6] C. Baffes et al., Proc. IPAC'12 (New Orleans, USA).

[7] J. Steimel, et al., Proc. IPAC'12 (New Orleans, USA), p. 3877.

[8] E. Vogel, et al., "Test and Commissioning of the Third Harmonic RF System for FLASH," IPAC10, Kyoto, Japan, THPD003.

[9] C. R. Prokop, P. Piot, and M. Church, "Beam dynamics simulation for a low-energy bunch compressor at ASTA," Fermilab technical memo FNAL-TM-2533-APC (2012). 


\subsection{Scientific Opportunities at ASTA}

\subsection{Overview of Research Thrusts}

As described above, the unique features and capabilities of ASTA will allow the exploration of a wide range of advanced accelerator R\&D topics. In this section we provide an overview of the major research thrusts.

\subsubsection{Accelerator R\&D for Particle Physics at the Intensity and Energy Frontiers}

The combination of a state-of-the-art superconducting linear accelerator and a flexible storage ring enables a broad research program directed at the particle physics accelerators of the future. The corresponding research program includes a number of proposals:

- Test of non-linear, integrable, accelerator lattices (the Integrable Optics Test Accelerator), which have the potential to shift the paradigm of future circular accelerator design

- Test of space charge compensation in high intensity circular accelerators

- Advanced phase space manipulations, including flat beam generation, transverse-tolongitudinal phase space exchange, and bunch shaping

- Demonstration of optical stochastic cooling

- Flat-beam-driven dielectric-wakefield acceleration in slab structures

- Investigation of acceleration and cooling of carbon-based crystal structures for muon accelerators

- Measurement of the electron wave function size in a storage ring

- Studies of beam relaxation and echo phenomena in a storage ring

- Development of a quadrupole pickup and study of the quadrupole mode dynamics in space-charge dominated beams

- High power targetry studies for LBNE

- A tagged photon beam at ASTA for Detector R\&D

- HEP applications of inverse Compton scattered photons.

\subsubsection{Accelerator R\&D for Future SC RF Accelerators}

High gradient, high power SC RF systems are critical for many accelerator facilities under planning for the needs of high-energy physics, basic energy sciences and other applications. ASTA offers a unique opportunity to explore the most critical issues related to SC RF technology and beam dynamics in SC RF cryomodules, such as:

- Demonstration of high power high gradient SRF Cryomodules with intense beams

- Demonstration of technology and beam parameters for the Project X pulsed linac

- Wakefield measurements in the SC RF cryomodules

- Demonstration of ultra-stable operation of SRF cryomodules with beam-based feedback

- Development and experimental test of neural network based control systems and optimization tools for SRF accelerators

- Exploration of long pule and CW modes of operation of the ILC-type SRF cryomodule. 


\subsubsection{Accelerator R\&D for Novel Radiation Sources}

High energy (up to $1 \mathrm{GeV}$ ), high peak and average power and high brightness electron beams have tremendous potential for generation of high-brightness high energy photon beams spanning the range from keVs to dozens of MeV. The high average power and brightness of the ASTA electron beam has unmatched potential for development of several novel radiation source ideas, such as:

- High brightness $X$-ray channeling as a compact $x$-ray radiation source

- Inverse Compton scattering gamma-ray source and its applications

- Demonstration of feasibility of an XUV FEL oscillator

- Production of narrow-band gamma-rays

- Laser-induced microbunching studies with high micropulse-repetition rate electron beams

\subsubsection{Accelerator R\&D for Stewardship and Applications}

With its high energy, high brightness, high repetition rate, and the capability of emittance manipulations built-in to the facility design, ASTA is an ideal platform for exploring novel accelerator techniques of interest for very broad scientific community beyond high energy physics. The proposed research program includes several proposals and expressions of interest for such explorations:

- Demonstration of techniques to generate and manipulate ultra-low emittance beams for future hard X-ray free-electron lasers

- Beam energy dechirper using corrugated pipes

- Development and test of a beam-beam kicker

- Coherent diffraction radiation measurements of bunch length

Subsequent sections in this proposal explore these research thrusts in greater detail. All the proposals have been discussed at the Fermilab's Accelerator Advisory Committee (February 6-8, 2013) and later thoroughly reviewed by the ASTA Program Advisory Committee (July 23-24, 2013). In this document, we first present most developed proposals based on our evaluation. Other expressions of interest and short conceptual proposals for experimental beam studies at ASTA are presented in the Attachment A. 


\subsection{Accelerator R\&D Proposals for Particle Physics at the Intensity Frontier}

\subsubsection{Experimental Demonstration of Integrable Optics Lattices at IOTA}

\subsubsection{Introduction}

What prevents us from building super-high intensity accelerators? The answer is case-specific, but it often points to one of the following phenomena: machine resonances, various tune shifts (and spreads), and instabilities. These three phenomena are interdependent in all present machines, which are built to have "linear" focusing optics (also called lattice). A path towards alleviating these phenomena can be opened by making accelerators nonlinear. This idea is not totally new: Orlov [1] and McMillan [2] have proposed initial ideas on nonlinear focusing systems for accelerators. However, practical implementations of such ideas proved elusive, until recently when Danilov and Nagaitsev proposed a solution for nonlinear integrable accelerator lattice that can be implemented with special magnets [3]. In this document we propose a proof-of-principle experiment for demonstration of the concept, and describe the design of a machine for this demonstration-the Integrable Optics Test Accelerator (IOTA).

The ASTA facility will offer a unique opportunity to carry out the proposed research toward demonstration of the feasibility of the integrable optics technique. That research requires construction and operation of a dedicated storage ring (IOTA). It cannot be carried out anywhere else (e.g., at the existing storage rings) as it involves very special insertions (highly nonlinear magnets) which extend over a significant fraction of the ring circumference, special arrangements of the optics lattice and precise control of the elements (strength, positions, etc.).

\subsubsection{Concept of Nonlinear Integrable Optics}

\subsection{Nonlinear Integrable Optics and Potential}

The lattice design of all present accelerators incorporates dipole magnets to bend particle trajectory and quadrupoles to keep particles stable around the reference orbit. These are "linear" elements because the transverse force is proportional to the particle displacement, $x$ and $y$. This linearity results (after the action-phase variable transformation) in a Hamiltonian of the following type:

$$
H\left(J_{1}, J_{2}\right)=v_{x} J_{1}+v_{y} J_{2} \text {, }
$$

where $v_{x}$ and $v_{y}$ are betatron tunes and $J_{1}$ and $J_{2}$ are actions. This is an integrable Hamiltonian. The drawback of this Hamiltonian is that the betatron tunes are constant for all particles regardless of their action values. It has been known since early 1960-s that the spread of betatron tunes is extremely beneficial for beam stability due to the so-called Landau damping. However, because the Hamiltonian (1) is linear, any attempt to add non-linear elements (sextupoles, octupoles) to the accelerator generally results in a reduction of its dynamic aperture, resonant behavior and particle loss. A breakthrough in understanding of stability of

Proposal for an Accelerator R\&D User Facility at Fermilab's Advanced Superconducting Test Accelerator 
Hamiltonian systems, close to integrable, was made by Nekhoroshev [4]. He considered a perturbed Hamiltonian system:

$$
H=h\left(J_{1}, J_{2}\right)+\varepsilon q\left(J_{1}, J_{2}, \theta_{1}, \theta_{2}\right),
$$

where $h$ and $q$ are analytic functions and $\varepsilon$ is a small perturbation parameter. He proved that under certain conditions on the function $h$, the perturbed system (2) remains stable for an exponentially long time. Functions $h$ satisfying such conditions are called steep functions with quasi-convex and convex being the steepest. In general, the determination of steepness is quite complex. One example of a non-steep function is a linear Hamiltonian Eq. (1).

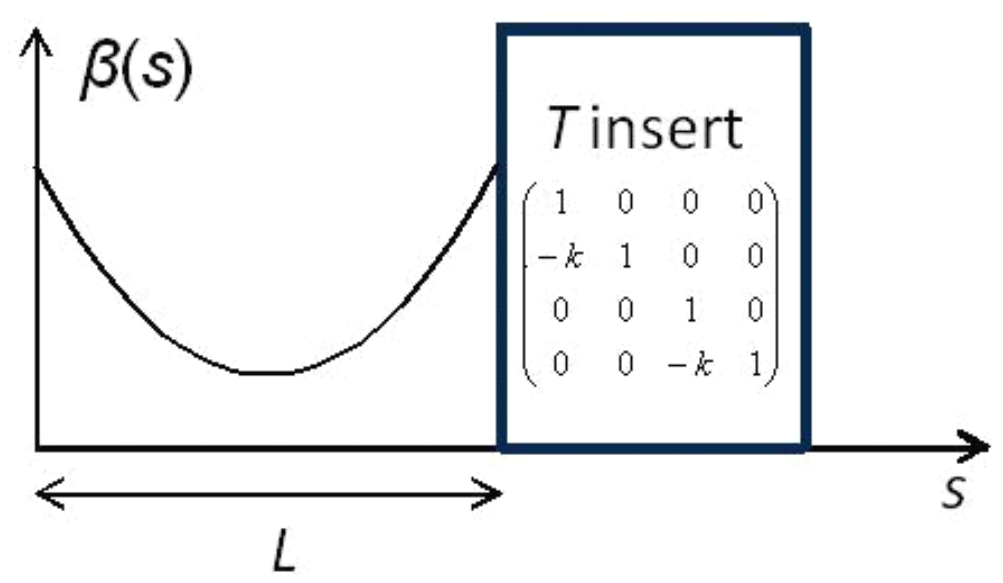

Figure 1: An element of periodicity: a drift space with equal beta-functions followed by a T-insert.

In Ref. [1] three examples of nonlinear accelerator lattices were proposed. Here we will concentrate on one of the lattices, which results in a steep (convex) Hamiltonian.

Consider an element of lattice periodicity consisting of two parts: (1) a drift space, $L$, with exactly equal horizontal and vertical beta-functions, followed by (2) an optics insert, $T$, which is comprised of linear elements and has the transfer matrix of a thin axially symmetric lens; see Figure 1.

Let us now introduce additional transverse magnetic field along the drift space $L$. The potential, $V(x, y, s)$, associated with this field satisfies the Laplace equation, $\Delta V=0$.

Now we will make a normalized-variable substitution [3] to obtain the following Hamiltonian for a particle moving in the drift space $L$ with an additional potential $V$ :

$$
H_{N}=\frac{p_{x N}^{2}+p_{y N}^{2}}{2}+\frac{x_{N}^{2}+y_{N}^{2}}{2}+U\left(x_{N}, y_{N}, \psi\right),
$$

Where 


$$
U\left(x_{N}, y_{N}, \psi\right)=\beta(\psi) V\left(x_{N} \sqrt{\beta(\psi)}, y_{N} \sqrt{\beta(\psi)}, s(\psi)\right),
$$

and $\psi$ is the "new time" variable defined as the betatron phase,

$$
\psi^{\prime}=\frac{1}{\beta(s)} \text {. }
$$

The potential $U$ in equation (3) can be chosen such that it is time-independent [1]. This results in a time-independent Hamiltonian (3). We will now choose a potential such that the Hamiltonian (3) possesses the second integral of motion. We will omit the subscript $N$ from now on.

Consider potentials [5] that can be presented in elliptic coordinates in the following way

$$
U(x, y)=\frac{f(\xi)+g(\eta)}{\xi^{2}-\eta^{2}},
$$

where $f$ and $g$ are arbitrary functions,

$$
\xi=\frac{\sqrt{(x+c)^{2}+y^{2}}+\sqrt{(x-c)^{2}+y^{2}}}{2 c} \quad \eta=\frac{\sqrt{(x+c)^{2}+y^{2}}-\sqrt{(x-c)^{2}+y^{2}}}{2 c}
$$

are elliptic variables and $c$ is an arbitrary constant.

The second integral of motion yields

$$
I\left(x, y, p_{x}, p_{y}\right)=\left(x p_{y}-y p_{x}\right)^{2}+c^{2} p_{x}^{2}+2 c^{2} \frac{f(\xi) \eta^{2}+g(\eta) \xi^{2}}{\xi^{2}-\eta^{2}}
$$

First, we notice that the harmonic oscillator potential $\left(x^{2}+y^{2}\right)$ can be presented in the form of Eq. (6) with $f_{1}(\xi)=c^{2} \xi^{2}\left(\xi^{2}-1\right)$ and $g_{1}(\eta)=c^{2} \eta^{2}\left(1-\eta^{2}\right)$. Second, we find the following family of potentials that satisfy the Laplace equation and, at the same time, can be presented in the form of Eq. (6):

$$
f_{2}(\xi)=\xi \sqrt{\xi^{2}-1}(d+t \operatorname{acosh}(\xi)) \quad g_{2}(\eta)=\eta \sqrt{1-\eta^{2}}(q+t \operatorname{acos}(\eta)),
$$

where $d, q$, and $t$ are arbitrary constants. Thus, the total potential energy in Hamiltonian (3) is given by

$$
U(x, y)=\frac{x^{2}}{2}+\frac{y^{2}}{2}+\frac{f_{2}(\xi)+g_{2}(\eta)}{\xi^{2}-\eta^{2}}
$$

Of a particular interest is the potential with $d=0$ and $q=\frac{\pi}{2} t$, because its lowest multipole expansion term is a quadrupole. Figure 2 presents a contour plot of the potential energy Eq. (10) for $c=1$ and $t=0.4$. 


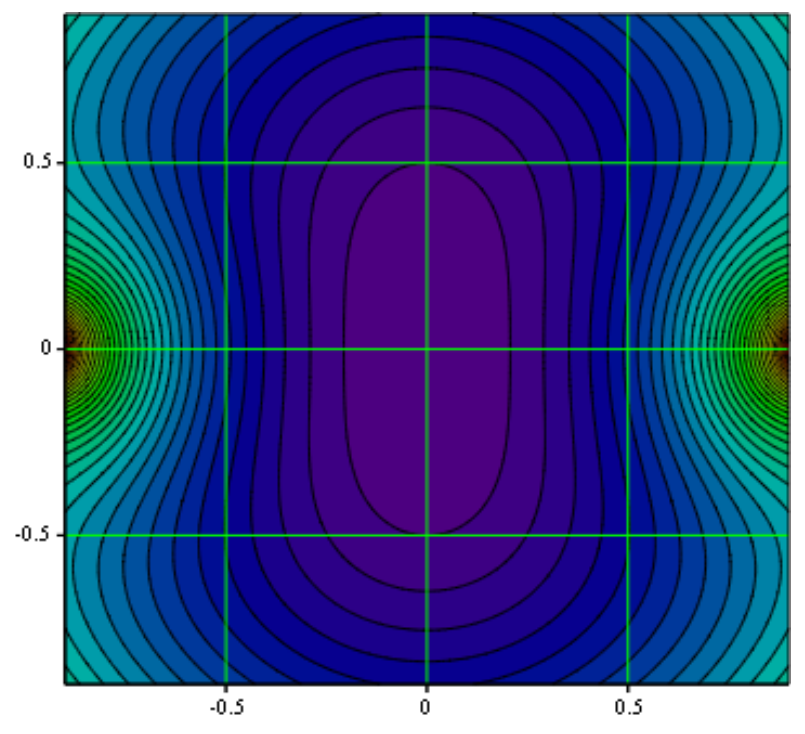

Figure 2: Contour plot of the potential energy Eq. (10) with $c=1$ and $t=0.4$. The repulsive singularities are located at $x= \pm c$ and $y=0$.

The multipole expansion of this potential for $c=1$ is as follows:

$$
U(x, y) \approx \frac{x^{2}}{2}+\frac{y^{2}}{2}+t \operatorname{Re}\left((x+i y)^{2}+\frac{2}{3}(x+i y)^{4}+\frac{8}{15}(x+i y)^{6}+\frac{16}{35}(x+i y)^{8}+\ldots\right)
$$

where $t$ is the magnitude of the nonlinear potential.

Since the 2D Hamiltonian with this potential has two analytic integrals of motion, it is integrable and thus can be expressed as an analytic function of actions:

$$
H=h\left(J_{1}, J_{2}\right) \text {, }
$$

where

$$
J_{1}=\frac{1}{2 \pi} \oint p_{\eta} d \eta \quad J_{2}=\frac{1}{2 \pi} \oint p_{\xi} d \xi
$$

\subsection{Maximum Nonlinear Tune Shift}

The potential (10) provides additional focusing in $x$ for $t>0$ and defocusing in $y$. Thus, for a small-amplitude motion to be stable, one needs $0<t<0.5$. This corresponds to the following small-amplitude betatron frequencies,

$$
v_{1}=v_{0} \sqrt{1+2 t} \quad v_{2}=v_{0} \sqrt{1-2 t},
$$

where $v_{0}$ is the unperturbed linear-motion betatron frequency. For arbitrary amplitudes the frequencies are obtained by

$$
v_{1}\left(J_{1}, J_{2}\right)=\frac{\partial h}{\partial J_{1}} \quad v_{2}\left(J_{1}, J_{2}\right)=\frac{\partial h}{\partial J_{2}}
$$


Figure 3 presents frequencies $v_{1}\left(J_{1}, 0\right)$ and $v_{2}\left(0, J_{2}\right)$, normalized by $v_{0}$ for $t=0.4$.
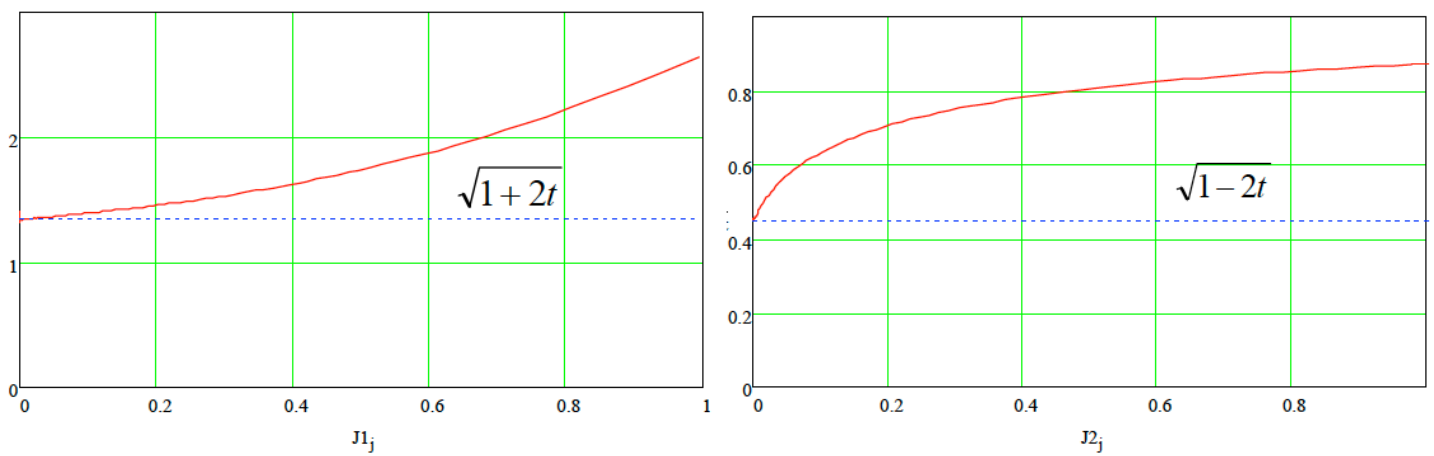

Figure 3: Betatron oscillation frequencies of the two modes - 1 (left) and 2 (right) normalized by $v_{0}$ as functions of actions. Nonlinearity strength parameter $t=0.4$.

The unperturbed linear motion tune $v_{0}$ - the betatron phase advance over the drift space $L$, is limited to 0.5 (in units of $2 \pi$ ). The phase advance in the $T$-insert must be a multiple of 0.5 . This makes the full tune of one element of periodicity $0.5+0.5 \mathrm{n}$. Thus, the theoretical maximum attainable nonlinear tune shift per cell is $\sim 0.5$ for mode 1 and $\sim 0.25$ for mode 2 . Expressed in terms of the full betatron tune per cell, this tune shift can reach $50 \%(0.5 /(0.5+0.5))$.

Numerical simulations with single and multi-particle tracking codes were carried out in order to determine the tune spread that can be achieved in a machine built according to the above recipe [6-8]. Various imperfections were taken into account, such as the perturbations of the $T$ insert lattice, synchrotron oscillations, and other machine nonlinearities. In Figure 4 a result of one of the simulations is presented. The tune footprint obtained with Frequency Map Analysis [9] demonstrates that vertical tune spread exceeding 1 can be achieved and very little resonances are caused by imperfections. No dynamic aperture was observed in the system. 


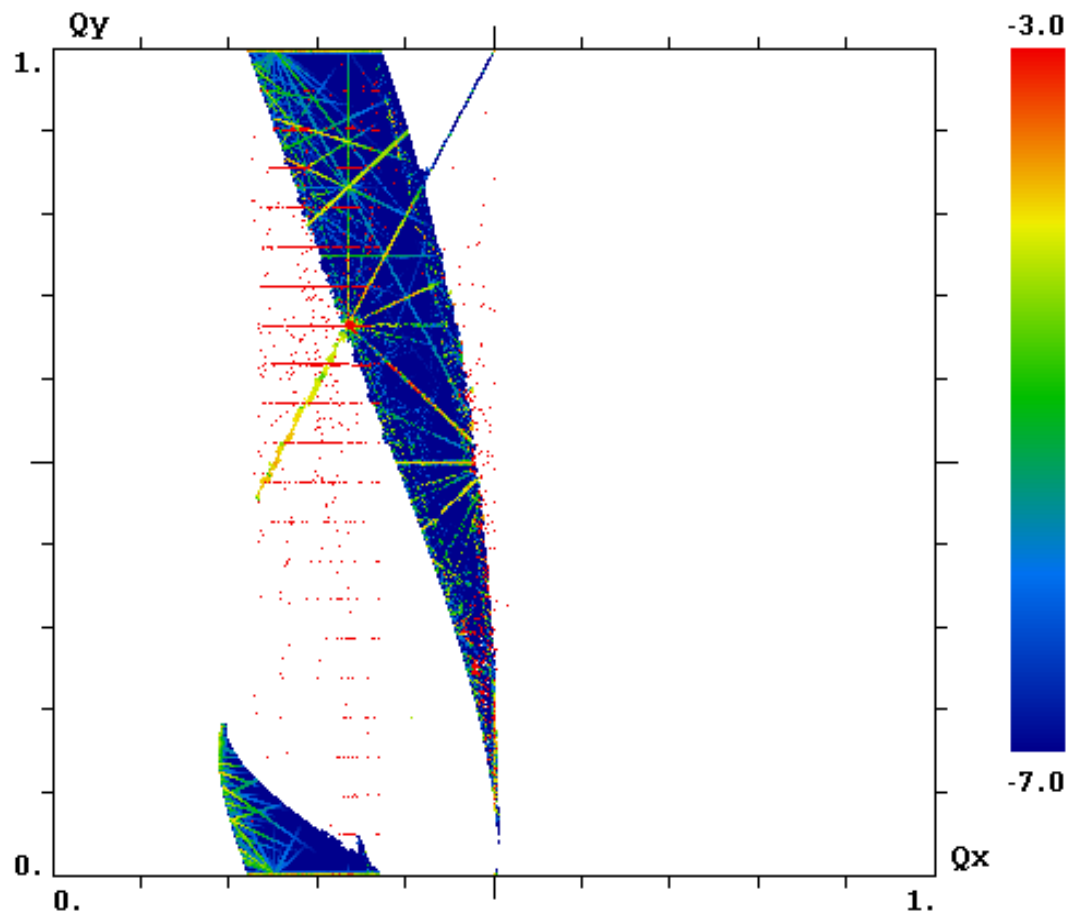

Figure 4: Beam tune footprint for $v_{0}=0.3$, four elements of periodicity, $t=0.4$. Simulation with Lifetrac Frequency Map Analysis.

\subsubsection{IOTA Goals and Scope}

In Section 8.2.1.2 we demonstrated that using conventional and special nonlinear focusing magnets it is possible to construct an accelerator lattice, in which the betatron motion is strongly nonlinear yet stable. The strong nonlinearity of betatron motion would result in a significant spread of betatron tunes of particles within the bunch (up to $50 \%$ of the nominal tune), thus providing strong Landau damping of coherent instabilities.

The superconducting RF linac test at Fermilab's ASTA will provide electron beam with energies up to $800 \mathrm{MeV}$. The high energy Experimental Area-3 at the end of the linac will be located in a $20 \times 15 \mathrm{~m}$ hall, which is large enough to house a small electron storage ring.

The proposed Integrable Optics Test Accelerator (IOTA) will get beam from ASTA's $1.3 \mathrm{GHz}$ SRF linac and will be used for the demonstration of the possibility to achieve very large nonlinear tune shifts in a realistic accelerator design. This proof-of principle experiment will initially concentrate on the single-particle motion stability in the nonlinear integrable system, leaving the studies of collective effects and attainment of high beam current to future research. 
Research at IOTA will include experiments on the following topics:

- Attainment of large nonlinear tune shift/spread without degradation of dynamic aperture

- Suppression of strong lattice resonances (e.g. by crossing the integer resonance by part of the beam without intensity loss)

- Stability of the nonlinear system to perturbations: chromatic effects, effect of synchrotron oscillations, lattice distortions

- Studies of different variants of nonlinear magnet design

In addition to the primary goal, the ring can accommodate other Advanced Accelerator R\&D experiments and/or users. This is possible because only a portion of the ring circumference will be occupied with nonlinear magnets and otherwise the machine is a conventional low-energy storage ring. One of the AARD experiments incorporated in the current design of the ring is the Optical Stochastic Cooling; see Section 8.3.2.

\subsubsection{IOTA Design}

\subsection{Machine Lattice}

The machine lattice must satisfy the following design criteria:

- Be periodic, with the element of periodicity comprised of a drift space with equal betafunctions, and a focusing and bending block with the betatron phase advance in both planes equal to $\pi$ ( $T$-insert in Figure 1 ).

- The drift space must be long enough to accommodate practical nonlinear magnets. We set the minimum drift length to $2 \mathrm{~m}$.

- The $T$-insert must be tunable to allow a wide range of phase advances (and betafunctions) in the drift space in order to study different betatron tune working points.

- It is preferable that the focusing block is achromatic in order to avoid strong coupling between the transverse and longitudinal degrees of freedom.

- The ring must have one long $(5 \mathrm{~m})$ straight section for the Optical Stochastic Cooling experiment.

- The machine must fit in the footprint of the experimental hall and be properly oriented with respect to the injection line.

In our design the ring is made of four cells. The cells are mirror-symmetric in pairs, and each consists of 8 quadrupoles and two dipole magnets bending by 30 and 60 degrees. With the betatron phase advance per cell of 0.8 , this makes the total betatron tune of 3.2. Hence, in the extreme case the maximum tune shift generated by the nonlinear magnets may reach 1.6, meaning some particles in the bunch will cross an integer resonance.

This design provides $2 \mathrm{~m}$ insertions for the nonlinear magnets, two $1 \mathrm{~m}$ long straight sections for $\mathrm{RF}$ and other systems, and two $5 \mathrm{~m}$ sections - one for injection/extraction and one for Optical Stochastic Cooling setup. The minimal number of quadrupole magnets required to implement 
an axially symmetric lens in a straight section is five. The large number of quadrupoles we use permits a wide range of tuning for the betatron tune, which can be varied between 2.4 and 3.6, and dispersion and momentum compaction.

Table 1 lists the main parameters of the machine. Figures 5, 6 show the machine layout in the experimental hall, and lattice functions of one cell.

Table 1: IOTA Ring Parameters.

\begin{tabular}{|l|c|c|}
\hline \multicolumn{1}{|c|}{ Parameter } & Value & Unit \\
\hline Beam Energy & 150 & $\mathrm{MeV}$ \\
\hline Circumference & 38.7 & $\mathrm{~m}$ \\
\hline Bending dipole field & 0.7 & $\mathrm{~T}$ \\
\hline RF voltage & 50 & $\mathrm{kV}$ \\
\hline Maximum $\beta$-function & 22 & $\mathrm{~m}$ \\
\hline Momentum compaction & 0.14 & \\
\hline Betatron tune & $Q_{x} Q_{y}=3.2(2.4$ to 3.6$)$ & $\pi \mu \mathrm{m}$ \\
\hline Equilibrium transverse emittance r.m.s. & 0.06 & (non-normalized) \\
\hline Synchrotron radiation damping time & & sec( $10^{7}$ turns) \\
\hline
\end{tabular}




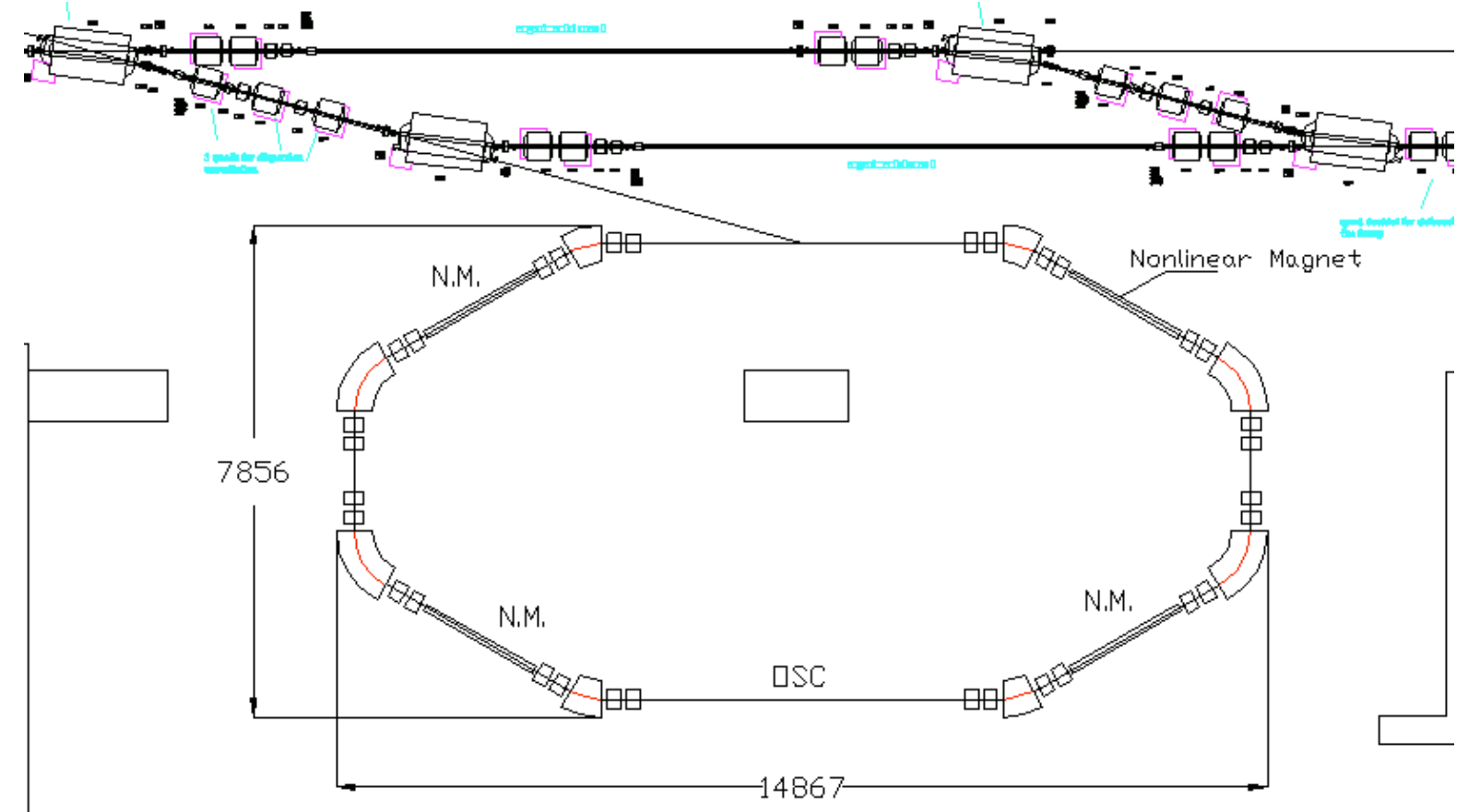

Figure 5: IOTA ring layout.

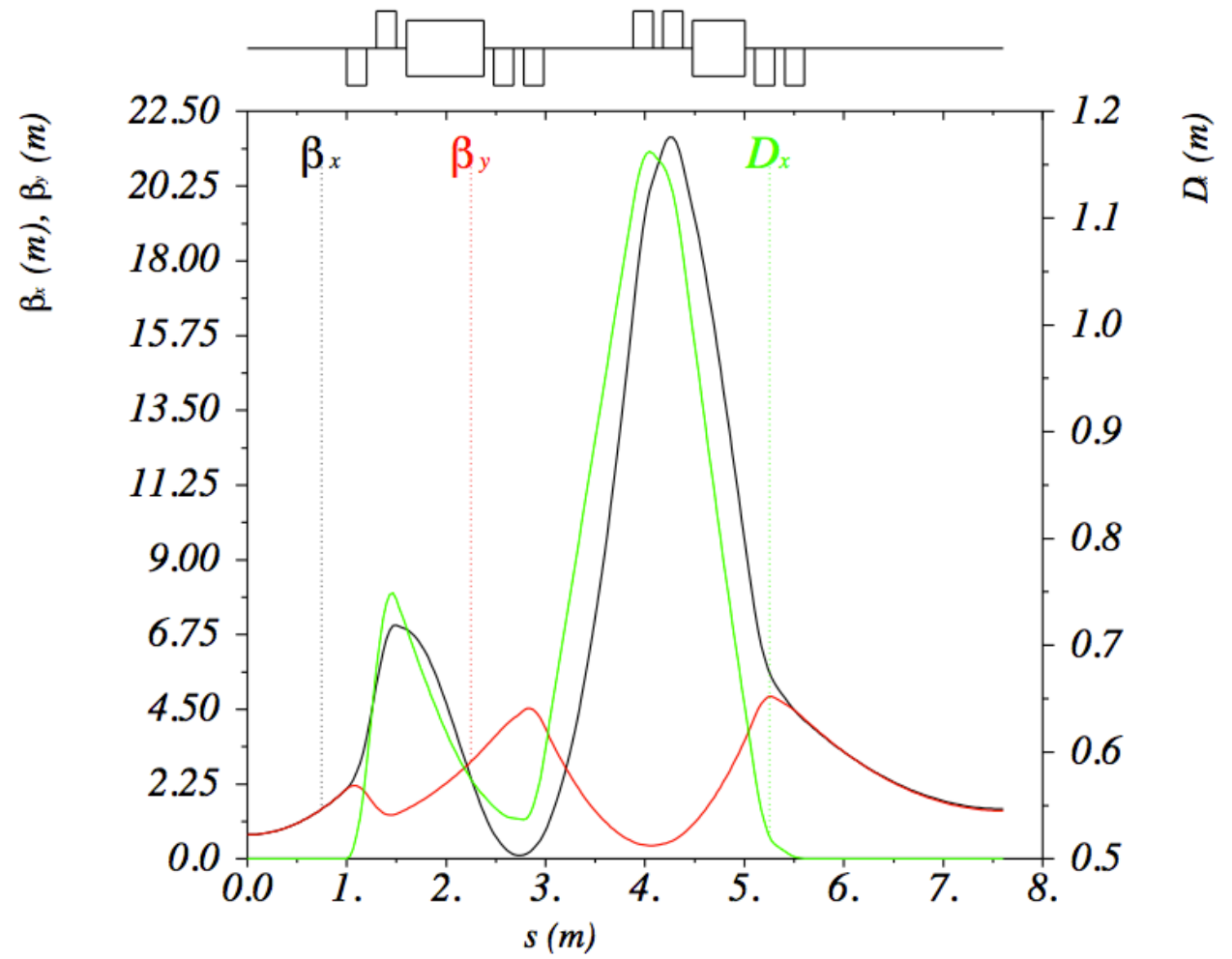

Figure 6: Lattice functions of the element of periodicity. 


\subsection{Nonlinear Magnets}

The condition of the Hamiltonian time-independence requires that the nonlinear potential must continuously change along the length of the nonlinear section (Eq. 4). The potential is defined by two parameters: the strength parameter $t$, and the geometric parameter $c$, which represents the distance between the singularities, or the element aperture (Eq. 10). The geometric parameter $c$ scales as the square root of $\beta$-function in the nonlinear straight section, and the strength parameter $t$ scales as $1 / \beta$. Since it's not practical to manufacture a magnet with complex varying aperture, we consider approximating the continuously varying potential with a number of thin magnets of constant aperture. Figure 7 shows the distribution of strength of the lowest (quadrupole) harmonic in 20 thin magnets.

The magnetic potential can be expanded into multipole series Eq. (11). However, this expansion is only valid inside the $r=\sqrt{x^{2}+y^{2}}<c$ circle. Vertical oscillation amplitudes $y>c$ are essential for achievement of large tune spread. By proper shaping of the magnetic poles we were able to achieve good field quality in the region $x<c, y<2 \times c$ (Figure 8). The magnet can be optimized further to include fringe-field effects and extend the good field region.

In order to demonstrate the high tune spread within the beam, the transverse beam size must be comparable to the distance between the poles of the magnet. For the chosen ring energy and equilibrium emittance, the beam size would be $\sigma_{\mathrm{x}}, \sigma_{\mathrm{y}} \approx 0.25 \mathrm{~mm}$, which would require impractically small transverse dimensions of the nonlinear elements. However, due to the very long damping time it is possible to "paint" a larger area with the small emittance linac beam. Hence, we considered nonlinear elements with the aperture $2 \times c \approx 2 \mathrm{~cm}$.

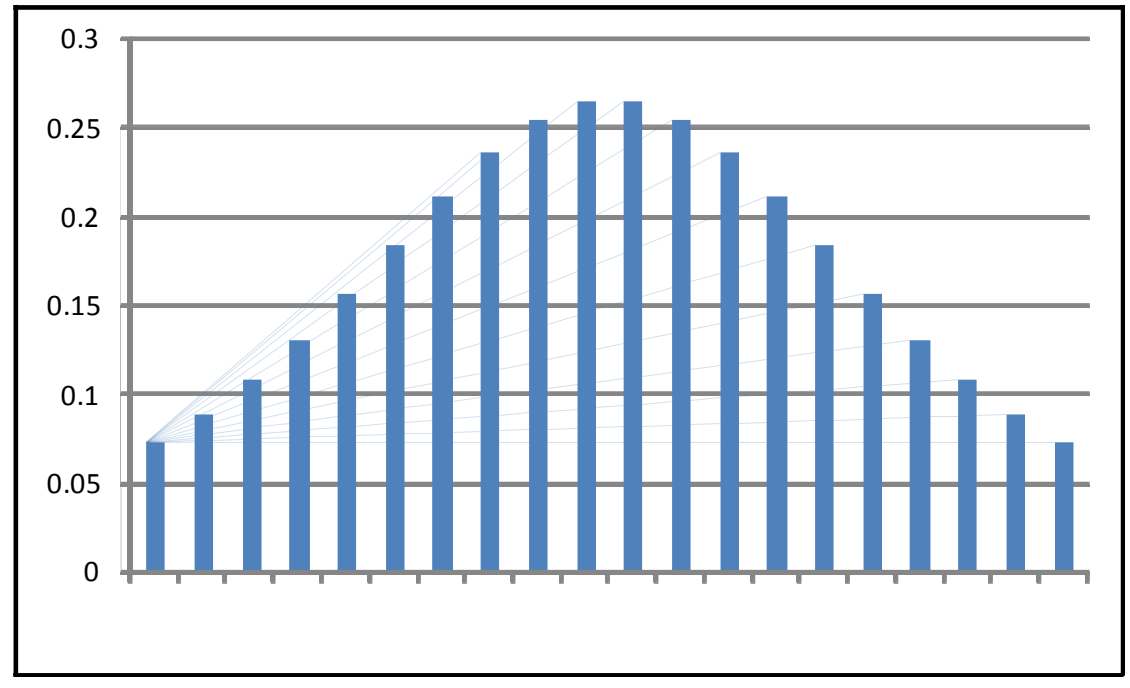

Figure 7: Distribution of quadrupole component $(\mathrm{T} / \mathrm{m})$ in the nonlinear magnet section. 


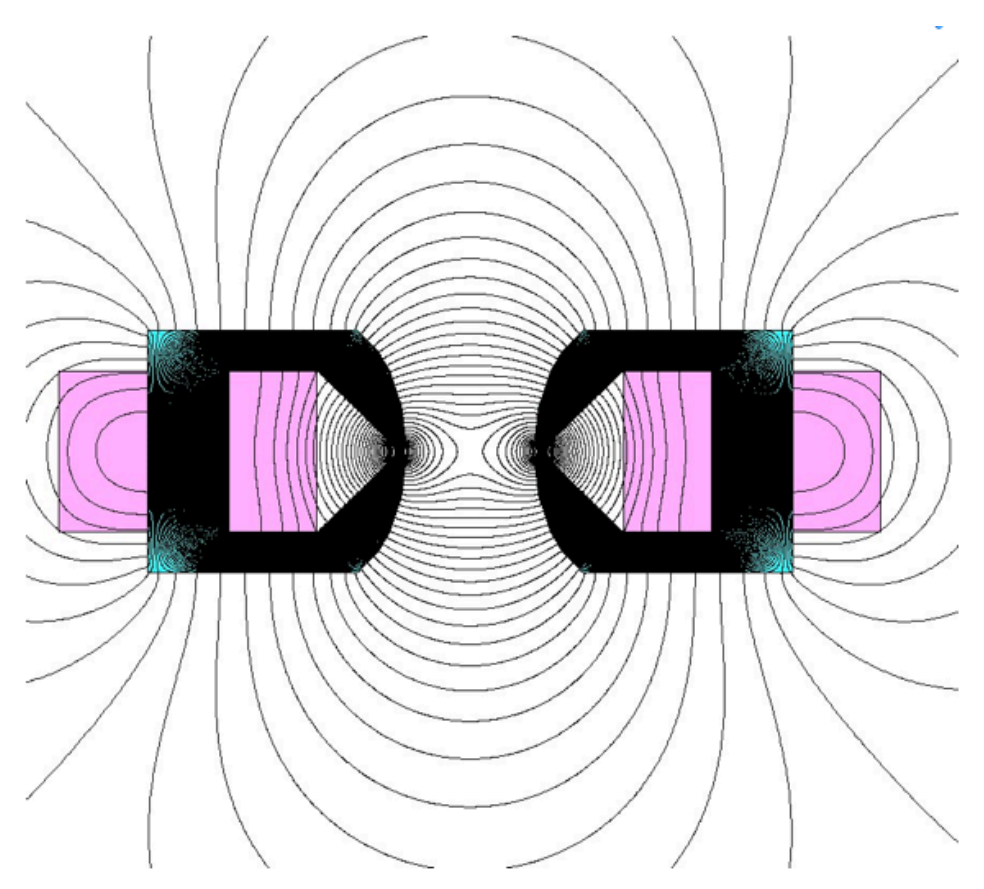

Figure 8: Cross-section of nonlinear magnet.

\subsection{Vacuum System}

Although studies of integrable optics require beam circulation only for 100,000 turns, other applications of the ring will demand beam lifetime of the order of 1 hour. This determines the specifications of the vacuum system, which must provide partial pressure of $\mathrm{CO}$ and $\mathrm{H} 2$ of $10^{-7}$ torr and $10^{-8}$ for Ar. The Aluminum beam pipe will have round cross-section with $50 \mathrm{~mm}$ diameter in quadrupoles and dipole magnets, and elliptical shape $15 \times 75 \mathrm{~mm}$ in the nonlinear magnet straight sections. Eight vacuum pumps will be attached to ports on the inside of dipole magnets.

\subsection{Beam Diagnostics}

Accurate tuning of the linear machine lattice is critical for proper functioning of the nonlinear integrable optics. The $\beta$-functions in the straight sections must be controlled to better than $5 \%$, and tolerance on the betatron phase advance in the $T$-insert is 0.02 . These requirements necessitate an extensive beam position monitoring system with 16 button-type pickups, capable of capturing the turn-by-turn beam position for 8-16 thousand turns with the precision of $100 \mu \mathrm{m}$.

Additionally, the ports in vacuum chamber in dipole magnets will provide points for synchrotron light based diagnostics. 


\subsection{RF System}

We plan to adopt the Project X $162 \mathrm{MHz}$ copper cavity design with some modifications. The RF cavity parameters are listed in Table 2.

Table 2: RF Cavity Parameters.

\begin{tabular}{|l|c|c|}
\hline \multicolumn{1}{c|}{ Parameter } & Value & Unit \\
\hline Aperture diameter & 50 & $\mathrm{~mm}$ \\
\hline Frequency & 162.45 & $\mathrm{MHz}$ \\
\hline Q factor & 30,877 & \\
\hline Shunt impedance & $4.5 \times 10^{6}$ & Ohm \\
\hline Maximum voltage & 50 & $\mathrm{kV}$ \\
\hline Power & 0.56 & $\mathrm{~kW}$ \\
\hline
\end{tabular}

\subsubsection{Status and Schedule}

As of the time of this document (2013), the machine design requirements are finalized. The layout was selected, and most numerical simulations of particle dynamics in the final lattice have been performed. Some components of beam instrumentation and vacuum system have been procured. The technical specification on elements of magnetic system and vacuum chamber were completed in 2012.

An external review of IOTA has been organized by Fermilab's Accelerator Physics Center (APC) and took place at Fermilab on 02/23/2012. The review committee was chaired by Prof A. Seryi of John Adams Institute (UK).

The committee reviewed all aspects of the IOTA experiment, including:

1) theoretical foundations of the IOTA method

2) numerical computer simulation of the beam stability in IOTA

3) overall design and plan of the experiment

4) technical preparedness of the design of key components of IOTA

5) schedule of implementation, cost and resources

6) plan of beam studies and experiments beyond Phase I.

Particular focus was on items 3 and 4 (magnets, vacuum, RF, injection, diagnostics, power supplies, mechanical support and alignment, beam dump, safety, controls and overall integration).

All presentations, summary report, and committee recommendation are available at

https://indico.fnal.gov/conferenceDisplay.py?confld=5151 
The committee recommended to proceed with procurement of the longest lead items (magnets for IOTA ring) in FY12 in anticipation that the ring assembly will take place during 2013-14, and beam commissioning will start in 2015-16. The committee report can be found in the Appendix at this URL given above.

We would like to thank V. Kashikhin, G. Romanov (FNAL), and I. Batalov (MIPT) for their contributions.

\subsubsection{References}

[1] V. V. Vecheslavov and Yu. F. Orlov, 1966 J. Nucl. Energy, Part C Plasma Phys. 8, p. 717.

[2] E. M. McMillan, in Topics in Modern Physics. A Tribute to E. U. Condon, edited by E. Britton and H. Odabasi (Colorado University Press, Boulder, 1971), pp. 219-244.

[3] V. Danilov and S. Nagaitsev, Phys. Rev. ST Accel. Beams 13, 084002 (2010).

[4] N. Nekhoroshev, "Behaviour of Hamiltonian systems close to integrable," Functional. Anal. Appl. 5 (1971), 338-339

[5] G. Darboux, "Sur un problème de mécanique," Arch. Néerlandaises Sci., Vol. 6, 371-376 (1901).

[6] S. Nagaitsev et al., in Proceedings of HB2010, Morschach, Switzerland (2010), THO1D01.

[7] A. Valishev et al., in Proceedings of PAC11, New York, NY (2011), WEP070.

[8] I. Batalov, A. Valishev, Fermilab Beams-doc-3959 (2011).

[9] D. Shatilov et al., Phys. Rev. ST Accel. Beams 14, 014001 (2011). 


\subsubsection{Space Charge Compensation in High Intensity Circular Accelerators}

We propose to explore a novel scheme of space-charge compensation that could lead to a significant increase of the beam intensity for future accelerator-based high-energy physics experiments and other sciences.

Through its past success in electron cooling of high-energy antiprotons [1], beam-beam compensation using the electron lenses [2], and controlled halo removal by hollow electron beams [3], Fermilab has gained extensive experience and resources in manipulating highenergy particle beams by means of well-controlled electrons. As the mission of US high energy physics program is pushing the Intensity Frontier, it is of great technical and scientific merit for the community if this remarkable tradition of Fermilab can be applied to overcome the beam intensity limit in the present accelerator technology. Hence, we propose to investigate a novel method of space-charge compensation to achieve very intense and stable beams in circular accelerators through trapping and controlling of the electrons generated from beam-induced residual gas ionization. The method has a great potential to improve performance of leading high-current proton accelerator facilities and experiments, such as LBNE with Project $X$ intensities, Mu2e and "g-2" after the intensity upgrades, compressor and accumulation rings envisioned in the Neutrino Factory and Muon Collider projects. The method may also offer a transformational technology for the next generation high-intensity proton sources, e.g., such as those needed for the Accelerator Driven Systems.

The main idea of this compensation method is based on the long-known fact that the negative effect of Coulomb repulsion can be mitigated if beams are made to pass through a plasma column of opposite charge. This idea has been successfully applied to transport high-current low-energy proton and $\mathrm{H}^{-}$beams into the RFQ in many linacs. In circular machines, partial neutralization by ionized electrons was attempted with notable improvements in beam intensity, namely one order of magnitude higher than the space-charge limit. However, the beam-plasma system was subject to strong transverse electron-proton (e-p) instability. In principle, this difficulty can be overcome if protons and electrons are immersed in a longitudinal magnetic field which is a) strong enough to freeze the electron density distribution; b) strong enough to suppress the e-p instability; c) weak enough to allow positive ions to escape transversely, in addition to longitudinal draining; and d) uniform enough to avoid betabeat excitations. In addition, we note that significant improvements have been made on the physics of non-neutral plasmas and on the stability of beam-plasma systems in the plasma physics community over the past decade, some of which could be readily adopted for the present project.

The scope of this proposal will be based on the resources and facilities available at Fermilab within the five year timeline. The existing ion source (proton and $\mathrm{H}^{-}$), LEBT system, and RFQ of High Intensity Neutrino Source (HINS) program will be reused as an injector for the ring with currents up to $20 \mathrm{~mA}$ and energy of $2.5 \mathrm{MeV}$. The Integrable Optics Test Accelerator (IOTA) ring, which is now under construction at Fermilab's ASTA with completion expected in 2015-16, will be used to accumulate protons through charge-exchange injection. The Tevatron electron lens

Proposal for an Accelerator R\&D User Facility at Fermilab's Advanced Superconducting Test Accelerator 
system, a nonlinear element to be installed in IOTA ring, can be used to trap electrons for the initial space-charge compensation experiments. The scientific program will consist of both extensive theoretical modeling, and installation and operation of the test accelerator, outlined as follows:

- Studies of the physics of electron column [4] formation and the stability of beam-plasma system

- Measurements of electron accumulation and beam-plasma stability at HINS beamline

- Design and construction of charge-exchange injection system for IOTA ring

- Installation of HINS front-end (with $\mathrm{H}^{-}$source) to ASTA hall

- Measurements of electron accumulation and beam-plasma stability at IOTA ring using the electron lens system [5]

- Upgrade of the electron lens system with dedicated diagnostic and control equipment.

The present proposal [6] perfectly fits the main thrusts of the Fermilab's accelerator R\&D plan, and it will create lots of synergies with other programs as well. For example, once the IOTA ring stores low energy proton beams, combined effects of space-charge compensation and nonlinear integral optics could be readily studied.

The ASTA facility will offer unique opportunities to carry out the proposed research toward demonstration of the feasibility of the space-charge compensation methods, such as with electron columns, electron lenses, or in combination of the two with the elements of the integrable optics technique. That research requires a dedicated storage ring (IOTA) and its operation with protons. It cannot be carried out anywhere else as there are no existing proton storage rings or synchrotrons which can afford the installation of special insertions (columns, lenses, etc.), and offer special arrangements of the optics lattice and precise control of the insertion devices and the ring elements.

\subsubsection{References}

[1] S. Nagaitsev et al., Phys. Rev. Lett. 96, 044801 (2006).

[2] V. Shiltsev et al., Phys. Rev. Lett. 99, 244801 (2007).

[3] G. Stancari et al., Phys. Rev. Lett. 107, 084802 (2011).

[4] V. Shiltsev, Proc. PAC'2007, page 1159 (2007).

[5] A. Burov et al., FERMILAB-TM-2125 (2000).

[6] M. Chung et al., Proc. NA-PAC'2013, TUOBB1(2013). 


\subsection{Accelerator R\&D Proposals for Particle Physics at the Energy Frontier}

\subsubsection{Advanced Phase Space Manipulations}

\subsubsection{Transformative Applications of Advanced Phase Space Manipulations}

Over the last decade, Fermilab has pioneered the experimental development of advanced phase space manipulations at the A0 photoinjector test facility. These manipulations include the generation of "flat" beams with high transverse-emittance ratio $[1,2,3,4]$ and the emittance exchange between the horizontal and longitudinal degrees of freedom $[5,6]$. The beamline used for the latter experiment was also shown to be capable of producing arbitrary-shaped current profile including the production of sub-picosecond bunch trains with variable spacing as needed to produce, e.g., tunable coherent radiation $[7,8]$. The combination of these two manipulations could enable the arbitrary repartitioning of emittances between the three degrees of freedom $[9,10]$. Given the local expertise, this type of experiments will be resumed at ASTA with the end goal of pushing their limits to new frontiers and possibly utilizing these phase-spacemanipulation concepts to advance the performances of accelerator-based light sources or new acceleration concepts.

Advanced phase space transformation techniques, flat beam generation, emittance exchange (EEX), and temporal pulse shaping could have transformative applications to advanced accelerator science. At ASTA some of these transformations were included in the baseline design (e.g. flat beam in the photoinjector) and will be readily available to the user. Most of the components and expertise required to assemble other phase space manipulation methods, e.g. emittance exchange, are available. The availability of these manipulation techniques to carry out experiments is unique to the ASTA facility and, when combined with the high-repetition rate, could be utilized to investigate, e.g., dynamical effect in plasma-wakefield accelerators.

\subsubsection{Flat Beam Transformation}

Under normal conditions, the electron beams generated in a photoinjector are round due to the cylindrical symmetry of the photocathode drive-laser as well as the rf acceleration and solenoidal focusing system. Several factors drive the bulk properties of an electron beam, such as space-charge, beam emittance and angular momentum. An electron beam in thermal motion inside a cathode is born into a region with an axial magnetic field $\boldsymbol{B}$, suddenly acquiring canonical angular momentum. The beam is then brought into a field-free region, and the kinetic angular momentum is removed by a set of three skew-quadrupole magnets. In this process the initially equal emittances are partitioned into unequal emittances $\left(\gamma \varepsilon_{+}, \gamma \varepsilon_{-}\right)$, preserving the product: $\gamma \varepsilon_{x} \times \gamma \varepsilon_{y}=\gamma \varepsilon_{+} \times \gamma \varepsilon_{-}$; see Figure 1 . 


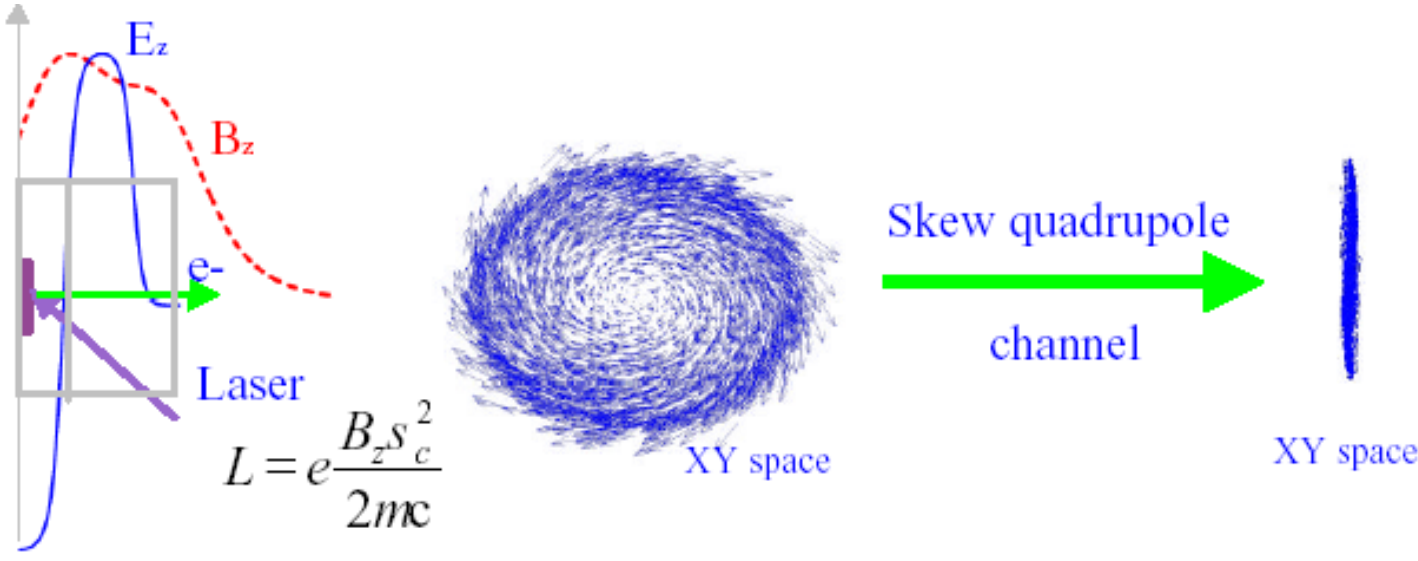

Figure 1: The round-to-flat beam transformation. The beam is produced in an rf gun (left), wherein the axial magnetic field is non-zero, thereby imparting a nonzero canonical angular momentum. Upon exit from the gun and the magnetic field, the canonical angular momentum is converted into kinetic angular momentum (middle). The angular momentum is removed by applying a torque on the beam using a system composed of three skew-quadrupole magnets. The beam is thereby made flat (right).

An unequal partitioning of equal emittances is not possible in a symplectic process; in a symplectic process the emittances in different directions can either remain the same or be exchanged among themselves. The flat-beam technique circumvents this limitation because the process of beam birth in a magnetic field is non-symplectic. The expected flat beam transverse emittances are [11]:

$$
\gamma \varepsilon_{ \pm}=\left[(\gamma \varepsilon)^{2}+(\gamma \Lambda)^{2}\right]^{\prime 2} \pm \gamma \Lambda \stackrel{\gamma L>\gamma \varepsilon}{\longrightarrow}\left\{\begin{array}{l}
2 \gamma \Lambda \\
\frac{\gamma \varepsilon}{2 \Lambda \gamma}
\end{array}\right.
$$

where $\Lambda$ is related to the beam's mean canonical angular momentum. The emittance ratio after the round-to-flat-beam transformation is given by

$$
\frac{\varepsilon_{+}}{\varepsilon_{-}} \approx\left(\frac{2 \gamma \Lambda}{\gamma \varepsilon}\right)^{2}=\left(\frac{e B\left(\sigma_{c}\right)^{2}}{m c \gamma \varepsilon}\right)^{2}>>1,
$$

where $e$ is the electron charge, $m$ is the electron mass, $c$ is the speed of light, and $\sigma_{c}$ is the transverse rms beam size of the laser on the photocathode. It was recently pointed out that the flat-beam generation from a CAM-dominated beam can be elegantly described using the concept of eigen-emittances; see Ref. [9]. Mathematically, the eigen-emittances are the eigenvalues of the beam matrix. Using this formalism, one can show that the transverse eigenemittances associated to a CAM-dominated beam are unequal and the RFTB effectively maps the eigen-emittances into the conventional beam emittances (i.e. the determinant of the $2 \times 2$ beam matrix sub-blocks associated to each degree of freedom). Therefore the eigen-emittance concept provides a powerful tool for perfecting the settings of a photoinjector. One can then 
optimize the performances of the RFTB by insuring the optimum eigen-emittances achieved in the photoinjector are fully transferred into the beam's emittances.

The flat-beam technique was experimentally demonstrated at Fermilab's A0 Photoinjector [4] where an emittance ratio of 100 was achieved; see Figure 2.

The ASTA photoinjector incorporates a set of three skew-quadrupole magnets, a round-to-flatbeam transformer (RFBT), to possibly convert an incoming magnetized round beam into a flat beam. Furthermore, the solenoidal lenses surrounding the $\mathrm{rf}$ gun can be axially moved. The bucking solenoid on the rf-gun was designed to provide a large axial field on the photocathode surface. Therefore, by properly positioning the solenoidal lenses and setting their currents, the axial magnetic field on the photocathode can be tuned from zero to thousands of Gauss. At bunch charge of $1.0 \mathrm{nC}$ and beam energy of $50 \mathrm{MeV}$, the normalized emittance is optimized around $\varepsilon_{\perp}{ }^{n}=2.3 \mu \mathrm{m}$ [12]. This includes the thermal emittance which is about $0.85 \mu \mathrm{m}$ for a residual kinetic energy of $0.55 \mathrm{eV}$ from CsTe cathode. Considering the case of a 1000-G axial magnetic field on the photocathode would result in a normalized angular momentum $\gamma L=29.64$ $\mu \mathrm{m}$; then the achievable flat-beam transverse normalized emittances partition is $\left(\gamma \varepsilon_{-}, \gamma \varepsilon_{+}\right) \approx$ $(0.1,59.4) \mu \mathrm{m}$ corresponding to an emittance ratio of $\sim 600$. The small emittance will be challenging to measure with the standard multislit method but should be properly diagnosed with the quadrupole scan method. Accelerating these flat beams to $250 \mathrm{MeV}$ while preserving the small emittance will also present interesting challenges and remains to be demonstrated.
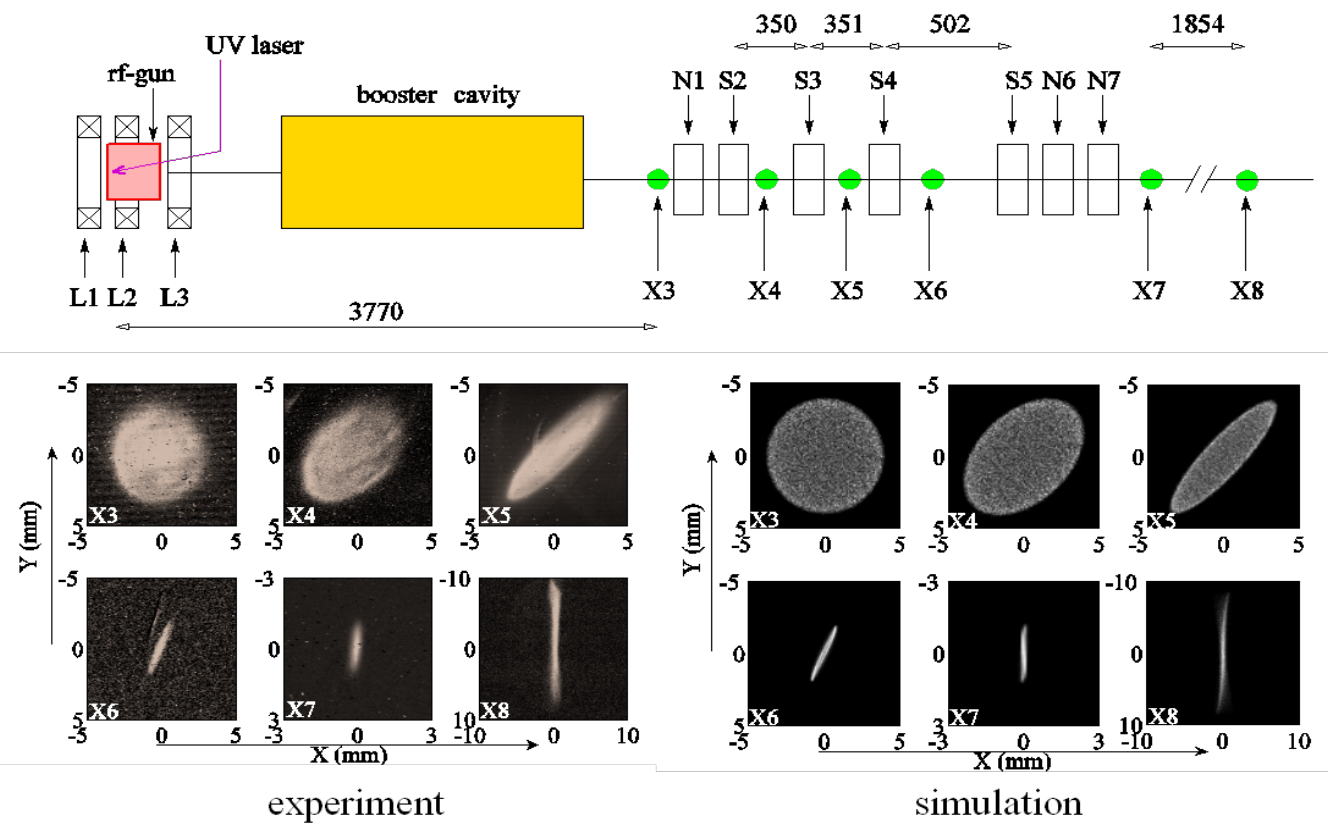

Figure 2: The round-to-flat beam transformation experiment at A0 photoinjector [3). Top: experimental configuration (the labels " $\mathrm{X}$ ", " $\mathrm{N}$ ", " $\mathrm{S}$ " respectively represent the locations of diagnostics, normal and skew quadrupole magnets). Bottom: transverse measured (left) and simulated (right) beam density at different axial locations along the accelerator beamline. 
We have recently used the concept of eigen-emittances to optimize the generation of flat beams at the ASTA. An example of evolution of eigen-emittance along the ASTA photoinjector for a 3.2-nC beam produced from a photocathode immersed in an axial magnetic field $B \simeq 800$ $\mathrm{G}$ appears in Figure 3 (left plot). The achieved beam parameters for the flat- and round-beam scenarios are summarized in Figure 3 (right table). This example confirms that the fourdimensional emittance achieved for round and flat beams are comparable when the beamline settings are adequately optimized. In this particular case, a transverse emittance ratio of 334 was simulated. The optimizations were carried using a Pareto-based multi-objective optimizer with main objectives of: (1) minimizing the four-dimensional emittance and (2) maximizing the eigen-emittance ratio. The conversion of these eigen-emittances to beam's emittance using the skew quadrupole magnets was also confirmed but found to be strongly dependent on the beam's fractional momentum spread.

Finally, compressing the produced flat beam could provide exciting opportunities: compressed flat beam can be used for image charge undulators or beam-driven acceleration techniques employing asymmetric structures. In addition, flat beams could also provide a way to mitigate deleterious effects in a magnetic chicane bunch compressor. Preliminary simulations of the compression of a flat beam with different incoming transverse emittance ratio have been performed [17]. The simulations indicate that the four-dimensional emittance $\varepsilon_{4} \equiv \sqrt{\varepsilon_{x} \varepsilon_{y}}$ degradation is mitigated for flat beams with transverse emittance ratio; see Figure 4 . The compression of flat beam at ASTA will be a significant improvement over the experiments carried at the $\mathrm{A} 0$ photoinjector.

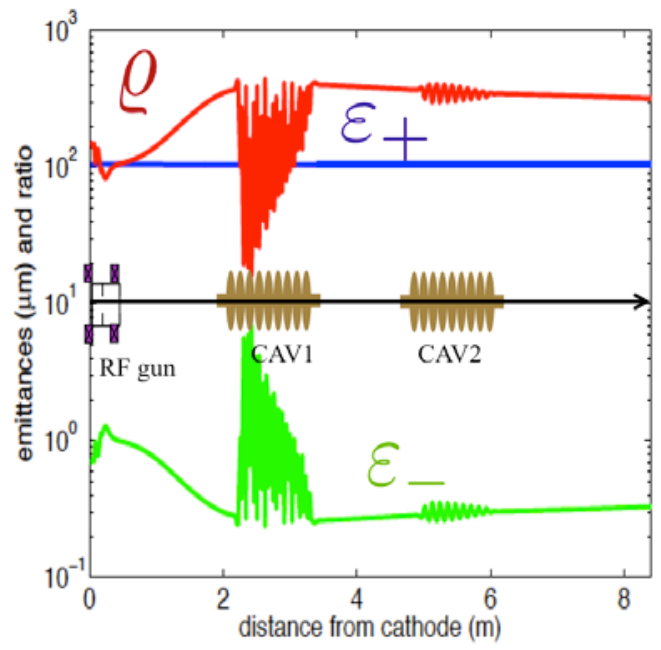

\begin{tabular}{cccc}
\hline parameter & $\begin{array}{c}\text { flat-beam } \\
\text { configuration }\end{array}$ & $\begin{array}{c}\text { round-beam } \\
\text { configuration }\end{array}$ & units \\
\hline \hline$Q$ & 3.2 & 3.2 & $\mathrm{nC}$ \\
$E$ & 47.18 & 48.77 & $\mathrm{MeV}$ \\
$\varepsilon_{z}$ & 105.04 & 5.43 & $\mu \mathrm{m}$ \\
$\varepsilon_{y}$ & 0.31 & 5.44 & $\mu \mathrm{m}$ \\
$\varepsilon_{4 D}$ & 5.53 & 5.44 & $\mu \mathrm{m}$ \\
$\rho$ & $\simeq 334$ & $\simeq 1$ & - \\
\hline
\end{tabular}

Figure 3: Evolution of eigen emittances (green and red trace) and their ratio (red trace) along the photoinjector beamline (left) and simulated performance of the ASTA photoinjector for the flat- and round-beam configurations (right). 


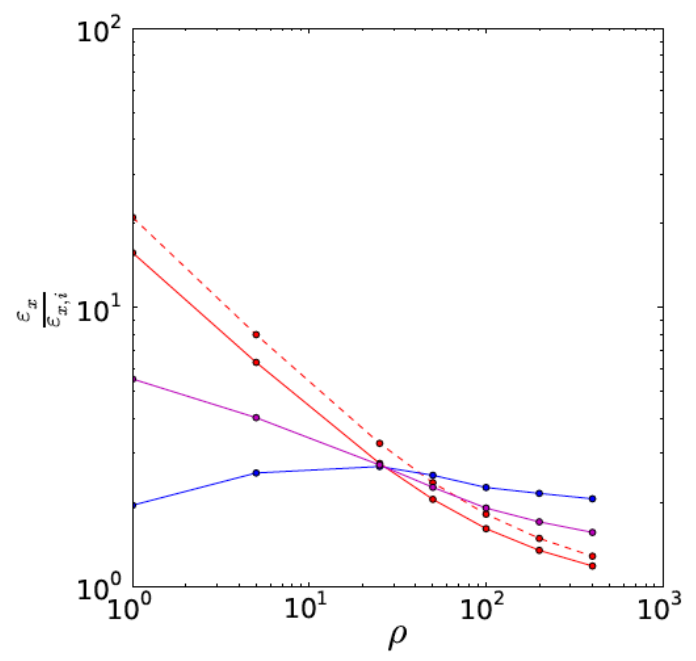

Figure 4: Transverse emittances dilution as a function of incoming flat-beam emittance ratio $\left(\rho \equiv \varepsilon_{x, i} / \varepsilon_{y, j}\right)$ through the bunch compressor (BC1) of the ASTA photoinjector. The bending-plane (horizontal) emittance dilution (red traces-correspond to the ratio of final over initial horizontal emittances) is simulated with CSRTrack (dashed line) and IMPACT-Z (solid line). The vertical (blue trace) and four-dimensional $\left(\varepsilon_{4} \equiv \sqrt{\varepsilon_{x} \varepsilon_{y}}\right.$, magenta trace) emittance dilutions are obtained from IMPACT-Z simulation. The bunch charge is $3.2 \mathrm{nC}[17]$.

The first-beam configuration of the ASTA photoinjector will readily include the round-to-flat beam transformation and will therefore support experimental studies pertaining to the compression of flat beams with high transverse-emittance ratio. We especially plan to perform parametric study of transverse and longitudinal phase space degradation as function of incoming beam flatness.

\subsubsection{Transverse-to-Longitudinal Phase Space Exchange}

Over the last decade beamlines capable of exchanging the transverse and longitudinal phase space coordinates have emerged. Typically such beamlines are composed of a deflecting cavity operating on the $\mathrm{TM}_{110}$ mode flanked by two dispersive sections. Such a beamline can serve as a phase space exchanger provided the conditions [5]

$$
D_{+}=\left(\begin{array}{ll}
R_{11,+} & R_{12,+} \\
R_{21,+} & R_{22,+}
\end{array}\right) D_{-} \text {, and } \kappa=-\frac{1}{\eta},
$$

are verified. Here the + and - signs refer to values associated to respectively the downstream and upstream dispersive sections, $D \equiv(\eta, d \eta / d s)$ is the dispersion vector, $R_{i j}$ are the usual transport matrix elements, and $\boldsymbol{K}$ is the TDC normalized deflecting strength. 
The simplest implementation devised to date is the "double-dogleg configuration" where the two dispersive sections upstream and downstream of the deflecting cavity are simple doglegs each composed of two dipoles; see Figure 5. When this condition is fulfilled and under the thin lens approximation, the horizontal and longitudinal phase space coordinates $\left(x, x^{\prime}, z, \delta\right)$ before and after the EEX are related via

$$
\left(\begin{array}{c}
x \\
x^{\prime} \\
z \\
\delta
\end{array}\right)_{\text {out }}=\left(\begin{array}{cccc}
0 & 0 & \frac{L+s}{\alpha L} & \alpha S \\
0 & 0 & \frac{1}{\alpha L} & \alpha \\
\frac{1}{\alpha L} & \frac{L+s}{\alpha L} & 0 & 0
\end{array}\right)\left(\begin{array}{c}
x \\
x^{\prime} \\
z \\
\delta
\end{array}\right)_{\text {in }}
$$

where the geometric parameters are introduce in Fig 5. The block anti-diagonal form of this matrix results in an exchange of phase-space coordinates between the horizontal and longitudinal phase spaces. As a consequence the corresponding emittances are also exchanged. It should be noted that other beamline configurations are also capable of exchanging the transverse and longitudinal phase space an alternative approach using a chicane-like configuration is investigated in Ref. [18].

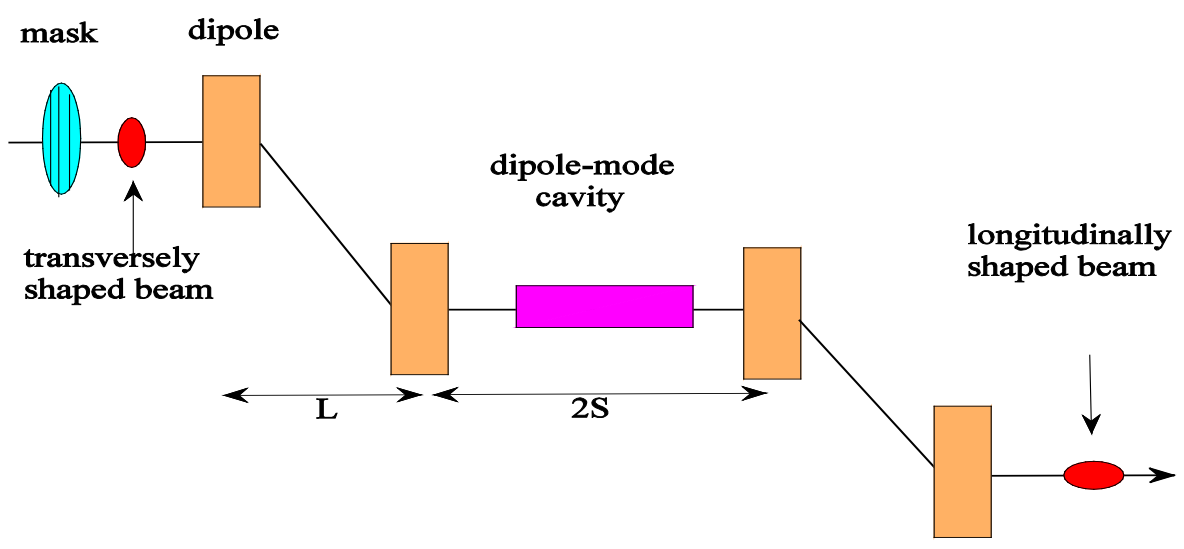

Figure 5: Overview of the double-dogleg transverse-to-longitudinal phase-spaceexchanging beamline.

Since this beamline exchanges the phase space coordinates, it also swaps the longitudinal and horizontal emittances. In fact this was the initial motivation for developing this class of beamlines [13]. A proof-of-principle experiment demonstrating the exchange of transverse and longitudinal emittances was carried at the $A 0$ photoinjector [6]. It was later realized that this phase space exchange beamline could also be used as a bunch-current shaper [14] and proof- 
of-principle experiment was carried at the A0 photoinjector; see Figure 6. A byproduct of this experiment was the generation of narrowband $\mathrm{THz}$ coherent transition radiation. The radiation was shown to have a 20-25 \% FWHM relative bandwidth and was tunable over the 0.4-0.9 THz range. These experiments were limited by the mask used to produce a transversely-segmented beam (the mask was indeed optimized to measure the beam emittance). At the A0 photoinjector, the deflecting cavity used was a LN2-cooled normal conducting cavity composed of five elliptical cells following the design of a superconducting cavity developed for kaon separation [15].

We plan on continuing phase space exchange experiments at ASTA. Several avenues to improve over the series of experiments performed at the $A 0$ photoinjector are under consideration. The overarching goals of this "second generation" phase space exchange experiments are:

1. To improve the performance of the first generation phase space exchange experiment, and

2. To find beamline configurations that would be more practical, e.g., that do not offset the beam's direction so that manipulated beams could be sent to the high-energy area and be used to support experiments.
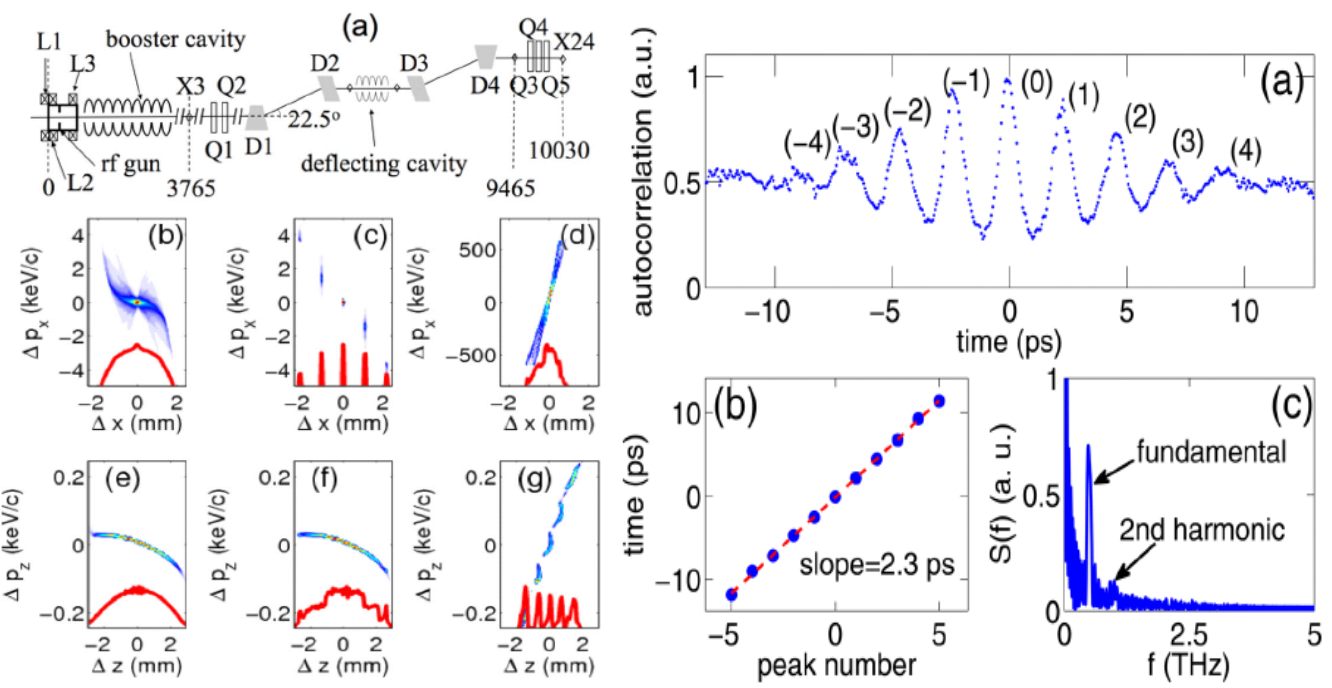

Figure 6: Left figures: (a) experimental configuration and simulated transverse $(b, c, d)$ and longitudinal $(e, f, g)$ phase spaces before $(b, e)$ and after $(c, f)$ the multi-slit mask and downstream $(d, g)$ of the emittance-exchanger beamline. Right figure: typical autocorrelation of transition radiation (a) and corresponding peak separation (b) and frequency analysis (c). 

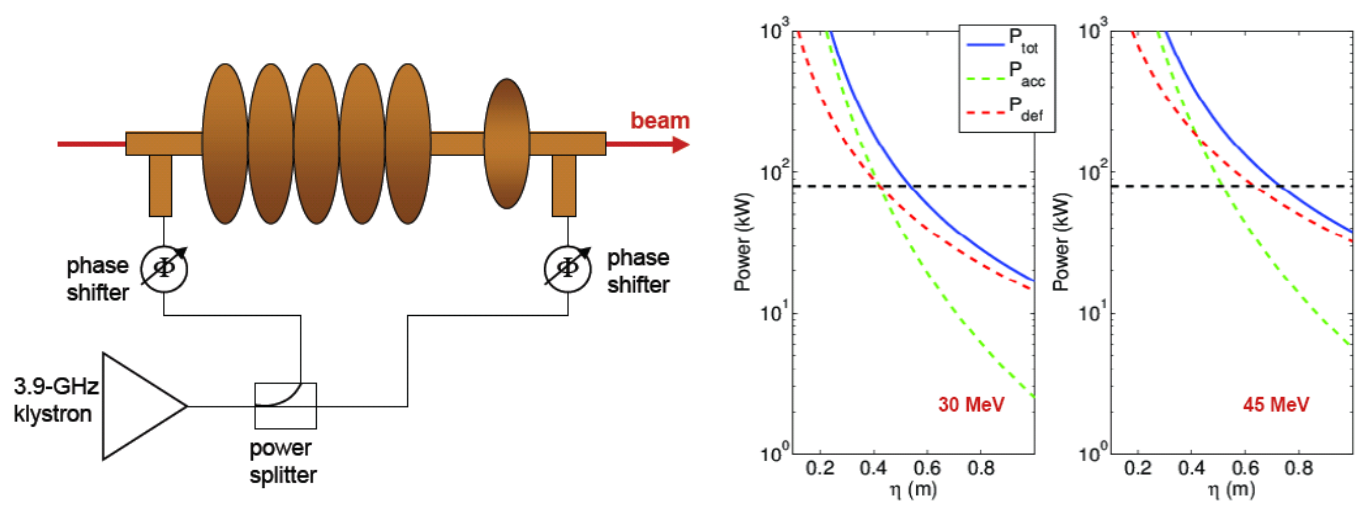

Figure 7: The "thin-lens" deflecting cavity consisting of a five-cell TM110 cavity followed by a single-cell TM010 cavity (left). Requirements on klystron power for ideal longitudinal to transverse phase space exchange as a function of the dispersion value at the cavity location. The calculations are performed for a 3.9-GHz klystron feeding an LN2-cooled system. The horizontal black line corresponds to the maximum power available from the available klystron.

Item (1) consists in improving the diagnostics downstream of the phase space exchanger beamline (the AO experiment did not have any viable longitudinal phase space diagnostics which precluded a direct measurement of the final longitudinal emittance) and possibly considering an "hybrid" deflecting cavity that will also have a $6^{\text {th }}$ cell operating on the $\mathrm{TM}_{010}$ mode. The latter improvement was shown to cancel some aberration associated the realistic (and not thin lens model) of deflecting cavities [16]. The current 3.9-GHz rf system should be able to drive such a hybrid cavity provided the dispersion at the cavity is close to $0.8 \mathrm{~m}$ (for a 50 $\mathrm{MeV}$ beam); see Figure 7. One option would consist in installing a double-dogleg beamline in the $50 \mathrm{MeV}$ experimental area; see Figure 8. However due to the limited 3.9-GHz klystron power $(80 \mathrm{~kW})$, the use of a LN2-cooled deflecting cavity forces us to increase the dispersion in order to lessen the deflecting strength required for full phase space exchange. In the current layout, the dipole magnets in each dogleg are separated by $1.07 \mathrm{~m}$ and the distance between the two central dipole magnets is $1.99 \mathrm{~m}$. Each dipole bends the beam by $22.5^{\circ}$ while each dogleg generates a dispersion of $44 \mathrm{~cm}$. The beamline was modeled using General Particle Tracer with 3D space-charge effects included for a $250 \mathrm{pC}$ and $50 \mathrm{MeV}$ electron bunch. The design is in essence very similar to the setup used at the AO photoinjector but would include better diagnostics. In addition it could support experiments aiming at repartitioning the emittances within the three degrees of freedom. In such experiments, the flat beam transform would be used to create a flat incoming beam and the phase space exchange would exchange the horizontal and longitudinal emittances. This versatility would allow for parametric studies of the phase space exchange mechanism. Finally the beamline could also support more advanced current shaping technique. Using triangular mask (or shaping the transverse profile of the laser on the photocathode) could be used to produce ramped bunches which have extremely important applications in beam driven wakefield acceleration technique as they enhance the transformer ratio. 


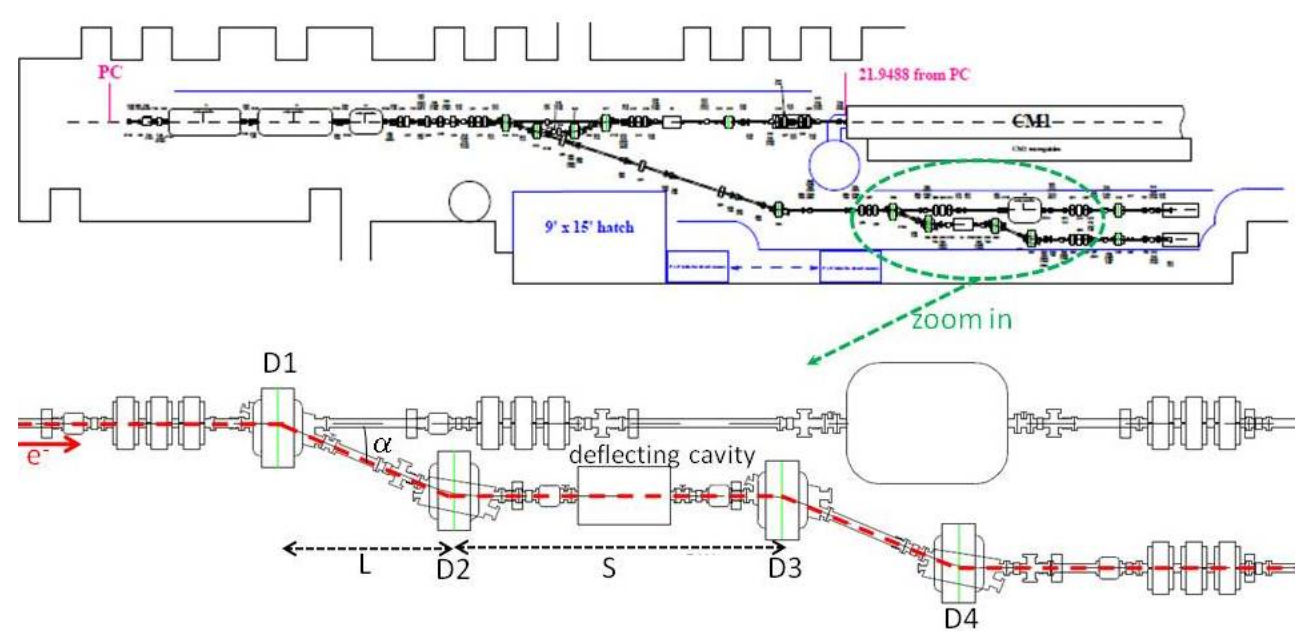

Figure 8: The NML injector and double dogleg beamline for the transverse-tolongitudinal phase space exchange.

Beside the double-dogleg beamline, other configurations are also possible, such as the magnetic chicane with quadrupoles inserted between the dipole. This latter setup has the main advantage of not translating the beam direction and could be a more suitable configuration for a higher energy phase space exchanger. It could also open the exploration of tradeoff between dispersion and deflecting strength. Numerical simulations are currently underway to explore possible layouts at ASTA and examples of preliminary results are displayed in Figure 9. In the latter it was demonstrated via numerical simulation that the double-dogleg and chicane-line configurations have very similar performances though their optical lattice has substantial difference (the double-dogleg beamline has a stronger compression ratio than the chicane-like configuration). 

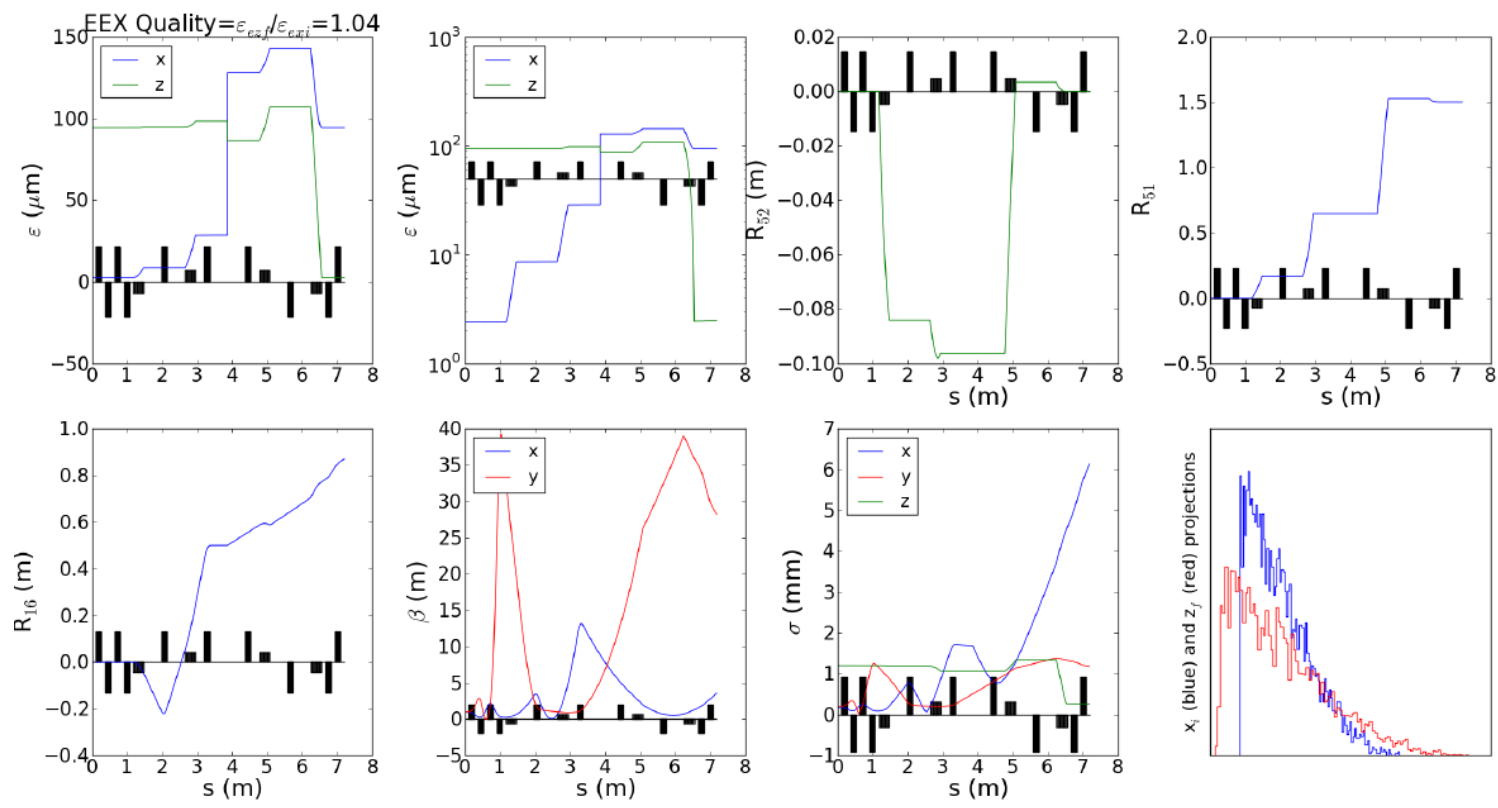

Figure 9: Horizontal (blue trace) and longitudinal (green trace) emittance evolution along the chicane-like emittance exchanger (top row, first two plots). Evolution of cumulated transfer matrix elements (top row last two plots and bottom left plot), beta functions $\left(2^{\text {nd }}\right.$ plot on bottom row). Horizontal (blue trace), vertical (red trace) and longitudinal (green trace) beam rms size evolution (second row $3^{\text {rd }}$ plot). Left plot on second row show the transformation of an incoming linearly ramped horizontal profile downstream of the exchanger to illustrate the production of linear-ramped current distribution [18].

\subsubsection{Transverse-to-Longitudinal Phase Space Exchange Downstream of Cryomodule(s)}

The chicane-type configuration described in the previous section could form the basis for a phase space exchange beamline downstream of the ASTA cryomodule(s). The available realestate would also enable tests of the double emittance exchange beamline suggested by $A$. Zholents. In this configuration, two phase-space exchangers are placed back to back and separated by an appropriate optical lattice; see Figure 10. Locating a mask between the two exchangers would enable beam shaping discussed earlier without, in principle, exchanging the horizontal and longitudinal emittance (an initially transversely round beam would have its final transverse emittances preserved). 


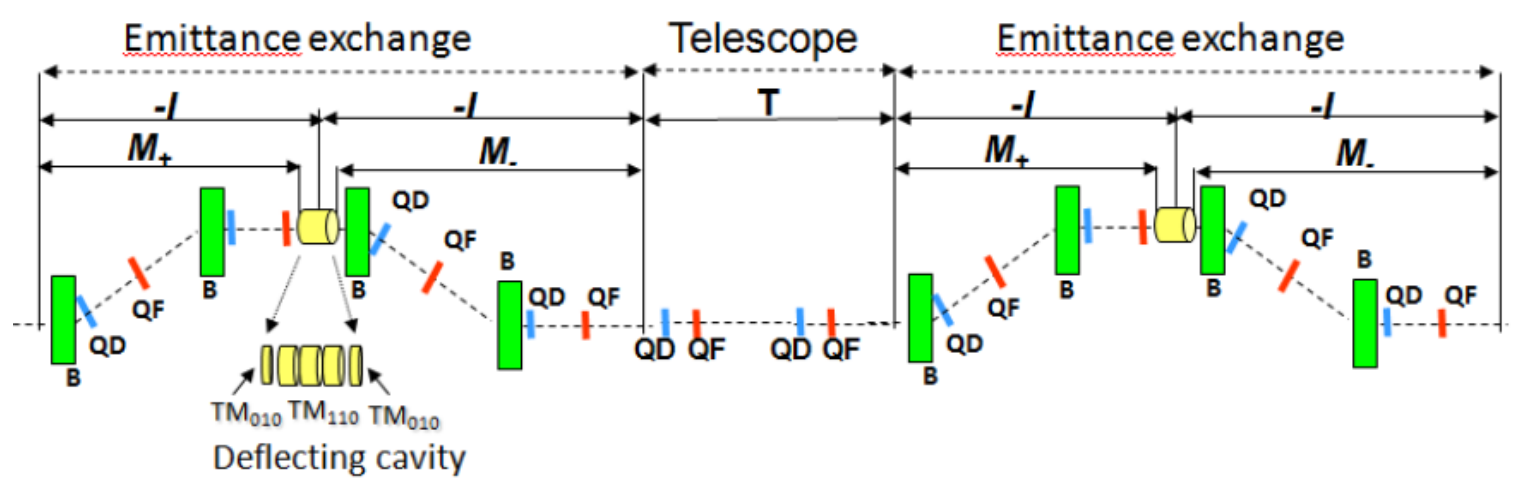

Figure 10: A possible configuration for a double phase space exchange [16].

This configuration could also serve has a bunch compressor that does not require the impression of a correlated energy spread on the incoming beam as needed in a conventional magnetic compressor. In such a scheme, the section between the two exchangers would demagnify the beam horizontally resulting in a shorter bunch length after phase-space exchange in the exchanger. This manipulation has been included in a variant of the compressed harmonic generation ( $\mathrm{CHG}$ ) scheme for seeding short-wavelength free-electron lasers and discussed elsewhere in this proposal.

The overall advantage of such a "double phase space exchanger" configuration compared to a single phase space exchanger is that it leaves the transverse emittances unaffected (under linear optics). This bunch compressor could also be used for frequency up-conversion of the energy modulation provide by the laser interaction with electron beam, and thus can possibly seeding a free-electron laser at a significantly higher harmonics; or it can also be used to do frequency down-conversion for generation of $\mathrm{THz}$ radiation.

The design of such a double EEX beamline would require a detailed study as our preliminary investigation indicate that second-order effects are detrimental and need to be mitigated by inclusion of sextupoles. On another hand, from an order-of-magnitude analysis, LN2-cooled cavities would put a high power demands (several MW) on the klystrons. Thus it is natural to consider a SRF system operating at $3.9 \mathrm{GHz}$ similar to the system developed at Fermilab for kaon separation and longitudinal phase space linearization of the FLASH accelerator at DESY. A possible configuration using the 3.9-GHz deflecting and accelerating mode cavities developed at Fermilab is diagrammed in Figure 11, other solution based on other types of cavities are also being considered. 


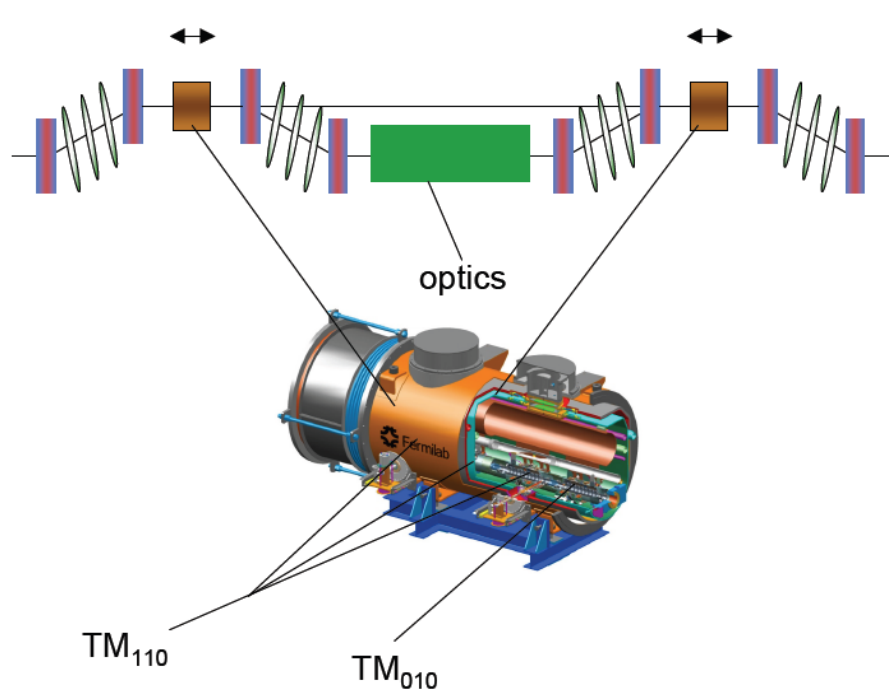

Figure 11: A possible configuration for a $3.9 \mathrm{GHz}$ deflecting/accelerating hybrid SRF structure for a double phase space exchanger in the high energy beamline of ASTA.

\subsubsection{References}

[1] D. A. Edwards, et al., "Status of the flat electron-beam production," Proceedings of the 2001 Particle Accelerator Conference, Chicago, p. 73 (2001).

[2] Y.-E Sun, "Angular-momentum-dominated electron beams and flat-beam generation," PhD Dissertation, University of Chicago (May 2005).

[3] Y.-E Sun et al., Phys. Rev. ST Accel. Beams 7, 123501 (2004).

[4] P. Piot, Y.-E. Sun, and K.-J. Kim, Phys. Rev. ST Accel. Beams 9, 031001 (2006).

[5] T. Koeth, PhD Dissertation, "An Observation of a Transverse to Longitudinal Emittance Exchange at the Fermilab A0 Photoinjector," Rutgers University (May 2009).

[6] J. Ruan, et al., Phys. Rev. Lett. 106, 244801 (2011).

[7] Y.-E Sun et al., Phys. Rev. Lett. 105, 234801 (2010).

[8] P. Piot, et al., Appl. Phys. Lett. 98, 261501 (2011).

[9] N. Yampolski, et al., "Controlling Electron-Beam Emittance Partitioning for Future X-Ray Light Sources," preprint arXiv:1010.1558 (2010).

[10] P. Emma, Z. Huang, K.-J. Kim, and P. Piot, Phys. Rev. ST Accel. Beams 9, 100702 (2006).

[11] K.-J. Kim, Phys. Rev. ST Accel. Beams 6, 104002 (2003).

[12] P. Piot, Proc. IPAC'10, Kyoto, Japan, (23-28 May 2010), p. 4316.

[13] M. Cornacchia and P. Emma, Phys. Rev. ST Accel. Beams 5, 084001 (2002).

[14] P. Piot, et al., Phys. Rev. ST Accel. Beams 14, 022801 (2011).

[15] M. McAshan and R. Wanzenberg, report FERMILAB-TM-2144 (2001).

[16] A. A. Zholents and M. S. Zolotorev, "A New Type of Bunch Compressor and Seeding of a Short Wave Length Coherent Radiation," report ANL/APS/LS-327 (2011).

[17] C. Prokop, P. Piot, B. Carlsten, M. Church, "Beam dynamics performances and applications of a low-energy electron-beam magnetic bunch compressor," Nucl Instrum. A 716, pp. 1728 (2013).

[18] C. Prokop, P. Piot, and B. E. Carlsten, Proc. IPAC13, Shanchai, Japan (12-17 May 2013), 3103 (2013). 


\subsubsection{Optical Stochastic Cooling Experiment at ASTA}

Besides the experiments on highly non-linear integrable optics, the $150 \mathrm{MeV}$ electron storage ring IOTA at ASTA will be used to carry out a test of the optical stochastic cooling (OSC) technique. This method is a novel approach to the beam dynamics paper and has potential serious implications for a range of heavier (than electron) particle accelerators, ranging from LHC and Muon Collider to other rings. Accelerator experts have called for an experimental demonstration of OSC for a long time.

The experiment will have two phases. At the first step the cooling will be achieved without an optical amplifier. It should introduce a damping rate higher than the cooling rate due to synchrotron radiation. At the second phase, an optical amplifier will be used.

The ASTA facility will offer unique opportunity to carry out the proposed research toward demonstration of the feasibility of the optical stochastic cooling technique. That research requires a dedicated storage ring (IOTA) and its operation with 100-150 MeV electrons. It cannot be carried out anywhere else as there are no existing electron storage rings in that energy range which can afford installation of special insertions (optical equipment, wigglers, etc.), and offers special arrangements of the optics lattice and precise control of the insertion devices and the ring elements. Previous attempts to identify such an existing facility were unsuccessful (e.g., the proposal to use the MIT-Bates storage ring was found to be very expensive as the ring nominal energy and size were significantly beyond what was needed for the OSC demonstration).

\subsubsection{Introduction}

The stochastic cooling suggested by Simon Van der Meer [1,2] has been successfully used in a number of machines for particle cooling and accumulation. However it was not helpful for cooling of bunched beams in proton-(anti)proton colliders due to very high phase density of the bunches. Only recently, bunched beam stochastic cooling has been introduced in operation at the Relativistic Heavy Ion Collider (RHIC) [3]. In the case of optimal cooling the maximum damping rate can be estimated as:

$$
\lambda \approx \frac{2 W \sigma_{s}}{N C}
$$

where $W$ is the bandwidth of the system, $N$ is the number of particles in the bunch, $\sigma_{s}$ is the rms bunch length, and $C$ is the machine circumference. For the LHC proton beam $\left(\sigma_{s}=9 \mathrm{~cm}, C=\right.$ $26.66 \mathrm{~km}$ ) and a system with one octave bandwidth and its upper boundary of $8 \mathrm{GHz}$ one obtains $\lambda^{-1}=12000$ hour. An effective cooling requires faster damping rates by least 3 orders of magnitude. The OSC suggested by Mikhailichenko, Zolotorev, and Zholents [4,5] can have a bandwidth of $\sim 10^{14} \mathrm{~Hz}$ and, thus, suggests a way to achieve required damping rates. The basic principles of the OSC are similar to the normal (microwave) stochastic cooling except that it uses optical frequencies, allowing an increase of system bandwidth by 4 orders of magnitude.

Proposal for an Accelerator R\&D User Facility at Fermilab’s Advanced Superconducting Test Accelerator 
In the OSC a particle radiates EM radiation in the pickup wiggler. Then, the radiation amplified in an optical amplifier makes a longitudinal kick to the particle in the kicker wiggler as shown in Figure 1. Further we will call these wigglers pickup and kicker. A magnetic chicane is used to make space for an optical amplifier and to bring the particle and the radiation together in the kicker wiggler. In further consideration we assume that the path lengths of particle and radiation are adjusted so that the relative particle momentum change is equal to:

$$
\delta p / p=-\kappa \sin (k \Delta s) .
$$

Here $k=2 \pi / \lambda$ is the radiation wave number, and $\Delta s$ is the particle displacement on the way from the pickup wiggler to the kicker wiggler relative to the reference particle which obtains zero kick:

$$
\Delta s=M_{51} x+M_{52} \theta_{x}+M_{56}(\Delta p / p)
$$

Here $M_{5 n}$ are the elements of $6 \times 6$ transfer matrix from pickup to kicker, $x, \theta_{x}$ and $\Delta p / p$ are the particle coordinate, angle and relative momentum deviation in the pickup.

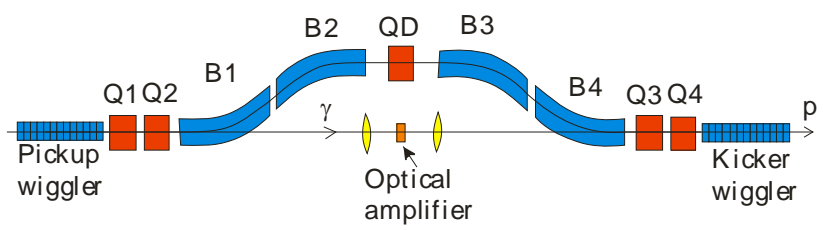

Figure 1: Schematic of Optical Stochastic Cooling (OSC).

For small amplitude oscillations the horizontal and vertical cooling rates are [6]:

$$
\left[\begin{array}{c}
\lambda_{x} \\
\lambda_{s}
\end{array}\right]=\frac{k \kappa}{2}\left[\begin{array}{c}
M_{56}-C \eta_{p k} \\
C \eta_{p k}
\end{array}\right],
$$

where $\eta_{p k}=\left(M_{51} D_{p}+M_{52} D_{p}^{\prime}+M_{56}\right) / C$ is the partial momentum compaction determined so that for a particle without betatron oscillations and with momentum deviation $\Delta p / p$ the longitudinal displacement relative to the reference particle on the way from pickup and kicker is equal to $C \eta_{p k} \Delta p / p$. Here we also assume that there is no $x-y$ coupling. Introduction of $x-y$ coupling outside the cooling area allows redistribution of horizontal damping rate into both transverse planes. The sum of damping rates, $\Sigma \lambda_{n}=k \kappa M_{56} / 2$, does not depend on the beam optics outside of the cooling chicane.

An increase of betatron and synchrotron amplitudes results in a decrease of damping rates [6]:

$$
\begin{aligned}
& \lambda_{x}\left(a_{x}, a_{s}\right)=F_{x}\left(a_{x}, a_{s}\right) \lambda_{x}, \\
& \lambda_{s}\left(a_{x}, a_{s}\right)=F_{s}\left(a_{x}, a_{s}\right) \lambda_{s},
\end{aligned}
$$

where the form factors are:

$$
\begin{aligned}
& F_{x}\left(a_{x}, a_{s}\right)=2 J_{0}\left(a_{s}\right) J_{1}\left(a_{x}\right) / a_{x}, \\
& F_{s}\left(a_{x}, a_{s}\right)=2 J_{0}\left(a_{x}\right) J_{1}\left(a_{s}\right) / a_{s} ;
\end{aligned}
$$


and $a_{x}$ and $a_{s}$ are the amplitudes of longitudinal particle motion due to betatron and synchrotron oscillations expressed in the units of e.-m. wave phase:

$$
\begin{aligned}
& a_{x}=k \sqrt{\varepsilon_{1}\left(\beta_{p} M_{51}{ }^{2}-2 \alpha_{p} M_{51} M_{52}+\left(1+\alpha_{p}{ }^{2}\right) M_{52}{ }^{2}\right)}, \\
& a_{s}=k C\left|\eta_{p k}\right|(\Delta p / p)_{\max } .
\end{aligned}
$$

Here $\varepsilon_{1}$ is the Courant-Snyder invariant of a particle, and $(\Delta p / p)_{\max }$ is its maximum momentum deviation. As one can see from Eqs. (4) and (5), the damping rate changes sign if any of amplitudes exceeds the first root of the Bessel function $\mathrm{J}_{0}(x), a_{x,} a_{s}>\mu_{0} \approx 2.405$.

The following conclusions can be drawn from Eqs. (3) and (6). $M_{56}$ depends only on focusing inside the chicane, while $\eta_{p k}$ additionally depends on the dispersion at the chicane beginning, i.e. on the optics in the rest of the ring. Consequently, the damping rates ratio,

$$
\lambda_{x} / \lambda_{s}=M_{56} / C \eta_{p k}-1
$$

and the longitudinal cooling range,

$$
n_{\sigma s} \equiv(\Delta p / p)_{\max } / \sigma_{p}=\mu_{0} /\left|k C \eta_{p k} \sigma_{p}\right|,
$$

depend on focusing and dispersion inside the chicane, but do not depend on the beta-function. Here $\sigma_{p}$ is the relative rms momentum spread. In contrast, the transverse cooling range,

$$
n_{\sigma x} \equiv \frac{\varepsilon_{\max }}{\varepsilon}=\frac{\mu_{0}{ }^{2} /\left(k^{2} \varepsilon\right)}{\beta_{p} M_{51}{ }^{2}-2 \alpha_{p} M_{51} M_{52}+\left(1+\alpha_{p}{ }^{2}\right) M_{52}{ }^{2}},
$$

does not depend on the dispersion but depends on the beta-function. Here $\varepsilon$ is the rms transverse emittance.

Below we consider two cooling schemes. The first one is passive cooling [7] where radiation is focused into the kicker wiggler but is not amplified; and the second one is active where an optical amplifier is used. Both of them have its advantages and drawbacks. In the case of passive cooling one does not need an amplifier and, consequently, can use higher optical frequencies and larger bandwidth which boost the gain. It also requires smaller path difference which considerably increases the cooling ranges, $n_{\sigma s}$ and $n_{\sigma x}$. In the case of an active system one can reduce the length and magnetic field of the wigglers, but it requires an additional delay in the chicane to compensate a delay in the optical amplifier ( $5 \mathrm{~mm})$. Making an amplifier at required power and wavelength can be a challenging problem too.

\subsubsection{Beam Optics}

The main parameters of the ring, called IOTA [6], are shown in the Table I. The OSC system will take one of four straight sections with length of $\sim 5 \mathrm{~m}$. The beta-function and dispersion in the section are presented in Figure 2. The optics was built for $800 \mathrm{~nm}$ radiation where an optical amplification is a feasible task. The following limitations were taken into account in the optics 
design. The chicane should separate the radiation and the beam by $40 \mathrm{~mm}$ making a sufficiently large separation between the electron beam and optical amplifier. The cooling ranges, $n_{\sigma s}$ and $n_{\sigma x}$, (before the OSC is engaged) have to be large enough so that the major fraction of the beam would be cooled. The path length difference acquired by electron beam in the chicane has to be large enough to compensate delay in optical amplifier. Note that the rectangular dipoles do not produce horizontal focusing. Therefore in the absence of other focusing inside chicane the partial slip factor is equal to $M_{56} / C$ and does not depend on the dispersion. Consequently, there is no transverse cooling. To achieve it a defocusing quad was introduced in the chicane center. The strength of this quad is limited by reduction of transverse cooling range, $n_{\sigma x}$ which requires sufficiently large dispersion in the chicane. The major parameters of the cooling section are presented in Table 2.

Table 1: Main Parameters of IOTA storage ring configured for OSC.

\begin{tabular}{|l|c|}
\hline Circumference & $38.7 \mathrm{~m}$ \\
\hline Nominal beam energy & $150 \mathrm{MeV}$ \\
\hline Bending field & $7 \mathrm{kG}$ \\
\hline Betatron tune & $3.5 \div 7.2$ \\
\hline Maximum $\beta$-function & $3 \div 9 \mathrm{~m}$ \\
\hline $\begin{array}{l}\text { Transverse vertical emittance, } \\
\text { non-normalized }\end{array}$ & $3 \mathrm{~nm} \mathrm{r.m.s}$ \\
\hline Rms momentum spread, $\sigma_{p}$ & $1.5 \cdot 10^{-4}$ \\
\hline SR damping rates (ampl.), $\lambda_{s} / \lambda_{\perp}$ & $4 / 2 \mathrm{~s}^{-1}$ \\
\hline
\end{tabular}

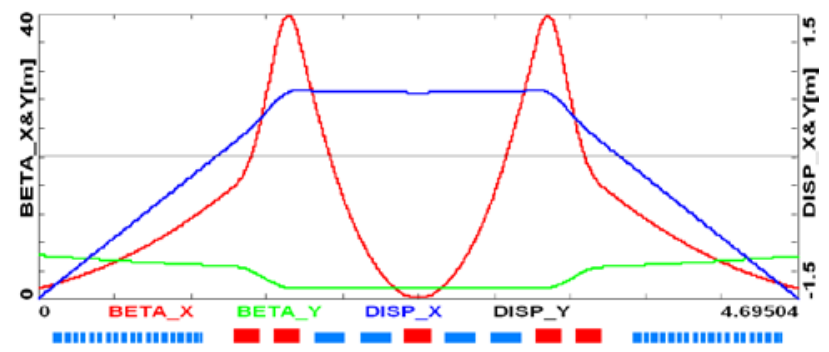

Figure 2: Optical lattice functions in the OSC section. 
Table 2: Major parameters of chicane beam optics.

\begin{tabular}{|l|c|}
\hline$M_{56}$ & $8.7 \mathrm{~mm}$ \\
\hline Cooling rates ratio, $\lambda_{x} / \lambda_{s}$ & 7.5 \\
\hline Horizontal beam separation & $40 \mathrm{~mm}$ \\
\hline Delay in the chicane & $4.5 \mathrm{~mm}$ \\
\hline Cooling ranges (before OSC), $n_{\sigma x} / n_{\sigma s}$ & $3.5 / 2$ \\
\hline Dipole magnetic field & $4 \mathrm{kG}$ \\
\hline Dipole length & $18 \mathrm{~cm}$ \\
\hline Strength of central quad, $\int G d L$ & $1.52 \mathrm{kG}$ \\
\hline Strength of central quad, $\int G d L$ & $1.52 \mathrm{kG}$ \\
\hline
\end{tabular}

The rms emittance and momentum spread are comparatively large for the chosen wavelength of $800 \mathrm{~nm}$. To accommodate it the optics was tuned to maximize the cooling ranges. In particular, we choose (1) the large cooling rates ratio to increase $n_{\sigma s}$ and (2) small betafunction in the chicane center $(2 \mathrm{~cm})$ to increase $n_{\sigma x}$. That resulted in high sensitivity of cooling parameters. Simulations show that relative accuracies should be $\sim 1 \%$ for the horizontal betafunction, $\sim 2 \mathrm{~cm}$ for the dispersion, and $\sim 2 \%$ for the focusing of central quadrupole (see Figure 3).
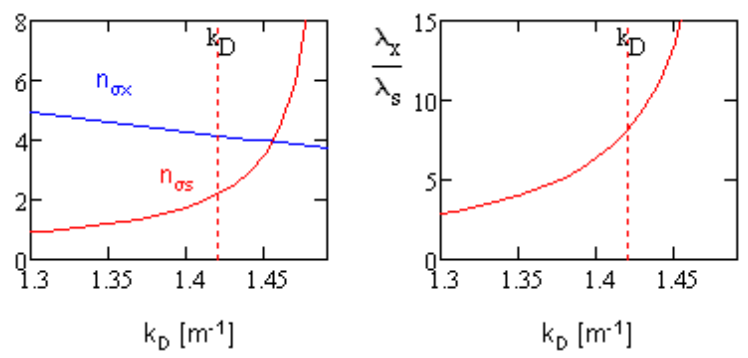

Figure 3: Dependencies of cooling ranges (left) and ratio of damping rates on focusing strength of central quadrupole. 


\subsubsection{Light Optics}

Let a particle move in the flat undulator so that its coordinates depend on time as following:

$$
\begin{aligned}
& \mathrm{v}_{x}=c \theta_{e} \sin \tau^{\prime}, \quad \mathrm{v}_{y}=0, \\
& \mathrm{v}_{z}=c\left(1-\frac{1}{2 \gamma^{2}}-\frac{\theta_{e}^{2}}{2} \sin ^{2} \tau^{\prime}\right), \tau^{\prime}=\omega_{u} t^{\prime}+\psi,
\end{aligned}
$$

where $\gamma$ is the particle relativistic factor $(\gamma>>1)$, and $\omega_{u}$ is the frequency of particle motion in the undulator. Substituting velocities of Eq. (10) to the Liénard-Wiechert formula [9] for the horizontal component of electric field in the far zone one obtains:

$$
\begin{aligned}
& E_{x}(r, t)=4 e \omega_{u} \gamma^{4} \theta_{e} \cos \tau^{\prime} \times \\
& \frac{1+\gamma^{2}\left(\theta^{2}\left(1-2 \cos ^{2} \phi\right)-2 \theta \theta_{e} \sin \tau^{\prime} \cos \phi-\theta_{e}^{2} \sin ^{2} \tau^{\prime}\right)}{c R\left(1+\gamma^{2}\left(\theta^{2}+2 \theta \theta_{e} \sin \tau^{\prime} \cos \phi+\theta_{e}^{2} \sin ^{2} \tau^{\prime}\right)\right)^{3}},
\end{aligned}
$$

where $\theta$ and $\phi$ are the angles for the vector from the radiation point, $\mathbf{r}^{\prime}$, to the observation point, $\mathbf{r}$, in the polar coordinate system, $R=\left|\mathbf{r}-\mathbf{r}^{\prime}\right|$, and $t-t^{\prime}=R / c$. In further calculations we will be leaving the radiation in the first harmonic only,

$$
\begin{aligned}
& E_{\omega}(r)=\frac{\omega(\theta)}{\pi} \int_{0}^{2 \pi / \omega(\theta)} E_{x}(r, t) \mathrm{e}^{-i \omega t} d t, \\
& \omega(\theta)=2 \gamma^{2} \omega_{u} /\left(1+\gamma^{2}\left(\theta^{2}+\theta_{e}^{2} / 2\right)\right),
\end{aligned}
$$

assuming that the radiation of higher harmonics is absorbed in the lenses and/or not amplified by optical amplifier. Then taking into account delay in the lens and applying Kirchhoff formula,

$$
E\left(r^{\prime \prime}\right)=\frac{1}{2 \pi i c} \int_{S} \frac{\omega(\theta) E_{\omega}(r)}{\left|r^{\prime \prime}-r\right|} \mathrm{e}^{i \omega\left|r^{\prime \prime}-r\right|} d s,
$$

one obtains the electric field in the focal point. For a large acceptance lens, $\theta_{m} \geq \theta_{e}+3 / \gamma_{\text {, }}$ located in the middle of pickup-to-kicker distance the results of numerical integration can be interpolated by the following equation:

$$
\begin{aligned}
& E_{x}=4 e \omega_{u}{ }^{2} \gamma^{4} \theta_{e} F\left(\gamma \theta_{e}\right) /\left(3 c^{2}\right), \\
& F(K) \approx 1 /\left(1+2.15 K^{2}+1.28 K^{4}\right), \quad K \leq 4,
\end{aligned}
$$

where $K=\gamma \theta_{e}$ is the undulator parameter, and $\theta_{m}$ is the lens angular size from the radiation point. Integrating the force along the kicker length one obtains the longitudinal kick amplitude:

$$
c \delta p_{\max } \equiv \kappa c p=2 e^{4} B_{0}^{2} \gamma^{2} L F(K) /\left(3 m^{2} c^{4}\right) .
$$

The bandwidth of the system is much more narrow $(\leq 10 \%)$ if an optical amplifier is used. In this case the kick value is: 


$$
c \delta p_{\max }=\frac{2 e^{4} B_{0}{ }^{2} \gamma^{4} L \theta_{\mathrm{m}}{ }^{2}}{m^{2} c^{4}\left(1+K^{2}\right)^{3}}=\frac{2 e^{4} B_{0}{ }^{2} \gamma^{4} L}{m^{2} c^{4}\left(1+K^{2}\right)^{3}} \frac{\Delta \omega}{\omega},
$$

where in the second equality we assumed that $\gamma^{2} \Delta \theta_{m}^{2}=\left(1+K^{2}\right) \Delta \omega / \omega$.

Above we assumed that the radiation coming out from the pickup is focused at the particle location throughout the entire course of the particle motion in the kicker. This can be achieved if the distance to the lens is much larger than the length of wiggler-a condition which is impossible to fully achieve in practice. A practical solution can be obtained with lens telescope which has the transfer matrix from the center of pickup to the center of kicker equal to $\pm \mathbf{I}$, where $\mathbf{I}$ is the identity matrix. The simplest telescope has 3 lenses as shown in Figure 4 . For symmetrically located lenses their focusing distances are:

$$
F_{1}=\frac{L L_{1}}{L+L_{1}}, \quad F_{2}=\frac{L_{1}^{2}}{2\left(L+L_{1}\right)}
$$

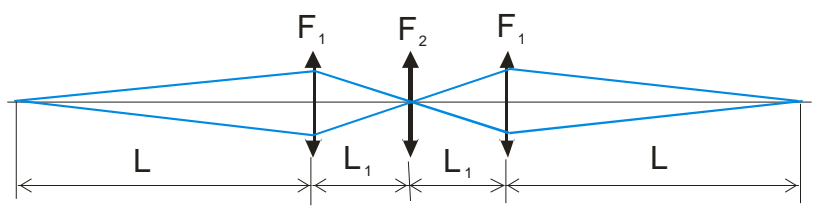

Figure 4: Light optics layout for passive cooling.

Table 3 presents the main parameters of undulators, light optics, and OSC damping rates for the passive and active OSC. The passive cooling requires about one octave band $(0.8-1.6 \mu \mathrm{m})$. The wave packet lengthening looks satisfactory for $4.5 \mathrm{~mm}$ light delay in magnesium fluoride. However a suppression of transverse focusing chromaticity looks to be an extremely challenging problem and needs additional study. Combination of glasses with normal and abnormal dispersions might be a good direction for study. A Ti:Sapphire optical amplifier is considered a good candidate capable of delivering $20 \mathrm{~dB}$ gain within the allocated signal delay. Technical details are presently under study. 
Table 3: Main parameters of Optical Stochastic Cooling (OSC).

\begin{tabular}{|l|c|}
\hline Undulator parameter, $\mathrm{K}$ & 1.5 \\
\hline Undulator period & $6.53 \mathrm{~cm}$ \\
\hline Number of periods & 14 \\
\hline Total undulator length & $0.915 \mathrm{~m}$ \\
\hline Distance between undulators & $3.6 \mathrm{~m}$ \\
\hline Telescope length, $2 L_{1}$ & $0.25 \mathrm{~m}$ \\
\hline Telescope aperture, $2 \mathrm{a}$ & $40 \mathrm{~mm}$ \\
\hline Lens focal distances, $F_{1} / F_{2}$ & $116 / 4.3 \mathrm{~mm}$ \\
\hline Damping rates of passive OSC $(x / y / s)$ & $100 / 100 / 25 \mathrm{~s}^{-1}$ \\
\hline Damp. rates $20 \mathrm{~dB}$ gain \& $10 \%$ band & $300 / 300 / 75 \mathrm{~s}^{-1}$ \\
\hline
\end{tabular}

\subsubsection{References}

[1] S. Van der Meer, "Stochastic damping of betatron oscillations," internal report CERN/ISR $\mathrm{PO} / 72 / 31$ (1972).

[2] J. Bisognano and C. Leemann, "Stochastic Cooling," in 1981 Summer School on High Energy Particle Accelerators, AIP Conf. Proceedings 87, Melville, NY, 1982, pp. 584-655.

[3] M. Blaskiewicz, et al., Phys. Rev. Lett. 105, 094801 (2010).

[4] A.A. Mikhailichenko and M.S. Zolotorev, Phys. Rev. Lett. 71, 4146 (1993).

[5] M. S. Zolotorev and A. A. Zholents, Phys. Rev. E, 50, 4, p. 3087 (1994).

[6] V. Lebedev, page 644, HB-2010, (2012).

[7] A. Mikhailichenko, Phys. Rev. ST Accel. Beams 11, 011302 (2008).

[8] S. Nagaitsev, et al., p. 16, IPAC-2012 (2012).

[9] L. D. Landau, "Classical theory of fields," Nauka (1973). 


\subsubsection{Flat-Beam-Driven Dielectric-Wakefield Acceleration in Slab Structures}

The availability of flat beams with high-repetition rate unique to ASTA would enable the testing of multi-bunch acceleration in dielectric-wakefield structures and the studying of possible dynamical effects. In the longer term, the inclusion of a transverse-to longitudinal phase space exchanger will add the capability to explore acceleration with enhanced transformer ratio.

Next generation multi-TeV high-energy-physics lepton accelerators are likely to be based on non-conventional acceleration techniques given the limitation of radio-frequency ( $\mathrm{rf}$ ) normaland superconducting cavities. Non-conventional approaches based on the laser-plasma wakefield accelerators have recently demonstrated average energy gradients one order of magnitude higher than those possible with state-of-the art conventional structures [1]. Another class of non-conventional accelerating techniques includes beam-driven methods which rely on using wakefields produced by high charge "drive" bunches traversing a high-impedance structure to accelerate subsequent "witness" bunches [2]; see Figure 1. Such an approach has the advantage of circumventing the use of an external power source and can therefore operate at $\mathrm{mm}$ and sub-mm wavelengths. Structures capable of supporting wakefield generation include plasmas [3], and dielectric-loaded waveguides (DLW's) [4].

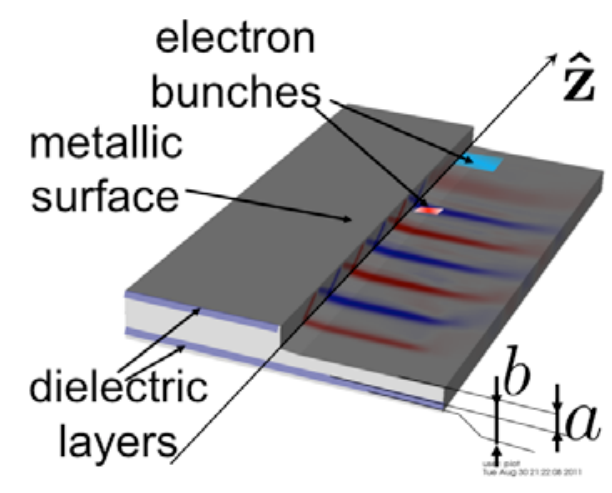

Figure 1: Configuration for beam-driven dielectric-wakefield acceleration in a slab dielectric-lined waveguide (DLW). The electron bunches include the drive (cyan) and witness (red) bunches. The darker red and blue field patterns represent the axial electric field in the structure's mid-plane. 


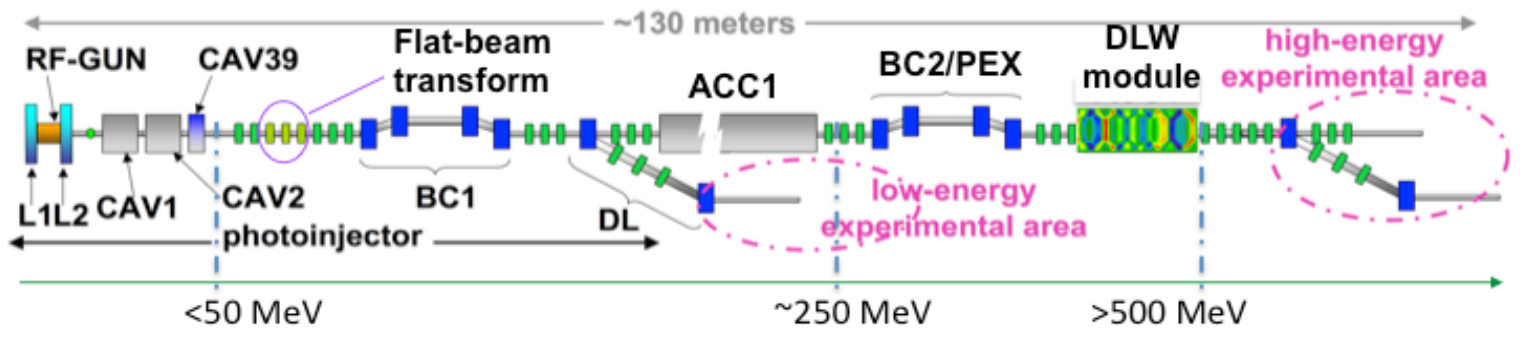

Figure 2: Overview of the ASTA facility with DLW module discussed in Ref. [10]. The beam energy could be boosted from 250 to $500 \mathrm{MeV}$ and eventually from $500 \mathrm{MeV}$ to 1 GeV. BC2/PEX represents an additional beamline insertion that could serve as a secondstage bunch compressor or phase space exchanger.

The proposal aims at testing asymmetric dielectric-loaded waveguides driven by flat beams with high-transverse emittance ratios. ASTA's capability to generate long bunch train will be used to explore limitations associated to the acceleration of bunch trains in DLW structures. Finally, at a later stage, the bunch-current tailoring capabilities of ASTA will also be used to demonstrate high-gradient acceleration with an enhanced transformer ratio.

\subsubsection{Past and Current Researches on Dielectric Wakefield Acceleration and Opportunities at ASTA}

Dielectrics can in principle sustain significantly higher electric surface fields. For example, in the case of fused silica tubes the onset for breakdown was observed when peak fields at the surface of the material reached approximately $13 \mathrm{GV} / \mathrm{m}$ [5]. Based on these observations it is tempting to replace the standard RF cavities with dielectric structures that can sustain much higher field gradients. Work on dielectric-lined waveguide (DLW) structures has been pioneered at Argonne in the late 1980s [6].

Until recently most of the work has been focused on cylindrical DLWs. Another class structure, the slab DLW (see Figure 1), have been proposed as better candidates for acceleration as they offer better stability, tunability and are easier to manufacture $[7,8]$. Because of the high transverse asymmetry of the structures, flat drive bunches that will be readily available at ASTA are better match to fully exploit the advantages of DLWs. In addition dynamical effects related to multi-bunch effects could be investigated because of available beam format unique to superconducting linacs. To date, and very recently, only preliminary studies on slab DLWs have been carried using round beams [9]. The ability to also investigate the performance of DLW for multi-bunch acceleration would possibly provide a demonstration of a multi-user shortwavelength-FEL concept recently proposed [11]. A successful completion of a proof-of-principle experiment demonstrating acceleration in a DLW, could also form the basis of future energy upgrades of ASTA that could eventually support some of the experiments demanding beam energies around $1 \mathrm{GeV}$ [10] using an implementation similar to the one detailed in Ref. [11] as outlined in Figure 2. Finally, the experimental techniques developed for this experiment will be beneficial to other beam-driven acceleration concepts that might be tested at ASTA (e.g. plasma wakefield acceleration). This proposal builds on R\&D being performed at Northern 
Illinois University under financial support from the Defense Threat Reduction Agency along with past or on-going collaborations between Northern Illinois University and the Argonne Wakefield Accelerator, Euclid Lab LLC, DESY, Tech X, and the University of Hamburg.
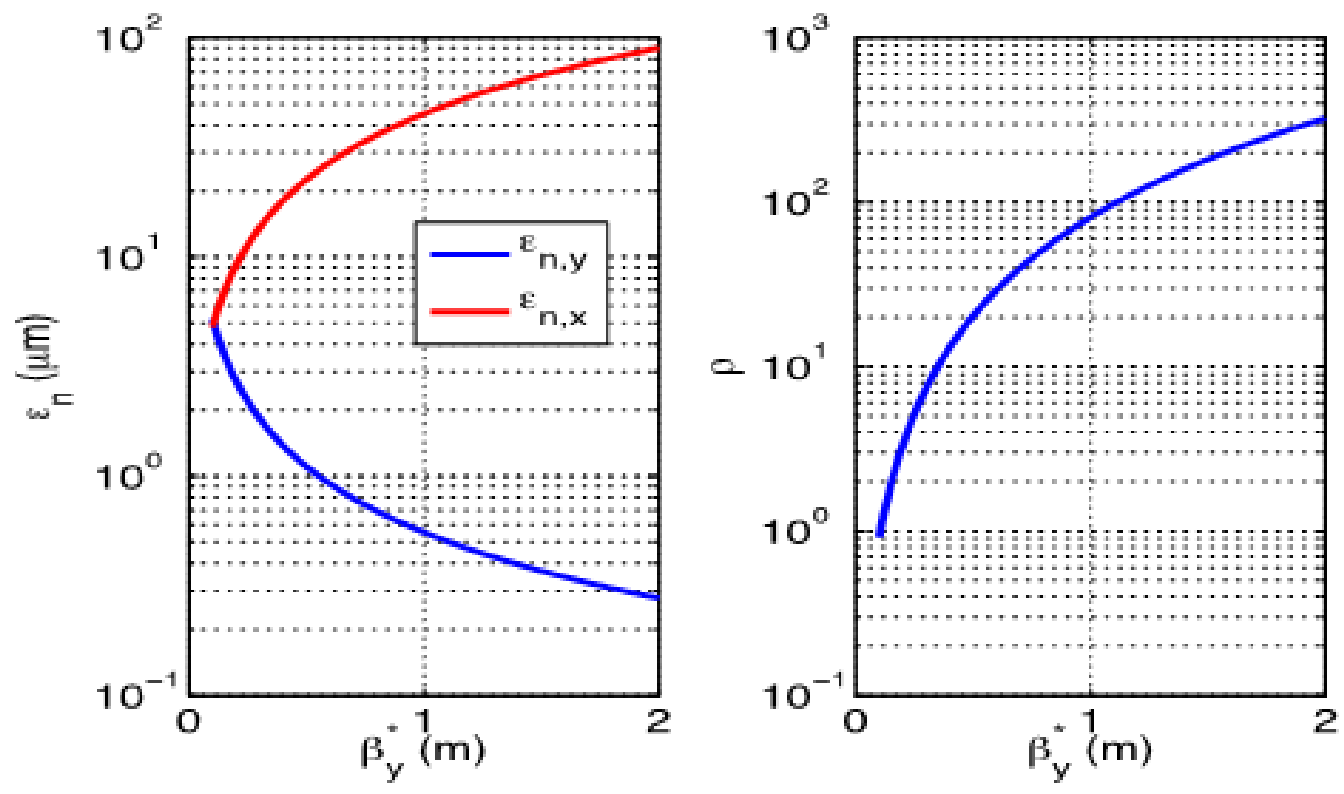

Figure 3: Required beam's transverse normalized emittances (left) and corresponding emittance ratio (right) as a function of the achieved minimum betatron function inside the DLW. The DLW's length and half-gap are respectively $L=5 \mathrm{~cm}$ and $a=100 \mu \mathrm{m}$.

\subsubsection{Technical Approach}

An issue associated to the use of cylindrical-symmetric DLW structures stems from the requirement on beam parameters and especially on the beam emittances. Given the DLW structure's inner radius $a$ and axial length $L$, the bunch normalized emittance has to satisfy

$$
\varepsilon_{n, \perp} \leq \frac{4 \gamma \beta^{*} a^{2}}{9\left(4 \beta^{*}+L^{2}\right)}
$$

where $\beta^{*}$ is the betatron function at the waist (located at the structure's center) and $\gamma$ the beam's Lorentz factor. The latter requirement is very stringent especially for moderate values of $\gamma$. For instance, considering acceleration through one cryomodule $\gamma \approx 500$, and taking $\beta^{*}=0.1 \mathrm{~m}, \mathrm{a}=100 \mu \mathrm{m}$ and $\mathrm{L}=5 \mathrm{~cm}$ would require a transverse emittance of $0.5 \mathrm{~mm}$. Such an emittance is challenging to achieve for bunch charge larger than $1 \mathrm{nC}$. Therefore, the flat beam technique developed at Fermilab combined with a slab DLW structure appears to be a viable path toward to generation of high-gradient electric field. 


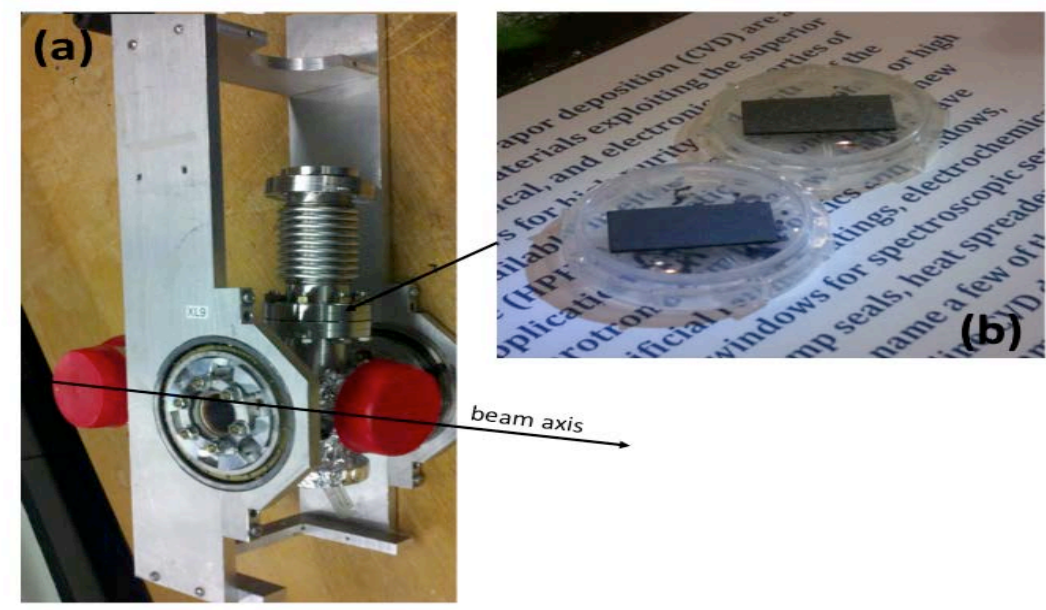

Figure 4: Assembled goniometer (a) and diamond slab structures (b) to be used in the proposed DWFA experiment. Photo (b) is courtesy of Euclid Lab LLC.

The previous equation sets a requirement for the normalized vertical emittance (assume to the smallest of the flat beam emittance)

$$
\varepsilon_{n, y} \leq \frac{4 \gamma \beta_{y}^{*} a^{2}}{9\left(4 \beta_{y}^{* 2}+L^{2}\right)}
$$

with $a$ now being the half-gap size of the structure and the subscript ${ }_{y}$ indicates the value is associated to the vertical degree of freedom. The product of the two transverse emittances is related to the four-dimensional emittance via

$$
\varepsilon_{n, x} \varepsilon_{n, y}=\varepsilon_{n, 4 D} \equiv \sqrt{\langle X \tilde{X}\rangle}
$$

where $X=\left(x, x^{\prime}, y, y^{\prime}\right)$, so that $\langle X \tilde{X}\rangle$ refers to the four-dimensional transverse beam matrix (the absolute-value sign indicates the determinant operator). Furthermore, we can write $\varepsilon_{n, 4 D} \equiv \varepsilon_{n, \perp}^{2}$. In the round-to-flat beam transformation transverse emittances with ratio $\rho \equiv \varepsilon_{n, x} / \varepsilon_{n, y}>1$ are produced which implies that the horizontal emittance has to fulfill the inequality

$$
\varepsilon_{n, x} \geq \frac{9 \varepsilon_{n, \perp}^{2}\left(4 \beta_{y}^{* 2}+L^{2}\right)}{4 \gamma \beta_{y}^{*} a^{2}},
$$

to be consistent with the requirement on $\varepsilon_{n, y}$. Finally, the minimum horizontal beam size inside the DLW structure is $\sigma_{x}=\sqrt{\beta_{x}^{*} \varepsilon_{n, x}}$ where $\beta_{x}^{*}$ refers to the horizontal betatron function. At ASTA we intend to take full advantage of the beam manipulations method that will be integrated in the facility. 
The flat-beam method discussed in Section 7.3.2 (Advanced Phase Space Manipulations) of this proposal can be used to tailor the beam emittances such as to match the aforementioned requirements [12]. Recent simulations have shown that a 3.2-nC bunch can have its transverse emittances partitioned to $\left(\varepsilon_{n, x}, \varepsilon_{n, y}\right) \approx(0.5,50) \mu \mathrm{m}$ consistent with typical optimized roundbeam emittances of $\varepsilon_{n, \perp} \approx 5 \mu \mathrm{m}$ [13]. The latter emittance partition with achievable minimum betatron functions of approximately $0.5 \mathrm{~m}$ would results in $\mathrm{rms}$ spot sizes of $\left(\varepsilon_{n, x}, \varepsilon_{n, y}\right)=(0.5,50) \mu \mathrm{m}$ at $250 \mathrm{MeV}(\gamma \approx 500)$. These beam sizes are compatible with a structure's half-gap value of $\boldsymbol{a}=\mathbf{1 0 0 \mu \mathrm { m }}$ which is necessary to produce $\mathrm{THz}$ wakefields as depicted in Figure 2.

Another important element to beam driven-acceleration would be the tailoring of the electron bunch's current profile. Several avenues are currently being explored to perform such bunchshaping technique. One of them directly builds on the use of a transverse-to-longitudinal phasespace-exchange technique pioneered at Fermilab's A0 photoinjector. As discussed in Ref. [14] and depicted in Figure 5, such a phase space exchanger could be used to tailor the profile of the drive bunch and create a low-charge witness bunch. Other methods based the impression of nonlinear longitudinal-phase-space correlation [16] are also under investigation.
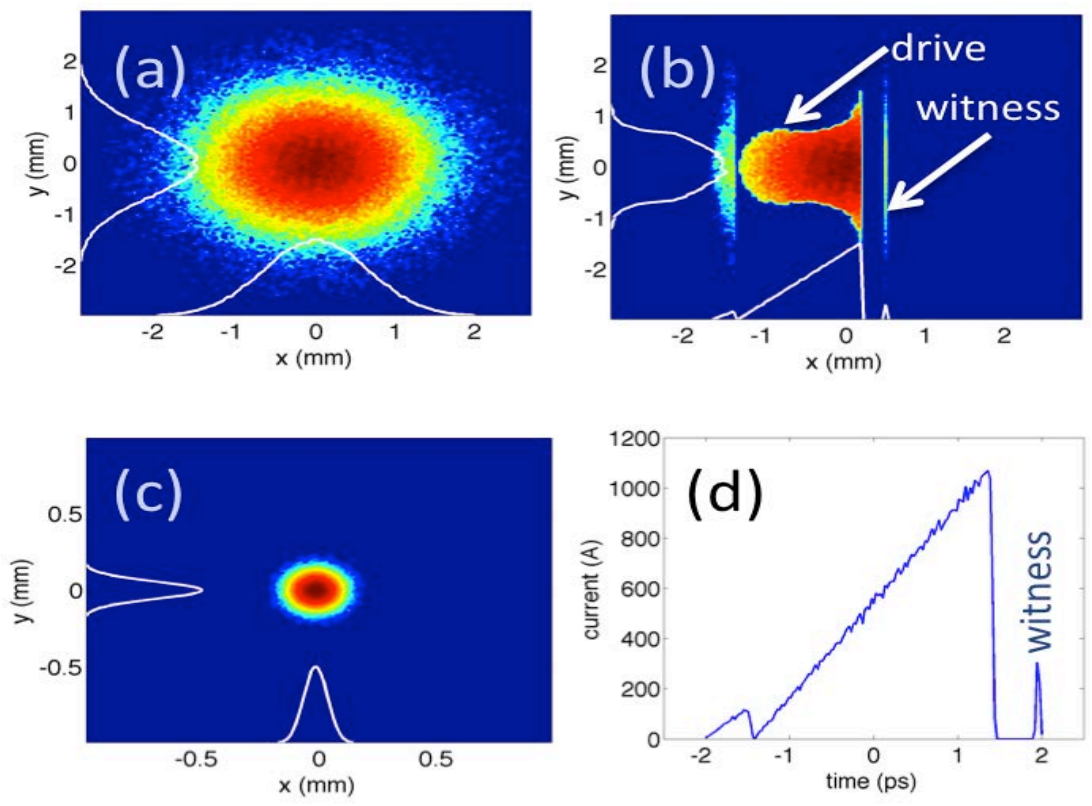

Figure 5: Double-triangular bunch generation using an EEX-based bunch shaper, where the transverse mask is tailored to generate a double-triangular bunch ["drive" in (b)] followed by a low-charge main bunch ["witness" in (b) and (d)]. (a) and (b) show the transverse beam density before and after the mask, respectively. (c)-(d) plot the beam density at the EEX exit, with (c) the transverse profile and (d) the current profile (positive time corresponds to the tail of the bunch [11]. The advantages of the doubletriangle distribution are detailed in Ref. [15]. 


\subsubsection{Experimental Plans}

We expect this proposal to be carried in several stages with requirements on beam parameters consistent with the incremental upgrade of ASTA. The experimental test of dielectric structure is in itself straightforward and most of the work will be related to shaping the beam to match the desired requirements. The following four-phase approach is proposed:

\section{Phase I: 50-MeV flat beams:}

The goal of this phase would be to demonstrate the propagation of a 50-MeV flat beam in a slab DLW structure. The structure would be located behind the bunch compressor (BC1 in the photoinjector) or in the off-axis low-energy user line. A downstream spectrometer would enable the measurement of energy loss and self-modulation (as the bunch would be longer than the wavelength of the mode supported by the DLW). The bunch charge would be varied. The structure would be located in a goniometer currently being assembled and to be tested at the HBESL facility in support to another experiment. The assembly is pictorially shown in Figure 4.

\section{Phase II: 50-MeV tailored flat beams:}

The goal of this phase would be to demonstrate some acceleration using a compressed flat beam with a tailored temporal profile using a method similar to Ref. [16] (but here the nonlinearities will be introduced using another, simpler, method rather than using a 3.9-GHz cavity [17]). In this phase the photocathode would be split to provide a highcharge $(1-2 \mathrm{nC})$ drive bunch followed by a low charge $(<100 \mathrm{pC})$ witness bunch and acceleration of the witness bunch would be explored. See Figure 6.

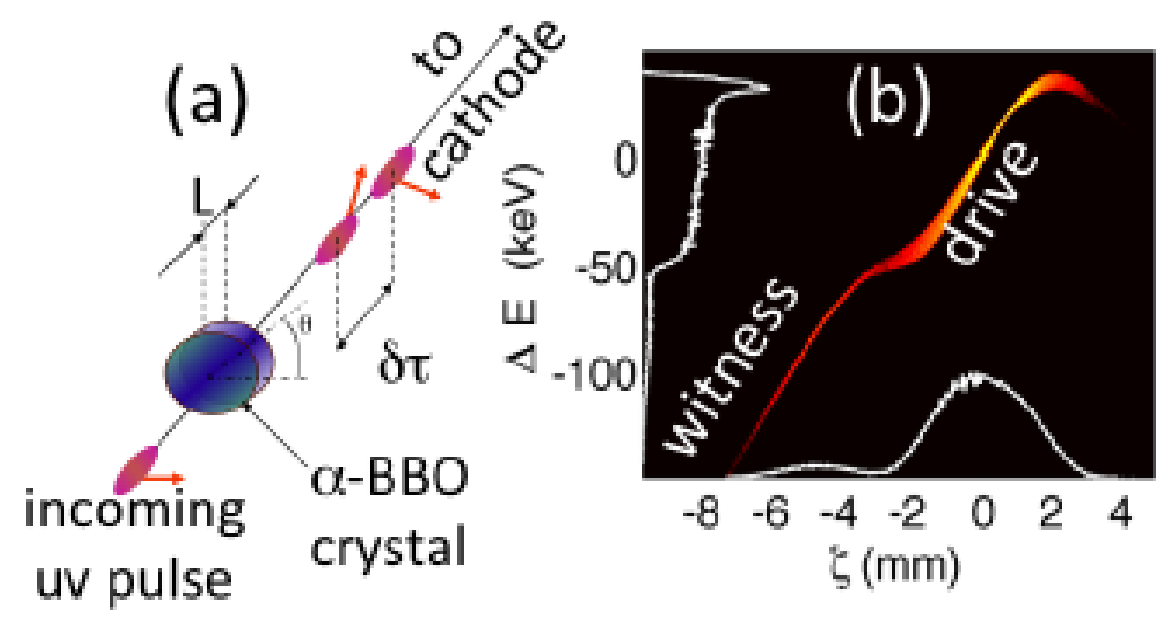

Figure 6: Photocathode drive-laser manipulation scheme to produce drive-witness bunch set (a): an alpha-BBO crystal is use to split the incoming ultra-violet photocathode laser pulse into two pulses. Corresponding simulated electron beam longitudinal phase space downstream of the RF gun [18]. 


\section{Phase III: High-energy tailored flat beam:}

Upon completion of Phase II and pending the availability of a higher energy at ASTA, the tailored flat beam would be accelerated to high energy (250 or $500 \mathrm{MeV}$ depending on the ASTA configuration) and a similar experiment as described in Phase II would be performed. The higher energy would enable smaller transverse beam sized and small structure aperture (thus higher accelerating field).

\section{Phase IV: A DWFA-module as an energy doubler for ASTA:}

Pending on favorable outcome of Phase III, a DLW-based accelerating module would be designed and implemented in ASTA to substantially boost the beam energy. In this configuration we could accelerate a $10-100 \mathrm{pC}$ electron bunch to $1 \mathrm{GeV}$. Details of the modules would depend on the lessons learnt from Phase III.

\subsubsection{References}

[1] W. Leemans et al., Nature Physics, 699 (2006).

[2] G. A. Voss and T. Weiland, "Particle Acceleration by Wake Field," DESY report M-82-10, April 1982; W. Bialowons et al., "Computer Simulations of the wakefield transformer experiment at DESY," Proceedings of the 1988 European Particle Accelerator Conference (EPAC 1988), Rome, Italy, 902 (1988).

[3] I. Blumenfeld et al., Nature 445, 741 (2007).

[4] M. Rosing, and W. Gai, Phys. Rev. D 42, 1829 (1990).

[5] M. C. Thompson, et al., Phys. Rev. Lett., 100, 214801 (2008).

[6] W. Gai, et al., Phys. Rev. Lett., 61, 2756 (1988).

[7] A. Tremaine, J. Rosenzweig, and P. Schoessow, Phys. Rev. E 56, No. 6, 7204 (1997).

[8] D. Mihalcea, P. Piot, P. Stoltz, Phys. Rev ST Accel. Beams 15, 081304 (2012).

[9] S. Antipov, C. Jing, A. Kanareykin, V. Yakimenko, M. Fedurin, K. Kusche, W. Gai, Appl. Phys. Lett. 100, 132910 (2012).

[10] F. Lemery, et al., "A DLW-based energy-doubling module for the Advanced Superconducting Test Accelerator at Fermilab," Proc. IPAC'13 (Shanghai), page 129 (2013).

[11] C. Jing, P. Schoessow, A. Kanareykin, J.G. Power, R.R. Lindberg, A. Zholents, P. Piot, "A compact soft X-ray free-electron laser facility based on dielectric wakefield accelerator," ANL/APS/LS-332 (2012).

[12] P. Piot, Y.-E Sun and K.-J Kim, Phys. Rev ST Accel. Beams 9031001 (2006).

[13] P. Piot, Y.-E Sun and M. Church, Proceedings of the 2010 International Particle Accelerator Conference (IPAC10), Kyoto, Japan, (23-28 May 2010), 4316 (2010).

[14] P. Piot, Y.-E Sun, M. Rihaoui, and J. G. Power, Phys. Rev ST Accel. Beams 14022801 (2011).

[15] B. Jiang, et al., Phys. Rev ST Accel. Beams, 15, 011301 (2012).

[16] P. Piot, C. Behrens, C. Gerth, M. Dohlus, F. Lemery, D. Mihalcea, P. Stoltz, M. Vogt, Phys. Rev. Lett. 108, 034801 (2012).

[17] F. Lemery, P. Piot, in preparation (2013).

[18] F. Lemery, P. Piot, C. Behrens, D. Mihalcea, C. Palmer, J. Osterhoff, B. Schmidt, P. Stoltz, Proc. IPAC'12, (New Orleans, May 20-25, 2012), page 2166. 


\subsection{Accelerator R\&D for Future SCRF Accelerators}

\subsubsection{Demonstration of High Power High Gradient SRF Cryomodules with High Intensity Beams}

The ASTA facility will be a unique test bed to carry out studies of high power, high gradient SRF cryomodules with high intensity beams. While high gradients have been demonstrated in many individual SRF cavities as part of the ILC R\&D program, they have not been demonstrated in operating cryomodules with very high intensity beams, e.g. with the full-scale ILC beam parameters and structure. ASTA is unique in its ability to bring together high-gradient cryomodule operation and realistic beam-based system tests.

Currently, there are no other test facilities in the world where this research can be carried out. These studies are all critical to determining the SRF cavity configurations for Project X, for the ILC, and for next generation light sources. In particular, whether HOM damping will be required and ramifications of long-pulse operation in the pulsed linac of Project $X$ are key questions that need to be answered.

Japanese interest in hosting the ILC brings to the forefront the remaining R\&D issues associated with ILC technology. A central element of that R\&D program is the system-level tests with high intensity beams that can be carried out at ASTA.

\subsubsection{ILC Cavity HOM Studies}

HOM Spectrum, Trapped Modes and Effects of HOM Excitation to Cryogenic Losses

High Order Modes (HOMs) is an issue for most superconducting accelerators: because of a beam interaction with the HOMs their resonant excitation is possible which in turn may lead to additional cryogenic loss in a cavity and emittance dilution. Transverse collective instabilities and beam break-up (BBU) may result because of HOM excitation as well. For ion and proton machines, or accelerators with the beam recirculation longitudinal collective instabilities may be possible. Some of the HOMs may be trapped in multi-cell cavities having high loaded $Q$ that may lead to the HOM excitation at high beam amplitude or long pulse trains. Careful measurements of the HOM excitation in ILC-type cavities at ASTA, investigation of resonance HOM excitation and its consequences and comparing these measurements to the computer models will provide valuable information for future superconducting accelerators. 


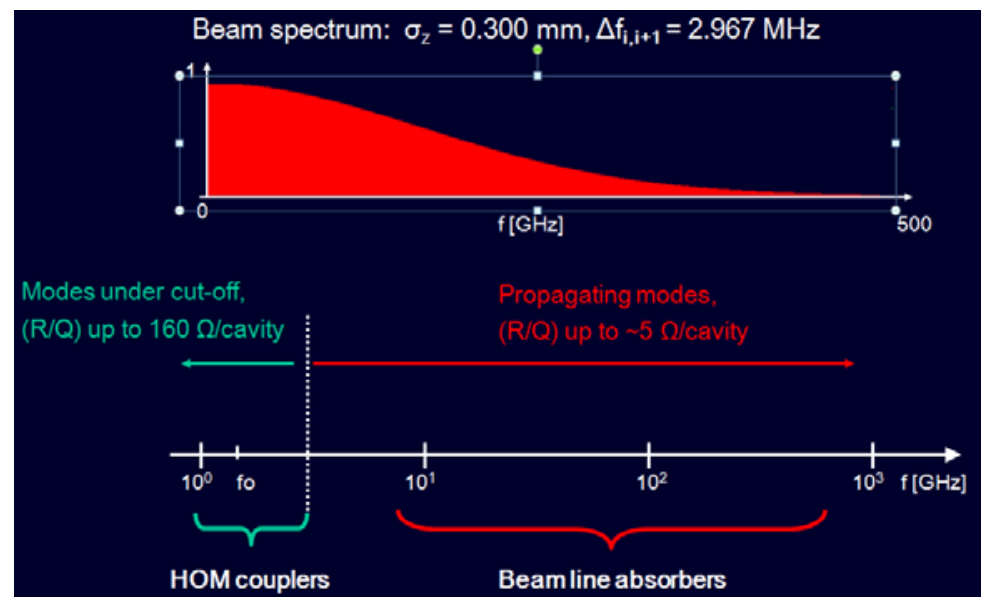

Figure 1: High order modes in ILC (J. Sekutowicz, DESY).

Lorentz Force Detuning for the HOMs

Lorentz force detuning is well investigated for the operating modes in the ILC-type cavity. However, there has not been any systematic investigation of the Lorentz force detuning for HOMs. These investigations are important for future superconducting accelerators, especially operating in the pulsed regime.

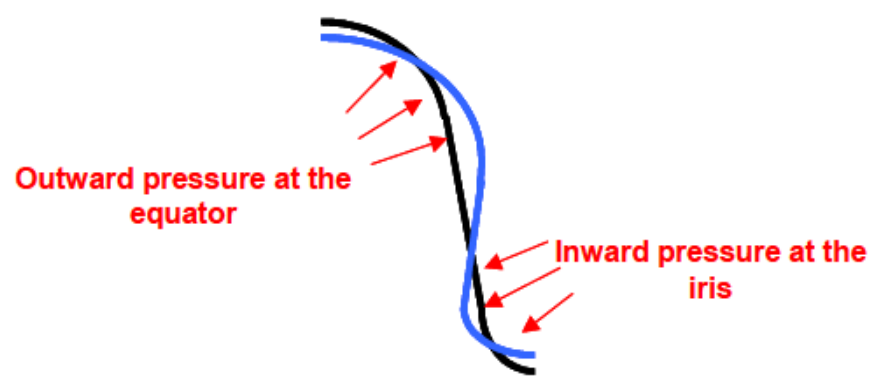

Figure 2: Illustration of cell deformation from Lorentz force detuning.

Range of detuning of the HOM modes due to plastic deformation

Special experiments performed at Fermilab Horizontal Test Stand (HTS) facility with the ILC-type 9-cell cavity showed that the HOM spectrum changes after detuning of the operating mode and tuning it back. This change is caused by small residual plastic deformations. This leads to the possible conclusion that in a pulse accelerator like ILC, where a special system provides the operating mode stabilization in the presence of Lorentz force, the HOM spectrum may change from pulse to pulse, decreasing the probability of resonant HOM excitation compared to CW machines. This effect has not been investigated yet. ASTA may be used for such investigations, which in turn, are very useful for future long-pulse accelerators such as Project $\mathrm{X}$. 


\begin{tabular}{|c|c|c|c|}
\hline $\mathrm{f}, \mathrm{MHz}$ & $\Delta \mathrm{f}, \mathrm{kHz}$ & $\delta \mathrm{f}, \mathrm{Hz}$ & Passband \\
\hline 1300 & 90 & 0 & 1Monopole \\
\hline 1600.093 & -218 & 360 & 1Dipole \\
\hline 1604.536 & -215 & 240 & 1Dipole \\
\hline 1607.951 & -214 & 360 & 1Dipole \\
\hline 1612.189 & -210 & 360 & 1Dipole \\
\hline 1621.344 & -211 & 240 & 1Dipole \\
\hline 1625.458 & -208 & 370 & 1Dipole \\
\hline 1830.836 & -185 & 370 & 2Dipople \\
\hline 1859.882 & -36 & 120 & 2Dipople \\
\hline 2298.807 & -278 & 480 & 1Quadrupole \\
\hline 2299.346 & -278 & 490 & 1Quadrupole \\
\hline 2372.333 & -224 & 490 & 2Monopole \\
\hline 2377.333 & -221 & 490 & 2Monopole \\
\hline 2383.575 & -213 & 240 & 2Monopole \\
\hline 2399.289 & -210 & 490 & 2Monopole \\
\hline
\end{tabular}

Figure 3: HOM frequency change $\delta f$ after the operation mode detuning and tuning back by $\Delta f$ ( $T$. Khabiboulline, Fermilab).

\section{Long Term Stability of the HOM Frequencies for Cavity in Cryostat}

During operation in a nominal regime, the tuning system maintains the operating frequency. However, Lorentz forces and microphonics may cause long-term changes of the frequencies of HOMs. This has never been investigated carefully. It is proposed to carry out a detailed investigation of a long-term stability of HOM frequencies for a $1.3 \mathrm{GHz}$ dressed ILC cavity in a cryomodule.

\section{Effect of HOM to the beam dynamics}

High-order modes excited in a cavity may create the following problems for a beam dynamics:

- Increased beam transverse emittance, or even cause the beam deflection and losses, due to the collective transverse beam-breakup instability, BBU. BBU may develop in ultra-relativistic beams, in contrast to the klystron-type longitudinal collective instability, and can be investigated at ASTA.

- Beam transverse and longitudinal emittance dilution caused by HOM resonance excitation. These effects can be investigated at ASTA, because it operates in pulsed mode-it is not possible for $\mathrm{CW}$ operation for regular beam timing structure.

\section{Using HOM signal for measurements of beam position in cavity (HOM based BPM)}

Measurements of the dipole HOMs excited by the beam may be used for measurements of the cavity off-set versus the beam axis. These experiments were performed successfully at DESY, with a $1.3 \mathrm{GHz}$ cavity, and with a $3.9 \mathrm{GHz}$ cavity. They showed that it is really possible to provide reliable and precise measurements using this technique. 

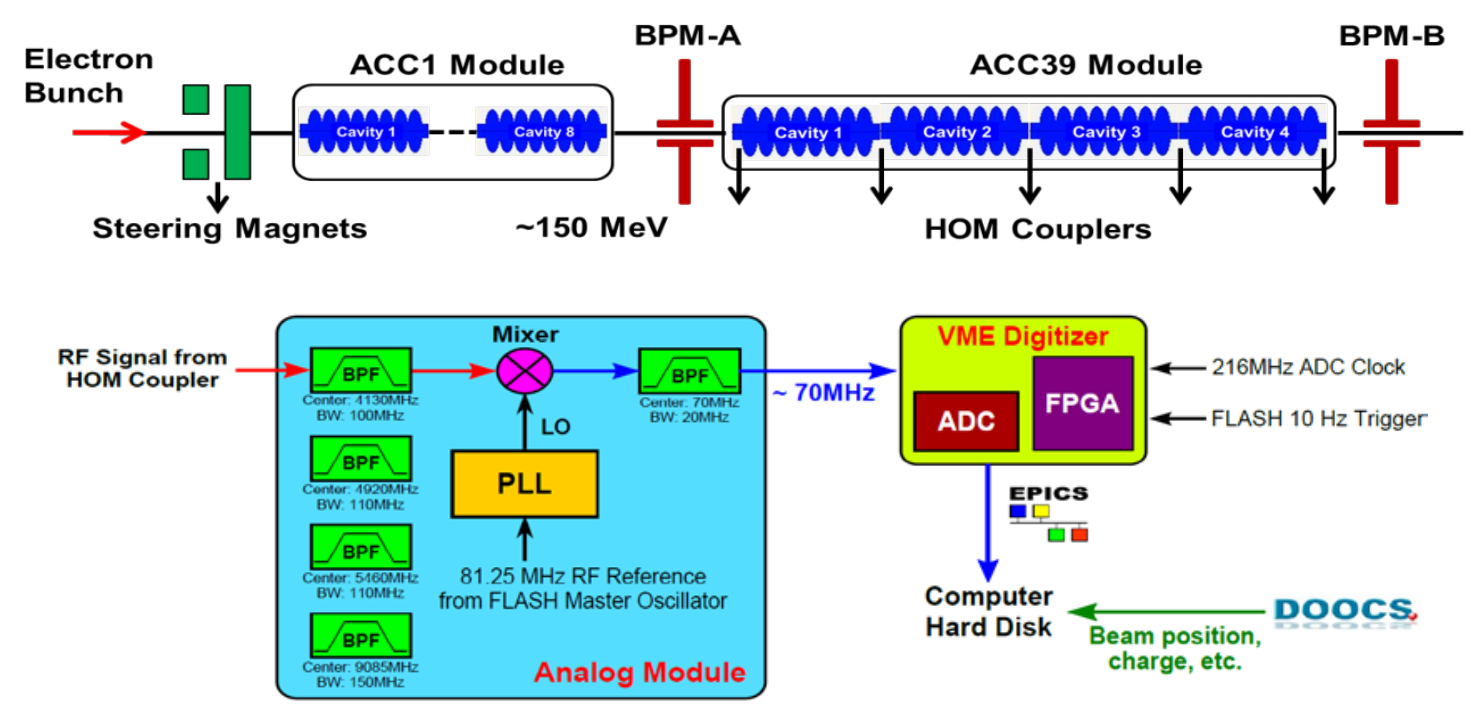

Figure 4: Schematic and electronics set up for cavity position measurement using HOMs in a $3.9 \mathrm{GHz}$ section (P. Zhang, N. Baboi, and R. Jones, FLASH/DESY).

\section{Effect of cavity tilts}

For collider operation, especially in the bunching system, not just cavity off-set is important, but also tilt of a cavity axis versus a beam axis, which may lead to significant emittance dilution. Dedicated experiments need to be done at ASTA in order to develop the technique to measure and correct for the tilt.

\subsubsection{Studies of Propagating High-Frequency HOM's (Wakefields)}

\section{Cryo-losses}

For short bunches (tens of microns) which are required not only for the ILC, but also for next generation light source, the beam current spectrum is very wide, up to hundreds of $\mathrm{GHz}$. These very high order modes can lead to additional cryo-losses. In order to prevent that, special HOM absorbers need to be developed and incorporated in the cavity design.

\section{Damping of the HOMs in absorber}

Design of efficient HOM absorbers requires careful investigation of the very high order mode excitation in a chain of cavities along the linac, dissipation in the SC cavity walls, radiation into the beam pipe and its interception by the absorbers. ASTA naturally allows all these experiments. 


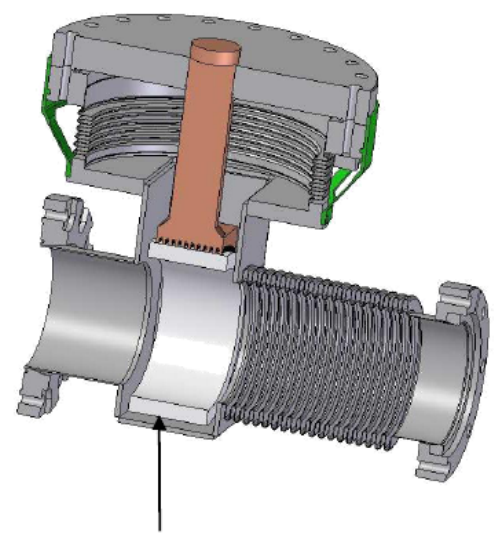

Lossy ceramic (final choice CA137)

Figure 5: XFEL HOM absorber (J. Sekutowic, DESY).

RF kick and wakefields in cavity caused by the RF and HOM couplers (asymmetry)

All RF cavities, being axisymmetrical, have perturbations of the symmetry caused by manufacturing errors and, more seriously, by the coupling elements (main power couplers, HOM couplers, etc.). These perturbations can cause the so-called RF kick and coupler wake, which can result in significant emittance dilution, especially in a bunching system, where the bunch is long.

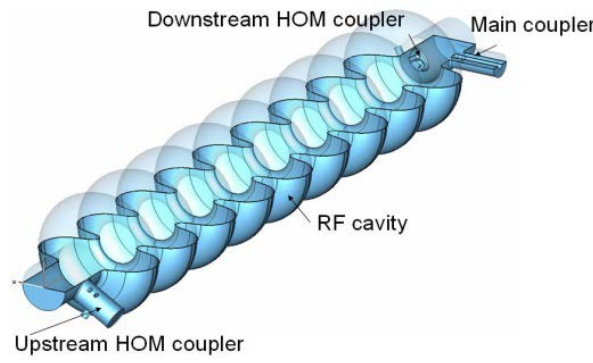

Figure 6: The ILC RF cavity with the main and HOM couplers.

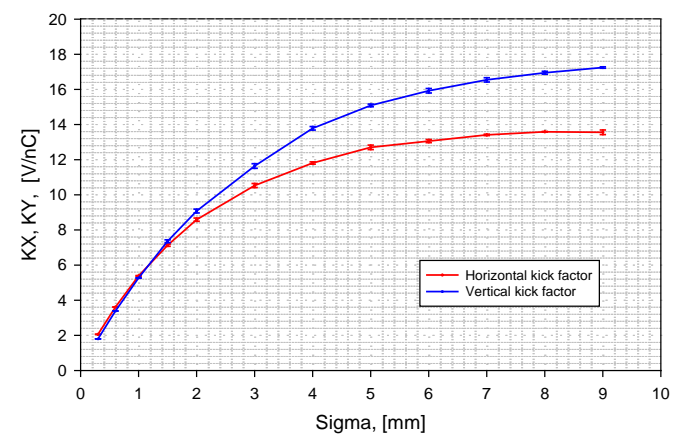

Figure 7: Horizontal and vertical wake kick-factors of the ILC type structure with couplers versus the rms bunch length (A. Lunin, Fermilab). 


\subsubsection{Lorentz Force Detuning Compensation (LFDC) Studies}

LFD and microphonics studies for short ( 1ms, ILC) and long pulse trains (8-30ms, Project X)

It is proposed to carry out a detailed investigation of Lorentz force detuning compensation for short pulses in the ILC operation regime, and for long pulses, which is essential for the highenergy pulse part of Project $X$. This work was already initiated at Fermilab, and impressive results have been achieved already. However, further investigations are needed in order to achieve reliable operation of the pulsed linac of Project $X$.
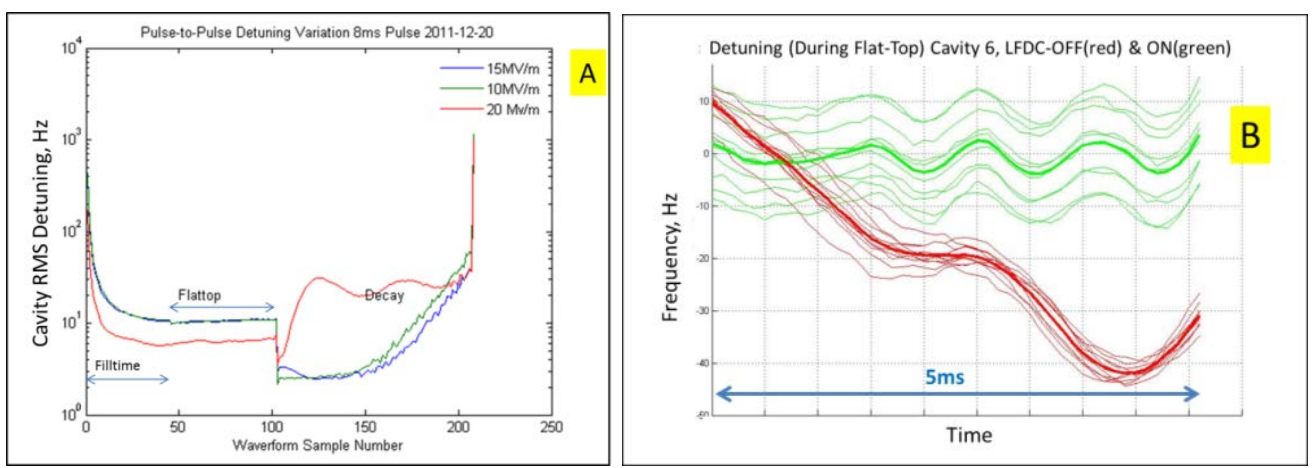

Figure 8: (A): Pulse-to-pulse detuning variation (RMS detuning) for $E_{a c c}=10 ; 15 ; 20 \mathrm{MV} / \mathrm{m}$. (B): Detuning (during $5 \mathrm{~ms}$ flat-top) for $10 \mathrm{RF}$ pulses with LFDC system ON (green) and OFF (red). Thick line is average of the 10 pulses. The gradient is $10 \mathrm{MV} / \mathrm{m}$. (W. Schappert, Yu. Pischalnikov, Fermilab).

Studies of sources of microphonics, spectrum, quantification of contributions from different sources and means to suppress microphonics

Another issue which determines the resonant frequency stability of a SC cavity is microphonics, i.e., the resonant frequency change caused by vibrations of the cavity wall. These vibrations may have different causes (mechanical resonances, improper design of a Helium vessel and cryo system, etc.). Studies at Cornell University have shown that the most serious source of the microphonics is Helium pressure fluctuations. We propose detailed investigations of the sources for the microphonics in ILC-type cryomodules, in order to quantify contributions from different sources, determine main reasons of microphonics, and thus, understand possible means for the suppression of microphonics. At Fermilab, this work has already been initiated, and initial encouraging results have been achieved for the SSR1 cavity.

Microphonics due to cavity cross-talking

Another important and poorly-investigated issue is microphonics due to cavity cross-talk, i.e. a cavity vibration influencing the frequency of a neighboring cavity and other cavities in the same 
cryo-module. Mechanical coupling of the cavities in a cryo-module may create additional difficulties for microphonics suppression and needs to be investigated.

Effects of the CM ends on microphonics

Mechanical coupling of a cavity to the environment depends strongly on the cavity position in a cryo-module. The end cavities are in a different position with different end constraints than the regular cavities. This may change microphonics amplitude and possibly create additional problems for their compensation.

\subsubsection{Field Emission and Dark Current}

\section{Energy and spectrum}

Field emission can cause high losses in SRF cavities, and thus, seriously limit the maximum acceleration gradient-the experience of ORNL's SNS is a good example. Thus, investigations of the dark currents, their sources, the energy spectrum, etc. are essential. ASTA provides a venue for experimental investigations of the dark currents which are important for many projects employing large SRF accelerators.

\subsubsection{Cavity Alignment}

\section{Effect of the cavity tilt on beam dynamics}

Experimental investigation of cavity mis-alignment - off-sets and especially tilts are very important for large-scale accelerators. The cavity tilt provides the beam acceleration in the transverse direction and introduces momentum spread along the bunch, which in turn causes dilution of the beam emittance. ASTA will allow these measurements in great detail.

\subsubsection{Cavity Gradient}

\section{Effect of CM ends to cavity gradient/degradation}

It has been noticed (FLASH and SNS experience) that frequently the end cavities in a cryomodule provide lower accelerating gradients than regular cavities. This limits the gradient of entire section. Sometimes degradation of the gradient in the end cavities takes place over time. We propose to investigate the reasons of the gradient limitation and degradation in the end cavities at ASTA cryomodules.

\section{LLRF and gradient spread in SRF CMs}

Achieving the design gradient of $31 \mathrm{MV} / \mathrm{m}$ on average is very challenging task for the SRF cryomodules and will be properly addressed at ASTA. Cavity performance spread may lead to different RF filling behavior during the pulse for different cavities when fed by a single RF source. This requires specially designed LLRF systems in order to provide amplitude and phase stability of the average field "seen" by the different bunches in the beam macro-pulse. Fermilab 
has participated in the long pulse experimental studies at the FLASH facility at DESY, Hamburg, which has the goal to provide reliable operation of the SRF linac in which a number of cavities are fed by a single RF source. ASTA allows for such a program at Fermilab. There should be more time available at ASTA to work on this long pulse study as FLASH has only limited time available for accelerator research due to operations for the users.

\subsubsection{Effects of the CM End (Cold to Warm Transition Between Cryomodules)}

Contamination of the end cavities (long term effects)

The end cavities in a cryo-module may be contaminated, which may in turn, cause long-term degradation. Investigation of the source of contamination may help to resolve this problem.

\section{Field emission and cavity degradation}

Another effect is a field emission in the end cavities caused by contamination, which may influence all the cavities in cryo-module.

Effect of thermal radiation from RT ends to cavities in $C M$

The end cavities are exposed to the thermal radiation from room-temperature gaps between the cryo-modules, which may lead to additional cryo-losses.

ASTA allows for all these investigations and may clarify the issues with the end cavities and improve their performance in future SRF linacs. 


\subsubsection{Proposed Long-Range Wakefield Measurements at ASTA}

\subsubsection{Introduction}

Next generation light sources will use a CW superconducting L-band linac to accelerate highbrightness electron beams up to $2.4 \mathrm{GeV}$ at $1 \mathrm{MHz}$ for injection into a farm of up to 10 freeelectron laser undulators for the production of high-brightness, coherent soft x-rays. Beam stability from bunch to bunch in all coordinates is critical for such a user facility, and the impact of beam dynamics issues such as long-range wakefields must be well understood. The LBNL team therefore proposes a series of beam-based and possibly RF-based measurements at the Advanced Superconducting Test Accelerator (ASTA) at Fermilab. The measurements require the installation of at least one 8-cavity cryomodule and a high-resolution electron spectrometer and beam diagnostics package to be located at the end of the Stage-I machine or similar (see Figure 1).

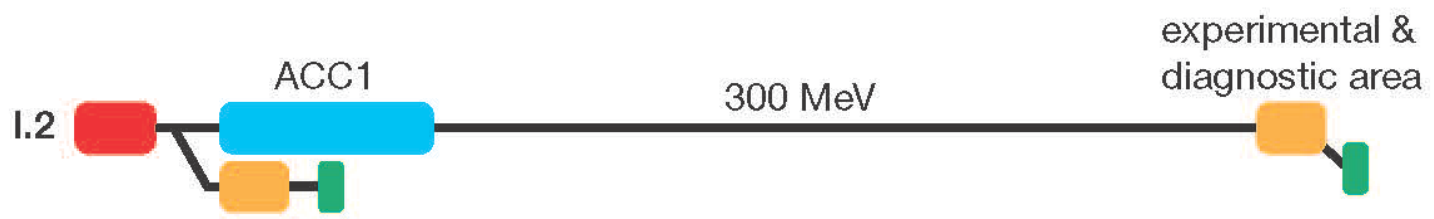

Figure 1: The configuration shown above (Stage 1.2, from the ASTA proposal) might be used in this 'Stage-I' ASTA layout to measure the long-range, longitudinal wakefields induced within one cryomodule (ACC1) using the electron spectrometer and diagnostics section shown at far right.

The proposal will attempt the measurement of long-range wakefields in the superconducting linac using an ILC-type cavity. The ASTA accelerator is uniquely suited for such an experiment due to its long bunch train.

\subsubsection{Beam-Based Measurements}

The experimental details need further definition, but the 3-MHz micropulse repetition rate and the planned bunch-by-bunch RF beam position monitor (BPM) system provide a unique opportunity for beam-based investigations in the USA. With a single 8-cavity cryomodule installed and a high-resolution electron spectrometer available at the terminal end (100-300 $\mathrm{MeV})$, it would seem quite possible to make beam-based measurements of the relative electron energy per bunch, over a bunch train of $1 \mathrm{~ms}$ or less, as a function of the bunch spacing (0.3-10 $\mu \mathrm{s})$ and the bunch charge $(0.1-1 \mathrm{nC})$, and for comparison to calculated long-range wakefields over a single cryomodule. Two BPMs are already planned before and after the downstream dipole to provide both entrance and exit angle of the beam for accurate relative energy measurements at up to the $3-\mathrm{MHz}$ rate. Using the first bunch in the train as a reference, the other bunches (up to 3000) will be measured using the BPM in the spectrometer bend plane. The relative electron energy per bunch is recorded by normalizing the measured beam position, 
$x$, to the spectrometer dispersion, $\eta_{x}$. A BPM resolution of $20 \mu \mathrm{m}$ and a dispersion function of $0.4 \mathrm{~m}$ (example) allow a possible resolution of $0.005 \%$. A more detailed layout drawing of ASTA (Stage-I) is shown in Figure 2, indicating the locations of a bunch compressor and 3.9-GHz RF linearizer cavity as well.

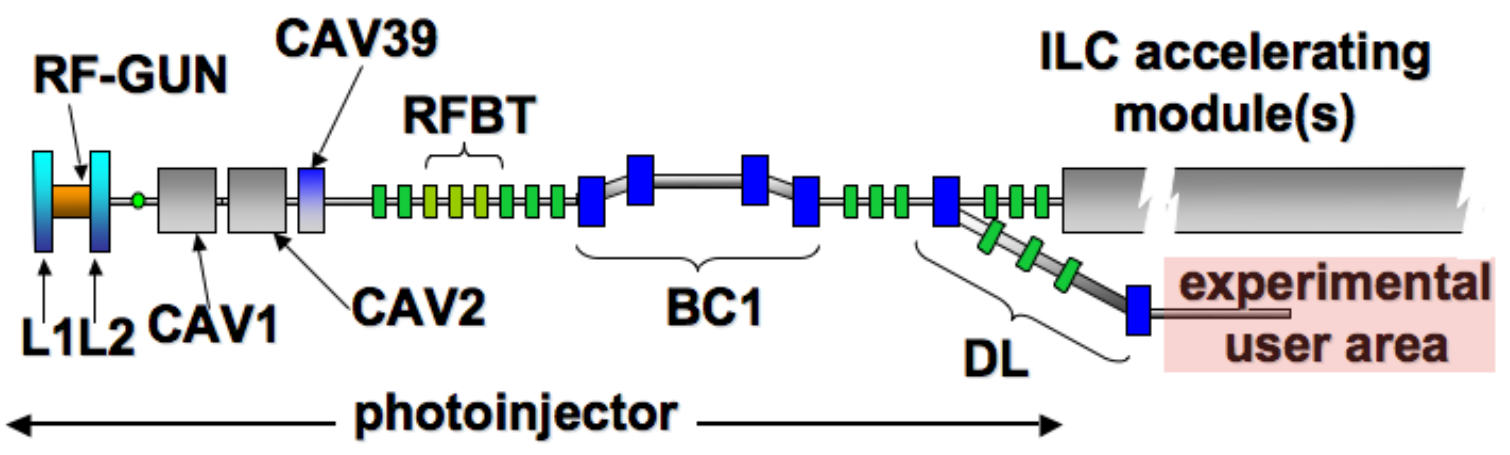

Figure 2: The layout used to measure wakefields, where the spectrometer after the "ILC accelerating module" is not shown here.

\subsubsection{RF-Based Measurements}

While the beam-based measurements described in the previous section can map the net effect of long-range wakefields on the beam, it is useful to directly monitor the fields excited in the corresponding higher order modes (HOMs) in the cavities themselves. This can be done using the two existing HOM coupling ports available per cavity and instrumenting them to observe the beam-induced signals in both time and frequency domain. Detailed characterization has been done on the cavities at FLASH [1].

In the study at FLASH, the authors developed a sophisticated technique for identifying the HOMs. For the dipole HOMs, they were able to use beam signals as beam position monitors and measure the alignment of individual cells within the cavity. They were also able to observe the monopole HOMs and proposed a technique to measure the relative beam phase with respect to the accelerating phase. A schematic of the electronics layout for recording the HOM signals is shown in Figure 3 along with an example concatenated signal from the up and downstream HOM couplers. Another example measurement shown in Figure 4 is the frequency spectrum of the monopole HOMs.

The resolution of the FLASH study was limited by the sensitivity of the charge measurement used for normalization of the signal and the phase of the local oscillator in the electronics. With use of a higher sensitivity charge measurement and better electronics, we propose to improve on this technique and use the measurement to correlate beam induced signals with the beam effects described in the previous section. 

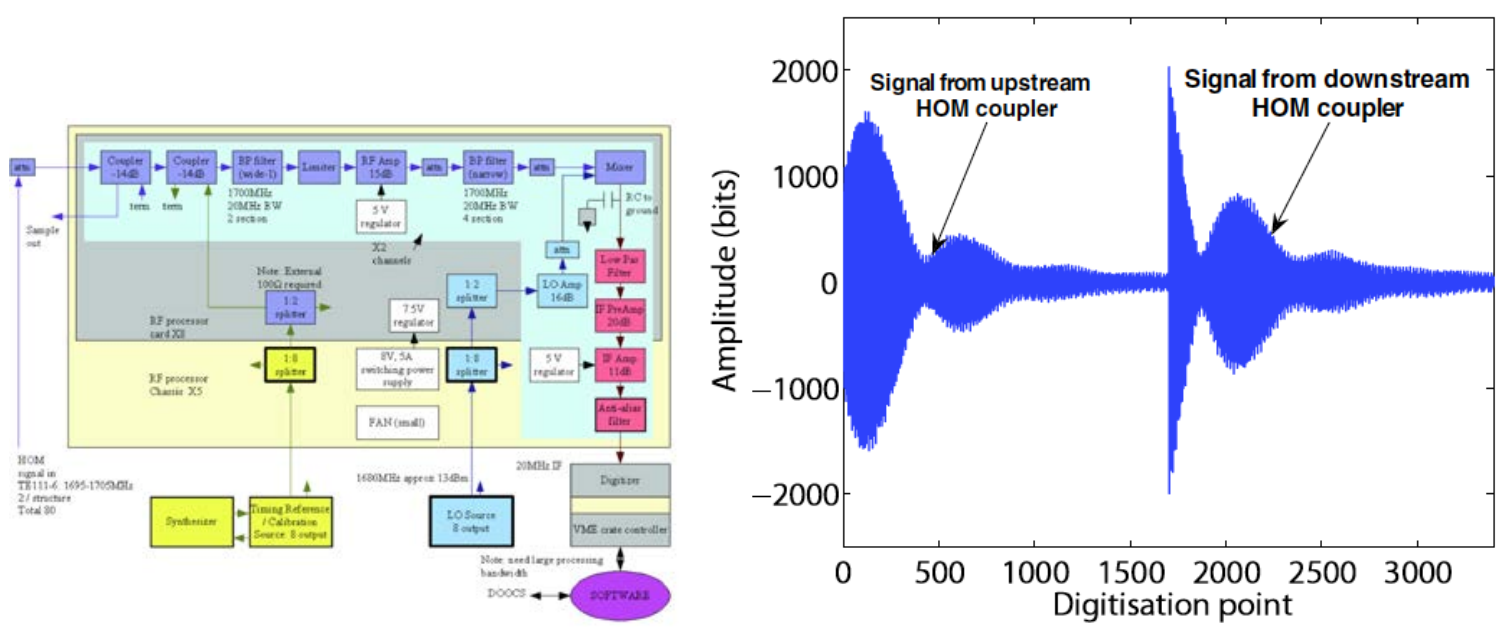

Figure 3: (Left) Detailed layout of electronics for recording beam-induced signals in the FLASH HOM couplers. (Right) Example signal of concatenated signal from up and downstream HOM couplers.

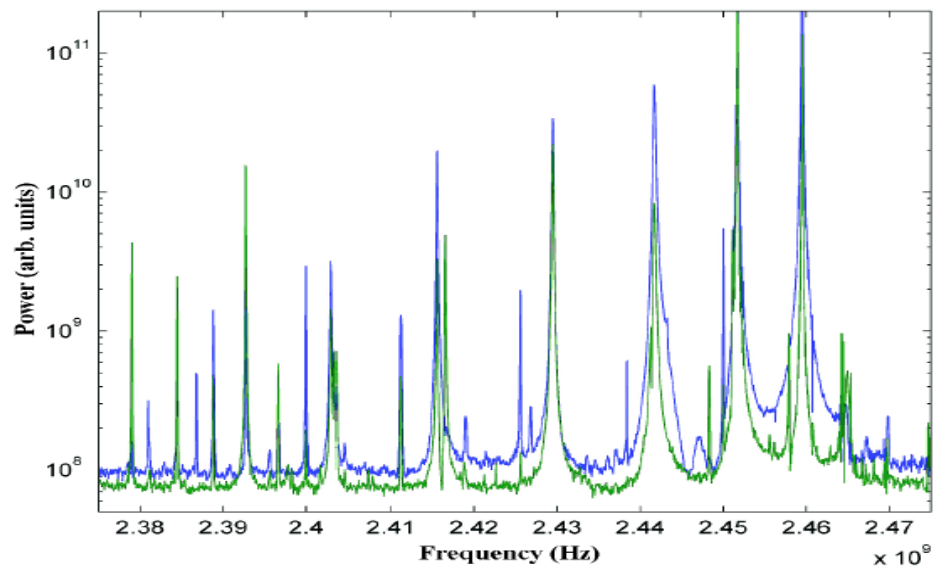

Figure 4: Frequency spectra of monopole HOMs measured on the up and downstream HOM couplers [1].

\subsubsection{Summary}

In summary, the transit of the ASTA 1-ms pulse train through a full 8-cavity cryomodule provides a unique opportunity for investigating beam dynamics effects such as long-range wakefields as a function of micropulse charge and temporal spacing that may impact beam stability in future systems. Elucidation of the effects and understanding their bounds should play a critical role in final design efforts.

\subsubsection{References}

[1] S. Molloy, et al. Phys. Rev. ST Accel. Beams 9, 112802 (2006). 


\subsubsection{Towards Ultra-Stable Operation of ASTA with Beam Based Feedback}

\subsubsection{Introduction}

Superconducting RF systems have been adopted in a variety of accelerator systems because of the ability to efficiently accelerate high power beams. However, because of the long RF and beam pulses used in these systems, it is also possible to use high precision feedback control of the RF systems to achieve extremely high stability of the beam parameters. In this section, we describe how such a feedback system can be implemented at ASTA, providing a unique capability for development of new accelerating techniques. For example, energy stability at the $10^{-5}$ level and timing stability below $10 \mathrm{fsec}$ would allow extremely tight synchronization with external laser systems and enable a range of advanced accelerator experiments which are either extremely difficult or impossible at existing facilities in the US.

The demonstration of a sub-femtosecond feedback system to stabilize the superconducting linac will be a very important test at ASTA.

Modern electron linacs consist of an injector and multiple accelerating sections with one or more interleaved bunch compressors. Jitter of the beam parameters such as energy, bunch length, and arrival time arises from two primary sources: 1) jitter of the beam from the injector and 2) amplitude and phase errors in the accelerating sections. In normal conducting linacs, the beam jitter is dominated by the second source because the RF pulses, typically a few microseconds long, are too short for intrapulse RF feedback to be applied. Therefore, uncorrelated errors between RF pulses cannot be corrected. In SRF systems, the RF pulses range from milliseconds to continuous and high precision feedback can been applied. Control of SC cavities has already demonstrated amplitude stability at the $10^{-5}$ level and phase stability of $\sim 0.01$ degrees.

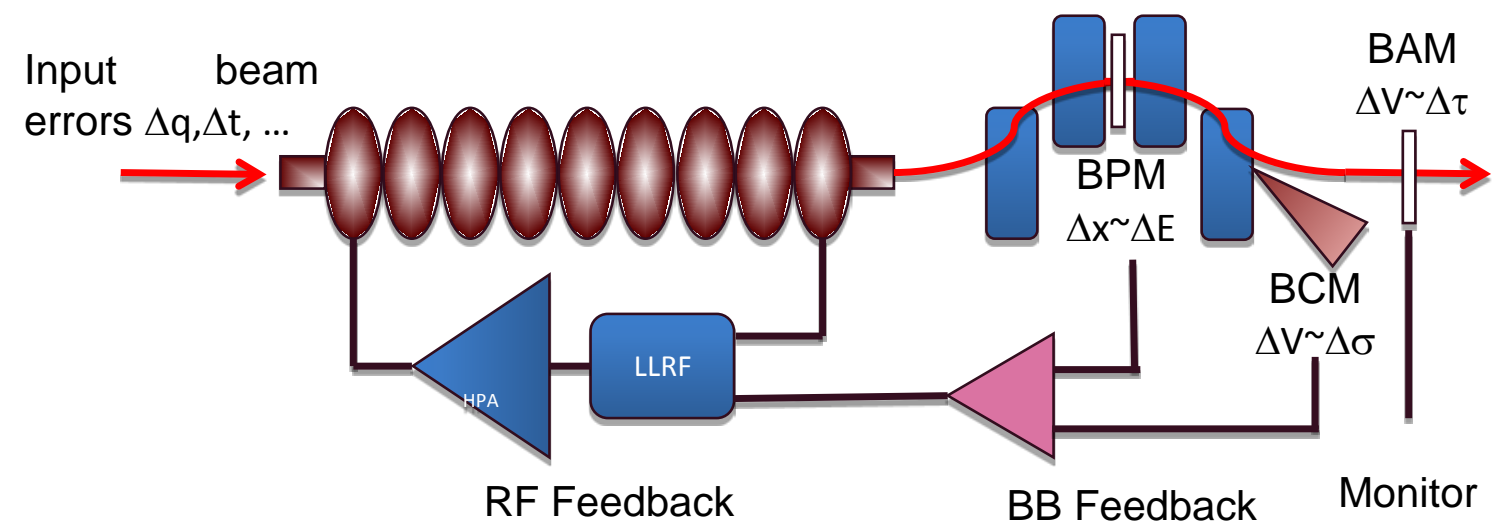

Figure 1: Conceptual schematic view of RF and beam-based feedback control. Energy, bunch length, and arrival time measurements can provide set point modulation to the RF sections preceding the bunch compressor. 
However, the beam parameters depend on the vector sum of the individual cavities and it is possible for beam parameters to drift from effects such as relative drift of the phase of individual cavities. The long beam pulses also allow the possibility of using beam-based feedback to provide modulation of the set point to the RF sections preceding the bunch compressor. The energy error is measured at each dispersive section by measuring the transverse beam position at a point of dispersion. Bunch length error is measured at each bunch compressor from the coherent synchrotron radiation emitted in the chicane dipole magnets. A linear combination of these measurements is then used to adjust the voltage and phase set points of the preceding RF sections. We can express this transformation as a matrix relating the measured beam errors with the changes to the RF voltages and phases. If we consider global RF and beam-based feedback for the entire linac, we can relate the measured beam parameters at several dispersion sections to the corresponding amplitude and phase correction in the different linac sections.

Experience with feedback control of SC linacs has been extremely positive. At FLASH, a combination of RF and BBF has been implemented. An example of the performance of this system is shown in Figure 2. The top plot shows the arrival time error at the entrance to the bunch compressor from the injector over the first 100 bunches in a train and the lower plot shows the reduction with feedback applied. The first few bunches are not affected because of delays in the feedback. The residual noise is limited by the bandwidth and noise floor of the feedback. This performance is excellent and has already enabled high precision pump-probe experiments using $\mathrm{x}$-ray free electron laser at the end of the linac. Further improvement could come from reducing the noise at the injector and improving the bandwidth and noise of floor of the feedback.

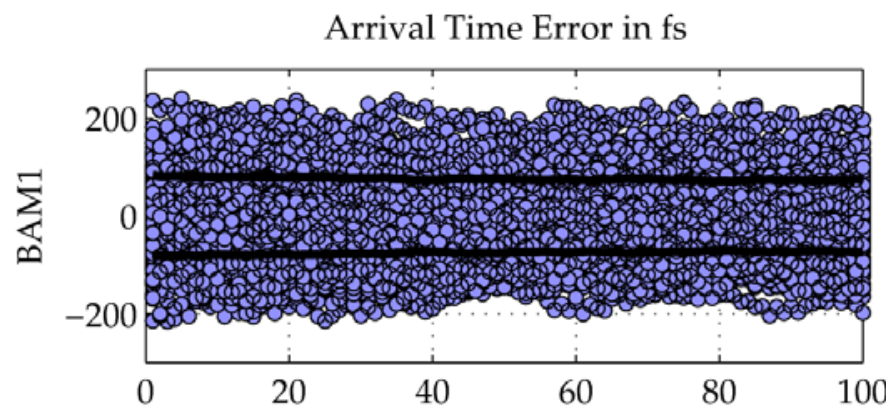

Histogram (normal distribution)
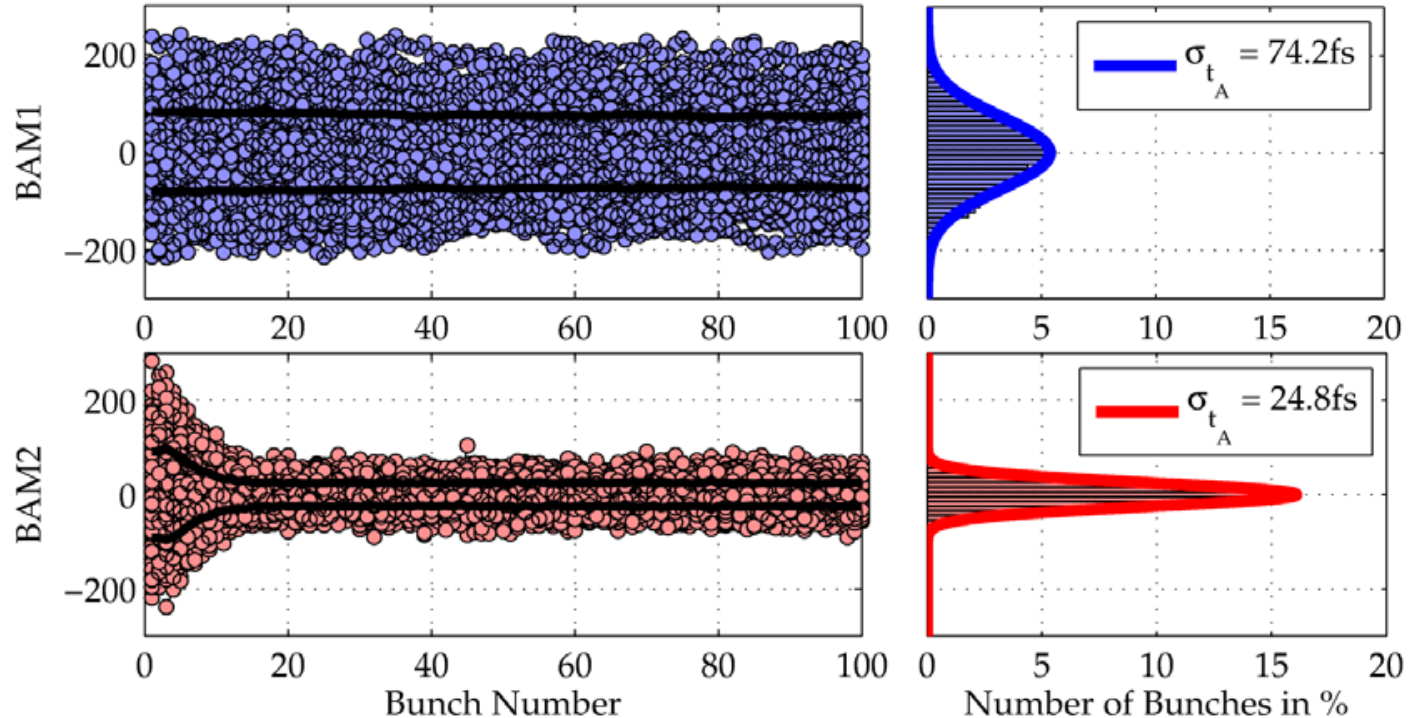

Figure 2: Example of RF and beam-based feedback control at FLASH. The top plot shows the arrival time error at the entrance to the bunch compressor from the injector over the first 100 bunches in a train and the lower plot shows the reduction with feedback applied. 


\subsubsection{RF Control and Feedback}

The Low Level RF System for ASTA shown in Figure 3 includes all controller hardware and software required providing high precision field and resonance control of the accelerating cavities. It also delivers the Master Oscillator RF through the Phase Reference Line used to synchronize RF, instrumentation, and experimental systems. The Field Controller is capable of combining the probe signals from 24 cavities from one ASTA RF station into a single vector sum. The vector sum is used as the feedback input signal to the control loop. The FPGA-based controller uses a combination of IQ feed-forward and proportional-integral feedback driving the klystron to minimize phase errors far below the $1 \%$ amplitude and 1 degree levels with a gain of 100 (see Figure 4).

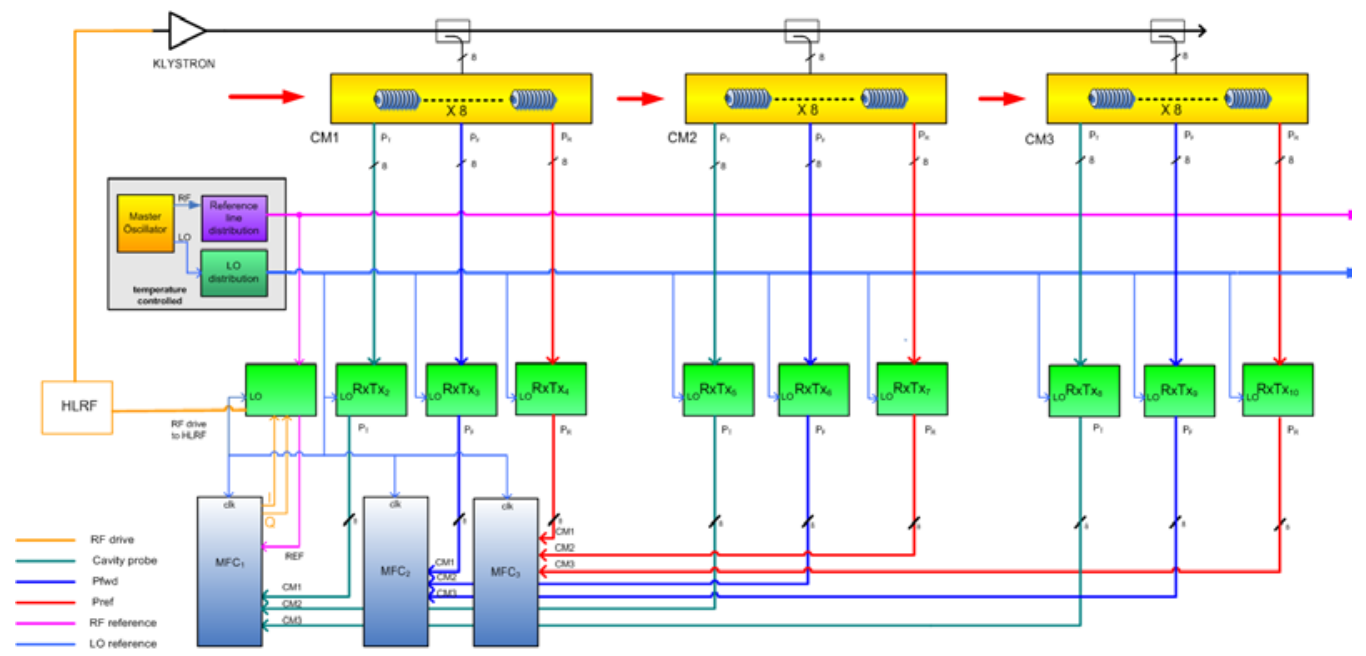

Figure 3: ASTA LLRF system level block diagram for 3 cryomodules driven by a single klystron. A similar system is planned for booster cavities CC1 and CC2 in the injector.
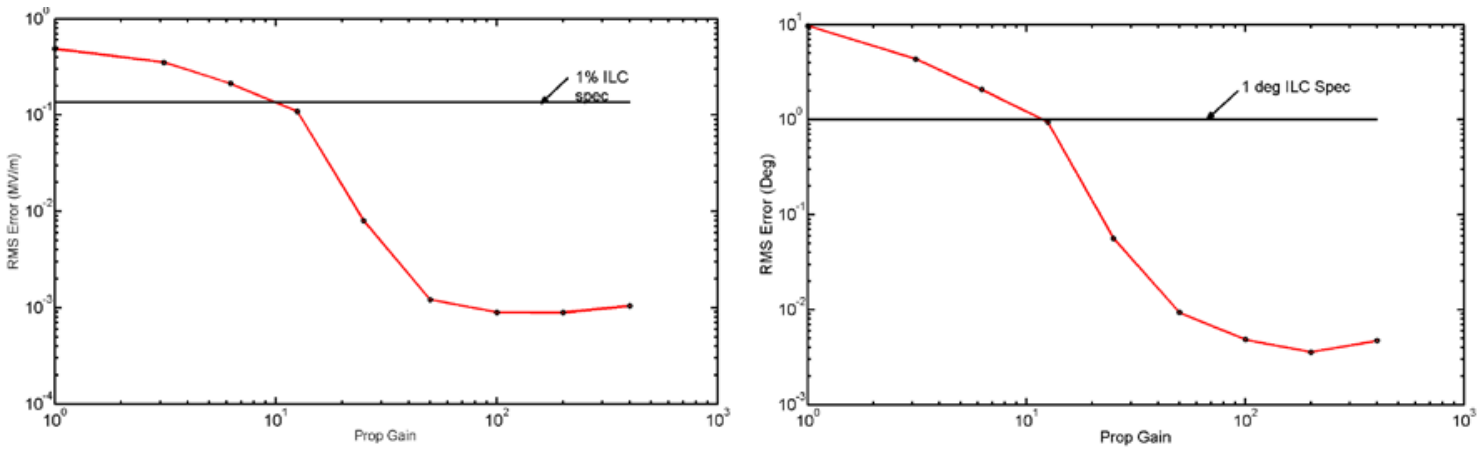

Figure 4: RMS magnitude and phase errors for Cryomodule 1 as a function of loop gain. Magnitude error is in $\mathrm{MV} / \mathrm{m}$ with a $22 \mathrm{MV} / \mathrm{m}$ gradient or $3.6 \mathrm{E}-5 \mathrm{rms}$. Phase error is $3 \mathrm{E}-3$ degrees rms. These measurements were made without beam through the cryomodule. 
The controller can easily be adapted to use the proposed error signal matrix from the beambased measurements with analog signals input into spare 65 MSPS digitizers or digitally with spare high speed serial links.

\subsubsection{ASTA Beam-based Feedback}

We propose to implement high precision feedback control at ASTA. The first stage of this work is to characterize and minimize the beam jitter from the injector. The beam energy, charge, timing and bunch length jitter can be characterized using the planned diagnostics. We expect that further control of the photocathode drive laser will be needed to reduce the charge and timing jitter. Characterization of the phase and amplitude control of the RF gun is also critical to minimizing the beam jitter. At a later stage, once a second bunch compressor is available downstream of the cryomodule(s), stabilization of the ASTA linac will be implemented.

\subsubsection{Beam Monitors}

To implement the first stage of beam-based feedback, we would implement a relative energy measurement using a beam position monitor in the BC1 bunch compressor along with a bunch length measurement following the bunch compressor. We expect the bunch length measurement to be based on detection of coherent radiation produced by either edge radiation (CER) or synchrotron radiation (CSR) from one of the chicane dipoles or from a dedicated coherent diffraction radiation (CDR) source. We also propose development of a femtosecond arrival time diagnostic to be initially used as an out-of-loop monitor of the feedback performance, but which could also be used in the loop. All of these diagnostics will be capable of operating with high precision at the bunch repetition rate of $3 \mathrm{MHz}$ or close to that.

\subsubsection{Arrival Time Diagnostics}

A Bunch Arrival Monitor (BAM) is in development for ASTA and will be installed just downstream of the bunch compressor (see Figure 5). The monitor is based on the conversion of time-to-voltage via the amplitude modulation of a pulsed laser via Electro-Optical Modulation (EOM). This scheme was first proposed by the DESY group [1]. Figure 5 (left) shows the basic principle of the bunch TOA monitor. The zero-crossing voltage of a broadband beam pickup is used to amplitude modulate a single 100 femtosecond wide laser pulse. Figure 5 (right) shows how the zero-crossing voltage changes as a function of the bunch arrival time. 

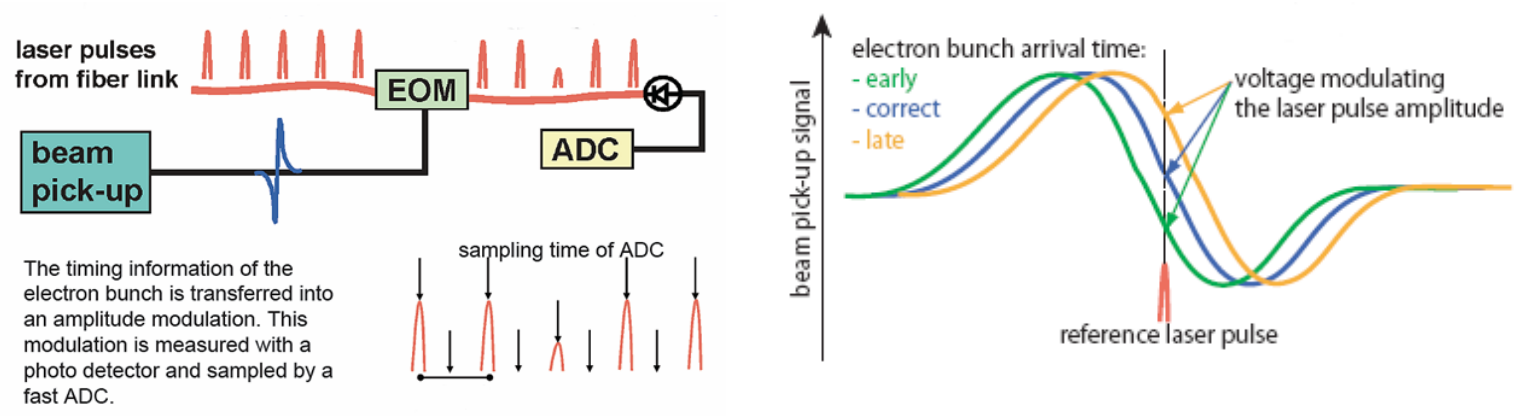

Figure 5: Left shows the basic principle of bunch time-of-arrival monitor. Right shows the relation between the modulating voltages to the bunch arrival time.

Figure 6 shows the BAM functional block diagram. The operation of the BAM starts with a wideband beam pickup, needed to permit femtosecond time-of-arrival measurements, that acquires the bunch electric field. The pickup signal is timed to arrive at the electro-optical modulator in coincidence with the RF-locked laser pulses. The zero-crossing of the pickup signal is used to modulate the amplitude of a single 100 femtosecond wide laser pulse. A photodiode converts the laser pulse into a voltage which is sampled by an ADC. The change in voltage then gives a measure of the time-of-arrival. The functional operation of the BAM allows for the measurement of arrival times for all bunches within the pulse train.

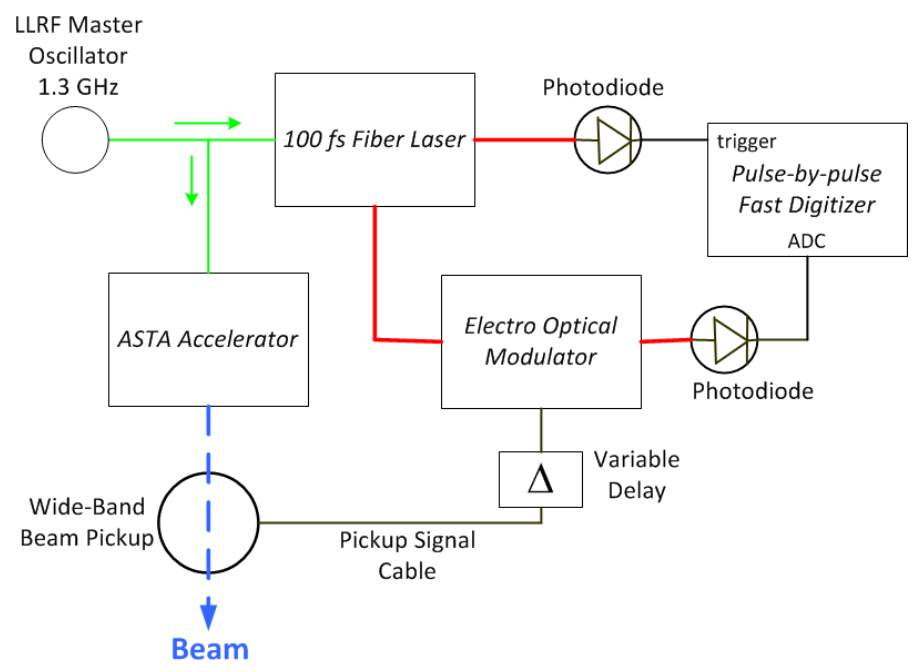

Figure 6: Functional block diagram of the proposed ASTA BAM.

For the proposed ASTA beam-based feedback system, the BAM can be used as (1) an out-ofloop monitor of the feedback performance from the bunch compressor energy and bunch length monitors or (2) as an in-loop part of the beam-based feedback system. DESY FLASH used the arrival-time information measured by a BAM to establish an intra-bunch-train arrival-time 
feedback acting on the amplitude of their first accelerating module. Figure 7 shows the bunchby-bunch effect of this feedback on the arrival-time stability [2].

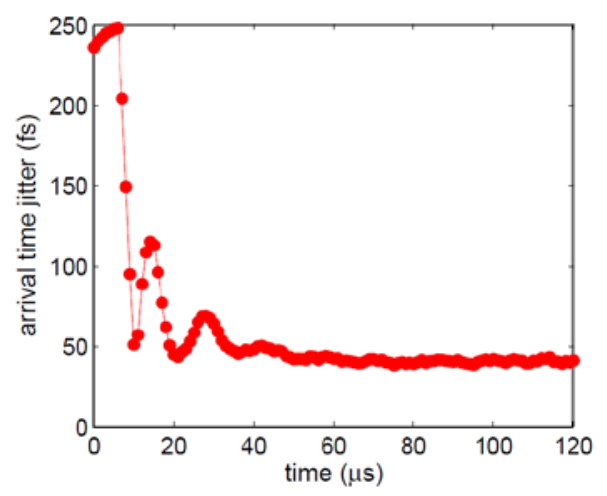

Figure 7: Effect of the intra-bunch train feedback on the arrival-time stability [2]. The first few bunches are not corrected by feedback.

One advantage of the proposed ASTA BAM system is that Fermilab already possesses a 100-fs Erbium fiber laser (MenloSystems C-Fiber femtosecond laser) and electro-optical modulators (Covega Mach-10 056). The Menlo Erbium fiber laser is designed to lock at $81.25 \mathrm{MHz}$ so we will use the $1 / 16^{\text {th }}$ sub-harmonic of the LLRF $1.3 \mathrm{GHz}$ master oscillator for the laser synchronization. The use of these pieces provides a significant cost savings toward the development of the BAM at ASTA.

One fundamental limitation of the BAM is that the slope of the beam pickup signal at the zero crossing defines the time resolution. For a high time-of-arrival resolution, the slope needs to be as steep as possible, and this requires a high bandwidth for the beam pickup [3]. As part of the ASTA BAM development, new beam pickup designs will be investigated to improve the time resolution.

\subsubsection{Bunch Compression Monitor}

The bunch compression measurement will utilize coherent radiation either in the form of synchrotron radiation from the center or edge of a bending magnet, or diffraction radiation from a metallic screen containing an aperture for the beam to pass through. The spectral intensity of these forms of coherent radiation is given by

$$
I(\omega)=I_{0}(\omega)\left[N+N(N-1)|\mathcal{F}|^{2}\right]
$$

where $I_{0}$ is the single particle radiation intensity, $N$ is the number of particles in the bunch, and $F$ is the Fourier transform of the longitudinal bunch distribution, ignoring the transverse distribution. The first term is the incoherent radiation while the second term is the coherent radiation. The Fourier transform implies that the $N(N-1)$ term will dominate when the wavelength of radiation is larger than the bunch length. This intensity dependence on the 
bunch length allows one to develop instrumentation that is sensitive to the bunch length. Figure 8 (taken from reference 4 ) shows the coherent synchrotron radiation intensity as a function of the phase of the accelerating cavity prior to a double bend in the A0 photoinjector. The varying phase corresponds to varying levels of energy chirp on the beam leading to varying levels of compression after the double bend.

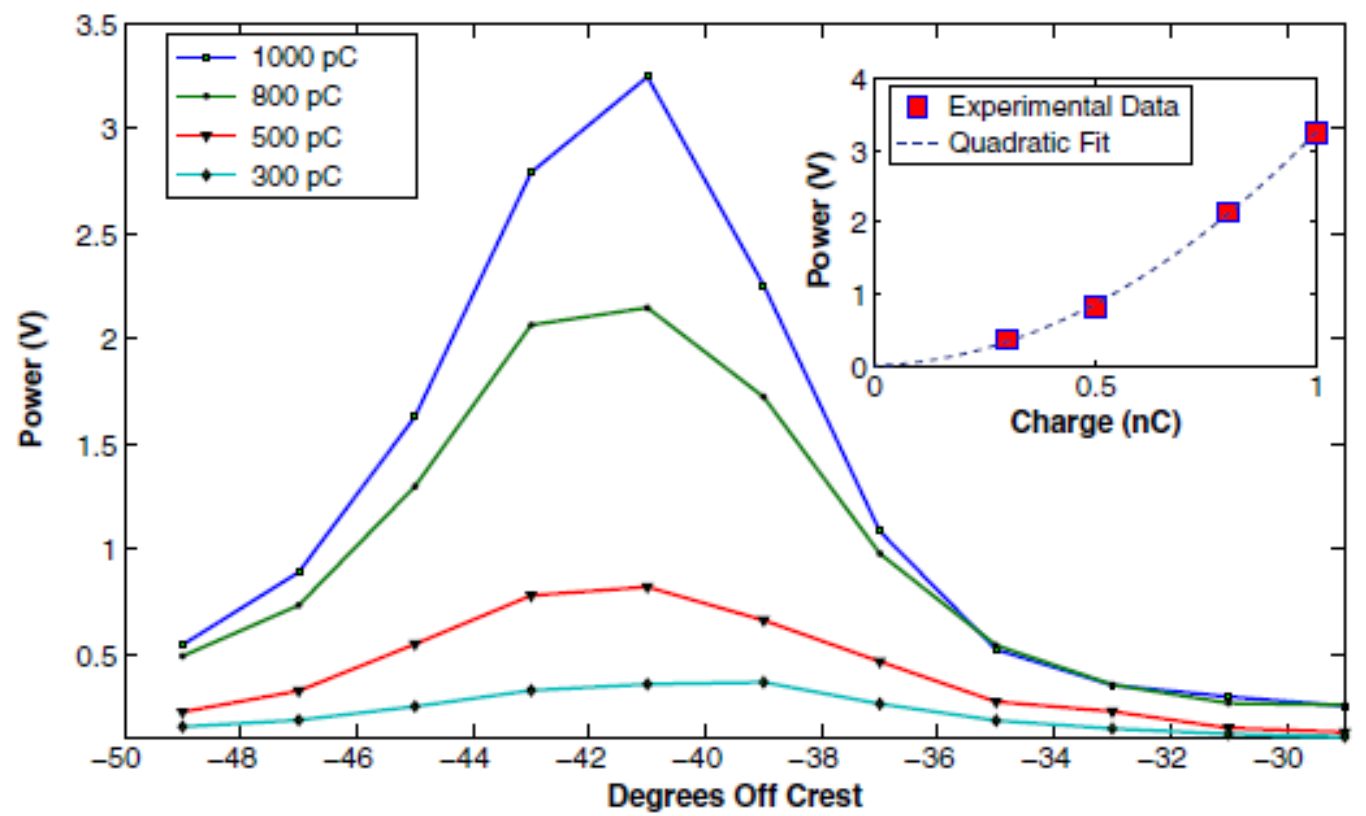

Figure 8: Coherent synchrotron radiation intensity as a function of phase of the accelerating cavity prior to a double bend.

Since typical bunch sizes are sub-picosecond to picoseconds, the frequencies of the coherent radiation is typically Terahertz. To use the coherent radiation intensity to make a bunch-bybunch measurement of the bunch length requires a somewhat broadband and relatively fast $\mathrm{THz}$ detector, since the bunch spacing can be as short as 300 ns. Several THz devices come to mind: a pyroelectric detector, a Golay cell, a bolometer, and a Schottky-diode antenna. There have been measurements made at the AO photoinjector with a broadband pseudo-optical Schottky-diode detector [5]. This device was able to measure individual bunches, although the construction of the device could prove difficult. FLASH has developed a compression monitor using a pair of pyroelectric detectors with different gains for a larger dynamic range [6]. Pyroelectric detectors are the simplest and least expensive to build of the above list and, given the success of the FLASH system, would probably be the detector of choice, with the layout of the instrument paralleling the FLASH compression monitor. Theirs is actually based on CDR, and we already plan to install a CDR monitor after the chicane at $50 \mathrm{MeV}$ for non-intercepting bunch length and relative bunch length measurements as described elsewhere in the proposal [7]. We will simply add a path to the THz detector designated for feedback. 


\subsubsection{Summary}

In summary, we propose the use of beam-based feedback to enhance the phase stability of the micropulse train to the $25 \mathrm{fs}$ regime or better and the energy stability at the $10^{-5}$ range in ASTA. We would take advantage of the 3-MHz micropulse repetition rate and the 1-ms macropulse length to implement the feedback with the planned LLRF system. This stable beam will enable experiments requiring these features including (but not limited to) emittance exchange, reversible beam heating, laser-beam interactions in electro-optical sampling or microbunching studies, and free-electron laser experiments. The facility would be unique in the HEP-supported realm in the USA.

\subsubsection{References}

[1] F. Loehl et al., "A sub 100 fs electron bunch arrival time monitor system for FLASH," Proceedings of EPAC 2006, Edinburgh, Scotland, 2006, pp. 2781-2783.

[2] F. Loehl et al., "Observation of $40 \mathrm{fs}$ synchronization of electron bunches for FELs," Proceedings of FEL 2008, Gyeongju, Korea, 2008, pp. 490-493.

[3] A. Angelovski et al., "Pickup design for high resolution bunch arrival time monitor for FLASH and XFEL," Proceedings of DIPAC 2011, Hamburg, Germany, 2011, pp. 122-124.

[4] J.C.T. Thangaraj, et al., "Experimental Studies on Coherent Synchrotron Radiation in the Emittance Exchange Line at the Fermilab A0 Photoinjector," PAC11, New York, New York, 2011.

[5] G.M. Kazakevich, et al., "Bunch Length Monitoring at the A0 Photoinjector Using a QuasiOptical Schottky Detector," PAC09, Vancouver, British Columbia, 2009.

[6] C. Behrens, B. Schmidt, S. Wesch, D. Nicoletti, "Upgrade and evaluation of the bunch compression monitor at the free-electron laser in Hamburg (FLASH)," IPAC10 Kyoto, Japan, 2010.

[7] A.H. Lumpkin, "Proposed Coherent Diffraction Radiation Measurements of Bunch Length at ASTA," Section 7.8 .5 of this ASTA proposal. 


\subsection{Accelerator R\&D for Novel Radiation Sources}

\subsubsection{High-Brightness X-ray Channeling Radiation Source}

The quest for a short-wavelength compact light source has been driven by applications ranging from fundamental science to homeland security. Recently, Vanderbilt and Northern Illinois University received funding to work on the development of a compact X-ray radiation source. The proposal aims at producing X-rays using a $50 \mathrm{MeV}$ electron beam. A proof-of-principle experiment is foreseen in the ASTA photoinjector area. This project aims at producing X-rays at $80 \mathrm{KeV}$ with high-average spectral brilliance. The proposal leverages on the ASTA's pulse-train format capabilities. Conversely, the possibility of an operating field-emission cathode in the ASTA rf gun would provide a bunch train with a $1.3-\mathrm{GHz}$ intra-bunch frequency. Such highrepetition rate train could be used to, e.g., explore high-order-mode excitation in the ASTA superconducting linac in a new regime. In addition, the exquisitely small emittance projected from the field-emission source will move RF-accelerator science into a regime where it has never played a role before. New diagnostics will be needed even to explore this region, and new effects will probably be discovered. Extension from a single field-emitter to an array will increase the total current, and used together with the beam-manipulation developments discussed in the following section may play a role in the development of coherent light sources. Thus, as an extra benefit, the channeling-radiation project will advance the basic objective of ASTA as an accelerator research tool. A major advantage of ASTA is its capability of supporting an X-ray source with high-average spectral brilliance. This section is based on the proposed concept detailed in Ref. [1].

\subsubsection{Introduction: Channeling Radiation as an Ultra-bright X-ray Source}

In a crystal, the ions in each crystal plane form a sheet of positive charge. When a relativistic electron travels through the crystal parallel to the crystal plane, Lorentz contraction increases the charge density by the factor $\gamma$ and the electron oscillates about the crystal plane in quantum states normal to the plane, as depicted in Figure 1. Radiation from transitions between the quantum states is called channeling radiation. Channeling radiation was predicted theoretically by Kumakhov [2] in 1974, and experimentally observed by Terhune and Pantell [3] in 1975. Since then, there has been extensive theoretical and experimental investigation of channeling radiation, and theory and experiment are in good agreement. 


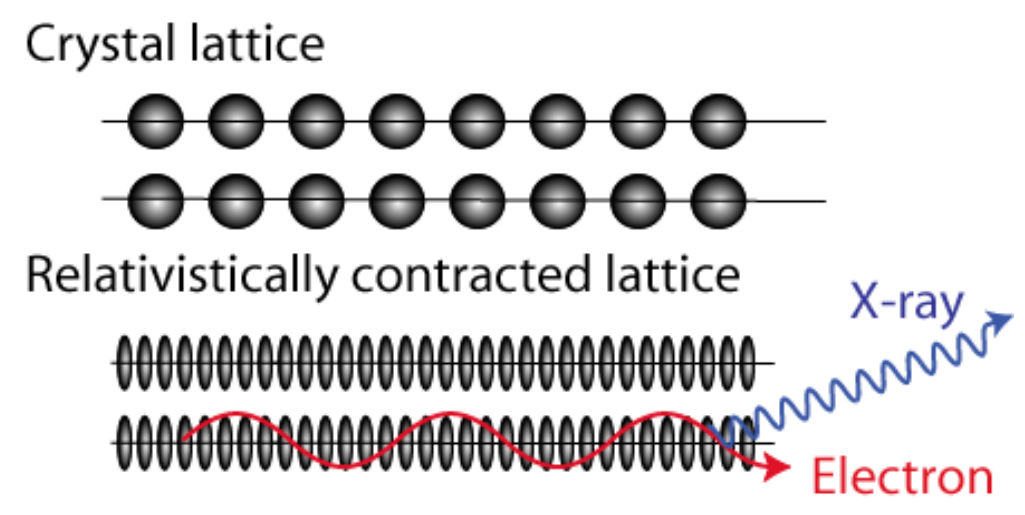

Figure 1: Mechanism of channeling radiation production [1].

The transverse forces experienced by an electron traveling along a crystal plane are comparable to those in a $10^{4}$-T magnetic undulator or a 1-TW laser undulator focused to a $10-\mu \mathrm{m}$ spot. The equivalent "undulator period" is on the order of $0.1 \mu \mathrm{m}$. The coherence length of the crystal "undulator" is limited by scattering to the order of $1 \mu \mathrm{m}$, so the effective number of "undulator periods" in channeling radiation is on the order of 10 . The photon yield is more than 10 photons per electron at high energy $(\mathrm{GeV})$, but on the order of $10^{-4}$ photons per electron in the $\mathrm{X}$-ray region [4]. The channeling-radiation peaks are typically an order of magnitude above the bremsstrahlung background [5]. The advantages of a channeling radiation source are clear. Compared with a conventional undulator, channeling radiation requires only a $50 \mathrm{MeV}$ electron beam, rather than a $10-\mathrm{GeV}$ beam to reach the hard X-ray region. Compared with a laser undulator, a channeling radiation source comprises a small diamond chip rather than a complex laser system circulating a kilowatt of laser power.

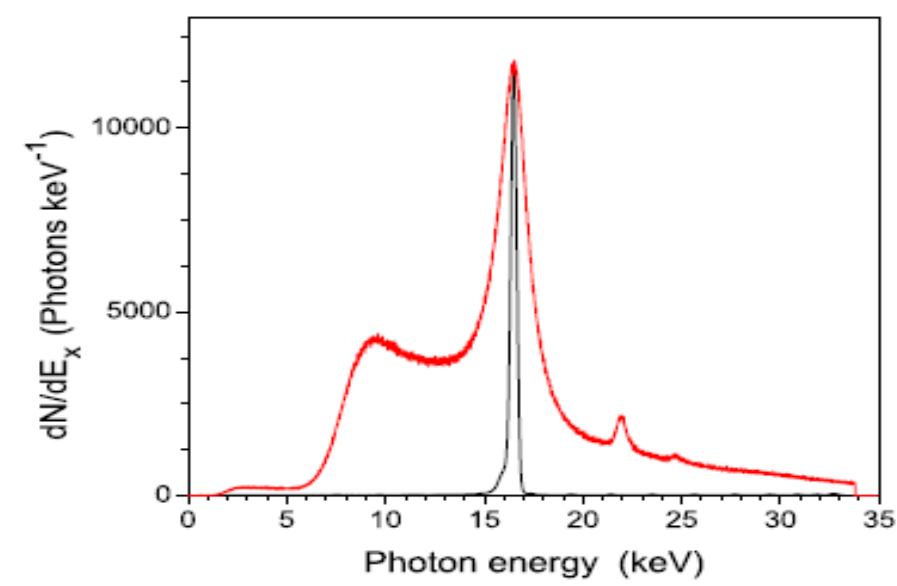

Figure 2: Observed spectrum of channeling radiation for transitions in (110) plane of diamond crystal at an electron energy of $14.6 \mathrm{MeV}$. Red: natural spectrum; black, monochromatized by Bragg reflection to remove the wings of the $C R$ line and the Bremsstrahlung background [6]. 
For electrons channeling in diamond, the best available measurements are those of Azadegan [6]. A typical channeling radiation spectrum is illustrated in Figure 2. The radiation is forward directed in a cone of angle $1 / \gamma$, and Doppler shifted by the factor $2 \gamma$. Including the Lorentz contraction of the crystal lattice, the photon energy scales roughly as $\gamma^{1.7}$, and spans the X-ray and gamma ray regions. For the $1 \rightarrow 0$ transition in (110) diamond, the photon energy may be tuned from 10 to $80 \mathrm{keV}$ by varying the electron energy as depicted in Figure 3. For a 30-MeV electron incident on a 42.5-micron thick diamond crystal, the yield on the $1 \rightarrow 0$ transition corresponds to about 0.028 photons-steradian-keV in a line 3-keV wide centered near $56 \mathrm{keV}$ [6]. In conventional units, this corresponds to $10^{-9}$ photons-mrad ${ }^{2}-0.1 \%$ bandwidth per electron.
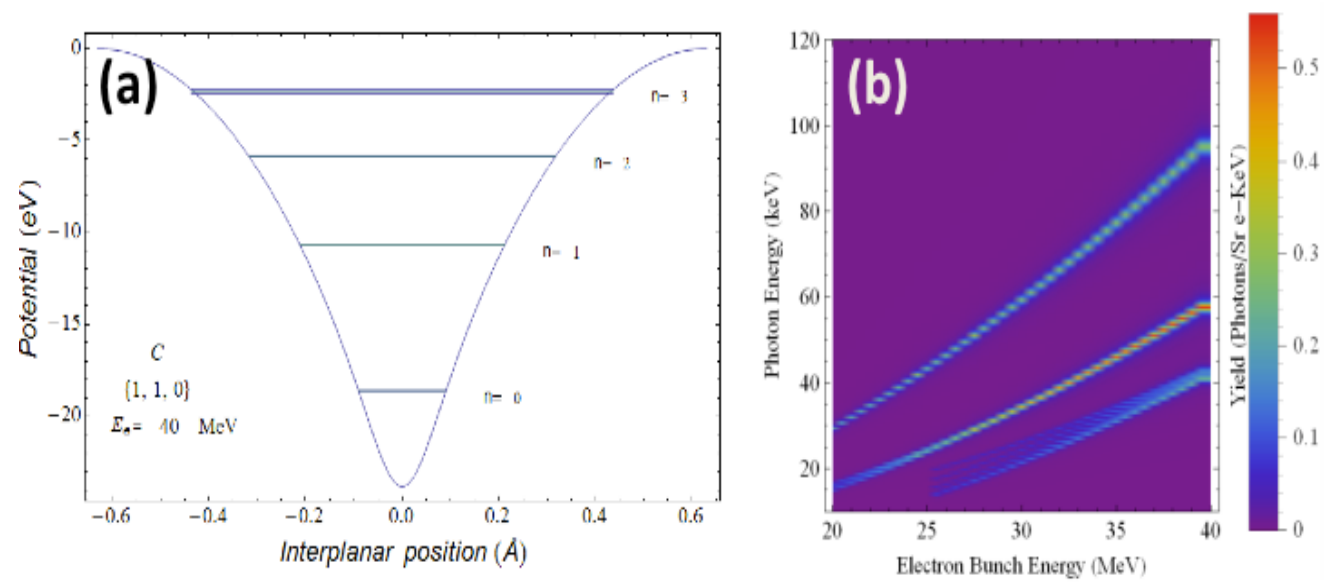

Figure 3: Simulated bounded states (horizontal line) and potential for the case of a 40MeV beam channeling in a $(1,1,0)$ diamond structure (a) and calculated yield as function of incoming electron bunch energy available in the ASTA photoinjector. The 3 traces in the density plot (b) correspond to the three allowed transitions between bounded states [17].

The spectral brilliance of the X-radiation depends on how tightly the electron beam can be focused, and this depends on the emittance of the beam. At the radiation source ELBE, in Dresden, a high-intensity channeling radiation source has been developed using a highbrightness electron beam incident on a diamond crystal [4]. Diamond is the best material for this application owing to its high thermal conductivity [7]. The normalized emittance of the beam after aperturing was $3 \mu \mathrm{m}$ rms at an average current of $100 \mu \mathrm{A}$ [8], which corresponds to a peak brightness on the order of $10^{9} \mathrm{~A} / \mathrm{m}^{2}$-sterad. The beam was focused to a $\mathrm{mm}$-size spot on the diamond. As much as $10^{11}$ photons/s were obtained in a $10 \%$ bandwidth, which corresponds roughly to an average spectral brilliance on the order of $10^{6}$ photons $/ \mathrm{s}-\mathrm{mm}^{2}$ $\mathrm{mrad}^{2}-0.1 \%$ bandwidth. 
The innovation being pursued by the Vanderbilt/NIU group is to use a single field-emitting tip as the current source, in place of the gridded thermionic gun used at ELBE. Simulations of a singletip field emitter yield a normalized emittance of $1.3 \mathrm{~nm}$ [9], which is an improvement of three orders of magnitude over the ELBE thermionic gun. In experiments at Vanderbilt, we have observed more than $10 \mu \mathrm{A}$ average current from a single field-emitting tip [10] with brightness approaching the quantum limit [11]. The current was limited by damage to the anode, which was in close proximity. The ultimate performance of diamond tips has yet to be determined. By using a single tip it may be possible to improve the transverse brightness of the electron beam by as much as six orders of magnitude, with a corresponding improvement of the spectral brilliance of the X-ray beam. The absolute emittance of the beam from a single tip, extrapolated to $30 \mathrm{MeV}$, is $\sim 40 \mathrm{pm}$. If we focus this at the critical angle for channeling radiation in diamond (about $1 \mathrm{mrad}$ ), we get a spot diameter of $40 \mathrm{~nm}$; the spectral brilliance of the X-ray beam is then $10^{12}$ photons $/ \mathrm{s}-\mathrm{mm}^{2}-\mathrm{mrad}^{2}-0.1 \%$ bandwidth at an average current of $200 \mathrm{nA}$.

\subsubsection{Production of X-rays at ASTA: Challenges and Methods}

The beam must be accelerated to high energy ( $50 \mathrm{MeV})$ to create hard X-rays. At ASTA, the first-stage acceleration will occur in the rf-gun to energies up to 4-5 MeV and further acceleration to $50 \mathrm{MeV}$ will happen in CAV1 and CAV2 cavities. We currently envision the fieldemitter cathode to be mounted on the tip of the inner conductor of a coaxial line. The coaxial line will be driven by 1.3 and $3.9-\mathrm{GHz}$ signals with solid-state amplifiers to bias the gate electrode and gate the field emission over duration shorter than the rf gun's fundamental period. Calculations indicate that such a biasing scheme will generate bunches with duration of approximately 20 ps [12]. Preserving the ultra-low transverse emittances produced by the tip after subsequent acceleration and manipulation will be challenging. Chromatic aberrations due to energy spread in the beam; emittance dilution due to nonlinearity in the rf fields, geometric aberrations in the electron beam transport lines, and collective effects can be mitigated according to start-to-end numerical simulations [13]. Numerical simulations performed for the ASTA configuration are summarized in Figures 4 and 5 . The simulated electron-beam parameters represent two orders of magnitude increase in electron beam quality. Other degrading effects which to date have been unnoticed, may also become important. One concern, for instance, is the extent to which Coulomb collisions at low energies (Boersch effect) will contribute to phase-space dilution [14]. 

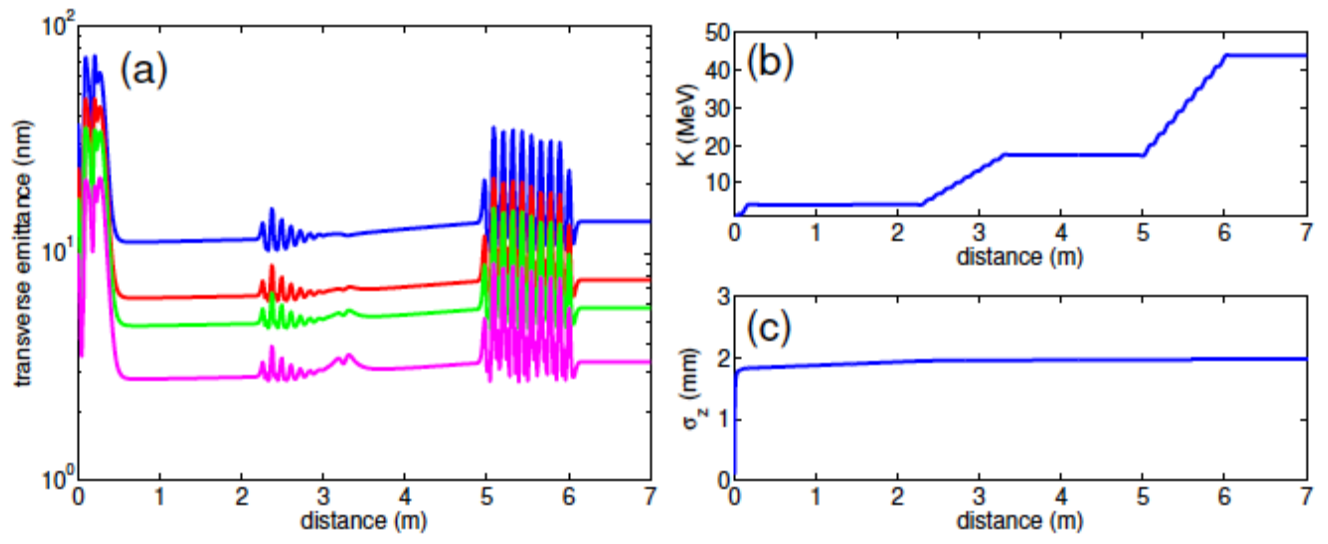

Figure 4: Transverse emittance (a), kinetic energy (b) and bunch length (c) evolution along the ASTA photoinjector accelerating section [comprising the RF gun $(0<z<30 \mathrm{~cm})$, CAV1 $(2.2<z<3.2 \mathrm{~m})$ and CAV2 $(5<z<6 \mathrm{~m})]$. The magenta, green, red and blue traces in plot (a) correspond to emittance respectively computed for the $85,90,95$, and 100 percentile of the particle distribution [13].
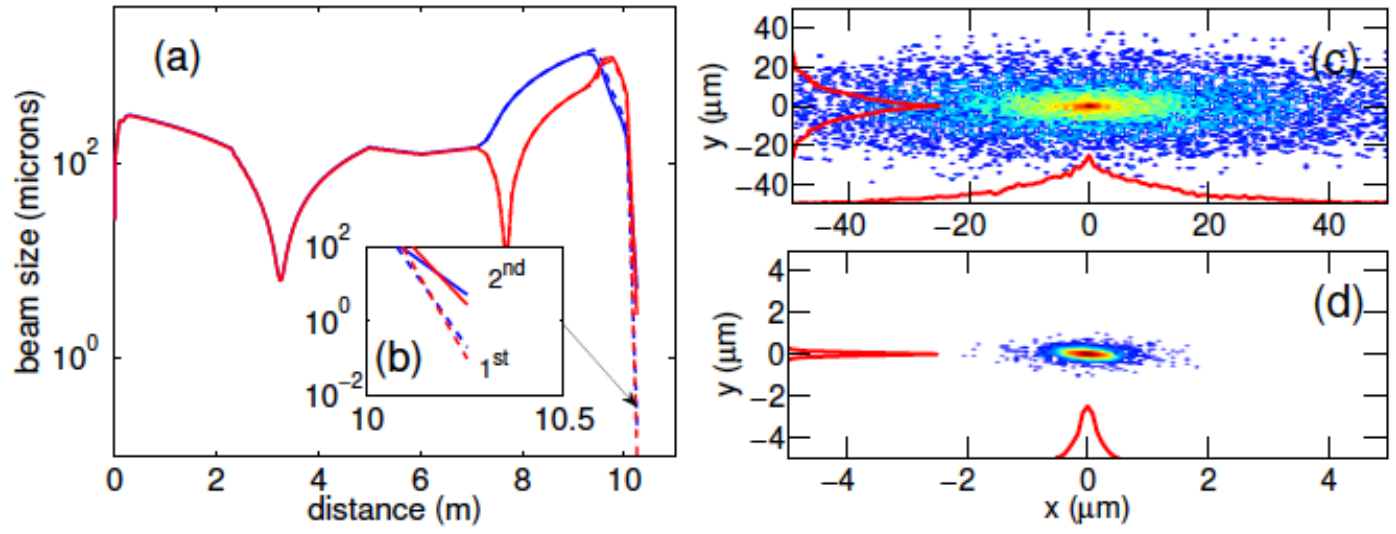

Figure 5: Horizontal (blue) and vertical (red) rms beam size evolution along the ASTA photoinjector beamline up to location of the diamond crystal (a). The solid and dashed line corresponds respectively to second- (" $\left.2^{\text {nd" }}\right)$ and first-order " 1 st”) particle-tracking simulations and inset (b) shows the rms beam sizes in the vicinity of the crystal. Density plots (c) and (d) are respectively the beam transverse density at the crystal location obtained from second and first order calculations. The red traces in plots (c) and (d) are the corresponding horizontal and vertical projections [13].

Because the x-rays are produced by the interaction of the electron beam with the crystal, the maximum current of the electron beam is limited by heating of, and radiation damage to, the crystal. Measurements and computations show that for diamond at room temperature the 
effects of heating are acceptable up to a few $\mathrm{mA}$ of beam current, so this will not be a limitation even for cw operation [7]. Measurements show that radiation damage becomes significant above a total beam fluence on the order of a few $C$ per square centimeter. Thus, the $40-\mathrm{nm}$ focal spot is destroyed in about $100 \mu \mathrm{s}$. The crystal must be moved at about $1 \mathrm{~mm} / \mathrm{s}$, and the crystal is destroyed at the rate of 0.1 square millimeters per hour at $100 \%$ duty factor. At the 1percent duty factor of ASTA, the diamond consumption rate will be much smaller.

\subsubsection{Experimental Plans at ASTA}

The production of channeling radiation will use the $50 \mathrm{MeV}$ beam produced by ASTA; see Figure 6. We plan to install the channeling radiation crystal downstream of a set of quadrupole magnets and just upstream of the first dipole of a chicane bunch compressor. This configuration will enable us to focus the beam on the crystal and separate the electron beam from the X-rays using the first dipole. In the straight-ahead line we plan on installing the energy-resolved CdTe detector needed to characterize the $\mathrm{X}$-ray radiation.
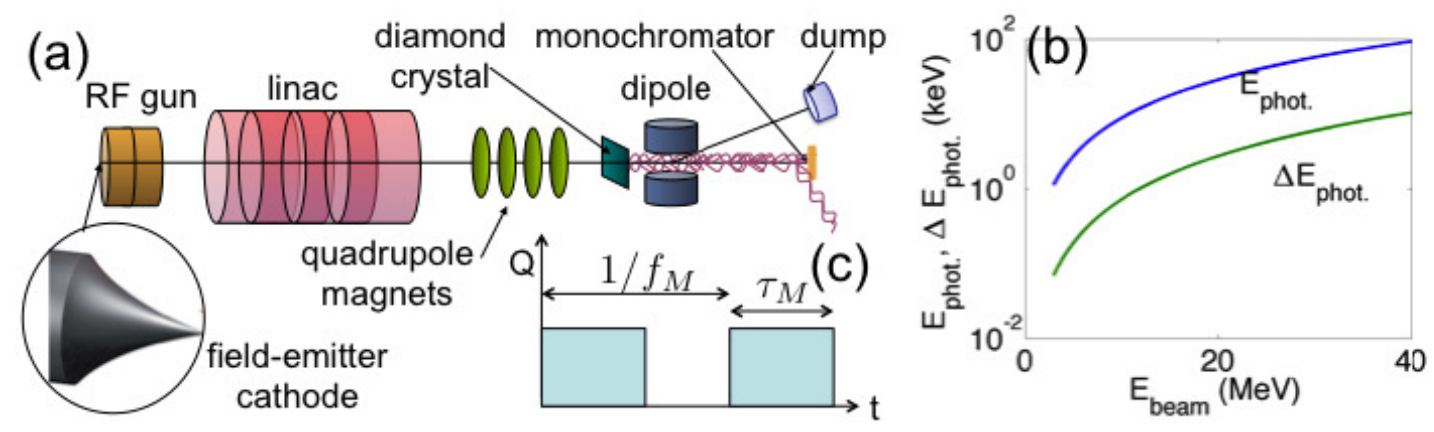

Figure 6: The layout of the X-ray channeling radiation source experiment in the $50 \mathrm{MeV}$ area of ASTA [13].

To minimize the risk, the approach will be staged. First the coaxial cathode holder will be developed and tested at the HBESL facility where the generation of field-emitted bunched beam will be demonstrated. Upon successful completion of this experiment the coaxial-line cathode holder will be installed at the ASTA. Because of the similarities between the HBESL and ASTA rf guns, we expect that the field-emitted cathode holder and associated subsystems developed during the test at HBESL will be integrally reusable without major changes. In parallel and independently from the success of the field-emission source R\&D, we will use the nominal electron beam produced via photoemission to gain experience with producing and detecting $\mathrm{X}$ rays from channeling radiation. It is expected that nanometer-level transverse emittance could also be produced by photoemission from the nominal Cesium-Telluride cathode using very small transverse laser spot [15]. Generation and characterization of ultra-low-emittance beams will be attempted during early commissioning of the ASTA photoinjector. 


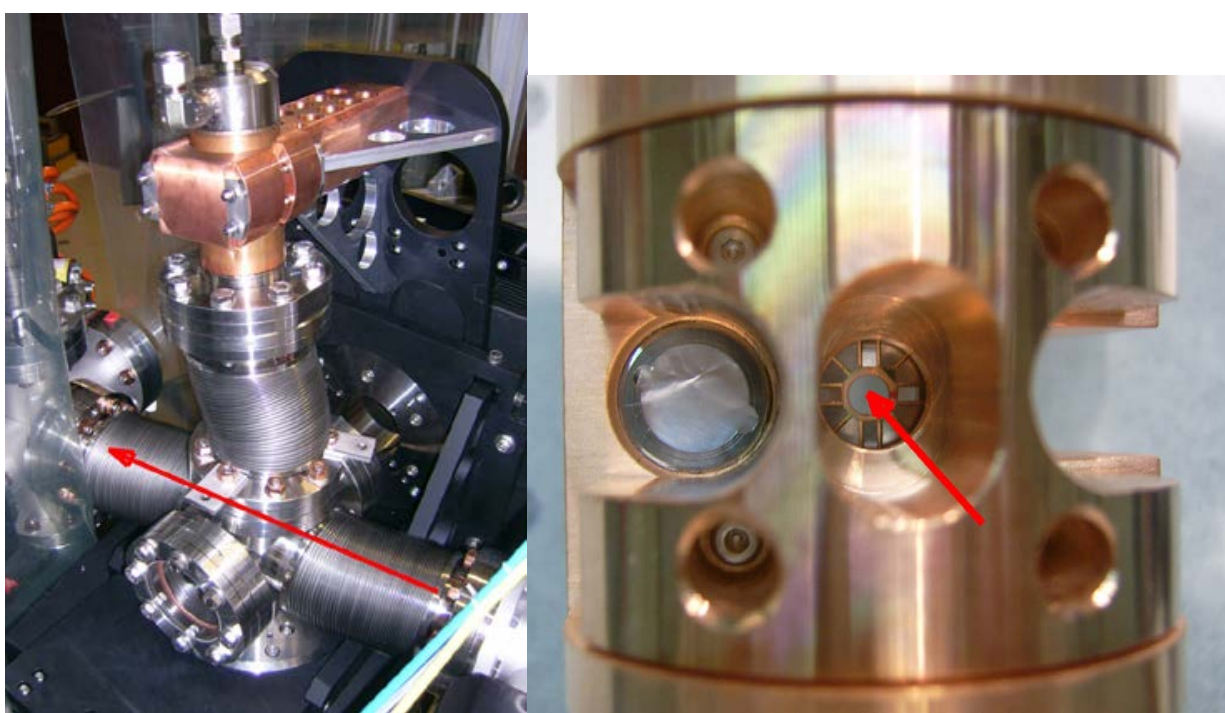

Figure 7: Photograph of the goniometer (left) and cooled crystal holder (right) to be installed in the ASTA photoinjector. The red arrow on the left photograph indicates the beam direction. The red arrow on the right photograph points to the diamond crystal location.

The goniometer needed to precisely control the orientation of the diamond crystal was provided by collaborators from the Helmoltz-Zentrum Rozendof; see photographs in Figure 7.

\subsubsection{Other Spin-off Opportunities}

The demonstration of the generation of bunched beam from field emission could find other applications especially since the principle could be applied to a field-emitter array cathode instead of a single-tip field emitter. The latter could produce much higher charge per bunch. For instance, if operated over the full duration of the macropulse, the total number of field-emitted bunches would be $1.3 \times 10^{6}$. Assuming a charge per bunch of $1 \mathrm{pC}$, this would results in a $1 \mathrm{mC}$ charge within the entire macropulse. This type of unprecedented bunch format could be used to investigate high-order mode excitation in the TESLA cavity or mimic the charge per macropulse anticipated in high-intensity linacs (e.g. Project X) proposal. In addition, the generation and acceleration of electron beams with exquisitely small emittance will extend accelerator technology to a new level. Alternatively a photo-field-emission cathode could be used and attosecond manipulation of the emitted bunches could be anticipated [16] thereby enabling new regimes for planned experiments in advanced acceleration technique or development of novel compact X-ray sources capitalizing on advanced phase space manipulations pioneered at Fermilab. 


\subsubsection{References}

[1] C. Brau, B. Choi, J. Jarvis, J. W. Lewellen and P. Piot, Synchrotron Radiation News, 25: 1-20 (2012); online at http://dx.doi.org/10.1080/08940886.2012.645419.

[2] M. A. Kumakhov, Phys. Lett. A 57, 17 (1976).

[3] R. W. Terhune and R. H. Pantell, Appl. Phys. Lett 30, 265 (1977).

[4] W. Wagner, B. Azadegan, M. Sobiella, J. Steiner, K. Zeil, and J. Pawelke, Nucl. Instrum. Meth. B 266, 327 (2008).

[5] M. A. Kumakhov, International Conference on Charged and Neutral Particles Channeling Phenomena, edited by S. B. Dabagov.

[6] B. Azadegan, Ph.D. thesis, Technischen Universität Dresden, 2007.

[7] C. K. Gary, R. H. Pantell, M. Ozcan, M. A. Piestrup, and D. G. Boyers, J. Appl. Phys. 70, 2995 (1991).

[8] W. Neubert, B. Azadegan, W. Enghardt, K. Heidel, J. Pawelke, W. Wagner, Nucl. Instrum. Meth. B 254, 319 (2006).

[9] C. Brau, Private communication (2011)

[10] J. D. Jarvis, H. L. Andrews, C. A. Brau, B.-K. Choi, J. Davidson, W.-P. Kang, and Y.-M. Wong, J. Vac. Sci. Technol. B 27, 2264 (2009).

[11] J. D. Jarvis, H. L. Andrews, B. Ivanov, C. L. Stewart, N. de Jonge, E. C. Heeres, W.-P. Kang, Y.-M. Wong, J. L. Davidson, and C. A. Brau, J. Appl. Phys. 108, 094322 (2010).

[12] J. W. Lewellen and J. Noonan, Phys. Rev ST Accel. Beams 8, 033502 (2005).

[13] P. Piot, C.A. Brau, W. E. Gabella, B. K. Choi, J. D. Jarvis, J. W. Lewellen, M. Mendenhall, D. Mihalcea, Fermilab-Conf-12-425-APC; Proceedings of the 2012 Advanced Accelerator Concept Workshop (AAC12), Austin TX, June 10-15, 2012.

[14] H. Boersh, Zeit. Physik, 139, 115 (1954).

[15] R. K. Li, K. G. Roberts, C. M. Scoby, H. To, and P. Musumeci, Phys. Rev. ST Accel. Beams 15, 090702 (2012).

[16] M. Krueger, M. Schenk, and P. Hommelhoff, Nature 475, 77 (2011).

[17] B. Blomberg, C. Brau, B. Choi, W. Gabella, M. Medenhall, D. Mihalcea, H. Panunganti, P. Piot, W. Wagner, "Channeling radiation with low-energy electron beams: experimental plans and status at Fermilab," Proceedings of the 2013 Free-Electron Laser Conference (FEL-2013). 


\subsubsection{Inverse Compton Scattering Gamma-Ray Source at ASTA}

\subsubsection{Introduction}

The unique electron beam parameters space of the ASTA facility at Fermilab offers the possibility to develop an inverse Compton scattering (ICS) gamma ray source (Figure 1) of unprecedented peak brilliance in excess of $10^{23}$ photons $/ \mathrm{s}-0.1 \% \mathrm{BW}-\mathrm{mm}^{2}-\mathrm{mrad}^{2}$ at $10 \mathrm{MeV}$. This is at least 4 orders of magnitude above the brilliance presently achieved anywhere else and in line with the most ambitious projects currently in planning; see Figure 2.

Such gamma ray source will enable a wide range of novel applications, such as probing the properties of nuclear matter [1] and nuclear physics research [2], synthesis of rare medical isotopes [3], and development of novel non-destructive testing techniques for industrial and homeland security needs [4]. With the high flux gamma rays ASTA facility may attract a significant interest from drug discovery and biotech communities [5]. Other important applications of tunable gamma ray beams include remote detection of concealed special nuclear materials [6], characterization of the nuclear waste and non-destructive evaluation of the internal condition of nuclear waste storage casks [7], non-destructive testing of civil construction elements, as well as high fidelity industrial systems. The goals of this project are (1) to demonstrate a proof-of-concept high duty cycle gamma-ICS system prototype, leveraged of the existing ASTA infrastructure; and (2) to conduct initial experiments with such source.

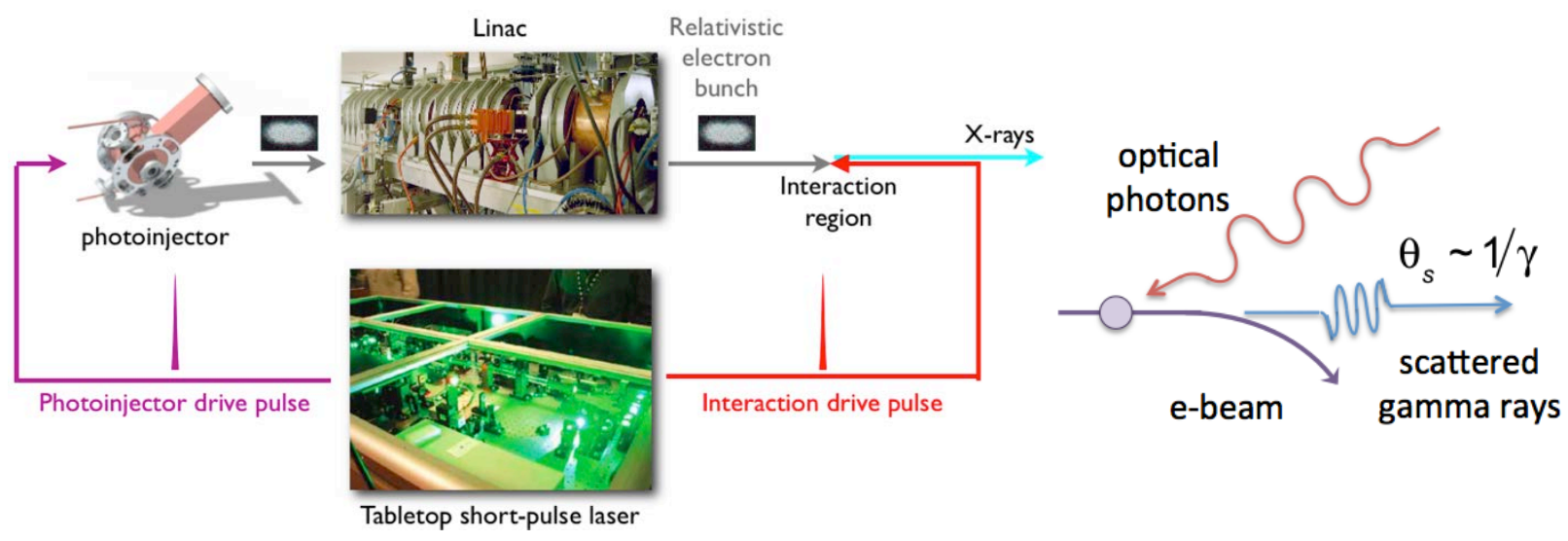

Figure 1: A pictorial diagram of the ICS process; picosecond relativistic electron and photon beams are focused and collided at the interaction point (IP), and scattered photons generate a narrow bandwidth, directional beam of gamma rays. 


\subsubsection{Anticipated Photon Parameters}

Preliminary beam parameters are displayed in Figure 2. Early in the design phase of the actual experiment, the parameters will be further optimized in collaboration with Fermilab. We note that the most challenging part of the proposed system is achieving optimal interaction efficiency over the long pulse train operations (up to 3000 bunches).

In this project RadiaBeam will collaborate with Fermilab on the design, engineering, and implementation of a recirculated ICS beamline at the ASTA facility [8], leveraging our recent experience conducting a pilot recirculated ICS experiment at the Accelerator Test Facility (ATF) at BNL [9], and the ongoing high duty cycle laser wire scanner development at Cornel ERL. The ICS beamline will be installed at the high-energy experimental area of the ASTA facility at Fermilab. The ICS beamline will use the reamplified, recirculated ASTA drive laser, and include: ICS electron beamline development; laser transport, reamplification/recirculation system including delay line; interaction chamber with e-beam final focus magneto-optics, laser beam in-vacuum optical system, and diagnostics and gamma ray beam collimator and detection system. The construction of the entire system is a collaborative effort between RadiaBeam and Fermilab RadiaBeam is currently seeking funding through the SBIR program for the design phase and construction of critical sub-systems (i.e. the interaction chamber).

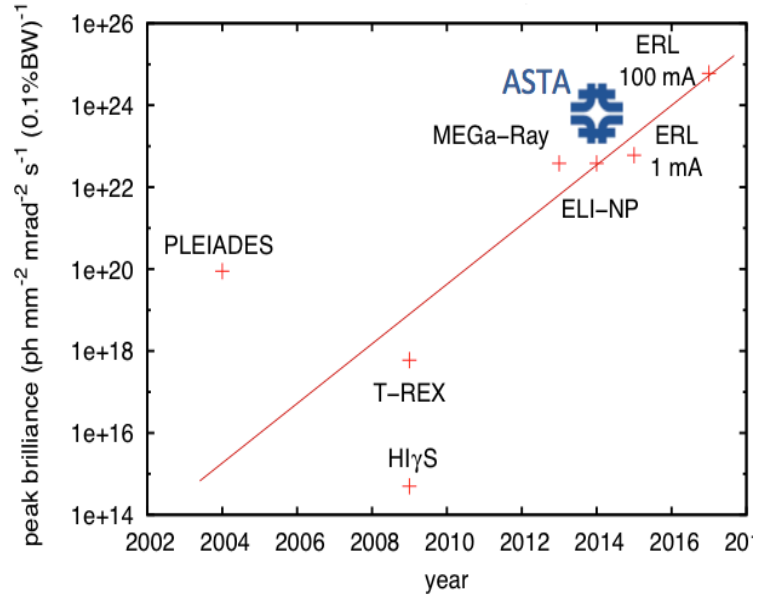

\begin{tabular}{l|c}
\hline \multicolumn{2}{c}{ E-beam } \\
\hline Charge & $3 \mathrm{nC}$ \\
\hline RMS bunch length & $10 \mathrm{ps}$ \\
\hline Normalized emittance & $5 \mu \mathrm{m}$ \\
\hline Energy & $500-1000 \mathrm{MeV}$ \\
\hline RMS spot size @ IP & $20 \mu \mathrm{m}$ \\
\hline \multicolumn{1}{c}{ Gamma rays } & $1 \mathrm{~J}$ \\
\hline Pulsed energy & $1 \mu \mathrm{m}$ \\
\hline Wavelength & $5-20 \mathrm{MeV}$ \\
\hline Peak energy & $3 \times 10$ \\
\hline Flux in 1 \% bandwidth & $3 \mathrm{MHz}$ \\
\hline Rep. rate & $10 \%$ \\
\hline Duty cycle & $10 \% \mathrm{cps}$ \\
\hline Average flux in 1\% BW & \\
\hline
\end{tabular}

Figure 2: Comparison of the proposed source at ASTA to other existing and planned ICS gamma sources normalized to $10 \mathrm{MeV}$ (plot is adapted from Ref.[5]), and the baseline parameters of the proposed ICS gamma ray source at ASTA.

\subsubsection{Experimental Outcome and Preliminary Simulations}

The design of the system and modeling of the ICS interaction will be first conducted analytically and also using the ICS computation code developed by Dr. Winthrop Brown [10] (MIT Lincoln Laboratory), which is a 3D time and frequency-domain code for analyzing the spatial, temporal, and spectral properties of an X-ray beam produced by relativistic Thomson scattering; see Figure 3. The code has been in use at RadiaBeam for 2 years and enables the use of realistic electron and laser beam models to obtain detailed characteristics of the ICS output. 

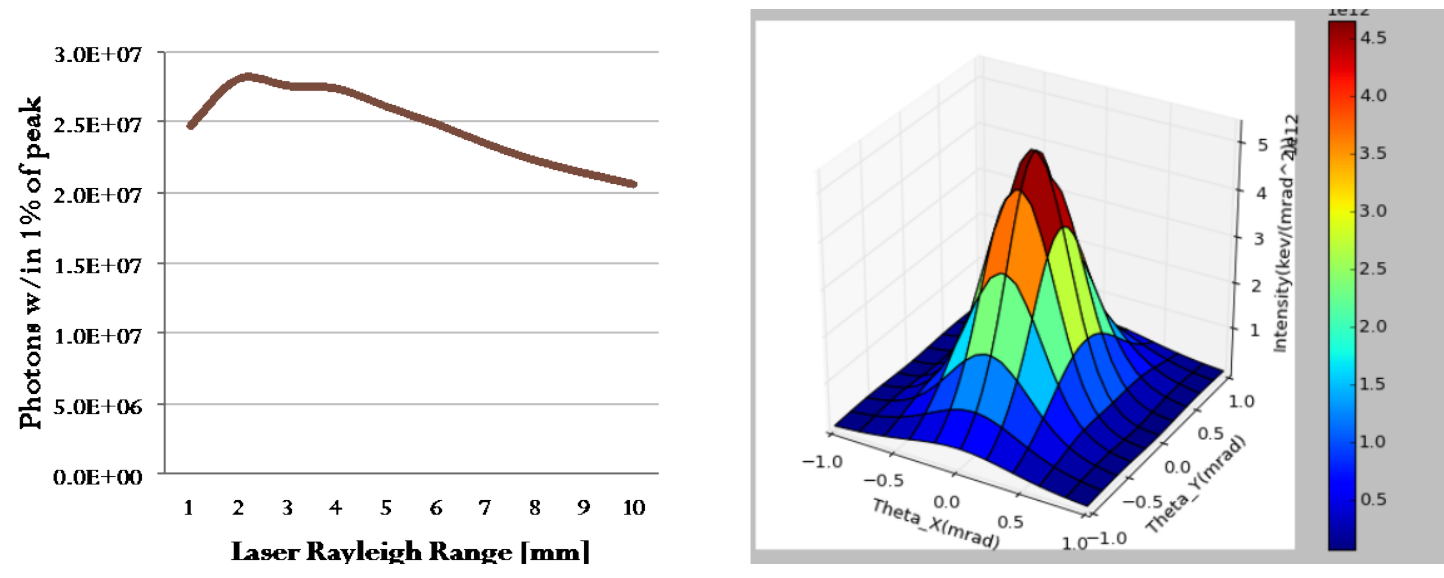

Figure 3: Numerical modeling of ICS ASTA source using W. Brown code. Left: preliminary optimization of the spectral brightness of ASTA ICS source as a function of laser spot size; right: ICS flux angular distribution.

Once the beam size is optimized at the IP, the final focus system will be designed based on PMQ magnetic optics. An important consideration is to keep the clearance aperture of PMQs large enough to allow the laser beam to propagate without clipping. Also, besides the final focus $P M Q$, a symmetric triplet will be added to re-magnify the e-beam for simpler handling downstream (transport to the beam dump or other experiments).

The experiment involves installation of the interaction chamber on the high-energy experimental area. The experiment is interceptive, i.e. it cannot be removed from the beam path without breaking vacuum. The interaction chamber is located around the interaction point (IP) and serves 3 purposes:

1. Hosting PMQ quadrupoles before and after the IP to focus the e-beam at the IP and then re-match it to the downstream beamline and into the beam dump.

2. Hosting laser input and output ports and maintaining alignment between laser axis and $P M Q$ axis.

3. Hosting diagnostics for e-beam, laser, and ICS interaction to ensure proper feedback and process optimization.

An example interaction chamber developed for the ICS experiment at BNL ATF is shown in Figure 4. The basic elements of design include alignment, permanent magnet quadrupoles (PMQ) with an adjustable holder, electron and laser beams diagnostics, optical components positioning, ports and windows location, mechanical and electrical feed throughs, UHV design, mechanical support hardware, and safe installation and handling practices. 


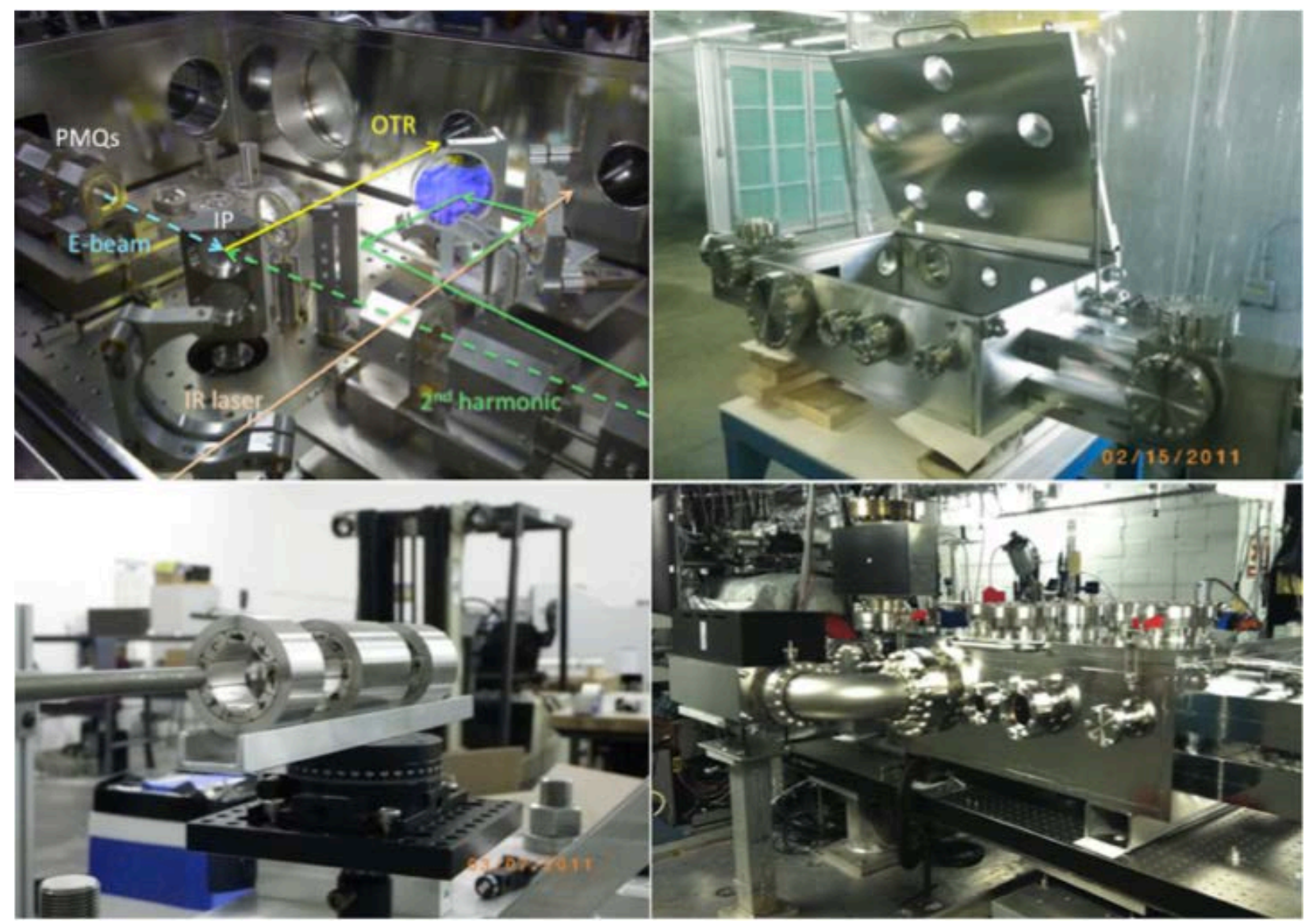

Figure 4: Interaction chamber developed at RadiaBeam for the ICS experiment at ATF BNL. From top right counterclockwise: UHV vessel at fabrication facility; inside the chamber view of the PMQ triplets and IP diagnostics station, RING optics, and beam trajectories; PMQ triplet on the alignment stand at RadiaBeam; and the chamber installed at the ATF beamline.

The chamber will be designed and fabricated to be compatible with ASTA's vacuum requirements $\left(10^{-9}\right.$ Torr). Other hardware modifications include upgrade of the Fermilab photocathode drive laser with amplification stages to reach 1-J energy at the input of the interaction chamber. Laser transport lines will have to be configured to bring the laser to the high-energy experimental area. The RING cavity [12] developed by RadiaBeam in prior ICS experiments $[9,13]$ is not very practical for ASTA's relatively slow pulse repetition rate $(3 \mathrm{MHz})$. So far we have considered an intracavity similar to the system proposed in Ref. [14] but with multiple mirrors to minimize the size of the system.

One important consideration in this project is the development of a detector system capable of accurately analyzing gamma ray flux. At full operational capacity, the detection system should be able to measure very high flux over a large energy range while also measuring spectral brightness of the gamma ray beam. For other experiments, such as NRF the system should be 
able to detect photon fluxes that are orders of magnitude lower with very high spectral resolution.

\subsubsection{References}

[1] T. Tajima, "Photonuclear Physics," The Fifth Blaise Pascal Lecture,Wednesday, March 10, 2010, Ecole Polytechnique, Palaiseau, France.

[2] D. Habs et al., Phys. Rev. Lett. 108, 184802 (2012).

[3] D. Habs et al., "Medical Applications Studies at ELI-NP," Proc. SPIE 8079, Prague, CZ, 80791H (2011).

[4] T. Tajima et al., "Science of Extreme Light Infrastructure," AIP Conf. Proc. 1228, 11-35 (2010).

[5] D. Habs and U. Köster, "Production of Medical Radioisotopes with High Specific Activity in Photonuclear Reactions with $\gamma$ Beams of High Intensity and Large Brilliance," Applied Physics B: Lasers and Optics 103, 501-519 (2011).

[6] S. Boucher et al., "High-Flux Inverse Compton Scattering Systems for Medical, Industrial and Security Applications," Proc. PAC'09, Vancouver, BC, Canada, TU6PFP046 (2009).

[7] R. Hajima, T. Hayakawa, N. Kikuzawa and E. Minehara, "Proposal of Nondestructive Radionuclide Assay Using a High-Flux Gamma-Ray Source and Nuclear Resonance Fluorescence," J. of Nucl. Sci. and Tech., 45 (5), 441-451 (2008).

[8] http://apc.fnal.gov/programs2/ASTA_TEMP/index.shtml.

[9] A. Murokh et al., "Inverse Compton Scattering Experiment in a Bunch Train Regime Using Nonlinear Optical Cavity," Proc. IPAC'12, New Orleans, LA, 3245-3248 (2012).

[10] W.J. Brown and F. V. Hartemann, Phys. Rev. ST Accel. Beams 7, 060703 (2004).

[11] P. Piot, “AARD@ASTA,” Presentation at Fermilab, Dec. 5, 2011

[12] I. Jovanovic et al., Nucl. Instr. and Meth. A 578, 160 (2007).

[13] R. Tikhoplav, A. Ovodenko and I. Jovanovic, "High-power pulse recirculation in a stable pseudo-confocal geometry," submitted for publication to Optics Letters (2012).

[14] C. Bruni, Y. Fedala, J. Haissinski, M. Lacroix, B. Mouton et al., "The ThomX X-Ray Source and its possible extension to 1-10 MeV for ELI Nuclear Physics Applications," AIP Conf. Proc. 1228, 68 (2010). 


\subsubsection{Application of High-Flux Inverse Compton Scattered Photons to Nuclear Astrophysics}

\subsubsection{Introduction and Goals of the Experiments}

The ${ }^{12} \mathrm{C}(\alpha, \gamma){ }^{16} \mathrm{O}$ reaction is a crucial reaction in nuclear astrophysics and for that reason has been studied extensively in the past using many different approaches. The standard techniques (i.e. bombarding ${ }^{12} \mathrm{C}$ targets with alpha beams or a ${ }^{4} \mathrm{He}$ gas target with ${ }^{12} \mathrm{C}$ beams) have reached levels of sensitivity that cannot easily be overcome. Using the terminology from high-energy physics the luminosity of these experiments (defined as $L=t I \varepsilon$, where $t$ is the target thickness, $I$, the beam intensity and $\varepsilon$ the detection efficiency) is typically around $10^{31} /\left(\mathrm{sec} \mathrm{cm}^{2}\right)$, which, for the current detection limit of $25 \mathrm{pb}$, gives count rates of about 1 count/hour [1].

We plan a different approach by measuring the inverse $(\gamma, \alpha)$ reaction using a bubble chamber for the detection of the reaction products. From the reciprocity theorem [2] we expect for the ${ }^{12} \mathrm{C}(\alpha, \gamma){ }^{16} \mathrm{O}$ reaction a gain of about 100 from the measurement of the time-inverse $(\gamma, \alpha)$ reaction. Furthermore since bubble chambers use liquids with much higher densities ( 5 $\mathrm{g} / \mathrm{cm}^{2}$ ) the target thickness is $10^{6}$ times higher than the ones used in standard nuclear physics experiments. This compensates the lower intensities available at $\gamma$-ray beam facilities. Altogether the luminosity in this experiment is about $10^{33} /\left(\mathrm{sec} \mathrm{cm}^{2}\right)$ and even higher values might be achievable with inverse Compton scattering (ICS) beams at the ASTA facility; see Section 8.5.2.

\subsubsection{Required Beam Parameters}

This proposal requires the inverse Compton scattering (ICS) $\gamma$-ray beam capabilities at ASTA. After we have shown in a proof-of-principle experiment at HlyS with the ${ }^{19} \mathrm{~F}(\gamma, \alpha){ }^{15} \mathrm{~N}$ reaction [3] that a bubble chamber can be used for quantitative measurements of very small cross sections we have started with the development of a new bubble chamber using a superheated oxygencontaining liquid. A first 'engineering' run with $\mathrm{N}_{2} \mathrm{O}$ was successfully performed very recently, providing information about the detection threshold which is needed to separate ${ }^{16,18} \mathrm{O}(\gamma, \alpha)$ from ${ }^{14} \mathrm{~N}(\gamma, p)$ events. Due to the high-photon flux, twelve 8 -hours shifts will be sufficient to measure the $(\gamma, \alpha)$ reactions on ${ }^{18} \mathrm{O}$ in the energy region $E_{\gamma}=7.5-10 \mathrm{MeV}$ using enriched $\mathrm{N}_{2}{ }^{18} \mathrm{O}$. Since the cross section for ${ }^{18} \mathrm{O}(\gamma, \alpha)$ is not well known in this region and is also the highest background for the planned ${ }^{16} \mathrm{O}(\gamma, \alpha){ }^{12} \mathrm{C}$ experiment (second set of twelve shifts) it is important to measure its energy dependence. This experiment will also provide information about the chemistry needed for the production of $\mathrm{N}_{2} \mathrm{O}$ using relatively inexpensive enriched ${ }^{18} \mathrm{O}$.

Proposal for an Accelerator R\&D User Facility at Fermilab's Advanced Superconducting Test Accelerator 


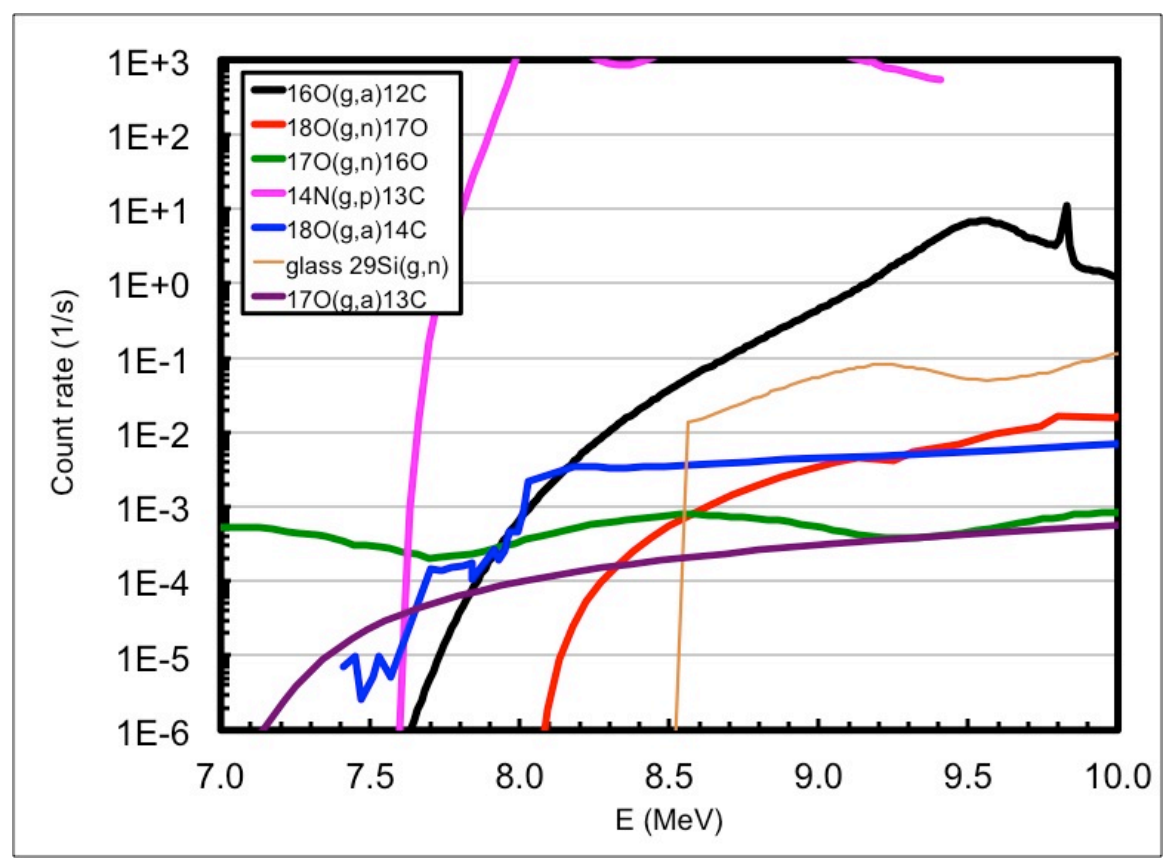

Figure 1: Expected yields for $\gamma$-induced reactions for a bubble chamber filled with enriched $\mathrm{N}_{2} \mathrm{O}$. The assumed incident flux is $10^{5} \mathrm{\gamma} / \mathrm{sec}$. The count rates scale with the available beam intensity.

\subsubsection{Experimental Technique and Expected Outcomes}

In order to gain experience with continuously operating bubble chambers, which are presently used mainly by the Dark Matter (DM) community, we have first developed in collaboration with members of the Fermilab Dark Matter group, a bubble chamber operating with fluorine containing liquids. The reason for that is that these liquids are superheated at room temperatures and considerable experience with building and operating these devices exist at FNAL. After an engineering run in 2010, we performed a first experiment in 2011 where we measured an excitation function of the ${ }^{19} \mathrm{~F}(\gamma, \alpha){ }^{15} \mathrm{~N}$ reaction in the energy region between $5-6$ $\mathrm{MeV}$. In this energy range two sharp resonances in ${ }^{19} \mathrm{~F}$ exist, whose widths and strengths have been well determined via the ${ }^{15} \mathrm{~N}(\alpha, \gamma){ }^{19} \mathrm{~F}$ reaction. The results from that experiment are shown in Figure 2. The solid points are the measured cross sections obtained in our experiment obtained from the number of bubbles and the incident $\gamma$-ray flux measured by a calibrated Ge detector located behind the bubble chamber. The solid line is the expected excitation function obtained by folding the calculated R-matrix cross section with the experimental distribution of the beam profile. There is an excellent agreement with the experimental data which means that the outgoing ${ }^{15} \mathrm{~N}+\alpha$ particles are detected with full efficiency in the bubble chamber. This is similar to the results obtained by the DM groups testing their detectors with similar reactions. It is worth mentioning that the incident gamma flux used at the maximum of the excitation functions where the ${ }^{15} \mathrm{~N}(\alpha, \gamma){ }^{19} \mathrm{~F}$ cross section is around $6 \mu \mathrm{b}$ was only $5 \times 10^{3} \gamma / \mathrm{sec}$, while the lowest cross section measured was around $3 \mathrm{nb}$ (using a flux of $2 \times 10^{6} \mathrm{\gamma} / \mathrm{sec}$ ). In both cases the count rate in the detector was about 400 counts/hour emphasizing the high luminosity of the experiment. 


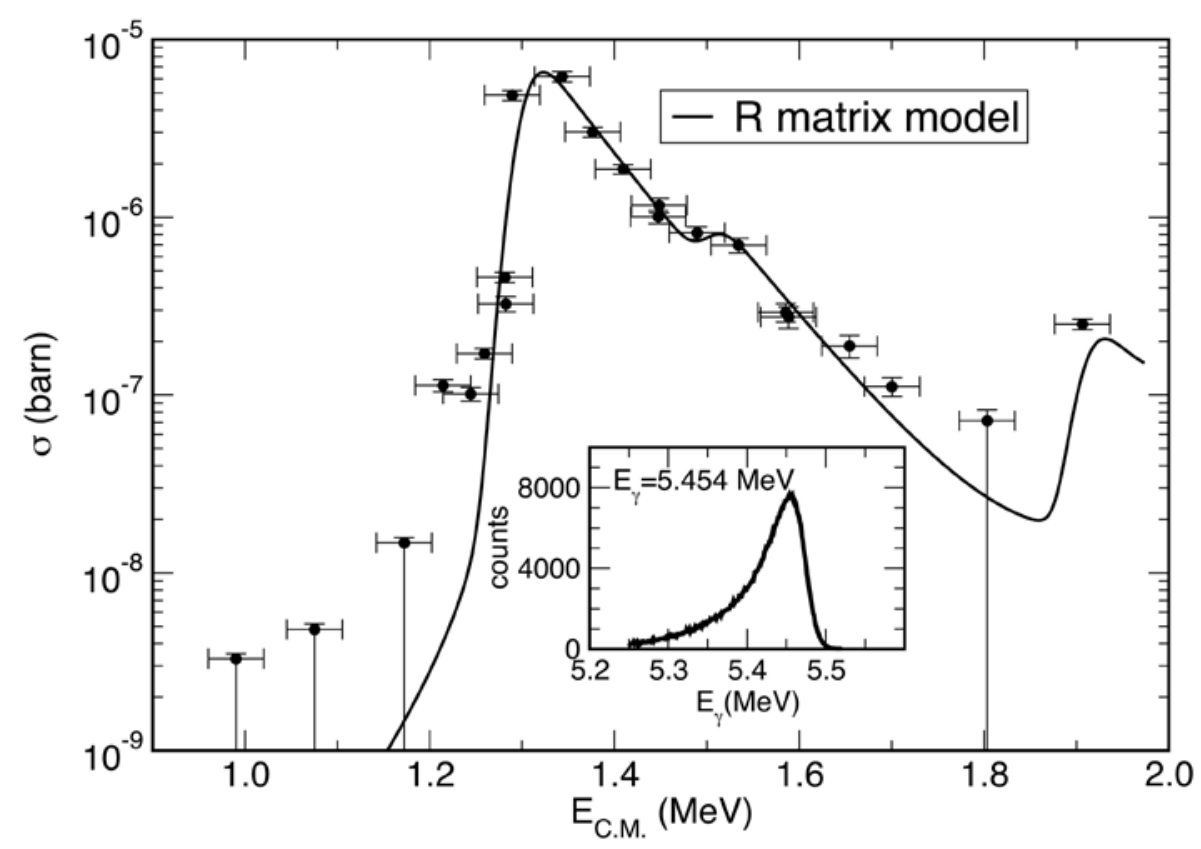

Figure 2: Solid points: Cross sections of the ${ }^{15} \mathrm{~N}(\alpha, \gamma){ }^{19} \mathrm{~F}$ reaction as measured via ${ }^{19} \mathrm{~F}(\gamma, \alpha)$ with a gamma beam from HlyS. The solid curve is the theoretical curve calculated using parameters from the literature folded with the energy distribution of the beam.

The observed cross section of $3 \mathrm{nb}$ is about a factor of 50 above the expected value of $100 \mathrm{pb}$. This limitation was probably caused by events originating from the Bremsstrahlung background $\gamma$-rays produced from electron-residual gas interaction of the $\sim 400 \mathrm{MeV}$ electron beam in the storage ring. Estimates of these background rates which depend on the vacuum of the electron ring are in agreement with the observed cross section limit at the lowest energy. Therefore, the design of a future ICS facility at ASTA would need to minimize the length of the section of the electron beam right before the photon-electron collision point. This could be achieved by bending the electron beam with a magnet after it is accelerated and right before it collides with the photons.

With the successful completion of the ${ }^{19} \mathrm{~F}(\gamma, \alpha){ }^{15} \mathrm{~N}$ experiment at $\mathrm{Hl} \gamma \mathrm{S}$ we have now focused on the ${ }^{16} \mathrm{O}(\gamma, \alpha)$ system. The first question concerns the choice of an oxygen containing liquid. The critical parameters in choosing the best liquid include the purity (i.e. presence of other nuclei such as $\mathrm{C}, \mathrm{N}, \mathrm{H}, .$. ), critical pressures, temperatures, flammability, etc. In addition one has to consider that in the final experiment highly enriched ${ }^{16} \mathrm{O}$ has to be used, since reactions on ${ }^{18} \mathrm{O}$ have large $(\gamma, \alpha)$ cross sections. Some of the liquids that were considered include liquid oxygen, $\mathrm{H}_{2} \mathrm{O}, \mathrm{CO}_{2}, \mathrm{~N}_{2} \mathrm{O}$, and $\mathrm{CH}_{3} \mathrm{OH}$. Toxicity and safety issues eliminated other possible choices such as $\mathrm{NO}, \mathrm{NO}_{2}, \mathrm{H}_{2} \mathrm{O}_{2}$. The most promising liquids are $\mathrm{CO}_{2}$ and $\mathrm{N}_{2} \mathrm{O}$. 
$\mathrm{CO}_{2}$ : Carbon dioxide becomes superheated at relatively low temperatures, but the presence of ${ }^{12} \mathrm{C}$ with a relatively low alpha breakup energy of $7.37 \mathrm{MeV}$, requires a separation of the ${ }^{16} \mathrm{O}(\gamma, \alpha){ }^{12} \mathrm{C}$ events from ${ }^{12} \mathrm{C}(\gamma, 3 \alpha)$. While the use of ${ }^{13} \mathrm{CO}_{2}$ eliminates interference from the ${ }^{12} \mathrm{C}(\gamma, 3 \alpha)$ breakup the low $(\gamma, \mathrm{n})$-threshold $(4.946 \mathrm{MeV})$ leads to neutron production that can generate additional backgrounds. As all other possible liquids it also requires high enrichment in ${ }^{16} \mathrm{O}$.

$\mathbf{N}_{2} \mathrm{O}$ : Nitrous oxide is similar to $\mathrm{CO}_{2}$ in its thermodynamic properties. The threshold for ${ }^{14} \mathrm{~N}(\gamma, p)$ is slightly higher than the triple-alpha threshold in ${ }^{12} \mathrm{C}$, but the cross sections above the ${ }^{14} \mathrm{~N}(\gamma, \mathrm{p})$ (threshold $7.55 \mathrm{MeV}$ ) are quite large. A reduction of this background is possible by choosing the right pressure and temperature conditions for the superheated liquid.

$\mathbf{H}_{2} \mathrm{O}$ : While $\mathrm{H}_{2} \mathrm{O}$ at $\mathrm{T}=200-250 \mathrm{C}$ worked well as a liquid for a continuously operating bubble chamber we observed after a few hours of operation an etching of the interior glass vessel (most likely caused by HF formed between the fluorine containing buffer fluid and the superheated water) which blocked the view for the CCD camera and furthermore lead to bubble formation at the glass surface.

Since all of these liquids have critical pressures above 40 atmospheres, a high pressure vessel was built as shown in Figure 3. It consists of a thick-walled stainless steel pressure vessel filled with mineral oil. Inside the pressure tank is a glass vessel containing the superheated liquid and a so-called buffer fluid, which minimizes the amount of the superheated oxygen-containing fluid, which, since it requires enriched isotopes, can be quite expensive. The pressure is transferred from the oil to the superheated liquid via a stainless steel bellows. Bubbles occurring in the superheated liquid are observed with a fast CCD camera (frame rate of $100 \mathrm{~Hz}$ ) mounted behind a $\sim 5 \mathrm{~cm}$ thick high-pressure glass window.

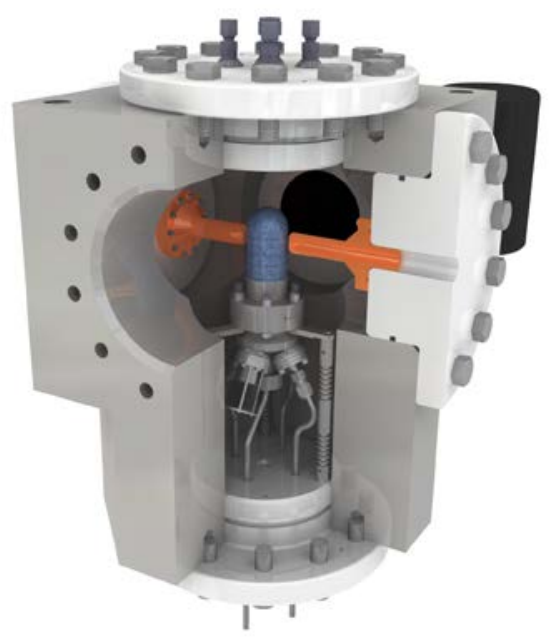

Figure 3: The new high-pressure bubble chamber showing the glass vessel (blue), the copper beam entrance and exit ports (orange) and the containment vessel (grey). 
By varying the amount of superheat in the $\mathrm{N}_{2} \mathrm{O}$ liquid, we were able to successfully suppress events from ${ }^{14} \mathrm{~N}(\gamma, p){ }^{13} \mathrm{C}$. As a result, we were able to observe $\mathrm{O}(\gamma, \alpha) \mathrm{C}$ events for the first time with a bubble chamber. An example is shown in figure 4 below.

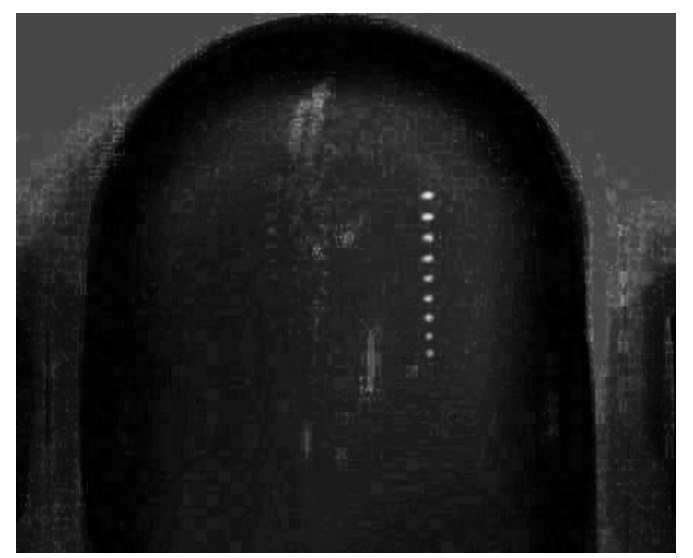

Figure 4: Composite of nine sequential photographs taken at $10 \mathrm{~ms}$ intervals for the first $\mathrm{O}(\gamma, \alpha) \mathrm{C}$ event in the $\mathrm{N}_{2} \mathrm{O}$ bubble chamber. The temperature was $11^{\circ} \mathrm{C}$ at $3.79 \mathrm{MPa}$ and the incident $\gamma$-ray energy was $9.66 \mathrm{MeV}$.

There are still several issues that need to be resolved before we can start with a measurement of the ${ }^{16} \mathrm{O}(\gamma, \alpha){ }^{12} \mathrm{C}$ reaction. In order to improve the visibility of the bubbles we plan to change the illumination from reflected light to back-light, which was used in our first $\mathrm{C}_{4} \mathrm{~F}_{10}$ bubble chamber run at HlyS. Judging from our present experience the most promising oxygencontaining liquid so far seems to be $\mathrm{N}_{2} \mathrm{O}$. The yield from the ${ }^{14} \mathrm{~N}(\gamma, \mathrm{p}){ }^{13} \mathrm{C}$ reaction, which has a large cross section when compared to the ${ }^{16} \mathrm{O}(\gamma, \alpha){ }^{12} \mathrm{C}$ process, can be eliminated by choosing the correct pressure/temperature conditions of the superheated liquid. For an $8.5 \mathrm{MeV}$ incident $\gamma$-beam the energy of the ${ }^{12} \mathrm{C}$ recoils from the ${ }^{16} \mathrm{O}(\gamma, \alpha){ }^{12} \mathrm{C}$ reaction is $300-400 \mathrm{keV}$ while the energy of the ${ }^{13} \mathrm{C}$ recoils from the ${ }^{14} \mathrm{~N}(\gamma, \mathrm{p}){ }^{13} \mathrm{C}$ reaction is only $50-90 \mathrm{keV}$. Since a bubble chamber is very effective in rejecting low-energy signals (e.g. from protons and minimum ionizing particles) by choosing the proper operating conditions we can eliminate the low-energy ${ }^{14} \mathrm{~N}(\gamma, \mathrm{p})$ events.

Also, we plan to determine the cross section of the ${ }^{18} \mathrm{O}(\gamma, \alpha){ }^{14} \mathrm{C}$ reaction, which is an important background in the energy range $E_{\gamma}=7.5-10 \mathrm{MeV}$. This reaction is estimated to provide the highest $(\gamma, \alpha)$ background towards the low energy region as shown in Figure 1 . Enriched ${ }^{18} \mathrm{O}-$ water for $\mathrm{N}_{2}{ }^{18} \mathrm{O}$ is available at Argonne and will provide a good test for producing $\mathrm{N}_{2}{ }^{16} \mathrm{O}$ with our enriched ${ }^{16} \mathrm{O}$-water in the future. Due to the large cross sections, the expected count rates for the ${ }^{18} \mathrm{O}(\gamma, \alpha){ }^{14} \mathrm{C}$ would be about $1 / \mathrm{sec}$ using enriched ${ }^{18} \mathrm{O}$ and an incident flux of $2 \times 10^{4} \gamma / \mathrm{sec}$.

On the other hand, for ${ }^{16} \mathrm{O}(\gamma, \alpha){ }^{12} \mathrm{C}$, an incident flux of $5 \times 10^{7} \gamma / \mathrm{sec}$ would produce count rates of $1 / \mathrm{sec}$ for this reaction at an energy of $8.5 \mathrm{MeV}$. For that experiment we ask for an additional 4 days of $\gamma$-ray beam with energies between 8.5 -10 MeV. Since the depletion factor for the heavier oxygen isotopes is at least $10^{-6}$ the ${ }^{17,18} \mathrm{O}$ yields in Figure 1 are upper limits. Thus a 
measurement of the ${ }^{16} \mathrm{O}(\gamma, \alpha){ }^{12} \mathrm{C}$ reaction in the energy region below $10 \mathrm{MeV}$ should be possible using the future ICS facility at ASTA.

\subsubsection{References}

[1] Kunz, R. et al., Phys. Rev. Lett. 86, 3244 (2001).

[2] J. M. Blatt, and V. F. Weisskopf, Theoretical Nuclear Physics, Springer-Verlag, 1979.

[3] C. Ugalde et al., Phys. Lett. B 719, 74 (2013). 


\subsection{Accelerator R\&D for Stewardship and Applications}

\subsubsection{Demonstration of Techniques to Generate and Manipulate Ultra-Low Emittance Beams for Future Hard X-ray Free-Electron Lasers}

\subsubsection{Abstract}

Next generation X-ray free-electron lasers (XFELs) will require significantly lower emittances than currently achievable to reach sub-Angstrom wavelengths. For example, the XFEL proposed for the Los Alamos Matter-Radiation Interactions in Extremes (MaRIE) experimental facility [1] will require $100-\mathrm{pC}$ to $250-\mathrm{pC}$ electron bunches with transverse emittances of about $0.15 \mu \mathrm{m}$ to produce $42 \mathrm{keV}$ photons. New beam-based seeding schemes have additionally been proposed to provide narrow $\mathrm{X}$-ray bandwidth and enhanced longitudinal coherence, also of potential use for MaRIE. The Advanced Superconducting Test Accelerator (ASTA) offers promise as a unique test bed to test and demonstrate emerging new concepts that may lead to meeting these types of requirements. Specifically, ASTA can be used to demonstrate novel emittance partitioning schemes, to test compressed harmonic generation (CHG) schemes, to verify our current understanding of coherent synchrotron radiation (CSR), and to benchmark CSR simulation models.

\subsubsection{Two-Stage Emittance Partitioning}

The technological limit on generating hard X-ray radiation is mainly determined by the availability of transversely bright electron beams. In order for an FEL to lase optimally, the normalized transverse beam emittance must be small, $\varepsilon_{n} \leq \beta \gamma \lambda_{x-r a y} / 4 \pi$, to ensure overlap between the electron and X-ray phase spaces, where $\beta$ and $\gamma$ are the beam's velocity normalized to the speed of light $c$ and its relativistic factor, respectively, and $\lambda_{x-\text { ray }}$ is the $\mathrm{X}$ ray wavelength. This condition is easier to satisfy at higher beam energies and this approach was used to design Linac Coherent Light Source (LCLS) [2] which generates $8 \mathrm{keV}$ photons using a $14 \mathrm{GeV}$ electron beam.

The next generation of hard X-ray FELs, producing photons well above $10 \mathrm{keV}$, cannot use the same approach due to increased beam energy spread caused by the single-particle synchrotron radiation at high energies. At the end of an undulator, this spread is equal to

$$
\frac{\Delta E_{r m s}}{E}=\sqrt{\frac{55}{48 \sqrt{3}}} \sqrt{\frac{\hbar e^{5} B^{3}}{4 \pi \varepsilon_{0} m^{5} c^{6}}} \gamma^{1 / 2},
$$

where $B$ is the rms undulator field strength, $L$ is the undulator length, and $e, m, \hbar$ and $\varepsilon_{0}$ are the electron charge, electron mass, normalized Planck's constant, and free-space permittivity. FEL performance is degraded when the total beam energy spread is greater than the gain

Proposal for an Accelerator R\&D User Facility at Fermilab's Advanced Superconducting Test Accelerator 
parameter. Estimates for generating $50-\mathrm{keV}$ photons (with a 100-meter long undulator with a 2.4-cm period and a 1-T rms field, driven by a 3.4-kA beam) show that the beam energy is limited to about $20 \mathrm{GeV}$. At this energy both the induced energy spread and the FEL gain parameter are close to $0.015 \%$ and the FEL efficiency rapidly drops at higher energies. This limitation on the beam energy puts a constraint on the normalized transverse beam emittance. Numerical simulations for this case indicate that emittances as high as $0.15 \mu \mathrm{m}$ are marginally acceptable (these transverse emittances are about a factor of two more relaxed than the optimal constraint, with some corresponding loss of efficiency), and with performance degrading significantly as the emittance is increased above that. In contrast, the longitudinal emittance for a $150-$ fsec bunch and $0.01 \%$ energy spread can be as high as $180 \mu \mathrm{m}$. A 250-pC electron bunch produced with currently available high-brightness photoinjectors would typically have normalized beam emittances $\varepsilon_{x, n} / \varepsilon_{y, n} / \varepsilon_{z, n}$ of $0.5 / 0.5 / 1 \mu \mathrm{m}$, with a total phase-space volume of $0.25 \mu^{3}$. At the same time, the electron bunch required for a $50 \mathrm{keV}$ XFEL must have normalized beam emittances not exceeding $\varepsilon_{x, n} / \varepsilon_{y, n} / \varepsilon_{z, n}$ of $0.15 / 0.15 / 25$, with a total phase-space volume of $0.6 \mu \mathrm{m}^{3}$. Therefore, currently available photoinjectors can generate bunches with sufficiently small phase-space volumes, but the partitioning of this phase space into longitudinal and transverse emittances is not ideal.

These considerations have led to the proposal of two-stage emittance partitioning schemes [3] which can be demonstrated for the first time at ASTA. The overall concept is shown in Figure 1.

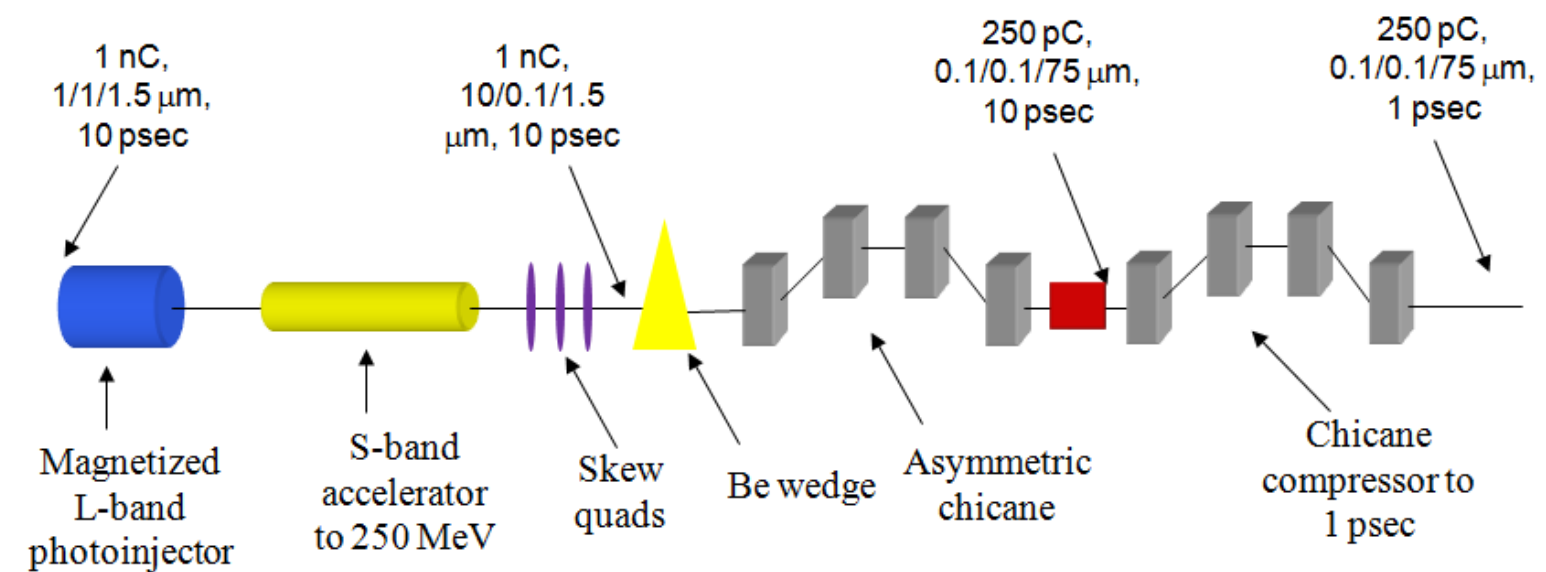

Figure 1: Overview of a two-stage emittance-partitioning scheme capable of producing an ultra-low transverse emittance beam.

A magnetized photoinjector (where the axial field at the cathode is non-zero) can lead to highly asymmetric transverse emittances, that can be recovered with a flat-beam transform (FBT), shown ideally here at $250 \mathrm{MeV}$. The larger transverse emittance (here $10 \mu \mathrm{m}$ horizontally) can be reduced at the expense of the axial emittance by using an element to generate a horizontalaxial coupling. A wedge-shaped piece of beryllium is shown as this element. This element 
should not be linearly symplectic in order to change the beam's eigen-emittances. In other words, while we can always define a linear part of the beam transformation across this element $R$ (from the quadratic part of the Hamiltonian obtained by expanding about a trajectory), the rms eigen-emittances are no longer necessarily conserved because $\sigma_{f} \neq R \sigma_{i} R^{T}$ if the Hamiltonian is higher order in phase space variables.

Although a wedge is one possible non-symplectic element, a canted undulator and inverse Compton scattering are other possibilities. For a canted undulator, the undulator gap varies horizontally across the beam, leading to an energy loss due to incoherent synchrotron radiation emission correlated with horizontal position. Alternatively, an offset high-power laser can lead to an equivalent correlated energy loss due to inverse Compton scattered photons. A wedge is the simplest version for demonstrating this concept, and can be used at energies as low as 50 $\mathrm{MeV}$, and with ASTA parameters. Remarkably, the eigen-emittances formed through this scheme can be recovered with a simple dogleg or asymmetric chicane.

Figure 2 shows numerical simulations illustrating features of this concept. These two figures below show the eigen-emittances produced from a magnetized injector for next generation light sources, through the first two traveling-wave structures (here to a total energy of 100 $\mathrm{MeV})$.
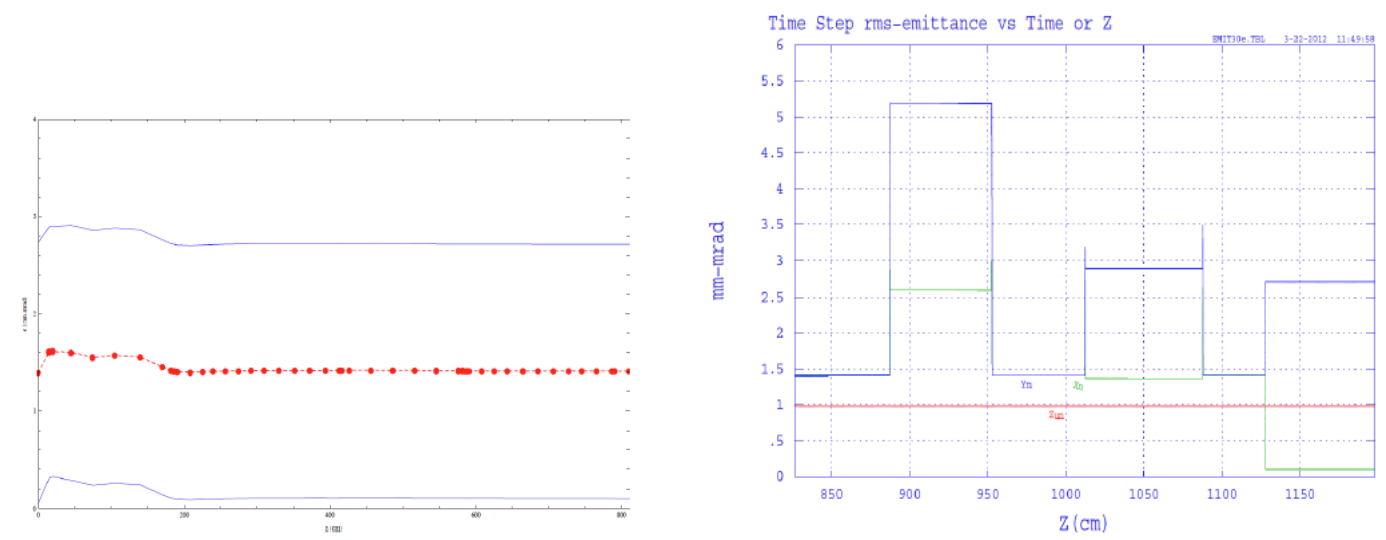

Figure 2: Evolution of the eigen (blue traces) and physical (red trace) emittances in next generation light sources photoinjector (left). Emittance evolution along the RFB converter (right).

The red lines in the above left figure are the physical emittances (about $1.35 \mathrm{~mm}$ ), and the blue lines are the eigen-emittances (about 0.1 and $2.7 \mu \mathrm{m}$ respectively). The emittance evolution in the FBT section is shown in the above right figure, and the eigen-emittances are recovered as the physical emittances, at the levels of 0.1 and $2.77 \mu \mathrm{m}$. There was $605 \mathrm{G}$ on the cathode for this simulation and the final product of the emittances is within $10 \%$ of the optimized emittances for the unmagnetized case. 

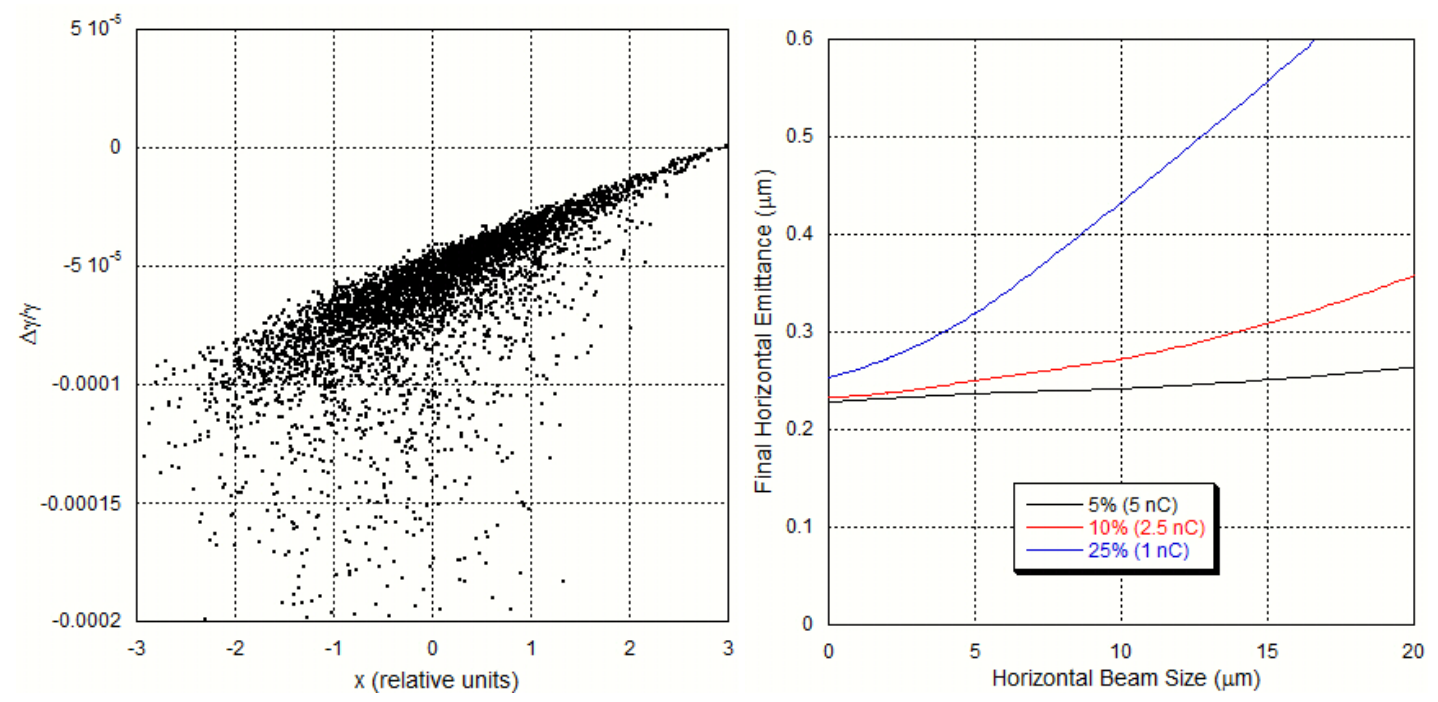

Figure 3: Energy-position correlation downstream of the wedge (left) and final horizontal emittance downstream of the asymmetric chicane (see Figure 1).

Figure 3 shows the basic concept of the second stage. After transmission through the wedge, an energy deviation is generated that is correlated with horizontal position, as shown in the above left figure. The dispersion of the dogleg or asymmetric chicane is adjusted so the energyhorizontal slew is rotated vertically, thereby decreasing the beam's horizontal size. The final normalized eigen-emittances are then (where $\varepsilon_{n+}$ becomes $\varepsilon_{z, n}$ and $\varepsilon_{n-}$ becomes $\varepsilon_{x, n}$ ):

$$
\varepsilon_{n+}=\gamma \beta\left(\frac{\Delta \gamma}{\gamma}\right)_{\text {slew }} \sigma_{z} \text { and } \varepsilon_{n-}=\gamma \beta \frac{\left(\left(\frac{\Delta \gamma}{\gamma}\right)_{\text {ind }}^{2}+\left(\frac{\Delta \gamma}{\gamma}\right)_{\text {int }}^{2}\right)^{1 / 2}}{\left(\frac{\Delta \gamma}{\gamma}\right)_{\text {slew }}}\left(\varepsilon_{x, \text { ind }}^{2}+\varepsilon_{x, \text { int }}^{2}\right)^{1 / 2}
$$

where here we have included the effects of both the intrinsic (int) beam emittance and energy spread as well as those induced (ind) by the interaction with the wedge. Due to these effects on the beam from the wedge, it is best to consider a larger initial bunch charge (even though that increases the initial emittances from the FBT) and to make the final $250 \mathrm{pC}$ bunch charge from best $10 \%$ to $25 \%$ of the initial bunch charge (shown in the above right figure).

Preliminary estimates indicate that a $2.5 \mathrm{nC}$ ASTA bunch can produce a $250 \mathrm{pC}$ bunch with a product of transverse emittances about an order of magnitude smaller than starting with an optimized $250 \mathrm{pC}$ bunch. 


\subsection{Compressed Harmonic Generation in Double Emittance Exchangers}

Theoretically, compressed harmonic generation ( $\mathrm{CHG}$ ) preserves harmonic seeding on a bunch better than other schemes like high-gain harmonic generation (HGHG) and even echo-enabled harmonic generation (EEHG) [4]. Conceptually, CHG is like the compression of an accordion bellows, where the modulation structure is the same as before, except the length scale has been compressed. Ratner [5] has proposed a multiple chicane based scheme, shown in Figure 4.

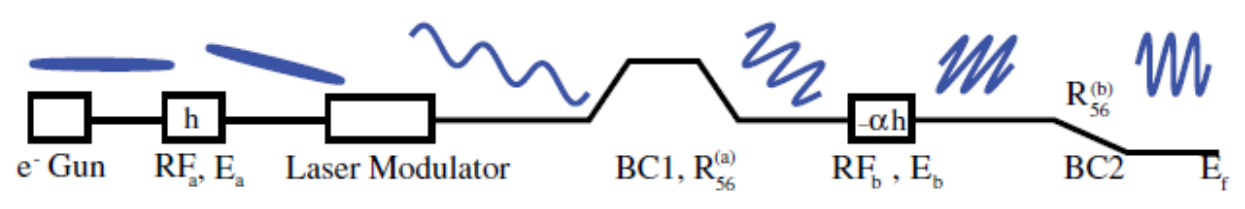

(a)

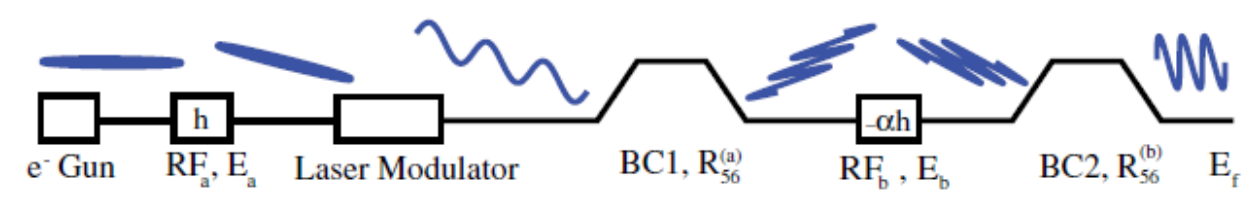

(b)

Figure 4: Principle of compressed harmonic generation (CHG).

Due to the simplicity of chicane compression, a relatively complex scheme has to be employed (otherwise the slight high-frequency modulation put on the beam gets dwarfed by the larger energy slew for the gross compression of the bunch).

A promising CHG scheme has been proposed by both Carlsten [6] and Zholents [7], where a double emittance exchanger (DEEX) is used to compress a bunch with an initial sinusoidal modulation on it. An example of such architecture is diagrammed in Figure 5.

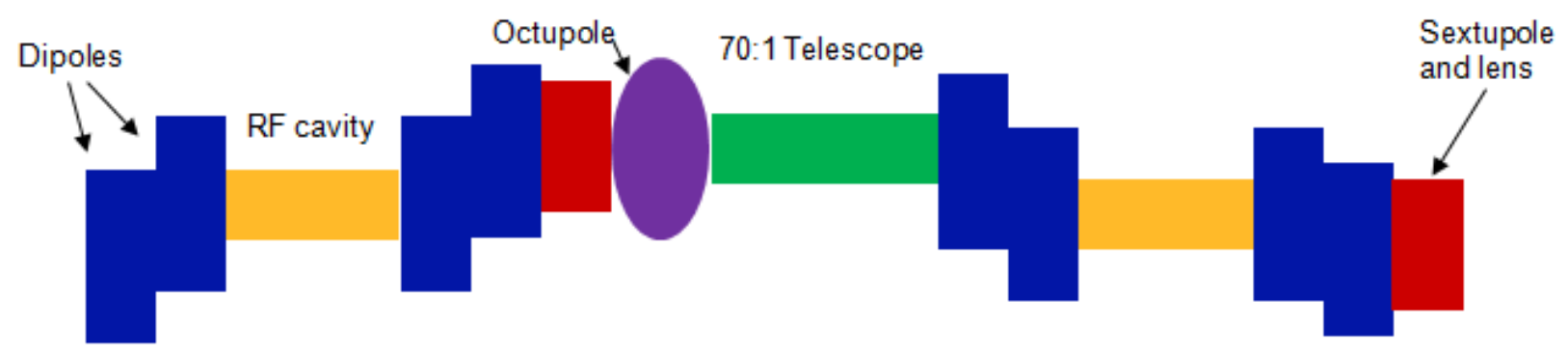

Figure 5: Principle of compressed harmonic generation (CHG) using a double-emittanceexchanger (DEEX) beamline. 
ELEGANT simulations not only show that such a compressor can be used to compress a beam without emittance growth, but that the optics in between the EEXs can also be used to straighten out the curvature induced from the beam's wakefields in the longitudinal phase space, shown in Figure 6 for a nominal $1 \mathrm{GeV}$ compression design.
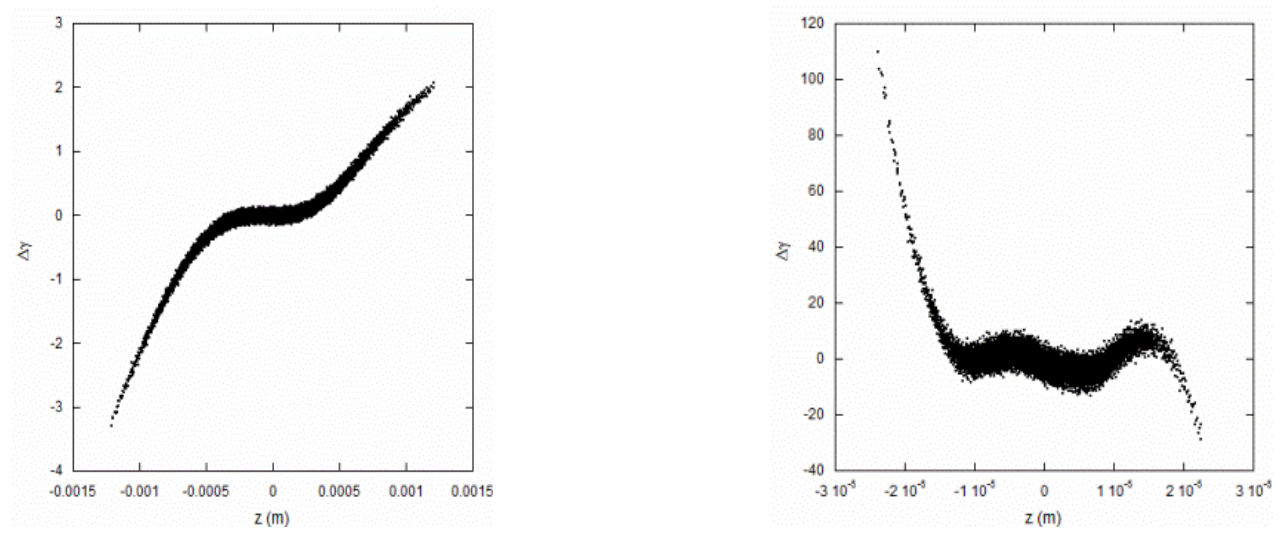

Figure 6: Longitudinal phase space before and after compression in a double emittanceexchange beamline.

Of particular importance for a CHG design is that the final axial position of a particle only depends the initial axial position, and that nonlinear effects are negligible. Various designs have been proposed and modeled, and the high energy ASTA beam would lead to a compelling demonstration of one or more optimized designs.

\subsubsection{Studies of CSR Fields Enhancement Due to Beam Microbunching}

CSR studies using exact particle fields (instead of the ultra-relativistic 1-D approximation) indicate that microbunching in beams result in much stronger energy-dependent CSR fields as compared to beams without bunching [8], see Figure 7.

For this $1 \mathrm{GeV}$ case, the peak CSR field from 100-nm microbunching (shown by the orange curve above) is nearly two orders of magnitude larger than the field for an unbunched Gaussian beam (shown by the light blue curve). This effect is expected to result in an additional energydependent growth of the microbunching instability which may significantly increase the beam energy spread and emittance in bunch compressors. Moreover, beam-based seeding schemes for FELs may be affected by this mechanism resulting in increased beam energy spread or increased bandwidth of the microbunching (this is why the CHG scheme described above uses an energy modulation but not a current modulation). 


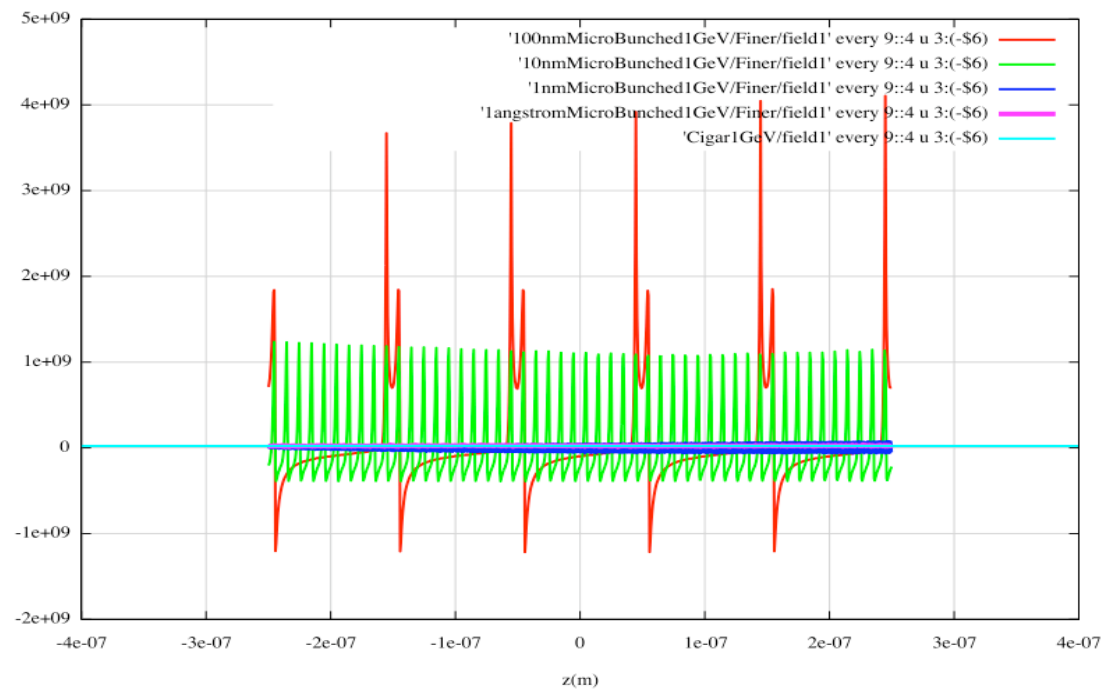

Figure 7: CSR electric field (vertical axis is in $\mathrm{V} / \mathrm{m}$ ) over a small fraction of the electron bunch.

Experimental studies of this effect can be performed at the ASTA facility. An upstream masked EEX optics can be used to create a longitudinally bunched beam in a controlled manner, as demonstrated earlier at Fermilab [9]. Specifically, the radiated power and power spectrum of the synchrotron radiation will provide enough information to determine if this enhanced CSR radiation exists, and will help benchmark numerical models used for simulating the microbunch instability.

The CSR radiation power is expected to scale quadratically with the bunch charge since CSR fields and beam current scale linearly with the total charge. In this way it will be possible to separate contributions from CSR and single-particle radiation from the total radiated power. Additionally, CSR generated by a microbunched beam is expected to be observed in two frequency domains determined by the wavelength of the microbunching and the rms bunch length. Therefore, it will be possible to determine whether beam microbunching significantly increases the effect of CSR on beam energy losses as the bunch charge and the microbunching wavelength can be varied separately from each other, which significantly simplifies the calibration of these measurements. 


\subsubsection{References}

[1] http://marie.lanl.gov.

[2] P. Emma, et al., Nature Photonics 4, 641 (2010).

[3] B. E. Carlsten et al., "Two-stage generation of beam correlations in electron beams to establish low transverse beam emittances," submitted to Physical Review Special Topics Accelerators and Beams, 2011.

[4] N. A. Yampolsky, "Description of modulated beam dynamics," submitted to Physical Review Special Topics - Accelerators and Beams, 2012.

[5] D. Ratner, A. Chao, and Z. Huang, Phys. Rev. ST Accel. Beams, 14, 020701 (2011).

[6] B. E. Carlsten, K. A. Bishofberger, S. J. Russell, and N. A. Yampolsky, Phys. Rev. ST Accel. Beams, 14, 084403 (2011).

[7] A. Zholents and M. Zolotorev, "Emittance exchange and bunch compression," Proc. 2011 Particle Accelerator Conference, New York (2011) TUOCN6.

[8] B. Carlsten, et al., "Three-dimensional coherent synchrotron radiation calculations," presentation to the $50^{\text {th }}$ ICFA Advanced Beam Dynamics Workshop on Energy Recovery Linacs, presentation available at http://erl2011.kek.jp/author information/oral/ index.html.

[9] Y.-E. Sun, et al., Phys. Rev. Lett., 105, 234801 (2010). 


\subsubsection{Beam Dechirper for Free-Electron-Laser Linacs}

\subsubsection{Introduction}

Lasing in X-ray FELs calls for electron beams with minimal energy spread, hence requiring that the energy-position correlation ("energy chirp") imparted for compression should be removed before the beams reach the undulators. The energy loss induced by the short-range RF wakefields in the accelerating section following the last compressor contributes to the removal of the energy chirp but, depending on the machine and beam parameters, may be insufficient to flatten the longitudinal phase space. Narrow-aperture, dielectric passive insertions ("dechirpers") have been proposed $[1,2,3]$ as a way to enhance the longitudinal wakefields and thus avoid the costly method of removing the residual chirp by operating the rf structures offcrest. In addition, dechirpers based on transverse deflecting cavity have also recently been explored. Availability of such dechirpers would benefit several proposed FEL projects, including next generation light sources [4], in terms of cost reduction, augmented design flexibility, and possibly higher performance.

Proof-of-principle demonstrations have been recently carried out [2] or are currently underway [5] but utilize electron beams that differ from those suitable for radiation production and employ devices with less than ideal design. The ASTA facility has the potential to offer a definitive demonstration of the practical feasibility of the concept and possibly the opportunity to do prototype testing for next generation light sources on beams that have characteristics close to those of interest (high brightness bunches with several hundred ampere peak current, $\sim 100 \mu \mathrm{m}$ bunch length or shorter, and $1 \mu \mathrm{m}$ or smaller normalized rms transverse emittance).

\subsubsection{Wakefield-Enhancing Insertion for Energy-Chirp Control}

A more precise assessment of ASTA full potential for this experiment will require a thorough understanding of the beam quality that can be delivered as well as the development path for the facility. We anticipate that extensive modeling of the beam dynamics through the injector, compressors, and dechirper will be needed before this proposal can be further refined. Ideally, the beam should be similar to that needed in FEL applications with bunch charge not to exceed the $\sim_{\mathrm{nC}}$ level to avoid large transverse emittance. The case study considered in our preliminary calculation, reported later in Sec. 4.0, assumes $Q=250 p C$, close to the nominal bunch charge [4] for next generation light sources. For this charge, recent simulations studies of the ASTA machine [6] show normalized transverse rms emittances on the order of $0.6 \mu m$ at exit of the injector at low $I \sim 15 \mathrm{~A}$ peak current. These studies also indicate that the transverse emittance can grow considerably through the low-energy compressor as a result of collective effects, particularly in the horizontal plane, hence posing some limitations the degree of compression. Peak currents of a few hundred amperes are desirable. Essential to the experiment will be the availability of the harmonic cavity linearizer in order to deliver beams with dominant linear energy chirp, high peak-current and possibly non-spiky current profiles. The option to compress at higher energy (i.e. after ACC1) in possible future ASTA upgrades as a way to reduce the emittance growth and allow for larger compression would be of course quite attractive.

Proposal for an Accelerator R\&D User Facility at Fermilab's Advanced Superconducting Test Accelerator 
Two kinds of insertions suitable for energy chirp control have been considered in the literature: narrow-aperture pipes with i) dielectric-lined or ii) corrugated metallic inner walls. In both cases the insertion geometry/material parameters are chosen so that passage of a bunch excites primarily a single mode with wavelength $\lambda$ of the same order as the bunch length $\sigma_{z}$ or longer. For this proposal we consider use of a corrugated, cylindrical pipe in the $\lambda \gg \sigma_{z}$ regime.

The magnitude of the longitudinal wakefields associated with the insertion scales as $Z_{0} c / \pi a^{2}$, where $Z_{0}$ is the vacuum impedance and $a$ the pipe radius. A schematic of the pipe longitudinal profile is shown in Figure 1 with the geometry of the corrugation defined by the parameters $g, p$, and $\delta$. For $\delta, p \ll a$ and $\delta \geq p$ the longitudinal wakefield potential is well approximated by [3] $(\mathrm{z}>0)$ :

$$
w(z)=\frac{Z_{0} c}{\pi a^{2}} \cos \frac{2 \pi z}{\lambda}
$$

with mode wavelength $\lambda=2 \pi \sqrt{a \delta g / 2 p}$. The convolution of the wake potential with the bunch current profile yields the energy loss along the bunch. For removal of the linear term of the energy chirp, $\lambda$ should be much longer than $\sigma_{z}$. In contrast, a shorter $\lambda$ could in principle be used to control higher order energy/position correlations [7]. Expanding the experimental program to explore the application of high-impedance insertions to the linearization of the longitudinal phase space will be worth considering in future revisions of this proposal.

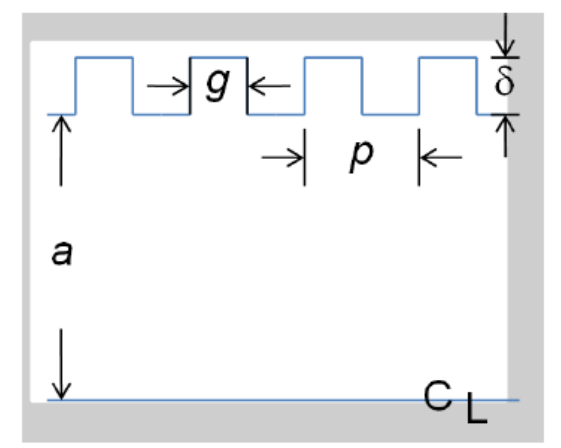

Figure 1: Sketch of the cylindrical-symmetric corrugated structure used to enhance wakefield effects.

For bunches with flattop current profile, peak current $I$ and length $L_{b}$ much shorter than $\lambda$, the energy loss by an electron at distance $z$ from the bunch head is about linear in $z$. In practical units:

$$
\Delta E[\mathrm{MeV}] \simeq 0.12 \times \frac{I[\mathrm{~A}] L[\mathrm{~m}] z[\mathrm{~mm}]}{a[\mathrm{~mm}]^{2}} \quad \text { or } \quad \Delta E[\mathrm{MeV}] \simeq 36 \times \frac{Q[\mathrm{nC}] L[\mathrm{~m}]}{a[\mathrm{~mm}]^{2}} \times \frac{z}{L_{b}}
$$


where $L$ is the insertion length. One meter long insertion with $a=3 \mathrm{~mm}$ aperture would cause about $1 \mathrm{MeV}$ energy spread on a $Q=250 p C$ bunch. The choice for the corrugation parameters summarized in Table 1 is a good match for next generation light source beams sand a good starting point to design an ASTA experiment.

Table 1: Corrugated-pipe parameters proposed for next generation light sources and the ASTA experiment

\begin{tabular}{|l|c|}
\hline Parameter & Value \\
\hline Pipe radius $a(\mathrm{~mm})$ & 3 \\
\hline Corrugation depth $\delta(\mathrm{mm})$ & 0.45 \\
\hline Corrugation period $p(\mathrm{~mm})$ & 1 \\
\hline Corrugation gap $g(\mathrm{~mm})$ & 0.75 \\
\hline Wakefield wavelength $\lambda(\mathrm{mm})$ & 4.5 \\
\hline
\end{tabular}

To be practical a dechirper installed in an operational machine should come with some degree of tunability. Mechanically, this could be easily realized by adopting a rectangular cross section for the insertion with adjustable upper and lower jaws, as for example in the PAL-ITF experiment [5]. Unfortunately this geometry is associated with fairly strong quadrupole-like transverse wakefields that could make integration into the machine optics complicated. A "revolver" style insertion with multiple openings of different apertures would be an interesting, although presumably mechanically more challenging, solution. Alternatively, the insertion could be broken into segments, with mechanisms in place to insert and extract the insertions at will. Clearly, the development of suitable mechanical designs would be a large component of the preparation work for this experiment.

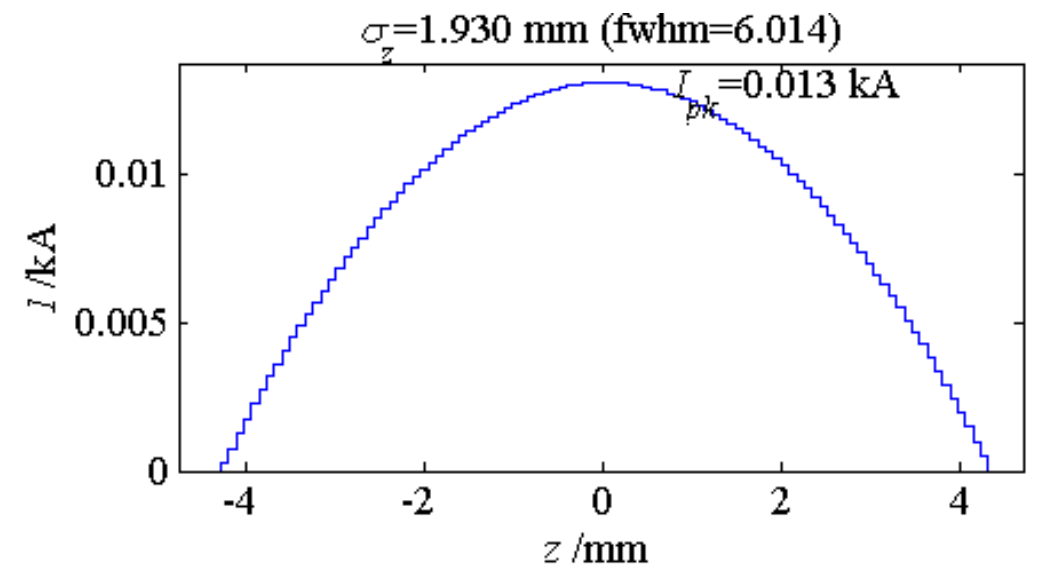

Figure 2: Parabolic current profile of the $Q=200 \mathrm{pC}$ beam model at the exit of the gun as used in the LiTrack simulations.

The ASTA experimental set-up could consist of one or two such insertions, for a total length of about 1 or $2 \mathrm{~m}$. They would be positioned downstream the bunch compressor and the first fulllength accelerating module ACC1 to make use of short-length high peak current beams. Control 
over the ACC1 rf phase and voltage could be exploited to conduct measurements over a range of beam energy and energy chirp.

We carried out preliminary LiTrack [8] simulations of the longitudinal dynamics of a 250 pC bunch through the dechirper including propagation through the injector two $1.3 \mathrm{GHz}$ cavities, a $3.9 \mathrm{GHz}$ cavity linearizer, and bunch compressor. We adopted a parabolic current profile (Figure 2) for the initial beam out of the gun at $5 \mathrm{MeV}$, which is not unrealistic [9]. However, the LiTrack simulation of the low-energy dynamics through the injector is very rough (no collective effects are included besides the wakefields of the rf structures and dechirper) and we shouldn't expect a very accurate prediction of the beam longitudinal phase space and pulse shape at the exit of the chicane. In fact, we have noticed that including a model of the $3^{\text {rd }}$ energy chirp induced by longitudinal space charge (as reported in [6]) would significantly affect the bunch shape suggesting, not surprisingly, that more accurate simulations are clearly needed.

Table 2: Settings of RF structures voltage and phases for the LiTrack simulations.

\begin{tabular}{|l|c|c|c|}
\hline & CAV1-2 & CAV39 & ACC1 \\
\hline $\mathrm{V}(\mathrm{MV})$ & 43 & 5.2 & 250 \\
\hline$\varphi(\mathrm{deg})$ & -10.6 & -180 & 0 \\
\hline
\end{tabular}

We tuned the rf structure settings to achieve nominal peak currents in the 300A range, avoiding larger compression that would likely come at the expense of brightness degradation (of course it remains to be seen whether this tentative compression factor would be compatible with an acceptable level of emittance growth). The settings are summarized in Table 2. The momentum compaction in the compressor chicane is $R_{56}=-0.19 \mathrm{~m}$.

To take advantage of the full potential of the experiment, diagnostics should be available for time-resolved measurements, including a transverse RF deflector, a spectrometer downstream of the dechirper to measure the longitudinal phase space, and a suitable set-up for quadrupole scans. This would be used to measure the projected emittance and slice of the emittance for at least one plane. This would preferably be in the vertical plane where the emittance of the incoming beam is smaller and in which plane the beam may be steered to excite transverse wakefields in the dechirper.

The results of the LiTrack run (Figure 3) show that the linear component of the correlated energy spread (about $0.6 \%$ over the full width of the bunch) carried by this beam at the exit of ACC1 is compensated by a $2 \mathrm{~m}$ long insertion. Notice that in this case the dechirper is also seen to add a noticeable cubic term to the chirp profile (the relative strength of which depends on the bunch shape). 

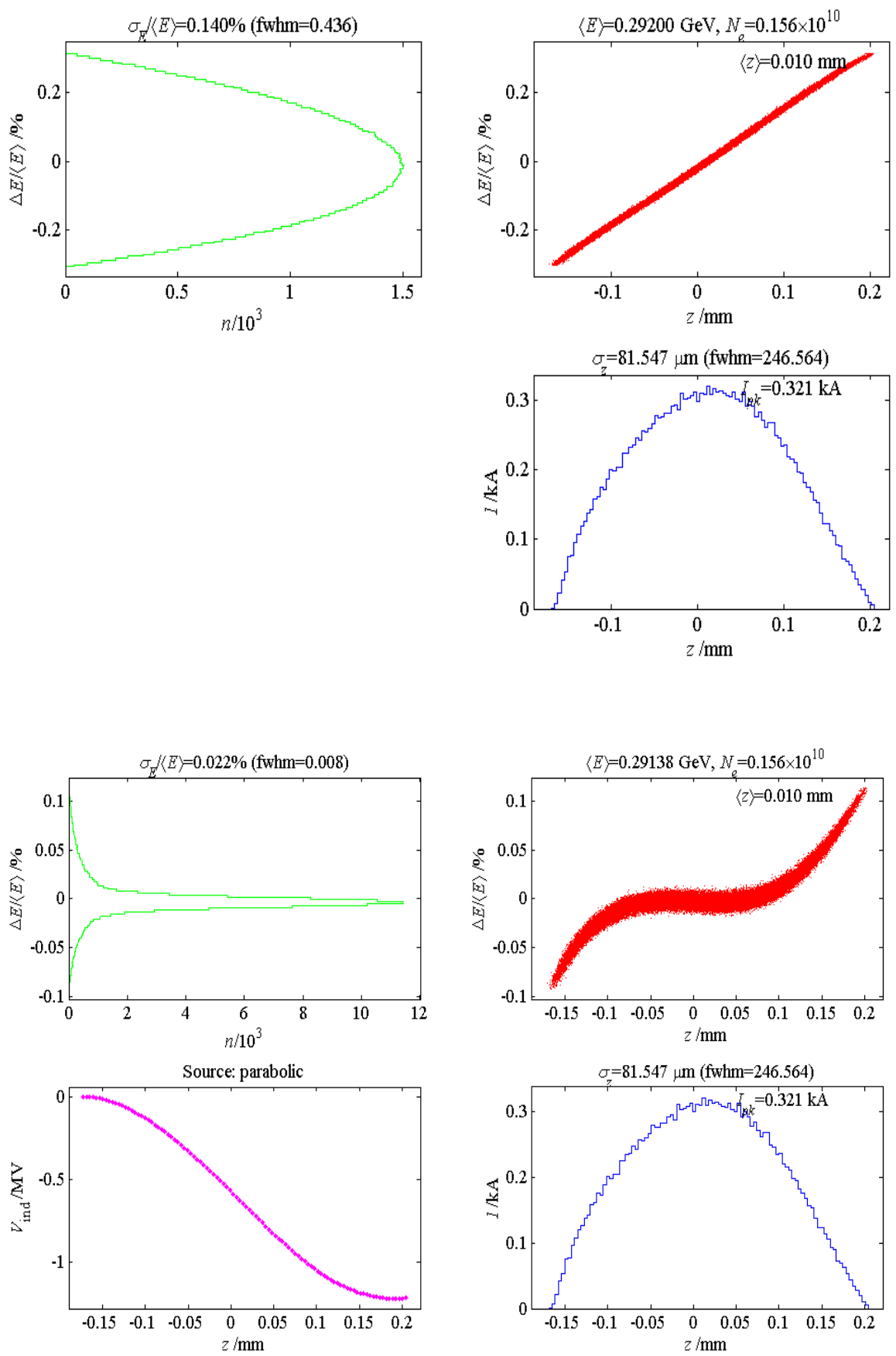

Figure 3: Top: beam longitudinal phase space and projections on the coordinate axes at the entrance of the dechirper after compression in the $\mathrm{BC}$ and acceleration through ACC1. Bottom: beam at the exit of the $2 \mathrm{~m}$ dechirper insertion (Table 2) showing complete removal of the linear energy chirp. The bottom left picture is the dechirperinduced voltage drop along the bunch. 


\subsubsection{Transverse-Deflecting-Cavity-Based Energy Dechirper}

Transverse deflecting cavities (TCAVs) can be used for efficiently imposing large energy chirps in short electron bunches. Each cavity modifies the beam in two ways: (1) imposes angular spread depending on the longitudinal position and (2) changes beam energy depending on the transverse position. The second property can be used for imposing large energy slews inside the bunch since its transverse size can be quickly changed using conventional focusing beam optics unlike the longitudinal size which requires expensive compressors. Strong correlation between the longitudinal and transverse positions in the bunch is required in order to use TCAV for imposing energy chirp depending on the longitudinal coordinate. Such a beam can be created using another TCAV and the following drift which create angular spread and following defocusing depending on the longitudinal coordinates.

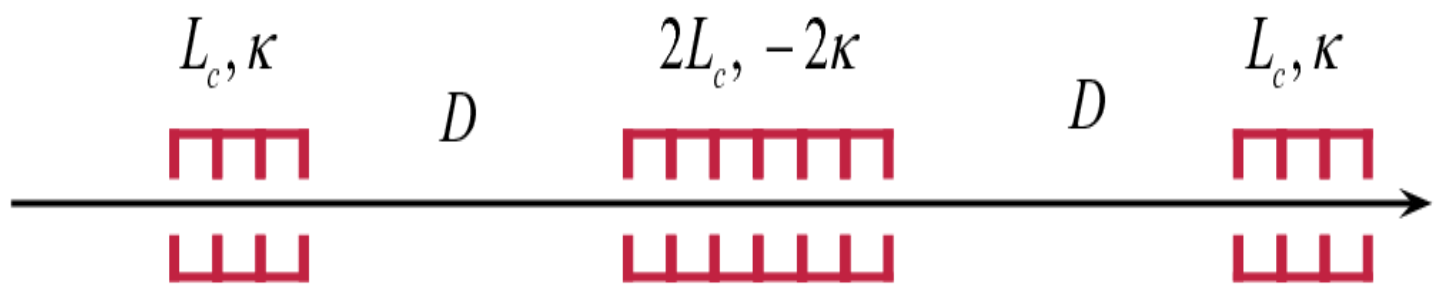

Figure 4: Schematics of the TCBC beamline consisting of three cavities with the same field gradient.

The schematics of the beamline are shown in Figure 4. The overall beamline for the transverse cavity based chirper (TCBC) imposes an energy slew but does not create other correlations inside the beam and consists of three TCAVs separated by two identical drifts. The strengths of the side cavities are the same and the strength of the middle cavity is twice of the side ones and has the opposite sign. The beam dynamics in such a beamline can be described as follows. The first cavity creates the angular spread in the beam depending on the longitudinal position. The beam drifts in vacuum and significantly increases its transverse size by the time it arrives to the middle cavity. At the same time, the beam shows strong correlation between the longitudinal and transverse coordinates. The middle cavity changes particles' energy depending on their transverse positions. This energy change manifests as a longitudinal energy chirp due to strong $x-z$ correlation in the beam. At the same time, the middle cavity imposes the angular spread in the beam which is the opposite from the spread imposed in the first cavity. As a result, the beam focuses in the second vacuum drift. It recovers its initial size upon its arrival to the third cavity which compensates the angular spread and removes all the correlations in the beam except for the energy chirp.

Linear analysis of the scheme allows one to find the transform matrix describing mapping of the longitudinal and transverse phase space coordinates: 


$$
\left(\begin{array}{l}
x \\
x^{\prime} \\
z \\
\delta
\end{array}\right)_{\text {out }}=\left(\begin{array}{cccc}
1 & 2 D+4 L_{c} & 0 & 0 \\
0 & 1 & 0 & 0 \\
0 & 0 & 1 & 0 \\
0 & 0 & -\frac{2}{3} \kappa^{2}\left(3 D+2 L_{c}\right) & 1
\end{array}\right)\left(\begin{array}{l}
x \\
x^{\prime} \\
z \\
\delta
\end{array}\right)_{\text {in }}
$$

The energy slew is mostly imposed in the middle cavity of the TCBC. The beam size increases dramatically at that point and can be made much larger than the longitudinal bunch length. $A$ strong $x-z$ correlation in the beam effectively substitutes the bunch length with its transverse size, so the scheme artificially increases the beam dimension for acquiring energy difference between different parts of the beam. This is the reason the TCBC scheme is more efficient compared to off-crest acceleration since the chirp gradient is similar for different cavity modes with the same frequencies but the beam size in TCBC is much larger as that compared to conventional off-crest acceleration. As a result, TCBC allows on-crest acceleration which results in higher accelerating gradient and leads reduced linac cost.

We plan on experimental demonstration of the TCBC scheme on ASTA. We will test the scheme in two setups. First, we test that compressing energy slews (low energy electrons in the head and high energy at the end of the bunch) can be imposed. Typical parameters of the experiment are presented in Table 3.

Table 3: Main parameters of TCBC.

\begin{tabular}{|l|c|}
\hline Beam energy & $250 \mathrm{MeV}$ \\
\hline rms bunch duration & $0.5 \mathrm{ps}$ \\
\hline RF frequency & $1.3 \mathrm{GHz}$ \\
\hline RF gradient & $22 \mathrm{MV} / \mathrm{m}$ \\
\hline Cavity length & $0.5 \mathrm{~m}$ \\
\hline Drift lengths & $5 \mathrm{~m}$ \\
\hline Imposed energy chirp & $\pm 0.5 \mathrm{MeV}$ \\
\hline
\end{tabular}

Preliminary ELEGANT simulations for parameters relevant for ASTA indicate that the beam quality will be well preserved in this scheme. Fig 5 shows the growth of the rms beam phase space volume (determinant of the rms beam matrix) at the end of the beamline. 


\section{Full Det Growth}

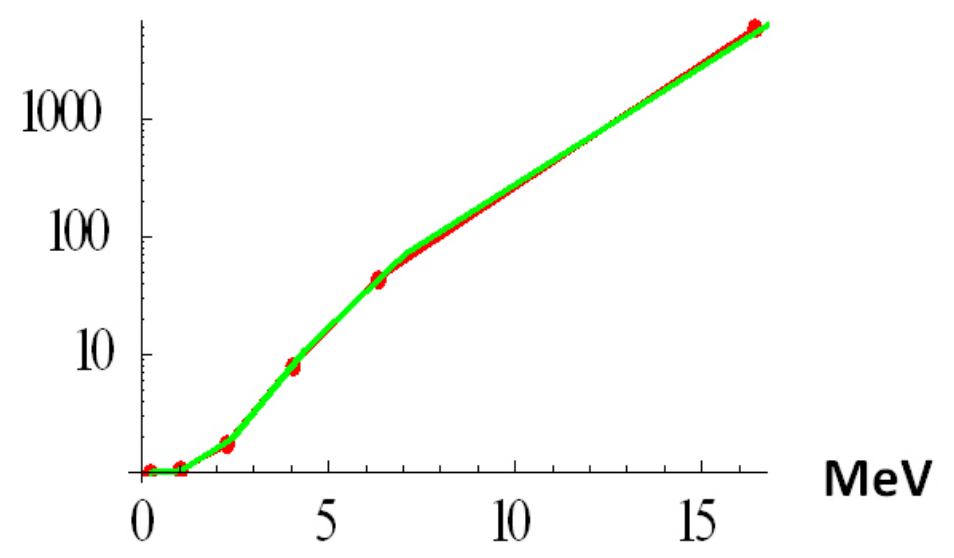

Figure 5: Growth of the rms beam phase space volume (determinant of the $6 \times 6$ beam's matrix) in the TCBC beamline as a function of the deflecting kick.

We will also experimentally investigate the possibility of using TCBC scheme to compensate for the compressing energy slews. The R65 element of the beamline matrix has a negative sign which allows one to impose only compressing chirps in the beam. However, the sign of the chirp can be flipped if drifts are substituted with FODO lattices which beam matrices are identical to drifts with negative drift lengths. In this setup the TCBC scheme can be used to eliminate the residual energy chirp after the final bunch compressor in linacs. That will allow the use of beams with larger energy chirps and reduce the strength of the compressor which will lead to smaller CSR effects and eventually brighter beams.

\subsubsection{Dielectric-Structure Dechirpers}

Dielectric structure can also be used to impart commensurate short-range wakefield which can be used to effectively remove correlated energy spread [1,2] or correct for nonlinear correlation within the longitudinal phase space [10]. Although dechirping was experimentally demonstrated [2] it extension to a long-macropulse bunch still remains to be demonstrated. In particular the technique proposed in [10] could provide a cheap alternative to a $3.9-\mathrm{GHz}$ accelerating cavity needed to linearize the longitudinal phase space prior to the BC1 bunch compressor in the ASTA photoinjector. Note however that the technique being charge dependent will not be as flexible as a 3.9-GHz cavity. 


\subsubsection{References}

[1] M. Rosing and J. Simpson, "Passive momentum spread reduction, the wakefield silencer," ANL Report WF-144 (1990).

[2] S. Antipov, et al., IPAC 2012 p. 598, (2012); S. Antipov, et al., Phys. Rev. Lett. 108, 144801 (2012);

[3] K. Bane and G. Stupakov, Nucl. Instr. And Methods A, 690, 106-110 (2012).

[4] J.N. Corlett et al., "Next Generation Light Source R\&D and design studies at LBNL," Proc .IPAC'12, New Orleans, TUPP070 (2012).

[5] H-S Kang, et al., PAL-ITF experiment, Proc. FEL13 Conference.

[6] C. Prokop, et al., Nucl. Instr. and Methods A, 719, 17-28 (2013).

[7] P. Craievich, PRST-AB 13034401 (2010).

[8] K. Bane and P. Emma, SLAC-PUB-11035 (2005).

[9] C. Prokop, et al., Start-to-end beam dynamics simulations for the SRF accelerator test facility at Fermilab, Proc. PAC'11, (2011).

[10] P. Craievich, Phys. Rev. ST Accel. Beams 13, 034401 (2010). 


\subsection{Technical Description of ASTA}

\subsection{Facility Overview}

The Advanced Superconducting Test Accelerator [1] is housed in the New Muon Lab (NML) building at Fermilab. It will be capable of testing 3 or more ILC-type SRF cryomodules under full ILC beam intensity and bunch structure. In addition, test beamlines and downstream beamlines will provide a venue for advanced accelerator R\&D (AARD). The original NML building had been previously used for a high energy physics experiment, but has recently been repurposed to house ASTA [2]. The original footprint has been extended to accommodate high energy beamlines, a high energy beam dump, and an experimental area for AARD. Figure 1 shows the plan view of the upstream half of the facility, and Figure 2 shows the plan view of the downstream half.

The electron beam is produced by a $1.3 \mathrm{GHz}$ RF photoinjector [3] and then accelerated to 50 MeV by two $1.3 \mathrm{GHz}$ SRF cryomodules, each containing a single 9-cell cavity, before being injected into the 1st 8-cavity cryomodule. Initial beam commissioning of the cryomodule string will take place with a single Tesla type III+ cryomodule [4] driven by a $5 \mathrm{MW}$ klystron. The next stage of commissioning will take place with two Tesla type III+ cryomodules and one ILC type IV cryomodule. The entire string will be driven by a $10 \mathrm{MW}$ multi-beam klystron, with its associated HV power supply, modulator, and waveguide distribution system. The high energy beamlines downstream of the cryomodules will provide transport to an AARD experimental area and to the high energy beam dump.

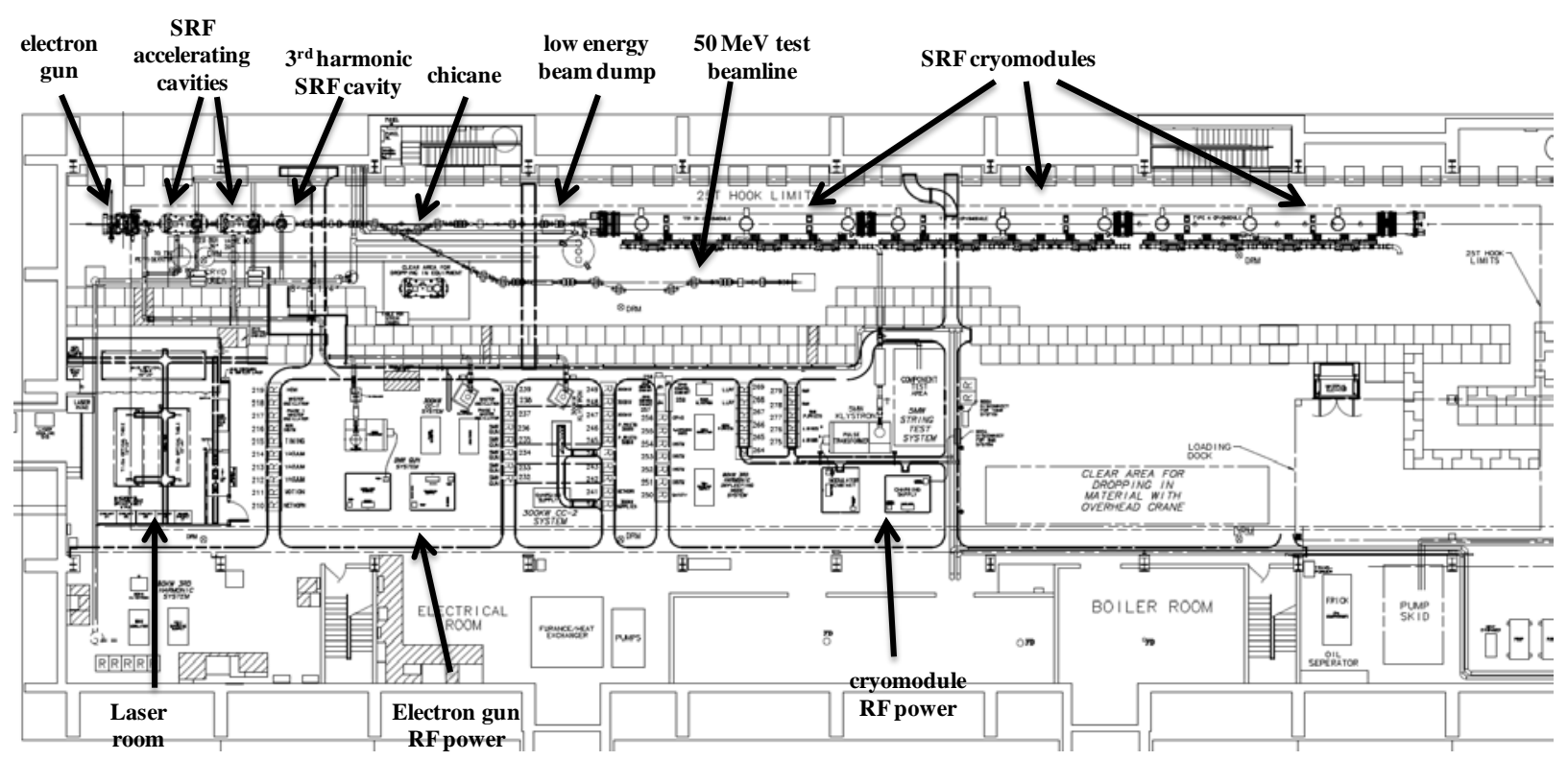

Figure 1: Upstream floor plan of the ASTA photoinjector and 3 SRF cryomodules in the original building footprint. The beamline is $1.2 \mathrm{~m}$ above the floor, the floor is $6.1 \mathrm{~m}$ below grade, and the building length is $74 \mathrm{~m}$. 
The cryogenic plant currently consists of 2 Tevatron-style satellite refrigerators capable of delivering a total of $110 \mathrm{~W}$ of $2 \mathrm{~K}$ helium and does not have the capacity to cool all 3 cryomodules at full beam intensity and at full pulse repetition rate. The Cryomodule Test Facility (CMTF) [3] is currently being built adjacent to NML and will provide the additional capacity to deliver $600 \mathrm{~W}$ of $2 \mathrm{~K}$ helium to NML and cryomodule test stands in CMTF.

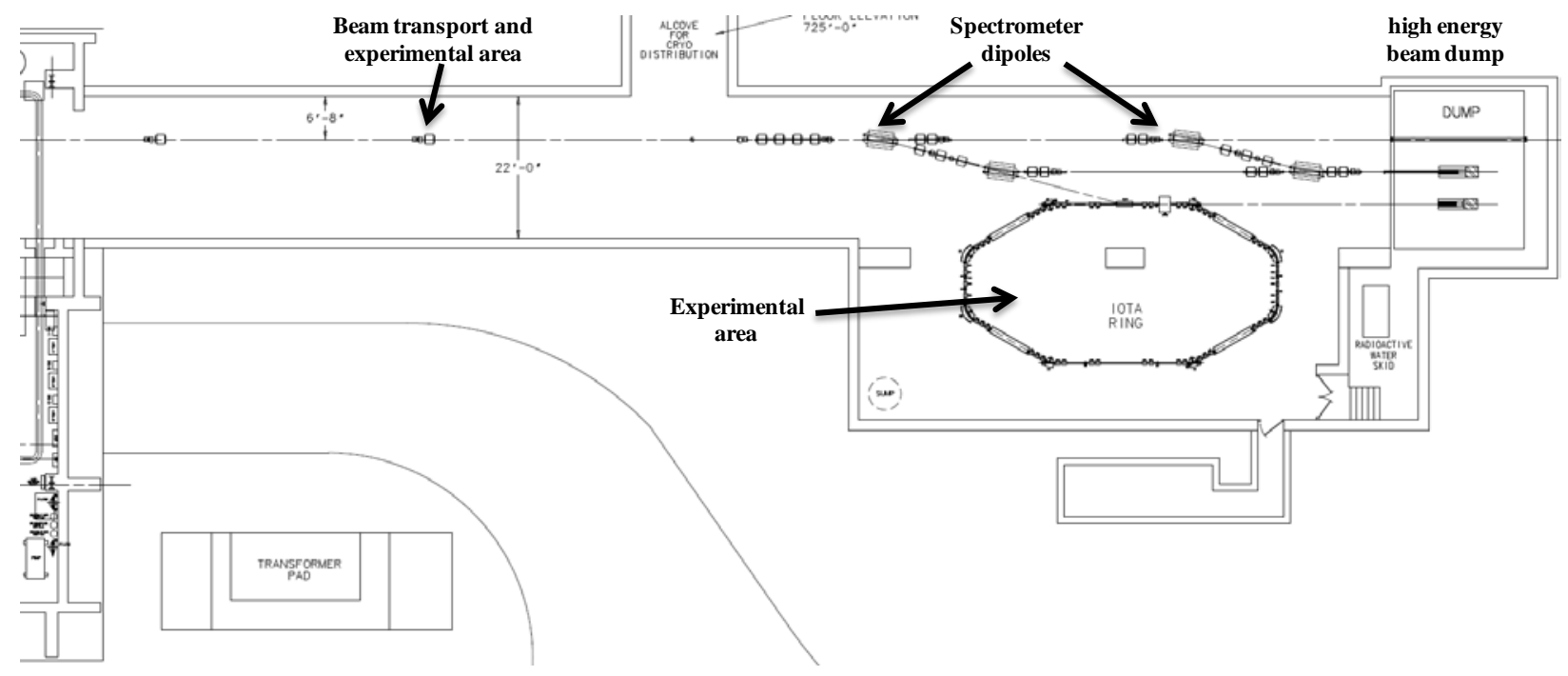

Figure 2: Downstream plan view of the ASTA high energy beamlines and experimental areas in the new underground tunnel. The tunnel extension is $70 \mathrm{~m}$ long. A service building is located above the experimental area.

The beam parameters for ASTA will have a wide range, depending on the particular application. Table 1 lists selected beam parameters for ILC-like conditions and the possible range for each parameter. As with all photoinjectors, many beam parameters are coupled, especially to the bunch intensity, because of space charge effects in the electron gun and low energy bunch compressor. 
Table 1: Beam parameters for ASTA. The ILC-like parameter values are listed and also the range of each parameter.

\begin{tabular}{|c|c|c|c|}
\hline Parameter & $\begin{array}{c}\text { ILC RF unit } \\
\text { test }\end{array}$ & Range & Comments \\
\hline bunch charge & $3.2 \mathrm{nC}$ & $10^{\prime} \mathrm{s}$ of $\mathrm{pC}$ to $>20 \mathrm{nC}$ & $\begin{array}{l}\text { minimum determined by diagnostic thresholds; } \\
\text { maximum determined by cathode QE and laser } \\
\text { power }\end{array}$ \\
\hline bunch spacing & 333 nsec & $<10$ nsec to $10 \mathrm{sec}$ & lower laser power at minimum bunch spacing \\
\hline $\begin{array}{l}\text { bunch train } \\
\text { length }\end{array}$ & $1 \mathrm{msec}$ & 1 bunch to $1 \mathrm{msec}$ & $\begin{array}{l}\text { maximum limited by modulator and klystron } \\
\text { power }\end{array}$ \\
\hline $\begin{array}{l}\text { bunch train } \\
\text { repetition rate }\end{array}$ & $5 \mathrm{~Hz}$ & $0.1 \mathrm{~Hz}$ to $5 \mathrm{~Hz}$ & $\begin{array}{l}\text { minimum may be determined by electron gun } \\
\text { temperature regulation and other stability } \\
\text { considerations }\end{array}$ \\
\hline $\begin{array}{l}\text { normalized } \\
\text { transverse } \\
\text { emittance }\end{array}$ & $\sim 25 \mathrm{~mm}-\mathrm{mrad}$ & $\begin{array}{c}<1 \mathrm{~mm}-\mathrm{mrad} \text { to }>100 \\
\mathrm{~mm} \text {-mrad }\end{array}$ & $\begin{array}{l}\text { maximum limited by aperture and beam losses; } \\
\text { without bunch compression emittance is } \sim 4 \mathrm{~mm} \text { - } \\
\mathrm{mrad} \text { at } 3.2 \mathrm{nC}\end{array}$ \\
\hline $\begin{array}{l}\text { RMS bunch } \\
\text { length }\end{array}$ & $1 \mathrm{ps}$ & $\begin{array}{l}\sim 10^{\prime} \text { s of fs to } \sim 10^{\prime} \text { s of } \\
\text { ps }\end{array}$ & $\begin{array}{l}\text { minimum obtained with Ti:Sa laser; maximum } \\
\text { obtained with laser pulse stacking }\end{array}$ \\
\hline $\begin{array}{c}\text { peak bunch } \\
\text { current }\end{array}$ & $3 \mathrm{kA}$ & $>10 \mathrm{kA}(?)$ & $\begin{array}{l}3 \text { kA based on CSRTrack simulations with low } \\
\text { energy bunch compressor }\end{array}$ \\
\hline injection energy & $50 \mathrm{MeV}$ & $5 \mathrm{MeV}$ to $50 \mathrm{MeV}$ & $\begin{array}{l}\text { may be difficult to transport } 5 \mathrm{MeV} \text { beam to the } \\
\text { dump; maximum determined by booster cavity } \\
\text { gradients }\end{array}$ \\
\hline high energy & $820 \mathrm{MeV}$ & $50 \mathrm{MeV}$ to $1500 \mathrm{MeV}$ & $\begin{array}{l}\text { radiation shielding issues limit the maximum; } \\
1500 \mathrm{MeV} \text { with } 6 \text { cryomodules }\end{array}$ \\
\hline
\end{tabular}

\subsection{Injector}

\subsubsection{Electron Gun}

The RF photocathode electron gun is identical to the guns recently developed at DESY Zeuthen (PITZ) for the FLASH facility [5]. It is a normal-conducting $1 \frac{1}{2}$ cell $1.3 \mathrm{GHz}$ gun operated in the $\mathrm{TM}_{010, \pi}$ mode, with a $\mathrm{Q}_{L}$ of 11,700 , and driven by a $5 \mathrm{MW}$ klystron. The power is coupled into the gun via a coaxial RF coupler at the downstream end of the gun. The gun is capable of average DC power dissipation of $\sim 20 \mathrm{~kW}$, and a temperature feedback system will regulate cooling water temperature to better than $\pm 0.02{ }^{\circ} \mathrm{C}$ for good phase stability. The gun will be routinely operated at peak gradients of $40-45 \mathrm{MV} / \mathrm{m}$, and output beam kinetic energy of $\sim 5$ $\mathrm{MeV}$. (For comparison, PITZ has successfully operated an identical gun at $60 \mathrm{MV} / \mathrm{m}$ for 700 usec pulse lengths.) The photocathode is a $10 \mathrm{~mm}$ diameter molybdenum disk coated with $\mathrm{Cs}_{2} \mathrm{Te}$ with $5 \mathrm{~mm}$ diameter photosensitive area. It is illuminated by $263 \mathrm{~mm}$ wavelength laser light which is directed onto the photocathode by a $45^{\circ}$ off-axis mirror downstream of the RF coupler.

The photocathodes are coated at a separate facility on the Fermilab site, transported under vacuum to the photocathode transfer chamber mounted on the upstream end of the gun, and inserted into the upstream end of the gun via external manipulators, all under vacuum. Several 
photocathodes have already been prepared and their quantum efficiency measured to be $\sim 10 \%$. The photocathode preparation, transport, and transfer chambers were developed and built by D. Sertore at INFN Milano [6] and commissioned at Fermilab.

For emittance compensation the gun is surrounded by 2 solenoid magnets built by Danfysik. Each magnet has a peak field of $0.28 \mathrm{~T}$ at $500 \mathrm{~A}$. Normally the magnet currents are set so the field at the photocathode is 0 in order to minimize the beam emittance; however, the field can be set to $>1 \mathrm{kG}$ at the photocathode for the production of angular-momentum dominated beams and flat beam production. ASTRA [7] simulations indicate that transverse normalized emittances of $4 \mu \mathrm{m}$ can be attained at a bunch charge of $3.2 \mathrm{nC}$, laser pulse length of $3.2 \mathrm{ps}$ RMS, and peak gun electric field gradient of $40 \mathrm{MV} / \mathrm{m}$. Stretching the laser pulse length will produce smaller transverse beam emittance out of the gun, as will higher gun gradients.

ASTA successfully produced its first photoelectron beam from the gun into a Faraday cup on June $20^{\text {th }}, 2013$; see Figure 3. The initial beam was 8-15 pulses at $1 \mathrm{~Hz}$ rep rate and electrons were observed on three of the primary diagnostics immediately downstream of the gun: loss monitor, resistive wall current monitor, and yttrium aluminum garnet (YAG) screen. Since this initial achievement, studies have been ongoing to characterize electron properties. Typical current operating parameters are $80 \mu \mathrm{s}$ RF pulse width, $31.5 \mathrm{MV} / \mathrm{m}$ peak field and $1 \mathrm{~Hz}$ repetition frequency.

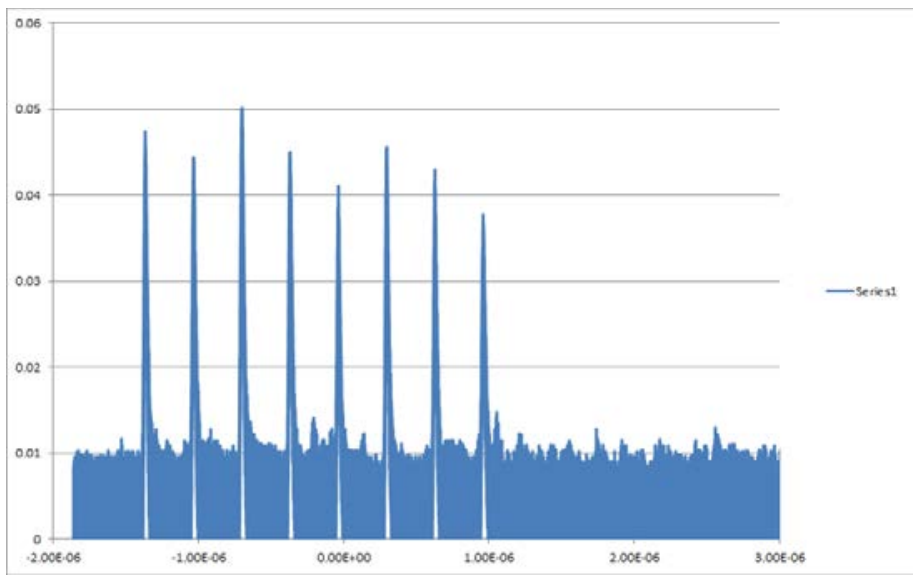

Figure 3: Response from Loss Monitor of First Photoelectrons out of ASTA Gun. Full horizontal scale is $5 \mu \mathrm{sec}$ showing 7 pulses at $3 \mathrm{MHz}$ frequency.

\subsubsection{Photocathode Laser}

The photocathode laser system is housed in an enclosed structure adjacent to the beam enclosure. A diagram of the laser system driving the photocathode is show in Figure 4 . The seed laser is a Yb-fiber laser acquired from Calmar, Inc. The $1.3 \mathrm{GHz}$ output is reduced to $3 \mathrm{MHz}$ by two pulse pickers, amplified by a multipass and two 2-pass amplifiers and frequency quadrupled by two beta barium borate (BBO) crystals. The UV pulse is transported to the gun 
through a vacuum pipe and directed to the photocathode via a $45^{\circ}$ off-axis mirror downstream of the gun RF coupler. The UV amplitude is sufficient to produce 10's of nC of bunch charge from the cathode, and the rms (gaussian) pulse length is $3.2 \mathrm{ps}$. In addition there will be a Ti:Sa laser for short pulse experiments ( 150 fs) and laser lab space for other laser R\&D efforts.

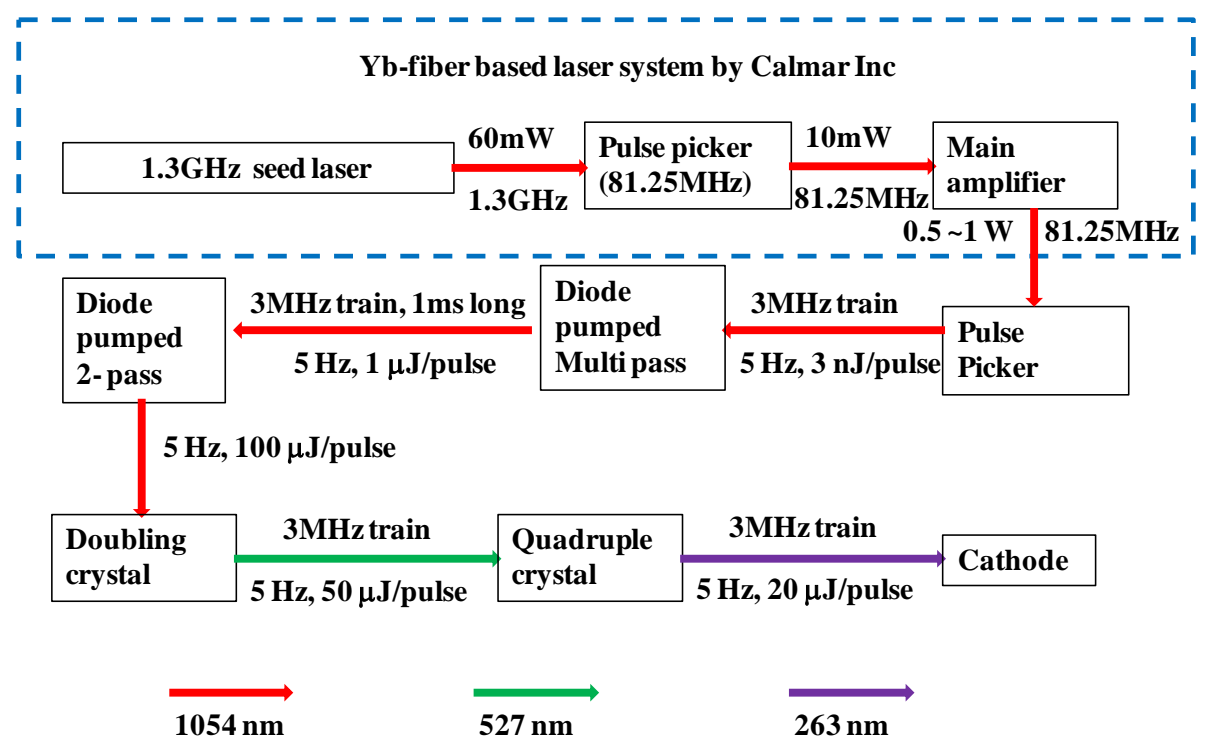

Figure 4: Schematic diagram of the photocathode laser system.

\subsubsection{Beamline Elements}

The injector beamline is shown in Figure 5. After a short instrumentation section, the electron gun is followed by two SRF cryomodules to accelerate beam to $50 \mathrm{MeV}$. Each of these cryomodules contains a 9-cell L-band cavity operating at $1.3 \mathrm{GHz}$, driven by a $300 \mathrm{~kW}$ klystron, and capable of peak accelerating gradients of $>22 \mathrm{MV} / \mathrm{m}$ [8]. These cavities will also be used to "chirp" the beam, i.e., generate a time-momentum correlation, in preparation for bunch compression in the downstream chicane. Downstream of these cavities is space allotted for a future SRF $3.9 \mathrm{GHz}$ cavity intended to be used for bunch linearization.

The SRF cavities are followed by a quadrupole doublet to control the beam size for the emittance measurement, 3 skew quads to generate flat beam, a matching section into the chicane, a 4-dipole chicane for bunch compression $\left(R_{56}=-0.18 \mathrm{~m}\right)$, a matching section into the vertically downward-bending dipole to the low energy beam dump, and finally a matching section into the first 8-cavity cryomodule. The $22.5^{\circ}$ dipole upstream of the dump will serve as the low energy spectrometer. The $50 \mathrm{MeV}$ beam dump will be capable of absorbing up to 400 $W$ of beam power, which is $15 \%$ of the full ILC intensity [9]. In addition, there is a dogleg to deliver $50 \mathrm{MeV}$ beam to a test beam area parallel to the $1^{\text {st }}$ cryomodule. This test area is intended for AARD experiments which utilize low-energy beam. 


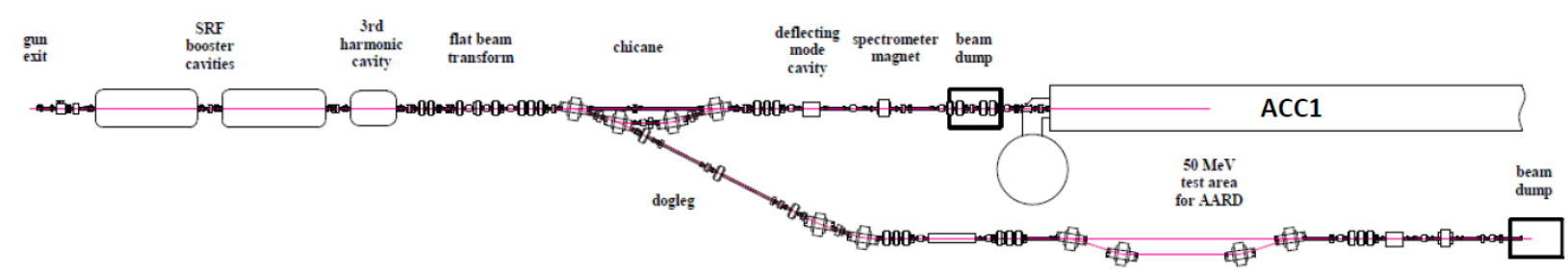

Figure 5: Injector layout.

\subsubsection{Diagnostics}

There are four primary types of diagnostics in both the injector and the high energy beamlines: BPM's to measure beam position, profile monitors to measure beam size, resistive wall monitors to measure beam current ( 4 in the primary injector beamline), and loss monitors ( $\sim 0$ total) to measure beam losses and serve as the primary protection element in the machine protection system.

Each BPM is a 4-button pickup measuring both $X$ and $Y$ position. The system will be capable of bunch-by-bunch measurements, and the anticipated accuracy is $<25 \mu \mathrm{m}$. There will be 23 BPM's in the primary injector beamline. Signals from HOM absorbers in the cryomodules can also be used for beam position monitoring.

The profile monitors are located in 6-way vacuum crosses and were fabricated by Radiabeam Technologies. A prototype was tested at the Fermilab A0 photoinjector [10] and a resolution of $<20 \mu \mathrm{m}$ has been obtained. They each contain a Ce-doped $100 \mu \mathrm{m}$ crystalline yttrium aluminum garnet (YAG) screen or a $100 \mu \mathrm{m}$ crystalline lutetium-yttrium oxyorthosilicate (LYSO) screen, a $1 \mu \mathrm{m}$ Al screen for OTR production, and a target screen for calibration. The screens are at right angles to the beam and are followed by a $45^{\circ}$ mirror to deflect optical light to an optical channel and a 5 megapixel camera. There will be 12 profile monitors in the primary injector beamline. Wire scanners may be installed at a later date.

In the injector beamline, transverse emittance will be measured by the "slits method" both upstream and downstream of the chicane. Beam energy will be measured by the $22.5^{\circ}$ spectrometer magnet. Bunch length will be measured by streak camera and interferometer both upstream and downstream of the chicane.

A $3.9 \mathrm{GHz}$ 5-cell deflecting mode RF cavity will be installed upstream of the low energy spectrometer magnet for measuring the longitudinal phase space of the beam. This cavity is normal-conducting and is operated at $80^{\circ} \mathrm{K}$. It has been in operation at the $\mathrm{AO}$ photoinjector for several years now. 


\subsection{Cryomodules}

\subsubsection{Description}

Figure 6 is a photograph of a single TTF type III+ cryomodule installed at ASTA. It is driven by a single $1.3 \mathrm{GHz} 5 \mathrm{MW}$ klystron, and RF power is distributed to the eight separate cavities by variable tap-offs (VTO) in the waveguide structure alongside the cryomodule. Each cryomodule consists of a string of eight 9-cell superconducting cavities. In addition, some cryomodules will contain a superconducting quadrupole doublet package, corrector package and BPM. The goal for these cavities is to reach an operational gradient of $31.5 \mathrm{MV} / \mathrm{m}$ at $2{ }^{\circ} \mathrm{K}$ under full ILC beam conditions. This will yield a total acceleration of $250 \mathrm{MeV}$ per cryomodule. The length of a single cryomodule is $11.9 \mathrm{~m}$, but the cold interfaces, HOM absorbers, and warm gate valves add an additional $1.7 \mathrm{~m}$ in length to each end.

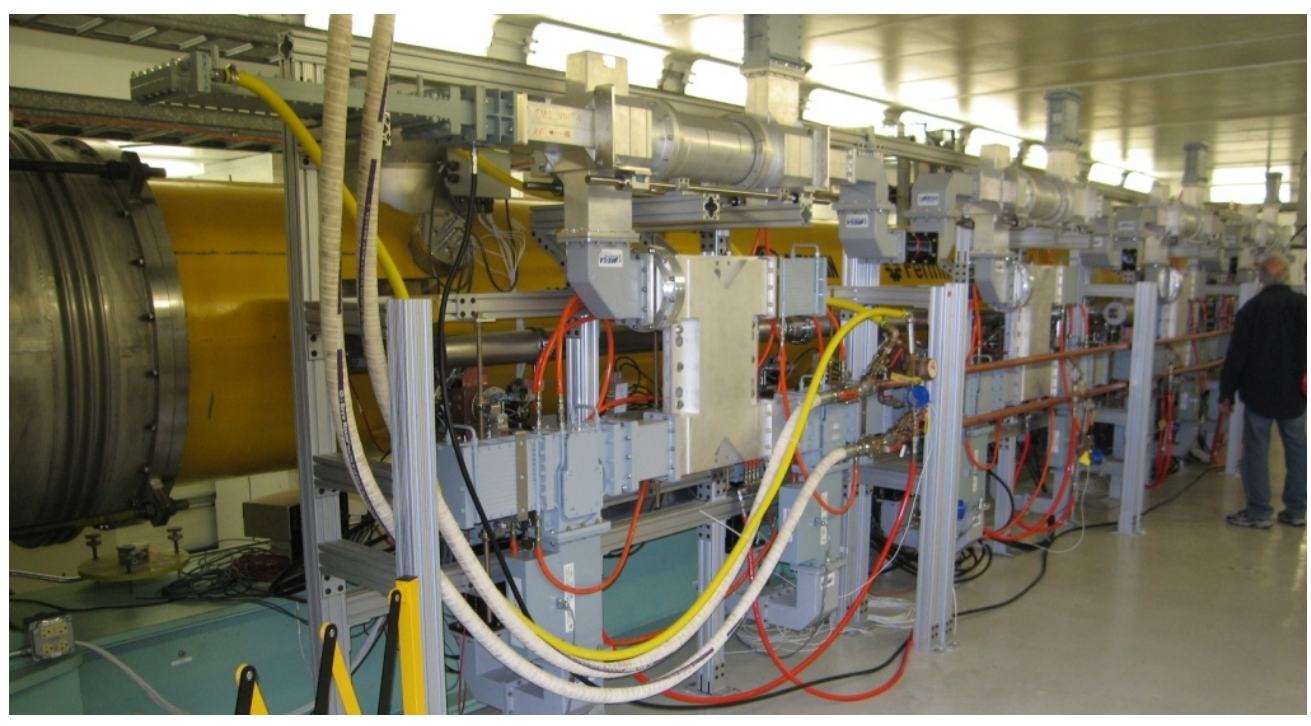

Figure 6: Photograph of cryomodule I installed in the ASTA beamline enclosure. View is looking downstream. Waveguide distribution structure is on the right of the cryomodule.

\subsubsection{Initial Operation}

The first TESLA Type III 8-cavity cryomodule, CM-1, was installed and operated in NML for approximately 15 months ending in March 2012. CM-1 came to Fermilab as a 'kit' and was assembled at Fermilab by the technical staff here with assistance from colleagues from DESY and LASA/INFN, Milano. Commissioning and testing followed the standard protocol developed worldwide for bringing cryomodules into full operation. The steps include: 
- RF Cable Calibration

- Technical Sensor/Interlock Check

- RF/Waveguide Check

- Warm Coupler Conditioning (off resonance)

- Cool down to $2{ }^{\circ} \mathrm{K}$

- Frequency spectra measurements

- Cavity Tuning to $1.300 \mathrm{GHz}$ via motorized slow tuner

- $\mathrm{Q}_{\mathrm{L}}$ adjustment to 3.0E6

- LLRF calibrations

- Cold Coupler Conditioning (on resonance)

- Performance Evaluation including

o Maximum gradient

o Dynamic Heat Load ( $Q_{0}$ vs. $\left.E_{A c C}\right)$

O Dark Current and Field Emission (X-rays vs. $E_{A C C}$ )

CM-1 was installed into its final position and aligned in January 2010 and final RF, cryogenics and vacuum connections were made thereafter. Warm off-resonance conditioning was performed with a single cavity at a time connected to the output of the $5 \mathrm{MW}$ klystron and took anywhere from 2 weeks for the first cavity to 4 days to complete. Once all eight couplers were conditioned final vacuum work was completed leading to initiation of cool down to $2{ }^{\circ} \mathrm{K}$. CM-1 first reached operating temperature on 22 November 2010.

Continuing with the sequence of commissioning steps, each cavity was then powered on resonance to complete coupler conditioning and determine cavity performance limitations. Again, each cavity was powered singly. All cavities were characterized by June 11, with gradients ranging from 20 to $28 \mathrm{MV} / \mathrm{m}$.

Full module testing was initiated on 6 July 2011. The waveguide distribution circuit was provided by SLAC and allows independent amplitude and phase control of adjacent pairs of cavities. VTO's were set based upon the gradient limits identified during cavity characterization. The bulk of the time spent powering the entire module was devoted to Low Level RF (LLRF) optimization and refining the Lorentz Force Detuning Compensation system. By the end of the run it was possible to control the RF amplitude and phase over 50 pulses to an RMS magnitude error of $6.0 \times 10^{-3} \%$ and RMS phase error of $0.005^{\circ}$. (The ILC specification for phase stability is $0.25^{\circ}$.) Much progress was made in developing a scheme to counteract the effects of Lorentz Force Detuning. An adaptive algorithm was deployed and improved which permits pulse to pulse correction.

In April, 2012 this first cryomodule was removed and a $2^{\text {nd }}$ cryomodule was installed. This cryomodule is anticipated to reach full ILC gradient in all of its cavities, based on cold cavity power tests in the horizontal and vertical test stands, as shown in Figure 7 below. 


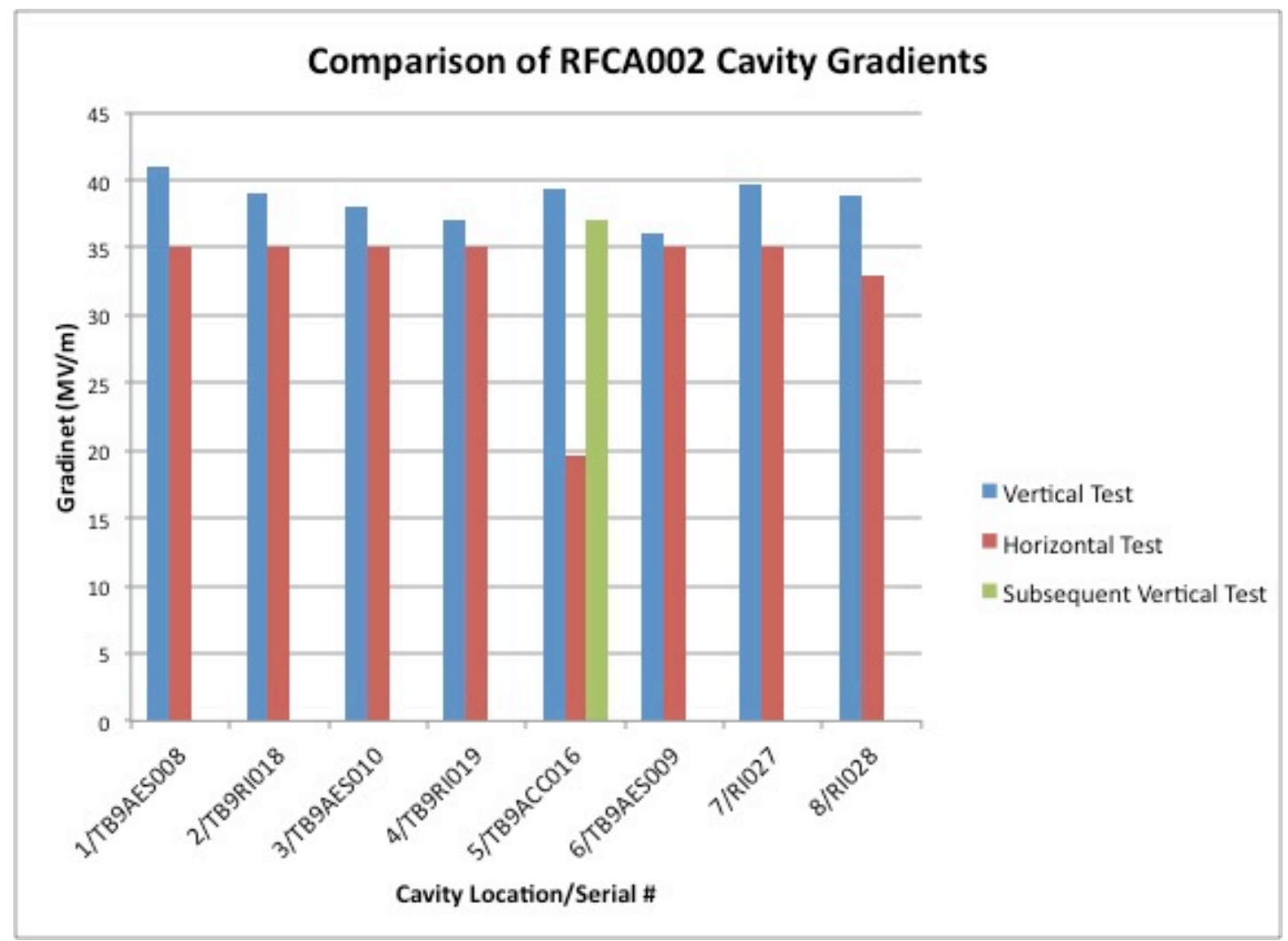

Figure 7: RFCA002 cavities test performance. For horizontal tests, an administrative upper limit of $35 \mathrm{MV} / \mathrm{m}$ was imposed. TB9ACC016 suffered from copper flaking in the coupler region during the horizontal test and was subsequently reprocessed and retested in the vertical test stand.

The second cryomodule (CM-2) is now installed, under vacuum and expected to be cooled down to 2 Kelvin in September 2013. Each cavity will be individually powered and characterized as was done with $\mathrm{CM}-1$ and then all cavities powered together.

\subsection{High Energy Beamlines}

\subsubsection{Beamline Elements}

The beam will be initially transported from the cryomodule string to the first high energy spectrometer magnet by a 4-quad matching section, a 4-quad FODO lattice, and an additional 4 quad matching section. This region of beamline is $45 \mathrm{~m}$ in length and is intended to house future AARD experiments, so it will be reconfigured from time to time to accommodate new experiments. The high energy beamlines downstream of this section are shown in Figure 8. There are 2 dispersion-cancelling doglegs which allow for two $10 \mathrm{~m}$ sections reserved for AARD experiments. The final 2 quads in the beamline are to be used to blow up the beam size to 
avoid damage to the beam dump core. A slow beam sweeping system is used for both the high energy and low energy dumps in order to distribute the beam power over the dump core.

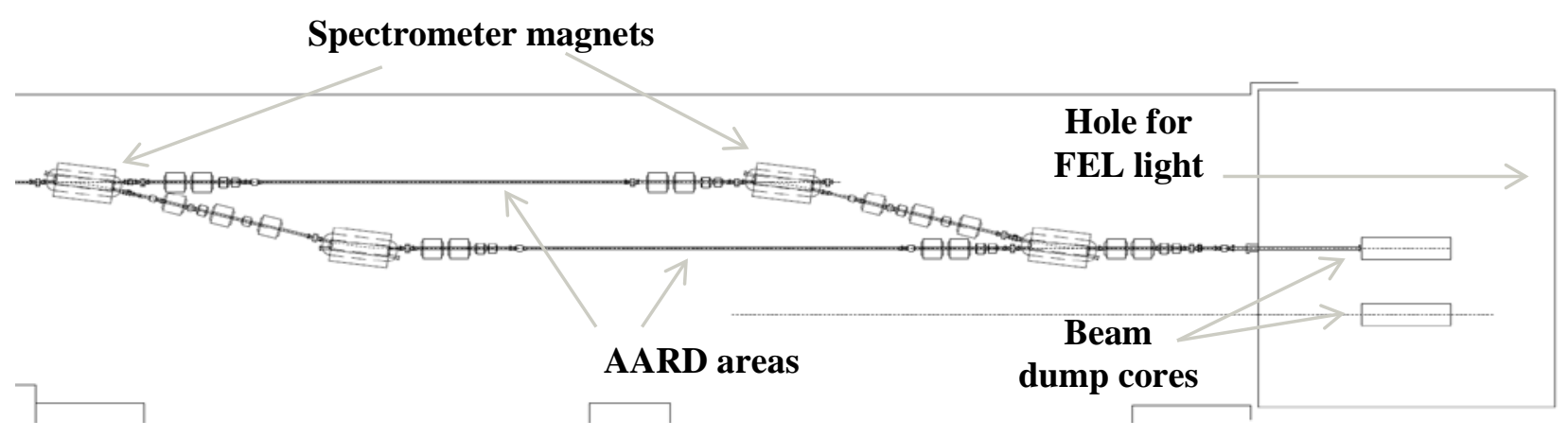

Figure 8: High energy beamline experimental area and beam dumps.

\subsubsection{Diagnostics}

Diagnostics in the high energy beamlines are identical to diagnostics in the injector beamline. There will be 27 BPM's, 9 profile monitors, 2 resistive wall monitors, and 20 loss monitors in the initial configuration. The "quad scan" method will be used for transverse emittance measurements, and energy measurements can be made at two of the $15^{\circ}$ dipole magnets.

\subsubsection{Beam Dump}

The high energy beam dump [11] consists of stacked graphite cores, surrounded by water cooled Al in thermal contact with the graphite, surrounded by steel, backed by a copper plug, all embedded in a $6.1 \mathrm{~m} \times 6.2 \mathrm{~m} \times 7.5 \mathrm{~m}$ concrete stack. Each graphite core is surrounded by an inert gas (argon) to prevent oxidation at elevated temperatures. There are two graphite cores within the beam dump, and a third core has been left out to allow FEL light (or beam) to pass through the dump in the straight-through beamline. Each beam dump core is designed to handle $80 \mathrm{KW}$ of beam power at beam energies up to $1500 \mathrm{MeV}$. The beam vacuum window consists of a $7 \mathrm{~mm}$ thick graphite disk enclosed by two $1 \mathrm{~mm}$ thick TZM disks (titanium, molybdenum, zirconium alloy).

\subsection{Beam Dynamics Calculations and Simulations}

Extensive lattice calculations and beam simulations have been performed to validate the ASTA beamline design under a wide range of beam parameters and configurations [12][13]. ASTRA [7] is used to simulate beam to the downstream end of the $2^{\text {nd }}$ acceleration cavity $(8.2 \mathrm{~m}$ from the photocathode); Elegant [14] is used to calculate single particle (low intensity) beam dynamics from $8.2 \mathrm{~m}$ to the high energy dump; Impact-Z [15] or CSRTrack [16] are used to calculate multiparticle (high intensity) beam dynamics from $8.2 \mathrm{~m}$ to the high energy beam dump and includes the effects of space charge and coherent synchrotron radiation. As an 
example, Figure 9 shows results from typical beam size calculations from Elegant and Impact-Z. As another example, Figure 10 shows an Impact- $Z$ simulation of the effects of low energy bunch compression on longitudinal phase space for varying bunch intensities.
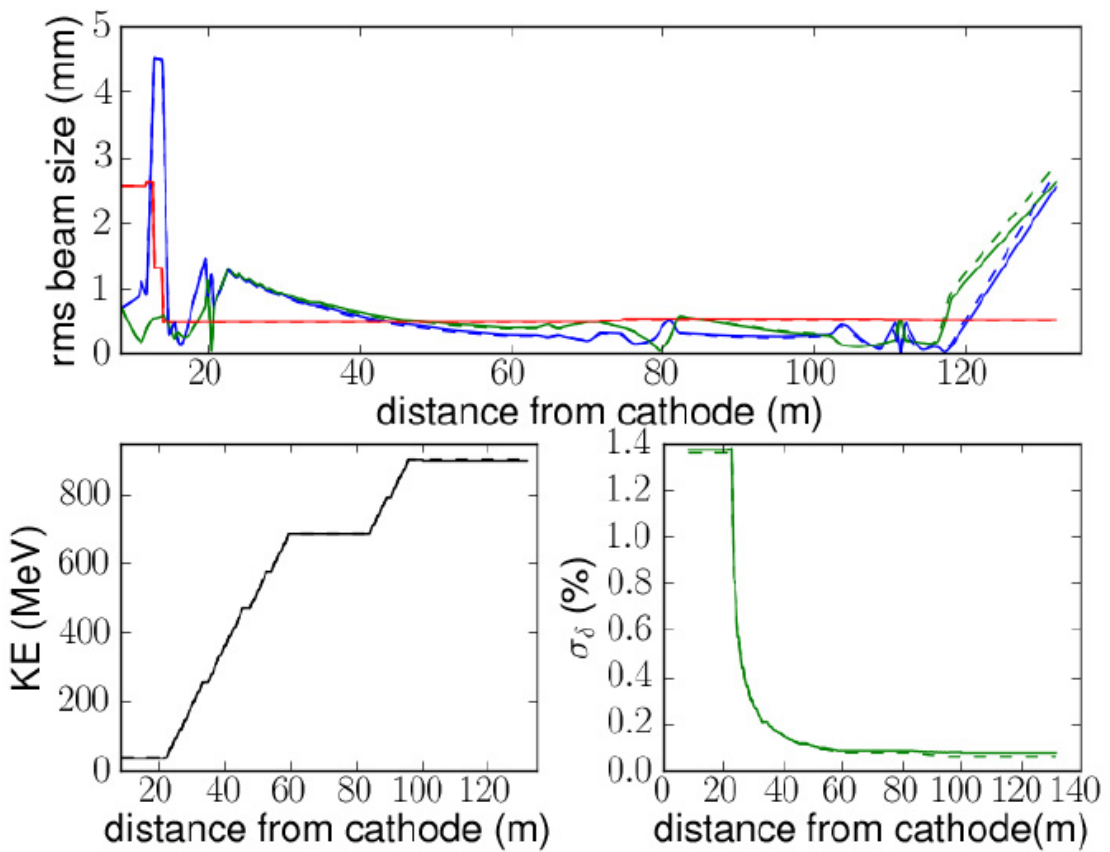

Figure 9: Top plot shows lattice functions from $8.2 \mathrm{~m}$ to the high energy dump in the 3cryomodule configuration followed by a 2nd high energy bunch compressor and 4th cryomodule. The solid red, blue and green traces in the upper plot correspond, respectively, to the bunch length and horizontal and vertical spot size evolution along the beam line obtained from simulation using IMPACT-Z. The corresponding dashed lines are the same parameters simulated with ELEGANT. The solid and dashed green trace on the bottom right plot shows results from simulation carried out with IMPACT-Z and ELEGANT respectively. 

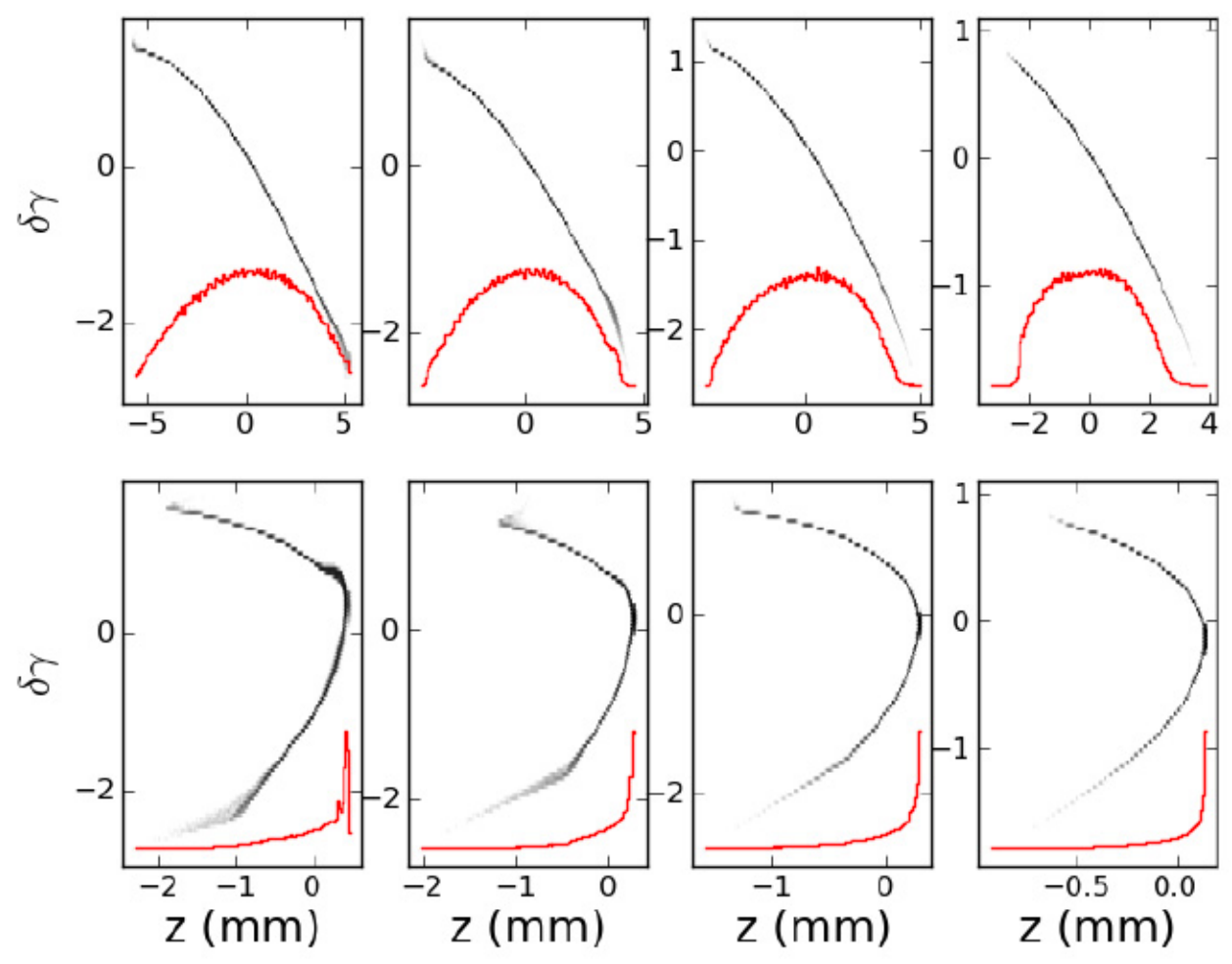

Figure 10: Longitudinal phase space plots, before (top row) and after (bottom row) low energy bunch compression. Columns are (from left to right) $3.2 \mathrm{nC}, 1 \mathrm{nC}, 0.25 \mathrm{nC}$, and $0.02 \mathrm{nC}$. Black trace is longitudinal phase space, and red trace is projection on $\mathrm{z}$ axis. For $3.2 \mathrm{nC}$, the peak beam current is $3.5 \mathrm{kA}$. These results are without a $3.9 \mathrm{GHz}$ linearizing cavity.

\subsection{References}

[1] M. Church, et al., "Design of the Advanced Superconducting Test Accelerator," Fermilab Beams-doc-4212; http://beamdocs.fnal.gov/AD/DocDB/0042/004212/004/ ASTA technical description.pdf.

[2] J. Leibfritz, et al., "Status and Plans for a Superconducting RF Accelerator Test Facility at Fermilab," MOOAC02 in IPAC2012, New Orleans, Louisiana, USA, May 2012; http://www.JACoW.org.

[3] P. Piot, Y.-E Sun and M. Church, "Beam dynamics simulations of the NML photoinjector at Fermilab," IPAC2010; http://epaper.kek.jp/IPAC10/papers/thpd020.pdf.

[4] E. Harms, et al., "RF Test Results from Cryomodule 1 at the Fermilab SRF Beam Test Facility," MOPO013 in $15^{\text {th }}$ International Conf. on RF Superconductivity (SRF2011), Chicago, IL, July 2011; http://Iss.fnal.gov/archive/2011/conf/fermilab-conf-11-352-adtd.pdf. 
[5] M. Otevrel, et al., "Conditioning of a New Gun at PITZ Equipped with an Upgraded RF Measurement System," WEPB05 in The Proceedings of FEL2010, Malmo, Sweden, Aug. 2010; http://www.JACoW.org.

[6] D. Sertore, et al., "Review of the Production Process of TTF and PITZ Photocathodes," MOPB009 in PAC05, Knoxville, TN, May 2005; http://www.JACoW.org.

[7] K. Flöttman, "ASTRA - A Space Charge Tracking Algorithm," http://www.desy.de/ mpyflo/.

[8] J. Branlard, et al., "Capture Cavity II Results at FNAL," WEPMN092 in PAC07, Albuquerque, NM, June 2007; http://www.JACoW.org.

[9] C. Baffes, "ASTA Low Energy Beam Dump Thermal Analysis," Fermilab Beams-Doc-4063, Feb. 2012; https://beamdocs.fnal.gov/ADpublic/DocDB/ShowDocument?docid=4063.

[10] A. Lumpkin, et al., "Initial Beam-profiling Tests with the NML Prototype Station at the Fermilab A0 Photoinjector," MOP219 in PAC11, New York, NY, Dec. 2011; http://www.JACoW.org.

[11] C. Baffes, "NML High Energy Beam Absorbers and Dump: Overview Talk," Fermilab beams-doc-3928, Oct. 2011; http://beamdocs.fnal.gov/ADpublic/DocDB/ShowDocument? docid $=3928$.

[12] C. Prokop, et al., "Start-to-end Beam Dynamics Simulations for the SRF Accelerator Test Facility at Fermilab," WEP036 in NA-PAC'11, New York, NY, March 2011, http://www.JACoW.org.

[13] C. Prokop, et al., "High energy Lattice for First-beam Operation of the SRF Test Accelerator at NML," Fermilab-TM-2516-APC, March 2012; http://Iss.fnal.gov/archive/test-tm/2000/ fermilab-tm-2516-apc.shtml).

[14] "Elegant - Electron Generation and Tracking," http://www.aps.anl.gov/Accelerator Systems Division/Accelerator Operations Physics/manuals/elegant latest/elegant.html.

[15] "Impact-Z - Integrated Map and Particle Accelerator Tracking Code," http://amac.lbl.gov/ jiqiang/IMPACT.

[16] “CSRTrack Version 1.1 User's Manual, http://www.desy.de/fel-beam/csrtrack/files/CSRtrack User Guide 1.100 01.pdf. 


\subsection{Accelerator Science Education at ASTA and Collaboration with the Program of Technology Development of the Illinois Accelerator Research Center (IARC)}

Accelerators have taken an increasingly important role in our Society and improving their performances, downsizing their footprints, or reducing their cost will be a long and challenging endeavor that will strongly rely on future generation of accelerator scientists. In addition, the multidisciplinary nature of Accelerator Science makes accelerators ideal platforms for student education over a vast range of topics.

New beam physics issues associated with future accelerators have emerged (single-bunch and multi-bunch collective effects in high brightness electron accelerators, simulations of large emittance beams in heavy ions and/or multi state charge accelerators) and more intricate accelerator physics tools capable of accounting for all these effects are being developed and need to be carefully validated at available facilities. Such models are mandatory to efficiently design and test the performance of these accelerators prior to their construction. Similarly, future accelerators will require the development of precise diagnostics and controls capable of measuring and/or correcting unprecedented beam parameters (sub-micrometer size, femtosecond duration, with high average current). Most of these topics will be explored to some extent at ASTA and could form the basis of research projects for PhD, Masters, or internships.

Interdisciplinary research projects involving the engineering discipline will also be possible at ASTA. Examples that come to mind are fabrication of state-of-the-art accelerator components requiring precision modeling, drafting, and manufacturing. $R F$ engineering $R \& D$ examples are ubiquitous in accelerator physics. Likewise, novel synchronization techniques and feedback systems will be needed, e.g. to insure that the accelerating fields in the superconducting linear accelerators are very stable.

Over the last decade the number of Educational Institutions that have taken on developing a curriculum in Accelerator Science has increased from a couple to about 10 [1]. None of these Universities currently operates a full-fledged accelerator complex and generally rely on facilities available at National Laboratories to carry most of their experiments. National laboratories have developed programs to foster partnership with Universities. Fermilab was actually the first laboratory to initiate such a selective program, which offers to financially support students to carry-out Accelerator-Science research at Fermilab's facilities [2]. This program-the Joint University-Fermilab Doctoral Program in Accelerator Physics and Technology-has graduated close to 40 students since its initiation in 1987. More than half of the program's graduates have remained in the field of Accelerator Science, and approximately one quarter of them are prominent scientists with leadership positions in national laboratories or universities.

Fermilab also created prestigious fellowships to attract promising scientists to carry-out research in Accelerator Science: the Peoples fellowship [3] was created in 2001 and the 
Bardeen-Engineering Leadership fellowship [4] was created in 2005. The John Bardeen Engineering Leadership Program is designed to provide entry-level opportunities for outstanding engineering graduates who are interested in working in a cutting edge research environment. Fermilab provides opportunities in the fields of electrical, electronics, radio frequency systems, power distribution, magnets, RF cavities, mechanical, materials science and cryogenic engineering. The Peoples Fellowship was created at Fermilab with the goal of attracting outstanding accelerator scientists early in their careers, both to enhance Fermilab's capabilities in accelerator science and related technologies, and to train and develop the accelerator scientists and technologists who will carry our field forward in the future. Together these fellowship programs have attracted and trained approximately 20 people within the last decade. Most of these fellows still carry-out research in Accelerator Science.

Finally, Fermilab has also been active in involving younger scientists (undergraduate students) through various summer programs. One of these programs, the Lee Teng Undergraduate Internship in Accelerator Science and Engineering established by Fermilab and Argonne, aims at attracting undergraduate students into the world of particle accelerator physics and technology. The selected students first attend a two-week general accelerator physics class at the summer US particle accelerator school thereby gaining a broad overview of Accelerator Science. They then work on a research project for the following weeks under the supervision of Fermilab and Argonne staff members. ASTA offers the possibility of significantly enhancing the Lee Teng program by incorporating a "hands-on" component to the students' summer research experience, making it a truly unique internship with the potential to attract future accelerator scientists at the critical undergraduate stage.

In addition to these laboratory-driven programs, Fermilab has also welcomed students from University groups carrying out research at Fermilab funded by grants to universities. Examples includes the development of photo-emission laser with University of Rochester, research on flat-beam generation with University of Chicago, or the investigation of beam-driven plasma wakefield acceleration with University of California, Los Angeles.

ASTA will be an excellent platform that could support and enhance the aforementioned education programs and support extramural research engaging students from Universities. Given the breath of the scientific program described in this proposal, we expect ASTA to provide support for topics of research in Advanced Accelerator R\&D, laser science, beam diagnostics and synchronization, accelerator-based light source, superconducting RF system, and Beam Physics. Finally, some of the proposed topics could also foster research beyond Accelerator Science such as the utilization of single-cycle Terahertz pulses for solid-state Physics research or the use of X-rays for developing phase-contrast imaging (with possible spin-off to medical imaging).

ASTA will also offer unique opportunities for the US Particle Accelerator School [5] sessions and classes. A number of hands-on, practical training laboratory sessions can use the ASTA accelerators - the photoinjector, the SRF cryomodule, and the IOTA ring. Among them, those which were very popular in the past US PAS sessions included:

Proposal for an Accelerator R\&D User Facility at Fermilab's Advanced Superconducting Test Accelerator 
- Modern RF systems class

- Beam Instrumentation Lab

- Fundamentals of Accelerator Instrumentation

- Beam Measurements and Diagnostics in Linacs and Rings

- Beam Dynamics Experiments at the IOTA ring

- Beam Measurements, Manipulation and Instrumentation in SRF Linac

Proximity of the Illinois Accelerator Research Center (IARC) will make these classes attractive for the industrialists and technologists.

At Fermilab's IARC [6], scientists and engineers from Fermilab, Argonne and Illinois universities will work side by side with industrial partners to research and develop breakthroughs in accelerator science and translate them into applications for the nation's health, wealth, and security. Besides a dedicated state-of-the-art facility that will house offices, technical and educational space to study cutting-edge accelerator technologies, it is anticipated that IARC's private industry partners will have access to the nearby ASTA facility to perform high power beam tests and collaborate with ASTA users from the US universities in training a new generation of scientists, engineers and technical staff in accelerator technology. These partnerships will make critical contributions to the technological and economic health of Illinois and the nation in general.

\section{References}

[1] W. Barletta, S. Chattopadhyay and A. Seryi, "Educating and training accelerator scientists and technologist for tomorrow," arXiv 1203:3065 (2012); available at http://arxiv.org/pdf/1207.3065.pdf.

[2] See http://apc.fnal.gov/programs2/joint university.shtml.

[3] See http://www.fnal.gov/pub/forphysicists/fellowships/john peoples/index.html.

[4] See http://www.fnal.gov/pub/forphysicists/fellowships/john bardeen/index.html.

[5] US Particle Accelerator School, http://uspas.fnal.gov/.

[6] Illinois Accelerator Research Center at Fermilab, http://iarc.fnal.gov/. 


\subsection{Scope, Cost, Schedule, and Management}

\subsection{Scope}

Construction of the ASTA facility at Fermilab began in 2006 as part of the International Linear Collider/SRF R\&D Program and later the American Recovery and Reinvestment Act (ARRA) within DOE OHEP, with the goal of developing and testing SRF technology in a fully functioning SRF accelerator with ILC beam parameters. Since that time, a great deal of infrastructure and equipment (approximately 80\%) has been procured, installed, and commissioned (Figure 1). This proposal represents the remaining $20 \%$ necessary to complete the facility, as well as an annual operating cost to support a user program at ASTA.

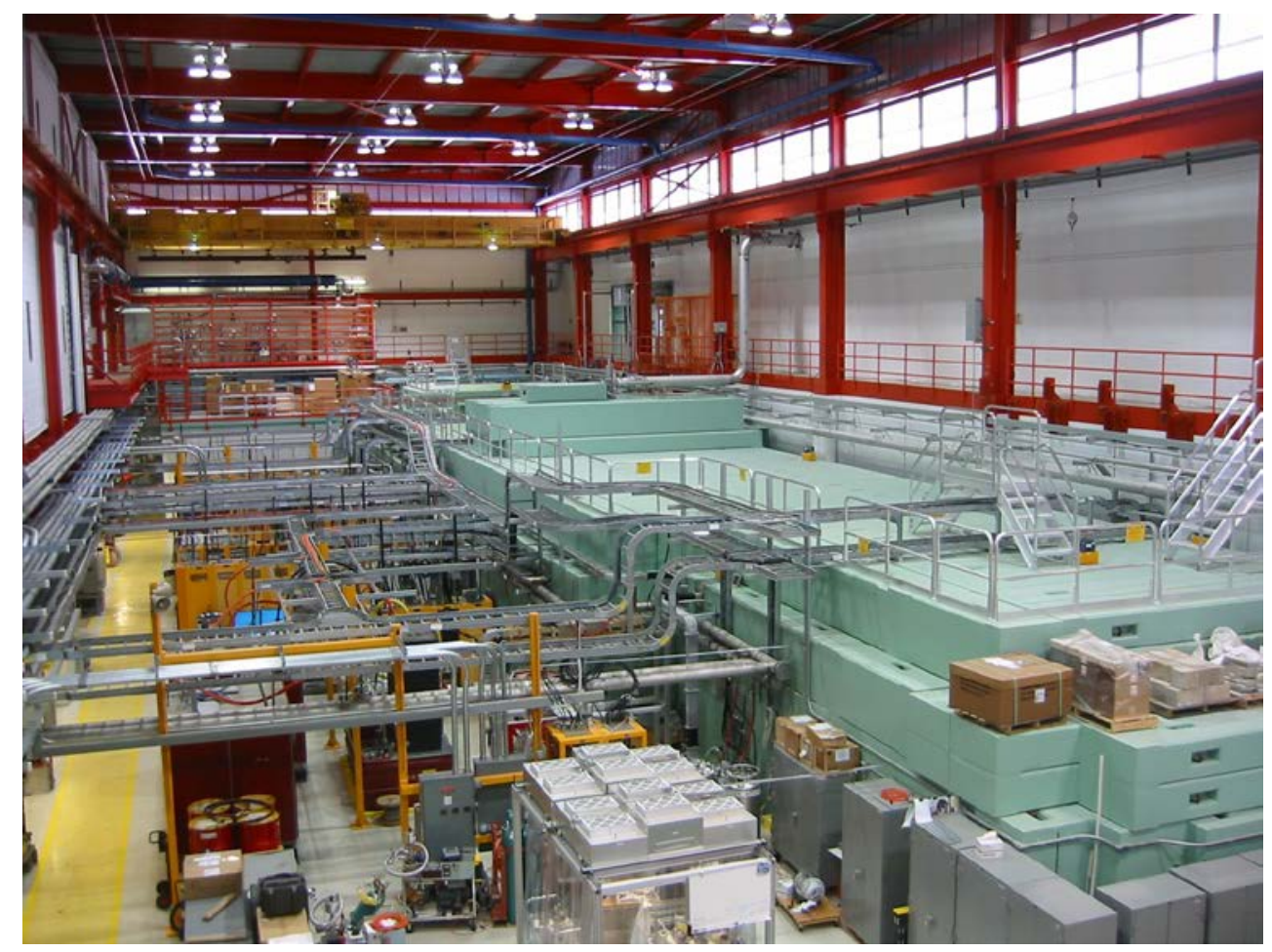

Figure 1: Photograph of ASTA facility at Fermilab.

(View is looking upstream with accelerator enclosure on the right.)

Many aspects of the facility are fully operational and have been in use for years. In addition, many of the technical components and systems needed to complete the ASTA facility have either already been procured or are in operation, such as:

- The recently constructed tunnel extension (\$4.9M)

- All the concrete blocks required to complete the accelerator enclosure (\$1.3M)

- All the RF power and distribution systems required for the full ASTA accelerator (\$8.0M) 
- The cryogenic systems needed for the entire facility $(\$ 16.7 \mathrm{M})$

- All magnets, power supplies, and vacuum equipment for the various beam lines (\$4.1M)

- The high-energy beam absorbers, dump, and water cooling system (\$2.0M)

- A fully operational RF gun and injector capable of providing the $50 \mathrm{MeV}$ beam in the low-energy portion of ASTA (first photoelectrons were generated in June 2013) (\$4.4M)

- The (3) cryomodules needed to complete the accelerator portion of ASTA (\$15M)

- The controls system infrastructure, a control room, and office space (\$2.6M)

- A fully operational laser room $(\$ 1.3 \mathrm{M})$

- Electrical, water cooling, HVAC, and compressed air systems capable of supporting the entire ASTA facility $(\$ 5.4 \mathrm{M})$

- Magnets, support stands, and vacuum equipment for IOTA storage ring (\$0.9M).

The completion of ASTA has been broken up into four distinct operational stages. The completion of Stage I will provide a fully operational facility, whereas Stages II, III, and IV add additional experimental capabilities to the facility.

\section{Stage I: Beam through a single cryomodule}

Stage I will provide a test area for low-energy (50 MeV) experiments (Section 9, Figures 1 and 4); a high-energy test area (Section 9, Figures 2 and 8) capable of providing (300 MeV) beam; and the IOTA storage ring (Section 8.2). The scope of work to complete Stage I will include the following principle components, of which most of the equipment has already been procured:

- Assembly of the cave extension, connecting the existing concrete accelerator enclosure to the newly constructed downstream tunnel extension

- Installation of the low-energy test beamline (magnets, instrumentation, vacuum system, and beam absorber)

- Installation of the high-energy beam transport line (magnets, instrumentation, and vacuum system) from downstream of the first cryomodule to the high-energy beam dump (Section 9, Figures 2 and 8 )

- Installation of the IOTA storage ring (magnets, instrumentation, and vacuum system).

\section{Stage II: Beam through three cryomodules}

Stage II will increase the energy of the ASTA accelerator facility and provide full test capability at high energy $(800 \mathrm{MeV})$. The scope of work to complete Stage II will include the following principle components:

- Installation of the $10 \mathrm{MW}$ RF system (components have already been procured using ARRA funds)

- Relocating the cryogenic end cap for the cryomodule string

- Installation and commissioning of two additional cryomodules (fabrication and assembly of the cryomodules is funded elsewhere). 


\section{Stage III: Addition of proton beam}

Stage III will include the installation of a low-energy proton injector (that already exists at the HINS experimental facility) into the IOTA storage ring. The scope of work to complete Stage III will include the following principle components:

- Relocating and installing the beamline (magnets, instrumentation, vacuum system, etc.) of the HINS injector into ASTA

- Relocating and installing the RF systems from the HINS experiment to the ASTA facility

- Installation of the necessary utilities to support the proton beamline (water, power, controls, etc.).

\section{Stage IV: $3.9 \mathrm{GHz}$ capability}

Stage IV will include the addition of a $3.9 \mathrm{GHz}$ superconducting cavity into the ASTA injector to expand the experimental capabilities of the facility. The scope of work to complete Stage IV will include the following principle components:

- Fabrication and testing of a superconducting linearizing $3.9 \mathrm{GHz}$ cavity and cryostat

- Construction of an $80 \mathrm{~kW}, 3.9 \mathrm{GHz}$ RF system

- Cryogenic modifications to the existing system to tie in the $3.9 \mathrm{GHz}$ cavity.

\subsection{Cost}

ASTA has been funded as an R\&D program since 2006. Approximately $80 \%$ of the equipment and infrastructure has already been procured and installed, including $\$ 15 \mathrm{M}$ of in-kind contributions and reused equipment from other experiments and Laboratories. The costs detailed in this proposal represent the remaining $20 \%$ of the total cost (labor and materials) to complete the facility. The Total Estimated Cost (TEC) of this proposal is $\$ 24.2 \mathrm{M}$, including contingency. This is the cost to complete the infrastructure, installation, and commissioning of all stages of the facility. Following completion of the facility, the annual operating cost is estimated to be $\$ 3.9 \mathrm{M} /$ year. These estimates include all indirect and overhead costs based on current laboratory rates (as of July 2013), and assumes funding will begin in fiscal year 2015.

\subsubsection{Cost Basis}

The cost estimates given in this proposal are based on detailed estimates of all components and systems required to finish each stage of the facility. The basis of estimates for these costs are primarily derived from actual unit cost quotes from vendors, historical costs of purchases previously made for the program, and estimates from experts in the specific area of interest.

All M\&S costs are given in FY2013 dollars and have an approximately $5-10 \%$ inherent contingency built into the estimates. 
The cost of labor is based on actual FY2013 fully burdened rates for the types of specific job categories required to carry out each stage of work. The average labor rate used is $\$ 230 \mathrm{k} / \mathrm{FTE}$. A $30 \%$ flat contingency was then applied to the overall total cost (labor and materials) to account for escalation, price fluctuations, and any uncertainties.

\subsubsection{Stage I Cost}

The cost to complete Stage I is broken down (Tables $1 \mathrm{a}, 1 \mathrm{~b}$ and $1 \mathrm{c}$ ) into the various operational capabilities associated with each sub-stage, as summarized in Figure 2. These costs include the infrastructure and commissioning costs (labor and materials) required to complete each respective stage of ASTA.

\section{Stage I}

I.1

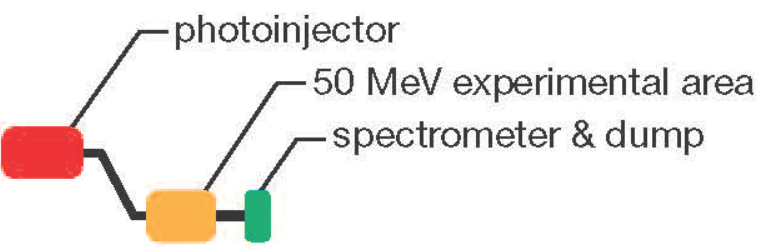

1.2

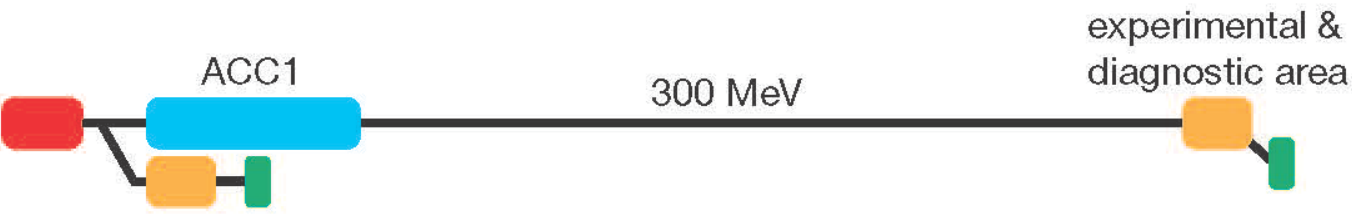

1.3

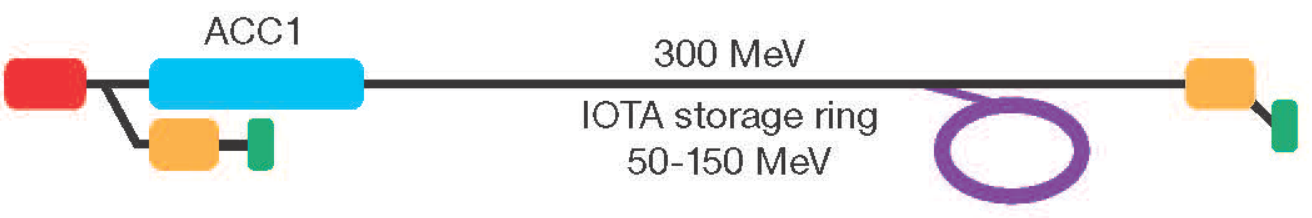

Figure 2: Stage I of the ASTA AARD User Facility construction and operational phases. 
Table 1a: Stage I.1 Cost (50 MeV Experimental Area).

\begin{tabular}{|l|c|c|}
\hline Description & FTE & Total Cost (K\$) \\
\hline \hline Infrastructure & & \\
\hline Materials \& Services/Equipment & - & 1279 \\
\hline Labor & 3.8 & 874 \\
\hline Commissioning & & \\
\hline Materials \& Services/Equipment & - & 241 \\
\hline Labor & 3.9 & 897 \\
\hline & & \\
\hline Contingency (30\%) & - & 987 \\
\hline \hline Total Cost of Stage I.1 & & \\
\hline
\end{tabular}

Table 1b: Stage I.2 Cost (300 MeV Experimental Area).

\begin{tabular}{|l|c|c|}
\hline Description & FTE & Total Cost (K\$) \\
\hline \hline Infrastructure & & \\
\hline Materials \& Services/Equipment & - & 136 \\
\hline Labor & 3.2 & 736 \\
\hline & & \\
\hline Commissioning & & \\
\hline Materials \& Services/Equipment & - & 482 \\
\hline Labor & 6.0 & 1380 \\
\hline & & \\
\hline Contingency (30\%) & - & 820 \\
\hline \hline Total Cost of Stage I.2 & & \\
\hline
\end{tabular}

Table 1c: Stage I.3 Cost (IOTA Ring).

\begin{tabular}{|l|c|c|}
\hline Description & FTE & Total Cost (K\$) \\
\hline \hline Infrastructure/Installation & & \\
\hline Materials \& Services/Equipment & - & 618 \\
\hline Labor & 1.7 & 391 \\
\hline Commissioning & & \\
\hline Materials \& Services/Equipment & - & 148 \\
\hline Labor & 1.2 & 276 \\
\hline Contingency (30\%) & - & 430 \\
\hline \hline Total Cost of Stage I.3 & & \\
\hline & & $\mathbf{1 8 6 3}$ \\
\hline Total Cost of Stage I (I.1+l.2+I.3) & & $\mathbf{9 6 9 5}$ \\
\hline
\end{tabular}




\subsubsection{Stage II Cost}

The cost to complete Stage II is shown in Table 2 and includes the infrastructure and commissioning costs (labor and materials) required to add two additional cryomodules, increasing the energy of ASTA to $800 \mathrm{MeV}$ (Figure 3).

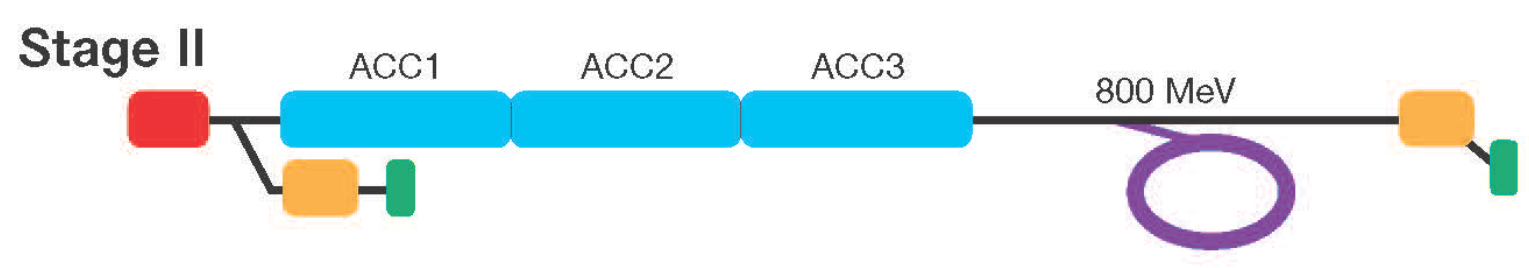

Figure 3: Stage II of the ASTA AARD User Facility.

Table 2: Stage II Cost (800 MeV Experimental Area).

\begin{tabular}{|l|c|c|}
\hline Description & FTE & Total Cost (K\$) \\
\hline \hline Infrastructure & & \\
\hline Materials \& Services/Equipment & - & 927 \\
\hline Labor & 6.9 & 1587 \\
\hline Commissioning & & \\
\hline Materials \& Services/Equipment & - & 1019 \\
\hline Labor & 10.0 & 2300 \\
\hline Contingency (30\%) & - & 1750 \\
\hline \hline Total Cost of Stage II & & $\mathbf{7 5 8 3}$ \\
\hline
\end{tabular}




\subsubsection{Stage III Cost}

The cost to complete Stage III is shown in Table 3 and includes the infrastructure and commissioning costs (labor and materials) required to install an existing proton/ $\mathrm{H}$ - injector into the IOTA storage ring (Figure 4).

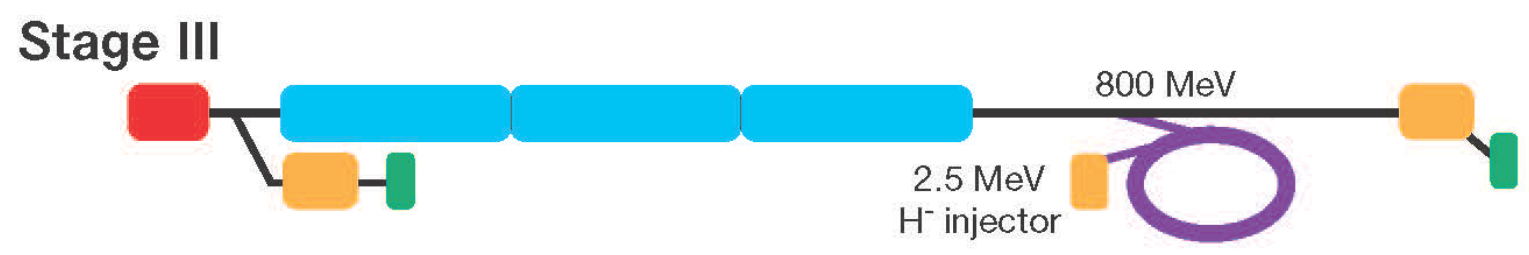

Figure 4: Stage III of the ASTA AARD User Facility.

Table 3: Stage III Cost (Proton/H- Injector into IOTA Ring).

\begin{tabular}{|l|c|c|}
\hline Description & FTE & Total Cost (K\$) \\
\hline Infrastructure & & \\
\hline Materials \& Services/Equipment & - & 741 \\
\hline Labor & 2.4 & 552 \\
\hline & & \\
\hline Commissioning & & \\
\hline Materials \& Services/Equipment & - & 136 \\
\hline Labor & 1.1 & 253 \\
\hline & & \\
\hline Contingency (30\%) & - & 505 \\
\hline & & \\
\hline \hline Total Cost of Stage III & & $\mathbf{2 1 8 7}$ \\
\hline
\end{tabular}




\subsubsection{Stage IV Cost}

The cost to complete Stage IV is shown in Table 4 and includes the infrastructure and commissioning costs (labor and materials) required to fabricate and install a superconducting 3.9 GHz linearizing cavity into the ASTA injector (Figure 5).

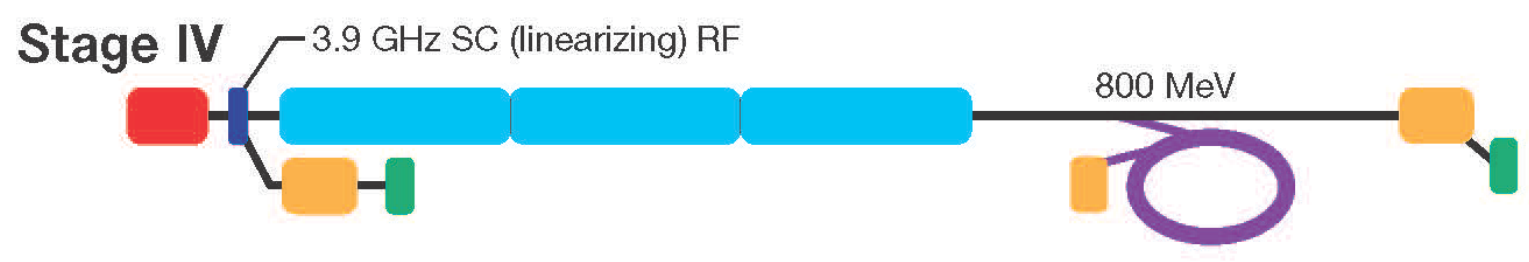

Figure 5: Stage IV of the ASTA AARD User Facility.

Table 4: Stage IV Cost (3.9 GHz System).

\begin{tabular}{|l|c|c|}
\hline Description & FTE & Total Cost (K\$) \\
\hline \hline Infrastructure \& Commissioning & & \\
\hline Materials \& Services/Equipment & - & 2109 \\
\hline Labor & 6.8 & 1564 \\
\hline & & \\
\hline Contingency (30\%) & - & 1102 \\
\hline & & \\
\hline \hline Total Cost of Stage IV & & $\mathbf{4 7 7 5}$ \\
\hline
\end{tabular}

After the Stage I and Stage II infrastructure is in place, the ASTA facility will be fully operational at full design energy (50 MeV and $800 \mathrm{MeV}$ ). The long-term operational cost of the facility is described in the following section.

\subsubsection{Operating Cost}

This estimate of the annual ASTA operating budget is the steady-state cost to operate the facility after the Stage I and Stage II infrastructure construction work has been completed. It includes the following assumptions.

- 9 months of operation and 3 months for shutdown, installation, and maintenance per year

- 2 shifts/day during operation

- 3 cryomodules installed

- ASTA Operations department of 8 people

Furthermore, it is assumed that the experimental groups will provide funding for all experimental equipment and that ASTA will provide only ancillary support such as standard 
instrumentation, beamline modifications, vacuum connections, infrastructure modifications, and other upgrades to accommodate new experiments. Labor and M\&S operating cost estimates are shown in Tables 5 and 6.

Table 5: Operational Labor Estimate.

\begin{tabular}{|l|c|c|}
\hline Labor Description & FTE & $\begin{array}{c}\text { Cost } \\
(\mathrm{K} \$)\end{array}$ \\
\hline \hline ASTA Operations Department and Mgt. & 7.6 & \\
\hline Mechanical Support & 1.2 & \\
\hline Controls \& Instrumentation & 1.6 & \\
\hline RF Dept. & 0.7 & \\
\hline Cryogenic Dept. & 1.5 & \\
\hline \hline Total & 12.6 & $\mathbf{2 8 9 6}$ \\
\hline
\end{tabular}

The cost of labor is based on actual FY2013 fully burdened rates for the types of job categories required to carry out the work. The ASTA Operations department consists of operators, engineering physicists, scientists and technical specialists whose principle responsibility is to manage the daily activities and operate the accelerator at the ASTA facility. The remaining categories are specialists (engineers, technicians, programmers, etc.) from various support departments that will be needed on a periodic basis to support and maintain the various systems of the ASTA facility. These estimates are based on the commissioning experience of ASTA over the past few years and the anticipated needs after the facility is completed.

Table 6: Operational M\&S Estimate.

\begin{tabular}{|l|c|}
\hline Item Description & Cost (K\$) \\
\hline \hline Infrastructure/Aux. Systems & 161 \\
\hline RF & 74 \\
\hline Laser & 37 \\
\hline Vacuum/Clean room & 124 \\
\hline Instrumentation & 86 \\
\hline Controls & 19 \\
\hline Experimental Support & 62 \\
\hline Cryogens & 457 \\
\hline \hline Total & 1020 \\
\hline
\end{tabular}

The M\&S cost estimate includes consumables, maintenance and upgrade items needed on an annual basis to support the operations and infrastructure of the ASTA facility. These estimates are based on recent historical costs over the past few years and the anticipated needs after the facility is completed and in full operation. 


\subsubsection{Cost Breakdown}

A summary of the ASTA costs is shown broken down by individual operational stage in Table 7 and a proposed funding profile by fiscal year is given in Table 8 and in Figure 6.

Table 7: ASTA Cost Breakdown by Stage.

\begin{tabular}{|c|c|c|c|}
\hline Stage & & $\begin{array}{c}\text { Infrastructure \& } \\
\text { Commissioning } \\
\text { Costs (\$k) }\end{array}$ & $\begin{array}{l}\text { Operations Cost/yr } \\
\text { (\$k) }\end{array}$ \\
\hline I.1 & add $50 \mathrm{MeV}$ experimental area, spectrometer, \& dump & $\$ 4,278$ & \\
\hline 1.2 & add $\mathrm{CM} \# 1$ ( $300 \mathrm{MeV}$ ), experimental area, and diagnostics area & $\$ 3,554$ & \\
\hline \multirow[t]{2}{*}{1.3} & add IOTA Storage Ring $(50-150 \mathrm{MeV})$ & $\$ 1,863$ & \\
\hline & Total Cost (Stage I): & $\$ 9,695$ & \\
\hline II & add $\mathrm{CM} \# 2$ and $\mathrm{CM} \# 3$ ( $800 \mathrm{MeV})$ & $\$ 7,583$ & \\
\hline Ops. & annual operating cost of ASTA after Stage I \& II infrastructure is complete & & $\$ 3,916 / y r$ \\
\hline III & add HINS $2.5 \mathrm{MeV} \mathrm{H-/proton} \mathrm{injector}$ & $\$ 2,187$ & \\
\hline \multirow[t]{2}{*}{ IV } & add superconducting $3.9 \mathrm{GHz}$ linearizing cavity & $\$ 4,775$ & \\
\hline & Total Project Cost (Stage I thru IV): & $\$ 24,240$ & \\
\hline & & & \\
\hline
\end{tabular}

Table 8: ASTA Cost Breakdown by Fiscal Year.

Cost by Fiscal Year and Stage (\$M)

\begin{tabular}{|c|c|c|c|c|c|c|c|c|c|}
\hline Fiscal Year & 1.1 & 1.2 & 1.3 & II & III & IV & Ops. & $\begin{array}{l}\text { Total Cost } \\
(\$ M)\end{array}$ & Details of Scope/Comments \\
\hline FY 2015 & 4.3 & 3.6 & 1.1 & - & - & - & 0.2 & 9.1 & Half of IOTA cost, begin operation of $50 \mathrm{MeV}$ \\
\hline FY 2016 & - & - & 0.8 & 5.0 & 0.8 & 1.3 & 1.0 & 8.9 & $\begin{array}{l}\text { Complete IOTA, begin HINS \& } 3.9 \mathrm{GHz} \text {, } \\
\text { operation of all Stage I }\end{array}$ \\
\hline FY 2017 & - & - & - & 2.6 & 1.4 & 2.6 & 2.0 & 8.6 & $\begin{array}{l}\text { Complete } 800 \text { MeV \& HINS, complete } 3.9 \\
\text { fabrication, operate Stage I }\end{array}$ \\
\hline FY 2018 & - & - & - & - & - & 0.9 & 3.9 & 4.8 & Install and commission 3.9, operate all stages \\
\hline $\begin{array}{c}\text { FY } 2019 \& \\
\text { Beyond }\end{array}$ & - & - & - & - & - & - & 3.9 & 3.9 & Operations of entire facility \\
\hline Cost per Stage & 4.3 & 3.6 & 1.9 & 7.6 & 2.2 & 4.8 & & & \\
\hline $\begin{array}{c}\text { Cumulative } \\
\text { Cost }\end{array}$ & 4.3 & 7.8 & 9.7 & 17.3 & 19.5 & 24.2 & & & \\
\hline
\end{tabular}




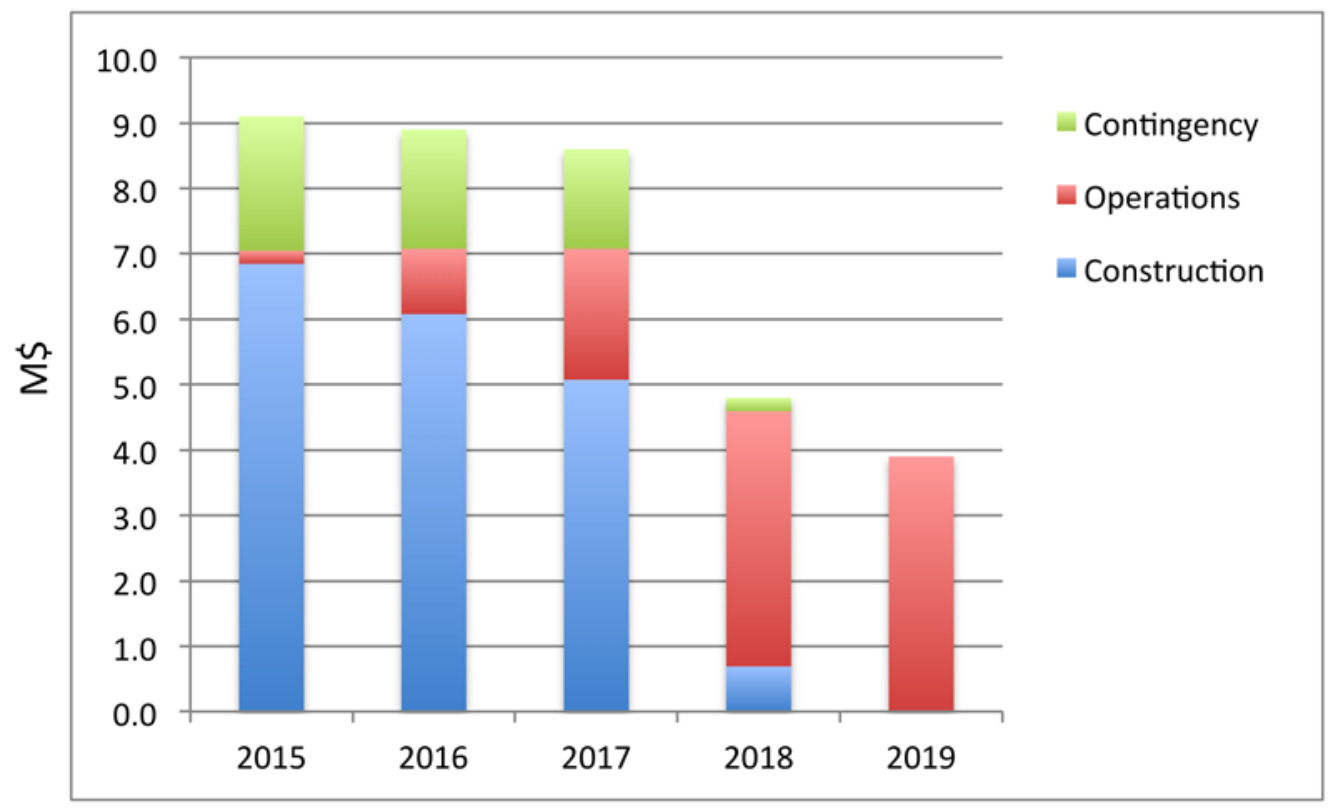

Figure 6: ASTA Costs by Fiscal Year (Construction, Operations, and Contingency).

\subsection{Schedule}

At the beginning of the program (2006), the overall scope of the ASTA facility was established, budget estimates were developed, and a schedule was created. However, as an R\&D program, funding for ASTA has varied significantly from year to year, which directly affected M\&S expenditures as well as labor levels. As a result of these budget fluctuations, it became impossible to maintain a long-term resource loaded schedule. Therefore, an alternative strategy was taken to manage the schedule of the facility development. The overall long-term scope of the program remained essentially fixed, which allowed planning for long-term procurements and future planning, but a schedule and associated progress milestones were established on an annual basis once the budget and labor-levels were known for a given year. This has proved to work well in an unstable budget environment and has allowed the flexibility to slow the program down when budgets were tight as well as accelerate the progress when the opportunity presented itself, such as when ARRA funds became available.

A proposed schedule and associated milestones are given in the following section, based on the proposed funding profile shown in Table 8 . Once a realistic funding plan is known, a detailed schedule and associated milestones will be created.

\subsubsection{Installation Schedule}

Stage I of ASTA is expected to take approximately one year to install at the funding and labor levels described above. At the completion of this Stage, ASTA will be a fully operational SRF 
accelerator capable of supporting a $50 \mathrm{MeV}$ low-energy and a $300 \mathrm{MeV}$ high-energy test program. The installation and commissioning of IOTA is also planned to occur during the Stage I schedule of ASTA.

After Stage $\mathrm{I}$ is complete, experimental operation of ASTA could begin on the schedule described in Section 11.2.6 (9 months of operation and 3 months of shutdown per year).

Stage II of ASTA is expected to take an additional 1.5 years to complete at the funding and labor levels given in Section 11.2.3. At the completion of this Stage, the capability of the high-energy test area will increase to $800 \mathrm{MeV}$.

The installation of Stages III and IV of ASTA can be scheduled to coincide with the annual 3month shutdown/maintenance intervals to minimize interruptions of the experimental program.

A summary of the proposed installation, commissioning and experimental schedule for the various Stages of ASTA build-out is shown in Figure 7 and a list of milestones based on this schedule is shown in Figure 8. This schedule assumes funding for Stage I (including IOTA) is available in FY 2015.

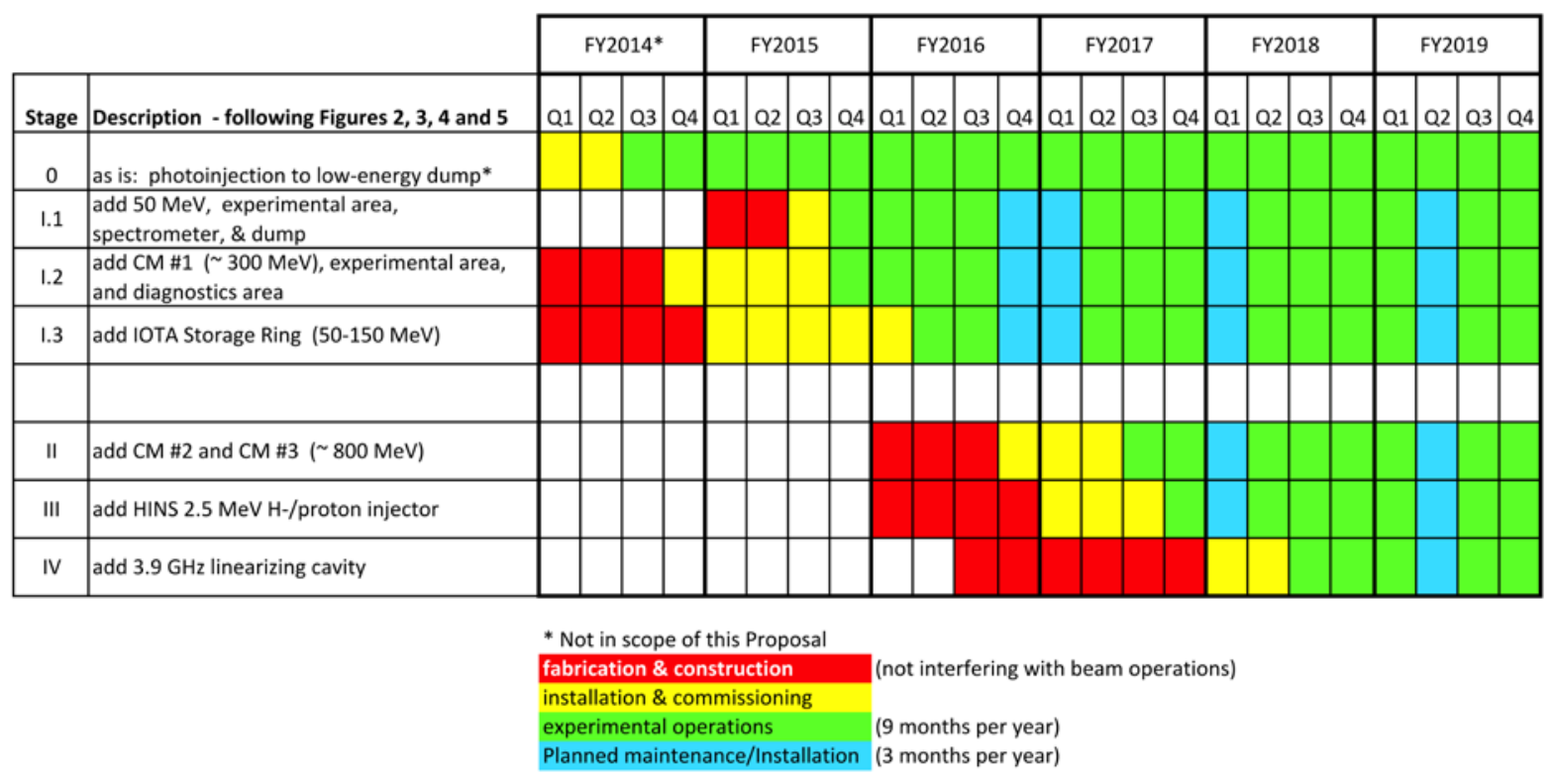

Figure 7: ASTA Schedule. 


\begin{tabular}{|c|c|c|c|c|c|c|c|c|c|c|c|c|c|c|c|c|c|c|c|c|c|}
\hline \multirow[b]{2}{*}{ Stage } & \multirow[b]{2}{*}{ Milestone Description } & \multicolumn{4}{|c|}{ FY2014* } & \multicolumn{4}{|c|}{ FY2015 } & \multicolumn{4}{|c|}{ FY2016 } & \multicolumn{4}{|c|}{ FY2017 } & \multicolumn{4}{|c|}{ FY2018 } \\
\hline & & Q1 & Q2 & Q3 & Q4 & Q1 & Q2 & Q3 & Q4 & Q1 & Q2 & Q3 & Q4 & Q1 & Q2 & Q3 & Q4 & Q1 & Q2 & Q3 & Q4 \\
\hline 0 & Photoinjection to low-energy dump* & & $\mathrm{X}$ & & & & & & & & & & & & & & & & & & \\
\hline \multirow[t]{2}{*}{1.1} & $50 \mathrm{MeV}$ experimental line installed & & & & & & $\mathrm{X}$ & & & & & & & & & & & & & & \\
\hline & $\begin{array}{l}50 \mathrm{MeV} \text { experimental line commissioned } \\
\text { (beam to dump) }\end{array}$ & & & & & & & $\mathrm{x}$ & & & & & & & & & & & & & \\
\hline \multirow[t]{2}{*}{1.2} & $\begin{array}{l}300 \mathrm{MeV} \text { beam line installed (from } \mathrm{CM} \# 1 \text { to } \\
\text { high-energy dump) }\end{array}$ & & & & & $\mathrm{x}$ & & & & & & & & & & & & & & & \\
\hline & $\sim 300 \mathrm{MeV}$ beam to high-energy dump & & & & & & & $\mathrm{x}$ & & & & & & & & & & & & & \\
\hline \multirow[t]{2}{*}{1.3} & IOTA Storage Ring Installed & & & & & & & & $x$ & & & & & & & & & & & & \\
\hline & Beam injected into IOTA Ring & & & & & & & & & $\mathrm{X}$ & & & & & & & & & & & \\
\hline & & & & & & & & & & & & & & & & & & & & & \\
\hline \multirow[t]{2}{*}{ II } & $\mathrm{CM} \# 2$ and $\mathrm{CM} \# 3$ installed & & & & & & & & & & & & $\mathrm{X}$ & & & & & & & & \\
\hline & $\begin{array}{l}\text { Beam accelerated to high-energy } \\
\text { experimental area ( 800 MeV) }\end{array}$ & & & & & & & & & & & & & & $\mathrm{X}$ & & & & & & \\
\hline \multirow[t]{2}{*}{ III } & HINS $2.5 \mathrm{MeV} \mathrm{H}$-/proton injector installed & & & & & & & & & & & & & & $\mathrm{X}$ & & & & & & \\
\hline & HINS beam injected into IOTA & & & & & & & & & & & & & & & $\mathrm{X}$ & & & & & \\
\hline \multirow[t]{2}{*}{ IV } & 3.9 GHz cavity assembled and tested & & & & & & & & & & & & & & & & $\mathrm{X}$ & & & & \\
\hline & 3.9 GHz cavity installed and commissioned & & & & & & & & & & & & & & & & & & $\mathrm{X}$ & & \\
\hline
\end{tabular}

* not in scope of this proposal (expected to be complete in FY 2014)

Figure 8: ASTA Milestones.

\subsubsection{Operations Schedule}

Once completed, the schedule for the facility is planned to be 9 months of operation and 3 months for shutdown, installation, and maintenance per year. During operations, the facility will run 2 shifts per day.

Since all of ASTA is housed in a common accelerator enclosure, the installation of the various stages of ASTA will have varying impacts on the ability to operate the other stages. For example, during the installation of the two cryomodules in the accelerator for Stage II, beam cannot be transported to the $300 \mathrm{MeV}$ experimental area (Stage I.2) or IOTA (Stage I.3). The schedule shown in Figure 7 takes this into account by using the annual 3-month scheduled maintenance periods as well as commissioning periods, to minimize the effect of installation on the experimental program.

Once ASTA construction is complete, all experimental areas can operate simultaneously. However, there will be a need to occasionally access the ASTA enclosure to install and maintain individual experiments and do general maintenance on the various portions of the accelerator. The general plan is to schedule this short-duration work by shifts as much as possible, to maximize experimental operations of ASTA. For example, maintenance work on the $50 \mathrm{MeV}$ experimental area could occur during the day shift, and operation of the $300 \mathrm{MeV}$ experiments and IOTA could occur on the second shift. 
It may also be possible to install a shield wall downstream of the cryomodule string, separating the low-energy and high-energy portions of ASTA (Figure 9). This could allow the operation of the low-energy (50 MeV) experimental facility while the high-energy (800 MeV and IOTA) portion is being accessed. This is not in the current plan, but can be added in the future, if deemed valuable.

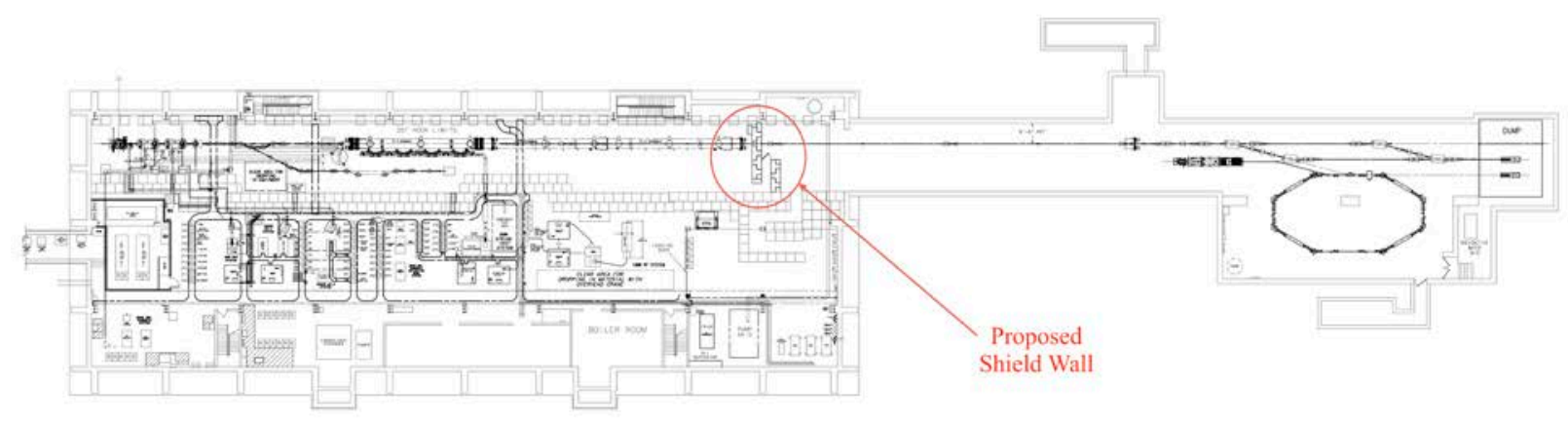

Figure 9: Proposed Shield Wall in ASTA Enclosure.

\subsection{Management}

\subsubsection{Organization}

Construction of the ASTA Facility at NML began in 2006 as part of the International Linear Collider/SRF R\&D Program and later the American Recovery and Reinvestment Act (ARRA) within the DOE OHEP. Funding for ASTA is managed through the Fermilab Directorate with specific responsibility for the management of the Scientific Program residing in the Accelerator Physics Center and the construction, operation and maintenance of the facility under the responsibility of the Accelerator Division. There is a dedicated team of scientists, engineers, technicians, and specialists from the various departments of these Divisions/Sections who currently support ASTA. The ASTA organization is shown in Figure 10. 


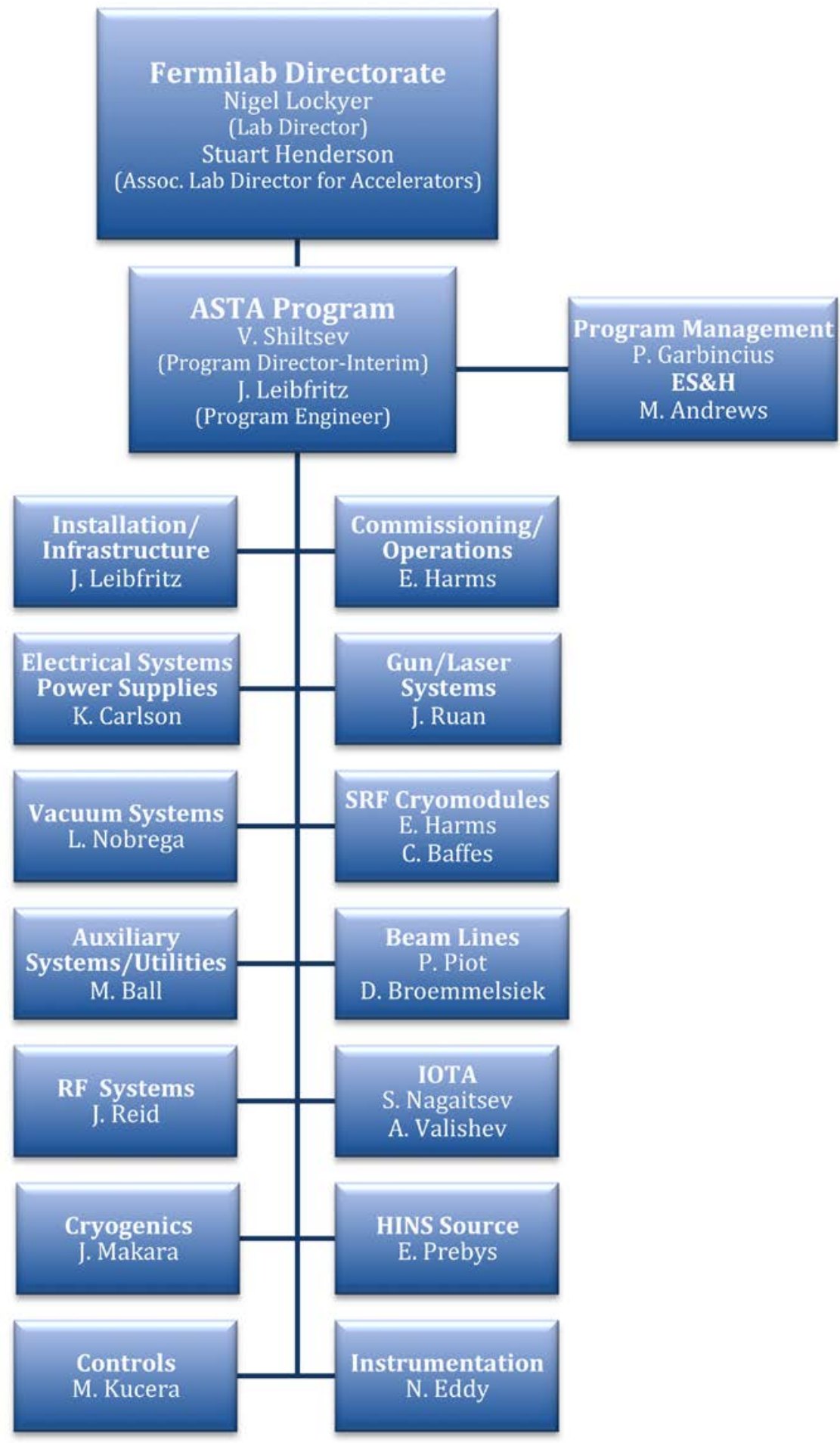

Figure 10: ASTA Organization. 


\subsubsection{Quality Control \& ES\&H}

The design, construction, installation, and operation of this facility will follow all established standards and procedures of Fermilab, will be in accordance with recognized engineering practices and design standards and will comply with the applicable portions of the U.S. Department of Energy and the State of Illinois codes, orders and regulations as incorporated into contract No. DE-AC02-07CH11359 between the U.S. Department of Energy and Fermi Research Alliance, LLC.

Fermilab has adopted the Necessary and Sufficient Process (NSP) for determining the Work Smart Set (WSS) of Standards, which are used to determine the appropriate environment, safety and health standards used to ensure the safe and environmentally responsible operation of the Laboratory.

\section{Integrated Safety Management (ISM)}

Fermilab subscribes to the philosophy of Integrated Safety Management (ISM), in accordance with Department of Energy Order 413.3b. ISM is a system for performing work safely and in an environmentally responsible manner. The term "integrated" is used to indicate that the Environment, Safety and Health (ESH) management systems are normal and natural elements of doing work. The intent is to integrate the management of ES\&H with the management of the other primary elements of construction: quality, cost, and schedule.

\section{ESH\&Q Management}

The Environment, Safety, Health and Quality (ESH\&Q) Section of Fermilab has the responsibility for providing safety coordination support and oversight of safety throughout the Laboratory. As with all Fermilab programs, attention to ESH\&Q concerns will be part of ASTA management and safety will be incorporated into all processes. 


\subsection{List of Acronyms}

Defined at first use in text.

AARD: Advanced Accelerator Research \& Development

ACCn: Superconducting RF Accelerating Module \#n of ASTA

ADC: Analog to Digital Converter

ADS: Accelerator Driven System for nuclear power generation

ALS: Advanced Light Source at Lawrence Berkeley National Laboratory (LBNL)

ANL: Argonne National Laboratory, Lamont, IL

APS: Advanced Photon Source at Argonne National Laboratory, Lamont, IL

Ar: Argon gas

ARIEL: future Advanced Rare IsotopE Laboratory at TRIUMF

ASTA: Advanced Superconducting Test Accelerator (this proposal for Fermilab)

ATF: Accelerator Test Facility, at Brookhaven National Laboratory, Upton, NY

AWA: Argonne Wakefield Accelerator at Argonne National Laboratory, Lemont, IL

A0: AZero - location at Fermilab of the Fermilab NICADD Photolnjector Laboratory

now called HBESL: High-Brightness Electron Source Laboratory

AOPI: A0 Photolnjector Laboratory at Fermilab

BAM: Bunch Arrival Monitor

BAT: Bormann Anomalous Transmission

BBO: beta barium borate

BBU: beam break-up

BC: bunch compressor

BELLA: Berkeley Laboratory Laser Accelerator at LBNL

BES: Basic Energy Sciences - a division of US DOE

BINP: Budker Institute of Nuclear Physics, Siberia, Russia

BLIP: Brookhaven Linear Isotope Producer

BNL: Brookhaven National Laboratory, Upton, NY

BPM: beam position monitor

BSM: new phenomena (outside the scope of) Beyond the Standard Model of particle physics

C-ADS: accelerator for Chinese Accelerator Driven System (for nuclear power generation)

CAVn: superconducting RF cavity \#n of the TESLA type

CCD: charge-coupled device, as used in a camera

CCR: Circulator Cooling Ring

CDR: Coherent Diffraction Radiation, also Conceptual Design Report

Ce: cesium, a photo-electron emitting metal

CdTe: Cadmium Telluride particle detector

CEBAF: Continuous Electron Beam Accelerator Facility - obsolete name for JLAB

CERN: European Center for Nuclear Research, Geneva, Switzerland

CER: Coherent Edge Radiation

Proposal for an Accelerator R\&D User Facility at Fermilab's Advanced Superconducting Test Accelerator 
CESR-TA: CESR-Test Accelerator: Cornell Electron-Positron Storage Ring-Test Accelerator, Ithaca, NY

CGH: Compressed Harmonic Generation

CHEF: A Framework for Accelerator Optics and Simulation

CM-n: Cyromodule \#n built at Fermilab

CNT: Carbon nanotube

CO: molecular Carbon Monoxide

COSR: coherent optical synchrotron radiation

CODR: coherent optical diffraction radiation

COTR: coherent optical transition radiation

CPU: computer (central) processor unit

CR: Channeling Radiation

Csl: Cesium lodide crystal

CSR: coherent synchrotron radiation

CST: Computer Simulation Technology (a company name)

CsTe: Cesium Telluride photocathode

CTR: coherent transition radiation

CUR: coherent undulator radiation

CUVTR: coherent ultra-violet transition radiation

CVD: chemical vapor deposition

CVUVTR: coherent vacuum ultra-violet transition radiation

CW: continuous wave $=$ constant or always active

CXTR: coherent $\mathrm{x}$-ray transition radiation

DAFNE: e+e- collider at the $\phi$ meson and synchrotron radiation source at INFN, Frascati, Italy

DESY: Deutsches Electronen-Synchrotron (Laboratory), Hamburg, Germany

DC: direct current, always on

DEEX: Double Emittance Exchanger

DL: dog-leg configuration of bending magnets

DOE: U.S. Department of Energy

DPAs: displacements per atom

DR: diffractive radiation

DWA: Dielectric Wakefield Accelerator

DWFA: Dielectric Wakefield Accelerator

EEHG: Echo-Enabled Harmonic Generation

EEX: emittance exchange

ELBE: synchrotron radiation source in Dresden, Germany

ELEGANT: simulation program for coherent synchrotron radiation

ELIC CR: Electron-Ion Collider Cooling Ring at JLab

EM: electromagnetic

EMMA: Electron Machine with Many Applications is a project at Daresbury Laboratory in the UK to build a linear non-scaling FFAG

EOM: Electro Optical Modulation 
EOS: Electro-Optical Sampling

e-p: electron-proton interaction

ERL: Energy Recovery Linac

ES\&H: Environmental, Safety, and Health

EUV: extreme ultra-violet

EO - name of a straight section in the Fermilab Tevatron

FACET: Facility for Advanced Accelerator Experimental Tests, at SLAC

FBT: Flat Beam Transform

FEL: Free Electron Laser

FELO: Free Electron Laser Oscillator

FELEX: Free-Electron Laser EXperiment simulation code from LANL

FERMI@Elettra: seeded Free-Electron Laser at ELLETRA, synchrotron light source, Trieste, Italy

FFAG: Fixed Field Alternating Gradient (accelerator)

FIR: far infra-red

FLASH: Free-electron LASer in Hamburg - (offspring of TTF) at DESY

FNAL: Fermi National Accelerator Laboratory, Batavia, IL

FODO: alternating Focusing-Defocusing beam transport channel

FPGA: field-programmable gate array

FWHM: full width at half-maximum

Ge: Germanium particle detector

GHz: Giga-Hertz frequency $=10^{\wedge} 9$ cycles $/ \mathrm{sec}$

GINGER: Free-Electron Laser simulation code from LBNL

g-2: Fermilab experiment to study the anomalous magnetic moment of the muon

HBESL: High-Brightness Electron Source Laboratory at Fermilab, formerly called AOPI, also Fermilab E-1023

HEP: High Energy Physics

HEPAP: US High Energy Physics Advisory Panel reporting to both DOE and NSF

HERA: Hadron Elecktron Ring Anlage (facility), an electron-proton collider at DESY, Hamburg DE

HGHG: High-Gain Harmonic Generation

Higgs: a particle which likely has been recently observed at CERN which provides other particles with mass, also the name of the theoretical physicist predicting its existence

HGHG: high gain harmonic generation

HHG: high harmonic generation

HINS: High Intensity Neutrino Source R\&D program at Fermilab

HOM: higher order mode

HSR: high strain rate

$\mathrm{H}^{-}$: a negative Hydrogen ion formed by a proton plus two electrons

IARC: Illinois Accelerator Research Center at Fermilab, Batavia, IL

ICCD: Intensified Charge-Coupled Device, a sensitive camera

ICL: Imperial College London, UK

Proposal for an Accelerator R\&D User Facility at Fermilab's Advanced Superconducting Test Accelerator 
ICS: Inverse Compton Scattering

IFMIF: International Fusion Materials Irradiation Facility, in Japan

IIT: Illinois Institute of Technology, Chicago, IL

ILC: International Linear Collider

ILCTA: ILC Test Accelerator

INFN: Istituto Nazionale di Fisica Nucleare - Italian government agency supporting HEP

IOT: Inductive Output Tube, a tube producing RF power output

IOTA: Integrable-Optics Test Accelerator proposed for the ASTA facility at Fermilab

IR: Infra-red

JLab: Thomas Jefferson National Laboratory, Newport News, VA

KAM: Kolmogorov-Arnold-Moser theory of toroidal orbits

KoRIA: Korean Rare Isotope Accelerator

LANL: Los Alamos National Laboratory, Los Alamos, NM

LASA: Laboratorio Acceleratori e Superconduttiva Applicatà, INFN, Milano, Italy

L-band: $1-2 \mathrm{GHz}$ range of RF frequencies, TESLA/TTF/FLASH and ILC are based at $1.3 \mathrm{GHz}$

LBNE: Long Baseline Neutrino Experiment - under development at Fermilab

LBNL: Lawrence Berkeley National Laboratory, Berkeley, California

LCLS: Linear Coherent Light Source at SLAC

LCLS-II: Linear Coherent Light Source-II - a proposed high-repetition-rate soft X-ray FEL

LCW: low conductivity (cooling) water

LDRD: Laboratory Directed Research and Development

LEBRA: Laboratory for Electron Beam Research and Applications, Nihon U., Tokyo, Japan

LFD: Lorentz Force Detuning

LFDC: Lorentz Force Detuning Compensation

LEBT: low energy beam transport

LHC: Large Hadron Collider at CERN

$\mathrm{LiH}$ : lithium hydride

LIM: laser-induced microbunching

LLRF: low-level RF controls

LN2: liquid Nitrogen

LPS: longitudinal phase space

LSCA: longitudinal space charge amplifier

LSCIM: longitudinal-space-charge-induced microbunching

LYSO: lutetium-yttrium oxyothosilicate

MAMI: Mainz Microtron, and electron accelerator in Mainz, Germany

MAP: Muon Accelerator Program to study feasibility of producing intense or colliding beams of muons

MaRIE: Matter-Radiation Interaction at the Extreme project at Los Alamos National Lab

MCP: micro-channel plate photo- or electron-multiplier

MDB: Meson Detector Building

Proposal for an Accelerator R\&D User Facility at Fermilab's Advanced Superconducting Test Accelerator 
MEIC: Medium-energy Electron-Ion Collider at JLab

MPa: Mega-Pascal, a unit of pressure

MPI: Martin-Puplett Interferometer

MRDL: NIU Microelectronic Research \& Development Laboratory

Mu2e: Fermilab experiment to search for direct conversion of muons into electrons

MW: Mega-Watt ( $10^{6}$ watts), a unit of beam power or electrical power

NC: normal conducting (as opposed to superconducting)

ND: Neutral Density optical filter to attenuate signal

NdFeB: a rare-earth Neodymium compound used in permanent magnets

$\mathrm{NI}$ : non-intercepting diagnostic technique

NIR: near infra-red

NIU: Northern Illinois University, DeKalb, IL

NGLS: Next Generation Light Source - a high-repetition-rate soft X-ray FEL proposed by LBNL

NML: New Muon Laboratory - site of the ASTA facility at the north part of Fermilab

NSF: US National Science Foundation

ODR: optical diffraction radiation

OHEP: Office of High Energy Physics of the U.S. Department of Energy

OPA: optical parametric amplifier

ORNL: Oak Ridge National Laboratory, Oak Ridge,Tennessee

OSC: Optical Stochastic Cooling

PAC: Program Advisory Committee

PC: photo-cathode

PECVD: plasma-enhanced chemical vapor deposition

PDR: Preliminary Design Report

PEP: Positron-Electron Project, an e+e- collider at SLAC, Menlo Park, California

PhD: Doctor of Philosophy

PIC: particle-in-crystal

PITZ: Photo Injector Test Facility at DESY, Zeuthen (near Berlin), Germany

PNNL: Pacific Northwest National Laboratory, Richland, WA

PoC: proof-of-concept

Project X: proposed future high intensity proton accelerator program at Fermilab

PXR: Parametric X-ray Radiati

P5: Particle Physics Project Prioritization Panel - a sub-panel of HEPAP

Q: quality factor of an RF cavity $=2 \pi *$ Energy stored in a cavity/Energy dissipated per RF cycle

$Q_{L}$ : loaded $Q$-value = quality factor of an RF cavity while loaded with (accelerating) beam

QA: Quality Assurance

Quadrupole: shorthand for a magnet with four poles, which is used to focus the beam

RaDIATE: collaboration to study Radiation Damage in $\underline{\text { Accelerator }}$ Target Environments RFBT: round-to-flat-beam transformer

RFQ: Radio Frequency Quadrupole magnet which accelerates and focuses low energy beams

Proposal for an Accelerator R\&D User Facility at Fermilab's Advanced Superconducting Test Accelerator 
RHIC: Relativistic Heavy Ion Collider at Brookhaven National Laboratory (BNL)

RMS: root-mean-square

RPI: Rensselaer Polytechnic Institute, Troy, New York

RTI: Real-Time Interferometer

RTFB: round-to-flat beam transformer

SARAF: Soreq Applied Research Accelerator Facility, in Israel

SBIR: Small Business Innovation Research - US government program (also refers to its grants)

SciDAC: Scientific Discovery through Advanced Computing, a DOE program

SRF: Super Conducting Radio Frequency

SDUV FEL: Shanghai Deep Ultra-Violet (seeded) Free Electron Laser, Shanghai, China

SER: spontaneous emission radiation

Sextupole: shorthand for a magnet with six poles, usually used to improve the momentum acceptance of a beamline or accelerator by compensating for chromatic aberrations.

SIM: SASE-induced microbunching

SINAP: Shanghai INstitute of Applied Physics, Shanghai, China

SLAC: Stanford Linear Accerator Center, Menlo Park, California

SLC: SLAC Linear Collider

SmCo: Samarium Cobalt

SNM: Special Nuclear Material

SNS: Spallation Neutron Source at Oak Ridge National Laboratory, Oak Ridge, TN

SPS: Super Proton Synchrotron at CERN

SRF: Superconducting RF

SSRn: Superconducting Spoke Resonator cavity \#n

SWCNT: single-wall carbon nanotube

TCBC: Transverse Cavity-Based Chirper

TESLA: $500 \mathrm{GeV}$ e+e- collider proposed by DESY using SRF cavities

THz: Tera-Hertz $\left(10^{12}\right.$ cycles/sec) frequency

T-insert: a beam optics element described in section 3.5.1.2.1 of this proposal

TM: Transverse EM Mode

TOA: Time of Arrival

TR: Transition Radiation

TRIUMF: Canada's particle physics laboratory in Vancouver, British Columbia

TTF: Tesla Test Facility (evolved into FLASH) at DESY

TW: Tera-Watt $\left(10^{12}\right.$ Watts) power level

UHV: ultra-high vacuum

UIUC: University of Illinois, Urbana-Champaign, IL

UMER: University of Maryland Electron Ring

UR: undulator radiation

UV: ultra-violet

VEPP-3: accelerating-storage complex at Budker Institute of Nuclear Physics (BINP)

Proposal for an Accelerator R\&D User Facility at Fermilab's Advanced Superconducting Test Accelerator 
VLHC: Very Large Hadron Collider, a machine design considered in the early 2000's, with energy beyond that of the LHC

VORPAL: Versatile Plasma Simulation Code (by the Tech-X company)

VUV: vacuum ultra-violet

VTO: variable tap-off (of power from an RF distribution system)

WBS: Work Breakdown Structure

XFEL: European X-ray Free Electron Laser being built at DESY, Hamburg, Germany XUV: extreme ultra-violet

YAG: yttrium aluminum garnet

YB: ytterbium

1-D: one-dimensional

2D: 2 dimensional 


\section{A.0 Other Expressions of Interest and Concepts for Experimental Studies}

\section{A.1 Studies Utilizing ASTA Capabilities in SCRF Technology}

\section{A.1.1 Artificial Intelligence-based LLRF Control}

State-of-the-art test accelerators such as ASTA are of profound importance for the advancement of accelerator science and technology, applications in nuclear and high-energy physics, and the needs of the light source user community. Novel experiments in these fields often require tight tolerances on beams with unprecedented parameters in the energy and intensity frontiers. In this context, the development and validation of robust, accurate control systems is absolutely critical and presents exciting challenges. Two of the initial research thrusts mentioned in the ASTA proposal are Accelerator R\&D for Future SC RF Accelerators and Accelerator R\&D for Particle Physics at the Intensity and Energy Frontiers [1]. These areas are particularly ripe with opportunity for development and testing of novel control schemes under demanding conditions. To name a few: control of beams in superconducting RF cryomodules, control of high-energy and space-charge dominated beams, high-average power beam propagation and control, and controls applications for studies in advanced phase space manipulation.

Currently, an effort is underway at Colorado State University to develop adaptive algorithms specifically for control of beam parameters in accelerators. Members of our group have previously used genetic algorithms and neural-network-based techniques to developed adaptive control and optimization schemes for accelerators. A subset of these was successfully implemented at Australian Synchrotron and the $\operatorname{LCLS}[2,3,4,5]$.

Neural networks may be more advantageous than classical control schemes in systems where there are many complex interactions between parameters, or where the governing dynamics of the physical system under study might be too complicated to model analytically. Adaptive neural-network based systems may be able to identify statistical correlations which might otherwise go unnoticed, as well as adjust for slow changes in operational conditions over time (due to equipment aging, etc.). This latter capability is particularly important when faced with high user demands on beam time. In the long run, we would like to design and implement accelerator control systems which are capable of continuously self-training and adjusting controller responses in real-time. 
Our group has already discussed with Brian Chase of the Accelerator Division at Fermilab some possible initial areas of focus for this work, namely:

- Adaptive field and resonance control of the copper gun

- Adaptive resonance control of the superconducting RF cavities to account for long-term changes in operational conditions

- Self-calibration and control of laser timing drift

- Superconducting RF cavity field control with advanced control approaches

- Efficient global system start up, mode control, and exception handling.

ASTA would be a challenging and valuable test bed for the development of novel accelerator control schemes. Development of novel controls techniques for superconducting RF systems and accelerators operating at the energy and intensity frontiers will be an essential asset to ensure tolerances in beam parameters are met for future high-energy physics experiments and future light sources.

\section{A.1.1.1 References}

[1] Proposal for an Accelerator R\&D Facility at Fermilab's Advanced Superconducting Test Accelerator (ASTA). March 2013.

[2] E. Meier, M. Morgan, S.G. Biedron, G. LeBlanc, J. Wu, "Development of a combined feed forward-feedback system for an electron Linac," Nucl. Instr. And Meth. A, 609 (2009) 79.

[3] E. Meier, S.G. Biedron, G. LeBlanc, M.J. Morgan, and J. Wu, "Electron beam energy and bunch length feed forward control studies using an artificial neural network at the Linac coherent light source," Nucl.Instr. and Meth. A, 610 (2009) 629.

[4] E. Meier, S.G. Biedron, G. LeBlanc, M.J. Morgan, "Artificial Intelligence Systems for Electron Beam Parameters Optimization at the Australian Synchrotron," Proceedings of IPAC'10, Kyoto, Japan, page 1770.

[5] E. Meier, S.G. Biedron, G. LeBlanc, M.J. Morgan, "Development of a novel optimization tool for electron linacs inspired by artificial intelligence techniques in video games," Nucl.Instr. and Meth. A: Accelerators, Spectrometers, Detectors and Associated Equipment, 632 (2011) 1. 


\section{A.1.2 Experimental Test of CW Operation of the ASTA SRF Cryomodule \#1}

\section{A.1.2.1 Introduction}

Superconducting accelerating technology becomes especially attractive in the CW regime. Low RF losses in accelerating cavities and magnets without losses provide much higher accelerating gradients, efficiency, and lower cost of the facility including both construction and operation costs. Several recently proposed projects in USA are based on SRF technology in CW operation mode: Energy Recovery Linac (ERL-Cornell, JLAB and BNL proposals), next generation light sources, and Project X. Even more projects outside of US will utilize advantages of a new technology: (XFEL-upgrade, International Fusion Materials Irradiation Facility (IFMIF), Soreq Applied Research Accelerator Facility (SARAF), Chinese accelerator for ADS (C-ADS), Korean Rare Isotope Accelerator (KoRIA), etc.).

ASTA is a unique facility which can be used for the demonstration of CW operation for an Lband SC Linac. Currently, the experimental program is based on beams generated in the pulse regime with 1-1.5ms pulse length operated up-to $10 \mathrm{~Hz}$ repetition rate. In this regime all SRF cavities in ASTA facility (one cryomodule with $3.9 \mathrm{GHz}$ cavity, two cryomodules with 9-cell 1.3 $\mathrm{GHz}$ cavities and several ILC type cryomodules with eight 9-cell $1.3 \mathrm{GHz}$ cavities each) are optimized to provide high gradient and high current operation. The $\mathrm{CW}$ regime of operation requires some modifications in cavity and CM design to better take advantages of the technology and improved performance. Nevertheless, even without these changes, operating in the CW regime is possible in ASTA with lower gradients.

\section{A.1.2.2 Gradient Limitations for SCRF Cavities in CW Regime}

All cavities installed in upstream ASTA cryomodule (CM serial \#2) were tested in vertical and horizontal test cryostats and demonstrated performance above the ILC requirements: quench limit $>35 \mathrm{MV} / \mathrm{m}$ and $\mathrm{Q}_{0}>10^{10}$ at $35 \mathrm{MV} / \mathrm{m}$. Two capture cavities installed in a separate cryostat are qualified for a gradient of $25 \mathrm{MV} / \mathrm{m}$. When installed in the cryostat, cavities had several limitations for CW operation:

- Cryogenic limit: Individual cavities cannot operate if losses exceed $30 \mathrm{~W} /$ cavity. This sets a gradient limit of $\sim 16 \mathrm{MV} / \mathrm{m}$. Some cavities with higher $Q_{0}$ can exceed this limit. The ASTA cryogenic capacity is limited, limiting the practical operating gradient to around $5-7 \mathrm{MV} / \mathrm{m}$.

- HOM couplers as designed in CM\#2 limit the accelerating gradient to 7-10 MV/m. Minor modification in the coupling antenna and cooling of HOM coupler proposed at DESY and $\mathrm{JLAB}$ will increase this limit up to $20 \mathrm{MV} / \mathrm{m}$ in the $\mathrm{CW}$ mode.

- Existing RF couplers are limited by average power level to $\sim 4 \mathrm{~kW}$. This means that average beam current will be limited to less than $1 \mathrm{~mA}$.

The proposed next generation light source project calls for SCRF L-band technology to provide $16 \mathrm{MV} / \mathrm{m}$ accelerating gradient in the CW regime. Within this R\&D program, Fermilab will 
modify the existing design of cavity, couplers, and cryomodule for reliable operation in the CW regime. We assume that additional cryomodules installed in the ASTA beamline will be better optimized for high gradient operation in CW regime.

A high duty cycle regime may be considered as an alternative operation. For many users, long pulses (10-100 ms or longer) will provide the same information as for the $\mathrm{CW}$ regime. High duty cycle $\sim 20-50 \%$ in a long pulse regime is an attractive option. This regime will relax cryogenic and gradient limitations coming from high average power without unacceptable performance for users.

\section{A.1.2.3 Proposal and Resources}

This proposal describes the path to CW operation in several steps. These steps (phases) are needed to understand system performance and limitations for high duty cycle and CW operations.

Phase-I: Test of individual cavities in long pulsed and CW regime.

- The spread in individual cavity parameters will limit it performance at CW or high duty cycle modes. In the first phase of this proposal, we will test cavities at different pulse lengths for different accelerating gradients $(5-20 \mathrm{MV} / \mathrm{m}), Q_{\text {loaded }}$ $\left(6 \times 10^{6}-5 \times 10^{7}\right)$ and operating temperature (1.7-2.0 K). Lorentz force detuning and microphonics compensation is one of the issues and limiting factors. Machine tunability and reliability of operation are key issues in these studies. The Low Level RF system should be adapted for CW operation. One $30 \mathrm{~kW} 1.3$ $\mathrm{GHz}$ Inductive Output Tube (IOT) power source will be required. Existing cryogenic capacity are adequate for these studies

1. Proposal for system upgrade to run in CW/long pulses regime.

- Based on studies done for phase I (described above), beam simulations, and analysis of user requests, we will define one or several modes of operation and required upgrades. Upgrades may include: RF sources, LLRF, electron beam source, pulse compressors, beam diagnostics, vacuum, control, protection system and other subsystems, if needed.

2. Operation in $\mathrm{CW}$ and long pulse regime.

- After commissioning for new operation regime, the ASTA facility will be able provide beam for users. 


\section{A.1.2.4 Timescale and Resources}

The time scale of this proposal is not defined and depends on the ASTA priorities and available resources.

- Phase-I: an optimistic time scale for this program is 1-1.5 years. Fermilab involvement in $R \& D$ for the next generation light source project may accelerate these studies. Rough estimates for resources are $2.5 \mathrm{FTE}$-years $+\$ 400 \mathrm{~K}$.

- Phase-II: Optimistic estimation for this proposal is $\sim 1 / 2$ years to create requirements and design reports. Minimum resources are 1.5 FTE-years.

- Phase-III will require some modifications to the ASTA facility. Timescale will depend on the proposal and funding. 


\section{A.1.3 A Magnetron Transmitter Test at the ASTA SRF Linac}

\section{A.1.3.1 Introduction and Goals of the Experiments}

We propose an experimental test of a $1.3 \mathrm{GHz}$ high-power RF source with a wide-band control in phase and power, based on injection-locked magnetrons to drive Superconducting RF (SRF) cavity of the ASTA linac. Utilization of the magnetron RF sources for the intensity-frontier project is intended to study capabilities of the RF sources based on injection-locked magnetrons to power the SRF cavities of intensity-frontier high-energy superconducting linacs allowing acceleration of the electron beam with minimized longitudinal and transverse emittances. To prevent enlargement of the emittance resulted from mechanical oscillations (microphonics and oscillations caused by Lorentz-force) which are inherent in the SRF cavities and disturb the beam dynamics, a rapid control of amplitude and phase of the accelerating field in each individual cavity by a closed wide-band feedback loop is necessary. This will allow the elimination of parasitic modulation caused by microphonics and phase modulation caused by dynamic tuning errors and beam loading. Thus feeding of each SRF cavity by individual RF source rapidly controlled in phase and power by a Low Level RF (LLRF) system with a wide-band closed feedback loop will allow to optimize phase and amplitude of the accelerating field in the SRF cavity minimizing emittances of the beam.

\section{A.1.3.2 Technical Approach}

The proposed magnetron based RF source parameters are determined by the following parameters of ASTA linac:

$\begin{array}{ll}\text { Bunch charge } & \mathrm{C}_{\mathrm{b}}=3.2 \mathrm{nC} \\ \text { Bunch rep. rate } & \mathrm{F}_{\mathrm{b}}=3 \mathrm{MHz} \\ \text { Train pulse duration } & \mathrm{t}_{\mathrm{Tr}}=1 \mathrm{~ms} \\ \text { Train rep. rate } & \mathrm{F}_{\mathrm{Tr}}=5 \mathrm{~Hz} \\ \text { Energy gain per cavity } & \mathrm{E}_{\mathrm{c}}=31.25 \mathrm{MeV} / \text { cavity } \\ & (250 \mathrm{MeV} \text { per cryomodule containing 8 cavities }) .\end{array}$

From the parameters follows that the accelerated charge in train is: $Q_{T}=C_{b} \cdot F_{b}=9.6 \mathrm{mC}$, thus the current accelerated in train is $\mathrm{I}_{\mathrm{Tr}}=9.6 \mathrm{~mA}$. That gives RF pulse power per cavity of $\mathrm{P}_{\mathrm{Pc}_{\mathrm{c}}}=\mathrm{I}_{\mathrm{Tr}} \cdot \mathrm{E}_{\mathrm{c}}=300 \mathrm{~kW}+$ power for overhead of $\sim 360 \mathrm{~kW}$ per SRF cavity. Since the accelerator uses pulsed mode with duty factor, $d=1 / 200(1 \mathrm{~ms} ; 5 \mathrm{~Hz})$ the average power per cavity is: $P_{\text {Avcav }}=P_{\text {Pcav }} \cdot d=1.8 \mathrm{~kW}$.

A conceptual block-diagram of the magnetron RF source with a rapid phase and power control based on injection-locked magnetrons is shown in Figure 1 [1]. 


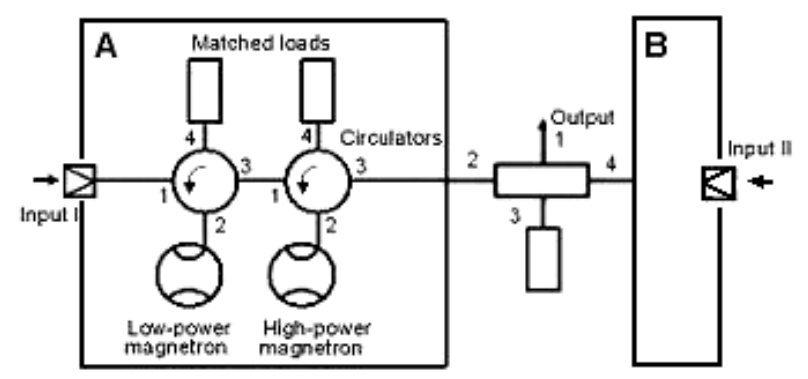

Figure 1: Block-diagram of the magnetron transmitter available for a fast control in phase and power.

The transmitter consists of two identical channels ( $A$ and $B$ ) of 2-cascade injection-locked magnetrons, combined in power by a $3-\mathrm{dB}$ hybrid. The phase management is provided by a phase-modulated signal controlling simultaneously and equally the phases at inputs of both 2cascade magnetrons. The power management is provided at a control by phase difference at the inputs of the 2-cascade magnetrons. The 2-cascade injection-locked magnetron system allows reducing the required locking power by 10-15 dB [2].

Proof of principle of the proposed transmitter was demonstrated in experiments verifying capability of the fast control by phase-modulated injection-locking signal providing the phase and power management of the transmitter active components at various setups. The experiments were conducted by MuPlus, Inc. in collaboration with Fermilab, using two 2.45 $\mathrm{GHz} 1 \mathrm{~kW}$ microwave oven magnetrons with free run frequencies differing by $\approx 5.7 \mathrm{MHz}$. The magnetrons were chosen to be injection-locked at the same frequency $(2.469 \mathrm{GHz})$ and were powered by a modulator with pulse duration of 2.5-15 ms operating at low repetition rate [3]. Because of dissimilar Volt-Amps characteristics of the magnetrons a compensated divider was used to power the magnetron with lower anode voltage. The magnetrons were installed in separate modules allowing commutation of inputs and outputs.

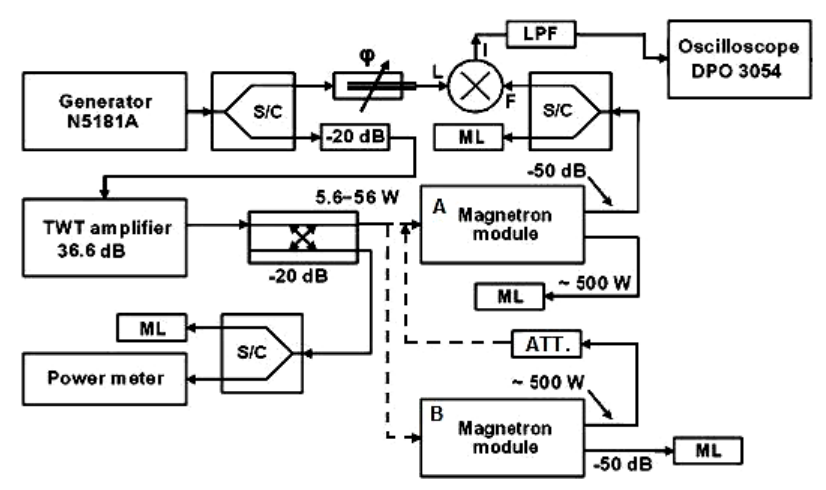

Figure 2: Setup to test operation of single and 2-cascade injection-locked magnetrons. $\mathrm{S} / \mathrm{C}$ is a splitter/combiner; LPF is a low pass filter; ML is a matched load; and ATT is an attenuator. Power of the magnetrons is depicted in this figure. 
Setup for tests of the phase performance of injection-locked single and 2-cascade magnetrons is shown in Figure 2 [4].

The single injection-locked magnetrons were tested in configuration using module $A$ with the magnetron locked by the TWT amplifier and fed by the modulator, while the module B was disconnected from the amplifier and the modulator. The 2-cascade magnetron was tested in a configuration in which the magnetron module B was locked by the TWT amplifier while the module A magnetron was connected via attenuator to the module B output. In this case the magnetron in the module $A$ was locked by the pulsed signal of magnetron $B$ lowered in the attenuator. The modulator fed both the magnetrons. Traces of a single and 2-cascade magnetron phase performance are plotted in Figure 5.

The setup to test the magnetron transmitter power performance, utilizing both experimental modules, is shown in Figure 3 [3]. The power control was realized by a vector summation of output signals of the injection-locked magnetrons controlled by the phase difference. The controlling phase difference was provided by a trombone-like phase shifter $\varphi_{\| I}$ and/or an analogue phase shifter JSPHS-2484 controlled by a voltage.

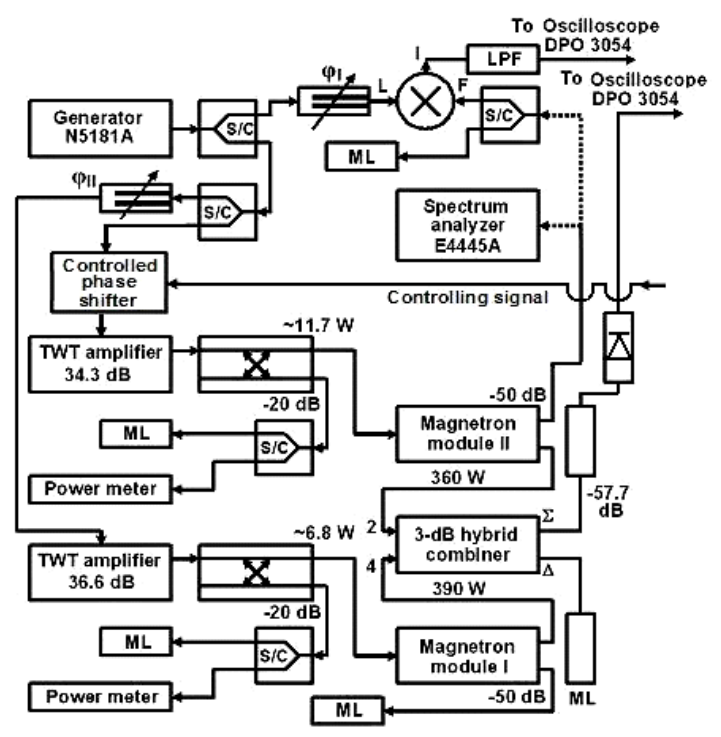

Figure 3: Setup with the injection-locked magnetrons and a 3-dB 180 degrees hybrid for test of the power control by power combining. A phase detector including phase shifter $\varphi_{1}$, double balanced mixer and Low Pass Filter (LPF) was used to measure intrapulse phase performance of the injection-locked magnetrons without and with the phase control; a calibrated detector was used to measure combined power at the fast power control. 
Performance of the injection-locked magnetrons at a fast power control first was demonstrated controlling the phase shifter JSPHS-2484 by a sequence of meander-like pulses with period of $\approx 30 \mu$ s, Figure 4, [3].

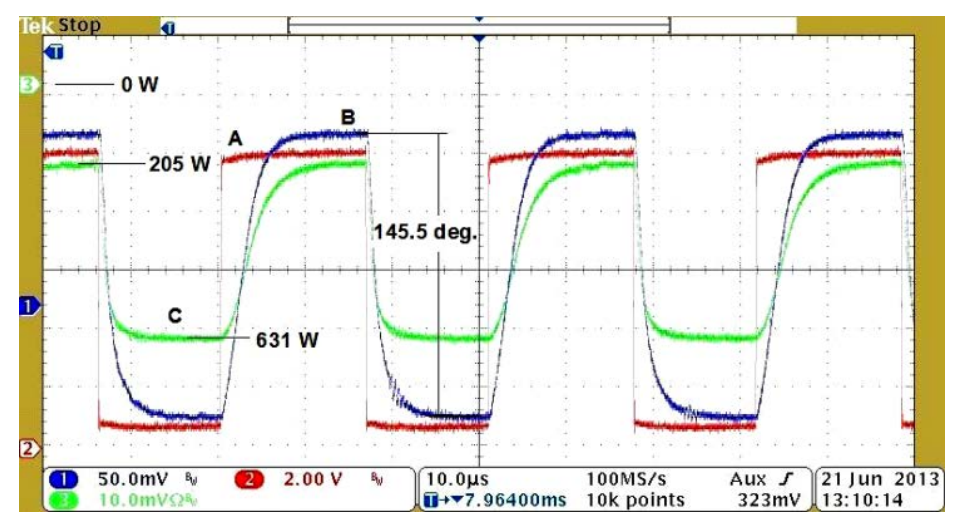

Figure 4: Trace A is shape of the voltage, controlling the phase shifter JSPHS-2484; trace $B$ is the magnetron II phase response on the shifter phase control; and trace $C$ shows the power variations measured by the calibrated detector at the port " $\Sigma$ " of the $3 \mathrm{~dB} 180$ degrees hybrid.

Note that bandwidth of control of the analogue phase shifter is $\approx 50 \mathrm{kHz}$. Plots $\mathrm{B}$ and $\mathrm{C}$ show that the bandwidth of the phase and power control of the combined magnetrons is limited by this value, but not by the bandwidth of the magnetron phase control, which is significantly wider, as noted below.

Performance of the transmitter setup for a fast phase control was evaluated measuring phase response of the combined magnetrons on phase modulation in generator at various modulating frequencies. The phase detector compared the phase-modulated signal of the generator with response of the single, 2-cascade or combined in power magnetrons. This allowed measuring the phase delay of the magnetrons, $\tau_{\mathrm{P}}=-\varphi\left(\omega_{\mathrm{PM}}\right) / \omega_{\mathrm{PM}}$, injection-locked by the phase-modulated signal vs. the angular frequency of the phase modulation, $\omega_{\mathrm{PM}}$. Obtained data were used to estimate the group delay, $\tau_{\mathrm{g}} \approx-\delta \varphi\left(\omega_{\mathrm{PM}}\right) / \delta \omega_{\mathrm{PM}}$. The group delay determined "velocity" of the phase control of the injection-locked magnetrons and, consequently, the bandwidth of the phase control. Determined in the measurements with single, 2-cascade and combined in power magnetrons $\left|\tau_{\mathrm{P}}\right| \leq 2.6 \mu$ s and $\tau_{\mathrm{g}}<40$ ns at the locking power $\mathrm{P}_{\mathrm{L}} \geq-13 \mathrm{~dB}$ (per a magnetron in 2cascade system) indicate available phase control bandwidth of $>>100 \mathrm{kHz}$.

Measured in pulsed mode phase performance of single, 2-cascade magnetron and the magnetrons with power combining operating in injection-locked mode (without the phase control), Figure 5, does not demonstrate significant perturbations of the magnetron phases; measured high-frequency noise rms magnitude is $\leq 0.7$ degrees. 


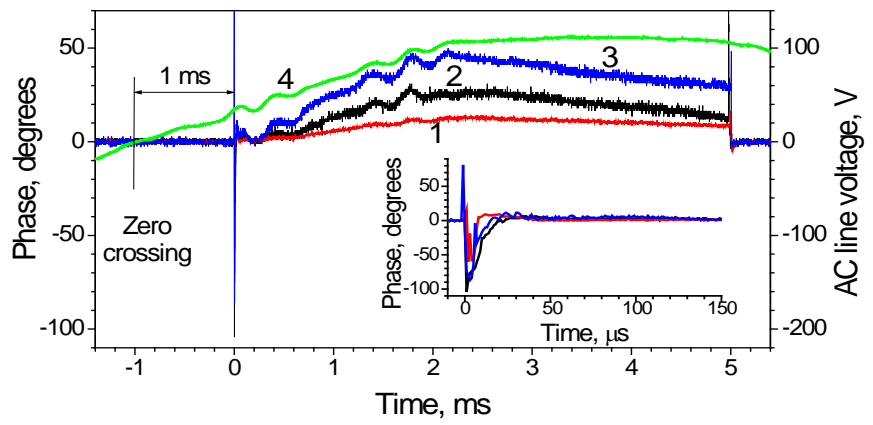

Figure 5: Phase perturbations of the injection-locked magnetrons: a single magnetron, trace 1, the magnetrons with power combining, trace 2, 2-cascade magnetron, trace 3. The measurements were performed without any phase control. Trace 4 shows measured distortions of AC line voltage.

The measured phase perturbations are caused in general by the phase pushing resulted from variation of the magnetron cathode current. The phase variations are caused by competitive processes at pulsed feeding: the cathode overheating because of back-streaming electrons, increasing the cathode current and a droop of the current associated with a discharge of the modulator storage capacitor. The last process dominates in the second half of the pulse. The alternating magnetic field induced by the AC magnetron filament circuitry also perturbs the magnetron phase stability. It is seen in synchronism of the magnetron phase performance and perturbations of the AC voltage. All the slow perturbations with the magnitude less than 1 radian can be easily and rapidly suppressed by the fast phase control of the injection-locked magnetrons due to a fast phase response.

Notable phase perturbations during $<20 \mu$ s on the leading edge of the modulator pulse may be caused by the phase pushing resulting from a multipactoring, when the pulsed HV is applied, and/or because of cleaning of the magnetron cathode by back-streaming electrons. Operation of SRF cavities will not suffer from these short perturbations.

\section{A.1.3.3 Experimental Tests}

Experimental modeling of the transmitter with various setups indicated capability of the proposed transmitter to power the ASTA SRF cavity suppressing parasitic modulation of the accelerating field caused by mechanical oscillation (microphonics and oscillations resulted from Lorentz-force), beam loading, dynamic tuning errors, and the low-frequency perturbations of the magnetron performance. By connecting the magnetron-based RF source to one of the ASTA cavities we will be able to explore behavior of the system in high-power, high-gradient operational conditions of the SRF cavity-first without, and later with high intensity ASTA beam. 


\section{A.1.3.4 References:}

[1] G. Kazakevich and V. Yakovlev," Magnetron option for a pulse linac of the Project X," Project X document 896, http://projectx-docdb.fnal.gov.

[2] G. Kazakevich et al., IPAC12 Proceedings, New Orleans, USA, page 2348 (2012).

[3] G. Kazakevich et al., TUOAT12 report at 11-th International Topical Meeting AccApp 13, Bruges, Belgium, 2013.

[4] G. Kazakevich et al., WEPH015, PAC13 Proceedings, Pasadena, USA. 


\section{A.1.4 Feasibility of an XUV FEL Oscillator at ASTA}

\section{A.1.4.1 Abstract}

The ultra-bright electron beam with a micropulse repetition rate of $3 \mathrm{MHz}$ and $\mathrm{GeV}$-scale energy of the Advanced Superconducting Test Accelerator (ASTA) facility could enable the first XUV free electron laser oscillator (FELO) experiments. The ultra-bright beam will provide sufficient gain to compensate for reduced mirror reflectances in the VUV-XUV regimes, the micropulse repetition rate will support an oscillator configuration, the SRF linac will provide energy stability, and the eventual GeV-scale energy will provide the resonance condition in the XUV. Concepts based on combining such beams with a suitable undulator and optical resonator cavity for an FELO are described. In addition preliminary single-pass studies using undulator radiation (UR) as a non-intercepting diagnostics technique for bright electron beams are presented. Initial studies at visible wavelengths would be done for $125 \mathrm{MeV}$ electron beams using an undulator with period of $4-5 \mathrm{~cm}$, magnetic structure length of $4.5 \mathrm{~m}$, and tunable field parameter, $K$. Standard time-resolved imaging techniques would be employed to evaluate beam size, position, emittance, bunch length, phase, and energy during the macropulse. Extension of the techniques to higher energy beams using the corresponding UV and VUV UR will also be described.

\section{A.1.4.2 Introduction}

One of the exciting challenges of the present-day and proposed superconducting linear accelerators with concomitant high-power beams is identification of unique new applications for those beams. The high-power electron beams for the Advanced Superconducting Test Accelerator (ASTA) facility involve up to 3000 micropulses at a $3 \mathrm{MHz}$ repetition rate with up to $3.2 \mathrm{nC}$ per micropulse in a 1-ms macropulse in the ILC-like mode [1]. With beam energies projected from 45 to $800 \mathrm{MeV}$, various beam dynamics issues will be addressed, but it is proposed such a beam in a lower-charge and low-emittance mode could also uniquely drive the first extreme ultraviolet (XUV or EUV) free-electron laser (FEL) oscillator experiments. These anticipated transform-limited photon pulses in wavelength and pulse length would address issues of limited bandwidth inherent to the self-amplified spontaneous emission (SASE) FELs that start from noise and should be competitive with seeded FELs as discussed in a recent LBNLSLAC workshop [2]. An existing undulator is combined with a previously suggested ring resonator optical configuration [3,4] or a concentric cavity optical configuration that in principle could fit the FELO in the ASTA tunnel. Preliminary estimates are reported for the FEL oscillator power saturation at $42 \mathrm{~nm}$ and $13.4 \mathrm{~nm}$ with scaling to shorter wavelengths with higher beam energies and shorter period undulators.

In preparation for the multi-pass FEL tests, single-pass experiments are also proposed that provide the non-intercepting diagnostics of the beam size, emittance, energy, bunch length, and phase. The acquisition of a comprehensive set of electron beam properties from a linear accelerator based on undulator radiation (UR) emitted from a 5-m long device was demonstrated over two decades ago on a visible wavelength free-electron laser (FEL) [5] driven by a pulse train of $100-\mu$ s extent. Comparable investigations were performed using $\mathrm{x}$ rays with a

Proposal for an Accelerator R\&D User Facility at Fermilab's Advanced Superconducting Test Accelerator 
dedicated diagnostics undulator on two storage ring projects [6,7]. The high-power electron beams of the ASTA facility with beam energies projected from 45 to $800 \mathrm{MeV}$ will need nonintercepting diagnostics. Besides the rf BPMs, optical synchrotron radiation (OSR), and optical diffraction radiation (ODR) techniques already considered, we propose the use of the properties of undulator radiation for electron-beam diagnostics from a dedicated device with a nominal period of $4-5 \mathrm{~cm}$, a tunable field parameter $K$, and a length of several meters. One proposes time resolving the e-beam properties within the macropulse by viewing the undulator radiation with standard electronic-shuttered CCDs or gated ICCD's (size and position) and a synchroscan streak camera coupled to an optical spectrometer (energy, bunch length, and phase). The feasibility of extending such techniques in the visible regime at a beam energy of 100-125 MeV into the UV and VUV regimes with beam energies of 250 and $500 \mathrm{MeV}$ will be presented.

After discussion of the single-pass mode aspects, we introduce considerations of taking full advantage of the 3-MHz micropulse repetition rate of the ASTA electron beam and 6dimensional ultra-bright beam quality to drive the first XUV FEL oscillator using an on-axis concentric optical cavity or a ring resonator optical configuration. This would be a unique opportunity to shatter the existing short-wavelength record of $176 \mathrm{~nm}$ in an FEL oscillator [8], and such a demonstration would impact the designs of the next generation of light sources.

The proposal to push the FEL oscillator concept to shorter VUV wavelengths requires a highrepetition rate to enable build-up of the radiation field within the FEL oscillator cavity. The 3$\mathrm{MHz}$, 1-ms bunch length train available only at ASTA and the anticipated single-bunch brightness are shown to be adequate to drive a short-wavelength FEL oscillator. The availability of such a high-average-brightness electron beam is unique to ASTA.

\section{A.1.4.3 Conceptual Aspects of Undulator Radiation}

The propagation of the electron beam through the alternating magnetic fields of an undulator results in electron beam trajectory oscillations and the generation of co-propagating photons as schematically shown in Figure 1. This is initially through the spontaneous emission radiation (SER) process, but under the right conditions in a long undulator a favorable instability evolves, and the electron beam is microbunched at the resonant wavelength leading to a self-amplified spontaneous emission (SASE) FEL. This intrinsic SASE-induced microbunching (SIM) is fundamental to all SASE FELs and analogous microbunching occurs in all FELs. For a planar undulator, the radiation generation process on axis is governed by the resonance condition:

$$
\lambda=\lambda_{\mathrm{u}}\left(1+K^{2} / 2\right) / 2 \mathrm{n} \gamma^{2},
$$

where $\lambda$ is the UR wavelength, $\lambda_{\mathrm{u}}$ is the undulator period, $K=e B_{0} \lambda_{\mathrm{u}} / 2 \pi m c=0.934 \lambda_{\mathrm{u}}(\mathrm{cm}) B_{0}$ $(T)$ is the undulator field strength parameter $\left(B_{0}\right.$ is the magnetic field in the gap, $e$ and $m$ are the electron charge and mass, and $c$ is the speed of light), $n$ is the harmonic number, and $\gamma$ is the relativistic Lorentz factor.

Proposal for an Accelerator R\&D User Facility at Fermilab’s Advanced Superconducting Test Accelerator 


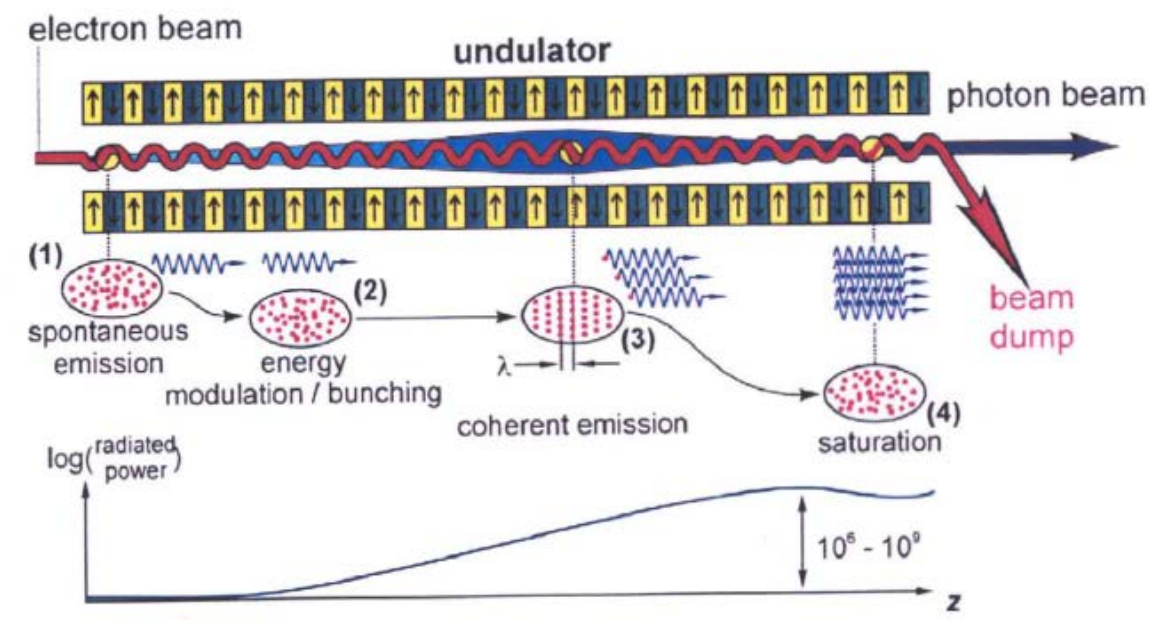

Figure 1: Schematic of the generation of undulator radiation for SER and SASE mechanisms [9].

Additionally, it has been calculated that there is coupling strength for energy exchange between the co-propagating electrons and the photon fields at the third harmonic of the planar undulators as shown in Figure 2 from reference [10]. The coupling coefficients $J_{n}$ for $n=1,3,5$ versus $K$ are reasonable, although with a rapid decrease in their values below $K=1.5$ for $n=3,5$. This indicates that the generation and measurement of these harmonics may in some cases be advantageous, particularly if the goal is to reach shorter wavelengths for a given beam energy.

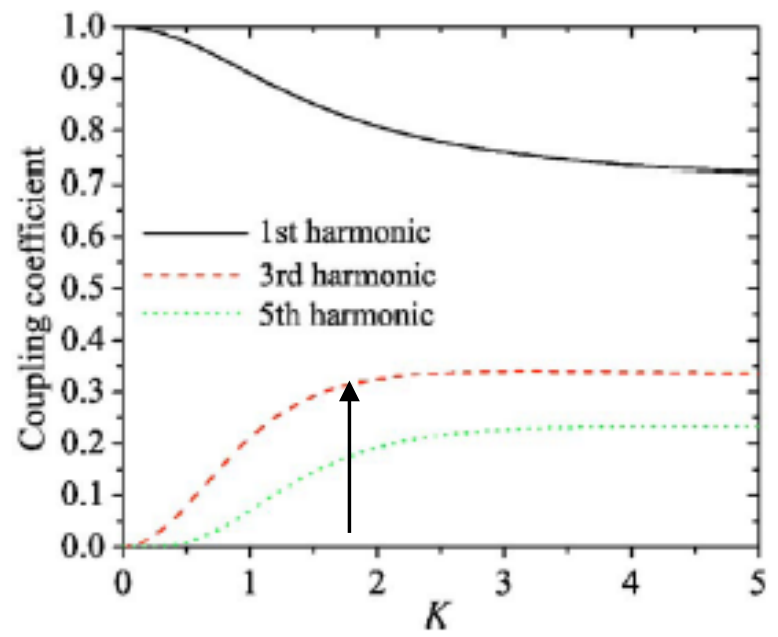

Figure 2: Coupling coefficients for odd harmonics for a planar wiggler as a function of $K$. The $\gamma=500$ case with $K=1.8$ is indicated by the arrow [10]. 


\section{A.1.4.4 Proposed Studies}

The ASTA linac (see Figure 4, Sec. 8.2.3) with photocathode (PC) rf gun, two booster L-band SRF accelerators (CC1 and CC2), and beamline will provide a $50 \mathrm{MeV}$ beam before the chicane, and an additional acceleration capability up to a total of $800 \mathrm{MeV}$ will eventually be installed in the form of three cryomodules with eight 9-cell cavities with average gradient of up to $31 \mathrm{MV} / \mathrm{m}$ after the chicane. The phase of the CC2 section can be adjusted to energy chirp the beam entering the chicane to vary bunch-length compression. Maximizing the FIR coherent transition radiation (CTR) in a detector after the chicane will be used as the signature of generating the shortest bunch lengths. Micropulse charges of 20 to 3200 pC will be used typically. The nominal pulse format for high power ILC-like beam is $3.2 \mathrm{nC}$ per micropulse at $3 \mathrm{MHz}$ for $1 \mathrm{~ms}$, and the macropulse repetition rate will be $5 \mathrm{~Hz}$. As schematically shown in Figure 3, this aspect is unique for test facilities in the USA and highly relevant to the next generation of FELs as will be discussed later in this paper. Basic applications of an undulator with ASTA beams are next described with the most significant being the feasibility of an XUV or EUV FEL oscillator being driven by such beams.

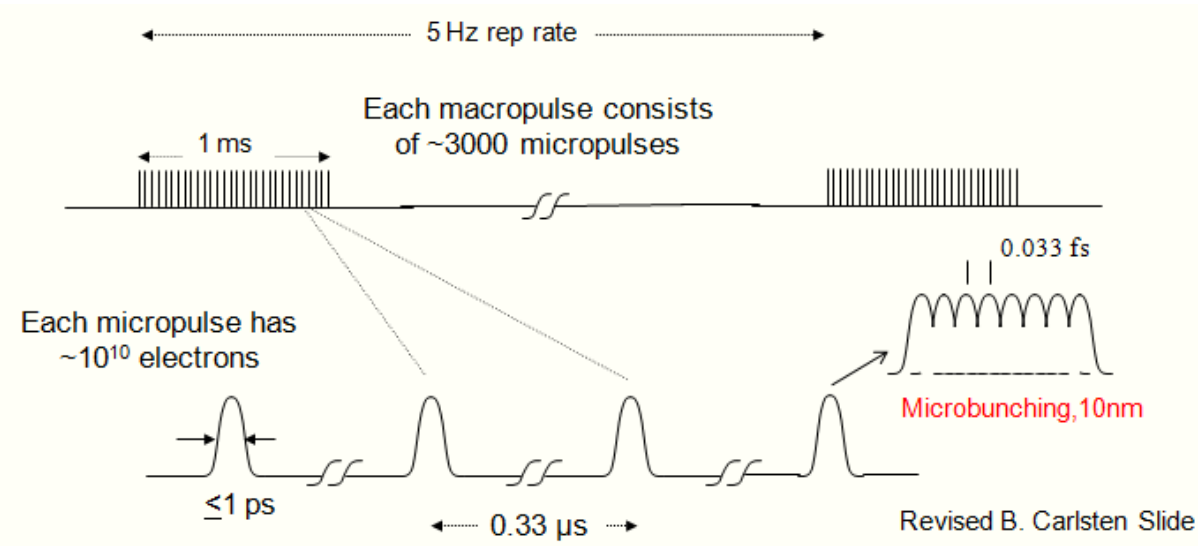

Figure 3: Schematic of the ASTA pulse structure showing the key time scales from ms to $\mu$ to $p s$ to fs.

\section{A.1.4.5 Application 1: Diagnostics Undulator}

Initially single-pass operations with an undulator would enable the use of UR properties for deducing time-resolved electron beam properties. The base image size and position will be seen in the UR spot size and transverse position (although the size will have depth-of-focus issues to address. A telescope with limited depth of focus may be used to emphasize a shorter $z$ length within the undulator's $4.5 \mathrm{~m}$ length.) The central wavelength of the emission is directly correlated with electron-beam energy, and the bandwidth from the UR will be about $1 / \mathrm{nN}$ (where $\mathrm{n}$ is the harmonic number and $\mathrm{N}$ is the number of periods) or $1 \%$ for 100 periods at the fundamental and $0.3 \%$ at the third harmonic. Signal strengths should allow tracking of a subset of the micropulses with standard imaging. The UR pulse length would be measured with the 
UV-visible C5680 streak camera, and this pulse length should be correlated with the electron beam bunch length subject to some narrowing when there is SASE gain. The synchroscan streak camera will also allow tracking of the relative phase within the macropulse of sets of micropulses to about $200 \mathrm{fs}$. At this time we anticipate one would provide optical transport for the signals to the high energy end laser lab where a small diagnostics suite of CCD camera, ICCD camera, streak camera, and optical spectrometer would be available on an optics table for characterizing the UR properties and the deduced electron-beam properties. Initial detection could be in the tunnel with local camera stations or detectors.

It is proposed to start studies during the commissioning of the first cryomodule at a beam energy of $125 \mathrm{MeV}$. As shown in Table 1 with the $5-\mathrm{cm}$ period and the magnetic gap adjusted for $K=1.2$, the fundamental UR would be at $680 \mathrm{~nm}$ with a third harmonic at $226 \mathrm{~nm}$. One could track the beam at higher energies by reducing the gap and increasing the $K$ value so that the resonance condition remains in the visible-UV regime to simplify the early tests. One can also consider 150, 200, and $250 \mathrm{MeV}$ cases as given in Table 1. At $150 \mathrm{MeV}$ the e-beam energy and bunch length via the central wavelength and pulse length of the UR, respectively, could be evaluated prior to injection of beam into the Integrable Optics Test Accelerator (IOTA) ring since the transport lines separate the paths at the high-energy end.

Table 1: Summary of possible SER/UR estimated wavelengths predicted with a 5-cm period undulator following the cryomodule(s) at ASTA at $125 \mathrm{MeV}$ and higher electronbeam energies. The last row is for a SC undulator with shorter period built for the ILC R\&D program in England [13].

\begin{tabular}{|c|c|c|c|c|}
\hline Phase \# & Beam Energy (MeV) & $\begin{array}{c}\text { Undulator } \\
\text { Fundamental } \\
(\mathbf{n m})\end{array}$ & Period (cm), $\boldsymbol{K}$ & $\begin{array}{c}\text { Undulator } \\
\text { Radiation } \\
\text { Harmonics } \\
\text { (nm) 3, 5 }\end{array}$ \\
\hline 1 & 125 & 680 & $5.0,1.2$ & 226 \\
\hline 1 & 150 & 472 & $5.0,1.2$ & 157 \\
\hline 1 & 200 & 265 & $5.0,1.2$ & 88 \\
\hline 1 & 250 & 170 & $5.0,1.2$ & 57 \\
\hline 2 & 250 & 262 & $5.0,1.8$ & 87 \\
3 & 500 & 42 & $5.0,1.2$ & $14,8.3$ \\
4 & 800 & 16 & $5.0,1.2$ & 5.3 \\
\hline
\end{tabular}

At these wavelengths and for the expected beam quality, the gain length should be sub-meter so sufficient SASE-induced microbunching should also occur in the 4.5-m length that could be accessed by demonstrated coherent optical transition radiation (COTR) techniques [11]. In the past these have allowed evaluation and adjusting of the critical electron-beam-photon-beam overlap [12]. 
In Phase 2 with two cryomodules and a beam energy of $500 \mathrm{MeV}$, one would need VUV diagnostics since the resonance condition for the fundamental would be at $\sim 42 \mathrm{~nm}$ with the third harmonic at $14 \mathrm{~nm}$ and the fifth at $8.3 \mathrm{~nm}$. An available in-vacuum flange-mounted 40$\mathrm{mm}$ diameter microchannel plate (MCP) manufactured by BURLE Electro-Optics has been identified as a candidate 2D imager that has sensitivity from $1 \mathrm{eV}$ to $50 \mathrm{keV}$. The output P43 phosphor would be viewed by a standard CCD camera, and two-dimensional images of the VUV UR angular distribution (and CVUVTR if one can block VUV UR) could be recorded. These should indicate laser/e-beam angular overlap which has been shown to impact SIM and FEL gain [12]. The bunch length and phase information could be tracked by collaborating with Dr. B. Yang on use of ANL's Hamamatsu VUV-X-ray streak camera mainframe with FNAL's M5676 synchroscan units tuned to $81.25 \mathrm{MHz}$. The intrinsic time resolution will not be as good as in the visible regime (sigma $<1 \mathrm{ps}$ ), but the flexibility for sub-macropulse studies would be maintained as well as system phase stability. Implementation of such a streak camera with a VUV spectrometer is also possible in principle.

Evaluation of the LBNL multilayer metal mirrors at various VUV-XUV wavelengths, and testing of their robustness to photon-beam pulse trains would be of interest. The multilayer mirrors would be located after the downstream dipole to eliminate e-beam bombardment. Development of VUV filtering schemes would also be tested. More detailed harmonic information should be obtained by use of imaging spectrometers in the UV and VUV (McPherson spectrometer version has a series of gratings to use and an imaging array for display). There also may be e-beam emittance effects detectable in the relative harmonic intensities and/or the spectral widths.

\section{A.1.4.6 Application 2: XUV FEL Oscillator}

In a separate proposal section (7.8.2) the possibility of laser-induced microbunching and high gain harmonic generation (HGHG) FEL R\&D at ASTA was explored [14]. In principle the 4.5-m long undulator could be the radiator for seeded FEL tests at 40-50 nm (but probably would not reach saturation). This would require an upstream modulator and chicane to be installed with the appropriate parameters. This is under evaluation, but we will defer further discussion of this aspect here in favor of the more intriguing application: the investigation of a VUV-XUV FEL oscillator configuration. The ASTA pulse train at $3 \mathrm{MHz}$ with a bright electron beam of nominal transverse emittances of $2 \pi \mathrm{mm}$ mrad, peak current of 300 to $800 \mathrm{~A}$, GeV-scale energy, energy spread of $5 \times 10^{-4}$ provides the enabling technology. Over two decades ago numerical studies using a ring resonator optical configuration were executed at Los Alamos with the FEL 3D simulation code, FELEX, in the VUV and XUV regimes [3]. This relied on two grazing incidence mirrors on-axis and two off-axis multifaceted mirrors (with 6 facets each) to obtain the individual mirror reflectivities needed in the XUV. This is schematically shown in Figure 4 . They considered operations at 100,50,12, and $4 \mathrm{~nm}$. At the time their $90 \%$ normalized emittance numbers, which I interpret as 4-sigma values, varied from 31 to $10 \pi \mathrm{mm}$ mrad (the latter being viewed as speculative at that time, but critical for the 4-nm case). They were using a photoinjected gun and normal conducting L-band rf linac in the plan with $8 \mathrm{nC}$ per micropulse of 20 ps duration and transverse emittances of about $25 \pi \mathrm{mm}$ mrad [4]. At this time, one can expect

Proposal for an Accelerator R\&D User Facility at Fermilab's Advanced Superconducting Test Accelerator 
to surpass at ASTA even their emittance speculations, albeit with lower charge per micropulse. Although they considered a 1.6-cm-period undulator with low $K$ value, we can use higher energies than their $261 \mathrm{MeV}$ to reach the resonance conditions in the 40-50 nm regime with only two cryomodules installed. One notes initial gain length evaluations could be done empirically in the single-pass mode before the final design, and a test of the resonator optical path tuning could be initiated with UV-Visible light with only one cryomodule operating.

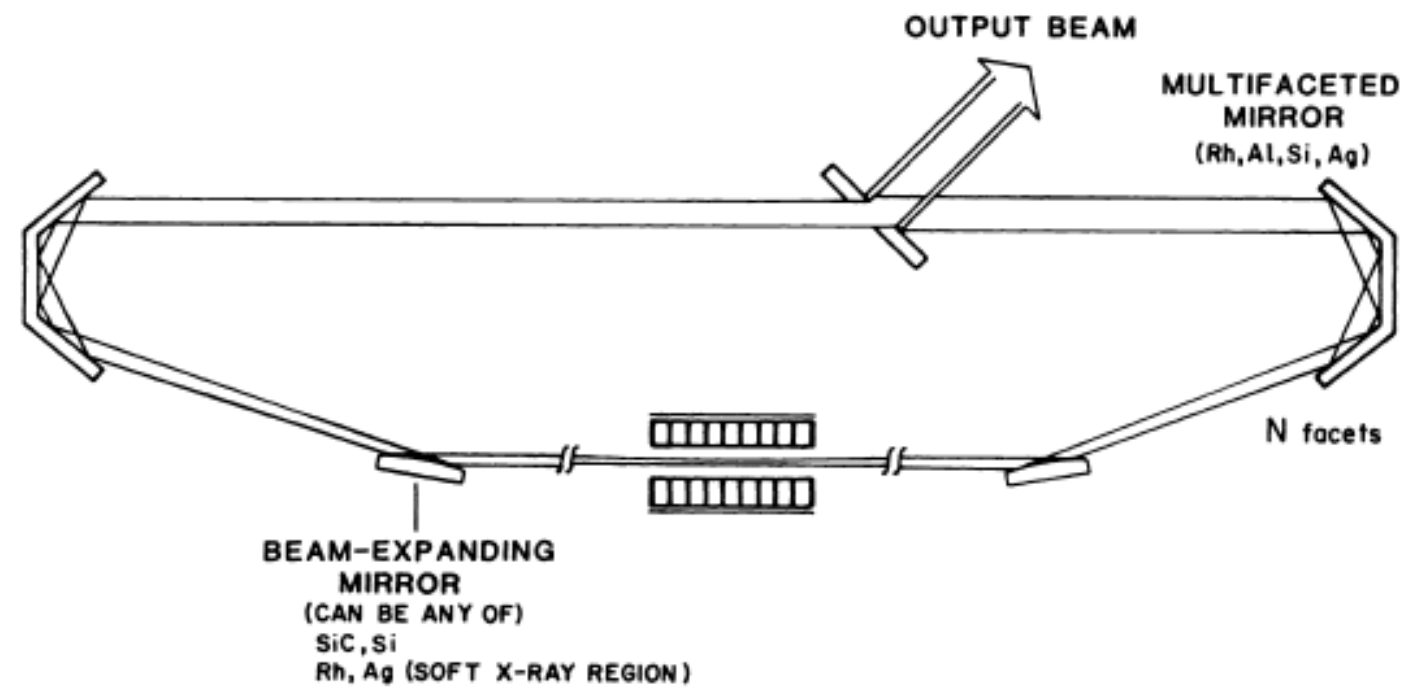

Figure 4: A schematic of the ring optical resonator for an XUV FEL oscillator using multifaceted mirrors and on-axis beam-expanding mirrors [3].

One can also consider, in principle, an on-axis concentric optical cavity based on two mirrors with z separation of $50 \mathrm{~m}$ (100 m round trip) with photons striking at 90 degrees to the surface of the multilayer metal mirrors. If each mirror has $\sim 70 \%$ reflectivity as reported for multilayer metal mirrors at LBNL in the XUV regime, the expected gain should exceed the losses. Examples at shorter wavelengths with $\mathrm{Mo} / \mathrm{Be}$ and $\mathrm{Mo} / \mathrm{Si}$ multilayers are shown in Figure 5 , and the second case is at the key lithography wavelength of $13.4 \mathrm{~nm}$. Some focusing element would still be needed to provide a Rayleigh range of about $2.2 \mathrm{~m}$ for the optical mode. Key issues of spherical aberrations and thermal distortions of the mirrors would be common for all of the designs since absorption of energy is expected in the mirrors. These issues may determine whether a simple concentric cavity would be actually practical. 


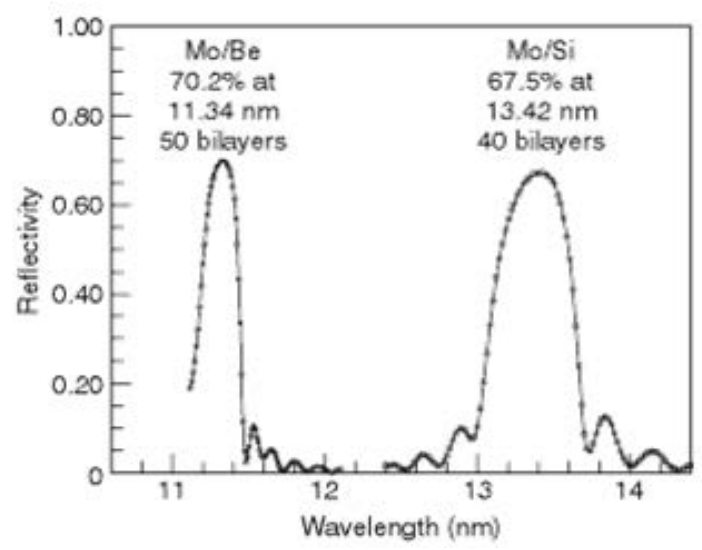

Figure 5: Examples of the reflectivities for multilayer metal mirrors with the incidence angle of 90 degrees to the surface. X-ray Data Booklet, Section 4.1, James $\mathrm{H}$. Underwood, CXRO-LBNL website.

In the ring resonator mode the resonator plane would be vertical (horizontal) so the circulating UR would pass just above (or left side of) the undulator. For a 100-m path length resonator, we would need one optical pass to time with the 100-m spacing of the electron beam micropulses at $3 \mathrm{MHz}$ (alternatively, one optical pass of $50 \mathrm{~m}$ length for a $6-\mathrm{MHz}$ micropulse rate). A single optical pass is definitely preferred so use of the full $50 \mathrm{~m}$ in $\mathrm{z}$ in the tunnel should be considered. The $z$ length in the beam line would be about $46 \mathrm{~m}$ with $2-\mathrm{m}$ distances to the respective off-axis mirrors. In the concentric cavity mode, only two on-axis mirrors are used. A chicane (not shown) could be used to transport the e beam around the left end on-axis mirror location in either case. Figure 6 shows the ring resonator with z-footprint of about $23 \mathrm{~m}$ and 50$\mathrm{m}$ optical path (invoking the 6-MHz rate) as it might fit into one high-energy AARD test area. Note this area is about $42 \mathrm{~m}$ downstream of the end of cryomodule 3 and where the chicane might be positioned for the 50-m spacing. (The initial Los Alamos design with two grazers and two multifaceted mirrors is only a little longer at $29.5 \mathrm{~m}$ in $\mathrm{z}$ for a $60-\mathrm{m}$ round trip path) Simplistically, the gain, $G$, should satisfy the relation $(1+G)$ (Total reflectivity) $>1$ so we need about three gain lengths in the undulator in this case [15]. Based on our previous calculations for SASE gain lengths of about $1 \mathrm{~m}$ at $26-40 \mathrm{~nm}$ with a different undulator with $3.3-\mathrm{cm}$ period [16], the 4.5-m undulator length should be enough to reach the needed gain and reach saturation in a few hundred passes. Output power coupling of FEL photons was by using a scraper mirror in one arm of the transport or by a mm-radius hole in one of the mirrors was previously proposed[3, 17]. 


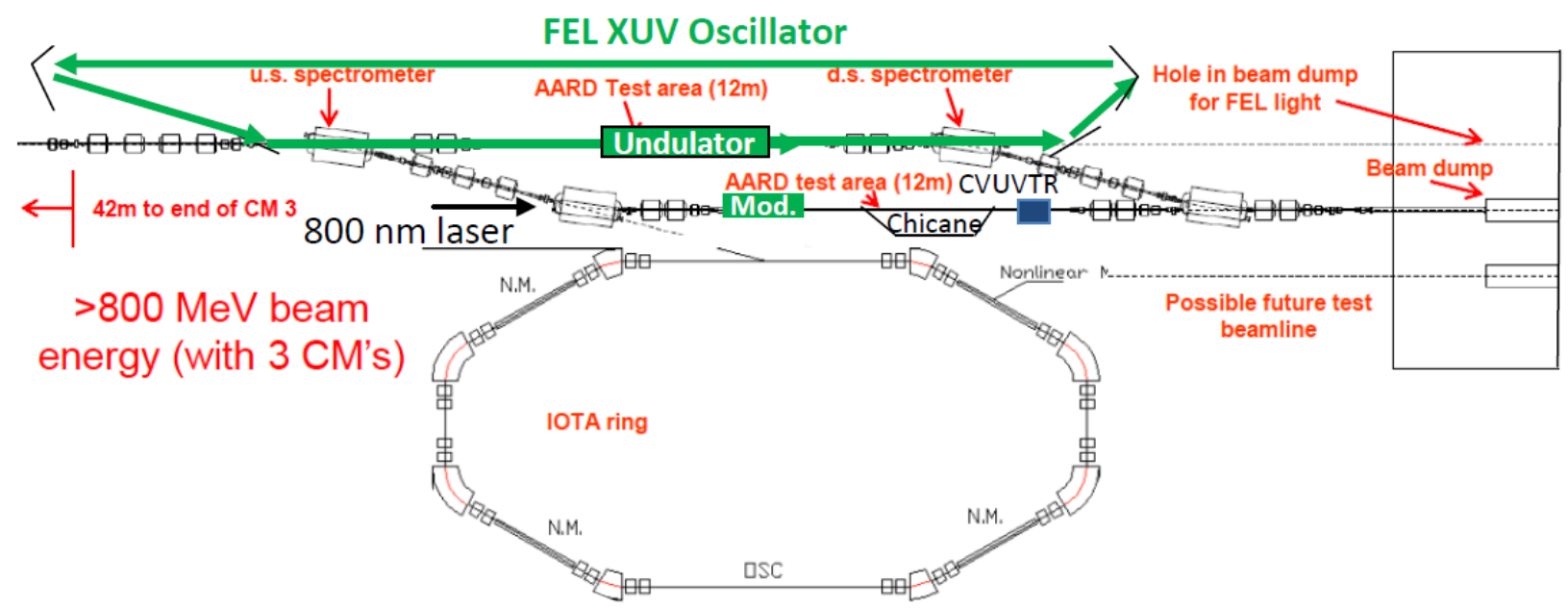

Figure 6: Schematic of the experiment layout in one high-energy AARD test area at ASTA for the undulator and XUV FEL oscillator. In the ring resonator mode the resonator plane would be vertical so the circulating UR would pass just above the undulator as if the green arrows in this figure are a side view.

The above are rough estimates so I recently requested modeling of the oscillator properties and parameter dependencies with an existing 1-D particle tracking code with support for oscillators at LBNL. The initial calculations were to be followed by GINGER-based simulations for a subset of conditions as performed at LBNL. The LANL paper had reported a 50-nm FEL case with just a 5-m undulator length [3] so this regime is where we started. The ASTA 42-nm case at $500 \mathrm{MeV}$ beam energy was just evaluated by $M$. Reinsch (LBNL), and the results are shown in Figure 7 for a 400-A peak-current value. The cavity was approximated with four reflections with a net power reflectance of $(0.7)^{4}$. This was a compromise on a single-pass ring resonator estimate. The Beta value assumed was $5 \mathrm{~m}$ for the electrons at the undulator. The encouraging results show power gain of $10^{5}$ in 62 passes and saturation after about 100 passes for the 400-A case. The power saturates even sooner for 500- and 600-A cases. The gain length was computed at 1.0 to $1.2 \mathrm{~m}$ in the exponential growth regime so the $4.5 \mathrm{~m}$ undulator length was needed. The issues of mirror quality, thermal distortions, and mirror damage need also to be addressed. Perhaps the old FELEX code can be used to assess the former issue and newer codes the latter. There is expected to be relevant expertise at LBNL for XUV mirror robustness issues, and the newer mirror cooling techniques may be needed. 


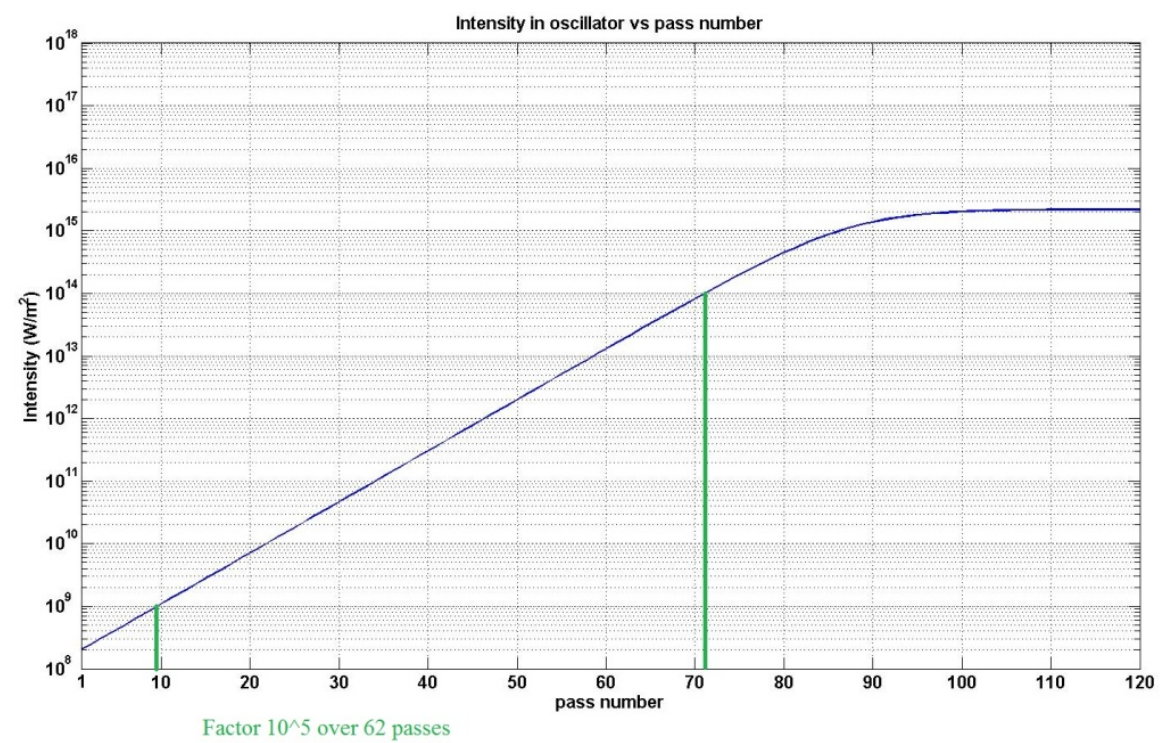

Figure 7: Preliminary FEL oscillator calculational results at $42 \mathrm{~nm}$, using $400 \mathrm{~A}$ and 500 $\mathrm{MeV}$ beam based on the 1-D particle tracking code with support for oscillators at LBNL. (courtesy of M. Reinsch)

More recently the first GINGER simulations for oscillator cases with projected ASTA beam parameters have been run at LBNL in consultation with $\mathrm{M}$. Reinsch. These calculations were done using the 4.5-m undulator length. After discussion with optics experts at LBNL, surprisingly the solutions for longer wavelengths of $40-50 \mathrm{~nm}$ with multilayer mirrors are estimated at only $40-50 \%$ reflectivity as compared to their reported $68 \%$ value at $13.4 \mathrm{~nm}$. It appears that in this full simulation shown in Figure 8, the effective gain length is less optimistic than the 1-D code, but one still can reach output power saturation at $40 \mathrm{~nm}$ and $13.4 \mathrm{~nm}$ in the concentric cavity cases invoking $50 \%$ and $68 \%$ reflectance, respectively, at 90 degrees using multilayer metal mirrors. This would occur after about 300 passes in the potential 3000-micropulse train with an 800-A peak current for $13.4 \mathrm{~nm}$ as shown in Figure 8b. These are only one example of the over 20 diagnostics plots from the simulations for each case. The multifacet mirror concept with comparable net reflectances may thus be the primary solution for broadband tunability of the FEL. In general, photon optics development for high reflectivity and at high power remains a critical R\&D topic that is ongoing at different light source and laser laboratories. 

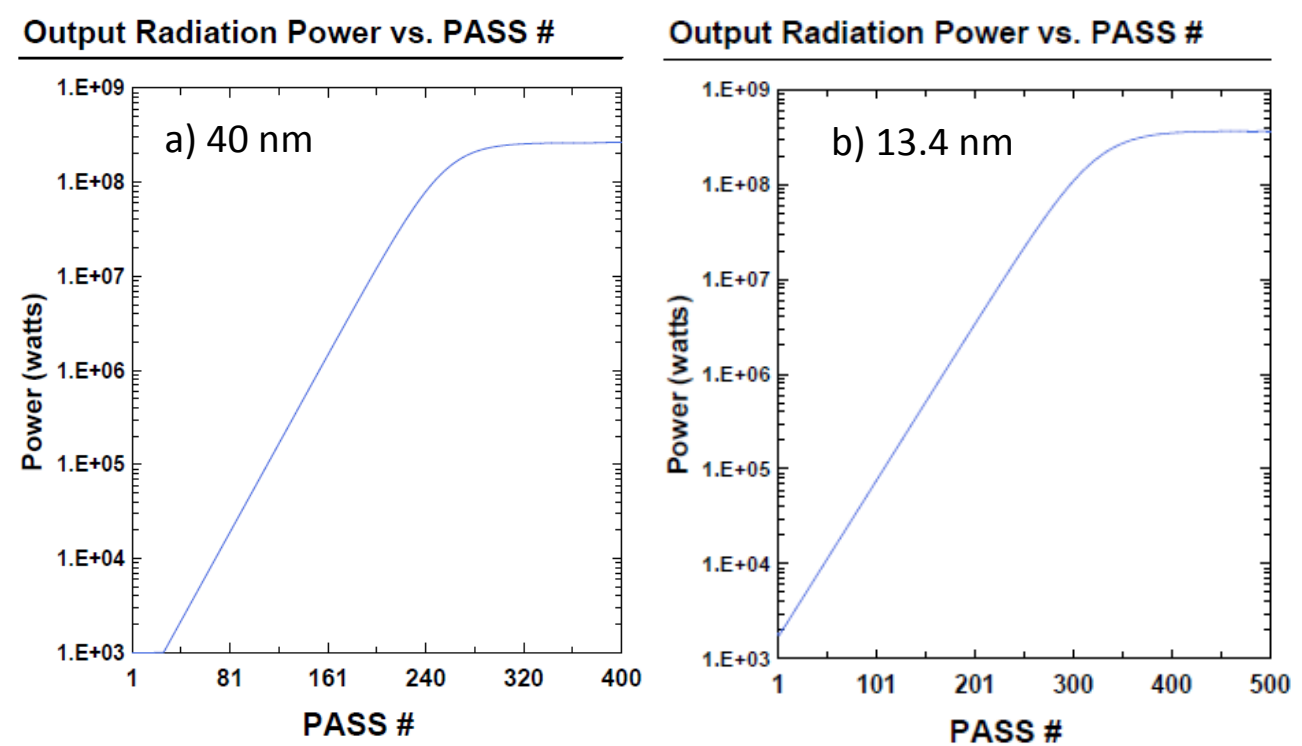

Figure 8: Initial GINGER simulations for output power saturation at the fundamental wavelength of a) $40.0 \mathrm{~nm}$ and b) 13.4-nm for a concentric cavity with multilayer mirror reflectances of $50 \%$ and $68 \%$, respectively. Peak currents of $700 \mathrm{~A}$ at $470 \mathrm{MeV}$ and 800 $A$ at $800 \mathrm{MeV}$ were used with $\mathrm{N}=90$ periods. The output power growth with pass number is shown. (Courtesy of M. Reinsch)

There is a significant opportunity for this FEL oscillator R\&D that can uniquely be done at ASTA. It is one possible configuration of the next generation of light sources with the potentially narrower bandwidths (transform limited) needed by some science cases, and should complement the seeded and self-seeded FEL configurations. Demonstration of an FEL oscillator at even 40-50 nm would shatter the existing world record of 180 nm [8]. With higher energies and shorter period undulators the few-nm regime should be attainable in principle as roughly scaled in the Phases 3 and 4 of Table 1, assuming the ring resonator concept with multifaceted mirrors is viable and/or cooled mirrors are used.

\section{A.1.4.7 Practical Considerations}

The two investigation areas provide a compelling case for consideration of installing such an undulator in ASTA. We currently have an option for the U5.0 undulator device [18] that is being retired from the ALS storage ring in January 2013. There are some practical considerations, however, mostly driven by the labeled weight of $47,000 \mathrm{lbs}$ which is close to the NML crane's 25-ton capacity. The undulator has a $5.0-\mathrm{cm}$ period, $4.5-\mathrm{m}$ magnetic structure length, a maximum field strength of $0.87 \mathrm{~T}$ at $1.4-\mathrm{cm}$ gap, and a remotely controlled adjustable gap from 1.4 to $21.4 \mathrm{~cm}$ leading to a range of $K$ values. The strong back and the specification to have the device's gap constant to about $50 \mu \mathrm{m}$ over the $4.5 \mathrm{~m}$ length leads to the very substantial support structure. Its total height is $2.4 \mathrm{~m}$ as compared to the ASTA tunnel height of $3.2 \mathrm{~m} \mathrm{(10.5}$ $\mathrm{ft}$.). The vacuum chamber is $5.1 \mathrm{~m}$ flange to flange and has an antechamber. As staged at ALS, the beamline centerline is at 55 inches, higher than our ASTA beamline center of 48 inches. 
There appears in reference [18] to be a base plate under the frame of about 15 in. height that might be shortened. These should be evaluated for modification, or we have to shift the beam trajectory upward with a dogleg in this area. Two photographs of the device in situ from M. Wendt's visit to LBNL in April 2012 are shown in Figure 9, and initial device characterizations were previously reported [19]. I don't believe there are any diagnostics or quadrupoles embedded within the structure's length. The mechanical, vacuum, and gap control aspects should be still evaluated by experts at FNAL.
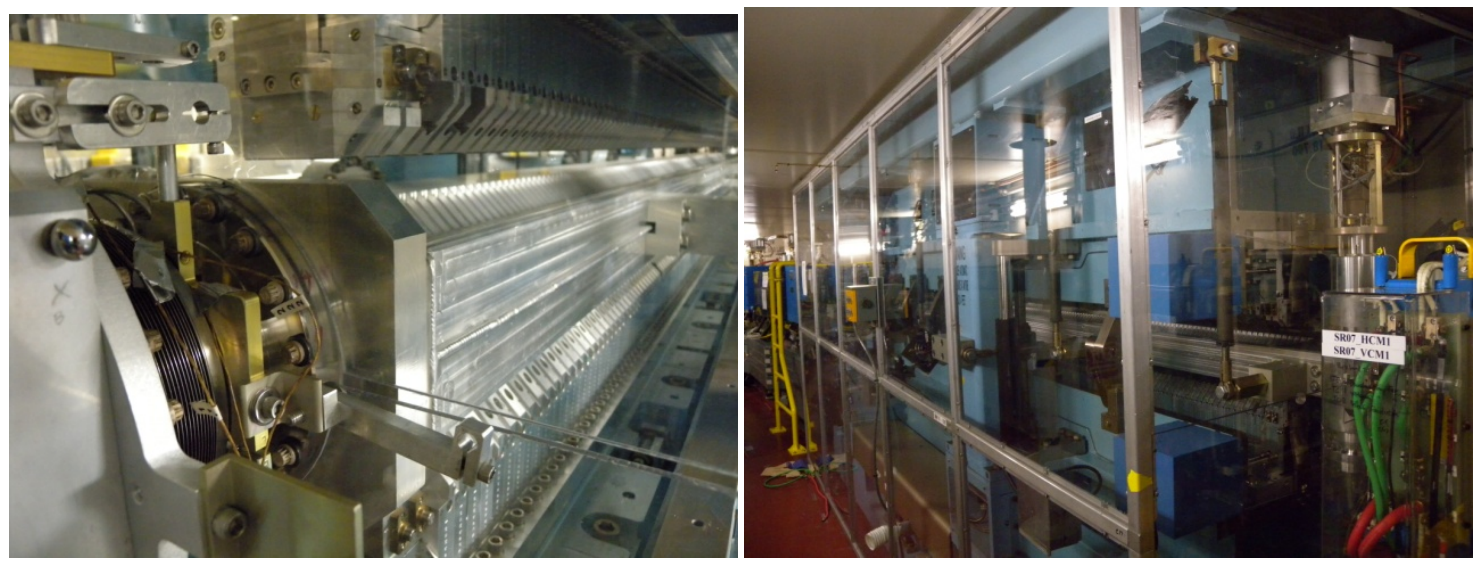

Figure 9: Photographs of the ALS U5.0 Undulator in the storage ring in April 2012.

Also, the estimated cost for a 1-m length permanent magnet device, 2-cm period, and with fixed gap from a vendor was $\$ 125 \mathrm{~K}$ with a 12-15 month lead time based on discussions with undulator-cognizant staff at ANL and LBNL in 2011. The permanent magnets themselves have been on a 12-month lead time from Japan for many months. There is a flexible magnet design with 2.4-cm period at Cornell [20], but at the moment no 4.5-m-long device to borrow. The ALS U5.0 option costs FNAL the shipping, installation, and controls time, but the device replacement value is over $\$ 1 \mathrm{M}$ (vendor's replacement estimate provided at FEL12).

\section{A.1.4.8 Summary}

In summary, we have described applications at ASTA of a $5-\mathrm{cm}$ period undulator with $4.5-\mathrm{m}$ length and tunable magnetic gap including: 1) non-intercepting e-beam diagnostics using UR during commissioning of the first cryomodule and 2) the magnetic structure for the first XUV FEL oscillator in the world. Although the ALS device may not be optimized for each application, its presence would jump start photon-related research at ASTA and support initial FEL oscillator tests at $\sim 40 \mathrm{~nm}$ and perhaps $13.4 \mathrm{~nm}$ later. In the future, use of shorter period $(1.8 \mathrm{~cm})$ permanent magnet devices or existing 1.1-cm-period SC devices and higher beam energies would allow us to push to few-nm wavelengths and soft $\mathrm{x}$-rays which should be of interest to the next-generation-of-light-source strategies (and consequently DOE/HEP, DOE/BES, and the National Science Foundation). The experiments should drive mirror technology implementations, XUV detector development, and preliminary basic science experiments that need transform-limited coherent photon sources of sub-ps duration as consistent with the 
identified mission needs of DOE. This would be a highly leveraged test program since the major infrastructure of the SRF linac is assumed as given and an undulator transfer negotiation is underway. The oscillator cavity and some detectors are the incremental costs and estimated as a fraction of the replacement value of the undulator. Finally, we note that FEL technology has been proposed recently at the Higgs Factory Workshop [21] as a possible high power photon source (when combined with Compton back scattering from $80-\mathrm{GeV}$ electrons) for future gamma-gamma colliders. Exploration of parameter spaces available with the SRF-linac-driven FEL configurations with the 1-ms pulse train could be initiated.

\section{A.1.4.9 Acknowledgements}

The authors acknowledge discussions with: M. Wendt, M. Church, H. Edwards, S. Nagaitsev, and S. Henderson of FNAL on the ASTA facility and the AARD program; R. Lindberg (ANL), M. Reinsch (LBNL) and J. C. Goldstein (LANL, retired) on FEL oscillators; and J. Ruan of FNAL on UV-Visible optical considerations.

Notes: Installation of 1-3 cryomodules assumed with transport of beam to the high energy beam dump. The details of the soon-to-be-retired ALS U5.0 undulator's transfer to FNAL are ongoing. The mirror chambers are estimated at $\$ 25 \mathrm{k}$ each for single mirrors with piezo adjustments for the initial concentric UV case. The ring resonator with multifacet mirrors needs further evaluations. A diagnostics suite would also be needed, but initially an existing invacuum MCP with standard camera, and YAG:Ce or LYSO:Ce scintillator crystals with photo detectors could be used. A VUV-XUV spectrometer would be needed to evaluate laser performance. 


\section{A.1.4.10 References}

[1] Sergei Nagaitsev, "ILC-TA at NML," ILC-TA Workshop at Fermilab, November 2006; J. Leibfritz, "Status of Plans for a Superconducting RF Test Facility at Fermilab," Proc. of IPAC12, May 2012.

[2] "Realizing the Potential of Seeded Soft X-ray FELs Workshop," hosted by LBNL and SLAC, Berkeley CA, Oct. 26-28, 2011.

[3] B.E. Newman et al., SPIE Vol. 1552, 154 (1991).

[4] John C. Goldstein, Brian. D. McVey, and Brian E. Newman, Nucl. Instr. and Methods in Phys. Research A296, 288 (1990); B.E. Carlsten, Nucl. Instr. and Methods in Phys. Research A285, 313 (1989).

[5] Alex H. Lumpkin, Nucl. Instr. and Methods in Phys. Research A296, 134-143 (1990).

[6] E. Tarazona and P. Ellaume, Rev. of Sci. Instrum. 66, 1974 (1995).

[7] Alex H. Lumpkin and Bingxin Yang, Phys. Rev. Letters, Vol. 82, No. 18, May 3, 1999, pp. 3605-3608.

[8] F. Curbis et al., Proc. of the $27^{\text {th }}$ FEL Conference, 473, JACoW econf, (2005).

[9] Based on D. Moncton slide at the WIFEL Workshop in Oct. 2007 and the original paper by R. Bonifacio, C. Pelligrini, and L. Narducci, Opt. Commun. 50,373 (1984).

[10] P. Musumeci, C. Pellegrini, and J.B. Rosenzweig, Phys. Rev. E 72, 016501 (2005).

[11] A.H. Lumpkin et al., PRL 88, 234801, (2002).

[12] A. H. Lumpkin et al., Proceedings of the $25^{\text {th }}$ FEL Conference, Tsukuba, Japan, Sept. 8-12, 2003, NIMA 528, pp. 179-183 (2004).

[13] E. Baynham et al., Proc. of PAC09, WE2RAIO1 (2009).

[14] A. H. Lumpkin, J. Ruan, J. M. Byrd, and R. Wilcox, "Potential for Critical Laser-Induced Microbunching Studies with the High-Micropulse-Repetition Rate Electron Beams at ASTA," DocDB Fermilab Dec. 2011.

[15] R. Lindberg (private communication, June 2012).

[16] A.H. Lumpkin, M. Church, H. Edwards, S. Nagaitsev, and M. Wendt, "Considerations on Fermilab's Superconducting Test Linac for an EUV/Soft X-ray SASE FEL," Proc. of FEL10, JACoW, (2010).

[17] Neil Thompson et al., "The 4GLS VUV-FEL," 29-03-07.

[18] E. Hoyer et al., Rev, Sci. Instrum. 63 (1), 359 (1992). This paper describes the ALS U5.0 undulator design.

[19] P. Heimann et al., Rev. Sci. Instrum. 66 (2), 1885 (1995).

[20] A. Temnykh, Proc. of PAC09, MO6PFP084, 324, JaCoW (2009).

[21] T. Raubenheimer, "SLC and NLC-type Higgs Factory," Higgs Factory Workshop, FNAL, Nov. 14-16, 2012. 


\section{A.1.5 LBNE Targetry Experiments at ASTA}

\section{A.1.5.1 Introduction}

LBNE (the Long Baseline Neutrino Experiment) uses solid targets to convert high-energy protons into pions and kaons to produce neutrinos [1]. These targets are subject to severe thermomechanical shock and radiation damage from the beam. These two effects limit the lifetime of the target and the precision of the experiment. The target must maintain its shape, size, and density to produce a consistent neutrino beam. Variations on the order of a percent can be unacceptable. The two effects operate on different timescales: thermo-mechanical shock occurs within a single pulse and radiation damage accumulates over millions of pulses. Still, the two are related as radiation damage changes the physical properties of the material that determine whether it will fail under thermo-mechanical shock. LBNE is actively pursuing several different materials for the composition of the target and target assembly. Graphite and beryllium are candidates for the primary target material. Numerous materials may be used in the target assembly, including beryllium, titanium, aluminum, stainless steel, and ceramic. Choosing among these materials requires experimental validation.

The ASTA beam can simulate some of the aspects of the thermo-mechanical shock and radiation damage that the LBNE target will experience. There are three foreseen modes of how ASTA can be used for these studies:

- High strain rates induced by beam heating

- Beam-induced thermo-mechanical shock of previously irradiated materials

- Direct radiation damage of materials

For the first two, the ASTA electron beam can be used to simulate the thermo-mechanical shock of the LBNE proton beam. The parameters will not be identical, but comparable stress and strain rates can be achieved. For the third the $\mathrm{H}^{-}$beam is likely best suited to induce radiation damage, though some can also be achieved with the electron beam. More detail follows in the below sections. The nominal LBNE beam parameters are listed in Table 1.

Table 1: Beam Parameters for LBNE.

\begin{tabular}{|l|l|}
\hline Species & Proton \\
\hline Energy & $120 \mathrm{GeV}$ \\
\hline Intensity & $4.9 \times 10^{13}$ protons \\
\hline Pulse Length & $10 \mathrm{microseconds}$ \\
\hline Repetition Rate & $0.8 \mathrm{~Hz}$ \\
\hline Size & $1.3 \mathrm{~mm} \mathrm{RMS} \mathrm{(horizontal} \mathrm{and} \mathrm{vertical)}$ \\
\hline Power & $700 \mathrm{~kW}$ \\
\hline Annual Beam & $\sim 6.5 \times 10^{20}$ protons \\
\hline Instantaneous Dose Rate & $5.2 \times 10^{-3} \mathrm{DPA} / \mathrm{s}$ \\
\hline Average Dose Rate & $5.2 \times 10^{-8} \mathrm{DPA} / \mathrm{s}$ \\
\hline Annual Dose & $\sim 1 \mathrm{DPA} / \mathrm{yr}$. \\
\hline
\end{tabular}

Proposal for an Accelerator R\&D User Facility at Fermilab's Advanced Superconducting Test Accelerator 


\section{A.1.5.2 High Strain Rates Induced by Beam Heating}

A central issue in the design of neutrino targets is whether they can withstand the thermomechanical shock of the beam impact. The central properties of the material are the strength (tensile/compressive), coefficient of thermal expansion, modulus of elasticity, specific heat, and energy deposition (determined by density, atomic number, and the beam properties). However, many of these parameters are valid only in the static limit. The rapidly pulsing nature of the particle beams will cause the material behavior to depart from that implied by the static material properties. Such departures are known as High Strain Rate (HSR) phenomena. HSR properties of materials are not well known, and are often measured only in idealized situations, not the heterogeneous situation of beam heating. Specifically, the tensile strength of beryllium could be as much as $40 \%$ greater for the strain rates relevant for LBNE [2].

For the LBNE, the goal will be to test candidate materials (chiefly graphite and beryllium) with comparable spatial and temporal profiles to that of the LBNE beam. The parameter space of total induced stress and strain rate will be explored to quantify the departure of material properties from static limits in regimes relevant to high-power targets. ASTA's capability to tune the pulse length and the focusing capabilities of the extraction line offer a wide area of coverage.

If the HSR effects can be established in a beam heating situation, it opens the door to building longer-lived targets. In particular, if beryllium's enhanced strength at high strain rate can be proven it becomes a more viable alternative to graphite. Beryllium is expected to experience radiation damage at a reduced rate as compared to graphite, and would thus have a longer lifetime. The static properties of typical graphite and beryllium are shown in Table 2.

Table 2: Static Properties of Graphite and Beryllium.

\begin{tabular}{|l|c|c|}
\hline & Graphite (POCO ZXF-5Q) & Beryllium (S-65C) \\
\hline Apparent density & $1.81 \mathrm{~g} / \mathrm{cc}$ & $1.82 \mathrm{~g} / \mathrm{cc}$ \\
\hline Compressive Strength & $195 \mathrm{MPa}$ & $260 \mathrm{MPa}$ \\
\hline Tensile Strength & $90 \mathrm{MPa}$ & $370 \mathrm{MPa}$ \\
\hline Modulus of Elasticity & $12.5 \mathrm{GPa}$ & $310 \mathrm{GPa}$ \\
\hline Thermal Conductivity & $70 \mathrm{~W} / \mathrm{m} / \mathrm{K}$ & $200 \mathrm{~W} / \mathrm{m} / \mathrm{K}$ \\
\hline Coefficient of Thermal Expansion & $8.1 \mathrm{um} / \mathrm{m} / \mathrm{K}$ & $10.7 \mathrm{um} / \mathrm{m} / \mathrm{K}$ \\
\hline Specific Heat & $710 \mathrm{~J} / \mathrm{kg} / \mathrm{K}$ & $1770 \mathrm{~J} / \mathrm{kg} / \mathrm{K}$ \\
\hline
\end{tabular}

\section{A.1.5.3 Beam-induced Thermo-mechanical Shock of Previously Irradiated Materials}

LBNE, as part of the RaDIATE collaboration [3], has amassed an inventory of accelerator materials that have been substantially irradiated at Fermilab and other locations. As a result of irradiation, it is expected that the material properties of the deviate from their un-irradiated values. This radiation damage is a potential failure mode for all high-power targets and must 
be investigated. At ASTA, these previously irradiated items can be tested under intense thermo-mechanical shock and the material properties investigated.

Most of these items are typical materials such as prototype targets and beam windows made of beryllium, tungsten, graphite, and other forms of carbon. They received substantial radiation dose, but often at reduced shock conditions. Some of these materials are activated, so transportation for study may prove difficult. For those materials stored at Fermilab, ASTA simplifies the issue of transport.

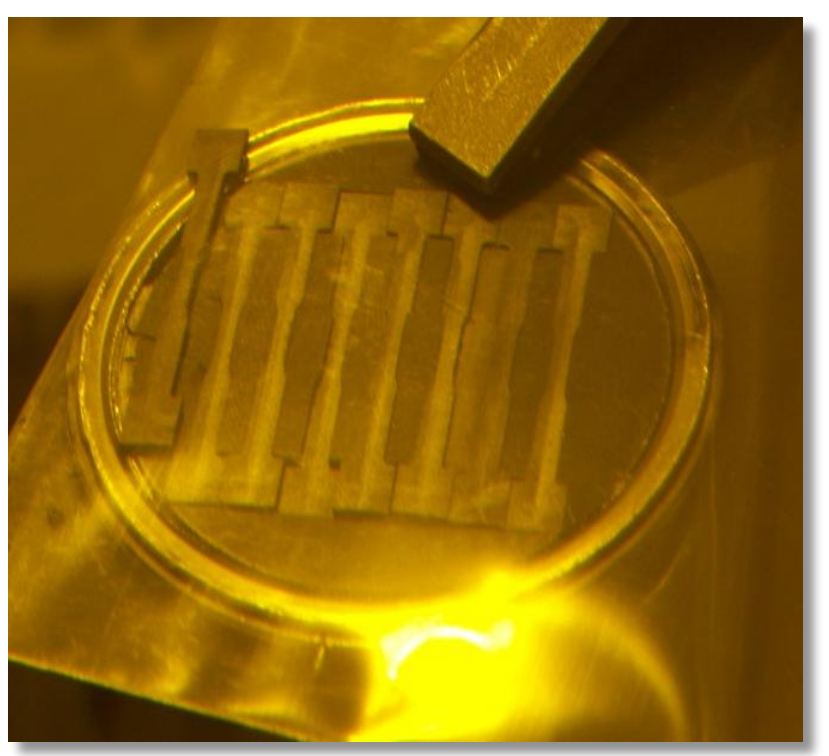

Figure 1: Graphite radiation damage samples irradiated in a previous beam test at the Brookhaven Linear Isotope Producer (BLIP).

\section{A.1.5.4 Direct Radiation Damage of Materials}

A portion of the RaDIATE collaboration allows the measurement of static material properties of very small irradiated samples. The role of ASTA here would be to produce a range of irradiated samples for this external testing. The nature of irradiation is relevant, and it is difficult to find a perfect proxy for any alternative beam. Still, some radiation effects can be simulated by the ASTA beams, specifically the $\mathrm{H}$ - (or proton) beam in addition the electron beam.

The ASTA beams will radiate the materials at various levels, with different beams and different irradiation conditions. Factors such as the temperature and environmental materials affect radiation damage. Joints between materials are a particular area of concern.

Comparing the various radiation techniques will be useful in understanding the radiation damage processes. Below are comments on three relevant mechanisms. 
- Ionization: the ASTA electron beam is well-suited to induce bulk ionization which can rearrange the crystalline structure of materials and exacerbate corrosive effects. This is the most common form of irradiation. There already exist substantial studies in this area; the other mechanisms are less well investigated.

- Atomic Displacement: the ASTA $\mathrm{H}^{-}$or proton beam would be well-suited to induce atomic displacements (measured in DPAs - Displacements Per Atom). This is one of the central mechanisms of radiation damage relevant to high-energy beams. It can have a drastic effect on the properties of any material. The samples in this case would be rather thin, $<1 \mathrm{~mm}$, because of the short range of MeV-scale protons in solid materials. However, this leads to much more concentrated production of DPAs. The electron beam is not very useful for this application. The $\mathrm{H}^{-}$beam can be used, though the energy is only just above threshold. The coulomb barrier is 1-2 MeV for the relevant nuclei, so an enhanced $\mathrm{H}^{-}$energy would be preferred.

- Gas Production: production of gasses (predominantly hydrogen and helium) is one of the unique challenges of high-energy particle irradiation. These gasses are created through spallation or other endothermic nuclear process. High-energy is needed to induce the spallation process or provide the extra energy to break up nuclei. These gasses can build up within bulk material leading to chemical changes and mechanical pressure contributing to failure. The $\mathrm{H}^{-}$beam is only capable of producing helium in beryllium, more than $8 \mathrm{MeV}$ would be required for carbon. The cross section will also be quite low at this energy. However, the $\mathrm{H}^{-}$can be a tool for direct implantation of gas into a sample, which can be combined with general ionization and atomic displacement to make a more accurate proxy for high-energy irradiation. Helium implantation would be an attractive addition.

\section{A.1.5.5 References}

[1] C. D. Moore et al., "Overview of the LBNE Neutrino Beam," Proc. IEEE PAC'11 (New York) page 1948 (2011); FERMILAB-CONF-11-086-AD (2011).

[2] Air Force Materials Laboratory "Mechanical Properties of Structural Grades of Beryllium at High Strain Rates," AFML-TR-75-168 (1975), http://www.dtic.mil/cgi-bin/GetTRDoc?AD=ADA020076.

[3] RaDIATE (Radiation Damage in Accelerator Target Environments) Collaboration, http://www-radiate.fnal.gov/index.html. 


\section{A.1.6 Potential for Critical Laser-Induced Microbunching Studies with the High-Micropulse-Repetition-Rate Electron Beams at ASTA}

\section{A.1.6.1 Abstract}

Investigations of the laser-induced microbunching as it is related to time-sliced electron-beam diagnostics and high-gain-harmonic generation (HGHG) free-electron lasers using bright electron beams are proposed for the ASTA facility. Initial tests at $50 \mathrm{MeV}$ with an amplified 800$\mathrm{nm}$ seed laser beam co-propagating with the electron beam through a short undulator (or modulator) tuned for the resonance condition followed by transport through a subsequent chicane will result in energy modulation and z-density modulation (microbunching), respectively. The latter microbunching will result in generation of coherent optical or UV transition radiation (COTR, CUVTR) at a metal converter screen which can reveal slice beam size, centroid, and energy spread. Additionally, direct assessment of the microbunching factors related to HGHG by measurement of the COTR intensity and harmonic content after the chicane as a function of seed laser power and beam parameters will be done. These experiments will be performed using the ASTA 1-MHz-rate micropulse train for up to $1 \mathrm{~ms}$ which is unique to test facilities in the USA.

\section{A.1.6.2 Introduction}

We have identified critical aspects of laser-induced microbunching (LIM) to be explored at the Advanced Superconducting Test Accelerator (ASTA) which relate directly to time-sliced electron-beam diagnostics and seeded free-electron laser (FEL) issues. In the first category the capability of evaluating the electron beam parameters such as beam size, centroid, and energy spread as well as the microbunching factors in sub-ps time slices should be possible by imaging the LIM coherent optical and UV transition radiation (COTR, CUVTR). In the second category, enhanced performance in gain length, spectral bandwidth, central wavelength stability, etc. of free-electron lasers (FELs) can be obtained by seeding the FEL either with electron beam microbunching at the resonant wavelength as in the case of high gain harmonic generation (HGHG), cascaded HGHG, and echo-enhanced harmonic generation (EEHG) [1-3] or by generating a photon beam at short wavelengths such as from a plasma as in high harmonic generation (HHG)[4]. These seeding processes are initiated by co-propagating a laser beam with the electron beam through a short undulator (modulator) tuned to the seed wavelength to generate an energy modulation which is then converted to a $z$ density modulation (microbunching) in a dispersive section such as a chicane. It has been found that the electron beam is also microbunched at the harmonics of the laser fundamental after the chicane, and the radiator undulator is tuned to one of them for the FEL [1]. Yu et al. have reported lasing on the $3^{\text {rd }}$ harmonic of the laser fundamental of $800 \mathrm{~nm}$, and the FERMI@Elettra staff has reported recent HGHG results out to the $13^{\text {th }}$ harmonic of $266 \mathrm{~nm}$ [5]. The direct measurement of the microbunching by looking at the coherent optical and UV transition radiation (COTR) was first done in a SASE FEL [6], but microbunching generated by LIM has rarely been measured [7]. Moreover, high harmonic content has not been directly observed, but it has been deduced from the HGHG radiator results [8]. Direct microbunching measurements have been proposed

Proposal for an Accelerator R\&D User Facility at Fermilab's Advanced Superconducting Test Accelerator 
on the SDUV FEL in Shanghai [9], and discussions are underway with FERMI@Elettra staff for VUV tests on their HGHG FEL. Elucidating the harmonic content, optimizing it, and benchmarking codes would be critical to present and future short wavelength (VUV-soft x-ray) FEL projects based on HGHG or EEHG. Only ASTA has the high-micropulse-repetition rate (1 $\mathrm{MHz}$ for $1 \mathrm{~ms}$ ) beam as proposed for the next generation of FELs [10], albeit with higher duty factor for the latter.

Our emphasis in this report is on the use of COTR (ultimately CVUVTR) as a direct microbunching diagnostic with the potential for time-resolved electron beam diagnostics and for benchmarking relevant FEL codes. As context, we point out that there are at least three mechanisms for generating optical-regime microbunching (which can be extended to the VUV) in an electron beam:

1. Longitudinal-space-charge-induced microbunching (LSCIM): This mechanism has been suggested by Saldin et al. [11] to contribute more in the visible wavelength regime than coherent synchrotron radiation (CSR) or wakefields. This effect can be considered as starting from shot noise in the charge distribution that couples through the longitudinal impedance of the transport line and linac into an energy modulation. This energy modulation will become a z-density modulation, or microbunching, after bunch compression in a chicane (or other $R_{56}$ lattice point) [12]. It is generally a broadband effect in wavelength, and most of the gain is in the FIR ( $>10-\mu \mathrm{m}$ regime). However, we have the LCLS/SLAC [13], APS/ANL [14], FLASH/DESY [15], and FERMI@Elettra [16] results on COTR in the OTR images and even scintillator screens. Spatially localized enhancements of 10-10,000 at visible wavelengths have been reported which prevent using the standard beam profiling techniques with optical beam images.

2. Laser-induced microbunching (LIM): (a) where an external laser beam is injected into the beamline so it co-propagates with the electron beam through a short undulator (the modulator) which interaction modulates the beam energy that then becomes a zdensity modulation after a dispersive element such as in a chicane and (b) where the seeding of an FEL with an external laser or harmonic at the long radiator undulator itself. The former results in narrowband microbunching and is used to prebunch or seed the beam for the FEL, and the latter is used as input to an FEL amplifier. The main objective of these proposed studies is measuring LIM.

3. SASE-induced microbunching (SIM) which is the fundamental mechanism of the selfamplified spontaneous emission (SASE) FEL process starts from noise when the SASE photon fields acting with the undulator fields on the electrons result in the growth of electron beam microbunching and concomitant exponential growth of SASE light at the resonant wavelength and harmonics. This is a narrowband effect. We note evidence for the LSCIM from the linac providing prebunched beam on experiments in the APS visible SASE FEL [17]. This demonstration actually links to the HGHG process via the principle of a prebunched beam's enabling FEL startup, as opposed to startup from noise as in a SASE FEL. Historically, LSCIM and SIM COTR were modeled after the first experiments, 
but in the LIM case we have more modeling in place before extensive experiments have been performed. We also expect to take advantage of techniques developed for the earlier modes.

This proposal would interact the electron beam with the laser inside an undulator to produce microbunching structure for the electron beam at harmonic frequency of the laser frequency. The microbunched beam is then expected to produce ultraviolet radiation. In the US, only ASTA has the pulse train to test the technique at $3 \mathrm{MHz}$ as needed, as well as the low energy jitter of an SC linac to enable generation and averaging of higher harmonics to greatly improve the signal-to-noise ratio and significantly increase the chance of detection of radiation-induced by the harmonic microbuching.

\section{A.1.6.3 Laser-Induced Microbunching}

The initial step in HGHG is co-propagation of a seed laser with the electron beam through a short undulator or modulator tuned to resonance as indicated schematically in Figure 1 . The laser pulse length can be used to modulate a time slice of the transverse distribution or it could be lengthened to provide modulation over the whole e-beam pulse length. At $50 \mathrm{MeV}$ it is impractical to satisfy the resonance condition for an 800-nm wavelength on the fundamental of an undulator based on permanent magnets. For a planar undulator, the FEL process is governed by the resonance condition:

$$
\lambda=\lambda_{\mathrm{u}}\left(1+K^{2} / 2\right) / 2 n \gamma^{2},
$$

where $\lambda$ is the FEL wavelength, $\lambda_{\mathrm{u}}$ is the undulator period, $K$ is the undulator field strength parameter, $\mathrm{n}$ is the harmonic number, and $\gamma$ is the relativistic Lorentz factor.

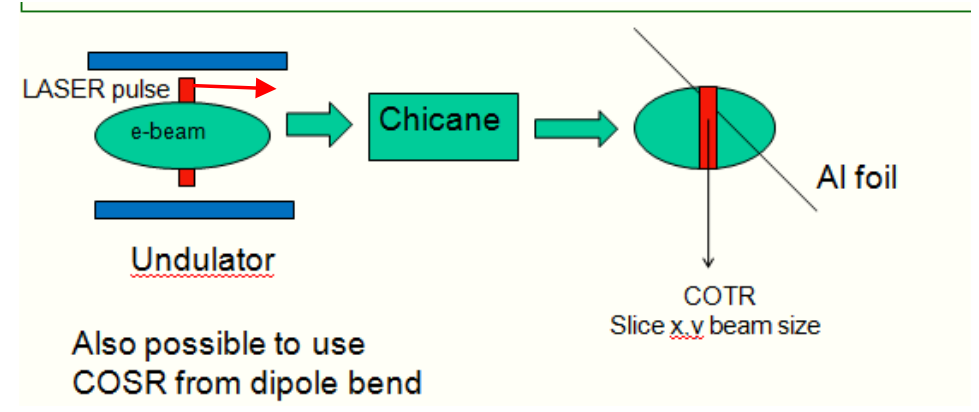

Figure 1: Schematic of laser-induced energy modulation followed by the dispersion in the chicane to produce a microbunched beam that can generate COTR or COSR in a slice. In principle the entire e-beam profile could be microbunched as well for HGHG.

However, it has been calculated that there is reasonable coupling strength at the third harmonic of the planar undulators as shown in Figure 2 from reference [18]. The coupling coefficients $\mathrm{JJ}_{\mathrm{n}}$ for $\mathrm{n}=1,3,5$ versus $K$ are reasonable, although with a rapid decrease in their values below $K=1.5$ for $n=3,5$ for the $780-n m$ case. We suggest with this concept we could 
modulate the energy at $800 \mathrm{~nm}$ in an undulator with period $1.8 \mathrm{~cm}$ using the third harmonic coupling of $\sim 0.25$ and then generate harmonics of the laser wavelength in the microbunching in the dispersive section. Initially, we would use visible-UV optics to detect $n=2,3,4$ at 400,266 , and $200 \mathrm{~nm}$, respectively, in the Phase 1a experiments. Subsequently we would look for the critical higher harmonics in the VUV with appropriate diagnostics in Phase $1 \mathrm{~b}$ as will be discussed in a later section. (Due to available 1054-nm lasers now at FNAL and LBNL, there may be some practical advantages for shifting to this wavelength. However the present S20 PC streak camera tube is insensitive at $1054 \mathrm{~nm}$, it is difficult to chirp sufficiently long IR pulses to overlap uniformly the entire few-ps electron beam longitudinally, and the harmonic wavelengths will be $20 \%$ longer than the reference case. Further evaluation of this aspect is needed.)

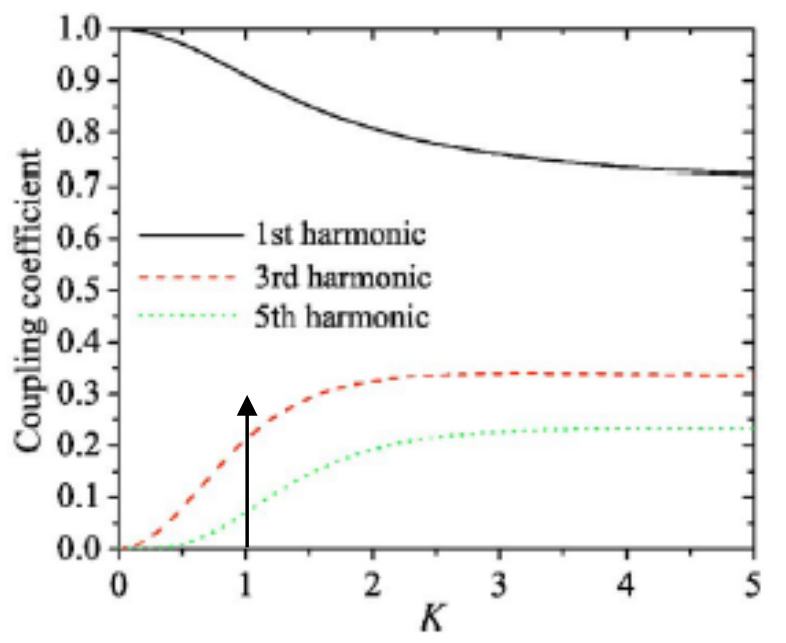

Figure 2: Coupling coefficients for odd harmonics for a planar wiggler as a function of $K$. The $\gamma=80$ case with $K=1.20$ is indicated by the arrow [18].

\section{A.1.6.4 Coherent Optical Transition Radiation}

A brief review of the source of the COTR, CUVTR, CVUVTR, etc. is in order. Transition radiation is emitted when a charged particle beam crosses the interface between two media with different dielectric constants, such as vacuum to a metal. It is a surface phenomenon, and the production time is estimated in the few-fs regime. Normally, incoherent OTR is a broadband phenomenon, but the special conditions of LIM lead to narrow band COTR. We propose that the simple modeling developed for SIM COTR [19] can be applied to LIM COTR. The coherent spectral angular distribution is given by the product of the reflection coefficients, the single electron spectral angular distribution, the interference term if applicable, and the coherence function which will be described below:

$$
\frac{d^{2} N}{d \omega d \Omega}=\left|r_{\perp, / /}\right|^{2} \frac{d^{2} N_{1}}{d \omega d \Omega} I(\mathbf{k}) \mathfrak{J}(\mathbf{k})
$$


where the single electron spectral angular distribution is given by

$$
\frac{d^{2} N_{1}}{d \omega d \Omega}=\frac{e^{2}}{\hbar c} \frac{1}{\pi^{2} \omega} \frac{\left(\theta_{x}^{2}+\theta_{y}^{2}\right)}{\left(\gamma^{-2}+\theta_{x}^{2}+\theta_{y}^{2}\right)^{2}}
$$

The coherence function is given by:

$$
\mathfrak{I}(\mathbf{k})=N+N_{B}\left(N_{B}-1\right)|H(\mathbf{k})|^{2}
$$

with the Fourier transform of the charge form factors being,

$$
H(\mathbf{k})=\frac{\rho(\mathbf{k})}{Q}=g_{x}\left(k_{x}\right) g_{y}\left(k_{y}\right) F_{z}\left(k_{z}\right)
$$

and where $Q$ is the total charge and $f_{B}=N_{B} / N$ is the bunching fraction. Note the coherence function reduces to just the number of particles, $N$, when the number of microbunched particles $N_{B}$ is zero in this formalism. We expect significant fractions from the LIM process, and hence order of magnitude enhancements of COTR.

The transverse form factors are for $i=x, y$,

$$
g_{i}\left(k_{i}\right)=\frac{1}{\sqrt{2 \pi}} e^{-\sigma_{i}^{2} k_{i}^{2} / 2}
$$

and the longitudinal form factor for a train of $M+1$ micropulses

$$
\widetilde{F}\left(k_{z}^{\prime}\right)=f\left(k_{z}^{\prime}\right) \sum_{m=0}^{M} e^{-i k_{z}^{\prime} m \ell}=f\left(k_{z}^{\prime}\right) \frac{\sin \left(M k_{z}^{\prime} \ell / 2\right)}{\sin \left(k_{z}^{\prime} \ell / 2\right)}
$$

with the longitudinal form factor for an individual microbunch

$$
f\left(k_{z}\right)=\frac{1}{\sqrt{2 \pi}} e^{-\sigma_{z}^{2} k_{z}^{2} / 2}
$$

Ratner, Chao, and Huang [18] have considered LSCIM COTR in their modeling, and related the effects to the bunching fractions in Eq. 9. The first term in the square bracket is the incoherent OTR, and the second term is the coherent contribution due to microbunching with bunching factor $b_{c}(\boldsymbol{k})$ where $\boldsymbol{k}$ is the wavevector of the microbunching. In their model the OTR spectral gain is the ratio of the angle-integrated second term over the angle integrated first term. The solid angle of their optics is given by $\vartheta_{m}$. One can obtain significant gain for bunching fractions of only a few per cent, needless to say, when $N$ is large as in a 250 pC micropulse. 


$$
\begin{aligned}
\frac{d W}{d \omega}= & \int_{-\theta_{m} / 2}^{\theta_{m} / 2} d \theta_{x} \int_{-\theta_{m} / 2}^{\theta_{m} / 2} d \theta_{y} \\
& \times\left(\frac{d^{2} W}{d \omega d \Omega}\right)_{1}\left[N+N^{2}\left|b_{c}(\boldsymbol{k})\right|^{2}\right] .
\end{aligned}
$$

They considered the longitudinal space charge impedance of their S-band linac, the chicane's $R_{56}$, the energy chirp, slice energy spread, and other factors. For the broadband instability and a 3-keV slice energy spread, the model predicts a gain factor as shown by the red curve intensity compared to the incoherent OTR (blue curve) intensity as represented in Figure 3 [14].

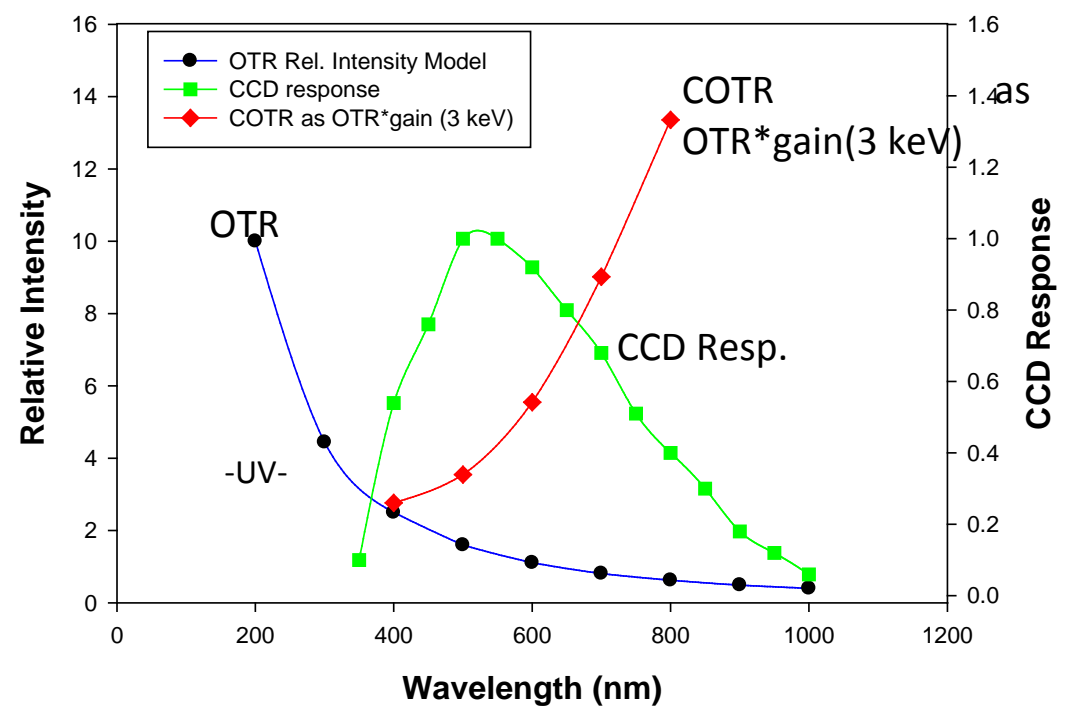

Figure 3: Plots of the nominal OTR intensity dependence on $1 / \lambda^{2}$, the COTR intensity using the calculated gain factor at LCLS for a $3-\mathrm{keV}$ slice energy spread $[14,20]$, and the nominal CCD camera relative response.

These considerations describe the gain expected with LSCIM, but the narrowband nature of LIM may be best illustrated by showing the results of the narrowband microbunching in the SIM experiments at APS/ANL in 2002. Figure 4 shows a composite of the spectra obtained for a visible FEL operating near $535 \mathrm{~nm}$ [6]. The COTR spectra on the left are narrowband, but not quite as narrow as the SASE also observed after undulator 5 of nine undulators. The bunching fraction at saturation was calculated at about $20 \%$ of the e-beam, and enhancements of $10^{4}$ for COTR and $10^{6}$ for SASE were obtained. As we understand it bunching fractions of 5-10 \% could be obtained in HGHG and EEHG configurations, even for harmonics of $n=10$ or 50 , respectively. The potential for measuring the resultant enhanced transition radiation and elucidating LIM is high. We note that the bunching fractions would also apply to enhancing coherent optical synchrotron radiation (COSR), coherent undulator radiation (CUR), and coherent optical 
diffraction radiation (CODR) which mechanisms provide the potential for non-intercepting beam diagnostics for the high power beam mode of ASTA.
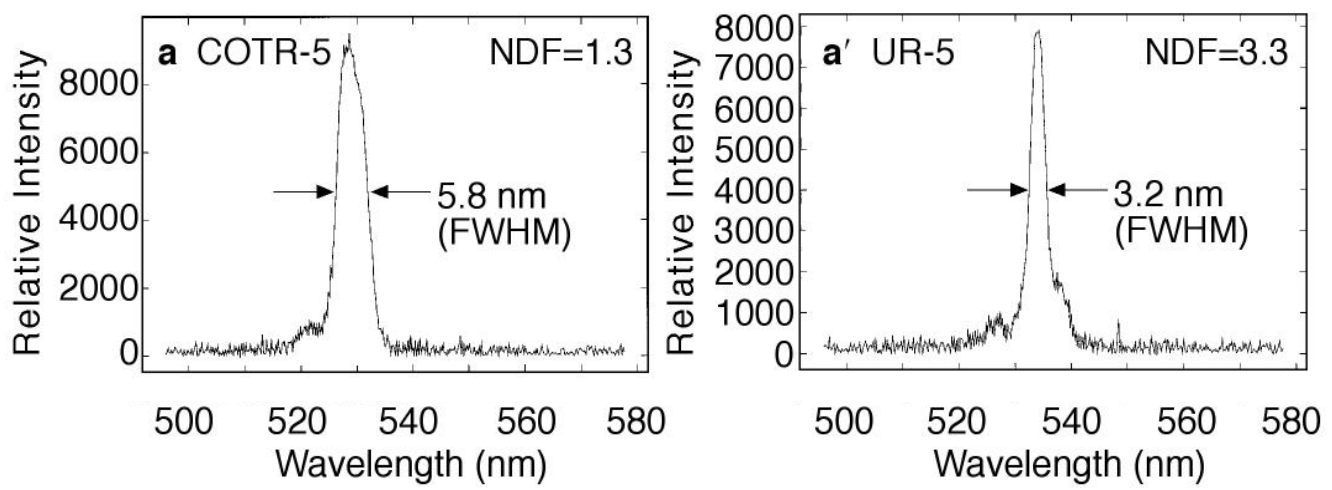

Figure 4: Example COTR (L) and SASE (R) spectra from the visible FEL experiments at ANL sampled after undulator 5 with SASE saturation occurring at about undulator 5 . The SASE is 100 times brighter than the COTR as indicated by the use of a neutral density filter (NDF) value of 3.3 versus the 1.3 NDF value indicated for the COTR. The SASE involved 365 undulator periods versus two transition radiation converter interfaces [6].

\section{A.1.6.5 Proposed Studies}

The ASTA linac with photocathode (PC) rf gun, two booster L-band SRF accelerators (CC1 and CC2), and beamline is schematically shown in Figure 5 . The L-band accelerating sections will provide a $50 \mathrm{MeV}$ beam before the chicane, and an additional acceleration capability up to a total of $800 \mathrm{MeV}$ will eventually be installed in the form of three cryomodules with eight 9-cell cavities with average gradient of $31 \mathrm{MV} / \mathrm{m}$ after the chicane. The phase of the CC2 section can be adjusted to energy chirp the beam entering the chicane to vary bunch-length compression. Maximizing the FIR coherent transition radiation (CTR) in a detector after the chicane will be used as the signature of generating the shortest bunch lengths. Micropulse charges of 20 to 3200 pC will be used typically. The nominal pulse format for high power ILC-like beam is $3.2 \mathrm{nC}$ per micropulse at $3 \mathrm{MHz}$ for $1 \mathrm{~ms}$. The macropulse repetition rate will be $5 \mathrm{~Hz}$. For the 1-ms period the pulse train micropulse spacing can be at a higher rate than a proposal of $100 \mathrm{kHz}$ in any one of ten FEL beamlines. This aspect is unique for test facilities in the USA and highly relevant to the next generation of FELs. 


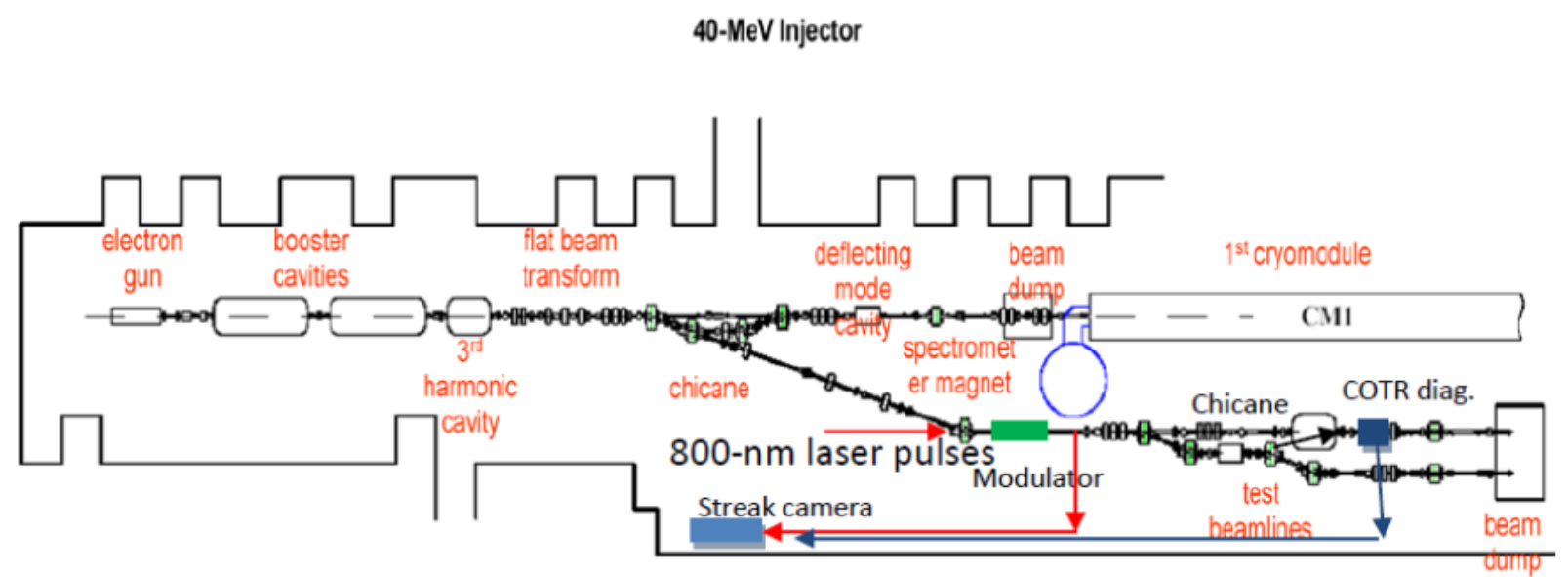

Figure 5: Schematic of the injector for the ASTA facility showing PC rf gun, booster accelerators, and beamlines. The first LIM tests are proposed in the test beamline as schematically indicated by the $800-\mathrm{nm}$ laser beam injection into the beamline, modulator, chicane, and COTR diagnostics station locations. The Visible-UV streak camera could be used for both laser synchronization and COTR measurements.

\section{A.1.6.6 Phase 1}

We would start Phase 1 at $50 \mathrm{MeV}$ with an 800-nm seed and a modulator with about 1.8 to 2.2 $\mathrm{cm}$ period, $K \sim 1.2$, and with length about $1 \mathrm{~m}$. (The loan from LBNL of two or three 0.5-m sections with $2.18 \mathrm{~cm}$ period is under discussion.) The experiment location is shown in Figure 5. We would need to use resonance at the 3rd harmonic of the planar undulator as explained earlier. This is a key, and confirmation of this approach and modeling of the expected microbunching is recommended. We would need an amplifier for the $100 \mathrm{kHz} 800-\mathrm{nm}$ laser beam from the ASTA Ti:Sa laser or doubled- frequency Erbium Fiber laser to provide about 200$500 \mu \mathrm{J}$ per pulse, although we could start with fewer pulses in the pulse train initially. An optical parametric amplifier (OPA) could do this in principle. A laser concept developed for the EOS experiments envisioned for ASTA is shown in Figure 6. The pump laser is an existing Nd:YLF operating at $1 \mathrm{MHz}$. One might obtain higher energy pulses at the lower repetition frequency of $100 \mathrm{kHz}$ and for longer pulses. The pulse train also might be shortened for initial tests. LBNL would collaborate on a solution for the OPA. The modulator should fit after the dogleg entering the low-energy test beamline at ASTA with diagnostics at both ends to align the laser and electron beam transversely. The FNAL Visible-UV streak camera would be used for timing the laser with the UR or OTR within the modulator to 1 ps or better. We have successfully done this in recent EOS tests at AOPI with the $800 \mathrm{~nm}$ Ti:Sa and incoherent OTR signals [21]. It also might be used to look directly at the LIM COTR within the micropulse as generated at the test station. (The system temporal resolution will be improved with narrow bandwidth COTR and also might be extended with a deflecting mode cavity [7].) The chicane configuration that is planned for an emittance exchange (EEX) could presumably be used with its tunable $R_{56}$ [22], but this needs to 
be checked. Visible-UV diagnostics would be needed after the Chicane to measure the harmonics $n=2,3,4$ of $800 \mathrm{~nm}$ at 400, 266, and $200 \mathrm{~nm}$, respectively.

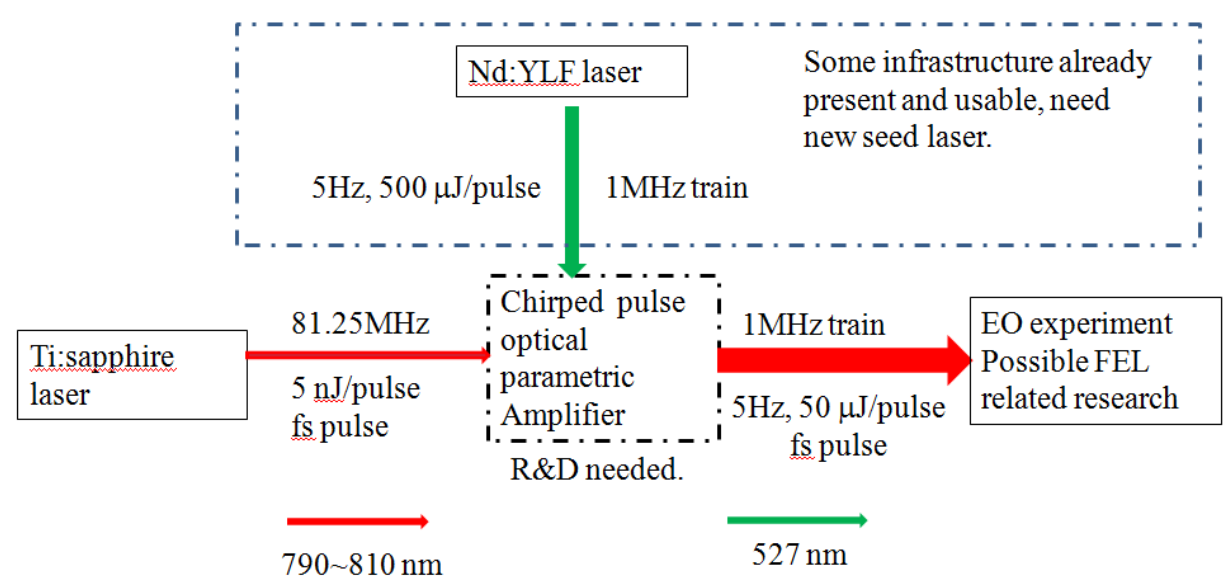

Figure 6: Schematic of the proposed ASTA diagnostics laser with OPA indicated. The pulse length and wavelengths need to be evaluated for microbunching tests. This laser system is being considered for the downstream diagnostics lab to support electro-optic tests.

In Phase 1b we would use VUV diagnostics since codes generally indicate harmonics of $\mathrm{n}^{\sim 10}$ should be generated. An available in-vacuum flange-mounted 40-mm diameter microchannel plate (MCP) manufactured by BURLE Electro-Optics has been identified as a candidate 2D imager that has sensitivity from $1 \mathrm{eV}$ to $50 \mathrm{keV}$. The output P43 phosphor would be viewed by a standard CCD camera, and two-dimensional images of the VUV angular distribution could be recorded. These should indicate laser/e-beam angular overlap which has been shown to impact SIM microbunching and FEL gain [23]. Evaluation of the LBNL multilayer metal mirrors at various VUV wavelengths, and testing of their robustness to electron-beam pulse trains would be of interest. As an alternative the multilayer mirrors might be located after the downstream dipole to eliminate e-beam bombardment. Development of VUV filtering schemes would also be tested. Additionally, one could use laser pulses shorter than the e-beam micropulse to probe the slice transverse beam size and centroids of the electron beam or map out the longitudinal profile as previously demonstrated at FLASH [24]. More detailed harmonic information should be obtained by use of imaging spectrometers in the UV and VUV (McPherson version has a series of gratings to use and an imaging array for display). This would provide the ultimate diagnostic of the microbunching harmonics' central wavelengths and spectral widths. LBNL would have or provide the OPA for the FNAL Ti:sapphire laser oscillator, the modulator system in principle (informal discussion), the VUV mirrors, and possibly the VUV spectrometer. They also have the needed modeling capability for the HGHG and EEHG FEL processes which should include calculation of microbunching factors entering the radiator. 


\section{A.1.6.7 Phase 2}

In Phase 2 we would move to the high-energy test lines, and the seed laser could be located in the downstream laser room. We may consider shifting to amplified $266 \mathrm{~nm}$ seed and look for $\mathrm{n}=10$, but we should be able to start at $800 \mathrm{~nm}$ on the fundamental with a 20 -cm-period undulator for an e-beam energy of $250 \mathrm{MeV}$. Depending on beam energy and undulator period we should be able to reach the VUV to soft x-ray regime in the microbunching. Only ASTA in the USA has the pulse train to test the technique at $100 \mathrm{kHz}$ as needed, as well as the low energy jitter of a SC linac to enable generation and averaging of higher harmonics. We estimate HGHG and EEHG might be explored in a high-energy test line within a 12-15 m zone, but cascaded HGHG could be tight. At one time $30 \mathrm{~m}$ of $\mathrm{z}$ space was indicated as usable, and various configurations should be explored. A schematic is shown in Figure 7 of the layout of the simplest. With $900 \mathrm{MeV}$ (4 cryomodules plus injector) and a short period undulator (maybe $\mathrm{SCU}$ ) we could ultimately reach a few $\mathrm{nm}$ in the beam and the radiator. This should have strong relevance for future FELs in general.

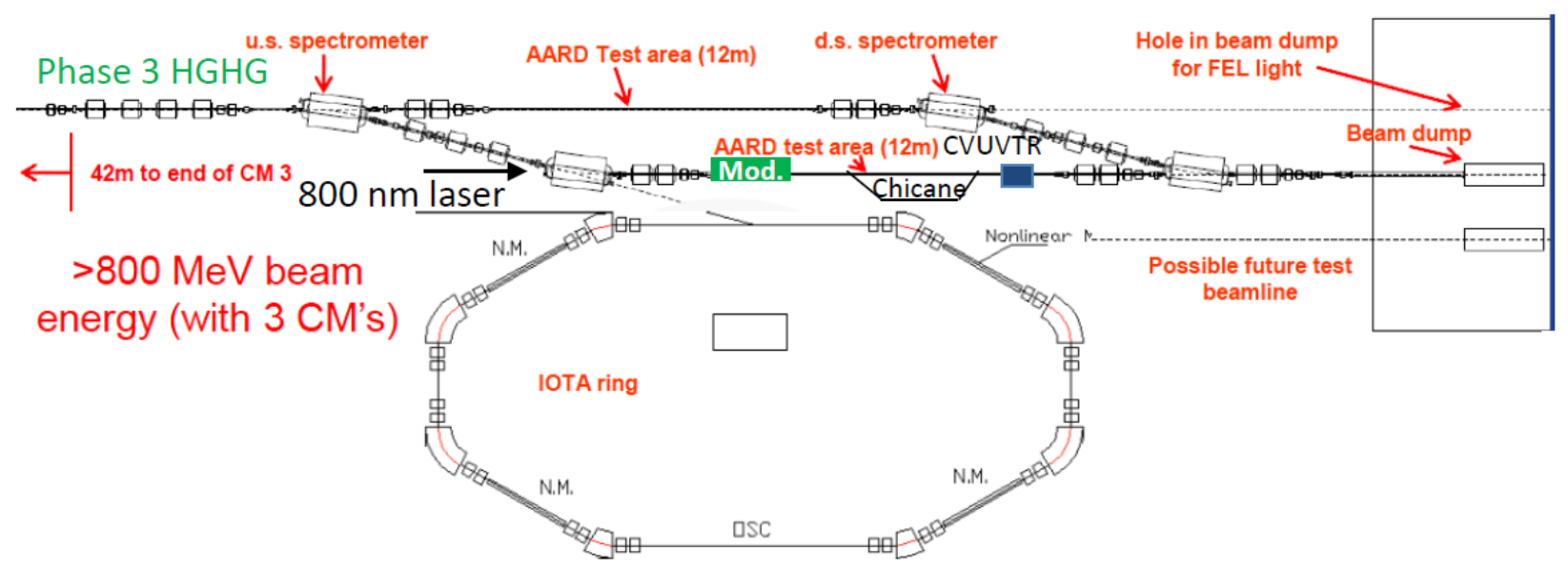

Figure 7: Schematic of the simplest microbunching experiment layout in the high-energy area. The possible configurations for HGHG and EEHG should also be considered.

Table 1 summarizes some of the configurations for the proposed studies. In some cases we are only concerned about looking at the microbunching in the electron beam post chicane so the radiator needed for HGHG is not a constraint. Phase 1 could be started in FY14 if agreements can be made soon so preparations can be made in FY13. The EEHG case is not determined yet, but we are looking for an $n=100$ demonstration where the radiator has a short period. The last SASE case at $900 \mathrm{MeV}$ invokes the use of a 4-m-long SC undulator [25] with period $1.1 \mathrm{~cm}$ and $K=0.92$ to generate radiation and microbunching at $3 \mathrm{~nm}$ as a test since we would not have 2.5 GeV available. Separating the CXTR and SASE sources is a technology challenge to be addressed for this case. 
Table 1: Summary of possible microbunching experiments at ASTA at $50 \mathrm{MeV}$ and higher energies following the cryomodules. The potential observation wavelengths of several of the harmonics are indicated.

\begin{tabular}{|c|c|c|c|c|}
\hline Phase \# & $\begin{array}{c}\text { Beam Energy } \\
\text { (MeV) }\end{array}$ & $\begin{array}{c}\text { Laser } \\
\text { Fundamental } \\
\text { (nm) }\end{array}$ & $\begin{array}{c}\text { Und. Period } \\
\text { (cm), } \boldsymbol{K}, \boldsymbol{n}\end{array}$ & $\begin{array}{c}\text { Microbunching } \\
\text { Harmonics (nm) }\end{array}$ \\
\hline 1a & 44.5 & 800 & $2.18,1.2,3$ & $400,266,200$ \\
\hline 1b & 44.5 & 800 & $2.18,1.2,3$ & $100,90,80$ \\
\hline 2 & 250 & 800 & $20.0,1.36,1$ & $400,266,200,80$ \\
\hline 2 & 200 & 266 & $5,1.2,1$ & $48,29,26$ \\
2-SASE & 500 & ---- & $5,1.2,1$ & 42,14 \\
3-HGHG & 900 & TBD & TBD & -- \\
4-SASE & 900 & -- & $1.1,0.9,1$ & 3 \\
EEHG & 900 & -- & TBD & \\
\hline
\end{tabular}

\section{A.1.6.8 Summary}

In summary, we have described how a series of direct LIM experiments with diagnostics in the optical, VUV, and soft x-ray regime could be implemented at the ASTA facility. This is the only facility in the USA that has the pulse train of at least $100 \mathrm{kHz}$ repetition rate to simulate proposed FEL configurations. These experiments should extend understanding of the critical phenomena of microbunching harmonic generation and preservation and allow benchmarking of codes. The potential use for HGHG and EEHG FELs at several $\mathrm{nm}$ with scalable results is targeted. In addition, the as-described sub-micropulse electron-beam diagnostics based on COTR could be developed into non-intercepting electron beam diagnostics if used with the COSR, CUR, or CODR mechanisms.

Notes: Assumed $50 \mathrm{MeV}$ User beamline for EEX installed with chicane with tunable $\mathrm{R}_{56}$ : FNAL streak camera and UV diagnostics; one meter long undulator with stand (loan from LBNL); FNAL in-vacuum MCP for VUV detection; seed laser transport line to modulator (\$25k); miscellaneous optics (15K).

Long Range: Propose VUV McPherson spectrometer (\$75k) with readout camera. Need cryomodules installed and beam transport to high energy end test locations. Re-locate 1-m long undulator or other.

\section{A.1.6.9 References}

[1] L.H. Yu et al., Phys. Rev. Lett. 91, 074801, (2003).

[2] Z. Zhao et al., "Seeding Experiments at the SDUV-FEL," WEOB2, Presented at FEL11, August 24, 2011.

[3] G. Stupakov, PRL 102, 074801, (2009). 
[4] G. Lambert et al., "High Harmonics from Gas," MOOBI2, Presented at FEL11, Aug.24-27, 2011.

[5] E. Allaria et al., "FEL Commissioning at FERMI@Elettra," Presented at FEL 11,WEOBI1, Shanghai, China, Aug.24-27, 2011.

[6] A.H. Lumpkin et al., PRL 88, 234801, (2002).

[7] D. Xiang et al., Phys. Rev. ST -AB, 14, 112801 (2011).

[8] Z. Zhao et al., "Seeding Experiments at the SDUV-FEL," WEOB2, Presented at FEL11, August 24, 2011.

[9] A.H. Lumpkin, seminar presented at SINAP, Shanghai, China, Aug. 17, 2011.

[10] See contributions to "Realizing the Potential of Seeded FELs in the Soft X-ray Regime Workshop," Berkeley, CA, Oct. 26-28, 2011.

[11] E.L. Saldin, E.A. Schneidmiller, and M.V. Yurkov, DESY Report No. TESLA-FEL-2003-02, 2003.

[12] Z. Huang et al., PRST-AB 7, 074401 (2004).

[13] D.H. Dowell et al., "LCLS Injector Commissioning Results," Proc. of FEL07, Aug. 26-30, 2007, Novosibirsk; R. Akre et al., Phys.Rev.ST-AB 11, 030703 (2008).

[14] A.H. Lumpkin, N.S. Sereno, W. Berg, M. Borland, Y. Li, and S. Pasky, "Characterization and Mitigation of Coherent Optical Transition Radiation Signals from a Compressed Electron Beam," Phys. Rev. ST-AB 12, 080702,(2009).

[15] M. Yan et al., "Beam profile Measurements Using a Fast Gated CCD Camera and Scintillation Screen to Suppress COTR," THPB16, presented at FEL 11, Shanghai China, Aug. 24-27, 2011.

[16] M. Veronese and E. Allaria, WEPB23, presented at FEL11, Shanghai, China, Aug.24-27, 2011.

[17] A.H. Lumpkin, R.J. Dejus, and N.S. Sereno, Phys. Rev. ST-AB, 12, 040704 (2009).

[18] P. Musumeci, C. Pellegrini, and J.B. Rosenzweig, Phys. Rev. E 72, 016501 (2005).

[19] D. W. Rule and A.H. Lumpkin, "Analysis of COTRI Patterns Produced by SASE-Induced Microbunches," Proc. of PAC2001,1288 (2001).

[20] D. Ratner, A. Chao, and Z. Huang, "Three-Dimensional Analysis of Longitudinal Space Charge Microbunching Starting from Shot Noise," Proc. of FEL08, Gyeongju, Korea, Aug. 24-29, 2008.

[21] T. Maxwell et al., "Single-shot Electro-optic Sampling of CTR at the AO Photoinjector," Proc. of IPAC11, Sept. 2011.

[22] P. Piot, FNAL AAC Review, Nov. 6, 2011.

[23] Y.-C. Chae, A.H. Lumpkin, M. Erdmann, J.W. Lewellen, and S.V. Milton, "An Experimental Study of the Beam-steering effect on the FEL Gain at LEUTL's Segmented Undulators," Proc. of FEL 2004, Trieste Italy, 100, (2004).

[24] G. Angelova et al., PRST-AB,11, 070702 (2008).

[25] E. Baynham et al., Proc. of PAC09, WE2RAI01, (2009). 


\section{A.1.7 Generation of Parametric X-rays at ASTA}

\section{A.1.7.1 Goals of the Experiment}

Our primary aim is to demonstrate that ASTA can be used as a compact source of very monochromatic, easily tunable, and bright source of "parametric" X-rays. Our specific goals are to demonstrate that parametric X-rays can be generated a) with a very narrow energy spread (significantly $<1 \%$ ), b) with high photon intensity using carbon nanotubes and c) to use for imaging objects and beam diagnostics.

\section{A.1.7.2 Description of PXR}

The passage of an energetic charged particle through a periodic crystalline structure results in the release of electromagnetic radiation by a variety of processes. These include transition radiation (TR), channeling radiation (CR), bremsstrahlung and parametric X-ray radiation (PXR). PXR is often interpreted as the Bragg reflection from the crystal planes of virtual photons accompanying the particle. It can also be understood as coherent transition radiation from different crystal planes. It was predicted by several authors more than four decades ago [1-3]. PXR from electron beams has been experimentally studied at several laboratories around the world, e.g. references [4-6] with electron beam energies ranging from a few MeV to nearly a $\mathrm{GeV}$ with X-rays observed in the energy range from 4-40 keV. More recently PXR has also been observed from $400 \mathrm{GeV}$ protons in the CERN SPS [7]. Like CR, PXR has the features of being a quasi-monochromatic, tunable, polarized source of X-rays, but unlike $C R$, it can be observed at large angles from the primary beam. The comparison of PXR with CR will be discussed in more detail in a later section.

The monochromaticity and energy tunability make PXR very well suited for imaging soft tissue since a lower dosage is required. For example, reference [8] showed that PXR generated X-rays created clearer images of a mouse kidney cell than conventional X-ray absorption images. Applied to accelerators, the use of PXR has been proposed and used $[9,10]$ to measure beam sizes and bunch lengths of very short electron beams where optical transition radiation cannot be used due to the appearance of coherent microstructure in the bunch.

\section{A.1.7.3 Characteristics of the PXR Spectrum}

The X-ray spectrum is characterized by its central energy, the angular spectrum, the photon yield and the energy spread of the spectrum. We consider each of these in turn.

\section{A.1.7.3.1 X-ray Energy}

The photon energy generated via the PXR mechanism can be written as

$$
\hbar \omega=\hbar c \frac{|\vec{\beta} \cdot \vec{g}|}{1-\sqrt{\varepsilon_{0}} \vec{\beta} \cdot \hat{k}}
$$


where $\vec{\beta}$ is the particle's relative velocity vector, $\vec{g}$ is the reciprocal lattice vector, $\hat{k}$ is the unit vector in the direction of photon emission and $\varepsilon_{0}$ is the mean dielectric constant. For X-rays, $\varepsilon_{0} \sim 1$ and assuming relativistic electrons $(\beta \sim 1)$, this expression can be simplified to

$$
\hbar \omega=\hbar c g \frac{\sin \left(\theta_{B}+\varphi\right)}{2 \sin ^{2}\left(\theta_{D}+\varphi\right) / 2}
$$

where $\theta_{B}$ is the angle between the beam trajectory and the crystal plane, $\theta_{D}$ is the angle of the $\mathrm{X}$-ray detector plane relative to the beam trajectory and $\varphi$ is the deviation of the individual electron's path from the beam trajectory. When $\theta_{D}=2 \theta_{B}$ and $\varphi=0$, this reduces to Bragg's law for reflection from a crystal plane. The photon energy does not depend on the beam energy but only on the lattice constant $g=2 \pi / d$ ( $d$ is the lattice spacing between the planes) and on the orientation of the crystal and detector. For example, using the (110) planes in diamond at a Bragg angle of $22.5^{\circ}$ and the detector at $45^{\circ}$ to the electron beam, this predicts a photon energy of $6.4 \mathrm{keV}$. At smaller observation angles, the X-ray energy would increase but with decreasing intensity. At a fixed angle of observation, the energy of the $\mathrm{X}$-ray beam can be changed by rotating the crystal relative to the beam trajectory.

\section{A.1.7.3.2 Angular Spectrum}

The angular distribution of the photon intensity in PXR can be written as

$$
\frac{d^{2} N}{d \theta_{x} d \theta_{y}}=\frac{\alpha}{16 \pi} \frac{\omega_{B}^{3}}{g^{2}}\left|\chi_{g}(\omega)\right|^{2} f_{g e o}(\hat{\beta}, \hat{g}, \hat{k})\left[\frac{\theta_{x}^{2} \cos ^{2} 2 \theta_{B}+\theta_{y}^{2}}{\left(\theta_{x}^{2}+\theta_{y}^{2}+\theta_{p h}^{2}\right)^{2}}\right]
$$

where $\theta_{x}, \theta_{y}$ are the deviations of the photon direction from $2 \theta_{B}$ (relative to the beam trajectory) in the plane and perpendicular to the plane determined by $(\hat{\beta}, \hat{g})$ respectively. $\alpha$ is the fine structure constant, $\omega_{B}$ is the Bragg frequency, $\chi_{g}(\omega)$ is the real part of the crystal's susceptibility which depends on the crystal structure factor and the atomic form factor; $f_{\text {geo }}(\hat{\beta}, \hat{g}, \hat{k})$ is a geometric form factor which depends on the angles between $(\hat{\beta}, \hat{g})$ and $(\hat{g}, \hat{k})$, on the photon absorption length $L_{a}$, and on the path length $L$ of the electron through the crystal. If the crystal thickness is much less than the absorption length, $f_{\text {geo }}(\hat{\beta}, \hat{g}, \hat{k}) \approx L$. For example, the absorption length of a $10 \mathrm{keV}$ photon beam in a diamond crystal is about $1.2 \mathrm{~mm}$ while typical crystal thickness are less than a $\mathrm{mm}$. The photon beam has an opening angle

$$
\theta_{p h}=\left[\frac{1}{\gamma^{2}}+\left(\omega_{p} / \omega\right)^{2}\right]^{1 / 2},
$$

where $\omega_{p}$ is the plasma frequency of the crystal. At a fixed Bragg frequency, the intensity increases quadratically with the lattice spacing $d$. 

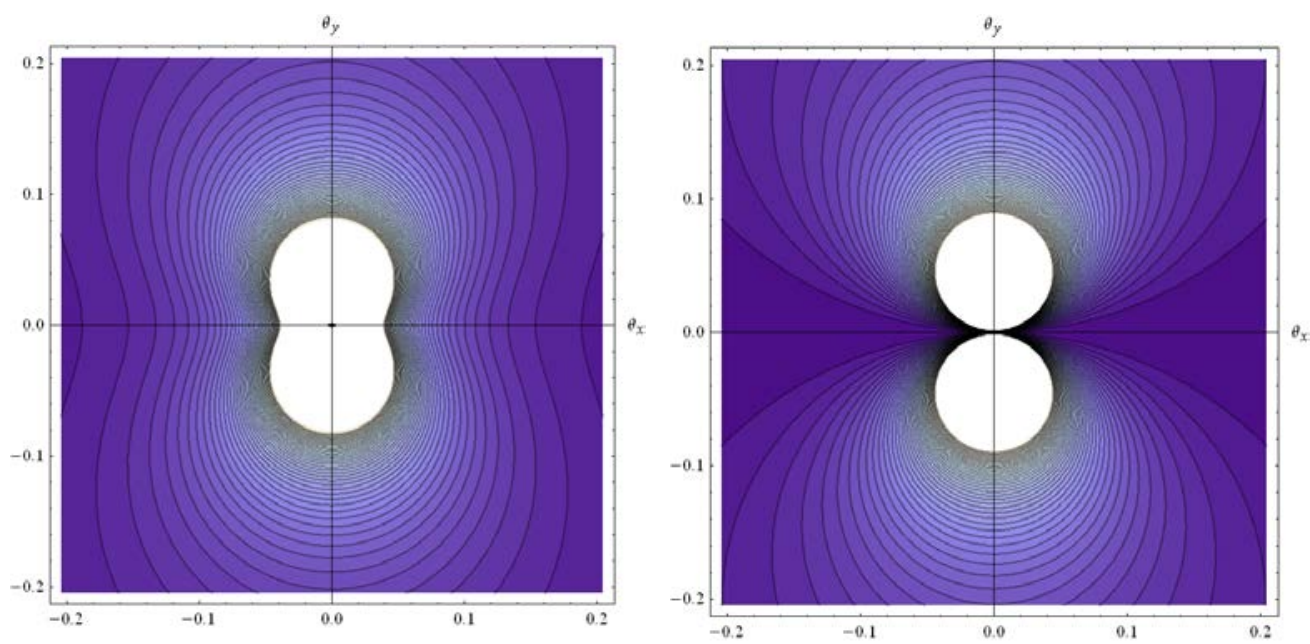

Figure 1: Contour plots of the angular spectrum as a function of $\left(\theta_{x}, \theta_{y}\right)$ for a $50 \mathrm{MeV}$ electron beam. The white areas are the most intense parts of the spectrum. Left: Bragg angle of $22.5^{\circ}$. Right: Brag angle of $45^{\circ}$. At this angle, the maximum photon intensity is at $90^{\circ}$ to the electron beam. In this case, the intensity vanishes along the line $\theta_{y}=0$.
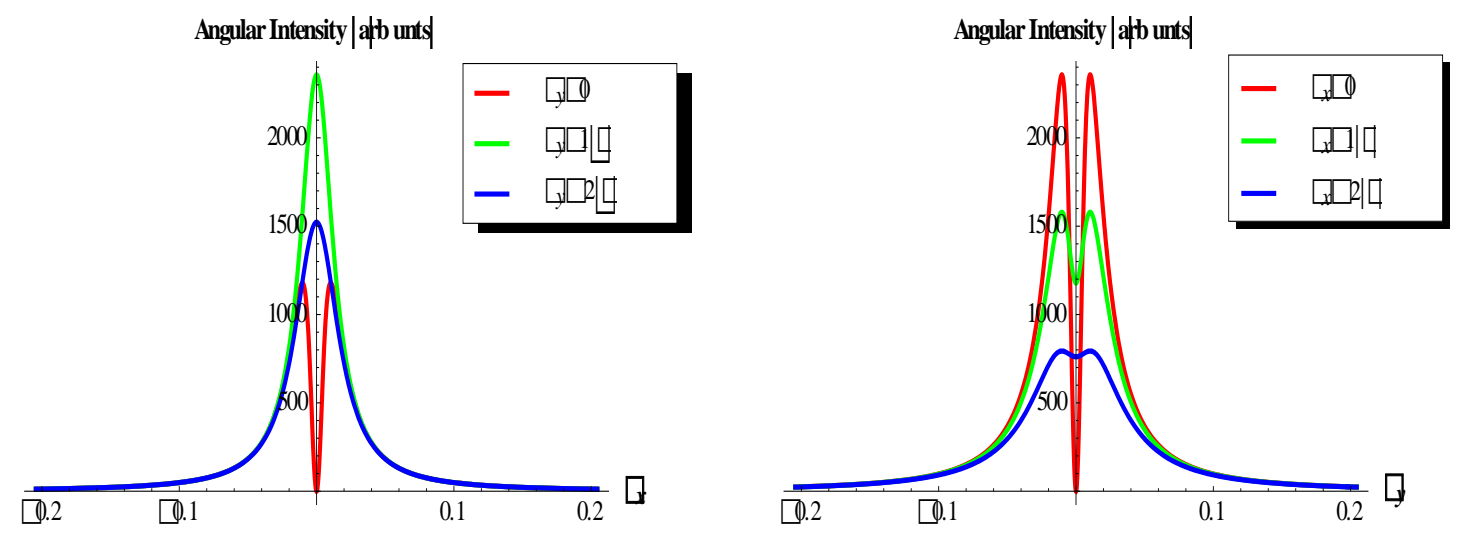

Figure 2: Variation of the angular intensity along $\theta_{x}$ (left) and $\theta_{y}$ (right) for a Bragg angle of $22.5^{\circ}$ with a $50 \mathrm{MeV}$ electron beam $(\gamma \approx 98)$. Along $\theta_{x}$ there is a double peak structure (with peaks at $\theta_{x}= \pm 1 / \gamma$ ) for $\theta_{y}<1 / \gamma$ and reaches a maximum intensity with a single peak at $\theta_{y} \approx 1 / \gamma$. However along $\theta_{y}$ the maximum intensity is obtained for $\theta_{x}=0$ and the double peak structure is maintained for all $\theta_{x}$. The double peak spectrum is similar to that seen in transition radiation.

Figure 1 shows contour plots of the angular spectrum for two choices of the Bragg angle while Figure 2 shows scans of the angular distribution along $\theta_{x}, \theta_{y}$ for the same Bragg angle of $22.5^{\circ}$. 


\section{A.1.7.3.3 Line Width}

A few effects contribute to the broadening of the PXR spectrum. There is a natural line width due to absorption of photons within the crystal, and in addition geometric effects and effects due to crystal imperfections. Geometric effects include contributions from a spread in observation angle at the detector due to a finite electron beam size and detector position resolution, from the angular spread in the electron beam and from the angular spread due to multiple Coulomb scattering of electrons within the crystal. The natural line width is given by $\Gamma_{\text {nat }}=\hbar c /\left(2 \sin ^{2} \theta_{B} L_{a}\right)$ where $L_{a}$ is the photon absorption length. At a photon energy of 10 $\mathrm{keV}$, the absorption length in diamond is $\sim 1.2 \mathrm{~mm}$ and the natural line width at a Bragg angle of $22.5^{\circ}$ is a mere $0.56 \mathrm{meV}$. This natural line width will be swamped by the geometric effects which can be minimized by using beams with a small emittance, lower $Z$ crystals to reduce multiple scattering and also using a backward Bragg geometry to detect the radiation. Indeed observations at the MAMI microtron in Mainz, Germany using electron beams of energy 855 $\mathrm{MeV}$, emittance $7 \pi \mathrm{nm}$ with Bragg geometry found PXR line widths of 20-40 meV [11]. Similar line widths may be observable at the ASTA facility but using much lower energy $50 \mathrm{MeV}$ electron beams.

\section{A.1.7.4 Comparison of PXR with Channeling Radiation}

Channeling radiation (CR) and PXR have many properties in common. Like PXR, the CR energy is also tunable but it requires either changing the crystal or changing the beam energy. The PXR energy can be changed by changing the crystal's orientation with the beam. PXR is observable at relatively large angles from the beam while $C R$ is emitted within $a \sim 1 / \gamma$ cone of the beam direction, so CR suffers from more background, particularly from incoherent and coherent bremsstrahlung. An experimental comparison of $C R$ and PXR using electrons with energy between 3 and $9 \mathrm{MeV}$ from a linac was reported in [11]. This study showed that in this energy range, the intensity of CR was about three orders of magnitude higher than PXR intensity in the Laue (forward) geometry. It also found that the line width of PXR was about an order of magnitude smaller than the $C R$ line width. Indeed there are several additional sources that contribute to the $C R$ line width. These include contributions from the finite lifetimes of the initial and final quantum states of the electrons in the transverse potential of the crystal, Bloch wave broadening which causes the transition energies to depend on the transverse momentum in the Brillouin zone and from the electron beam energy spread. The experiment reported in [12] found CR line widths of a few keV for CR lines between 3 and $21 \mathrm{keV}$. The PXR spectrum observed in the forward Laue geometry had line widths around $50 \mathrm{eV}$ with multiple scattering alone contributing about $40 \mathrm{eV}$ and the detector resolution about $18 \mathrm{eV}$. Observing the PXR spectrum in the backward Bragg geometry significantly reduces the effects of multiple scattering. As another example, the channeling experiment at ELBE [13], which forms the basis for the planned channeling experiment at ASTA, also found line widths of a few keV in a 16.4 $\mathrm{keV}$ CR line generated by a $14.6 \mathrm{MeV}$ electron beam. After monochromization by Bragg reflection from another crystal (and resulting loss of intensity), the width was reduced to 0.4 keV.

Proposal for an Accelerator R\&D User Facility at Fermilab’s Advanced Superconducting Test Accelerator 
To summarize, at a given electron beam energy the advantage of $C R$ is a significantly higher intensity while PXR offers greater ease of tunability, lower background and a more monochromatic spectrum. With PXR, a small energy spread of the electron beam is not a critical factor as it is with CR. The challenge with PXR is to find ways to increase the intensity while maintaining the advantages to make it a more useful source of X-rays.

\section{A.1.7.5 Carbon Nanotubes for PXR Generation}

Equation (3) above shows that the PXR intensity is proportional to the square of the lattice spacing $\left(1 / \mathrm{g}^{2}\right)$. Compared to typical crystals with spacing of an $\AA$, carbon nanotubes (CNTs) with $10 \AA$ or more have been manufactured. Use of these CNTs could result in significantly higher photon yields from PXR. Figure 3 shows a calculation (ref [15]) comparing yields from different materials. This calculation predicts that a $(10,10)$ armchair style CNT (diameter $=1.4 \mathrm{~nm}$ ) will have a photon yield larger than two orders of magnitude over diamond. Chemical synthesis of CNTs with diameters of several $\mathrm{nm}$ has been reported in the literature and use can be made of the fabrication facility at Northern Illinois University. Other properties such as the very high tensile strength and excellent thermal conductivity make CNTs a promising source of PXR generation.

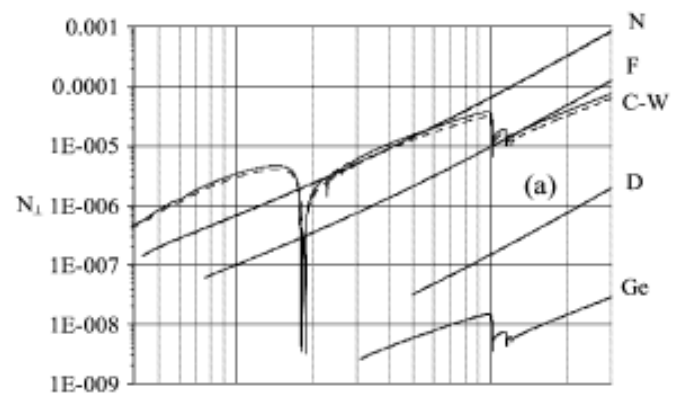

Figure 3: Comparisons of PXR photon yield per electron $(\gamma=1000)$ with different crystal materials including: $N=(10,10)$ armchair style carbon nanotube, $F=$ fullerene, $C$ $\mathrm{W}=$ Carbon-tungsten multiple layer crystal, $\mathrm{D}=$ diamond and $\mathrm{Ge}=$ Germanium [15].

\section{A.1.7.6 PXR at ASTA}

Recently a number of experiments have used electron beams from linacs to generate PXR and used the X-ray beam for imaging biological samples and electronic circuits. Possibly the first imaging experiment was done at RPI, New York with $56 \mathrm{MeV}$ electron beams and LiF crystals to image objects with a $16.6 \mathrm{keV}$ PXR beam with a FWHM of 3\% [6]. Improved image quality was reported from more recent experiments $[8,13]$ at the LEBRA facility in Japan. X-rays were lgenerated in the energy range of 5-34 keV with a $100 \mathrm{MeV}$ electron beam from a linac and photon fluxes of $10^{6}-10^{7} / \mathrm{s}$ per $\mu \mathrm{A}$ of electron beam current. These experiments reached the conclusion that PXR is a promising source for medical imaging but further improvements in beam and image quality are needed. 
Channeling experiments are already planned at ASTA using very small emittance electron beams. This offers a unique opportunity to improve the state of the art in PXR generation and imaging with relatively minor changes to the channeling experiment. Table 1 compares the beam parameters in LEBRA with the low emittance design parameters of ASTA. The smaller emittance and higher macropulse current in ASTA should result in a PXR spectrum with a narrower line width, higher photon yield and better suited for imaging than the beam used in the LEBRA facility.

Table 1: Comparison of beam parameters in the LEBRA facility linac, taken from Ref [14], with ASTA's beam parameters for the X-ray radiator experiment [16].

\begin{tabular}{|l|l|l|}
\hline Parameter & LEBRA & ASTA design \\
\hline Electron energy & $100 \mathrm{MeV}$ & $50 \mathrm{MeV}$ \\
Rf frequency & $2.856 \mathrm{GHz}$ & $1.3 \mathrm{GHz}$ \\
Macropulse duration & $\sim 10 \mu \mathrm{s}$ & $1 \mathrm{~ms}$ \\
Electron bunch length & $\leq 10 \mathrm{ps}$ & $\sim 20 \mathrm{ps}$ \\
Macropulse repetition rate & $2 \mathrm{~Hz}$ & $5 \mathrm{~Hz}$ \\
Average macropulse current & $\sim 2 \mu \mathrm{A}$ & $\sim 48 \mu \mathrm{A}$ \\
Normalized emittance & $\leq 20 \pi \mu \mathrm{m}$ & $0.01 \pi \mu \mathrm{m}$ \\
Energy spread & $\leq 1 \%$ & $\leq 1 \%$ \\
\hline
\end{tabular}

\section{A.1.7.7 Experimental Plan and Required Resources}

The experiment will use the same set up as needed for the proposed channeling experiment [16]. No additional resources will be required in the initial stage.

Stage 1: The goal will be to first observe PXR using the same $40 \mu \mathrm{m}$ crystal used for CR. Next we will observe PXR in a backward (Bragg) geometry to obtain the narrowest line width possible. Background from bremsstrahlung will also be reduced in this configuration. Spatial coherence of the X-ray beam will be measured.

Stage 2: The goal here will be to significantly increase the PXR yield by using large diameter CNTs in place of the diamond crystal. These CNTs are commercially available at very little cost.

Stage 3: The goal will be to image objects (including organic samples if available) using the generated PXR. We will also use the PXR to measure the transverse beam size and bunch length of the electron beam. 


\section{A.1.7.8 References}

[1] M.L. Ter-Mikaelian, "High energy electromagnetic processes in condensed media," Wiley, 1972.

[2] G.M. Garibyan and S. Yan, Sov. Phys. JETP, 34, 1756 (1971).

[3] V.G. Baryshevsky \& J.D. Feranchuk, Sov. Phys. JETP, 34, 178 (1971).

[4] S. Asano et al., Phys. Rev. Lett. 70, 3247 (1993).

[5] K-H. Brenzinger et al., Phys. Rev. Lett. 79, 2462 (1997).

[6] B. Sones et al., Nucl. Instr. \& Meth. A 560, 589 (2006).

[7] W. Scandale et al., Phys. Lett. B, 701, 180 (2011).

[8] Y. Takahashi et al., X-Ray Spectrom. 41, 210 (2012).

[9] Y. Takabayashi, Phys. Lett. A, 376, 2408 (2012).

[10] G. Kube et al., Proceedings of IPAC13, p 491 (2013).

[11] W. Lauth et al., Eur. Phys. J. A 28, 185 (2006).

[12] J. Freudenberger et al., Nucl. Instr. \& Meth. B 119, 123 (1996).

[13] W. Wagner et al., Nucl. Instr. \& Meth. B 266, 327 (2008).

[14] Y. Hayakawa et al., Nucl. Instr. \& Meth. B 266, 373 (2008).

[15] X. Artru et al., Phys. Rep. 412, 89 (2005).

[16] C.A. Brau et al., "High Brightness X-ray Channeling Radiation Source", Section 8.5.1. 


\section{A.2 Studies Utilizing IOTA}

\section{A.2.1 Electron Wave Function Size Measurements in IOTA Ring}

The electron wave function size and its behavior in accelerators is a longstanding problem that does not yet have a solution yet. Simple quasi-classical estimates for the centroid motion were obtained long ago [1], but a theory for the wave function size has not been developed and it turned out to be too small to measure (see [2] and references therein). The reason for this could be that the electron wave function undergoes a continuous measurement process and its localization could be related to its collapse under acts of measurement. Or there are some other random processes that lead to the localization of the wave function. For next generations of electron machines it could be important to obtain insights into this problem-it may lead researchers to new physics of radiation, particle dynamics, etc.

The first dedicated experiments were started in Novosibirsk about two decades ago and described in $[2,3]$. The experiments showed that the wave function of an electron in a storage ring is very localized, and its motion is similar to the motion of a classical particle with random kicks without any sign of phase space dilution due to rf potential well nonlinearities.

The experiments were performed in the VEPP-3 storage ring with a single circulating electron [3] and the light from an undulator that was detected by photomultipliers. The standard BrownTwiss intensity interferometer scheme used a splitter to send the photons to two photomultipliers. The basic idea to measure the longitudinal wave function size was to detect two photons by different photomultipliers during one passage of an electron through the undulator and the rms difference in time, multiplied by velocity of light, was supposed to give the wave function size. Unfortunately, the photomultipliers were slow-their response time (called the dispersion in [3]) was around 160 ps. The signal time difference from two photomultipliers was well within this number, therefore it was concluded that the wave function size is much shorter than that resolution. This experiment characterized the fourthorder correlation function of the radiation field, and quantum effects could be seen in it. Another high-order correlation experiment was the measurement of the photon arrival times during a long time interval. Fitting of amplitude and phase of synchrotron oscillation gave the electron trajectory in the longitudinal phase plane. The trajectory appeared to be continuous (with tens-picosecond precision) and chaotic, demonstrating the Brownian motion in a phase plane. In contrast to the classical Brownian motion, which may be predicted in principle by knowing the motion of molecules, surrounding the Brownian particle, the electron chaotic motion is fundamentally unpredictable (at least, according to the standard quantum theory), and gives us a rare example of the true random process.

The IOTA ring will have an undulator for the optical stochastic cooling experiments [4]. It is straightforward to repeat similar experiments with the VEPP-3 setup but with a much faster and advanced detection system-systems with a psec resolution are under development by Fermilab, ANL, and the University of Chicago [5]. It corresponds to a $0.3-\mathrm{mm}$ wave function size for electrons. If it is shorter than this we have to use or develop a different technique for the

Proposal for an Accelerator R\&D User Facility at Fermilab's Advanced Superconducting Test Accelerator 
measurement. Having well-defined initial conditions and time structure, the radiation of a single electron may be a "standard candle" source for various kinds of quantum optics experiments with the high-order field correlation function, like, for example, quantum cryptography and teleportation. The experimental and theoretical investigation of the stationary state of the electron in a storage ring is useful for the development of nonperturbative methods of quantum electrodynamics, and, in general, the foundations of the quantum theory.

The ASTA facility will offer a unique opportunity to carry out the proposed measurement of the electron wave function size. That research requires a dedicated storage ring (IOTA) and its operation with 100-150 MeV electrons. It cannot be carried out anywhere else as there are no existing electron storage rings in that energy range which can afford installation of special insertions (wigglers, etc.), and which offer special instrumentation needed to perform such measurement. In addition, the high availability of IOTA for the experiment is extremely advantageous.

In summary, the proposed experiment with wave function measurements is practically an unknown territory with possibilities of scientific breakthroughs and the IOTA ring would be well suited to do these measurements.

\section{A.2.1.1 References}

[1] M. Sands, Phys. Rev. 97 (1955) 470.

[2] N. Vinokurov, in Proceedings of Joint US-CERN-Japan-Russia accelerator school, World Scientific (1988) 108.

[3] T. Shaftan, PhD thesis, BINP, Novosibirsk (1997), in Russian.

[4] V. Lebedev, "Optical Stochastic Cooling Experiment at ASTA," Section 8.3.2.

[5] http://psec.uchicago.edu/. 


\section{A.2.2 Multi-Pickup Beam Profile Monitor for IOTA}

\section{A.2.2.1 Introduction and Goals of the Experiments}

The University of Maryland Accelerator Physics Group has recently submitted (September 9, 2013) a proposal to the Department of Energy for support of a collaborative research program with Fermi National Accelerator Laboratory's Advanced Superconducting Test Accelerator's (ASTA) Integrable Optics Test Accelerator (IOTA).

The University of Maryland Accelerator Physics Group will design, simulate, and construct a proof of principle experiment at the University of Maryland Electron Ring (UMER) to demonstrate the feasibility of achieving high beam intensities through the use of nonlinear optics. This effort is motivated by recent theoretical and simulation works and will be in collaborative concert with the developing program at Fermilab's ASTA on IOTA. Our proposed experiment will investigate non-linear optics by incorporating a Chaotic Bounded Octupole lattice into the existing UMER lattice. We will explore the resulting nonlinear betatron motion using a probe beam of negligible space charge and UMER's extensive diagnostic capabilities to characterize the features that would suppress emittance growth and halo formation. Implementing this proof of principle experiment on UMER, we will be able to quickly and cost effectively investigate the recent theoretical predictions and provide guidance to the initial electron studies at IOTA.

In tandem, we will also develop a new multi-element diagnostic for use at both UMER and Fermilab's IOTA ring. The development of a Multi-Pickup Beam Profile Monitor (MPBPM), because it is non-intercepting, permits the necessary quantification of the beam size evolution in halo/resonance experiments with intense beams at both the UMER and IOTA rings. Plans for the IOTA ring include an upgrade from 150 MeV electron beam studies to $2.5 \mathrm{MeV}, 20 \mathrm{~mA}$ proton experiments, which will enable high intensity testing of the nonlinear integrable optics lattice which establishes a need for fast, non-destructive measurement of beam size and distribution. In collaborative contribution, we will deliver the design of a MPBPM diagnostic to Fermilab's IOTA ring, which intends to achieve full nonlinear integrability.

\section{A.2.2.2 Required Beam Parameters}

The MBPM diagnostic will be used at IOTA with the $2.5 \mathrm{MeV}$ proton beam in the entire range of the operating parameters. Since the device is non-destructive, the commissioning will not require dedicated beam study time and can be done concurrently with normal machine operations. 


\section{A.2.2.3 Personnel Involved}

We expect the following Fermilab personnel to be involved in the experiment:

Table 1: Personnel involved from Fermilab.

\begin{tabular}{|l|l|l|l|l|l|}
\hline & Planning & Experiment & Diagnostics & Analysis & Simulation \\
\hline S. Nagaitsev & & & & & \\
\hline A. Valishev & & & & & \\
\hline
\end{tabular}

Kiersten Ruisard is a second-year graduate student at the University of Maryland who will incorporate the simulations of and data taken with the octupole lattice during this experiment in her doctoral dissertation. A second graduate student at the University of Maryland will participate in the designing, commissioning, and data taking gathering with the MPBPM diagnostic for his or her doctoral dissertation. Dr. Irving Haber is a senior research scientist at the University of Maryland and will contribute his simulation expertise to both the beam dynamics and beam diagnostics. Prof. Rami Kishek will similarly provide his simulation expertise. Dr. Brian Beaudoin will participate in the development of the data acquisition electronics as well as the design, commissioning, and data taking gathering with the MPBPM diagnostic.

\section{A.2.2.4 Experimental Technique and Expected Outcomes}

A quadrupole pickup is a noninvasive device that measures the quadrupole moment of the transverse beam distribution, which is very sensitive to variations in the beam size. The beam size variations may be a sign of a mismatch at injection or a signature of the space-charge induced quadrupole oscillations. The quadrupole moment can be measured by sampling the electromagnetic fields produced by a charged particle beam moving through the beam pipe.

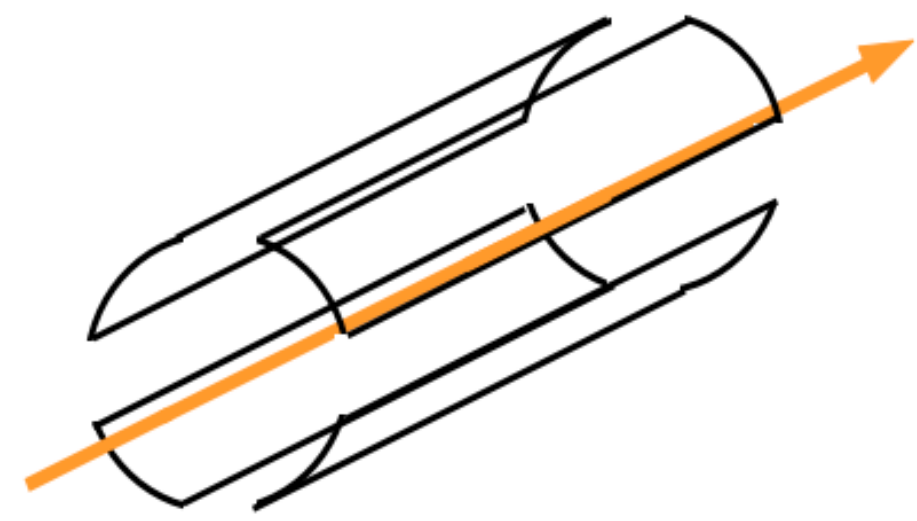

Figure 1: Schematic of a quadrupole. Arrow shows the direction of beam propagation. Image courtesy of A. Jansson. 
A number of designs have been developed over the years [3-5], for example Figure 1 shows the schematic of a magnetic quadrupole pickup that consists of four induction loops oriented to be sensitive to the magnetic flux in the radial direction.

The UMD group will develop a Multi-Pickup Beam Profile Monitor hardware including the pickup chassis and the signal acquisition electronics. The pickup body will be compatible with the IOTA operating vacuum of 10-8 Torr, and baking temperature of 150 degrees $C$.

The hardware will be tested at UMER and transferred to IOTA. The necessary commissioning work at IOTA will include software integration and beam-based calibration. Since the instrumentation device is non-destructive, all beam tests can be conducted concurrently with the machine operation.

The addition of a quadrupole pickup diagnostics will add an essential capability to the IOTA instrumentation.

\section{A.2.2.5 References}

[1] K. Poorrezaei et al., "New technique to measure emittance for beams with space charge," Phys. Rev. ST-AB, 16, 082801 (2013).

[2] A.V. Burov et al., "Space-Charge Compensation in Proton Boosters," Proc. IEEE PAC'2001, Chicago, IL (2001), page 2896.

[3] R. H. Miller et al., "Nonintercepting Emittance Monitor," in Proceedings of the 12th International Conference on High Energy Accelerators, Batavia (1983).

[4] A. Jansson et al., "Measurements with the Magnetic Quadrupole Pick-Up in the CERN PS," Proc. EPAC'2000, Vienna, Austria (2000), page 1744.

[5] A. Jansson, "Noninvasive single-bunch matching and emittance monitor," Phys. Rev. ST$A B, 5,072803$ (2002). 


\section{A.2.3 Conservative Relaxation and Echo Studies with IOTA}

\section{A.2.3.1 Background}

Is it possible to have some kind of decay without dissipation in the evolution of certain physical systems? The short answer is yes, and the title alludes to this fact: intense charged particle beams can relax conservatively. However, the details of conservative relaxation mechanisms under realistic initial and boundary conditions, over considerable lengths of time, remain elusive. IOTA will be an ideal test bed to systematically study these phenomena. The insight gained can be utilized to optimize the design and operation of accelerator systems, thereby pushing the intensity frontier of beam physics towards new realms.

In order to understand the underlying ideas and where the challenges and opportunities lie, let us start by recalling that charged particle beams are collections of point-like objects interacting through pair-wise electrostatic Coulomb forces. At the intensity frontier, we can safely assume that the number of particles is very large. Therefore, the dynamics of the corresponding beams can be thought of as transitioning from single particle to multi-particle to infinitely many particles, as the number increases. Several interesting things happen in this progression. From our point of view, probably the most important one is that collisionality goes away in the infinite particle limit, and the beam behaves like a fluid on time scales of interest to us. It is worthwhile to mention that no actual particle beam is intrinsically collisionless or collisional; it all depends on the time frame of observation. Hence, more precisely, we are interested in the long time bulk behavior of particle beams of finite, but very large number of particles, but over time intervals over which collisional effects do not influence significantly the dynamics. Over this period of time, of interest are the beam stability under perturbations, and properties of the resulting dynamical equilibria as a function of initial and boundary conditions.

Since the beams do not reach thermodynamic equilibrium over this time interval, the question arises if some other-conservative-relaxation mechanisms can play a (de)stabilizing role. Heuristically, in the limit of infinitely many particles, the evolution of the empirical (Klimontovich) distribution tends towards the Vlasov equation (although not established rigorously for the Coulomb potential, it is most likely just a technical difficulty), the so-called mean field limit. As a consequence, the natural framework to study conservative relaxation is the Vlasov equation.

The Vlasov equation has been studied for over 60 years in the plasma, beam, and other communities. However, most studies have been done using its linearized version. In this approximation several results are known, that more or less boil down to the following statement: an initially spatially homogeneous plasma, having a linearly stable velocity distribution, will relax to another homogeneous equilibrium exponentially fast following a perturbation (such as an external electric field). In this process, the electric field perturbation decays. However, no information is lost, no entropy produced, since all initial information is stored in the velocity distribution. This phenomenon is called the (linear) Landau damping. It is a well-known mechanism that often acts against collective beam instabilities-the beam's 
immune system. Despite the successes of the linear theory, bothersome shortcomings were obvious: linearizing the Vlasov equation spoils some symplectic invariants that the full nonlinear equation must obey, and there is no intrinsic mechanism that would assure convergence to equilibrium due to the conservative nature of the equation. Hence, a priori there is no reason to believe that the linear Landau damping survives nonlinearity as an asymptotic limit in time.

Significant progress was achieved recently in extending Landau damping results into the nonlinear regime: C. Mouhot and C. Villani proved that Landau damping survives a small perturbation in the nonlinear regime, that is, the same statement is true for the full, nonlinear Vlasov equation as it is true for its linearized version [1]. Therefore, Landau damping is essentially a linear phenomenon. This proof was worth a Fields Medal to Villani. While a great result indeed, the enormous mathematical difficulties encountered make the application of these results directly to intense charged particle beams at the intensity frontier unrealistic.

Nevertheless, they provide great insight, some of which are collected here:

- In the single-particle limit, transition from integrability to near-integrability is mediated by resonances

- This is described by Kolmogorov-Arnold-Moser (KAM) theory

- Linear Landau damping can be considered integrable in a certain sense

- Transition from linear to nonlinear Landau damping is mediated by echoes

- This is described by Mouhot and Villani's theory which is to infinite dimensional Hamiltonian systems what KAM theory is to low dimensional Hamiltonian systems

- Echos are similar to resonances

- There are important differences between the mean field limit and single-particle limit, but we omit them here for the purpose of this discussion.

These insights guide us towards the following:

- Nonlinear Landau damping survives nonlinearity, and it is not helped by particle interactions, but works despite them

- Proven to work in the perturbative nonlinear regime, and numerical evidence suggest that only in the perturbative regime

- Some kind of confinement is necessary; it does not work in unbounded spaces

- Long time evolution depends on the history of the system through initial conditions, boundary conditions and explicitly on the dynamics

- Landau damping implies orbital stability (a posteriori justification for numerical studies).

Therefore, we summarize the shortcomings of the theory with respect to intense particle beams in realistic systems:

Proposal for an Accelerator R\&D User Facility at Fermilab's Advanced Superconducting Test Accelerator 
- Landau damping is proven to work only in the perturbative regime

- No applied external fields were included

- Only spatially homogeneous initial distributions were allowed

- Realistic boundary conditions have been circumvented with periodic boundary conditions obtained from restricting the problem domain to tori.

Collecting these facts, we are lead to consider that, strictly speaking, Landau damping is what has already been proven, and its generalization to more realistic conditions (like in the list above) is the subject of a slightly different phenomenon, which we may call violent relaxation (if it exists; borrowed from astrophysics). Landau damping could be part of it, related to it, or linked to it since probably all relaxation methods must depend on mixing and filamentation. The purpose of a piece of the experimental IOTA program would be to explore these conservative relaxation phenomena.

As a slight detour, let us mention the current state-of-the-art of the associated computational efforts. As mentioned in the preceding paragraphs, the particle beams have their own specialized theories in the single-particle, multi-particle, and mean field limit cases. The situation is similar in the computational realm. Specialized methods are employed in the different subdomains. In the single-particle case there is the Hamiltonian theory and associated symplectic integration methods; the solution of the equation of the motion through tracking, Differential Algebraic [2] and Lie Algebraic methods [3], and analysis of the resulting motion through transfer maps, normal forms [4] and frequency maps [5]. In the multi-particle limit, there are particle-particle codes, particle-mesh codes and hierarchical spatial subdivision codes. Best known examples are the Particle-in-Cell-type solvers and the Fast Multipole Method [6]. In the mean field limit, there is the basis function method [7], softened N-body solvers [8] and direct Vlasov solvers. Recently developed, transfer map methods can be successfully combined with Fast Multipole and basis function methods. With the advent of supercomputers, currently the pair-wise interactions of more than three trillion particles can be computed quite accurately in minutes, reaching near-realistic populations of intense charged particle beam bunches.

As a consequence, realistic setups that are beyond the reach of theory can only be explored by systematic computational experiments. However, due to the sheer size and amount of computations necessary, the observation of the long term behavior is problematic. Hence, while theory gives insight, and computational studies provide guidance, experiments are the only realistic way to date to access the asymptotic limits in time.

\section{A.2.3.2 Possible Experiments at IOTA}

Combining our line of thought so far, we conclude that experiments are necessary to advance the intensity frontier. IOTA is ideal for such a purpose [9]. Especially the low energy, high intensity proton operation fits our goals. Ability to tailor initial distributions, modify applied fields and shape the boundary conditions would be advantageous. Observations would detect the stability properties of the beams as a function of these parameters. Insight into microscopic behavior can be gained by excitation, detection, and measurement of beam echoes. To this 
end, an anti-damper and a quadrupole pick-up are required. Intensity, position and emittance measurements are necessary. Behavior as a function of energy would be a plus.

First proposed experiment: given that the easiest parameters to vary are the initial beam intensity and the applied fields, study qualitatively and quantitatively beam echoes excited by an anti-damper and detected by quadrupole pickups. Determine behavior as a function of intensity and applied fields, where there are many possibilities limited by the power supply configurations. Long time behavior should be characterized to the extent possible (stability/instability, growth rate, emittance growth, tunes, chromaticities, etc.) Correlate results with computational studies.

Later experiments would focus on studies as a function of other parameters, as features are put in place: initial distribution tailoring and boundary condition shaping.

To summarize, this conceptual framework points to experiments for the:

- Observation of long term beam behavior, and

- Observation and measurement of various beam echoes, by

- Modifying initial conditions (some distributions might be more stable than others, i.e. have larger "damping basins"), and

- Modifying confinement mechanisms, i.e. different boundary conditions and applied fields.

\section{A.2.3.3 References}

[1] C. Mouhot and C. Villani, "On Landau Damping," Acta Mathematica, vol. 207, no. 1, pp. 29-201, 2011.

[2] M. Berz, Modern Map Methods in Particle Beam Physics, London: Academic Press, 1999.

[3] A. Dragt, Lie Methods for Nonlinear Dynamics with Applications to Accelerator Physics, College Park: unpublished, 2013.

[4] M. Berz, "Differential Algebraic Formulation of Normal Form Theory," in Proc. Nonlinear Effects in Accelerators, 1992.

[5] J. Laskar, "Frequency Map Analysis of a Hamiltonian System," in Workshop on Nonlinear Dynamics in Particle Accelerators, AIP Conf. Proc., Arcidosso, 1995.

[6] S. Abeyratne, B. Erdelyi,and S. Manikonda, "A Novel Differential Algebraic Adaptive Fast Multipole Method," Proc. IPAC'13, Shanghai (2013), page 1055.

[7] B. Erdelyi, E. Nissen and S. Manikonda, "A Differential Algebraic Poisson Solver for Charged Particle Beams," Communications Computational Physics, 2013.

[8] W. Dehnen, "Towards Optimal Softening in Three-Dimensional N-Body Codes," Monthly Notices of the Royal Astronomical Society, vol. 324, no. 2, pp. 273-291, 2001.

[9] S. Nagaitsev, A. Valishev, V. Danilov, and D. Shatilov, "Design and Simulation of IOTAA Novel Concept of Integrable Optics Test Accelerator," Proc. IPAC'12, New Orleans (2012), page 16. 


\section{A.3 Studies Utilizing ASTA Capabilities for Phase Space Manipulation}

\section{A.3.1 Miniaturized Magnetic Undulators for X-Ray Generation}

\section{A.3.1.1 Introduction and Goals of the Experiments}

The University of Florida and Georgia Institute of Technology research team has recently developed miniaturized magnetic undulator structures with magnetic periods 100x to $1000 \mathrm{x}$ smaller than the state-of-the-art $[1,2]$. Reducing the spatial period $\left(\lambda_{u}\right)$ of magnetic undulators is one way for increasing the photon energy level and spectral purity of synchrotron radiation. Peak magnetic fields up to $0.25 \mathrm{~T}$ have been demonstrated for a 50-period undulator with $\lambda_{u}=$ $400 \mu \mathrm{m}$ and an air gap of $200 \mu \mathrm{m}$. It should be noted that because permanent magnets are used, no power source is required to create such magnetic fields. Depending on the electron energy, such structures are adaptable for radiation ranging from terahertz to gamma rays.

The basic undulator structure employs two separable periodic arrays of north-south magnetized permanent magnets, which are sandwiched face-to-face forming a slit-like air gap. Laser-micromachining and precision assembly of bulk rare-earth magnetic materials (SmCo and $\mathrm{NdFeB}$ ) are used to form the so-called "mini" undulators with magnetic periods of 100's of micrometers. A first-generation prototype undulator has been made using $\mathrm{Sm}_{2} \mathrm{Co}_{17}$ with 50 periods and a magnetic period $\lambda_{u}=400 \mu \mathrm{m}$, as shown in Figure 1). The total length of a magnetic array is $20 \mathrm{~mm}$ with a transverse width of $2 \mathrm{~mm}$. The magnetic field generated by the magnet arrays, representing each one half of the structure, was characterized by a highresolution scanning Hall probe system and magneto-optical imaging techniques. Figure 2 presents the B-field from a single magnet array: the scanning height was $100 \mu \mathrm{m}$ and the B-field was then scaled to display what would it would at $200 \mu \mathrm{m}$. These measured fields are then used to estimate the field in the fully assembled undulator structure. Using 100- $\mu$ m-thick lasermachined $\mathrm{Cu}$ shims, we can adjust the air gap between 100 and $400 \mu \mathrm{m}$, resulting in an undulator peak B-field varying from $0.53 \mathrm{~T}$ to $0.05 \mathrm{~T}$, respectively. As presented in Figure 1.a), the top and bottom frames and the $\mathrm{Cu}$ shims are then assembled with screws to an adapter allowing for precise alignment of the undulator gap with the electron beam.
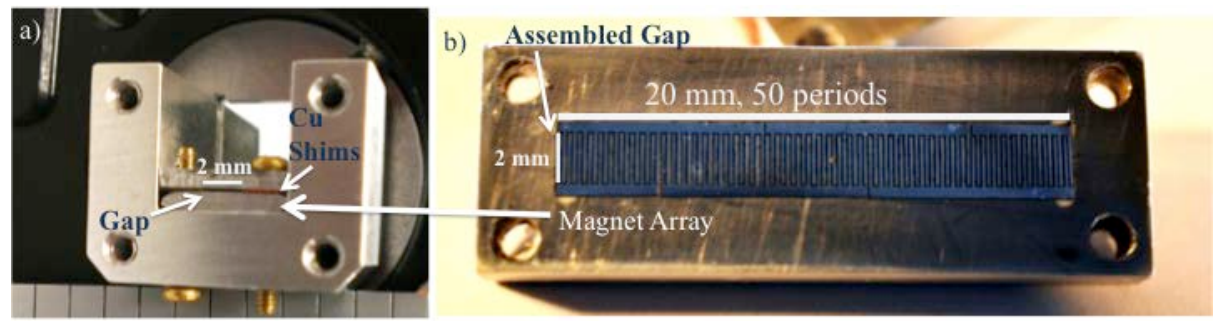

Figure 1: (a) Photograph of the front of an assembled undulator in an adapter; (b) Top view photograph of a magnet array; another magnet array placed directly above creates an undulator.

Proposal for an Accelerator R\&D User Facility at Fermilab's Advanced Superconducting Test Accelerator 


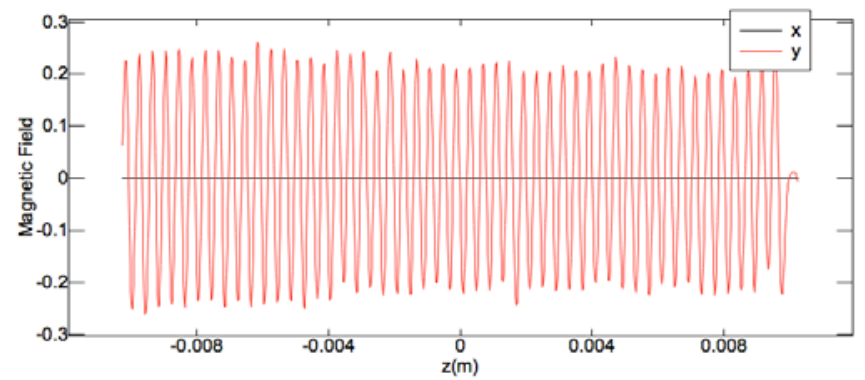

Figure 2: B-field of one of the two magnetic arrays at $200 \mu \mathrm{m}$ above the surface.

Our goal of using the ASTA facility is to demonstrate the possibility of generating photons with the mini undulator. The major obstacle to overcome is to inject the electron beam through the thin air gap. However, contrary to the electron beams produced in the other accelerator facilities, the ASTA facility has the ability to produce round-section beams and to convert them to flat beams. Flat beams have been developed and experimentally tested before at Fermilab [3]. Flat beam manipulation allows beam of high aspect ratio (large horizontal emittance vs. small vertical emittance) to be generated. They could then be injected into small air gap undulators making beam injection and transport through the mini undulators easier. Our proposal marries the flat beam technique with mini-undulator technology for potentially compact light sources.

If successful, these experiments will demonstrate for the first time the generation of photons with an undulator in the micron-size period range.

\section{A.3.1.2 Required Beam Parameters}

The electron velocity and the spatial period of the undulator magnetic field determine the wavelength of the electromagnetic radiation. A shorter magnetic period translates to shorterwavelength radiation. Hence, a major opportunity of our mini undulator is reducing the period from typical centimeter length scales down to hundreds of micrometers to enable very shortwavelength (high-frequency) radiation. The mini undulator has 50, 400- $\mu \mathrm{m}$-long magnetic periods resulting in an overall length of $20 \mathrm{~mm}$.

With the $50 \mathrm{MeV}$ ASTA beam, the undulator is expected to generate UV light, photons having an energy of $59 \mathrm{eV}$. If available, higher beam energies at $800 \mathrm{MeV}$ would yield hard x-rays.

Table 1 lists the beam parameters that would be required for our experiments in order to reach our goal. 
Table 1: Required beam parameters.

\begin{tabular}{|l|c|l|}
\hline \multicolumn{1}{|c|}{ Parameter } & Value/Range & \multicolumn{1}{c|}{ Units } \\
\hline Beam Energy & $20-50$ & $\mathrm{MeV}$ \\
\hline Bunch Charge & $0.02-3.2$ & $\mathrm{nC}$ \\
\hline Bunch repetition rate & 3 & $\mathrm{MHz}$ \\
\hline Number of bunch/macropulse & $1-3000$ & \\
\hline Transverse emittance/spot size & 50 & $\mu \mathrm{m}$ \\
\hline Bunch length & 3 & $\mathrm{ps}$ \\
\hline Fractional momentum spread & $2 \mathrm{e}-4$ & \\
\hline
\end{tabular}

\section{A.3.1.3 Personnel Involved}

Brock Peterson is a second-year PhD student from Georgia Tech. The data taken during this experiment will be incorporated in his dissertation. Dr. Alexandra Garraud is a postdoctoral research associate from University of Florida. They will both travel to the Fermilab to perform the experiments.

As for the Fermilab team involvement in the experiments, Jayakar C. Thangaraj will be our primary person involved in this project and the point of contact of the experiment. Dr. Philippe Piot will also be involved. The distribution of tasks is summarized in Table 2.

Table 2: Personnel involved, from University of Florida and Georgia Tech, and from Fermilab.

\begin{tabular}{|l|l|l|l|l|l|}
\hline & Planning & Experiment & Diagnostics & Analysis & Simulation \\
\hline A. Garraud & & & & & \\
\hline B. A. Peterson & & & & & \\
\hline M. G. Allen & & & & & \\
\hline D. P. Arnold & & & & & \\
\hline J. C. Thangaraj & & & & & \\
\hline P. Piot & & & & & \\
\hline
\end{tabular}

\section{A.3.1.4 Experimental Technique and Expected Outcomes}

The software SPECTRA [4] was first used to evaluate what photon output we would obtain. A $50 \mathrm{MeV}, 1-\mathrm{nC}$ electron beam was used as the source. The mini-undulator is expected to produce per bunch $6.1 \times 10^{5}$ photons of $59 \mathrm{eV}$, as shown in Figure 3. Figure 4 presents the angular flux density in order to indicate how many photons will reach the detector. 


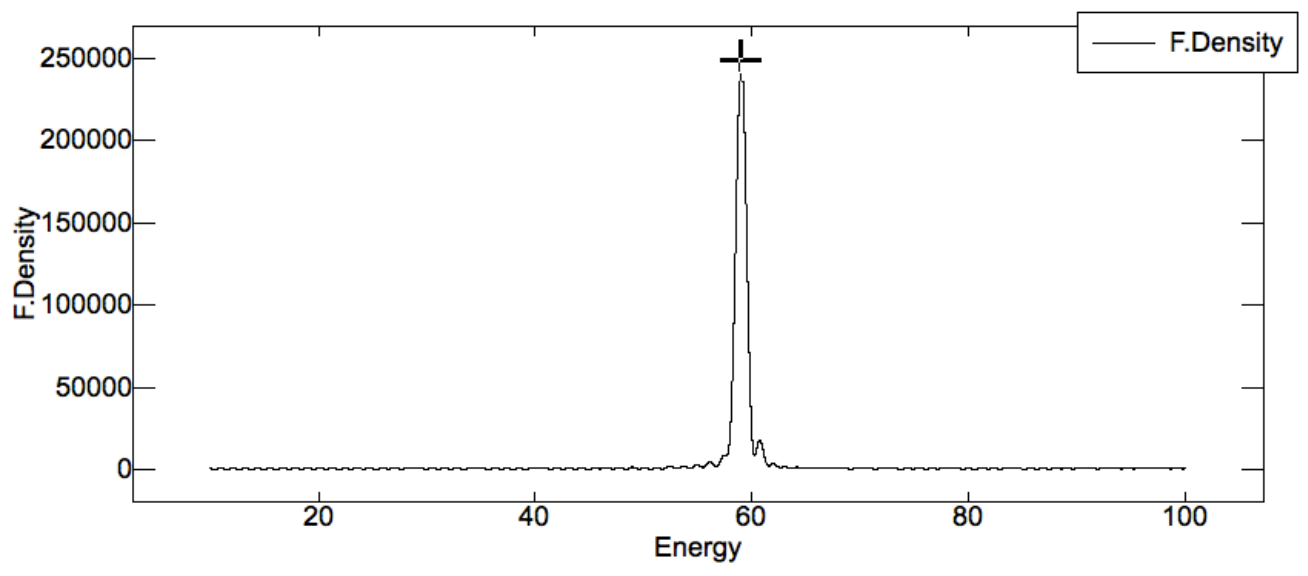

Figure 3: Simulated flux density [photons $\left./\left(\mathrm{s} . \mathrm{mm}^{2} .0 .1 \mathrm{BW}\right)\right]$ vs. the output energy [eV] from the mini undulator, for a $50 \mathrm{MeV}$ electron beam input. The cross is located at (58.994; 248,500).

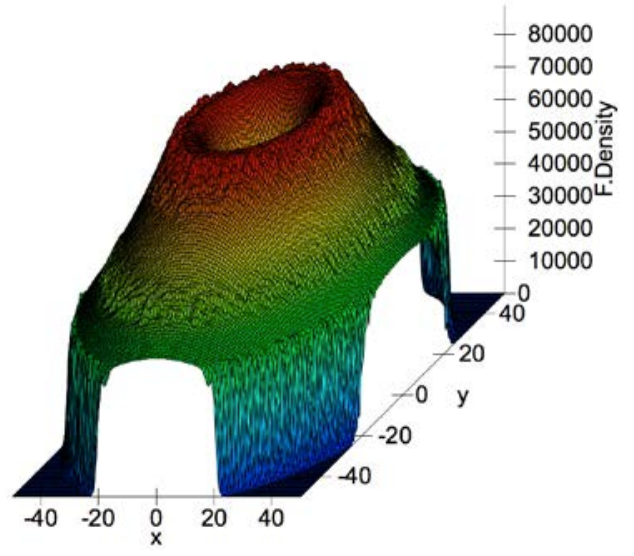

Figure 4: Angular flux density expected. Note that the distances are in $\mathrm{mm}$.

Figure 5 gives a schematic drawing of the experimental setup. The mini undulator would be placed in the magnetic bunch compressor (BC1) during the experiments. The ASTA beamline was adapted from this ASTA proposal, Section 7.1, Figure 4.

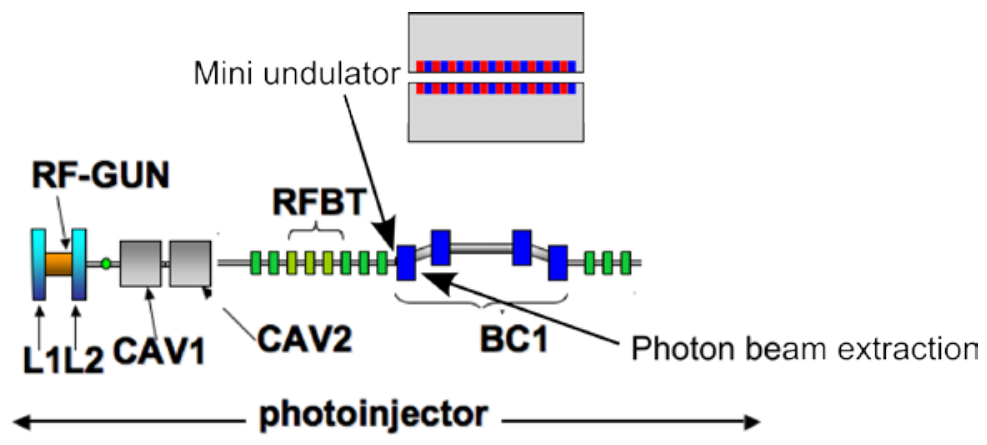

Figure 5: Location of the mini-undulator in the electron beam path. In the sketch, red and blue colors represent the magnetic pole arrays, facing each other (not to scale). 
The mini undulator is composed of SmCo comb-like structures assembled in aluminum frames with parylene as described in Figure 1. It has already been proven that the undulator can be placed in a vacuum down to $5 \times 10^{-5}$ torr without degradation of the magnetic properties (remanence, saturation magnetization). Also, we expect no limitation due to high vacuum level $\left(10^{-9}\right.$ torr) required with the ASTA beamline.

A first test would be to turn off the superconducting radio-frequency (SRF) TESLA-type cavity named CAV2, in order to inject a $20 \mathrm{MeV}$ electron beam into the mini undulator. This will confirm the generation of photons in the V-UV range. Afterwards, the CAV2 would be turned on, enabling a $50 \mathrm{MeV}$ electron beam: the mini undulator is then expected to generate UV light, photons having an energy of $59 \mathrm{eV}$. If available at the time of the experiments, higher beam energies, up to $850 \mathrm{MeV}$, would yield soft to hard x-rays. Regardless of the photon energy, the main objective of the experiments is to prove the ability of the mini undulator to generate photons and to explore the utility of the high-aspect-ratio flat electron beams.

The alignment of the electron beam with the undulator will be controlled using two beam position monitors (BPMs) and positioning the undulator on a multi-axis positioning. The bottom and top parts of the mini undulator can be easily separated during this stage to facilitate the positioning. The air gap height is determined at the beginning of the experiment by the number of $100-\mu \mathrm{m}$ Cu shims stacked together. Once assembled and aligned, the air gap is fixed for the duration of the experiments.

After exiting the mini undulator, the resulting beam, photons, would reach a parabolic mirror, presenting a slit in its center. This opening would allow the photons to reach a UV detector.

We estimate that two periods of 5 shifts each would be necessary to perform our experiments.

\section{A.3.1.5 References}

[1] O. D. Oniku, W. C. Patterson, A. Garraud, E. Shorman, P. Ryiz, D. P. Arnold, B. A. Peterson, F. Herrault, M. G. Allen, "Progress Toward Mini and Micro Magnetic Undulators," Presentation at the HBEB 2013 conference, Puerto Rico.

[2] B.A. Peterson, W.C. Patterson, F. Herrault, D.P. Arnold, and M.G. Allen, "Lasermicromachined permanent magnet arrays with spatially alternating magnetic field distribution," PowerMEMS 2012, p 319-322, Dec. 2012.

[3] P. Piot, Y.-E. Sun, K.-J. Kim, Photoinjector generation of a flat electron beam with transverse emitance ratio of 100, Phys. Rev. ST Accel \& Beams, 9031001 (2006).

[4] http://radiant.harima.riken.go.jp/spectra/ 


\section{A.3.2 Electron Beam Shaping and High Gradient, High Transformer Ratio Acceleration in a Dielectric Tube}

\section{A.3.2.1 Introduction and Goals of the Experiments}

Our team at Los Alamos National Laboratory (LANL) is funded at 1 FTE level for 3 years by LANL's Laboratory Directed Research and Development (LDRD) program do execute a project with an ultimate goal to demonstrate operation of a high-brightness Dielectric Wakefield Accelerator (DWA) with an acceleration gradient above $100 \mathrm{MV} / \mathrm{m}$ and less than $0.1 \%$ induced energy spread in the accelerated beam. In the long term, we project the DWA concept as a performance upgrade for the future LANL signature facility Matter Radiation Interaction in Extremes (MaRIE). The pre-conceptual design for MaRIE is underway at LANL, with the design of the electron linear accelerator being one of the main research goals. The cost of the linac is significant and the existing space constraints of the mountainous terrain dictate that the final energy of the electron beam for the X-ray Free-Electron Laser (XFEL) is no higher than $12 \mathrm{GeV}$. The number and the energy of photons produced by the XFEL is however strongly dependent on the electron beam's energy with the more energetic beam delivering more energetic photons to the user. Although generally the baseline design needs to be conservative and rely on existing technology, any future upgrade would immediately call for looking into the advanced accelerator concepts capable of boosting the electron beam energy up by a few GeV in a very short distance without degrading the beam's quality. This work has the potential to advance the DWA technology to a level that would make it of relevance for MaRIE and also for a number of other national security applications.

In this particular project we plan to combine the two proven concepts of the Dielectric Wakefield Accelerator (DWA) [1,2] and the Emittance Exchanger (EEX) [3] in a unique way to experimentally demonstrate a high-brightness DWA with the gradient of above $100 \mathrm{MV} / \mathrm{m}$ and less than $0.1 \%$ induced energy spread in the accelerated beam. We have realized that it is possible that an accelerated beam in a DWA can achieve the needed small energy spread if its profile is properly customized. A trapezoidal bunch shape can be used to reduce the witness beam energy spread, and, in doing so, preserve the beam brightness. The proposed research builds upon LANL's capabilities in advanced beam optics, relying on the Laboratory's strong numerical simulation base, and previous experimental work on advanced accelerator research and millimeter-waves.

We plan to use the $50 \mathrm{MeV}$ user area of ASTA facility. We have shipped the $1.3 \mathrm{GHz}$ transverse deflecting cavity to ASTA which should complement the set of available components to finish the assembly of the Emittance Exchanger in the $50 \mathrm{MeV}$ user area. With the EEX in operation, we plan to perform the following experimental demonstrations: (1) simultaneous generation of a drive bunch and a main beam from a single Gaussian bunch produced at ASTA, (2) acceleration of the main beam with a wakefield excited in a dielectric fiber and significant increases in a DWA transformer ratio, and (3) low the measured energy spread in the main beam accelerated through a wakefield process.

Proposal for an Accelerator R\&D User Facility at Fermilab's Advanced Superconducting Test Accelerator 


\section{A.3.2.2 Required Beam Parameters}

To conduct the proposed experiment, LANL would require a $50 \mathrm{MeV}$ electron beam in single bunches with a bunch charge of $1 \mathrm{nC}$ or above. Higher bunch charges would provide us with higher excited gradients in dielectric fibers. To demonstrate more than $100 \mathrm{MV} / \mathrm{m}$ accelerating gradient in a $300 \mathrm{GHz}$ fiber we need a single bunch with a charge of $5 \mathrm{nC}$ or above. We require spot sizes of the order of a few millimeters to a few centimeters at the beam mask and emittances less than 10 micron. The preferable bunch length should be of the order of $1 \mathrm{~mm}$ or less, since longer bunches experience more non-linear effects in the emittance exchanger. The momentum spread needed is less that $1 \%$ with less than $0.1 \%$ preferred for the accurate measurement of the induced energy spread in the witness bunch. The required parameters are summarized in Table 1.

Table 1: Required beam parameters.

\begin{tabular}{|l|c|}
\hline \multicolumn{1}{|c|}{ Parameter } & Value/Range \\
\hline Beam Energy & 20-100 MeV (50 MeV desired) \\
\hline Bunch Charge & $1-10 \mathrm{nC}(5 \mathrm{nC}$ desired) \\
\hline Bunch repetition rate & Single pulse \\
\hline Number of bunch/macropulse & 1 \\
\hline Transverse emittance & $<10$ micron \\
\hline Spot size & $2-20 \mathrm{~mm}$ \\
\hline Bunch length & $<1 \mathrm{~mm}$ \\
\hline Fractional momentum spread & $<0.01(<0.001$ desired $)$ \\
\hline
\end{tabular}

We ask for 3 weeks of beamline time (each consisting of 5 8-hour shifts) in FY14 and 3 weeks in FY15.

\section{A.3.2.3 Personnel Involved}

The only required Fermilab manpower consists of beamline operators and technicians. We collaborate with Philippe Piot's research group at Fermilab with respect to design of the Emittance Exchanger and beam shaping experiments. At LANL we have 2 technical staff members continuously working on the project at about 0.33 FTE each (E. Simakov and D. Shchegolkov) and might be able to bring a postdoc on board in FY14 and FY15. Both LANL staffs will be involved with planning, experiment, diagnostics, analysis and simulations. Both LANL staffs will travel to Fermilab on as-needed basis.

\section{A.3.2.4 Experimental Technique and Expected Outcomes}

In FY14 we plan to conduct a number of beam shaping experiments to investigate possibilities for generation of shaped electron bunches of correct shapes. To conduct this experiment, we will need to assemble an emittance exchanger in either double-dogleg or chicane geometry. 
The simplest configuration of the emittance exchanger (shown in Figure 1) needs the following components: beam shaping mask, two quadruple magnets, four dipole magnets, a deflecting cavity, and a fundamental mode cavity to compensate for dispersive energy gain in the deflecting cavity. Installation of the beam mask and the cavities is interceptive. LANL has shipped to Fermilab a $1.3 \mathrm{GHz}$ deflecting cavity. LANL folks plan to assist Prof. Piot's team with the high power testing of the cavity as needed. LANL will supply different beam shaping masks as the experiment requires. The deflecting cavity and beam masks are compatible with ultralow pressure requirements of ASTA. We expect other hardware to be supplied by FNAL.

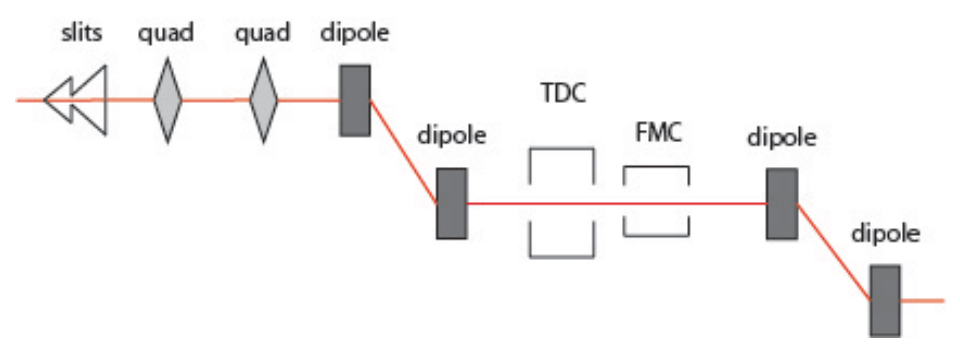

Figure 1: The simplest design of the EEX beamline for the beam shaping experiment.

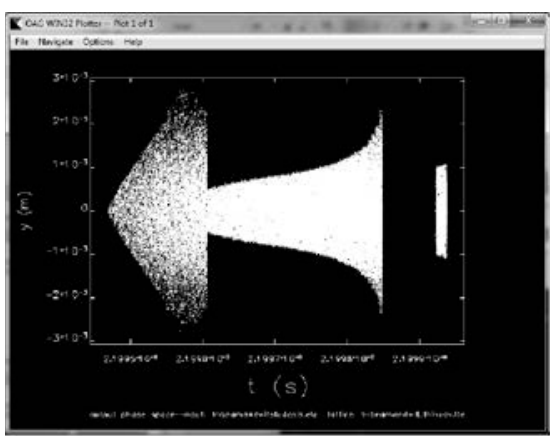

(a)

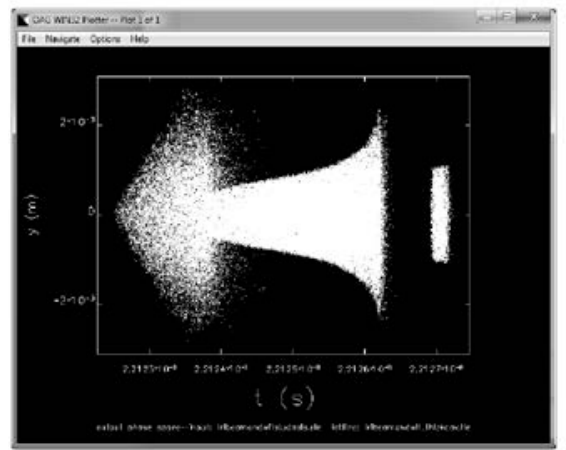

(c)

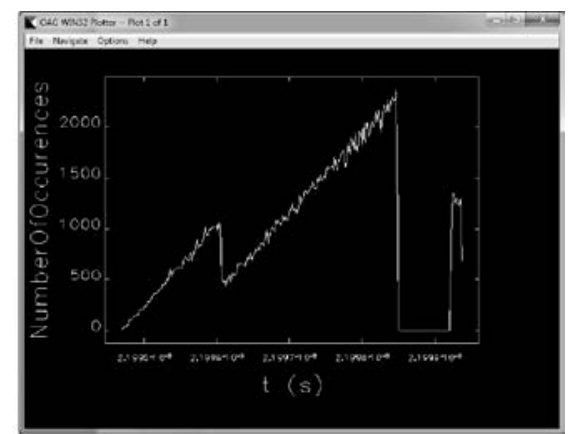

(b)

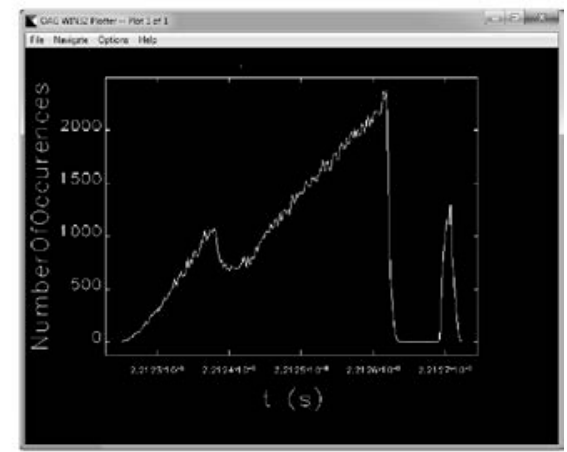

(d)

Figure 2: Elegant simulations of a pair of drive and witness bunches produced by the EEX: (a) distribution of particles as computed in the $1^{\text {st }}$ order; (b) current in the bunch as computer in the $1^{\text {st }}$ order; (c) distribution of particles as computed in the $2^{\text {nd }}$ order; (d) current in the bunch as computer in the $2^{\text {nd }}$ order. 
In the first experiment using the simplest EEX configuration shown in Figure 1 we expect to observe simultaneous production of a double-triangular drive bunch and a trapezoidal witness bunch. We will need to measure the temporal profiles of the bunches. We will require diagnostics to measure the profiles. The fast deflecting cavity will be a preferable diagnostics. We expect the diagnostics to be supplied by FNAL. We simulated the performance of the EEX with Elegant [4]. Figure 2 shows distribution of particles which we may expect to produce in the proposed experiment. Figures 2 (a) and (b) show the particle distribution and the current in the beam as computed with the $1^{\text {st }}$ order transfer matrices only. Figures 2(c) and (d) show computations with the $2^{\text {nd }}$ order effects taken into account. We can see that nonlinearities in the beam line elements may produce significant aberrations in the shape of the drive and especially witness bunches. In the experiment, we plan to study how closely we can reproduce simulated shapes of the bunches.

We plan to add more corrective elements to the beamline as shown in Figure 3 to reduce the effects of nonlinearities and produce better bunch shapes.

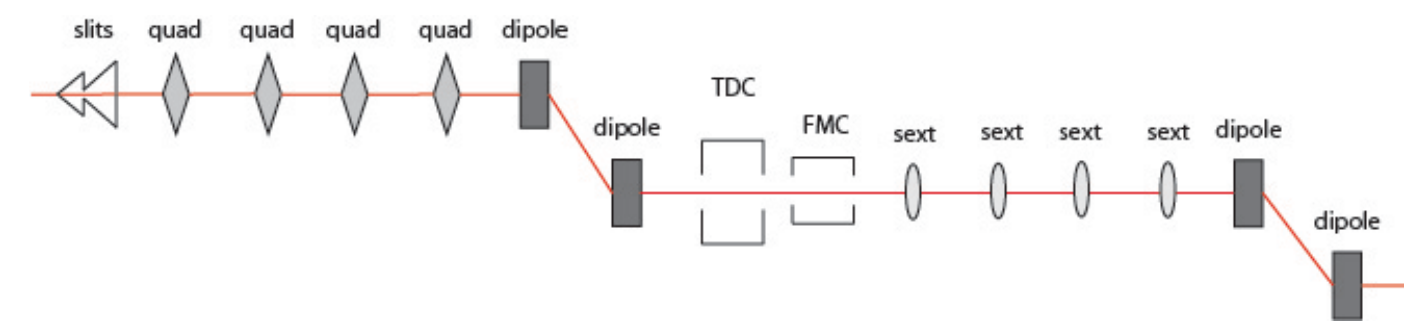

Figure 3: A more sophisticated design of the EEX beamline with quadruples and sextupoles capable to compensate for non-linearities in the beamline.

In FY15 we plan to conduct demonstration of the wakefield generation by a double triangular bunch in a dielectric (silica) fiber and acceleration of the shaped witness bunch with the high transformer ratio. The proposed experiment will be similar to the diamond structure wakefield mapping experiment conducted by Euclid Techlabs at the ATF in 2011 [5]. The dielectric fibers and the test chamber will be supplied by LANL. We will also supply a new mask which would allow us to vary the distance between the double triangular drive bunch and a witness bunch. Installing the test chamber and the new mask is interceptive. 


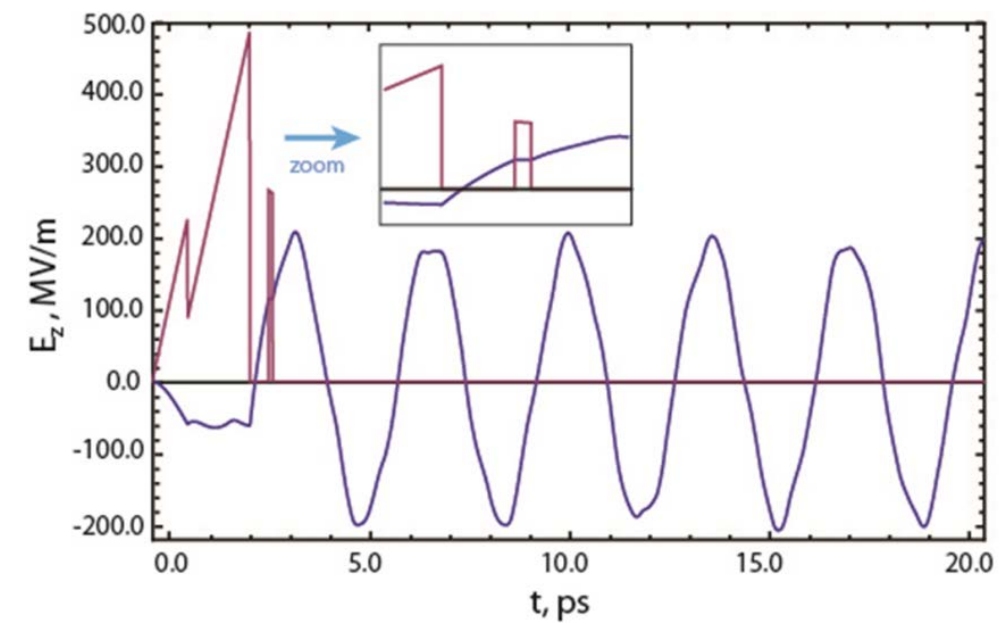

Figure 4: The wakefield (blue) from a double-triangular drive bunch, showing a large transformer ratio (the ratio of the peak accelerating gradient to the decelerating gradient experienced by the drive bunch, in red). The wakefield inside of a trapezoidal witness bunch (also zoomed in) showing a flat gradient observed by the witness bunch.

The double triangular bunch will be passed through the fiber and the wake will be sampled by a small witness beam at variable distance from the drive. This measurement will explicitly show enhanced transformer ratio (acceleration of the witness vs. deceleration of the drive) and high accelerating gradients. Simulations of the wake excited by a $5 \mathrm{nC}$ double triangular drive bunch in a $300 \mathrm{GHz}$ silica fiber is shown in Figure 4. The transformer ratio in this simulation is about 3.5 and the maximum gradient $200 \mathrm{MV} / \mathrm{m}$. Figure 4 also shows the shaped witness bunch which experiences uniform accelerating gradient. In the experiment we will measure the momentum spread in the shaped witness bunch and expect to observe the small induced momentum spread. We will require a spectrometer to measure the momentum spread and expect the spectrometer to be provided by FNAL.

\section{A.3.2.5 References}

[1] W. Gai, P. Schoessow, B. Coley, R. Konecy, J. Norem, J. Rosenzweig, and J. Simpson, Phys. Rev. Lett., 61, 2756 (1988).

[2] M. Rosing and W. Gai, Phys. Rev. D, 45, 1829 (1990).

[3] Y.-E. Sun, P. Piot, A. Johnson, A. H. Lumpkin, T. J. Maxwell, J. Ruan, and R. Thurman-Keup, Phys. Rev. Lett., 105, 234801 (2010).

[4] Elegant accelerator code distributed by ANL, http://www.aps.anl.gov/ Accelerator Systems Division/Accelerator Operations Physics/software.shtml.

[5] S. Antipov, C. Jing, A. Kanareykin, J. E. Butler, V. Yakimenko, M. Fedurin, K. Kusche, and W. Gai, Appl. Phys. Lett. 100, 132910 (2012). 


\section{A.3.3 Beam-Beam Kicker for an Electron-Ion Collider}

\section{A.3.3.1 Letter of Intent}

August 14, 2012

Dr. Vladimir Shiltsev, Director

Accelerator Physics Center

Fermi National Accelerator Facility

Dear Vladimir,

As you know, Jefferson Laboratory is conducting study of possibilities for future high luminosity polarized Medium Energy Electron-Ion Collider (MEIC) at JLab: [see Ref.[1] or http://casa.jlab.org/meic/files/MEIC_Report.pdf].

Crab-crossing beams of low charge very short (up to 5 - $10 \mathrm{~mm}$ ) bunches at high repetition rate (up to .75 - $1.5 \mathrm{GHz}$ ) is the luminosity concept. Collider design includes use of ERL-based Electron Cooling for $20-100 \mathrm{GeV}$ hadron beam. In order to reduce the required electron current in ERL, the cooler scheme is complemented with a Circulator-cooler ring (CCR) operated by a fast kicker in a CW mode. Creation of fast kicker of raise time in range of a fraction of 1 ns is the central challenge of the CCR and cooler design.

As a clearly envisioned and promising version of such a kicker, we considering your kicker beam idea you proposed in 90s [2]. As an adjustment for more efficiency of your concept, we consider implementation of round to flat transformations of both beams in the kick sections. The imagined scheme is described in more detail and illustrated in numbers in the attached note.

It is our understanding that, in order to proceed firmly with these developments toward a viable cooler design and creation of a test device, we have to incorporate and rely essentially on collaboration with professionals of Fermi Laboratory and, specifically, with you and your Accelerator Physics Center which has experts and experience over many aspects of the related beam physics and techniques. I also would like to add that, our cooperation certainly could be extended to the joint efforts on whole the cooler design, building and tests for MEIC and other facilities with high energy hadron beams.

I would appreciate very much your reply, would you and your colleagues been interested in the subjects and cooperative work in these directions.

If you find it timely to start with connections on the matters, I can suggest the following names (CASA members) for communications:

Dr. Yuhong Zhang

MEIC Project Manager. Yzhang@jlab.org

Dr. Eduard Nissen.

"Nissen@jlab.org"

Dr. Geoff Krafft,

CASA Director. Krafft@jlab.org

Prof. Y. Derbenev

Scientist, derbenev@jlab.org 


\section{A.3.3.2 Beam-Beam Kicker}

An innovative idea recently under active investigation for MEIC project [1] utilizes a nonrelativistic sheet beam for providing transverse kicking to a flat electron bunch [3]. This idea of a beam-beam kicker was first proposed by Shiltsev [3] for two round Gaussian beams. Here we consider a case of two flat beams as shown in Figure 1, though analytical treatments for these two cases are quite similar. We present here final results plus considerations of cooling electron bunch requirement in order to have an effective kicker scheme. Two technical issues will be also briefly discussed.

Since the target flat (cooling) beam is moving at the speed of light, it passes through the nonrelativistic kicking flat beam in a period of time determined by the length of the kicker beam $l_{k}$. We consider ejection/injection of cooling bunches in the horizontal plane by kicks in $x$-direction, so both beams should be flattened in the kick sections to have a small horizontal size while relatively large the vertical sizes. This can be achieved by use of magnetized beams and performing flattening gymnastics, specific for each of two beams. At a close distance to the kicking beam, an electron in the target beam receives an instant horizontal angle kick determined by integration of the transverse force over that passing time

$$
\theta_{k}=\frac{2 \pi N_{k} r_{e}}{\gamma l_{y k}}
$$

under conditions

$$
\theta_{x} \ll \theta_{k}
$$

and

$$
\sigma_{x}, \sigma_{y}, h \ll l_{y k}
$$

where $N_{k}$ and $l_{y k} \sigma_{\mathrm{xk}}$ are number of electrons and vertical size of the kicker bunch, $\theta_{x}$ and $\sigma_{x}$ is the angle spread and transverse size of the cooling bunch in horizontal plane, $\gamma$ is its Lorentz factor, and $r_{e}$ is the electron classical radius. 


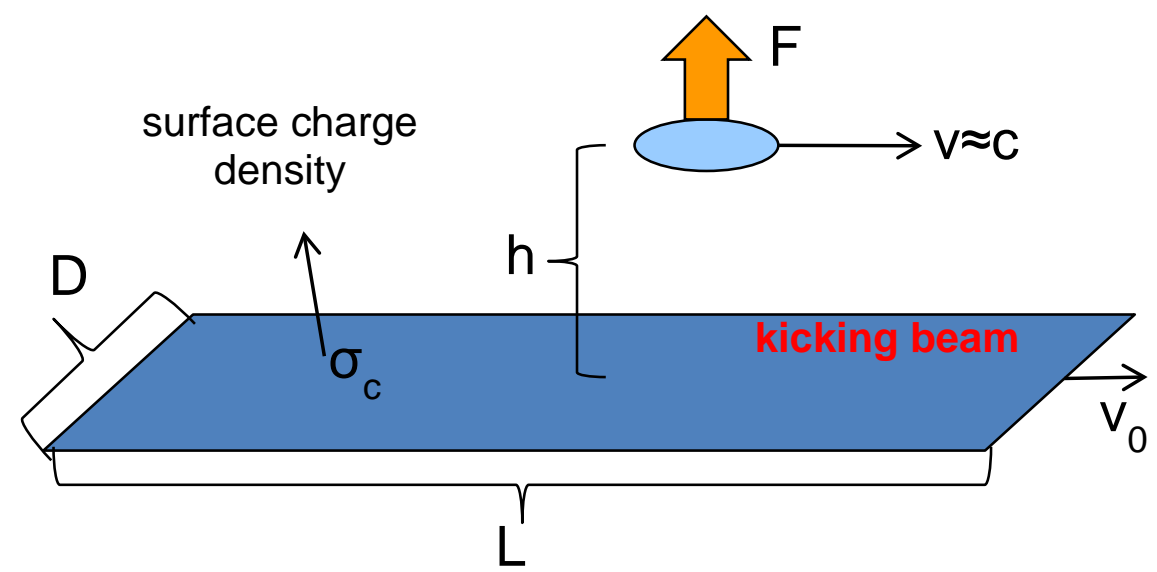

Figure 1: Schematic drawing of a fast beam-beam kicker.

In order to reduce the required kick, the cooling beam should have a small horizontal emittance and possibly large horizontal beta function at the kicker section. Combining this with the flatness condition, we find the following requirements to the cooling beam:

$$
\begin{gathered}
\theta_{k} \gg \sqrt{\frac{\varepsilon_{x}}{\gamma \beta_{x}}} \rightarrow \frac{2 \pi N_{k} r_{e}}{\gamma l_{y k}} \gg \sqrt{\frac{\varepsilon_{x}}{\gamma \beta_{x}}} \\
\sigma_{y}=\kappa l_{y k} ; \quad \kappa \ll 1 \\
\sigma_{x}=\sqrt{\frac{\varepsilon_{x}}{\gamma} \beta_{x}} \ll l_{y k}=\frac{1}{\kappa} \sqrt{\frac{\varepsilon_{y}}{\gamma} \beta_{y}}
\end{gathered}
$$

Table 1 summarizes the design parameters for a beam-beam kicker for the ELIC CR cooler design. It should be noted that, with 100 to 300 revolutions of cooling electron bunches in the $\mathrm{CR}$, the repetition frequency of the kicking beam can be a factor of 100 to 300 smaller than the repetition rate of the $\mathrm{CW}$ cooling beam.

\section{A.3.3.3 Making of a Flat Kicker Beam}

A flat kicker beam can be produced utilizing a grid-operated DC (thermionic) electron gun with a round magnetized cathode. While maintaining the beam in solenoid, one can impose a constant quadrupole field that causes beam shrinking in one plane while enlarging in the other plane due to the drift motion of particles. The process should be adiabatic relative to the particles' cyclotron motion in the solenoid [3]. The beam current density could be specifically profiled at the cathode to create uniform distribution in a homogenous field in a direction transverse to in the "plane" of flattened beam. 


\section{A.3.3.4 Obtaining a Flat Cooling Beam}

While the magnetized state of a cooling beam is transplanted from the gun to the solenoids in the cooling section, the beam can be made flat at a kicker section of the circulator ring applying round-to-flat beam transformation proposed for an angular momentum dominated beam [4]. Such transformation can be performed by a special group of skew-quadrupoles matched with optics of the circulator ring. This will create flat beam with two very different emittances [4-6]:

$$
\frac{\varepsilon_{x}}{\varepsilon_{y}}=\frac{\varepsilon_{c}}{\varepsilon_{d}}=\frac{r_{c}^{2}}{a_{0}^{2}}
$$

here $r_{c}$ and $a_{0} \varepsilon_{0}$ are the characteristic cyclotron radii of electrons and beam area radii in solenoid of cooling section, respectively.

Table 1: Design parameters for MEIC beam-beam kicker.

\begin{tabular}{|c|c|c|}
\hline Circulating beam energy & MeV & 33 \\
\hline Kicking beam energy & $\mathrm{MeV}$ & $\sim 0.3$ \\
\hline Kicking Repetition frequency & $\mathrm{MHz}$ & $5-15$ \\
\hline Kicking angle & $\mathrm{mrad}$ & 0.2 \\
\hline Kicking bunch length & $\mathrm{cm}$ & $10-20$ \\
\hline Kicking bunch width & $\mathrm{cm}$ & 0.5 \\
\hline Kicking bunch charge & $\mathrm{nC}$ & 2 \\
\hline
\end{tabular}

\section{A.3.3.5 Uniqueness of ASTA}

The beam-beam kicker concepts proposed aim at demonstrating the deflection of a flat beam using a non-relativistic sheet beam at $\mathrm{MHz}$ repetition rate. The availability of bunch trains with comparable intra-bunch frequency along with the round to flat beam transformation make ASTA uniquely suited to carry out the beam-beam kicker experiments.

The experiment would be carried out in the photoinjector user area with energy comparable to the circulating beam energy in the MEIC. A low-energy ( $300 \mathrm{keV}$ ) MHz repetition rate gun would have to be installed. Standard beam position monitor available at ASTA would be used to quantify the kicker performance. Additionally a variable bunch produced by the laser could be used to scan the respond time of the proposed kicker. 


\section{A.3.3.6 References}

[1] S. Abeyratne, et al., "Science Requirements and Conceptual Design for a Polarized Medium Energy Electron-lon Collider at Jefferson Lab" (JLAB, 2012), http://casa.jlab.org/meic/files/MEIC Report.pdf.

[2] V. Shiltsev, NIM, A374 (1996) 137.

[3] Ya. Derbenev and Y. Zhang, "Electron Cooling For Electron-Ion Collider at JLab," Proc. of Proc. of COOL 09, Lanzhou, China, 10-14 Sept. 2009.

[4] Ya. Derbenev, University of Michigan Report No. UM-HE-98-04, 1998.

[5] A. Burov, S. Nagaitsev, and Ya. Derbenev, Phys. Rev. E 66, 016503 (2002).

[6] Y.-E Sun, et al., "Generation of angular-momentum-dominated electron beams from a photoinjector," Phys. Rev. ST Accel. Beams 7, 123501 (2004). 


\section{A.3.4 Measuring Coherent Synchrotron Radiation (CSR) Effects on High Brightness Electron Beams}

Both next generation linear colliders and $\mathrm{x}$-ray free-electron lasers incorporate magnetic compression chicanes in their baseline configurations. Therefore, chicanes will continue to be the workhorse for bunch compression. With the high peak current requirement of current and proposed FELs, CSR can drive instabilities and thus reduce the beam quality. A complete map of CSR and its limits are much needed. The unique combination of a multi-MHz class superconducting linac with high brightness electron beams at ASTA and its diagnostics will allow us to reach a wide dynamic range in our measurements.

\section{A.3.4.1 Planned Work and Method of Measurement}

The heart of the project consists of a 4-dipole chicane followed by a vertically deflecting dipolemode cavity and a spectrometer - bending in x-plane. The schematic of the complete beamline is shown in the Figure 1 below. The beam which enters the beamline can be imprinted with an energy-position correlation by using the RF-accelerating cavity. The dipole compresses the beam longitudinally and most of the CSR is emitted at D3 and D4 where the bunch length is short[1]. After compression, the projected emittance $\left(\xi_{\mathrm{x}}\right.$ and $\left.\xi_{\mathrm{y}}\right)$ of the beam is measured using a standard multi-slit-method (shown as dotted vertical lines in Figure 1 ). The $x$-slice-emittance is measured using a vertical deflecting mode cavity and a quad scan. The combination of the vertical deflecting cavity (TDC) and the x-plane spectrometer is then used to measure the longitudinal phase space and slice-energy spread.

The emittance of the beam before entering the chicane will be measured by using a straight beamline by turning off the chicane. Both the $x$-slice emittance and slice energy spread will be measured. The longitudinal phase space will also be measured before the beam enters the chicane. After it traverses the chicane, a complete measurement of the $x$-slice emittance, $y$-emittance and longitudinal phase space will be done. This will complete the diagnostic of the systems and should give us the major electron beam parameters that we are pursuing in this project. 


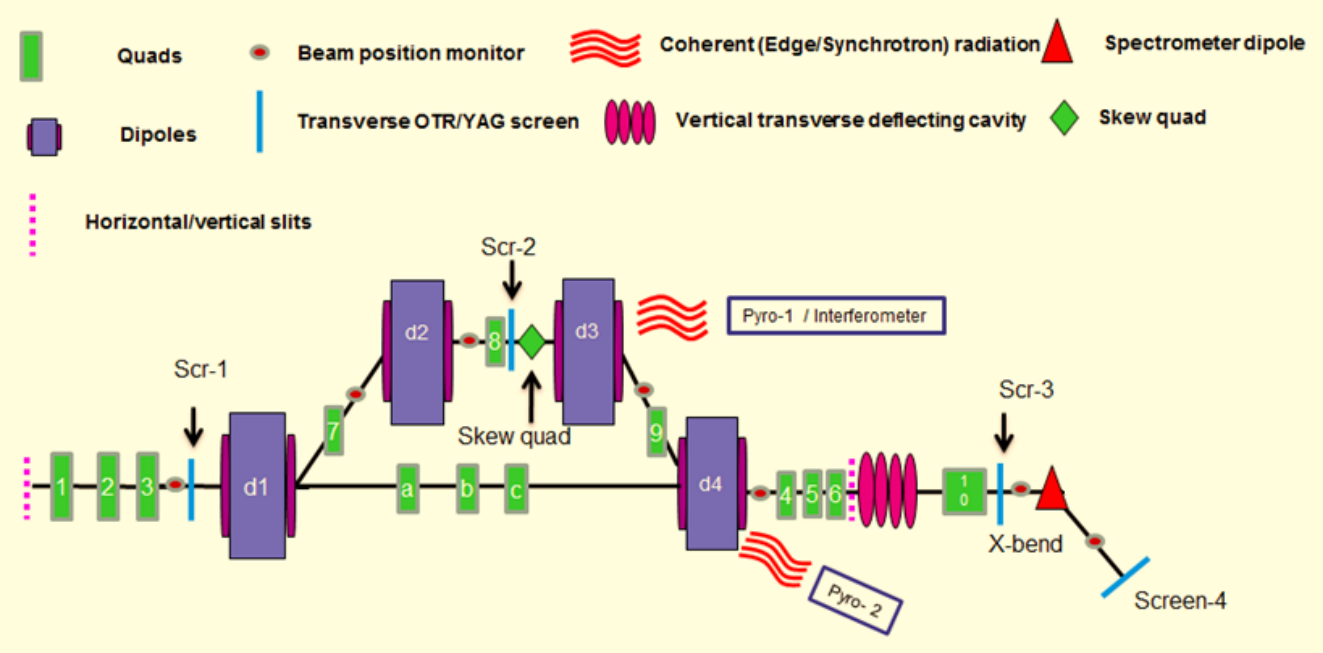

Figure 1: The beamline that will be built to study all possible aspects of CSR. The beam from the left can either be directed to a straight section (through quads $a, b, c$ ) or could be sent through the chicane. The beamline will consists of diagnostics that will also precise and complete measurement of beam and machine parameters. The skew quad in the middle of the chicane will function as a vertical deflector during the starting phase of the project. The combination of the vertical deflecting cavity followed by the $\mathrm{X}$-bend will reveal the longitudinal phase space of the bunch. The projected $x$ and $y$ emittance will be measured using $x / y$ multi-slits (shown as dotted vertical lines). THz radiation from dipole 3 and dipole 4 will be captured by pyros and the bunch length can be determined using an interferometer as well.

\section{A.3.4.2 Three-Dimensional Particle-in-Cell Simulation Showing Transverse Energy Loss Dependence}

In order to show the importance of 3-D effects of CSR inside a dipole, we present preliminary results from a full 3-D electromagnetic simulation of the proposed chicane that takes into account all aspects of the electromagnetic fields inside the chicane such as transverse space charge, longitudinal space charge and, finite dimensions of the beam in all co-ordinates (6-dimensions). The non-bending plane is not ignored [2]. We report on both the transverse and the longitudinal effect of CSR on the beam for our case as shown in Figure 2 below. The main message of the simulation is that the transverse size of the electron beam plays a significant role in affecting CSR energy loss. Particles of energy loss/gain are diagonally opposite to each other indicating that particles have quite different energy loss/gain histories. While 1D CSR treatments such as ELEGANT can reveal overall energy loss, a full 3D simulation can resolve transverse energy loss and the increase in energy spread. Our project will unlock such details with both simulation and the experiment. Given that CSR typically happens in the scale lengths of bunch length (100's of microns) while the interaction length can occur over orders of meters, $\mathrm{BF}$ modeling is a great fit for our problem[3]. The BF allowed us to resolve transverse dynamics 
of coherent synchrotron radiation in our chicane within a reasonable computational time ( 2 hours in an 8 core CPU). The simulations were done using Particle-In-Cell code WARP[4]. The normalized transverse emittance was 0.5 microns, with peak current $100 \mathrm{~A}$, the bunch length was $1 \mathrm{ps}(\sim 0.3 \mathrm{~mm})$ and the initial slice energy spread was $10 \mathrm{keV}$.

\section{A.3.4.3 Anticipated Benefits}

Upon completion of these studies, beamline designers will have an accurate understanding on the behavior of CSR in a chicane in all of its aspects. We will deliver a complete parametric study of a chicane in a superconducting linac with most of the beam parameter space measured with precision. Most of the parameters (emittance in all three planes, spot size, bunch length, charge, beta-function, slice x-emittance, slice energy spread, and RF chirp) will be captured before and after the chicane. The spot size inside the chicane, bunch length inside the chicane, $\mathrm{THz}$ radiation and the energy loss will also be made available. A relevant comparison with the existing CSR simulation codes will be carried out and presented. ASTA has a unique feature that sets it apart from other accelerator facilities for our proposal: record high average electron beam power and beam brightness, which allows us to reach wide dynamic-range of beam parameters.

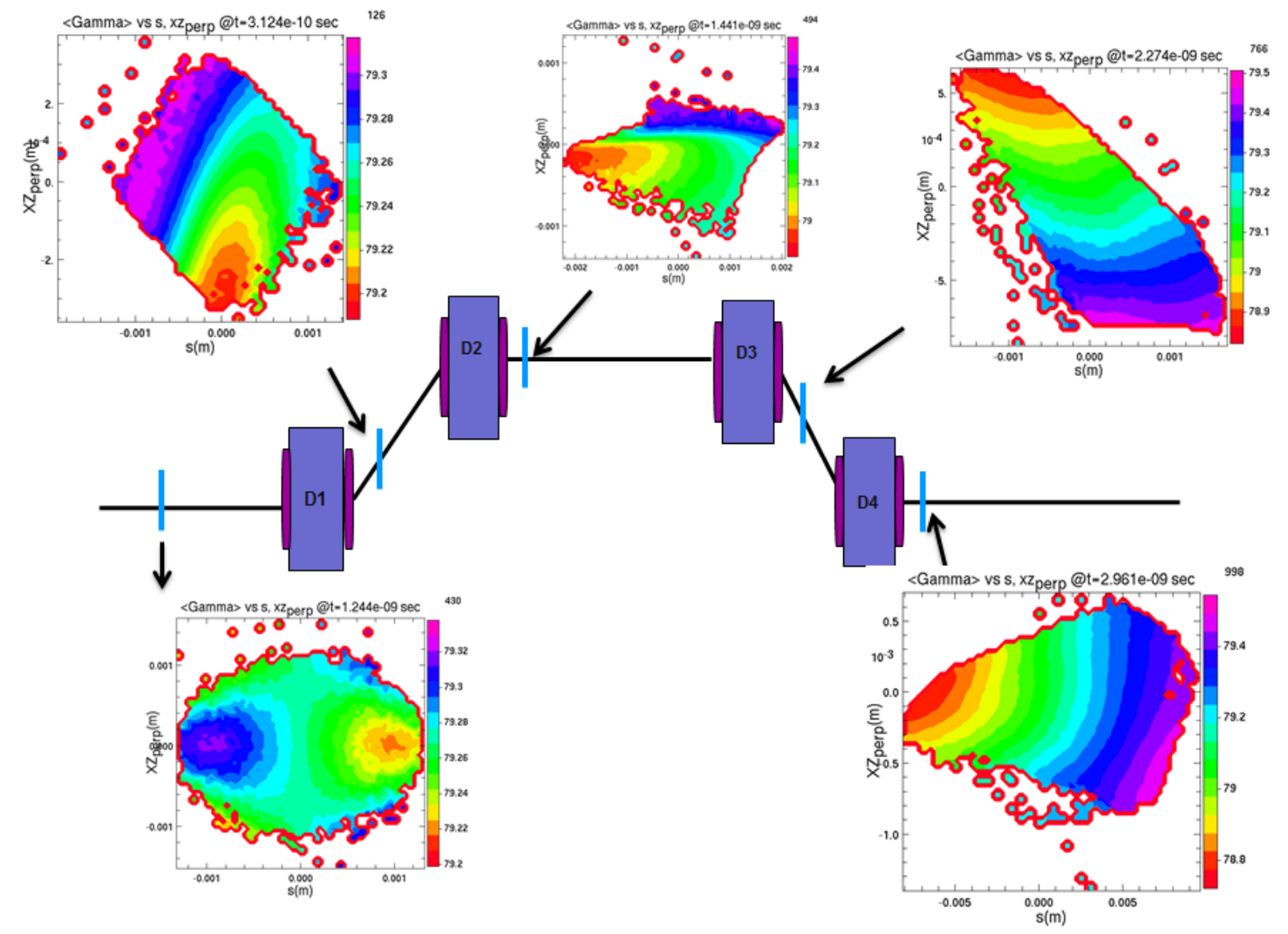

Figure 2: Overview of the four-bend chicane bunch compressor. The density plots correspond to the simulated evolution of the beam density distribution along the chicane in the bending plane [horizontal and vertical axis on the density plots correspond respectively to axial and horizontal coordinates]. 
False color image showing mean energy of the beam at various locations inside a chicane. The $x$-axis of each plot is the longitudinal position $(z)$ in $m$ and the $y$-axis of each plot is beam transverse radius $(x)$ in $m$. The key message of this plot is the transverse distribution of energy loss and transverse beam size plays a crucial role in CSR induced transverse emittance growth. The red colored particles are at a lower energy compared to magenta colored particles. The regions of red and magenta are diagonally opposite to each other implying that outer and inner particles (with respect to the arc motion) have different energy loss/gain profile. At the

entrance just before D1, the space-charge induced energy distribution due to the drifting of the particle beam is seen.

\section{A.3.4.4 References}

[1] C. Prokop, P. Piot, and M. Church, "Beam Dynamics Simulations for the low-energy magnetic bunch compressor at ASTA," FERMILAB-TM-2533-APC (2012).

[2] J. Thangaraj, and J. L. Vay, "3-D Electromagnetic Simulation of Coherent Synchrotron Radiation inside a Chicane using Lorentz-Boosted modeling," to appear in the Proc. IPAC'13, Shanghai (2013), page .

[3] J. L. Vay, et al., "Full electromagnetic simulation of coherent synchrotron radiation via the Lorentz-boosted frame approach," Proc. IPAC'10, Kyoto (2010), page 1874.

[4] D. P. Grote et al., "The warp code: modeling high intensity ion beams," AIP Conference Proceedings 749 (2005). 


\section{A.4 Other Studies at ASTA}

\section{A.4.1 Nano-Crystal Channeling Acceleration Experiment at ASTA}

\section{A.4.1.1 Introduction and Goals of the Experiment}

Plasma acceleration provides the highest acceleration gradient $\left(E_{0}=m_{e} c \omega_{\mathrm{p}} / e \approx 100 \times n_{0}{ }^{1 / 2}\right.$ $[\mathrm{GeV} / \mathrm{m}]$, where $n_{0}$ is the ambient plasma density $\left(n_{0}\left[10^{18} \mathrm{~cm}^{-3}\right]\right)$, corresponding to $30-100$ $\mathrm{GeV} / \mathrm{m}$. The density of charge carriers (conduction electrons, $n_{0} \sim 10^{20-23} \mathrm{~cm}^{-3}$ ) in solid media is $3 \sim 5$ orders of magnitude higher than those in gaseous plasma, so in principle, crystals can create an accelerating gradient of $\sim 300 \mathrm{GeV} / \mathrm{m}-30 \mathrm{TeV} / \mathrm{m}$. In the solid plasma, particles, as escaping from a driving field due to fast pitch-angle diffusion resulting from increased scattering rates, must be accelerated along major crystallographic directions. This is called "channeling acceleration". Wakefields in crystals can be excited by two sorts of driving source: x-ray laser (Fig. 1(a)) or short electron bunch (Fig. 1(b)). With the x-ray pumping method [1], a crystal channel can hold $>10^{13} \mathrm{~V} / \mathrm{cm}$ transverse and $10^{9} \mathrm{~V} / \mathrm{cm}$ longitudinal fields of diffracted traveling EM-waves. However, to hold the ultimate gradients, the acceleration requires coherent hard $x$-ray ( $\hbar \omega \approx 40 \mathrm{keV}$ ) of $\geq 3 \mathrm{GW}$ to compensate for radiation losses, which exceeds those conceivable as of today. The x-ray driving method thus fits for heavy particles, e.g. muons, protons, that have relatively smaller radiation losses. For electrons, the beamdriven acceleration is more favorably applicable to channeling acceleration as their energy losses can be transformed to acceleration energy [2,3]. The highly intensive plasma interaction in a crystal channel induces thermal radiations and collisional impacts accompanying with a large amount of heat energy, which would exceed the ionization thresholds and may even possibly destroy the atomic structure. Only disposable forms of crystals such as fibers or films are possibilities to be used for channeling acceleration. Also, lattice structures of crystals have fixed atomic dimensions, which thereby have some limits in designing acceleration parameters to mitigate physical constraints in solid plasma channel. Carbon nanotubes (CNTs) have various advantages for channeling acceleration over crystals [4]; a size of the channel is readily controllable up to sub-micron. The larger size of the unit-cell can also decrease de-channeling rates and increase acceptance angles. CNTs are comprised entirely of $s p 2$ bonds, which are extremely stable and thermally and mechanically stronger than crystals, steels, or even diamonds ( $s p 3$ bond). Nanotubes thus have a higher probability of surviving in extremely intense channeling, radiation, and acceleration environment. SLAC-FACET already demonstrated an energy gain of more than $42 \mathrm{GeV}$ in a $85 \mathrm{~cm}$ plasma accelerator [5] (Fig. 1(c)). The similar experiment, but with solid plasma channels, can be executed at the ASTA. The main goal of this experiment is to detect a measurable energy gain from the electron bunches passing through CNT structures (Fig. 1(d)). Successful demonstration of the experiment will verify the possibility of the ultra-high gradient acceleration scheme and pave the way of developing the laser-driven channeling acceleration as well. The experimental setup will be accommodated in the $50 \mathrm{MeV}$ main stream beam line, but the $50 \mathrm{MeV}$ user area and higher energy (300 MeV) beamline are also under consideration.

Proposal for an Accelerator R\&D User Facility at Fermilab’s Advanced Superconducting Test Accelerator 


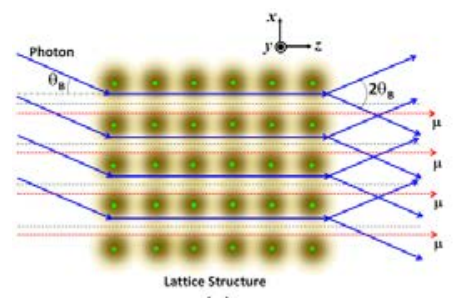

(a)

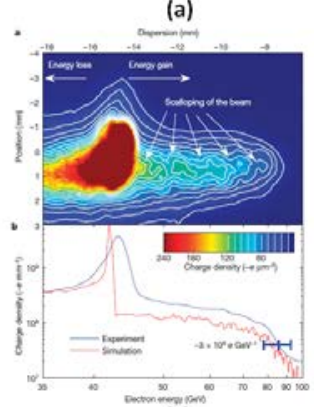

(c)

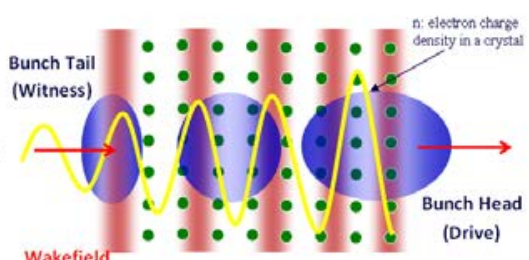

(b)

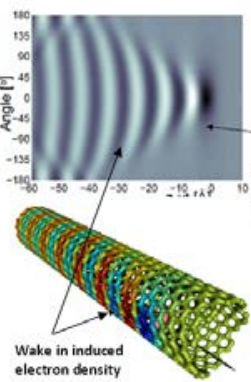

electron density

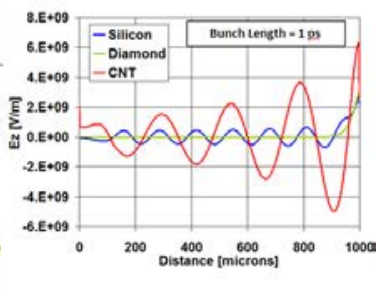

(d)

Figure 1: (a) Laser (x-ray) driven acceleration (muon, proton); (b) beam-driven acceleration (electron); (c) energy spectrum of the electrons (FFTB @ SLAC) (top) energy spectrum of the electrons in the $35-100 \mathrm{GeV}$ range. (head: $43 \mathrm{GeV}$, tail: $85 \mathrm{GeV}$ ) (bottom) projection of the image in (top), shown in blue (the simulated energy spectrum is shown in red); and (d) electron density distributions in (top) phase-space and (bottom) on the 3D CNT model (R) the simulated wakefields in silicon, diamond, and CNT with a 1 ps bunch.

\section{A.4.1.2 Required Beam Parameters}

A plasma density $\left(n_{\mathrm{p}}\right)$ of $10^{18} \sim 10^{22} \mathrm{~cm}^{-3}$, readily obtainable from CNTs, generates $0.1-10$ $\mathrm{TeV} / \mathrm{m}$ acceleration gradients with a plasma frequency of $10 \sim 1000 \mathrm{THz}(1-100 \mathrm{fs})$, which corresponds to $0.3 \sim 30 \mu \mathrm{m}$ plasma wavelength $\left(\lambda_{\mathrm{p}}\right)$. Nanotubes can be readily fabricated to be perfectly straight within a hundred micrometer range. We will first test $50 \sim 100$ micron long nanotubes with $n_{\mathrm{p}} \sim 10^{19} \mathrm{~cm}^{-3}$ that will offer $\sim 10 \mathrm{MeV}$ energy gain in principle. If an energy gain is not observed with the sample thickness, the test will be repeated with increasing sample thickness. For the initial experiment, we will test a nano-holed substrate embedded with 50 $100 \mu \mathrm{m}$ long multi-wall CNTs $\left(\lambda_{\mathrm{p}} \sim 10 \mu \mathrm{m}\right)$, which will lead to wakefields of $\sim 0.3 \mathrm{TeV} / \mathrm{m}$. The plasma wakefield generation in the linear regime requires a bunch-to-bunch distance $(\Delta z) \leq \lambda_{p}$. The 1 ps bunch is 30 times longer than the plasma wavelength, which may lower the transformer ratio and efficiency. We will test $\leq \sim 10 \mu \mathrm{m}$ long micro-bunches and compare it with test results of the 1 ps electron bunch. 


\begin{tabular}{|c|c|c|}
\hline Beam Parameter & Value/Range & Units \\
\hline Beam Energy & 50 & $\mathrm{MeV}$ \\
\hline Bunch Charge & 3.2 & $\mathrm{nC}$ \\
\hline Bunch repetition rate & $1 \sim 10$ & $\mathrm{~Hz}$ \\
\hline Number of bunch/macropulse & $1 \sim 100$ & \\
\hline Number of microbunch/bunch & $10 \sim 20$ & $\mathrm{ps}$ \\
\hline Bunch duration & 1 & $\mu \mathrm{m}$ \\
\hline Bunch length & 300 & $\mu \mathrm{m}$ \\
\hline Microbunch Length & $<10$ & $\mu \mathrm{m}$ \\
\hline Microbunch spacing & 10 & $\mathrm{~mm}-\mathrm{mrad}$ \\
\hline Transverse emittance & $\leq 1$ & $\mathrm{mrad}$ \\
\hline Divergence & $\leq 1$ & $\mu \mathrm{m}$ \\
\hline Spot size & 10 & $\%$ \\
\hline Fractional momentum spread & $0.01 \sim 0.05$ & \\
\hline
\end{tabular}

\section{A.4.1.3 Personnel Involved}

Currently five team members are involved in the proposed experiment. Y. M. Shin will be overseeing schedules and preparing experiment with data analysis and simulation. Tao Xu will develop CNT fabrication processes and provide test samples. J. C. Thangaraj will be engaged in the design/test of a micro-buncher, including beam diagnostics and data analysis, and $\mathrm{V}$. Shiltsev will assist to schedule/plan the experiment and data analysis with technical consultation. D. Still will support the system setup and instrumentation. A. Grabenhofer, a second year graduate student at Northern Illinois University, will assist in the experimental setup and simulation analysis, including data acquisition, which may be a part of a dissertation topic for his master degree.

\begin{tabular}{|c|c|c|c|c|c|}
\hline Personnel & Planning & Experiment & Diagnostics & Analysis & Simulation \\
\hline Y. M. Shin & $\mathrm{V}$ & $\mathrm{V}$ & & $\mathrm{V}$ & $\mathrm{V}$ \\
\hline Tao Xu & & $\mathrm{V}$ & & & \\
\hline J. C. Thangaraj & & & $\mathrm{V}$ & & \\
\hline V. Shiltsev & $\mathrm{V}$ & & & $\mathrm{V}$ & \\
\hline D. Still & & $\mathrm{V}$ & & & \\
\hline A. Grabenhofer & & & & $\mathrm{V}$ & $\mathrm{V}$ \\
\hline
\end{tabular}




\section{A.4.1.4 Experimental Technique and Expected Outcomes}

As we plan to conduct the first experiment at $50 \mathrm{MeV}$ beamline, test equipment will be installed downstream the Capture Cavity-2 and bunch compressor before the $1^{\text {st }}$ cryomodule. Figure 2 shows the beam line drawing, describing the currently planned location of the proposed experiment. The boxed areas (Area-1 and Area-2) indicates prospective locations of the vacuum-compatible goniometer to be loaded with a test sample. With the given schematic in our plan, $1 \sim 2$ ps photo-electron bunches with $\sigma_{\mathrm{x}}=1 \sim 2 \mathrm{~mm}$, emitted from $1.3 \mathrm{GHz}$ RF-gun, are compressed by the quadrupoles through the bunch compressor (BC). Once the beam is compressed down to $\sigma_{\mathrm{x}}=10 \mu \mathrm{m}$, it will be injected into a CNT test sample in the goniometer. While passing through the $B C$, the slit-mask placed in the $B C$ produces microbunch trains by imprinting the shadow of a periodic mask onto a bunch with a correlated energy spread. Once passing through a test device in the goniometer, beam energies will be measured by a spectrometer with a dipole magnet and YAG screen positioned in the beamline toward a beam dump. Two goniometers, previously used for the T980 experiment at Tevatron, are currently available and have already been moved to ASTA from DO and are waiting for the radioactive cleaning procedure. Their control system, including electronics for remote control and measurement, will be moved from the Tevatron EO area and installed at the $50 \mathrm{MeV}$ control deck. Most probably, the first test will use another goniometer (Fig. 2, Area-1) that is scheduled to be installed for the ASTA first experiment (x-ray channeling radiation test) [6]. We will continuously test samples with one of our goniometers, after being completely set up at the 50 MeV beamline, which will require two additional vacuum gate valves and two transition beampipe adapters. They all should go through radioactive-UHV cleaning procedure and be stored in a particle-free atmosphere in order to be compatible with vacuum level $\left(<10^{-9}\right.$ Torr) required for low energy beamline operation. Also, a cross for a slit-mask will need to be installed in the $\mathrm{BC}$ beamline.

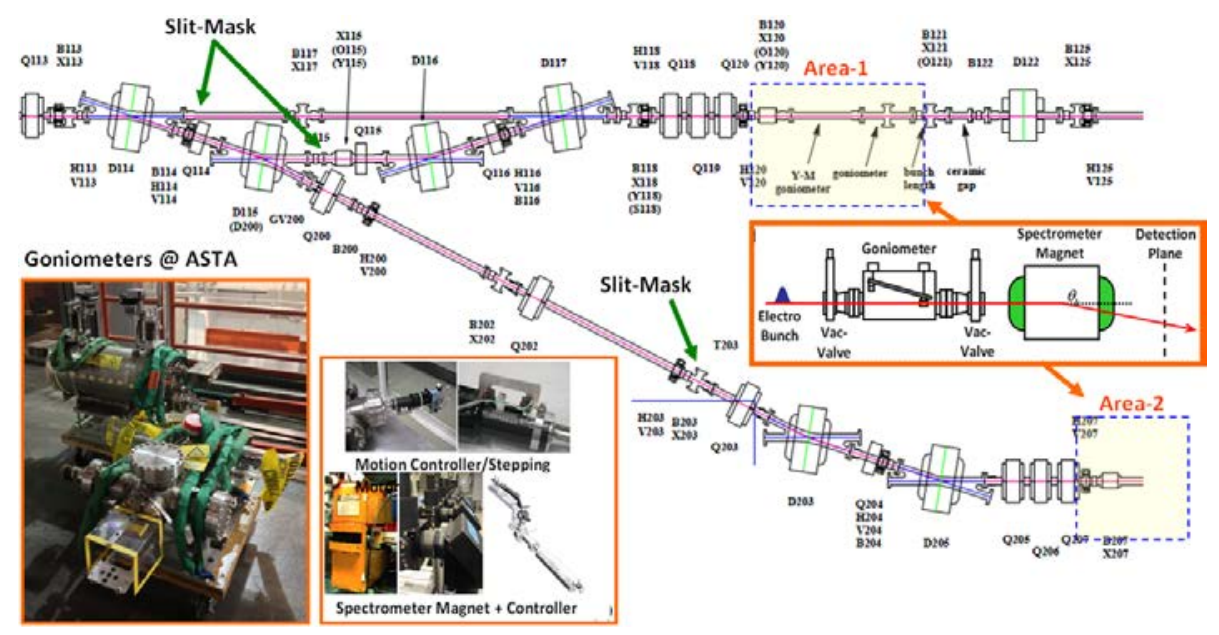

Figure 2: ASTA $50 \mathrm{MeV}$ beamline drawing with planned locations of goniometers (Area-1 or Area-2) and slit-mask micro-buncher, including experiment layout, and equipment/hardware: $\mathrm{H}-/ \mathrm{V}$-goniometers, control system, and spectrometer magnet. 


\section{(a) Test Device Preparation and Acceleration Test Configuration}

Two types of channeling accelerator structures, crystals (silicon or diamond) and arrayed CNT bundles, will be manufactured and tested as a first experiment, for comparison. Crystal samples will be possibly obtained by the similar fabrication technique used for silicon samples in the T980 experiment: cleaved and cut silicon wafers [7]. The sample holder, used for the T980 experiment, will also be re-used for crystal acceleration test, but only with the straight section (no bending). A test device with a straight multi-wall CNT bundle will be prepared by Tao Xu (NIU Department of Chemistry/Biochemistry) using anodic aluminum oxide (AAO) template (Fig. 3(a)) [8]. The template carbonization method consists of carbonization of an organic gas or polymer in the nano-space of an inorganic template and liberation of the deposited carbon from the template. The AAO film has uniform and straight nano-size channels with a tailored length and diameter (Fig. 3(b)). The length, diameter, and density of the as-synthesized CNTs can be uniformly tailored because of the controllability of the pore texture of AAO template. Furthermore, the wall thickness and crystallinity of the CNTs can be controlled by adjusting CVD conditions. We plan to test a $100 \mu \mathrm{m}$ long, $200 \mathrm{~nm}$ wide CNT structure first, which may possibly be manufactured within $2 \sim 3$ months. It is expected that these types of crystal channel induce plasmonic waves of $\lambda_{p} \leq \sim 10 \mu \mathrm{m}$. A bunch length of a driving beam should be less than $\lambda_{p}$, so multiple bunch acceleration in the nano-channel will be tested to investigate improvement of acceleration gradient and efficiency. When using multiple bunches (i.e. micro-bunches), by controlling their position and charge, the accelerator can be tuned to either maximize wakefield, transformer ratio, or efficiency of the system. Figure 4 shows schematic wakefield graphs of three different options with micro-bunches for maximum (a) wakefield $\left(R_{\text {tot }}=1.11\right.$, $13 \%)$, (b) transformer ratio $\left(R_{\text {tot }}=7.89,64 \%\right)$, and (c) efficiency $\left(R_{\text {tot }}=5.14,84 \%\right)$. The $10 \mu \mathrm{m}$ long micro-bunches will induce maximum wakefield of $\sim 300 \mathrm{GeV} / \mathrm{m}\left(E_{\max }=m_{e} c \omega_{p} / e\right)$ in the nano-channel $\left(\mathrm{n}_{0} \sim 10^{19} \mathrm{~cm}^{-3}\right)$. The longitudinal and transverse components of the wakefield accelerates and focuses the bunches respectively. With maximum longitudinal field transformer ratio $R_{z, \max } \sim 13 \%\left(W_{z} \sim 40 \mathrm{GeV} / \mathrm{m}\right)$, this wakefield will lead to $\sim 4 \mathrm{MeV}$ energy gain from the $100 \mu \mathrm{m}$ long channel. Taking into account radiation losses, $d E / d s \cong 5.7 \mathrm{MeV} / \mathrm{m}\left[\gamma / \gamma_{\mathrm{f}}\right]^{3 / 2} \sin ^{2} \phi$, where $\phi$ is the phase along the plasma wave, and all other losses from collisions and scatterings, an ultimate energy gain $(\sim 5 \mathrm{MeV})$ will be $\leq 10 \%$ of injection beam energy (50 $\mathrm{MeV})$. This is within the measurable range of the magnetic spectrometers to be installed in the ASTA beamline.

Proposal for an Accelerator R\&D User Facility at Fermilab's Advanced Superconducting Test Accelerator 


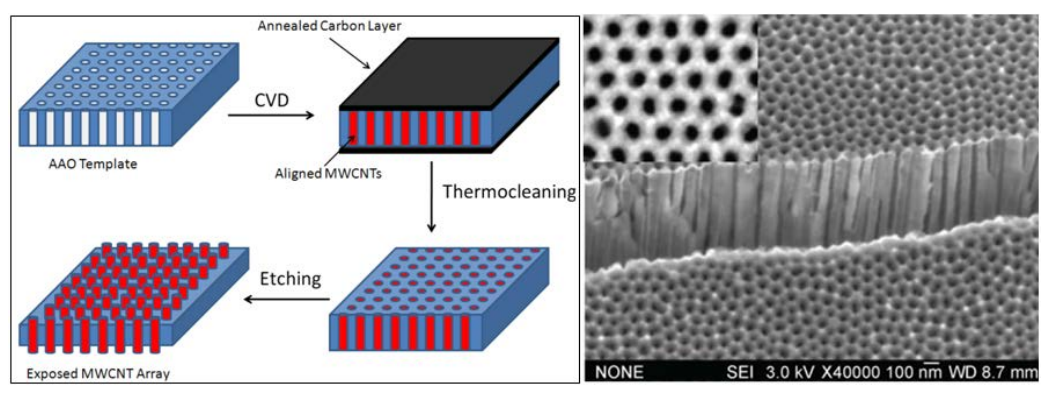

(a)

(b)

Figure 3: (a) Schematic diagram of an AAO template process; and (b) SEM image of an AAO.
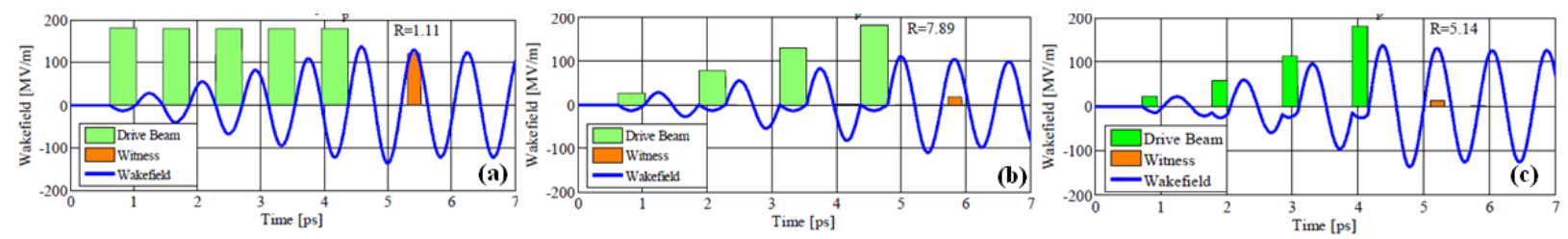

Figure 4: Plasma-beam phase matching conditions for (a) maximum wakefield (bunch separation $\sim \lambda_{\mathrm{p}}$ ); (b) maximum transformer ratio (bunch separation $\sim 1.5 \lambda_{\mathrm{p}}$ ); and (c) maximum efficiency (bunch separation $\sim 1.28 \lambda_{p}$ ).

\section{(b) Generation of Multiple Micro-Bunches}

For micro-bunch generation, we mainly consider the slit-mask technique, which is the simplest way to create multiple bunches within sub-picosecond (ps) scale. This idea is a proven technique as the Brookhaven National Laboratory (BNL) Accelerator Test Facility (ATF) has already demonstrated the generation of a stable train of microbunches with a controllable subpicosecond delay (Fig. 5(a)) [9]. The shadow of the mask is converted into a time pattern when entering the dispersion-free region of the beam line. We will measure this time pattern using coherent transition radiation (CTR) interferometry. This kind of simple method can be very easily implemented either in the $50 \mathrm{MeV}$ chicane beam line (D114 - D115) or in the $50 \mathrm{MeV}$ user beam line (D115 - D203), depending on a location of the goniometer. In the bunch compressor in the $50 \mathrm{MeV}$ beamline, designed with bending angle $(\alpha)$ of $18^{\circ}$ and $R_{56} \sim-0.18 \mathrm{~m}$ (bending radius $\sim 0.78 \mathrm{~m}$ ), the mask with multi-slit array is designed with $D$ (period) $=100 \mu \mathrm{m}$ and $d$ (aperture width) $=50 \mu \mathrm{m}$ (50\% transparency). In the linac, the beam is accelerated off the crest of the RF wave in order to impart a correlated energy spread on the bunch $(\Delta E / E \sim \pm$ $(0.01-0.05) \%)$. The bunch length, $\sigma_{z, \text { in }}=\left(\left(1+\kappa R_{56}\right)^{2} \sigma_{z 0}{ }^{2}+\left(R_{56}\left(\Delta E / E_{0}\right)\right)^{2}\right)^{1 / 2}(\kappa$ : linear chirp), compressed from the $50 \mathrm{MeV}$ (mean) and $300 \mu \mathrm{m}$ long electron beam, is divided by the number of microbunches $\left(N_{\mathrm{b}}=\sigma_{\mathrm{x}} / D\right.$, where $\sigma_{\mathrm{x}}=\left(\sigma_{\mathrm{x} 0}{ }^{2}+\left(\eta_{\text {mask }}\left|\Delta E / E_{0}\right|\right)^{2}\right)^{1 / 2}: \eta_{\text {mask }}$ is the 
dispersion at the mask) in the full bunch train. With $\leq \sim 1 \mathrm{~mm}$-mrad $\varepsilon_{\mathrm{n}}$, the designed mask generates a bunch-to-bunch spacing $\left(\Delta \mathrm{z}=\sigma_{\mathrm{z}} / N_{\mathrm{b}}\right)$ larger than the micro-bunch length, $\sigma_{\mathrm{z}, \text { out }}=((1$ $\left.\left.+\kappa R_{56}\right)^{2} \sigma_{\mathrm{z}, \mathrm{slit}}{ }^{2}+\left(R_{56}\left(\Delta E / E_{0}\right)\right)^{2}\right)^{1 / 2}$, where $\sigma_{\mathrm{z}, \mathrm{slit}}=\sigma_{\mathrm{x}, \mathrm{slit}}=D-d=50 \mu \mathrm{m}$. It will convert the $300 \mu \mathrm{m}$ long bunch to $\sim 10 \mu \mathrm{m}$ spaced microbunches after the chicane, which is injected to a nanochannel in the goniometer.

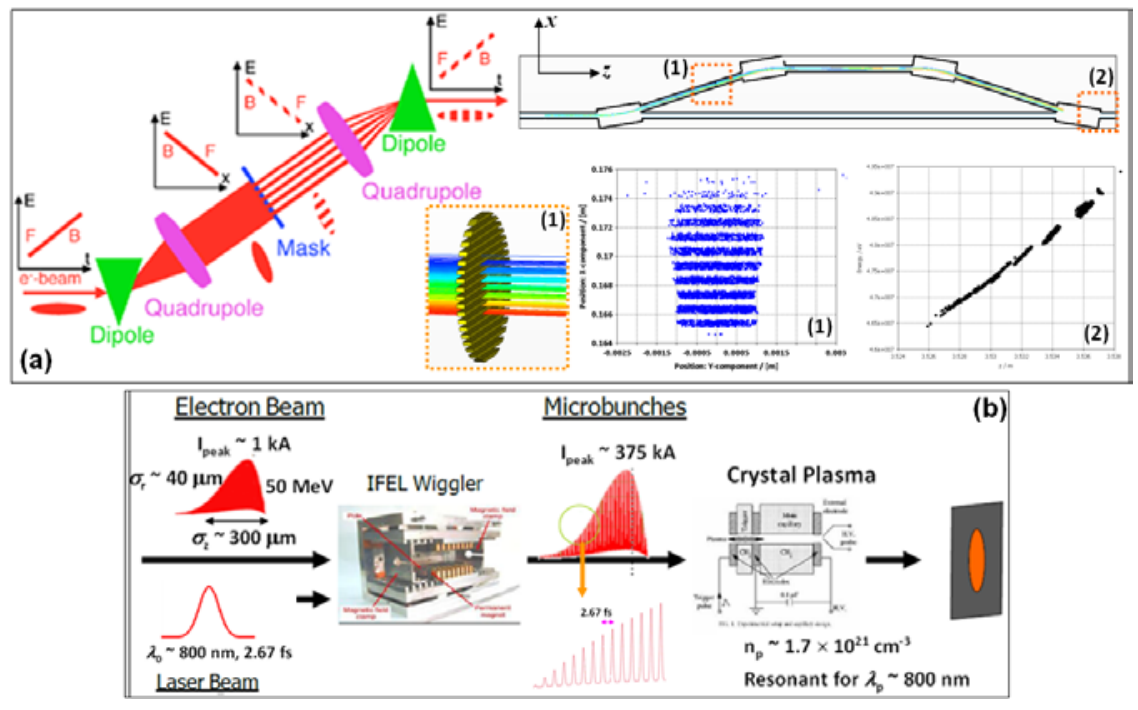

Figure 5: (a) Schematic drawing of slit-mask micro-buncher and beamline simulation (Energy versus $x$ and $z$ at (1) and (2); and (b) inverse-FEL undulator micro-buncher.

We also consider utilizing the inverse free electron laser process to create microbunches. The longitudinally sinusoidal-dependent static magnetic field inside the wiggler causes a perpendicular force on the beam electrons and initiates electron motion in the transverse plane. Using this transverse motion, the electron beam can be coupled to the laser pulse (by tuning the wiggler field period) such that some electrons feel the decelerating electric field of the laser and some electrons feel the accelerating field. This picture of half-acceleration and half-deceleration repeats over every laser period along the beam length and results in a velocity modulation of the beam electrons. After the exit of the wiggler the beam is left to propagate in vacuum over some specific distance, after which the low-velocity electrons have been overtaken by the high-velocity electrons thus creating microbunches, separated at the laser's wavelength. Currently, a laser-induced microbunching (LIM) scheme with the FEL undulator is proposed in the ASTA accelerator stewardship program [10], which may be potentially utilized to provide microbunches to the channeling acceleration test. 


\section{(c) Diagnostics and Measurement System}

The proposed experimental scheme will need several positions to monitor bunch profiles and measure their spatiotemporal radiation pattern and energy spectrum of accelerated beams. We measure time pattern of a masked beam entering the dispersion-free region of the beam line using coherent transition radiation (CTR) interferometry. The broadband transition radiation emitted by the electrons when entering a copper mirror placed after the dogleg, near the experimental region, is sent to a Martin-Puplett interferometer. Figure 6 shows a schematic diagram of the experimental arrangement used for acceleration measurements. After the energy-analyzed and collimated beam of electrons is transported through the bunch compressor into the experimental area, it is defocused by an asymmetrically split quadrupole triplet to give a low-divergence (nearly parallel) beam incident upon the crystal in its goniometer. A critical factor in performing channeling acceleration experiments is the divergence of the incident beam. Since the characteristic angle for the process is $1 / \gamma$, an angular resolution at least an order of magnitude smaller is required in order to obtain data of sufficient precision to compare with the results of theoretical calculations; for $\gamma \sim 100$, a beam divergence larger than $1 \mathrm{mrad}$ is inadequate. Moreover, the critical angle for channeling is a few mrad for electrons of a few tens of $\mathrm{MeV}$, and varies as $\gamma^{-1 / 2}$; therefore, in order that a large fraction of the beam be channeled, a beam divergence $\leq 1 \mathrm{mrad}$ is required. The experimental arrangement is used for obtaining a very-low-divergence beam, which is used as well for measurements of the transmission of electrons through crystals. The $22.5^{\circ}$ dipole upstream of the dump will serve as the low energy spectrometer. The $50 \mathrm{MeV}$ beam dump will be capable of absorbing up to 400 Watts of beam power.

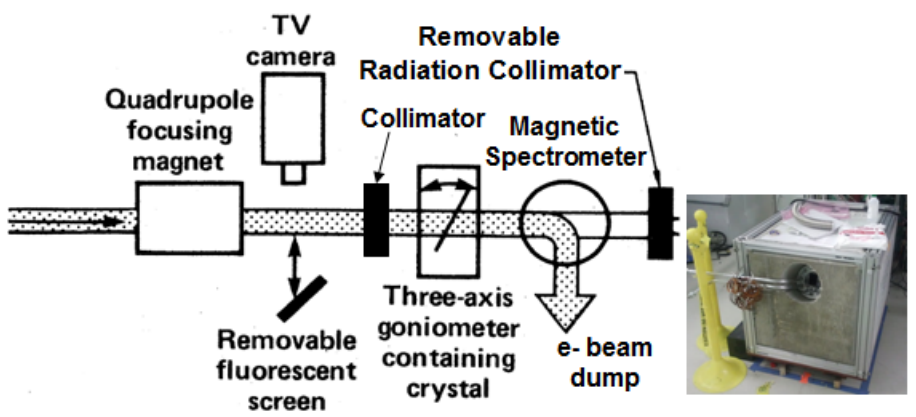

Figure 6: Schematic diagram of the experimental arrangement for the measurement of channeling-acceleration spectra from electrons. 


\section{A.4.1.5 References}

[1] T. Tajima, and M. Cavenago, PRL 59, 1440 (1987).

[2] P. Chen and R. J. Noble, AIP. Conf. Proc. 156, 2122 (1987).

[3] SLAC-PUB-4042 (1986).

[4] Ian Blumenfeld, et. al. SLAC-PUB-12363.

[5] M. Murakami, and T. Tanaka, APL 102, 163101 (2013).

[6] Philippe. R. G. Piot, ASTA-Proposal.

[7] http://accelconf.Web.cern.ch/accelconf/IPAC10/talks/tuoamh03 talk.pdf

[8] H. Peng Xiang, L. Chang, S. Chao, and C. Hui Ming, Chinese Science Bulletin 57, 187 (2012).

[9] P. Muggli, et. al., PRL 101, 054801 (2008).

[10] See Section A.1.6. 


\section{A.4.2 A Tagged Photon Beam at ASTA for Detector R\&D}

The efficient detection of photons in the energy range $10 \mathrm{MeV}-100 \mathrm{MeV}$ is a challenge for many experiments envisioned in the future at Fermilab. For instance, one of the most important backgrounds for a Project X experiment designed to measure the rate of $K^{0} \rightarrow \pi^{0} v \bar{v}$ [1] is the decay $K^{0} \rightarrow \pi^{0} \pi^{0}$ in which one $\pi^{0}$ decays asymmetrically and the high energy photon is lost down the beamline. A related experiment, ORKA [2], which plans to run before Project $X$, will measure the rate of $K^{+} \rightarrow \pi^{+} v \bar{v}$ using a stopped $\mathrm{K}^{+}$beam. One of the most problematic backgrounds is the decay $K^{0} \rightarrow \pi^{0} \pi^{0}$, with an asymmetric $\pi^{0}$ decay. In this case, the event kinematics ensures that the low energy gamma is at least $20 \mathrm{MeV}$. A tagged source of $\sim 20 \mathrm{MeV}$ photons would be an important resource during the development of instrumentation for these experiments.

Tagged bremsstrahlung photon beams have been used at electron accelerators for many years. The intensity of a tagged photon beam at ASTA would be limited by the requirement that the system operate in "singles mode." As shown in Figure 1 below, the spectrum of forward bremsstrahlung photons peaks at low energy. The rate of low energy photons needs to be kept low enough so that they do not create a significant background for measurements made with tagged high energy photons. An optimized tagger could require a customized magnetic spectrometer. However, it appears likely that a very inexpensive tagger could be designed using a standard ASTA dipole magnet and a small system of either scintillation counters or silicon detectors. During $50 \mathrm{MeV}$ accelerator operations, this system could tag a modest, but useful, flux of photons in the 10-50 MeV range. A wider range of photon energies would become accessible with a $300 \mathrm{MeV}$ electron beam. 


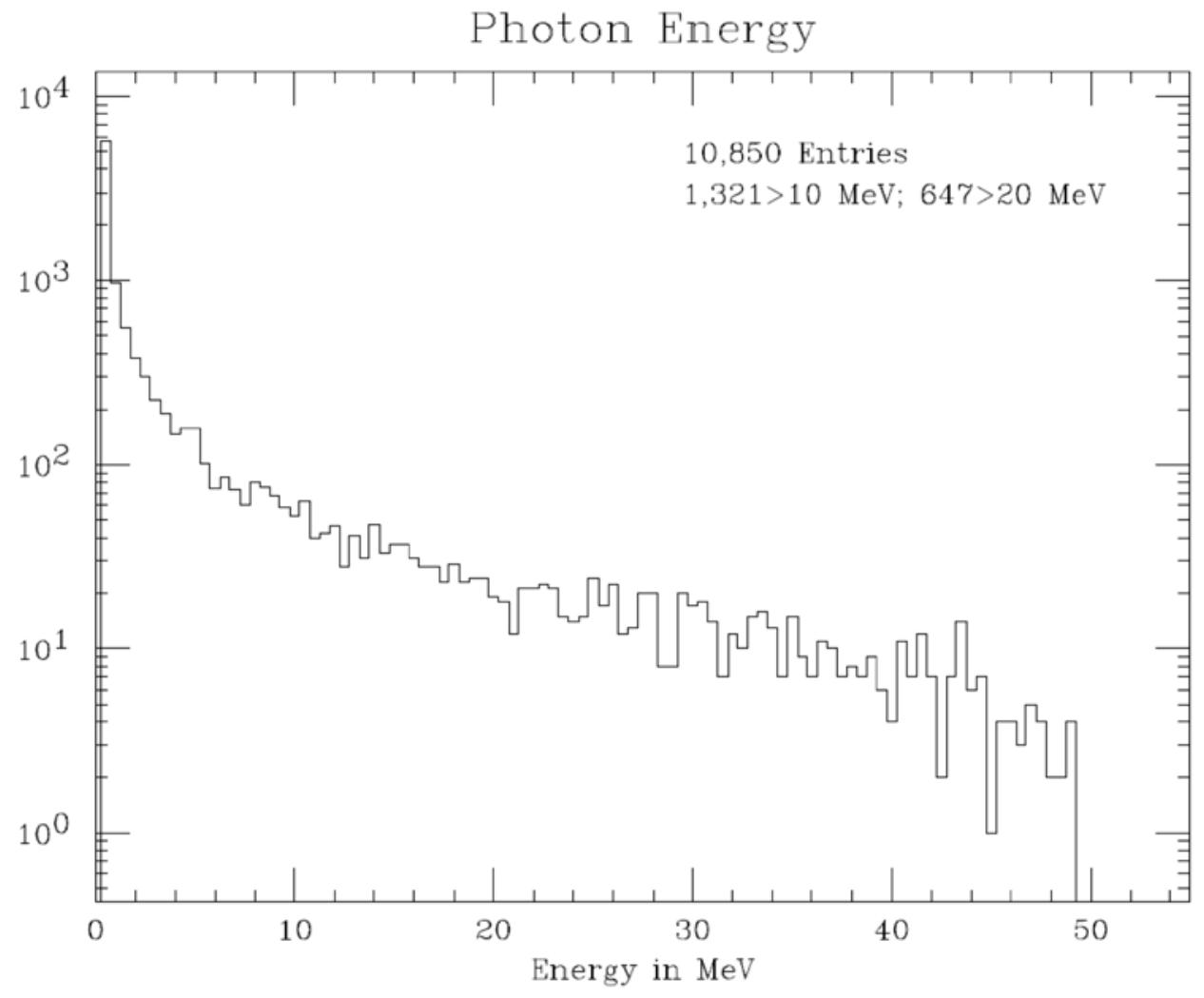

Figure 1: The energy distribution of photons produced into a forward 10 mradian cone in a G4beamline [3] simulation of 5 million $50 \mathrm{MeV}$ electrons passing through 20 microns of aluminum ( $2 \times 10^{-4}$ of a radiation length).

\section{References:}

[1] June 2012 Project X Physics Study: https://indico.fnal.gov/conferenceDisplay.py? confld=5276, "The Project X Kaon Physics Program," accessible from the Project X web site: https://indico.fnal.gov/getFile.py/access?resld=1\&materialld=0\&confld=3579.

[2] Fermilab proposal P1021, "ORKA: Measurement of the $K^{+} \rightarrow \pi^{+} v \bar{v}$ Decay at Fermilab," https://projects-docdb.fnal.gov:440/cgi-bin/ShowDocument?docid=1365.

[3] G4beamline, http://www.muonsinternal.com/muons3/G4beamline. 


\section{A.4.3 Tunable, Nearly Monoenergetic Gamma Ray Beams for Special Nuclear Material (SNM) Interrogation}

\section{A.4.3.1 Introduction and Goals of the Experiment}

We propose to use an electron beam striking a high-Z target to produce positrons. A dipoleplus-wedge monochromator then makes the positrons nearly mono-energetic. After passing through a beam transport system, the positrons annihilate in flight in a converter to produce nearly mono-energetic gammas of tunable energy. Simulation results show that the proposed system will meet the goals of this solicitation: enough intensity and flexibility to meet the scanning speed requirements for fissile materials while greatly reducing the useless dose from low-energy photons that a bremsstrahlung beam delivers. The inherent flexibility allows implementation of multiple scanning modes; for example, the system could start with a broadarea scan for fissile materials detected by delayed photo-fission gammas and/or neutrons, followed by a high spatial resolution radiographic imaging scan to "zoom in" on suspect areas of the previous scan. Due to its simplicity, the proposed system competes favorably, in terms of size, mobility, cost, ease of operation and expected reliability, with a system using Compton back-scattering to produce a beam of the same energy. Its transformational impact derives mainly from the use of positrons as an intermediary, thereby enabling the sophisticated beam manipulations that are possible with a charged-particle beam. We plan to reduce the cost of the proof-of-concept ( $\mathrm{PoC}$ ) experiment by performing it at an existing electron accelerator facility; our collaborators at PNNL will provide much of the nuclear detector expertise and will loan appropriate detector equipment and detector support paraphernalia for the experiment.

\section{A.4.3.2 Technical Approach}

Our initial work in this topic has previously been supported by contracts with Pacific Northwest National Laboratories (PNNL) to investigate the capabilities of a system based on our design concepts, using simulation and performance modeling methods. At the completion of the PNNL funding period we had produced:

- An evaluation that determined the optimum incident electron energy

- A series of results for the performance of a dipole-plus-wedge monochromator

- A preliminary design of a simple transport system

- Studies of the characteristics of the gamma ray beam for several converter materials and thicknesses

- The effect of imposing material in the gamma beam ("hardening") to further reduce the residual low energy tail in the gamma spectrum

- A layout of a preliminary version of the system that could be housed in two semitrailers.

Our objectives are now to refine our design and to prepare and complete a proof of concept experiment to evaluate the validity of our simulations and to uncover and address any issues that emerge from the experiment. 


\section{Positron Production Studies}

The positron production subsystem used in simulation studies was a $75 \mathrm{MeV}$ electron beam impinging on a $4.4 \mathrm{~mm}$ long tungsten (W) target. Those design choices were based on simulation studies that sought to optimize the production rate of useful positrons normalized to the electron beam power.

\section{Dipole-plus-Wedge Monochromator Concept}

The dipole-plus-wedge monochromator reduces the momentum spread of the positron beam based on the following ideas: (1) positrons produced near the forward direction with a given momentum in a uniform dipole field converge after a 180 degree bend to a horizontal focus, (2) positions of these horizontal foci are dispersed in momentum, with higher momentum positrons farther from the production target, and (3) a wedge-shaped energy absorber can be placed such that each positron will emerge with approximately the same momentum after it passes through the absorber. This will produce a spatially wider, but nearly monoenergetic positron beam. The concept is illustrated in Figure 1.

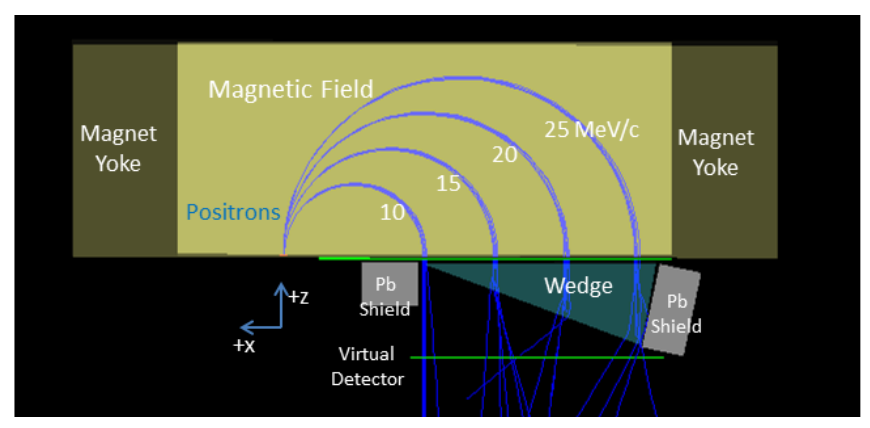

Figure 1: Illustration of dipole-plus-wedge concept. Positrons of four different momenta enter a uniform magnetic field, are bent $180^{\circ}$, and pass through a beryllium (Be) wedge that provides momentum loss proportional to distance along the wedge. The emerging positron momenta are concentrated near $10 \mathrm{MeV} / \mathrm{c}$.

The initial positron spectrum ranges up to $30 \mathrm{MeV} / \mathrm{c}$, while the spectrum after the wedge is peaked at $10 \mathrm{MeV} / \mathrm{c}$, with a small low momentum tail (which is eliminated by the transport system). Comparisons were made with momentum selection by a conventional collimator. The yields from the dipole-plus-wedge were typically a factor of 5 to 10 greater than from a collimator that provides a comparable momentum spectrum.

\section{Positron Beam Generation and Transport System}

Figure 2(a) shows a layout of the elements for the production of the gamma beam: the primary electron beam, the positron production target, the dipole-plus-wedge, the positron transport 
system elements, the positron annihilation converter, and the magnets that sweep the residual positrons away from the emerging gamma ray beam. The trajectories shown were for positrons generated by a G4beamline simulation. The transport system contains and steers the positrons emerging from the dipole-plus-wedge to the converter plate at which the gammas are produced. Positrons emerging from the wedge are rendered more parallel by the quadrupole triplet magnets Q1-Q3 and bent by the bending magnet triplet R1-S1-R2 and impinge on the gamma converter plate. After the gamma converter plate, the remaining positrons are swept away by S2 and R3 sweeper magnets. The gammas emerge from the converter plate toward the right.

Figure 2(b) shows the evolution of the positron spectra produced by $75 \mathrm{MeV}$ electrons on a 4.4 $\mathrm{mm} \mathrm{W}$ target, from the positron production target to the gamma converter plate. The curves are from a G4beamline simulation. The initial positron spectrum is somewhat similar to a bremsstrahlung spectrum, peaked at low momenta and ranging out to beyond $30 \mathrm{MeV}$. After emerging from the dipole-plus-wedge, which is set for $10 \mathrm{MeV} / \mathrm{c}$, the spectrum is peaked at 10 $\mathrm{MeV} / \mathrm{c}$, with a small residual low momentum tail. The transport system essentially eliminates the tail and narrows the $10 \mathrm{MeV} / \mathrm{c}$ peak, thus producing a nearly monochromatic positron spectrum at the gamma converter. Note that the vertical scale is logarithmic.

(a)

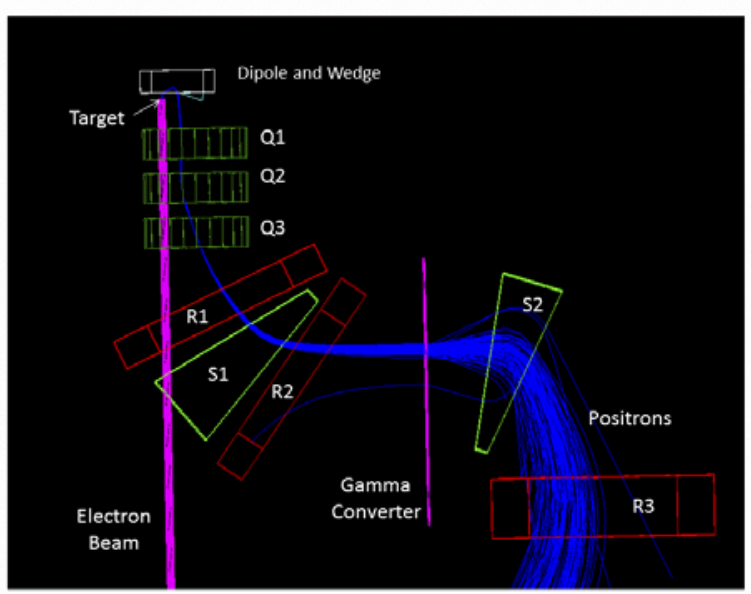

(b)

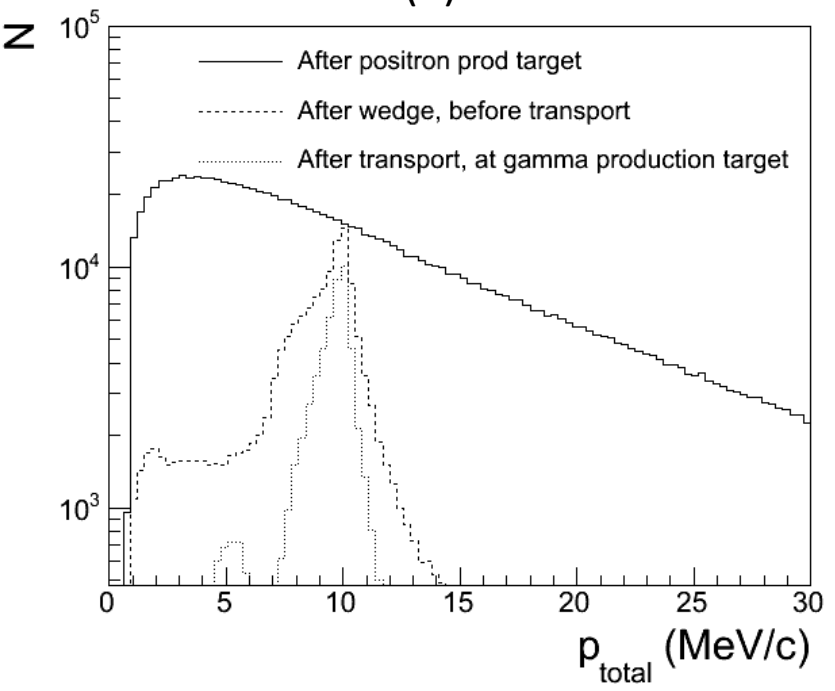

Figure 2: (a) Layout of the positron production system. Simulated positron trajectories are shown in blue. (b) Positron momentum spectra at three locations: After the positron production target, after the wedge, and at gamma converter. 


\section{Positron Annihilation Gamma Spectrum}

Figure 3 shows the expected gamma spectra for gammas resulting from the annihilation of $10 \mathrm{MeV}$ positrons and the gamma spectrum from $11 \mathrm{MeV}$ electrons striking a $\mathrm{W}$ target. The positron annihilation spectra have a much smaller number of unwanted (< $8 \mathrm{MeV}$ ) gammas than the bremsstrahlung (W) spectrum. Note again that the vertical scale is logarithmic.

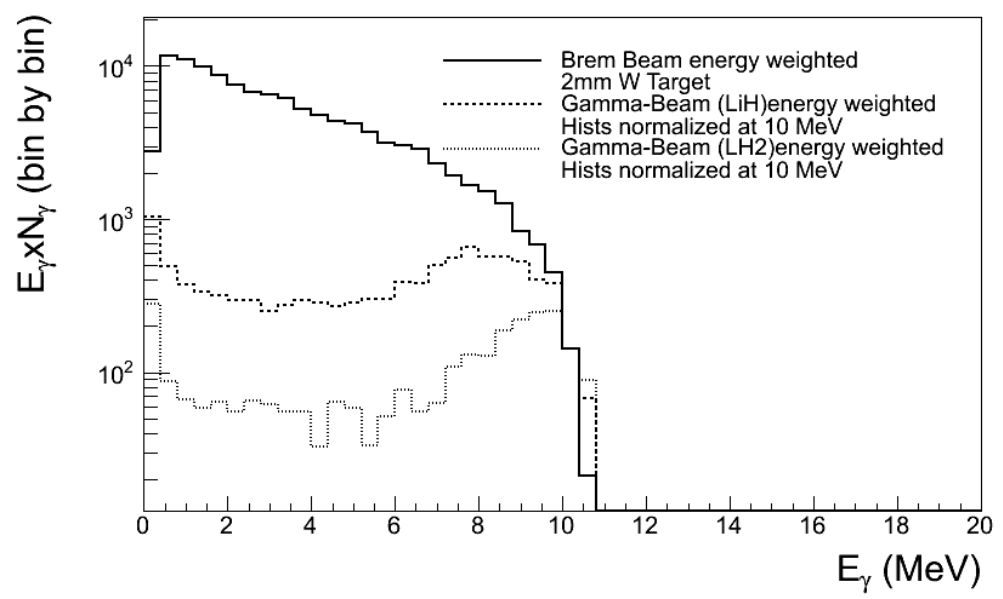

Figure 3: Gamma spectra weighted by gamma energy for $10 \mathrm{MeV}$ positrons impinging on a lithium hydride ( $\mathrm{LiH}$ ) converter, $10 \mathrm{MeV}$ positrons impinging on a liquid hydrogen (LH2) converter and for $11 \mathrm{MeV}$ electrons impinging on a tungsten (W) target, normalized at $10 \mathrm{MeV}$.

The results of Figure 3 are summarized as follows. The events are weighted by gamma energy and normalized to have equal yields at $10 \mathrm{MeV}$. We consider yields of gammas with energies $>8 \mathrm{MeV}$ to be useful, and those with energies $<8 \mathrm{MeV}$ to be unwanted. The ratios $\mathrm{R}$ of useful to unwanted are the following:

1. For electrons incident on $\mathrm{W}, \mathrm{R}=0.0456$

2. For positrons incident on $\mathrm{LiH}, \mathrm{R}=0.338$

3. For positrons incident on $\mathrm{LH} 2, \mathrm{R}=0.838$

Thus the positron annihilation useful yield to unwanted yield is a factor of 7.4 better for positrons on $\mathrm{LiH}$ than for electrons on $\mathrm{W}$, and the corresponding improvement factor for liquid hydrogen is 18 .

The design of a PoC experiment will be different from a design of a field-deployable system. The experiment will be done at an existing electron accelerator that has an appropriate energy beam, and costs will be reduced by the use of existing beam elements, detectors, and electronics as much as possible. The design of the experiment will require simulations specific to the experimental layout, and the results of the experiment will be used to refine the design of the field-deployable system. 


\section{A.4.3.3 Project Phases}

Many of the personnel of Muons, Inc. have considerable experience doing high energy physics experiments and/or developing accelerator systems, and so inevitably we will approach this experiment as if it were a combination of a small HEP experiment and an accelerator system. Our technical implementation plan is based on a systematic approach, with a logical series of development and testing phases applied separately to each functional subsystem. In this section our technical implementation plan is presented in two ways: first as a bulleted list of major tasks, with considerable detail provided for the Phase I tasks, then as a Work Breakdown Structure (WBS).

\section{- Phase I work}

o Phase I will begin by analyzing requirements, critical issues, and technical risks. The requirements that need to be defined start with the gamma beam requirements for a field-grade system and for a Proof-of-Concept (PoC) experiment. Working backwards, those requirements then dictate corresponding requirements on the positron beam, the monochromator, the target, and the electron accelerator. Other considerations include the level of backgrounds that are tolerable; that will determine the required level of shielding around the subsystem components and emanating from the beam stop.

o To determine whether the proposed experiment is feasible, concepts about how to do the experiment must be developed. So the most important deliverable in Phase I is a Conceptual Design Report (CDR) for the experiment. The CDR will not only allow assessment of the feasibility of the experiment, but will also provide the basis for the fully developed design report to be produced in Phase II. The overriding technical question to be addressed is whether a system like the one proposed can meet the performance requirements for a field-deployable system. Our previous design and simulation work has gone a long way toward establishing technical feasibility of the system design concept. The purpose of the proof-of-concept experiment is to provide engineering and physics information that will allow evaluation of the practicality of a field-deployable system.

o A critical engineering issue is the design of the positron production target. That subsystem will be engineered in Phase III, but a preliminary evaluation of its feasibility will be carried out in Phase I.

o There are two critical physics questions to be addressed by the PoC experiment: (1) How do the real rates, spectra, and distributions compare with the simulation results? (2) Can the backgrounds be reduced to a tolerable level? We also want to design a positron beam transport system that will allow demonstration of some of the flexibility and tunability that are possible.

o A major technical risk is the possibility that the fluxes of background particles (presumably mainly neutrons and gammas) are intolerably high. That suggests two questions: (1) How high is too high? (2) How can that risk be mitigated? In our previous work with Pacific Northwest National Laboratory, we put a modest 
effort into the design of shielding and simulated its effects. We need further work in that area, both to establish the limits of tolerable backgrounds (for personnel safety, for reduction of induced radioactivity in scanned objects, and for the feasibility of detection strategies we will plan to employ) and to optimize the shielding design. Pacific Northwest National Lab will take the lead in developing the detection strategies.

o Our previous work concentrated mainly on providing a beam that would be useful for a broad-area scan to detect delayed gammas and neutrons resulting from photo-fission processes. Such a system would provide some information about the location of suspect materials, but it may not provide the desired spatial resolution of $1 \mathrm{~cm}$. In preparation for the proposed proof-of-concept experiment, we need to develop the best strategy for achieving the desired position resolution via transmission radiography. One possibility is to collimate the positron beam and focus it upon the converter to provide a small source size for the emerging gammas. That, in combination with a position-sensitive gamma detector, may provide the needed resolution. Other concepts are also under consideration.

o Phase I will include a preliminary investigation of potential facilities at which to carry out the PoC experiment. If electron beam from Fermilab's Advanced Superconducting Test Accelerator (ASTA) is available at the appropriate time, ASTA will be an attractive candidate site; in that case, Fermilab's Illinois Accelerator Research Center (IARC) could provide valuable logistical support.

\section{- Phase II work}

o Phase II will focus on design of the overall PoC experiment and the design of the component subsystems. The main output is a Preliminary Design Report (PDR) containing design specifications for the component subsystems.

\section{- Phase III work}

o Phase III will develop the engineering design of the subsystem components and the overall system, including determining the availability of existing subsystem components such as the monochromator dipole magnet and positron beam transport magnets, which can reduce the costs of the experiment. In Phase III the determination of the host facility is to be completed.

\section{- Phase IV work}

o Phase IV is directed at development, fabrication, assembly and testing of components for the PoC experiment.

0 At the end of Phase IV, the experiment should be ready to install and run at the host facility.

\section{- Phase V work}

0 Phase $V$ entails the installation of equipment at the host facility, the running of the experiment, and the analysis of the data.

o Among the studies will be beam tuning studies and measurements of rates, spectra, and distributions of positrons and gammas as well as measurements of background rates. 


\section{A.4.4 Proposed Coherent Diffraction Radiation Measurements of Bunch Length at ASTA}

\section{A.4.4.1 Abstract}

The feasibility of using the autocorrelation of coherent diffraction radiation as a nonintercepting diagnostics technique for bunch length and indirectly rf phase measurements is evaluated and proposed for the Advanced Superconducting Test Accelerator (ASTA) facility. Previous experiments on an rf thermionic cathode gun beam at $50 \mathrm{MeV}$ provide a proof-ofprinciple reference for the ASTA injector. The technique would also be applicable at high energy in straight transport lines after the cryomodules.

\section{A.4.4.2 Introduction}

The high-power electron beams for the Advanced Superconducting Test Accelerator (ASTA) facility involve up to 3000 micropulses with up to $3.2 \mathrm{nC}$ per micropulse in a 1-ms macropulse [1]. With beam energies projected from 50 to $800 \mathrm{MeV}$, the need for non-intercepting diagnostics for beam size, position, energy, and bunch length is clear. In this note, we address non-intercepting (NI) diagnostics of the bunch length and inferred rf phase by using coherent diffraction radiation (CDR) techniques [2,3]. Previous evaluations of incoherent optical synchrotron radiation indicated that the source strength for visible photons is quite weak for a single micropulse at $50 \mathrm{MeV}$ from the chicane dipoles, but synchroscan streak techniques to determine the bunch length after the chicane should be viable by synchronous summing of the long pulse train's micropulses [4]. This would be complemented by optical transition radiation (OTR) and coherent transition radiation (CTR) measurements of the tune-up beam with an intercepting metal screen after the chicane. It is proposed that at this station, the metal screen would also have an insertion position where a 4-5 $\mathrm{mm}$ tall slit/aperture is centered on the beam position to provide a non-intercepting source of CDR from the surrounding metal surface. The autocorrelation of the far infrared radiation (FIR) CDR would then be processed for the bunchlength information, and the potential for rf phase feedback for the injector cavities would also be possible based on the signal intensity monitored by the FIR detector, whether Golay cell or pyroelectric detector. An early measurement of CDR from a screen with a circular aperture was actually an intercepting configuration because the beam subsequently struck a 45-degree metal mirror to redirect the CDR (and CTR) to the FIR detector [2]. The first non-intercepting proof-ofprinciple demonstration was done with an rf thermionic cathode gun beam at $50 \mathrm{MeV}$ with an integrated charge of only $4 \mathrm{nC}$ using a slit geometry [3]. This means that potentially one would only need a few micropulses of the ASTA injector beam at the full $3.2 \mathrm{nC}$ per micropulse for $1 \mathrm{ps}$ rms bunch length to obtain reasonable signal levels with a Golay cell, and the full pulse train could be integrated to obtain information on even longer bunch lengths where FIR generated per $\mathrm{pC}$ is lower in the detector-response regime.

The implementation of the CDR converter screen at $50 \mathrm{MeV}$ will take advantage of the planned $\mathrm{THz}$ detection system and staff expertise, and the long pulse train allows CDR to be applied to the beam-based feedback concept uniquely in HEP test facilities. In principle, this could also be

Proposal for an Accelerator R\&D User Facility at Fermilab’s Advanced Superconducting Test Accelerator 
applied at the ASTA GeV-scale energies in a high power beam uniquely since it is a nonintercepting technique.

\section{A.4.4.3 Conceptual Aspect}

It is proposed that a multi-purpose station should be located after the chicane for incoherent OTR, CTR, and CDR generation and studies. As schematically indicated in Figure 1, the OTR will be transported to a Hamamatsu C5680 synchroscan streak camera (transferred from A0PI) [5], and the CTR and CDR will be transported to the Martin-Puplett interferometer (MPI) for measuring the autocorrelation of the FIR radiation [6]. A metal screen can be used for the first two mechanisms interceptively, and by centering the $5-\mathrm{mm}$ slit/aperture machined in the screen on the beam axis the CDR will be generated non-interceptively as the beam transits through the aperture. In principle, this latter configuration will work for the full pulse train. Alternatively, a single plane screen with its horizontal edge positioned above or below the beam axis might be used. In this case, a beam abort would initiate the withdrawing of the screen away from the beam. The electric field scaling parameter, $\gamma \lambda / 2 \pi$, where $\gamma$ is the Lorentz factor and $\lambda$ is the wavelength, sets the practical scales. For example with FIR $628-\mu \mathrm{m}$ radiation and $\gamma=100$, this parameter is $10 \mathrm{~mm}$ so a $5-\mathrm{mm}$ slit height is reasonable. One prefers a radiating surface of $100 \mathrm{~mm}$, however, this is unlikely to be attained in our beam pipe so some finite screen effects may be involved.

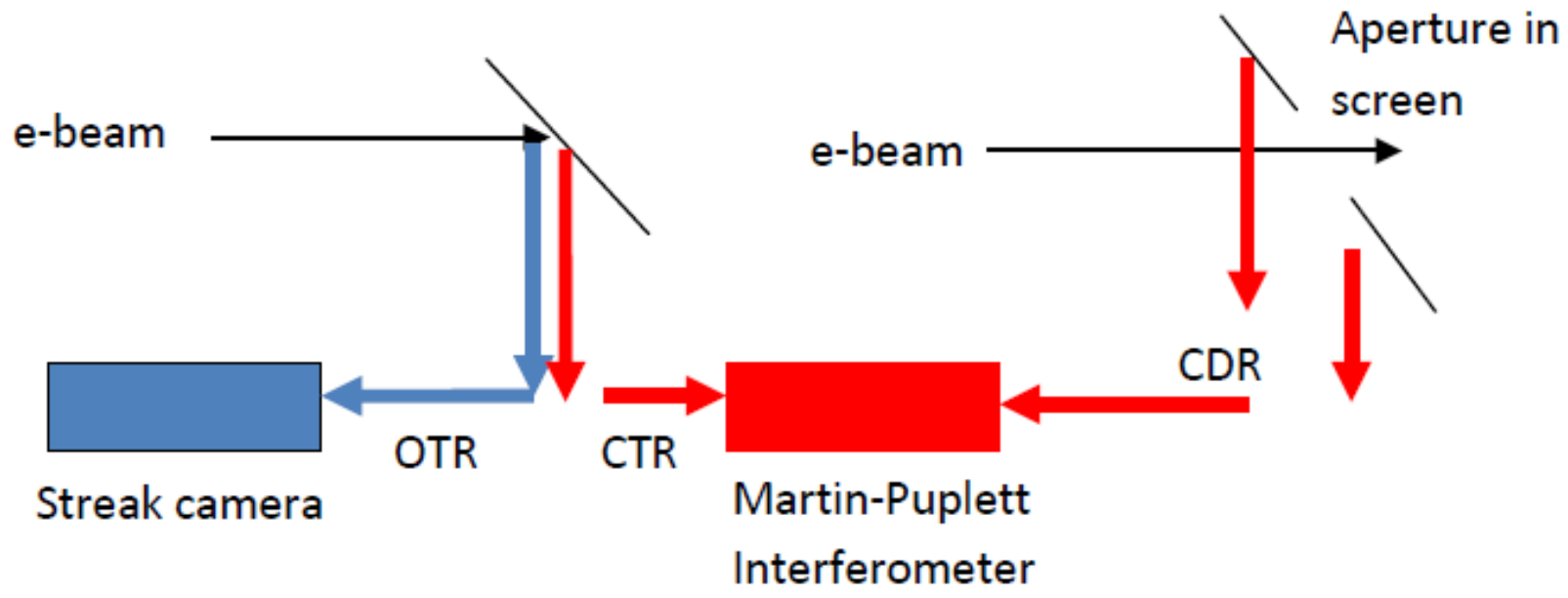

Figure 1: Schematic of a three-source option for OTR, CTR, and CDR diagnostics with the streak camera and MPI indicated. The OTR/CTR screen is at the left, and the CDR screen with aperture is at the right.

\section{A.4.4.4 Coherent Radiation Analytics}

A brief review [3] of the source of the CTR and CDR is in order. Coherent radiation generated by a bunch of electrons can be expressed as a product of a term representing the radiation process due to a single particle and a term which takes into account how much of the total 
charge in the bunch radiates together constructively, in phase. Thus the spectral, angular distribution of the radiation can be expressed as,

$$
\frac{d^{2} W}{d \omega d \Omega}=\left|r_{\perp, / /}\right|^{2} \frac{d^{2} W_{1}}{d \omega d \Omega} \mathfrak{I}(\mathbf{k})
$$

where $\frac{d^{2} W_{1}}{d \omega d \Omega}$

is the spectral angular distribution for the single particle radiation process, whether transition radiation (TR), synchrotron radiation, or diffraction radiation (DR), for example. In the present case we take it as for DR. $\mathfrak{I}(\mathbf{k})$ is the coherence factor for a bunch of $N$ electrons, and this term is related to the square of the Fourier transform of the spatial distribution of the bunch:

$$
\mathfrak{I}(\mathbf{k})=N+N(N-1)|H(\mathbf{k})|^{2}
$$

with the Fourier transform of the charge form factors using a simple Gaussian model being,

$$
H(\mathbf{k})=\frac{\rho(\mathbf{k})}{Q}=g_{x}\left(k_{x}\right) g_{y}\left(k_{y}\right) F_{z}\left(k_{z}\right)
$$

and where $Q=N e$ is the total charge. Note the first term in Eq. (2) yields the incoherent radiation produced by $N$ electrons in the bunch, while the second term gives the coherent production, which is proportional to $N^{2}$. The transverse form factors are for $i=x, y$,

$$
g_{i}\left(k_{i}\right)=\frac{1}{\sqrt{2 \pi}} e^{-\sigma_{i}^{2} k_{i}^{2} / 2}
$$

with the longitudinal form factor for an individual micropulse,

$$
F\left(k_{z}\right)=\frac{1}{\sqrt{2 \pi}} e^{-\sigma_{z}^{2} k_{z}^{2} / 2}
$$

Note that for the coherence factor to be sizable, the beam rms radius and the wavelength of interest $\lambda$ must be less than $1.4 \gamma \lambda / 2 \pi$ for angles of order $1 / \gamma$. The wavelengths of interest are determined by the longitudinal part of $\mathfrak{I}(\mathbf{k})$ such that the rms bunch length $\sigma_{z}<\lambda / 2 \pi$. The results of the calculations of CDR for several cases of $\sigma_{t}$ from 0.2 to 6 ps are summarized in Figure 2 for a $50 \mathrm{MeV}$ beam with $8 \mathrm{nC}$ of charge transiting the center of a $5-\mathrm{mm}$ tall slit in a metal plane [3]. (This was approximated as two infinite metal strips separated by $5 \mathrm{~mm}$ in the model.) Note the form factor results in significant enhancements of radiation at wavelengths about three times longer than the bunch length. For the ASTA case much higher total charges are anticipated, so the signals should be even stronger if a similar geometry be used. These are basically the same wavelengths expected for the CTR measurements in the MPI, and could in principle also be used with the real-time interferometer (RTI) to generate on-line autocorrelations as demonstrated recently at AOPI [7]. 


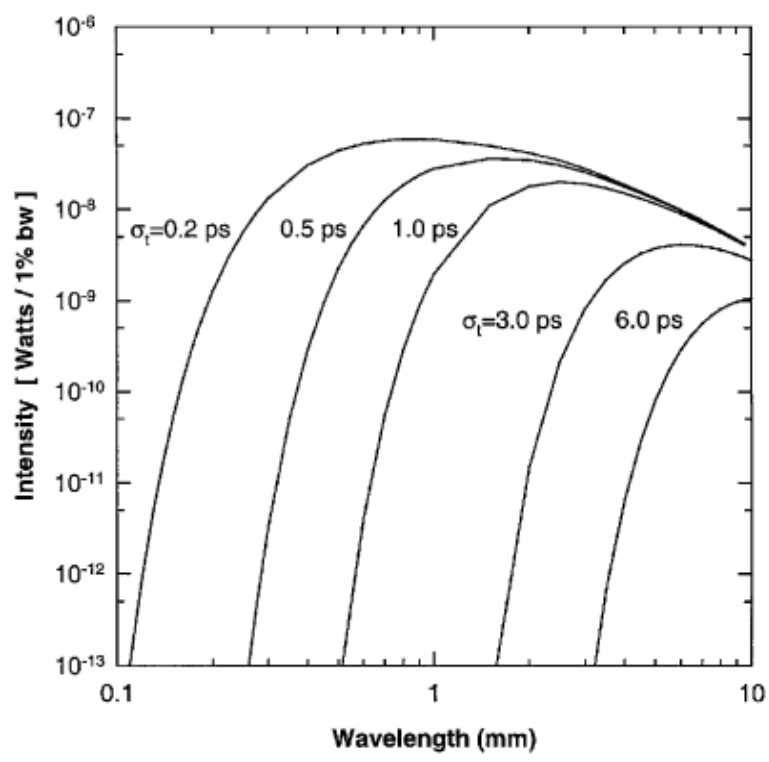

Figure 2: Calculated spectra of CDR for a $50 \mathrm{MeV}$ electron beam passing through a $5-\mathrm{mm}$ slit with $8 \mathrm{nC}$ total charge and bunch lengths $\sigma_{\mathrm{t}}$ of $0.2,0.5,1.0,3.0$, and $6.0 \mathrm{ps}$ [3].

\section{A.4.4.5 Proposed Implementation}

\section{Low Energy Station (50 MeV)}

The ASTA linac with photocathode (PC) rf gun, two booster L-band SRF accelerators (CC1 and CC2), and beamline is schematically shown in Figure 3. The L-band accelerating sections will provide $50 \mathrm{MeV}$ beam before the chicane, and an additional acceleration capability up to a total of $800 \mathrm{MeV}$ will eventually be installed in the form of three cryomodules with eight 9-cell cavities with average gradient of $31 \mathrm{MV} / \mathrm{m}$ after the chicane. The phase of the CC2 section can be adjusted to energy chirp the beam entering the chicane to vary bunch-length compression. Maximizing the FIR coherent transition radiation (CTR) in a detector after the chicane can be used as the signature of generating the shortest bunch lengths. An alignment laser should be planned to inject at a location after CC2 and through the straight ahead line to the bunch length monitor station and into the streak camera and FIR MPI or RTI. This will facilitate optical alignments of the transport systems to the detectors. Micropulse charges of 20 to $3200 \mathrm{pC}$ will be used typically. The nominal pulse format for high power ILC-like beam is $3.2 \mathrm{nC}$ per micropulse at $3 \mathrm{MHz}$ for $1 \mathrm{~ms}$. This aspect is unique for test facilities in the USA and highly relevant to the next generation of free-electron lasers. The macropulse repetition rate will be 5 $\mathrm{Hz}$. 


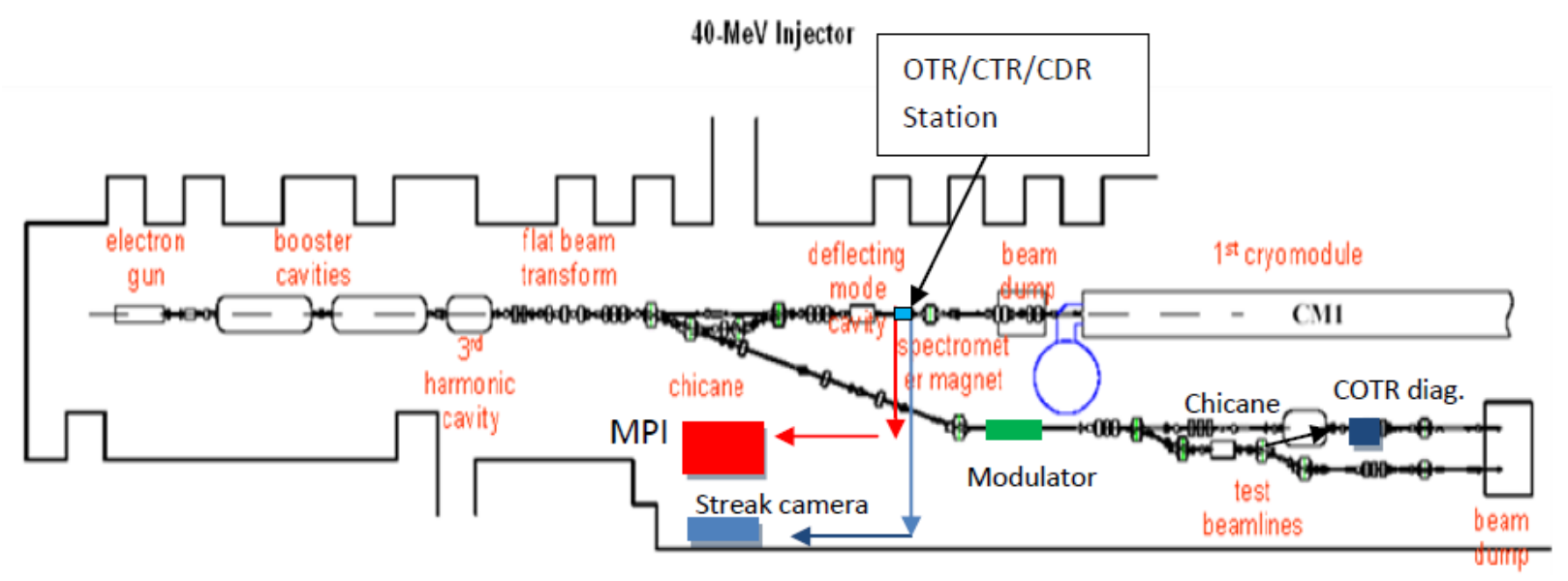

Figure 3: Schematic of the injector for the ASTA facility showing PC rf gun, booster accelerators, and beamlines. The visible-UV streak camera could be used for UV laser and OTR measurements, and the MPI for FIR CTR and CDR measurements. (modified M. Church schematic).

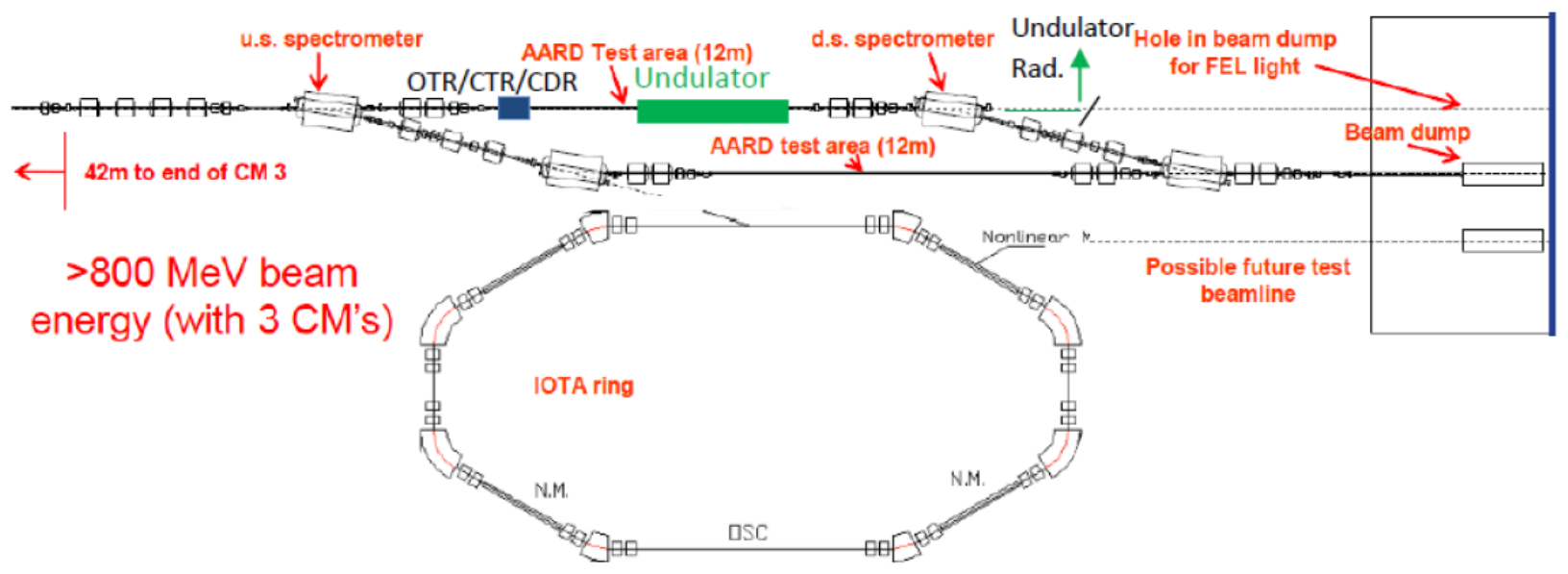

Figure 4: Schematic of the OTR/CTR/CDR monitor location in one high-energy AARD test area. This could be located just upstream of the proposed diagnostics undulator. (modified M. Church schematic).

\section{High-Energy Station (250-800 MeV)}

These OTR/CTR/CDR techniques also scale to use at higher energies as will be found after the cryomodule string. The extension of the CDR technique to higher energies should be straight forward as the CDR signal strengths will be even closer to the CTR signal strengths for a given bunch length and similar effective apertures. This could be used after a proposed second bunch compressor located downstream of the cryomodules. A possible location would be in the AARD section after the upstream dipole as indicated in Figure 4. The streak camera would need to be located in the high energy diagnostics laser lab upstairs, and the MPI would need to be in a 
locally shielded area. The EOS technique used at AOPI with CTR [8] might be employed with CDR signals to make that also a non-intercepting technique.

\section{A.4.4.6 Summary}

In summary, we have described how a combination of intercepting OTR and CTR techniques and non-intercepting CDR techniques could be implemented after the injector chicane to provide bunch length measurement capability for the $50 \mathrm{MeV}$ electron beam. The bunch length and deduced phase variation could be used in combination with the beam-arrival monitors in feedback and feed forward systems for rf phase for and during the macropulse. These OTR/CTR/CDR techniques also scale to use at higher energies found after the cryomodule string. Additionally, such a spectral range with 3000, 1-ps pulses of CDR might be used as a parasitic $\mathrm{THz}$ source for some applications.

\section{A.4.4.7 Acknowledgements}

The author acknowledges discussions with R. Thurman-Keup on the MPI and M. Wendt, M. Church, V. Shiltsev, S. Nagaitsev, and S. Henderson of FNAL on the ASTA facility and AARD program.

\section{A.4.4.8 References}

[1] S. Nagaitsev, talk presented at ILC-TA Workshop, Fermilab, November 28, 2006.

[2] Y. Shibata et al., Phys. Rev. E 52, 6787 (1997).

[3] A. H. Lumpkin, N.S. Sereno, and D.W. Rule, NIM A 475, 470 (2001).

[4] Evaluations presented at NML meetings by A.H. Lumpkin and R. Thurman-Keup (2011).

[5] A. H. Lumpkin, J. Ruan, and T.W. Koeth, "Initial Commissioning of a Dual-Sweep Streak Camera on the AO Photoinjector," Proceedings of Linac08, JACoW (2008); and A.H. Lumpkin et al., NIMA 2012.05.068.

[6] R. Thurman-Keup, R. Fliller, and G. Kazakevich, "Bunch-length Measurement at the Fermilab A0 Photoinjector Using a Martin-Puplett Interferometer," Proceedings of BIW08, Lake Tahoe, CA (2008).

[7] J. Thangaraj et al., "Demonstration of a real-time interferometer as a bunch length monitor in a high current electron beam accelerator," accepted for publication in Rev. Sci. Instr. (2012).

[8] Timothy Maxwell, "Measurement of Sub-picosecond Electron Bunches via Electro-optic Sampling of Coherent Transition Radiation," NIU Dissertation, May 2012. 


\section{B.0 Letters of Institutional Interest}

B.1 Argonne National Laboratory

B.2 Brookhaven National Laboratory

B.3 CERN

B.4 Colorado State University

B.5 Community Petascale project for Accelerator Science and Simulation (ComPASS)

B.6 Illinois Accelerator Research Center (IARC)

B.7 Illinois Institute of Technology

B.8 Indiana University

B.9 International Linear Collider (ILC)

B.10 John Adams Institute for Accelerator Science

B.11 Joint Institute for Nuclear Research

B.12 US LHC Accelerator Physics Program (LARP)

B.13 Lawrence Berkeley National Laboratory

B.14 US Muon Accelerator Program (MAP)

B.15 Northern Illinois University

B.16 Oak Ridge National Laboratory

B.17 Princeton Plasma Physics Laboratory

B.18 RadiaBeam Technologies, LLC

B.19 Tech-X Corporation

B.20 Thomas Jefferson National Accelerator Facility

B.21 US Particle Accelerator School (USPAS) 


\section{Argonne}

December 4, 2012

\author{
Alexander Zholents \\ Director \\ Accelerator Systems Division \\ Argonne National Laboratory \\ 9700 South Cass Avenue, Bldg. 401 \\ Argonne, IL 60439 \\ 1-630-252-4810 phone \\ 1-630-252-7369 fax \\ azholents@aps.anl.gov
}

Dr. Vladimir Shiltsev

ASTA Interim Director

Fermi National Accelerator Laboratory

Dear Vladimir,

I would like to express my strongest support for Fermilab's proposal to establish an Accelerator R\&D User Facility at FNAL Advanced Superconducting Test Accelerator (ASTA). The proposed facility offers unique opportunity to enable a broad range of research in beam physics and accelerator technology at its three experimental areas (50 MeV electrons, 300-800 MeV electron, and 50-150 MeV/c electron/proton ring) and has promise to become one of the most valuable and relevant places for accelerator scientists and engineers to come and do accelerator R\&D. Outstanding electron beam average and peak brightness, acceleration in superconducting RF structures and unmatched versatility of IOTA ring - all these features make ASTA a perfect testbed for many promising accelerator ideas for this country's future, such as high power beam acceleration in SC RF cryomodules, new beam optics concepts, advanced phase space manipulation, space-charge compensation and optical stochastic cooling. The range of applications of the ASTA research seems to be unmatched by any existing AARD facility in the US, and in the world, in that matter.

Argonne has long history of collaboration with Fermilab in the field of accelerators and we will be happy to see this connection further developed thanks to ASTA. Of a particular interest for ANL accelerator physicists are the opportunities which ASTA brings for the beam phase space manipulations (shaping, tailoring, microbunching) which can help to build low-cost high-efficiency FELs. Personally, I am very interested in seeing at IOTA a convincing demonstration of the optical stochastic cooling and a concept of nonlinear integrable optics. As this new facility is going to be built we will continue thinking of many other opportunities for collaborative research at ASTA which could be of importance for future beam-based facilities at Argonne.

Summarizing, I wish to express my support for the ASTA proposal at Fermilab and firm belief that such User facility will be of great value for Argonne, Fermilab, and entire US accelerator community.

Sincerely,

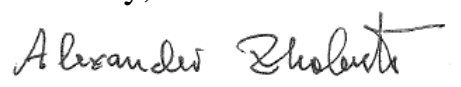

Alexander Zholents 
Dr. Vladimir D. Shiltsev

December 6, 2012

Director, Accelerator Physics Center

Fermi National Accelerator Laboratory

P.O. Box 500, MS 221

Batavia, IL 60510-0500

\title{
Letter of Support for an Accelerator R\&D User Facility at Fermilab's Advanced Superconducting Test Accelerator (ASTA)
}

\author{
Dear Dr. Shiltsev:
}

I write to support the proposal for an Accelerator R\&D User Facility at Fermilab's Advanced Superconducting Test Accelerator (ASTA).

Accelerator R\&D in the US is being performed both at existing accelerator facilities and at dedicated user facilities. Both approaches have been necessary to accomplish the goal of a vibrant R\&D effort to provide the basis for future high performance accelerator facilities for the nation as well as educate the next generation of accelerator scientists. The existing dedicated facilities, pioneered by the Brookhaven Accelerator Test Facility (ATF), have focused on advanced and novel accelerating structures with very high accelerating gradients.

The proposed facility at ASTA plans to use existing equipment at Fermilab to provide very unique capabilities that can address accelerator issues ranging from limits on beam intensities and space charge dominated beams to novel beam sources. Of particular interest is the small storage ring IOTA to study beam dynamics in a ring with fully integrable optics that can accommodate very large betatron tune spread. The accelerator R\&D at this facility at ASTA is of great interest to the high luminosity colliders RHIC and eRHIC at BNL and will be complementary to the work performed at the existing dedicated accelerator R\&D facilities.

Please do not hesitate to contact me if you need any further information.

Sincerely,
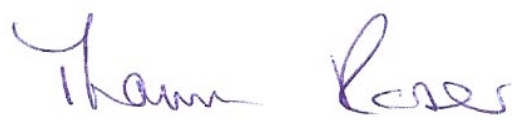

Thomas Roser

Chair

Collider-Accelerator Department

Brookhaven National Laboratory 
Laboratoire Européen pour la Physique des Particles / European Laboratory for Particle Physics

GENEVE, SUISSE

GENEVA, SWITZERLAND

Mail address:

Dr Stephen Myers

Director of Accelerators and Technology

CERN

CH-1211 GENEVE 23

Switzerland

Téléphone/Telephone:

Direct:

+41227673406

+41227678705

Central/Exchange:

E-mail:

+41227676111

Steve.Myers@cern.ch

Oliver.Bruning@cern.ch

Votre référence/ Your reference:

Notre référence/Our reference: OB_SM_SD/2012-004

Geneva, $11^{\text {th }}$ December 2012

\author{
Dr. Vladimir Shiltsev
Director, Accelerator Physics Centre \\ Director, Accelerator Physics Centre \\ FNAL, PO Box 500, MS221, Batavia, IL \\ 60510 USA
}


In light of the various potential applications and relevance for understanding space charge effects we therefore welcome the ASTA initiative and provide our full moral support for this new project proposal.

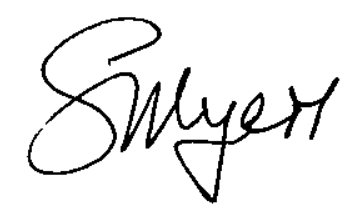

\section{Dr. Steve Myers}

Director of Accelerators and Technology

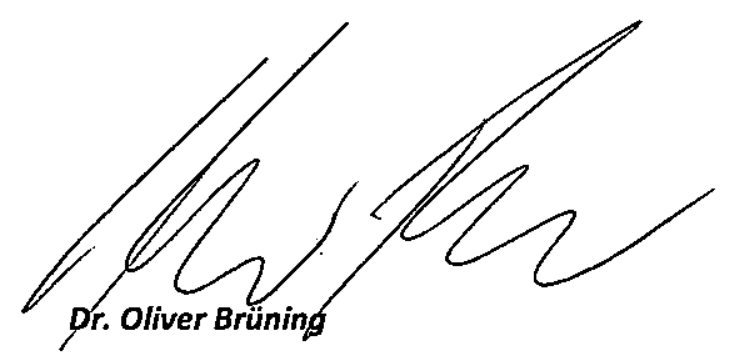

Head of the Accelerator Beam Physics (ABP) CERN-USLARP contact for Accelerator Systems studies. Deputy Project Leader of the HL-LHC upgrade project 


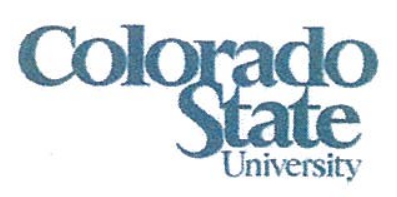

Electrical and Computer Engineering

1373 Campus Delivery

Fort Collins, Colorado 80523-1373 USA

(970) 491-6796

MOBILE: (708) 638-6813

biedron@engr.colostate.edu

December 10, 2012

www.engr.colostate.edu/ece

\author{
Dr. Piermaria Oddone \\ Director \\ Fermilab \\ P.O. Box 500 \\ Batavia, IL 60510-5011 \\ Dear Dr. Oddone,
}

On behalf of the Colorado State University's accelerators and beams community, we are writing to formally indicate our support for the Advanced Superconducting Test Accelerator (ASTA) proposal.

As you are aware, individuals from the Electrical and Computer Engineering Department, the Physics Department, and the Radiation and Oncology groups of the School of Veterinary medicine at Colorado State University have professional links to Fermilab in many areas, including accelerator and beam physics, highenergy physics, detector, and radiation safety, to name a few.

In the area of high-average power accelerator and beam physics, necessary to enable new science and technologies, we are enthusiastic that ASTA will facilitate much needed research including testing of:

- niobium cavities fabricated and polished with different protocols and subsequently tested with beam,

- novel cathode robustness and beam quality performance,

- high-average power beam propagation/dynamics and control,

- high-energy, space-charge dominated beams,

- non-intercepting, real-time diagnostics.

The operation of accelerators, specifically, superconducting cavities at a high repetition rates, are of interest for accelerator science and technology, for the light source user community in atomic, molecular and materials science, and for applications in nuclear and high-energy physics. For instance, a so-called "complete experiment" (reference Nature Physics, Jahnke et al, 2010) requires simultaneous detection of several particles in coincidence, thus relying upon high-average power beams.

Not only does the community require these tests for the realization of future user facilities and industrial devices for our Nation, we require ASTA for the training of our interns (high-school and undergraduate) and our graduate students as well as retention of our staff.

We applaud your proposal and are hopeful it will be funded in the near future.

Very truly yours,

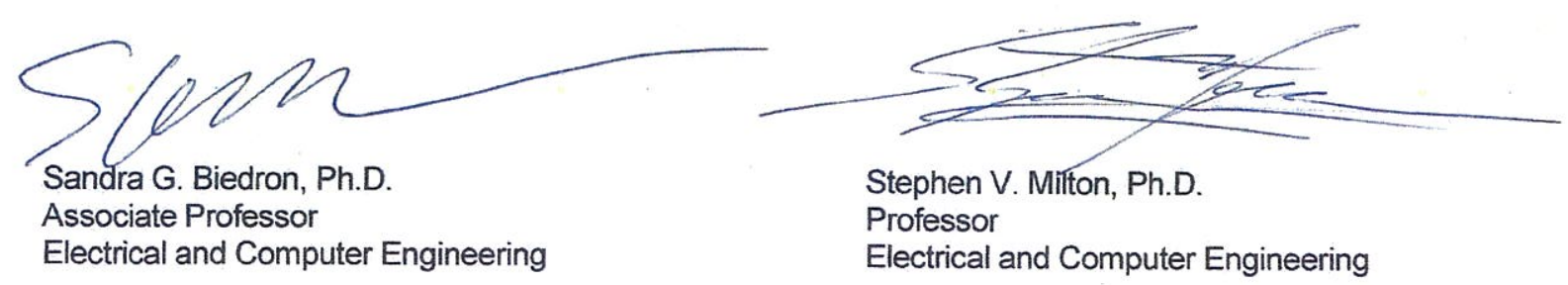




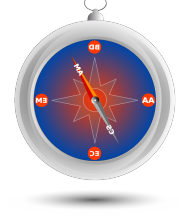

Dr. Vladimir Shiltsev,

Director, Accelerator Physics Center

Fermilab

P.O. Box 500, MS221

Batavia, IL 60510

Dear Vladimir,

I strongly support your proposal to obtain funding for the completion of the Advanced Superconducting Test Accelerator (ASTA) facility and for establishing a user accelerator R\&D program there. ASTA is unique among Advanced Accelerator R\&D (AARD) facilities because it focuses on the specific needs of accelerator science in support of Intensity Frontier accelerators. Researchers from the Community Petascale project for Accelerator Science and Simulation (ComPASS), which I direct, are heavily engaged in Intensity Frontier activities though our support of Project-X design. We also have a long tradition of supporting research in AARD facilities, most recently FACET and BELLA. We are excited about the prospect of collaboration on ASTA experiments, and engaging in the design and commissioning of both the superconducting $\mathrm{rf}$ linac and the IOTA storage ring. In particular, we are very interested in utilizing the IOTA ring to study space-charge effects and validate our numerical models. Even more importantly, we would like to model mitigation techniques for space-charge effects and verify these experimentally. In addition, we are very interested in supporting and guiding, via numerical simulation, experiments that test the ability of non-linear accelerators to reduce or eliminate beam instabilities. ASTA is the only facility that provides the infrastructure to study such instabilities and design and test techniques to mitigate them. Understanding how to control or eliminate beam instabilities and minimize beam losses is imperative for the successful operation of high-intensity accelerators.

ComPASS researchers are looking forward to working with you and your team to support and enable the planned research activities of this facility. We hope to pursue experiments and studies that will advance accelerator science and technology for the Intensity Frontier program.

Sincerely,

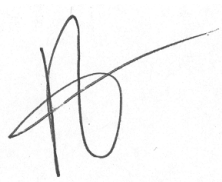

Panagiotis Spentzouris Director, ComPASS 


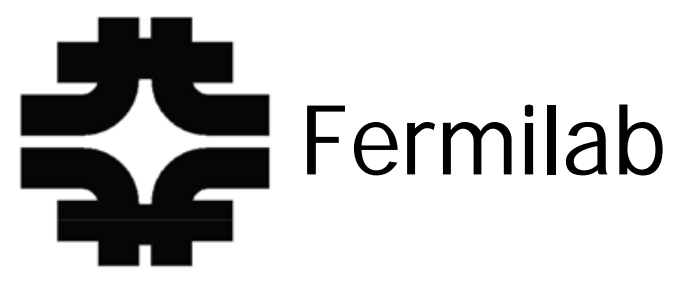

Fermi National Accelerator Laboratory

Directorate

P.O. Box 500 MS 105

Batavia, IL 60510

Fax: (630) 840-2900 Ph: (630) 840-3211

September 9, 2013

Dr. Vladimir Shiltsev

Interim Director

Advanced Superconducting Test Accelerator

Dear Vladimir,

This is a letter of support intended to indicate the importance of the proposed Advanced Superconducting Test Accelerator (ASTA) to the accelerator applications program planned at the Illinois Accelerator Research Center (IARC).

Particle accelerators are an enabling technology not only utilized in their traditional role for fundamental research, but also in such diverse fields as medicine, industrial processes, environmental mitigation, and national security. In recognition of this large impact on the US economy and the many future applications envisioned, the Fermi National Accelerator Laboratory (FNAL) has partnered with the Illinois Department of Commerce and Economic Opportunity (DCEO) and the Department of Energy's Office of High Energy Physics (DOE/OHEP) to build IARC. Located on the Fermilab campus, this state-of-the-art facility will provide outstanding capabilities for accelerator development and serve as a portal allowing access by industry to Fermilab's powerful accelerator staff and extensive infrastructure. In this way IARC will promote and enable new high technology businesses, a high tech workforce, and U.S. industrial competitiveness.

Even in the absence of a formal announcement launching the IARC program, interest from industry has been strong. I have already received 3 expressions of interest from industry to use capabilities at ASTA. It is clear that ASTA will be a key component in the suite of capabilities that IARC can offer to industrial partners.

I fully support the ASTA proposal and completion of the facility as soon as is feasible. If you need additional information in support of this recommendation please do not hesitate to contact me.

Sincerely,

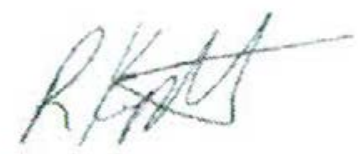

Dr Robert Kephart

Director

Illinois Accelerator Research Center 


\section{ILLINOIS INSTITUTE \\ OF TECHNOLOGY}

Transforming Lives. Inventing the Future.www.iit.edu
Biological, Chemical and

Physical Sciences

Life Sciences, Room 182

3105 S. Dearborn St.

Chicago, Illinois 60616

Phone: 312.567 .3480

Fax: $\quad 312.567 .3494$
Dr. Vladimir Shiltsev,

Director, Accelerator Physics Center

Fermilab

P.O. Box 500, MS221

Batavia, IL 60510

Dear Dr. Shiltsev,
Prof. Linda Spentzouris

IIT, 182 LS, Physics

3101 South Dearborn

Chicago, IL 60616

I strongly support your proposal to obtain funding for the completion of the ASTA User's Facility at Fermilab. There is a real need for facilities that have advanced accelerator research included in their mission. It is impractical or impossible to use accelerators serving a user community to test design innovations or investigate beam dynamics under unusual conditions. Universities such as the Illinois Institute of Technology (IIT) with accelerator physics programs, but no machine on campus, rely on the availability of accelerator facilities at the national laboratories. The existence of university programs in accelerator physics and technology, in turn, contributes to the development of improved machine components. IIT is an example of this synergy; material science, engineering, and chemistry faculty who have other research options are involved with cutting edge accelerator challenges due to the proximity of resources at Fermilab.

IIT has a long history of collaboration with Fermilab in the area of accelerator development. Several of our faculty have long been involved with the research effort in muon beam production and processing. We also have faculty members collaborating with Fermilab on the development of superconducting RF structures. Condensed matter and chemistry faculty have been studying the reasons for breakdown in SRF structures, as well as investigating the production of novel SRF structures using the technique of atomic layer deposition. We also have significant research going on in the area of photocathode development. Several IIT graduate students are, or have been, in the Fermilab graduate student program in accelerator physics.

We are eager for the completion of the ASTA facility, particularly the IOTA ring. One of our faculty members is already looking at the IOTA design. The capability to run both electrons and protons in the ring is very attractive; the prospect of doing experiments using IOTA should help us recruit more accelerator physics graduate students to IIT. We look forward to the new opportunities for research and the further collaboration enabled by the availability of ASTA.

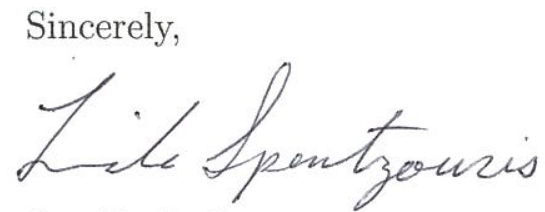

Dr. Linda Spentzouris 


\title{
$\Psi$ \\ DEPARTMENT OF PHYSICS \\ INDIANA UNIVERSITY \\ College of Arts and Sciences \\ Bloomington
}

\author{
Dr. V. Shiltsev \\ Fermi National Accelerator Laboratory
}

Dear Dr. Shiltsev,

I am happy to write a letter in support of your application of funding from the Department of Energy for the ASTA project. As you have pointed out well in your proposal that accelerator physics research is an integral part of the US High energy and nuclear physics research and development. Without accelerator physics research, it would not be possible to achieve "the particle physics today." Accelerator physics development has also great impacts on US industries and technology.

The ASTA is an interesting project that includes Photoinjector source; Linear accelerator based on superconducting RF cryomodules; Electron storage ring (IOTA); Low- and high-power lasers; and Experimental areas for research with low-energy and high-energy beams. The completion of such a project will provide decade of interesting physics research. The project will provide a research base for US accelerator physics for decades.

In summary, I write this letter to express my support to the importance of the ASTA proposal on the accelerator physics research in the US. I am sure that the Department of Energy realizes the importance of your project to high energy physics, to the United States high technology industries, and National Security. I support your efforts without reservation and hope to see your successful project.

Sincerely Yours,

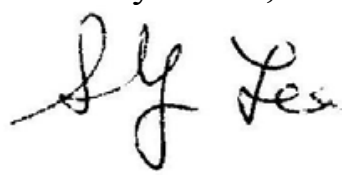

SY Lee, Professor of Physics, Indiana University

12/9/2012 


\section{ite}

January 22,2013

In support of ASTA

On behalf of the Linear Collider Collaboration and the ILC program, we would like to offer strong support for the ASTA Accelerator R\&D Project as described in the Fermilab proposal to the DoE. The superconducting linac technology is very similar to that proposed for the ILC and a dedicated R\&D facility will offer the kind of user access that it difficult if not impossible to obtain in an operating machine and as such is of great interest to the ILC program.

The GDE R\&D program to establish technical viability of an SRF based ILC had several elements but two of the most important were realistic beam-based system tests and the development of high gradient SRF technology. To date, beam based system tests have been performed primarily at the FLASH facility at DESY which uses ILC-like cryomodules but operates at significantly lower gradients than the ILC design. High gradients have been demonstrated in many SRF cavities but not in operating cryomodules. The ASTA proposal would represent a unique opportunity to combine these two features in a single facility. The ASTA cryomodules, from an SRF perspective, are essentially the ILC baseline design. The HLRF power systems are again very similar to the ILC baseline for a mountainous site. With the flexibility of the proposed beam parameters spanning the ILC range, many aspects of the hardware operation can be both verified and optimized. In addition, exercising the ILC hardware in an operating environment will provide valuable experience in terms of hardware reliability that would be difficult to collect elsewhere in the world on this time scale.

In conclusion, we see the ASTA project as a window of opportunity to provide realistic testing of the ILC hardware and we give enthusiastic support to the Fermilab proposal.

Sincerely,

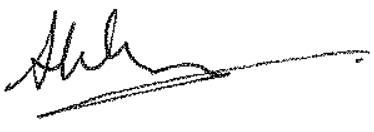

Lyn Evans

Linear Collider Collaboration

Director

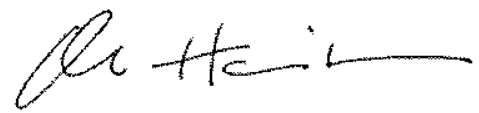

Mike Harrison

Linear Collider Collaboration

Associate Director for the ILC 


\section{Imperial College

\section{The John Adams Institute for Accelerator Science}

Dr Vladimir Shiltsev

10 December 2012

ASTA Interim Program Director

Fermilab

Prof. Andrei Seryi, Director

Denys Wilkinson Building

Keble Road, Oxford

OX1 3RH, UK

Mobile: [+44][0] 7722474701

Tel: [+44][0] 1865273595

Fax: [+44][0] 1865273601

e-mail: Andrei.Seryi@adams-institute.ac.uk

Dear Vladimir,

With this letter I would like to express my strongest support for the plans to establish an Accelerator R\&D User Facility at Fermilab's - Advanced Superconducting Test Accelerator (ASTA).

The ASTA facility will allow Fermilab to be at the forefront of the research and technology development in the areas of superconducting RF, creation of new optics concepts, investigation of promising method of space-charge compensation, developments of advanced techniques for Free Electron Lasers.

The ASTA user facility will build up very efficiently on the years of investments into advanced equipment at Fermilab, and will bring the arising opportunities close to the scientific and industrial user communities, enhancing the positive societal impact of Fermilab research.

The John Adams Institute for Accelerator Science (JAI) - a Centre of Excellence in the UK for advanced and novel accelerator technology - has a good tradition of fruitful collaborative research with Fermilab. Creating the ASTA user facility will open even more opportunities for collaborations of worldwide centres with Fermilab.

Sincerely,

Andrei Seryi

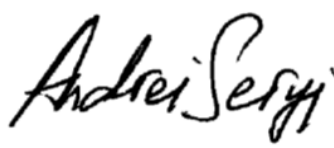

The John Adams Institute for Accelerator Science is jointly hosted by the Departments of Physics of the University of Oxford, the Royal Holloway University of London and the Imperial College London 


\section{ОБЪЕДИНЕННЫЙ ИНСТИТУТ ЯДЕРНЫХ ИССЛЕДОВАНИЙ JOINT INSTITUTE FOR NUCLEAR RESEARCH}

Дубна, Московская обпасть, Россия 141980 Dubna Moscow Region Russia 141980

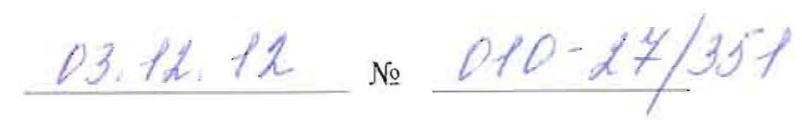

на № OT

Dr. Stuart Henderson

Associate Laboratory Director for Accelerators

Fermi National Accelerator Laboratory

Dear Dr. Henderson,

As you may know, the Joint Institute for Nuclear Research (Dubna, Russia) is developing the project of Nuclotron based Ion Collider fAcility (NICA). The facility will consist of three cyclic accelerators including the last stage - the collider of heavy ions $(1 \div 4.5 \mathrm{GeV} / \mathrm{u}$ kinetic energy) and polarized protons and deuterons $(5 \div 12.6 \mathrm{GeV}$ kinetic energy for protons). The NICA project is one of the subjects of Fermilab-JINR collaboration. We, the NICA team, are extremely interested in a progress of this collaboration and, above of all, are supporting the accelerator R\&D proposal at Fermilab's Advanced Superconducting Test Accelerator (ASTA). Many of the accelerator physics research topics addressed in this proposal are of great interest to us and also overlap with issues relevant to NICA project.

Of particular interest to us are the proposed IOTA ring and its associated research program in nonlinear integrable optics, optical-stochastic cooling, and space-charge compensation. We would like to be part of this research program and to this end JINR is contributing 32 high-quality quadrupole magnets for the IOTA focusing lattice. In addition, this ASTA proposal continues our strong cooperation in the area of ILC R\&D, as well as other topics, such as electron and stochastic cooling.

In the area of accelerator physics education, ASTA research program would offer many exciting opportunities which we are eager to exploit through graduate student and junior scientist exchange visits.

With best regards,

Prof. Igor Meshkov

JINR Directorate Adviser

Scientific Leader of NICA project

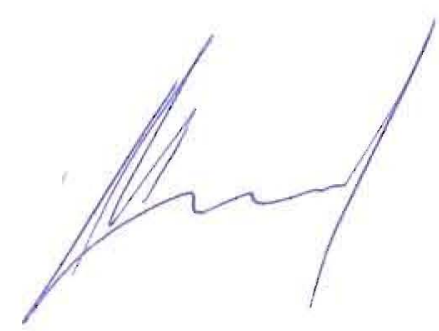




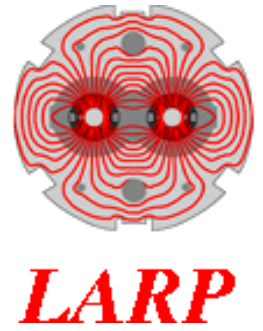

Dr. Vladimir Shiltsev, Director, Accelerator Physics Center

MS 221, Fermilab

Batavia, IL 60510
Dr. Eric Prebys

Program Director, US LHC

Accelerator Research Program

(LARP)

MS 221, Fermilab

Batavia, IL 60510

630.840 .8369

prebys@fnal.gov

December 10, 2012

Dear Vladimir,

I'm writing this letter in strong support of your proposal for the Advanced Superconducting Test Accelerator (ASTA). Although the LHC is not traditionally considered an "intensity frontier" machine, the ultimate luminosity is limited by beam brightness, which in turn is limited by intensity related effects at several points in the injector chain. The ASTA facility - and the IOTA ring in particular - will offer a powerful and unique experimental test bed to investigate these effects. The space charge compensation and optical stochastic cooling experiments, which have been proposed for ASTA, are already of great interest and it is quite likely that other experimental opportunities will present themselves. Tests done in the ASTA facility will provide invaluable data which will aid in effectively allocating resources to further tests at the LHC or the injector chain.

In addition, one of the most likely US contributions to the LHC luminosity upgrade will be the development of crab cavities, to compensate for the crossing angle of the colliding beams. It's possible that valuable R\&D could be done at the ASTA facility into the affect of crab cavities on beams, which would complement that tests planned at the SPS and in the LHC.

We enthusiastically support the project, and look forward to taking part in the research opportunities it will present.

Regards,

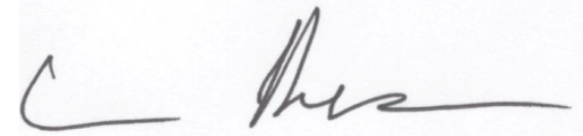

Eric Prebys

Program Director, LARP 


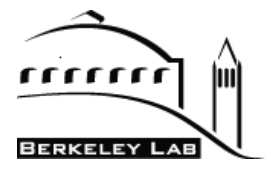

ACCELERATOR \& FUSION RESEARCH DIVISION

Director

December 13, 2012

Dear Vladimir,

The proposed Accelerator R\&D User Facility at Fermilab's Advanced Superconducting Test Accelerator (ASTA) will provide the Office of High Energy Physics with a state-of-the-art accelerator R\&D platform of unique versatility. The expected beam quality and broad capabilities of the facility will be of great interest in applications outside of HEP as well, offering an opportunity for expanding the role of OHEP in accelerator R\&D Stewardship. Several of our scientists in AFRD are eager to contribute to what we believe will be a diverse and exciting accelerator research program. The US, in my opinion, is severely lacking in dedicated accelerator test facilities that can focus on the improvements and generate the breakthroughs required to support a vibrant and competitive sceintific community. Investement in facilities such as ASTA are vital to developing a robust $R \& D$ portfolio that will create the tools of the future. Here at LBNL we wish you great success and stand ready to assit in any way we can, now and in the future.

Sincerely,

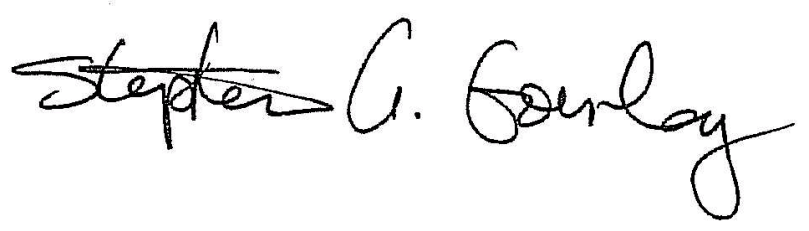

Steve Gourlay

Director, Accelerator and Fusion Research Division

Lawrence Berkeley National Laboratory

Ernest Orlando Lawrence Berkeley National Laboratory

One Cyclotron Road | MS50R4049 | Berkeley, California 94720

Tel: 510.486.7156 | Fax: 510.486.6003 


\section{U.S. Muon Accelerator Program Memorandum}

From: Mark A. Palmer, Director, U.S. Muon Accelerator Program

To: Vladimir Shiltsev, Director, Accelerator Physics Center, and ASTA User's Program Interim Director, Fermilab

Subject: Proposal for an Accelerator Research User's Facility at the Advanced Superconducting Test Accelerator

December 17, 2012

\section{Dear Vladimir,}

I am writing to express the support of the U.S. Muon Accelerator Program for the proposal to host an Accelerator Research User's Facility at the Advanced Superconducting Test Accelerator (ASTA) at Fermilab. The capabilities of ASTA provide critical R\&D reach for techniques and technologies that could be of great benefit in the construction of an intensity frontier Neutrino Factory (NF) and an energy frontier Muon Collider (MC). A foundational element for the NF and MC is the proton driver. Thus the demonstration of the operating parameters and the study of the high intensity beam dynamics relevant for the Project X pulsed linac represent critical steps in determining our preparedness for pursuing these challenging machines. In addition, the research program will enable the study of advanced technologies that have the potential to greatly improve the performance parameters and cost effectiveness of a facility supporting either the NF or MC. Two proposed experiments have the potential of demonstrating new methods for adequately cooling high intensity muon beams: the optical stochastic cooling experiment and the investigation of acceleration and cooling in carbon-based crystal structures. Studies of space charge compensation using an electron lens or or electron columns may provide new opportunities for handling the space charge limits of an ionization cooling channel. Finally, the study of non-linear, but integrable, optics designs in IOTA has the potential to provide important advances in the optics designs that can be used in these facilities.

Beyond these specific benefits in the development of new techniques and technology, the ability to utilize this facility in the training of the next generation of accelerator physicists offers a tremendous potential to maintain the leading role that the U.S. accelerator physics community has played in support of the world-wide high energy physics research effort. 


\section{U.S. Muon Accelerator Program Memorandum}

Thus, the MAP would like to express its very strong support for this proposal.

With Best Regards,

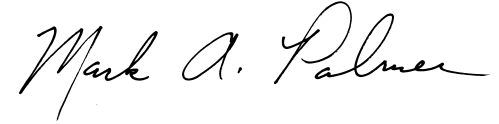

Mark A. Palmer

Director, U.S. Muon Accelerator Program

cc: Stuart D. Henderson, Associate Laboratory Director for Accelerators, Fermilab 
Northern Illinois University (NIU) enthusiastically supports the Fermilab proposal to the Department of Energy for construction and operation of the Advanced Superconducting Test Accelerator (ASTA). NIU has a strong history of collaboration with Fermilab in high energy and beam physics, and the University looks forward to strengthening the partnership through sustained research collaboration and investment at ASTA.

ASTA will establish a unique resource for research and development (R\&D) towards Energy Frontier facilities and a test-bed for SRF accelerators and high-brightness beam applications. FermiLab has been an active contributor to advanced accelerator R\&D over the past 15 years. NIU has been a strong partner, as evidenced by the establishment of the Northern Illinois Center for Accelerator \& Detector Development (NICADD) in 2001 and the subsequent shared investment in research projects and personnel. Currently, two faculty members in NICADD enjoy joint positions with the Accelerator Physics Center at Fermilab and the Department of Physics at NIU, including Dr. Phillipe Piot. Dr. Piot led the first ASTA User's Meeting at FermiLab, and he has been instrumental in developing the vision and priorities outlined in the submitted proposal. NIU supports the vision of ASTA as a powerful resource that will advance innovation in accelerator and beam physics, and provide new opportunities for joint investment by NIU and FermiLab in research and graduate training.

ASTA will also provide new opportunities for student training in advanced accelerator R\&D. A number of students have pursued or are pursuing graduate-level degrees with research in accelerator physics or radiofrequency (RF) engineering involving Fermilab projects. Moreover, the broader community of NIU undergraduate and graduate students has benefited through the established collaboration, as Fermilab scientists have taught courses on campus in the Departments of Physics and Electrical Engineering.

As a public research university that embraces engagement and economic development as key elements of its mission, NIU is very enthusiastic about the ways in which the ASTA proposal incorporates opportunities for application of accelerator science beyond discovery science. For instance, we appreciate that the development of novel accelerator-based sub-picosecond x-ray, single-cycle $\mathrm{THz}$ sources and research on free-electron lasers could offer new research opportunities to NIU faculty with established research interests in condensed matter, radiation biology, and medical physics. Some of the advanced acceleration methods discussed in the 
ASTA proposal could also have application in medical physics, an area of increasing interest at NIU.

To ensure that our faculty and students can realize the opportunities presented by locating the ASTA facility at the nation's particle physics laboratory, and in close proximity to the NIU campus, the senior leadership of NIU is committed to co-investing with FermiLab to add three faculty scientists with expertise in beam and laser source development and use, over the course of the next three years. The shared investment will promote the mission of ASTA, and also leverage the previous investment made by the Illinois Department of Commerce and Economic Opportunity and the U.S. Department of Energy in the Illinois Accelerator Research Center at FermiLab. Public-private partnerships focused on commercialization of accelerator technology are already forming. ASTA offers unique capabilities that will enable a range of industrialrelated R\&D.

NIU looks forward to the establishment of ASTA. We are enthusiastic about continued, focused collaboration with FermiLab to ensure that this powerful facility not only supports breakthroughs in accelerator science, but also translates these discoveries into applications that advance our nation's health, wealth and security.

Sincerely,

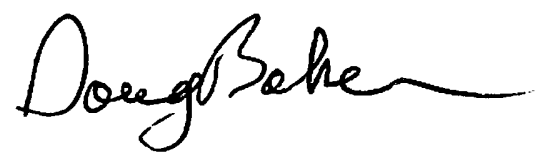

Douglas D. Baker

President

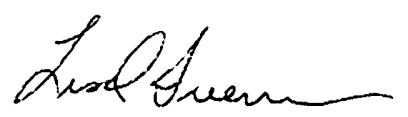

Lisa C. Freeman

Vice President, Research \& Graduate Studies

cc: Dr. Vladimir Shiltsev, Interim Director, ASTA 
November 20, 2012

Dr. Stuart Henderson

Associate Laboratory Director for Accelerators

Fermi National Accelerator Laboratory

Dear Stuart,

On behalf of the Spallation Neutron Source (SNS) accelerator effort, I wish to express support for the accelerator R\&D proposal at Fermilab's Advanced Superconducting Test Accelerator (ASTA). Many of the accelerator development areas addressed in this proposal overlap with issues faced in high power proton accelerators used for spallation sources at BES neutron scattering facilities.

For instance the ASTA proposed nonlinear integrable lens tests (in the IOTA ring) is of direct interest. This technique offers potential for fundamentally improved high intensity beam storage, with avoidance of resonances, reduced possibilities of halo growth - which are major drivers in high intensity storage rings required to provide short pulse $(\mu \mathrm{s})$ neutron sources such as SNS. The nonlinear lens technique also offers promise in eliminating the problematic e-p instability, that we already see evidence of in SNS present operation. This instability could be a major obstacle for planned intensity upgrades. Additionally the proposed ASTA space charge compensation (with plasmas / electron column) tests are directly applicable to the SNS ring, as we already have a significant space charge induced beam broadening. Any possibility of reducing beam broadening can reduce beam loss, a major consideration in SNS operations.

These intensity related issues are important for both SNS upgrade plans and in the design of future high power spallation neutron sources. Additionally there will be ancillary areas of ASTA development that are applicable to SNS such as halo beam instrumentation and stable superconducting RF operation. As such we consider many of the ASTA targeted research areas as being mutually beneficial with the long-term neutron scattering scientific program within BES.

Please let me know if I can be of any further help.

Sincerely

$$
\text { pun Aalumb }
$$

John Galambos 


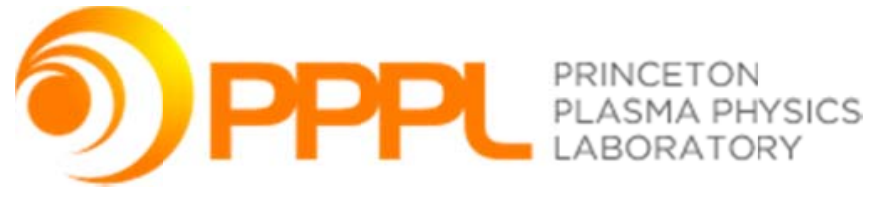

Plasma Physics Laboratory

Princeton University

P.O. Box 451

Princeton, New Jersey 08543

Dr. Vladimir D. Shiltsev

December 10, 2012

Director

Accelerator Physics Center

Fermi National Accelerator Laboratory

Batavia, IL 60510

Re: Proposal for an Accelerator R\&D Facility at Fermilab's Advanced Superconducting Test Accelerator (ASTA)

Dear Dr. Shiltsev:

It is a great pleasure to write this letter of strong support and participation for your proposal, "Proposal for an Accelerator R\&D Facility at Fermilab's Advanced Superconducting Test Accelerator (ASTA)." The breadth and depth of the proposed research activities is quite impressive. Our PPPL Non-neutral Plasmas and Beam Dynamics Group shares many common interests with your co-authors, contributors, and you. Specifically, we are very interested in developing an improved understanding of the physics of intense beam propagation, stability, and neutralization.

In addition to our experimental expertise, our research group has significant experience in the theory and modeling of intense charged particle beams and beam neutralization by background plasma. We are also currently developing a laboratory to study the role of plasma in the formation and behavior of nanoparticles. Therefore, we look forward to collaborating with the research you are proposing in sections 7.2.2 [Integrable-optics test accelerator (IOTA) ring construction and operation], 7.2.3 [Space charge compensation in high intensity circular accelerators], and 7.2.5 [Investigation of acceleration and cooling of carbon-based crystal structures for muon accelerators].

One collaboration would include the development of supporting experiments on the Princeton Paul Trap Simulator Experiment to simulate nonlinear, integrable, transport lattices in a compact laboratory Paul trap. We will apply our expertise in pure-electron plasmas trapped in MalmbergPenning traps to participate in the design of electron plasma diagnostics to characterize the properties of the trapped electron column used in the space charge compensation experiments. We will carry out experiments in our nanotechnology laboratory to explore the interaction of beams with carbon-based nanostructures. Further, we will apply our extensive theory and modeling tools, including fully self-consistent 3D particle-in-cell codes, to contribute to understanding topics such as emittance growth in nonlinear lattices, and two-stream instabilities in the trapped-electron/circulating proton system. 
Thank you very much for the opportunity to participate.

Sincerely

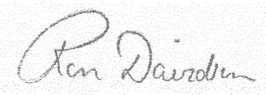

Ronald C. Davidson

Professor

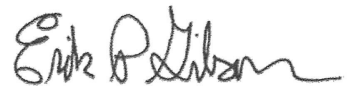

Erik P. Gilson

Research Physicist

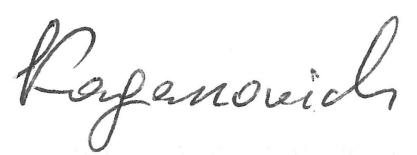

Igor D. Kaganovich

Principal Research Physicist 
December 7, 2012

Vladimir D. Shiltsev

Director, Accelerator Physics Center

ASTA Users' Program, Interim Director

Fermilab

PO Box 500, MS221

Batavia, IL 60510 USA

Dear Dr. Shiltsev,

This letter is written in enthusiastic support of your proposal to establish an Accelerator R\&D User Facility at Fermilab's Advanced Superconducting Test Accelerator (ASTA). This facility will fill an important role in providing high-energy, high average power beams for testing novel accelerator-related technologies and applications.

My company, RadiaBeam Technologies, has been active in accelerator R\&D and directed accelerator component and instrumentation development since its founding in 2004. We often require access to accelerator beamlines to test out new $\mathrm{R} \& \mathrm{D}$ concepts or new products. On the low-energy end $(<100 \mathrm{MeV})$, we have used the UCLA Pegasus lab, the BNL ATF, and the Idaho Accelerator Center. However there is no facility with $>100 \mathrm{MeV}$ beams that will readily provide us access to the beamline. The impressive beam parameters to be available at ASTA, including the high energy, average power and brightness, will certainly enable a host of new experiments to be performed by users like us, and will no doubt spur development of new technologies with commercial applications.

For example, as you know, we have already proposed to use the ASTA beam to generate highintensity gamma rays via the inverse Compton scattering (ICS) process. We originally became interested in an ICS gamma ray source as a probe to search for hidden Special Nuclear Materials over a long range ("active interrogation"). Our previous work resulted in the construction of a novel laser recirculator and laser-electron beam interaction system. Such a system, if configured at ASTA, would produce a beam of $5-20 \mathrm{MeV}$ gamma rays with more than $10 \mathrm{~W}$ of power in a narrow bandwidth ( 1\%). This would be an exciting source for homeland security applications, medical imaging and therapy, nuclear physics research, isotope production and non-destructive testing.

ASTA could also provide a test bed for the vendors to develop the accelerator instrumentation and components that will be needed for new high-intensity accelerators, such as ILC and CLIC, or a Higg's Factory. As an example, we are currently developing laser wire scanners for transverse profile measurement of high intensity beams, which would ideally be tested at a ASTA. Other companies would certainly be interested in testing their accelerator-related product at ASTA as well. 
Finally, I am also excited about the development of the integrable optics accelerator as part of the ASTA program. The accelerator applications enabled by IOTA is impressive, with impact found in the areas from basic science to medicine. For example, a multi-MW proton driver is a potential workhorse in scientific applications in the intensity frontier, with applications such as: providing high fluxes of primary particles for muon production in the context of a muon collider and/or neutrino factory; spallation neutron production; transmutation of nuclear waste; and accelerator-driven sub-critical reactors (i.e. thorium cycle fission). RadiaBeam has already started to look into the design of the novel nonlinear magnets required by IOTA, and we look forward to finding more opportunities to work on this exciting technology.

In conclusion, I strongly urge DOE to fund your proposal, as it would serve as a vital resource for both scientific and industrial users to develop and test novel accelerator technologies. I wish you the best of luck with this proposal, and look forward to working with you at ASTA.

Sincerely,

Salime Boucher, President

RadiaBeam Technologies, LLC 


\section{Tech-X Corporation}

February 22, 2012

Dr. Stuart Henderson

Fermi National Accelerator Laboratory

MS 105

P.O. Box 500

Batavia, IL 60510-5011

Dear Stuart:

This letter is to support the proposed Advanced Superconducting Test Accelerator. For the record, as you know, I have been an accelerator physics researcher for nearly 30 years, since I first joined the faculty at the University of Colorado. (I am still employed there, but I spend most of my time now at Tech-X, which has a strong accelerator computation group.) Over the years I have worked in nonlinear dynamics of accelerator lattices, beam equilibria and stability, laser-plasma acceleration, and most recently in advanced accelerator cavities involving dielectrics as well as dielectric laser acceleration. For all of these, I have been involved in the development of algorithms.

The Advanced Superconducting Test Accelerator would allow researchers from all over the country to have access to facilities to test the many advanced acceleration ideas that have been developed. I personally would like to see at least two areas developed. The first is the development of nonlinear accelerator lattices that show promise of permitting high-intensity beams without deleterious instabilities that create halos. Such a development would be invaluable to the intensity frontier. Said nonlinear accelerator lattices have been extensively studied theoretically. For further progress, a physical realization (experiment) is needed. That would be provided by the IOTA ring of ASTA.

A second important area is the exploration of new cavity concepts. These can be as modest as shape modifications of existing designs to radically new designs, such as cavities involving dielectrics or metamaterials. Many of these designs are coming out of smaller groups at Universities, which do not have the infrastructure for testing them. ASTA would provide the facilities for doing so, thus helping to advance accelerator physics research.

Of course, ASTA has many other aspects to it that would be useful for accelerator research in other areas. Especially as a theorist who is interested in these new accelerator physics concepts, I highly support the funding of ASTA.

Sincerely,

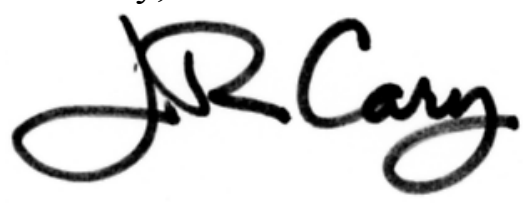

Dr. John R. Cary

CEO, Tech-X Corporation 
To whom it may concern,

It is with great pleasure I write this letter in support of Fermilab's Advanced Superconducting Test Accelerator (ASTA).

As Jefferson Lab's Associate Director responsible for Accelerators, I am very involved in furthering accelerator technologies and the training of future accelerator scientists and engineers.

Jefferson Lab has extensive experience in high current and high brightness electron sources used in the Nuclear Physics accelerator (CEBAF) and the Free Electron Laser (FEL). Jefferson Lab is a world leader in Superconducting Radio Frequency (SRF) accelerating structures and the interaction of electron beams with the RF fields. Due to the interest in high average currents in the present and future FELs and through our collaborations with ERL X-ray source advocates, we have worked extensively on many aspects of SRF technology suitable for high average current beams, such as cryomodules, highly HOM-damped cavities, damping couplers, high average power fundamental couplers, advanced cryogenics systems, to name a few. We will be very happy to share our expertise and the results of our continuing R\&D in these areas with the ASTA effort and provide our high beam current SRF technology for use in ASTA. In order to eliminate duplication of effort, and as ASTA's future plans become more concrete, it will be advantageous to both our programs to make use of the technologies which are already here and to collaborate on mutually beneficial future research. We look forward to suitable divisions of labor with Fermilab.

As noted in the ASTA proposal itself, an area of particular interest to Jefferson Lab is developing cooling technology for our polarized Medium Energy Electron Ion Collider (MEIC). Both Brookhaven and Jefferson Lab have concluded that the luminosity of any future electron-ion collider will be high enough only through beam cooling. Brookhaven is pursuing coherent electron cooling for this purpose.

The MEIC design includes the use of Energy Recovery Linac (ERL) based Electron Cooling for the hadron beam. In order to reduce the required electron current in ERL, the cooler scheme is complemented with a Circulator Cooler Ring (CCR) operated by a fast kicker with sub-nanosecond rise times. We would like to conduct experiments with ASTA's fast kicker as part of our MEIC studies and look forward to the opportunity of collaborating with Fermilab's scientists in the cooler design at ASTA. Operating the iota ring to mimic the circulator cooler is very attractive to us.

A number of high level scientific committees have emphasized educating and training the next generation of accelerator scientists and engineers as a national priority. Fermilab's 
ASTA would be an excellent facility which supplements class room teaching with handson training. Our experience at Jefferson Lab with accelerator physics students, (both undergraduate and graduate), is that the hands-on training enhances the understanding of critical concepts and contributes to the sustained enthusiasm for the field.

I strongly believe that ASTA will make significant contributions to accelerator science and engineering.

Sincerely,
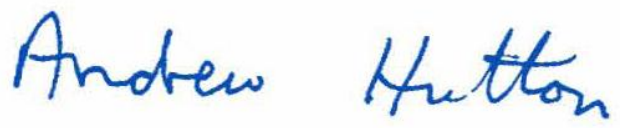

Andrew Hutton

Associate Director for Accelerators

Jefferson Lab 


\section{United States Particle Accelerator School}

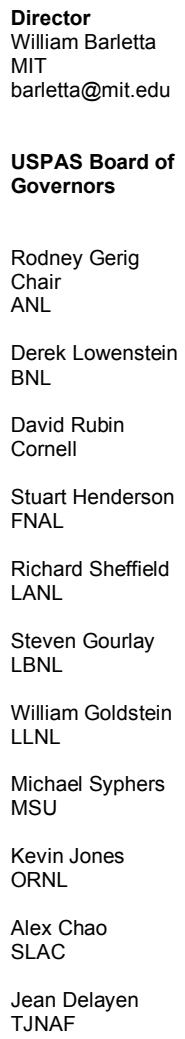

Dr. Vladimir Shiltsev

Director, Accelerator Physics Center

Fermi National Accelerator Laboratory

Batavia IL

Dear Vladimir,

I have read with interest your draft proposal for a test accelerator, ASTA, dedicated to beam physics studies. The opportunities for beam physics research on state of the art equipment are highly unusual. Of particular interest is the difficult task of attracting undergraduate physics and engineering students to our field. Therefore, it is natural to consider how ASTA can be used to train students in accelerator science and technology.

A highly cost-effective first use of ASTA for formal education would be an expansion of the already successful collaboration of USPAS, FNAL, and ANL in the Lee Teng Undergraduate Internship Program. In that program students take a formal course at USPAS and then spend the remainder of the summer doing research at FNAL and ANL. Were their first week of research transformed into a week of hands-on experience at ASTA, the Lee Teng Internship could be transformed into a truly unique educational experience with a strong potential to attract pre-graduate scientist

Students could spend their first week at the laboratories doing real hands-on experiments at ASTA. That would give everyone a chance to have an experimental experience even if their internship is with a theorist. As FNAL and ANL have already worked out affordable housing and transportation for the student interns, the additional costs of LeeTeng at ASTA would be minimal.

Such a program would be a great first step and also allow the ASTA staff to work out a set high quality instructional experiments for an ASTA-based curriculum that could be used on an annual basis. The USPAS would be pleased to work with you and the Lee Teng program coordinators to make this a model hands-on program.

Sincerely yours,<smiles>C=C1CC2CCC1CC2</smiles>

William A. Barletta

Director 
애, 말

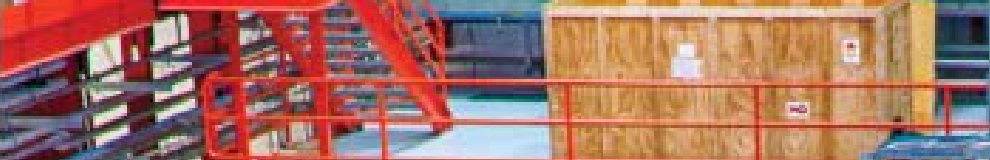

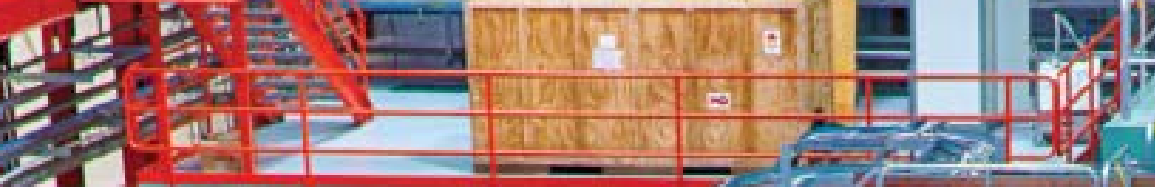

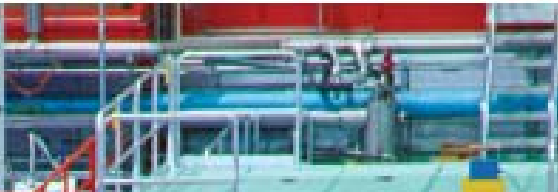

$-4=1$

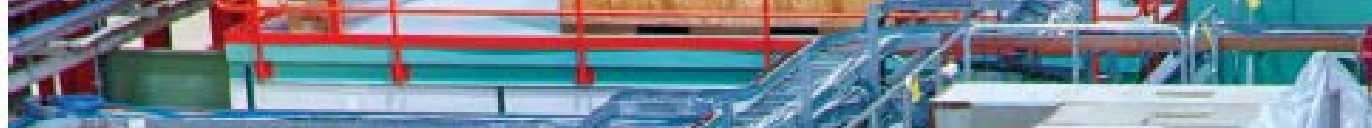

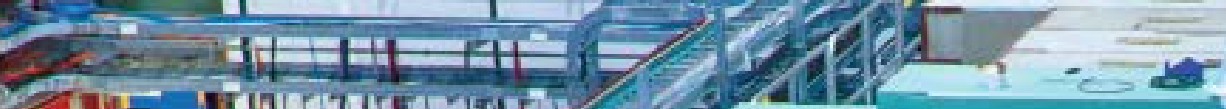

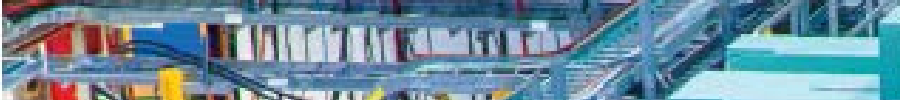

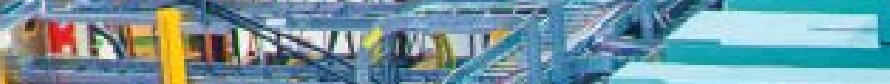
1.mins?

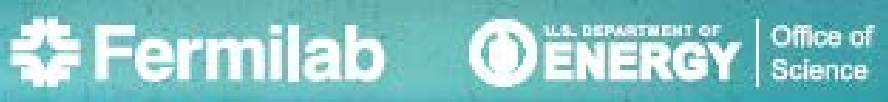

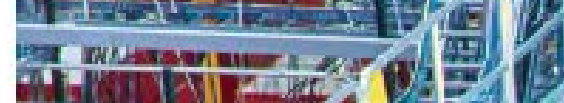

in

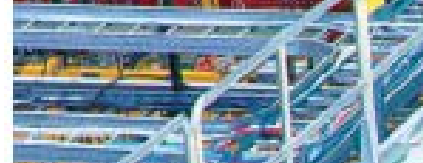

sxy -9 an

\section{灰}

